\title{
Science, technologie
}

et industrie : Tableau de bord de l'OCDE 2017

\section{LA TRANSFORMATION NUMÉRIQUE}

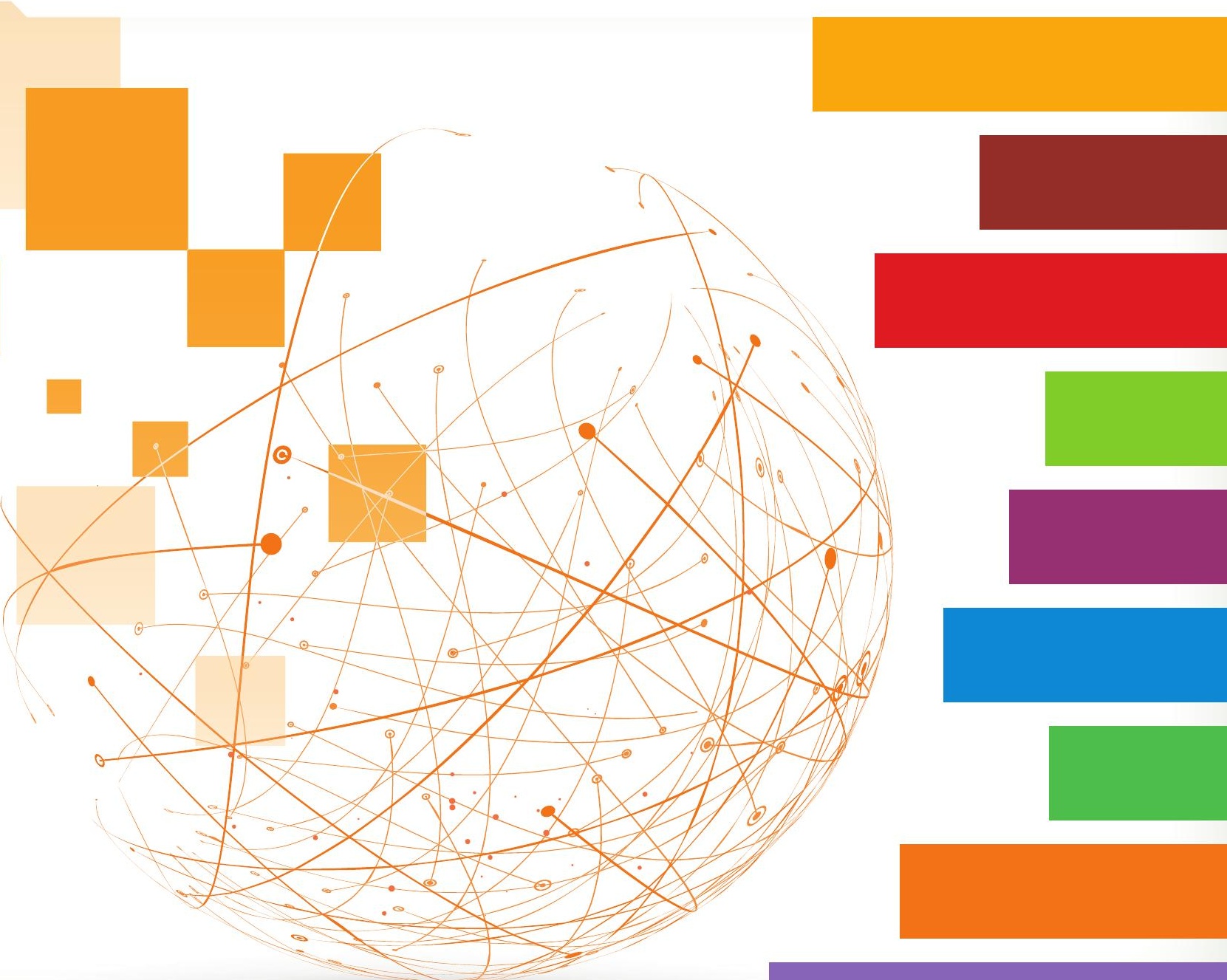





\section{Science, technologie et industrie : Tableau de bord de l'OCDE 2017}

LA TRANSFORMATION NUMÉRIQUE

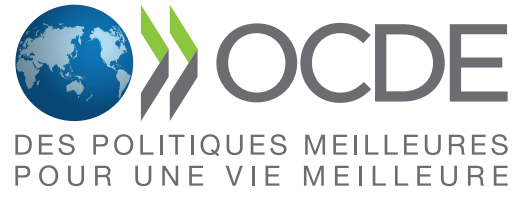


Cet ouvrage est publié sous la responsabilité du Secrétaire général de l'OCDE. Les opinions et les interprétations exprimées ne reflètent pas nécessairement les vues de l'OCDE ou des gouvernements de ses pays membres.

Ce document et toute carte qu'il peut comprendre sont sans préjudice du statut de tout territoire, de la souveraineté s'exerçant sur ce dernier, du tracé des frontières et limites internationales, et du nom de tout territoire, ville ou région.

\section{Merci de citer cet ouvrage comme suit :}

OCDE (2018), Science, technologie et industrie : Tableau de bord de l'OCDE 2017 : La transformation numérique, Éditions OCDE, Paris.

http://dx.doi.org/10.1787/sti_scoreboard-2017-fr

ISBN 978-92-64-29724-1 (print)

ISBN 978-92-64-29727-2 (PDF)

ISBN 978-92-64-30017-0 (epub)

Annuel : Science, technologie et industrie : tableau de bord de l'OCDE

ISSN 2074-7209 (imprimé)

ISSN 2074-7217 (en ligne)

Les données statistiques concernant Israël sont fournies par et sous la responsabilité des autorités israéliennes compétentes. L'utilisation de ces données par l'OCDE est sans préjudice du statut des hauteurs du Golan, de Jérusalem-Est et des colonies de peuplement israéliennes en Cisjordanie aux termes du droit international.

Crédits photo : Cover ๑ Pykha Studio graphique, inspirée par une image de Liu zishan/Shutterstock.com.

Les corrigenda des publications de l'OCDE sont disponibles sur : www.oecd.org/about/publishing/corrigenda.htm.

(c) OCDE 2018

Vous êtes autorisés à copier, télécharger ou imprimer du contenu OCDE pour votre utilisation personnelle. Vous pouvez inclure des extraits des publications, des bases de données et produits multimédia de l'OCDE dans vos documents, présentations, blogs, sites Internet et matériel d'enseignement, sous réserve de faire mention de la source OCDE et du copyright. Les demandes pour usage public ou commercial ou de traduction devront être adressées à rights@oecd.org. Les demandes d'autorisation de photocopier une partie de ce contenu à des fins publiques ou commerciales peuvent être obtenues auprès du Copyright Clearance Center (CCC) info@copyright.com ou du Centre français d'exploitation du droit de copie (CFC) contact@cfcopies.com. 


\section{Avant-propos}

Le présent rapport Science, technologie et industrie : Tableau de bord de l'OCDE 2017 est établi à partir des dernières données comparables au niveau international. Il identifie les atouts des pays de l'OCDE, ainsi que d'autres grandes économies, et montre comment la transformation numérique rejaillit sur la science, l'innovation, l'économie, l'organisation du travail et les modes de vie. Son objectif est d'aider les pouvoirs publics à concevoir des politiques de la science, de l'innovation et de l'industrie plus efficaces compte tenu des mutations de l'ère numérique.

Ce document présente les indicateurs traditionnels relatifs aux avancées de la science, de la technologie, de l'innovation et de l'industrie. Il comprend également de nouveaux indicateurs expérimentaux qui abordent, sous un nouvel angle, des domaines intéressant l'action publique.

Le Tableau de bord n'a pas pour objet de classer les pays ni d'établir des indicateurs composites, mais de fournir aux décideurs et analystes les moyens de comparer des économies de taille ou de structure similaire et de suiure les progrès accomplis dans la réalisation des objectifs à l'échelon national ou supranational. Ce rapport s'inscrit dans le prolongement de l'action menée par l'OCDE pour bâtir une infrastructure de données reliant acteurs, résultats et répercussions. Il met en lumière les potentialités et les limites de certains indicateurs et propose des pistes à explorer pour de futurs travaux.

Simples repères, les indicateurs ne traitent pas des liens de causalité. Leur validité dépend par ailleurs de l'usage qui en est fait. Ceux retenus ici ont été mis au point selon les critères suivants :

- Un indicateur doit reposer sur des statistiques de qualité et des principes analytiques solides, être mesurable dans le temps au niveau international avec des marges d'amélioration possibles.

- Un indicateur doit être pertinent, en particulier pour l'aide à la décision.

- Les indicateurs expérimentaux, qui complètent ceux plus aboutis, ouvrent de nouvelles perspectives et font progresser le travail sur la mesure. Ils ont pour but de stimuler les débats de fond et de dégager de nouvelles dynamiques.

Le premier chapitre, Économie du savoir et transformation numérique, donne une vue d'ensemble des tendances observées en lien avec la science, l'innovation et la croissance dans le paysage en pleine mutation des technologies numériques. La section 1 ("Science, innovation et révolution numérique ") présente l'évolution récente et les principaux acteurs de l'intelligence artificielle (IA), entre autres TIC de rupture, passe en revue le paysage scientifique global et analyse la concentration de la R-D d'entreprise. La section 2 ("Croissance, emploi et transformation numérique ») renseigne sur la place des pays dans les chaînes de valeur mondiales, en particulier dans les réseaux mondiaux de production TIC, étudie la nature évolutive des emplois et présente les actifs intellectuels qui constituent les clés de voûte de l'innovation et de la productivité. La section 3 ("Agir maintenant au service de l'innovation ") éclaire sur les mesures à prendre pour combler le fossé numérique tout en favorisant l'innovation et l'entrepreneuriat.

Cinq chapitres thématiques sont consacrés à des domaines clés de l'action publique :

- Le chapitre intitulé Le savoir, les talents et les compétences traite des actifs intellectuels que beaucoup d'entreprises et d'États considèrent comme la source d'une croissance durable à long terme, 
aujourd'hui et demain. Des indicateurs du capital intellectuel y sont proposés, comme la formation formelle et en cours d'emploi ou encore les actifs organisationnels, pour le secteur marchand et non marchand. Les compétences requises dans le nouvel environnement de travail façonné par les TIC, ainsi que les rendements des compétences en TIC sont analysés également sur la base d'une nouvelle série d'indicateurs.

- Le chapitre Excellence de la recherche et collaboration vient étayer la réflexion en proposant une série d'indicateurs concernant la variété et la nature des mécanismes de diffusion des connaissances à l'ère du numérique. Il y est question de la performance des pays qui suivent différentes voies de spécialisation scientifique, de la mobilité internationale des personnes hautement qualifiées, de l'innovation transnationale et de la collaboration inter-entreprises dans les processus d'innovation.

- Le chapitre L'innovation dans les entreprises étudie le dynamisme du secteur des entreprises et les conditions-cadre indispensables à l'innovation. Il passe en revue les caractéristiques de la R-D par secteur ainsi que les portefeuilles de propriété intellectuelle, en accordant une attention particulière au fait que les entreprises combinent brevets, marques, modèles et dessins industriels liés aux TIC pour protéger leurs innovations. Des estimations des incitations fiscales en faveur de la R-D et du financement direct de la R-D donnent un tableau plus complet de l'action déployée par les pouvoirs publics pour promouvoir la R-D d'entreprise, tandis que les données des enquêtes sur l'innovation permettent d'analyser le rôle des entreprises innovantes dans les marchés publics.

- Le chapitre Leadership et compétitivité s'intéresse aux moyens mis en ouvre par les pays pour consolider leurs atouts concurrentiels ainsi qu'au degré d'intégration et de spécialisation affiché par les économies dans les chaînes de valeur mondiales. Les indicateurs analysés concernent la spécialisation en $R-D$, les avantages technologiques et atouts relatifs, la diffusion du commerce électronique dans les entreprises et les secteurs, ainsi que la dynamique des start-ups du secteur des TIC par rapport au reste de l'économie. Les indicateurs établis à partir de la base de données OCDE-OMC sur les échanges en valeur ajoutée (TiVA) permettent d'appréhender la participation des économies aux échanges internationaux et aux chaînes de valeur mondiales, ainsi que les retombées qui en découlent pour l'emploi et la consommation.

- Le chapitre Société et transformation numérique s'appuie sur des indicateurs de l'inclusion numérique pour éclairer le débat sur l'action des pouvoirs publics. Une série d'indicateurs clés permet d'étudier l'accès des individus aux technologies et l'utilisation qu'ils en font dès le plus jeune âge, leur niveau de cyberactivité, de cyberconsommation et de recours aux services de l'administration électronique. Enfin, des indicateurs de la confiance donnent une idée des craintes que les entreprises et les individus nourrissent en matière de sécurité et de respect de la vie privée dans un monde de plus en plus irrigué par le numérique.

Le Tableau de bord s'adresse principalement aux analystes familiers du maniement des indicateurs et aux personnes qui produisent ce type d'informations à des fins d'analyse ou d'élaboration des politiques. Chaque indicateur fait l'objet d'un texte introductif de quelques paragraphes, assorti d'éléments d’interprétation. Les encadrés "Définitions ", "Mesurabilité " et «Le saviez-vous ? " apportent des précisions sur la méthodologie employée et résument les lacunes, difficultés et initiatives récentes propres à l'indicateur considéré, tout en attirant l'attention sur des faits ou des chiffres dignes d'intérêt, fondés sur les conclusions des cinq chapitres thématiques.

L'ensemble des graphiques et données y afférentes sont téléchargeables via StatLink (hyperliens vers une page web). De plus amples données, en termes de couverture géographique et chronologique, sont accessibles de la même manière. Par ailleurs, les lecteurs trouveront, sur le site web du Tableau de bord (www.oecd.org/fr/sti/science-technologie-industrie-tableau-de-bord.htm), plusieurs synthèses thématiques et notes par pays, ainsi que des outils en ligne leur permettant de visualiser les indicateurs et de réaliser des analyses à leur gré. 


\section{Remerciements}

C et ouvrage est le fruit d'un effort collectif de la Division des analyses économiques et des statistiques (EAS) de la Direction de la science, de la technologie et de l'innovation (DSTI) de l'OCDE. Il a été élaboré par Ali Alsamawi, Silvia Appelt, Brigitte van Beuzekom, Brunella Boselli, Frédéric Bourassa, François Chantret, Agnès Cimper, Alessandra Colecchia, Chiara Criscuolo, Taro Daiko, Hélène Dernis, Isabelle Desnoyers-James, Timothy Destefano, Fernando Galindo-Rueda, Robert Grundke, Joaquim Guilhoto, Peter Horvát, Takashi Inaba, Eugénie Joltreau, Daniel Ker, Elif Köksal-Oudot, Guillaume Kpodar, Alejandro Manríquez-Rangel, Luca Marcolin, Carlo Menon, Pierre Montagnier, Vincenzo Spiezia, Mariagrazia Squicciarini, Fabien Verger, Colin Webb et Norihiko Yamano.

Brigitte van Beuzekom et Elif Köksal-Oudot en ont coordonné l'élaboration avec le concours de Brunella Boselli, Agnès Cimper, Hélène Dernis, Isabelle Desnoyers-James et Fabien Verger, comme chefs d'équipe. Le secrétariat a été assuré par Celia Valeani. Alessandra Colecchia, Dirk Pilat et Andrew Wyckoff ont défini les orientations générales et formulé leurs commentaires.

Cette édition du Tableau de bord a également bénéficié de l'expertise de Véronique Gindrey, Corinne Heckmann, Massimo Loi, Mariarosa Lunati, Frédéric Parrot, Joris Ranchin, Cláudia Sarrico et Giovanni María Semeraro.

Certains des indicateurs présentés ici découlent de la dernière version de la Base de données COR\&DIP construite pour le rapport World Corporate Top R\&D Investors: Industrial Property Strategies in the Digital Economy, que l'OCDE et le Centre commun de recherche Institut de prospective technologique de la Commission européenne ont conjointement publiée en 2017.

L'OCDE remercie l'UE des fonds alloués, dans le cadre du programme H2020, aux travaux sur l'incidence et l'impact des incitations fiscales en faveur de la R-D et du soutien public à l'innovation d'entreprise. Il y a également lieu de saluer le précieux concours apporté par les réseaux d'experts sur les micro-données relatives aux DIRDE et les incitations fiscales en faveur de la R-D.

Les délégués des pays participant aux travaux du Groupe de travail de l'OCDE sur l'analyse de l'industrie (GTAI) et les autres membres du réseau DynEmp ont contribué de manière décisive à l'avancée du projet DynEmp, fondé sur des données récoltées au niveau des entreprises.

Enfin, la présente édition n'aurait pu voir le jour sans les délégués du Groupe de travail des experts nationaux sur les indicateurs de science et de technologie (GENIST), du Groupe de travail sur l'analyse de l'industrie (GTAI) et du Groupe de travail sur la mesure et l'analyse de l'économie numérique (GTMAEN) de l'OCDE, qui ont prêté leur concours et leur temps à son élaboration. Nous remercions tous les délégués de cette étroite collaboration. 



\section{Table des matières}

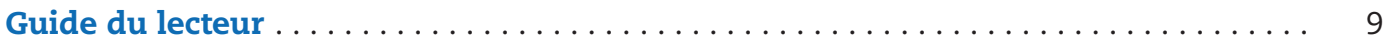

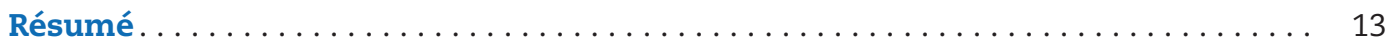

1. Économie du savoir et transformation numérique $\ldots \ldots \ldots \ldots \ldots \ldots \ldots \ldots \ldots$

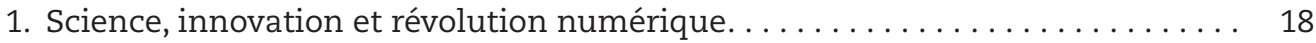

2. Croissance, emploi et transformation numerique............... 38

3. Agir maintenant au service de l'innovation .................... 64

Notes.............................................. 80

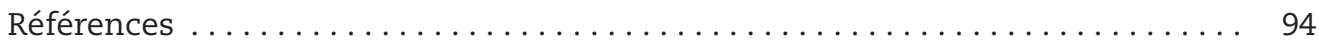

2. Le savoir, les talents et les compétences ..................... 97

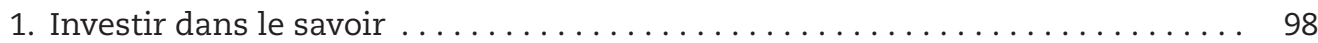

2. Enseignement supérieur et recherche fondamentale $\ldots \ldots \ldots \ldots \ldots \ldots \ldots \quad 100$

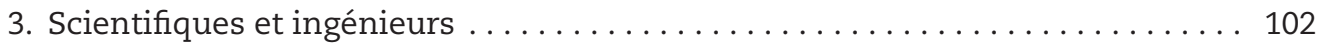

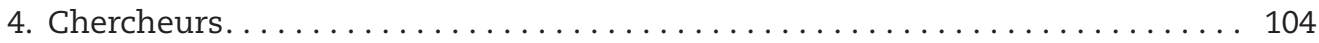

5. Compétences dans le monde numérique .................... 106

6. Rendements des compétences en TIC . . . . . . . . . . . . . . . . . 108

7. Capital intellectuel . . . . . . . . . . . . . . . . . . . . . 110

Notes . . . . . . . . . . . . . . . . . . . . . . . . . . . . . . . . . . 112

Références .............................................. 119

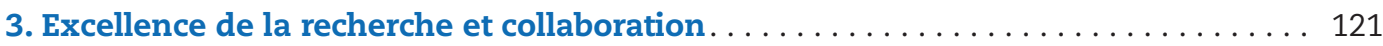

1. Excellence de la recherche et spécialisation..................... 122

2. Excellence de la collaboration scientifique..................... 124

3. Mobilité internationale des personnes hautement qualifiées ............ 126

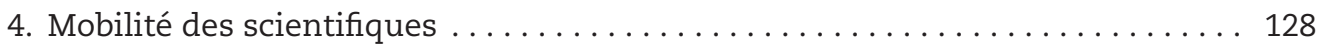

5. Mondialisation de la R-D . . . . . . . . . . . . . . . . . . . . . . . . 130

6. Inventions internationales . . . . . . . . . . . . . . . . . . . . . . . . 132

7. Collaboration en matière d'innovation . . . . . . . . . . . . . . . . 134

Notes . . . . . . . . . . . . . . . . . . . . . . . . . . . . . . . . . . . . . . . . . 136

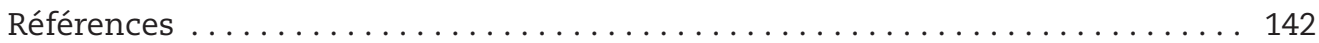

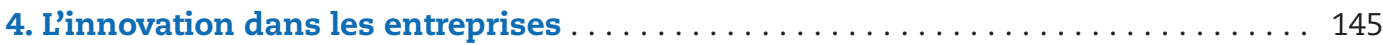

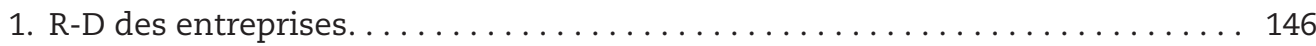

2. Principaux acteurs de la R-D . . . . . . . . . . . . . . . 148

3. Portefeuilles de PI ... . . . . . . . . . . . . . . . . . . . . . . . 150

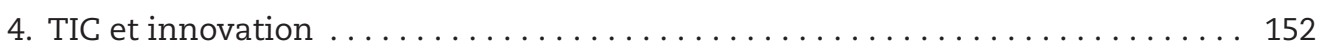

5. Panachage des modes d'innovation....................... 154 
6. Incitations fiscales en faveur de la R-D . . . . . . . . . . . . . . . . 156

7. Cadre de la politique économique et demande d'innovation $\ldots \ldots \ldots \ldots \ldots 158$

Notes . . . . . . . . . . . . . . . . . . . . . . . . . . . . . . . . . . . . . . . . . 160

Références ........................................... 167

5. Leadership et compétitivité . . . . . . . . . . . . . . . . . . . . . . . . . . . . . . . 169

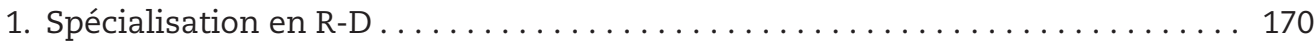

2. Adoption du commerce électronique . . . . . . . . . . . . . . . . . 172

3. Le commerce électronique à travers les secteurs et les applications . . . . . . . 174

4. Dynamique des start-ups . . . . . . . . . . . . . . . . . . . . . . 176

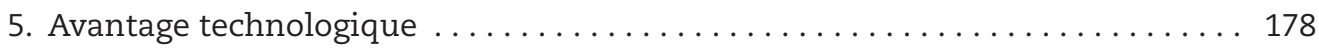

6. Participation aux chaînes de valeur mondiales . . . . . . . . . . . . 180

7. Échanges et emploi................................. 182

Notes . . . . . . . . . . . . . . . . . . . . . . . . . . . . . . . . . . . . . . . . . . . . . 184

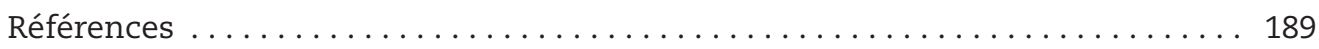

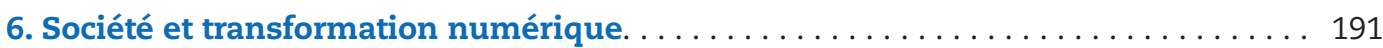

1. Connectivité . . . . . . . . . . . . . . . . . . . . . . . . . . . . . . . 192

2. Les enfants du numérique. . . . . . . . . . . . . . . . . . . . . . . . . . . . 194

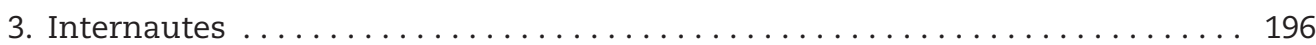

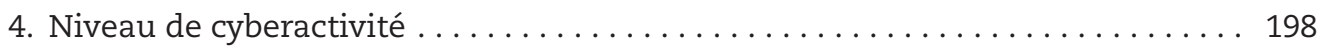

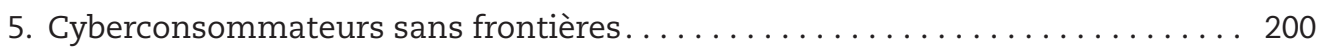

6. Administration électronique. ........................... 202

7. Confiance............................................. 204

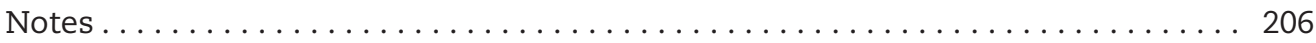

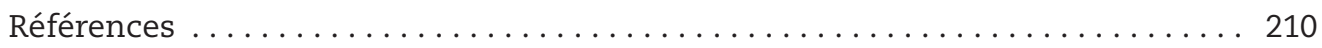

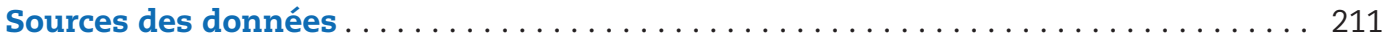

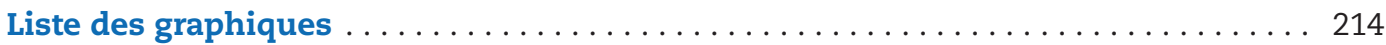

\section{Suivez les publications de l'OCDE sur:}

http://twitter.com/OECD_Pubs

http://www.facebook.com/OECDPublications

http://www.linkedin.com/groups/OECD-Publications-4645871

http://www.youtube.com/oecdilibrary

http://www.oecd.org/oecddirect/

\section{Ce livre contient des...}

En bas des tableaux ou graphiques de cet ouvrage, vous trouverez des StatLinks. Pour télécharger le fichier Excel ${ }^{\circledR}$ correspondant, il vous suffit de retranscrire dans votre navigateur Internet le lien commençant par : $h$ ttp://dx.doi.org, ou de cliquer sur le lien depuis la version PDF de l'ouvrage. 


\section{Guide du lecteur}

\section{Sigles et abréviations}

$\begin{array}{ll}\text { API } & \text { Interface de programmation } \\ \text { ASJC } & \text { All Science Journal Classification } \\ \text { ATR } & \text { Avantage technologique révélé } \\ \text { CBPRD } & \text { Crédits budgétaires publics de R-D } \\ \text { CIB } & \text { Classification internationale des brevets } \\ \text { CIS } & \text { Enquête communautaire sur l'innovation } \\ \text { CITE } & \text { Classification internationale type de l'éducation } \\ \text { CITI } & \text { Classification internationale type, par industrie, de toutes les branches } \\ & \text { d'activité économique } \\ \text { CITP } & \text { Classification internationale type des professions } \\ \text { CPC } & \text { Classification coopérative des brevets } \\ \text { CVM } & \text { Chaîne de valeur mondiale } \\ \text { DIRD } & \text { Dépenses intérieures brutes de R-D } \\ \text { DIRDE } & \text { Dépenses intérieures de R-D des entreprises } \\ \text { DIRDES } & \text { Dépenses intérieures de R-D de l'enseignement supérieur } \\ \text { DIRDET } & \text { Dépenses intérieures de R-D de l'État } \\ \text { DOI } & \text { Identifiant numérique d'objet } \\ \text { DPI } & \text { Droit de propriété intellectuelle } \\ \text { EAD } & \text { Échange automatique de données } \\ \text { EDI } & \text { Échange de données informatisé } \\ \text { EES } & \text { Établissements d'enseignement supérieur } \\ \text { EFT } & \text { Enquête sur les forces de travail } \\ \text { ETP } & \text { Équivalent temps plein } \\ \text { EUIPO } & \text { Office de l'Union européenne pour la propriété intellectuelle } \\ \text { EUR } & \text { Euro } \\ \text { FBCF } & \text { Formation brute de capital fixe } \\ \text { FGU } & \text { Fonds généraux des universités } \\ \text { FTTB } & \text { Fibre jusqu'à l'immeuble } \\ \text { FTTH } & \text { Fibre jusqu'au domicile } \\ \text { GRC } & \text { Gestion de la relation client } \\ \text { GSMA } & \text { Groupe Spéciale Mobile Association } \\ \text { IA } & \text { Intelligence artificielle } \\ \text { IDE } & \text { Investissement direct étranger } \\ \text { IdO } & \text { Internet des objets } \\ \text { IP5 } & \text { Oinq offices de la propriété intellectuelle (OEB, JPO, KIPO, SIPO, USPTO) } \\ \text { JPO } & \end{array}$




\begin{tabular}{|c|c|}
\hline KIPO & $\begin{array}{l}\text { Office coréen de la propriété intellectuelle } \\
\text { (Korean Intellectual Property Office) }\end{array}$ \\
\hline M2M & Communication entre machines \\
\hline NACE & $\begin{array}{l}\text { Nomenclature statistique des activités économiques dans } \\
\text { la Communauté européenne }\end{array}$ \\
\hline oaDOI & Open-access DOI \\
\hline OEB & Office européen des brevets \\
\hline OMPI & Organisation mondiale de la propriété intellectuelle \\
\hline OSE & Objectif socio-économique \\
\hline PGI & Progiciel de gestion intégré \\
\hline PI & Propriété intellectuelle \\
\hline PIB & Produit intérieur brut \\
\hline PME & Petites et moyennes entreprises \\
\hline PMF & Productivité Multi-Factorielle \\
\hline PPA & Parité de pouvoir d'achat \\
\hline PSBL & Secteur privé sans but lucratif \\
\hline PTF & Productivité totale des facteurs \\
\hline R-D & Recherche-développement \\
\hline RFID & Radio-identification \\
\hline roaDOI & Repository-based open-access DOI \\
\hline SCN & Système de comptabilité nationale \\
\hline SIPO & $\begin{array}{l}\text { Office d'État de la propriété intellectuelle de la République populaire } \\
\text { de Chine }\end{array}$ \\
\hline STI & Science, technologie, innovation \\
\hline STIM & Sciences, technologies, ingénierie et mathématiques \\
\hline TIC & Technologie de l'information et des communications \\
\hline TIES & Tableaux internationaux des entrées-sorties \\
\hline TivA & Échanges en valeur ajoutée \\
\hline UIT & Union internationale des télécommunications \\
\hline UNESCO & Organisation des Nations Unies pour l'éducation, la science et la culture \\
\hline USD & Dollar des États-Unis \\
\hline USPTO & $\begin{array}{l}\text { Office des brevets et des marques des États-Unis } \\
\text { (United States Patent and Trademark Office) }\end{array}$ \\
\hline vo & Vidéo à la demande \\
\hline
\end{tabular}




\section{Codes pays}

Les codes ISO des pays et économies ci-après apparaissent dans nombre des graphiques de la présente publication.

\begin{tabular}{|c|c|c|c|}
\hline ARG & Argentine & ISL & Islande \\
\hline AUS & Australie & ISR & Israël \\
\hline AUT & Autriche & ITA & Italie \\
\hline BEL & Belgique & JPN & Japon \\
\hline BGR & Bulgarie & KHM & Cambodge \\
\hline BMU & Bermudes & KOR & Corée \\
\hline BRA & Brésil & LTU & Lituanie \\
\hline BRB & Barbade & LUX & Luxembourg \\
\hline CAN & Canada & LVA & Lettonie \\
\hline CHE & Suisse & MEX & Mexique \\
\hline $\mathrm{CHL}$ & Chili & MLT & Malte \\
\hline $\mathrm{CHN}$ & République populaire de Chine & MYS & Malaisie \\
\hline CRI & Costa Rica & NLD & Pays-Bas \\
\hline CYM & Îles Caïmans & NOR & Norvège \\
\hline CYP & Chypre & NZL & Nouvelle-Zélande \\
\hline CZE & République tchèque & PHL & Philippines \\
\hline DEU & Allemagne & POL & Pologne \\
\hline DNK & Danemark & PRT & Portugal \\
\hline ESP & Espagne & ROU & Roumanie \\
\hline EST & Estonie & RUS & Fédération de Russie \\
\hline FIN & Finlande & SAU & Arabie saoudite \\
\hline FRA & France & SGP & Singapour \\
\hline GBR & Royaume-Uni & SVK & République slovaque \\
\hline GRC & Grèce & SVN & Slovénie \\
\hline HKG & Hong Kong (Chine) & SWE & Suède \\
\hline HRV & Croatie & TUR & Turquie \\
\hline HUN & Hongrie & TWN & Taipei chinois \\
\hline IDN & Indonésie & USA & États-Unis \\
\hline IND & Inde & VGB & Îles Vierges britanniques \\
\hline IRL & Irlande & ZAF & Afrique du Sud \\
\hline
\end{tabular}

\section{Groupes de pays}

\begin{tabular}{ll}
\hline ASEAN & Brunei Darussalam, Cambodge, Indonésie, Laos, Malaisie, Myanmar, Philippines, Singapour, Thaïlande et Viet Nam. \\
ALENA & Canada, États-Unis et Mexique. \\
BRICS & Brésil, Fédération de Russie, Inde, Chine et Afrique du Sud. \\
BRIICS & Brésil, Fédération de Russie, Inde, Indonésie, Chine et Afrique du Sud. \\
G20 & Afrique du Sud, Allemagne, Arabie saoudite, Argentine, Australie, Brésil, Canada, Chine, Corée, États-Unis, Fédération de Russie, \\
G7 & France, Inde, Indonésie, Italie, Japon, Mexique, Royaume-Uni, Turquie et Union Européenne. \\
OCDE & Allemagne, Canada, États-Unis, France, Italie, Japon et Royaume-Uni. \\
ROW & Total OCDE \\
UE28 & Reste du monde \\
WLD & Union européenne \\
Zone euro & Monde \\
& Allemagne, Autriche, Belgique, Chypre, Espagne, Estonie, Finlande, France, Grèce, Irlande, Italie, Luxembourg, Malte, Pays-Bas, \\
& Portugal, République slovaque et Slovénie.
\end{tabular}





\section{Résumé}

$\mathrm{M}$ obilité, cloud computing, internet des objets (IDO), intelligence artificielle (IA) et analytique de données massives font partie des piliers de l'économie numérique d'aujourd'hui. Ensemble, ils rendent possible le scénario d'avenir du « tout intelligent » et donnent des moyens d'agir aux entreprises, aux consommateurs et à la société en général. Le rapport Science, technologie et industrie : Tableau de bord de l'OCDE 2017 montre comment la transformation numérique rejaillit sur la science, l'innovation, l'économie, l'organisation du travail et les modes de vie. Il a pour objet d'aider les pouvoirs publics à concevoir des politiques de la science, de l'innovation et de l'industrie plus efficaces compte tenu des mutations de l'ère numérique. Les principaux messages à retenir, du point de vue des tendances numériques, dans les différents domaines abordés sont résumés ci-après.

\section{La révolution numérique suit son cours}

Au cours de la période $2012-15$, entre $70 \%$ et $100 \%$ des 20 plus grandes TIC de pointe ont été développées en Chine, en Corée, aux États-Unis, au Japon et au Tapei chinois, l'activité d'innovation du Japon et de la Corée touchant tous les domaines. Pour ce qui des technologies d'IA, leur évolution est mesurée par le nombre d'inventions liées à l'IA ayant donné lieu à un dépôt de brevet auprès des cinq principaux offices de propriété intellectuelle (IP5) : entre 2010 et 2015, il a augmenté de $6 \%$ par an en moyenne, ce qui représente le double de la moyenne calculée pour la totalité des brevets. En 2015, sur les 18000 inventions liées à l'IA brevetées dans le monde, 62 \% émanaient de la Corée, des États-Unis et du Japon. Jusqu'à $30 \%$ des brevets déposés qui concernent le diagnostic médical contiennent des éléments d'IA.

\section{Les grandes puissances scientifiques tirent l'innovation numérique}

Ces 15 dernières années, la Chine a triplé sa production scientifique à fort impact, telle que mesurée par son poids dans les $10 \%$ des publications les plus citées (14 \%), devenant ainsi la deuxième puissance scientifique, après les États-Unis (25\%). La Chine suit également les États-Unis dans le domaine de l'apprentissage automatique. Il faut désormais aussi compter avec l'Inde, qui représente un tiers des publications liées à ce domaine, mais se place toutefois au quatrième rang derrière le Royaume-Uni lorsque sa production est corrigée des variations de qualité. La communication entre machines (M2M) est indispensable à l'IDO. En juin 2017, la Chine concentrait 44 \% des connexions par carte SIM M2M, soit trois fois plus que les États-Unis.

\section{Les frontières technologiques se caractérisent par une forte concentration}

La R-D est une activité fortement concentrée : au sein des économies, une grande partie de la R-D exécutée par les entreprises l'est par un petit nombre d'entre elles. Les 50 principaux exécutants de la R-D locale se partagent $40 \%$ de cette activité au Canada et 
aux États-Unis, 55 \% en Allemagne et au Japon. Les 2000 entreprises du classement mondial de la R-D sont domiciliées dans quelques pays seulement - au premier rang desquels figurent les États-Unis, le Japon et la Chine - et environ 70 \% du total de leurs dépenses de R-D sont réalisés par les 200 premières. Ces entreprises sont les fers de lance de l'évolution technologique du numérique puisqu'elles détiennent quelque $75 \%$ des brevets liés aux TIC, 55 \% des modèles et dessins liés aux TIC et $75 \%$ des familles de brevets IP5 liées à l'IA.

\section{L'impact de la transformation numérique diffère d'un secteur à l'autre}

La création de valeur ajoutée qui découle de la production de biens et de services TIC intervient en grande partie dans d'autres secteurs que celui des TIC proprement dit. À l'échelle mondiale, la part imputable au reste de l'économie (par exemple, le verre utilisé dans la fabrication des écrans de smartphone) représente entre $19 \%$ et $34 \%$ de la valeur ajoutée totale, et atteint même 41 \% en Chine. La transformation numérique touche désormais tous les secteurs de l'économie, quoiqu'à des degrés divers. Selon la nouvelle taxonomie employée pour classer les activités en fonction de leur intensité numérique, les télécommunications et les services liés aux technologies de l'information arrivent systématiquement en haut du classement ; l'agriculture, les activités extractives et les activités immobilières à l'autre extrémité. D'autres secteurs se révèlent plus hétérogènes selon l'indicateur considéré, ce qui témoigne du rythme variable de la transformation. Si, aujourd'hui, pour ainsi dire aucune entreprise ne peut exercer son activité sans les TIC, l'impact de celles-ci dépend du type et du degré de sophistication des outils intégrés dans les processus métiers. Par exemple, la plupart des entreprises implantées dans la zone OCDE disposent d'une connexion haut débit, mais un quart seulement ont déclaré avoir recouru aux services de cloud computing en 2016 : $22 \%$ parmi les petites entreprises et $47 \%$ parmi celles de grande dimension.

\section{Les compétences requises couvrent un large éventail}

Créer, adopter et utiliser efficacement une technologie nouvelle suppose de posséder des compétences particulières. Les économies dans lesquelles l'exécution des tâches professionnelles fait le plus appel aux TIC (par exemple, la Norvège, la Nouvelle-Zélande et les Pays-Bas) sont également celles où la part des emplois à caractère non répétitif, qui impliquent des tâches relativement complexes, est plus élevée. Les actifs qui occupent un emploi dont la composante TIC est supérieure de $10 \%$ à la moyenne perçoivent un salaire horaire jusqu'à $4 \%$ plus élevé. Cependant, les compétences en TIC ne suffisent pas pour tirer son épingle du jeu dans l'économie numérique. Ceux dont l'emploi requiert également de posséder des compétences en gestion et en communication bénéficient d'un surcroît de rémunération. Les actifs employés dans les activités à forte intensité numérique affichent un niveau supérieur de compétences cognitives (aptitude à lire, écrire, compter et résoudre des problèmes), mais aussi de compétences sociales et non cognitives (par exemple, la communication et la créativité).

\section{Malgré la généralisation de l'internet, des écarts demeurent}

L'internet et les appareils connectés font désormais partie intégrante du quotidien du plus grand nombre et de la quasi-totalité de la population de plusieurs pays de l'OCDE. En Afrique du Sud, au Brésil et en Chine, plus de la moitié des 16-74 ans utilisent l'internet, réduisant ainsi l'écart avec les pays de l'OCDE. La tendance va s'accentuer à mesure que le coût des technologies d'accès à l'internet continuera de reculer et que les enfants du numérique atteindront l'âge adulte. La part des élèves ayant accédé à l'internet pour la première fois à l'âge de six ans ou plus jeunes encore est de 17 \% pour l'ensemble de la 
zone OCDE et atteint $30 \%$ au Danemark. Le tableau reste toutefois très contrasté dans la majorité des pays de l'OCDE, selon que l'on considère la généralisation et l'utilisation des technologies numériques chez les plus jeunes et les plus âgés, en milieu urbain et rural, en fonction du niveau d'études ou encore de la taille des entreprises.

\section{La transformation numérique touche moins les femmes}

Dans la zone OCDE, les femmes représentent environ 30 \% des diplômés en sciences naturelles, en ingénierie et en TIC. Elles ne représentent que $22 \%$ des auteurs scientifiques, voire moins encore si l'on considère les sous-catégories d'auteurs (par exemple, les personnes qui mènent une activité rémunérée de recension et de contrôle rédactionnel et celles qui se consacrent pleinement à la recherche). La part des brevets citant des femmes parmi les inventeurs oscille entre $4 \%$ environ (Autriche) et plus de $15 \%$ (Portugal). Dans la vie active, les femmes gagnent souvent nettement moins que les hommes, même à profil et à travail égal. Cet écart tient en partie aux compétences, notamment en TIC. D'après les estimations, toutes choses égales par ailleurs, les rendements des compétences en TIC sont plus élevés chez les femmes que chez les hommes. Former les femmes et les doter de compétences en TIC supplémentaires peut donc contribuer à accroître leur niveau de rémunération et à combler les écarts de salaire existant avec leurs homologues masculins. 


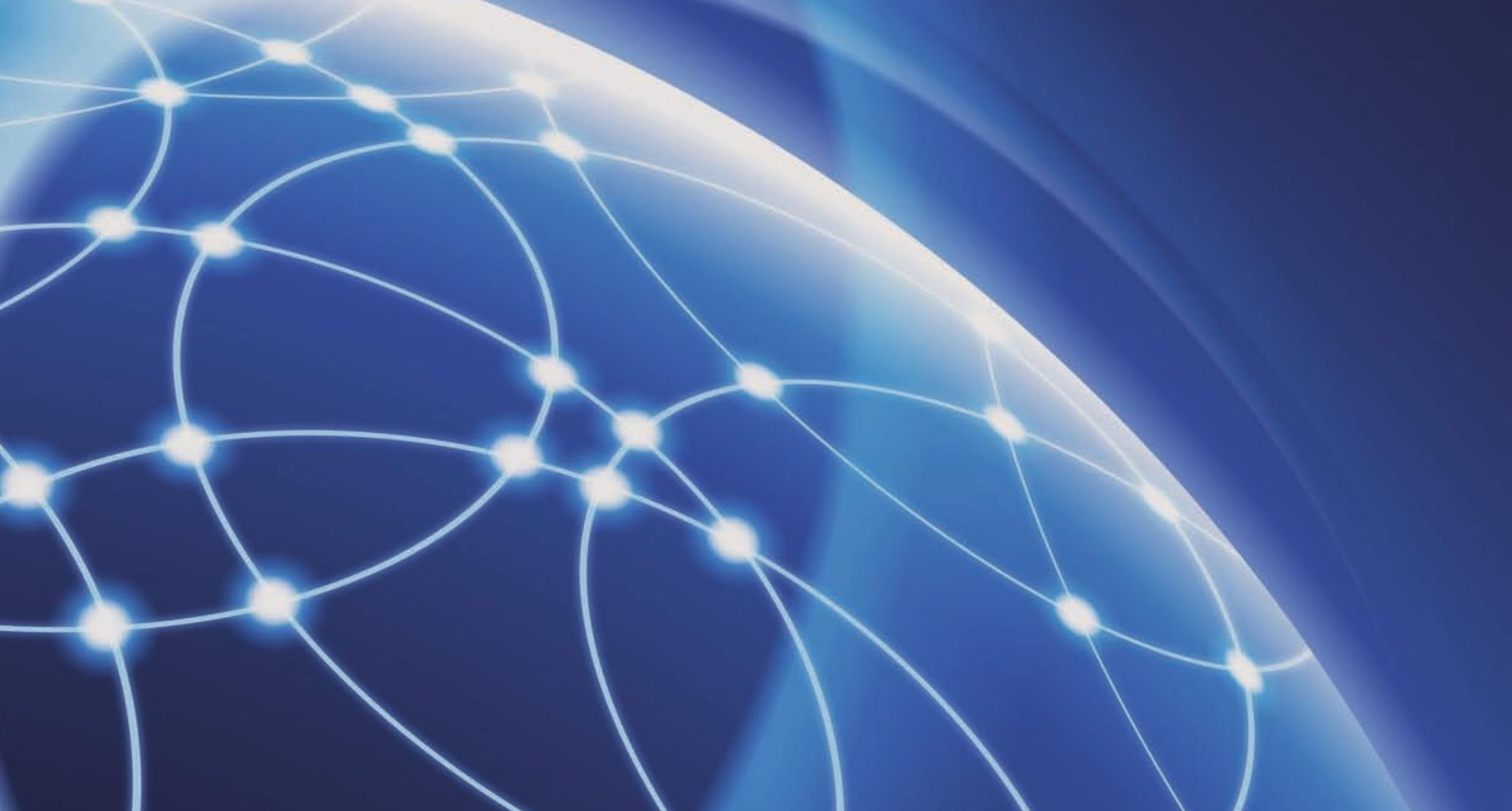




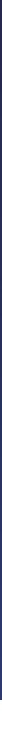

\section{1. ÉCONOMIE DU SAVOIR ET TRANSFORMATION NUMÉRIQUE}

1. Science, innovation et révolution numérique

2. Croissance, emploi et transformation numerique

3. Agir maintenant au service de l'innovation

Notes et références 


\section{1. ÉCONOMIE DU SAVOIR ET TRANSFORMATION NUMÉRIQUE}

\section{Science, innovation et révolution numérique}

\section{Infrastructure haut débit}

Les abonnements au haut débit fixe et mobile continuent de progresser à un rythme soutenu. À l'échelle mondiale, le nombre d'abonnements au haut débit fixe a bondi de $72 \%$ au cours des dix dernières années, passant de 531.8 millions en 2010 à 916.7 millions en 2016. Dans les pays de l'OCDE, on en dénombrait 386.8 millions en 2016, contre 307.3 millions en 2010 , soit une augmentation de $26 \%$. La croissance du haut débit mobile s'est révélée encore plus fulgurante : les abonnements sont passés, à l'échelle mondiale, de 824.5 millions en 2010 à 3864 milliards en 2016. Fin 2016, un peu plus de la moitié de la population mondiale disposait d'un abonnement au haut débit mobile, tandis que la moyenne de la zone OCDE s'élevait à $99.3 \%$. La situation est susceptible d'évoluer rapidement. Le nombre d'abonnements au haut débit mobile dans les pays non membres de l'OCDE a été multiplié par neuf au cours des dix dernières années, et l'Inde a enregistré 100 millions de nouveaux abonnements pour la seule année 2016.

\section{Pénétration du haut débit fixe et mobile dans le monde, 2010 et 2016}

Nombre total d'abonnements et nombre d'abonnements pour 100 habitants

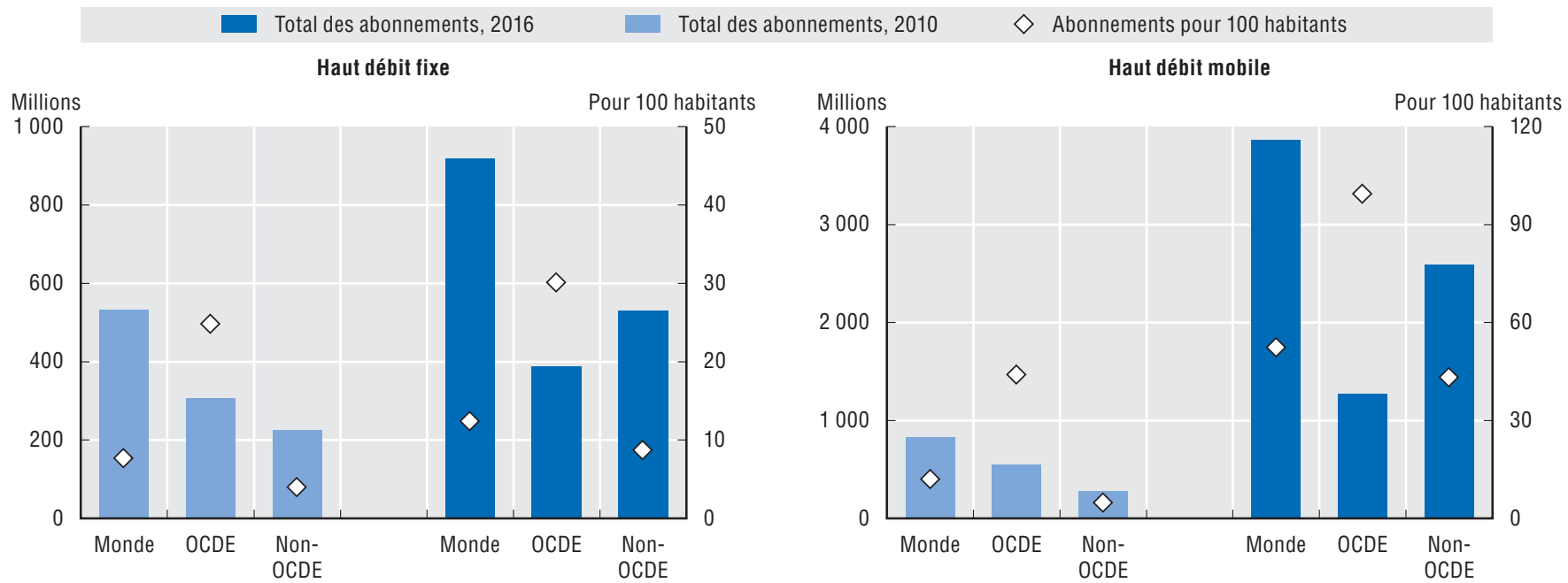

Sources : OCDE, Portail de l'OCDE sur le haut débit, http://oe.cd/265 et UIT, Base de données Indicateurs des télécommunications/TIC dans le monde, juillet 2017

2. Pénétration du haut débit mobile, OCDE, G20 et BRIICS, 2016

Nombre total d'abonnements et nombre d'abonnements pour 100 habitants

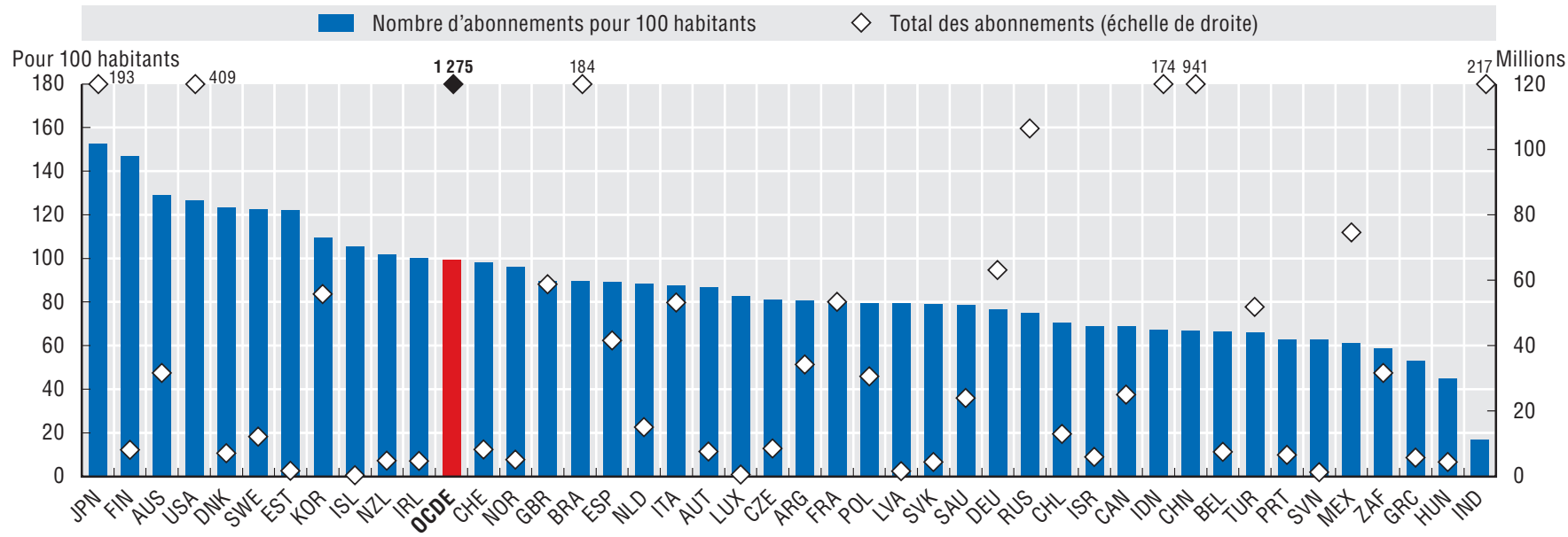

Sources : OCDE, Portail de l'OCDE sur le haut débit, http://oe.cd/265 et UIT, Base de données Indicateurs des télécommunications/TIC dans le monde, juillet 2017. Voir notes de chapitre. 


\section{Communication entre machines}

L'internet des objets (IdO) désigne un écosystème dans lequel applications et services exploitent des données collectées à partir d'appareils tenant lieu de capteurs et interagissant avec le monde extérieur. Cet écosystème pourrait rapidement s'imposer comme une composante à part entière du quotidien des habitants de la zone OCDE et au-delà. L'IdO trouve des applications dans presque tous les grands secteurs de l'économie, de la santé à l'éducation, en passant par l'agriculture, les transports, la production manufacturière, les réseaux électriques, et bien d'autres encore. La communication entre machines (M2M en anglais) fait partie intégrante de l'infrastructure sous-jacente. La GSMA (Groupe Spéciale Mobile Association) assure le suivi du nombre d'abonnements M2M dans le monde. Ces données révèlent le nombre de cartes SIM qui équipent des machines, telles que des automobiles ou des capteurs, et leur permettent de communiquer entre elles. Parmi les économies du G20, les États-Unis affichaient, en juin 2017, le taux de pénétration le plus élevé (exprimé en nombre de cartes SIM M2M par habitant) ; suivis de la France et du Royaume-Uni. Entre 2012 et le deuxième trimestre de 2017, le nombre d'abonnements a progressé de $131 \%$ dans les pays de l'OCDE et de $272 \%$ dans les économies du G20, bien que partant d'un niveau plus bas. La République populaire de Chine (ci-après dénommée "la Chine ") est le pays qui compte le plus d'abonnements M2M (44\% du total mondial), avec 228 millions d'abonnements M2M en juin 2017, soit le triple de la part des États-Unis.

\section{Pénétration des cartes SIM M2M, OCDE, Monde et pays du G20, juin 2017}

Pour 100 habitants

$2017 \diamond 2012$

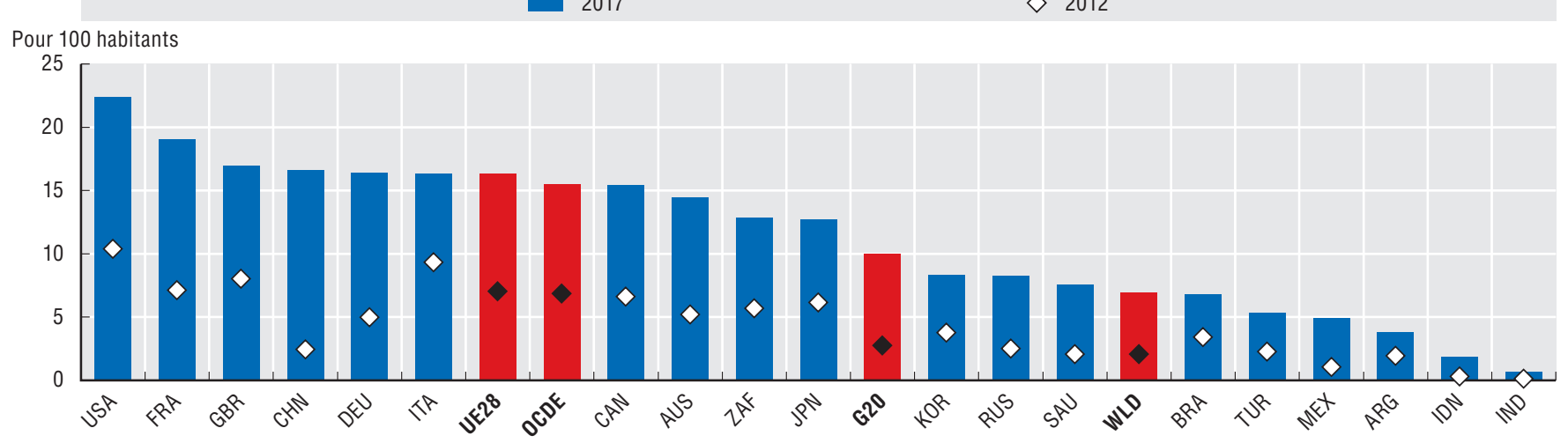

Source : Calculs de l'OCDE, d'après GSMA Intelligence, septembre 2017. Davantage de données via StatLink. Voir notes de chapitre.

StatLink - नilst $h$ ttp://dx.doi.org/10.1787/888933719483

\section{Pays comptant le plus de connexions par carte SIM M2M, juin 2017}

Nombre total de connexions et nombre de connexions en pourcentage du total mondial

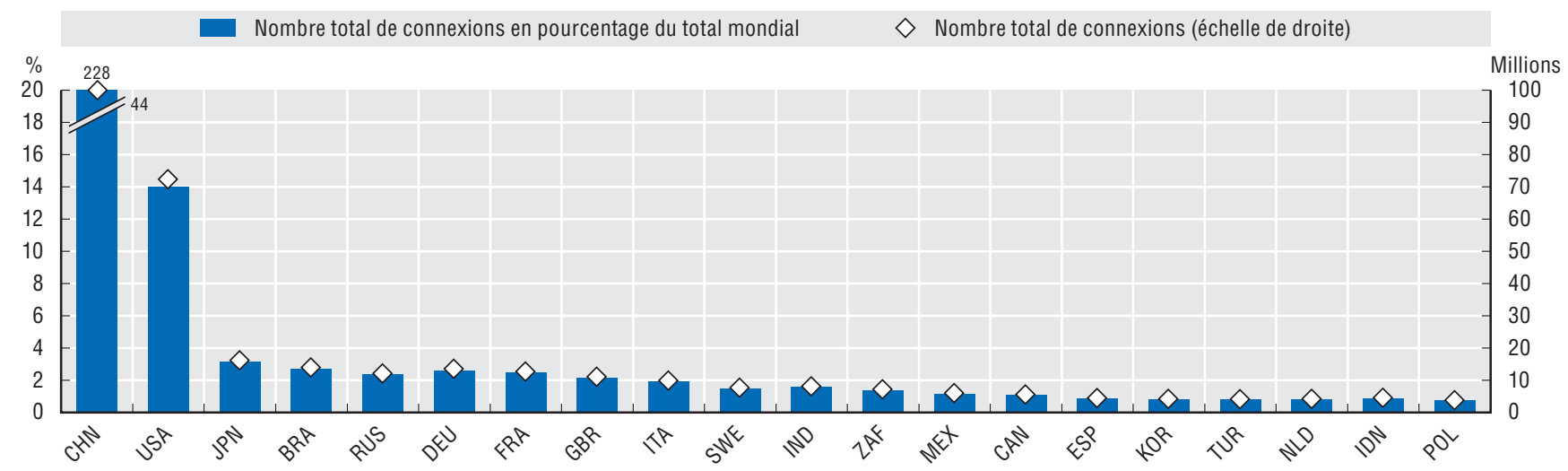

Source : Calculs de l'OCDE, d'après GSMA Intelligence, septembre 2017. Davantage de données via StatLink. Voir notes de chapitre.

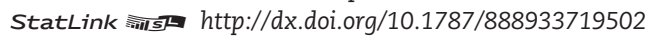

\section{Mesurer l'infrastructure de l'Ido à l'aide des données de la GSMA sur les communications entre machines}

Selon la définition établie par la GSMA, les communications entre machines désignent l'ensemble des cartes SIM uniques enregistrées sur un réseau mobile à la fin de la période considérée, permettant la transmission de données mobiles entre au moins deux machines. Ne sont pas compris les équipements informatiques relevant de l'électronique grand public comme les liseuses, les smartphones, les dongles et les tablettes. La GSMA collecte des informations librement accessibles sur les opérateurs mobiles ayant mis en œuvre des déploiements commerciaux de services de communication entre machines. Elle utilise ensuite un modèle fondé sur des données historiques de connexions M2M, transmises au fil du temps par les opérateurs mobiles et les régulateurs, ainsi que des hypothèses de marché fondées sur des enquêtes à grande échelle menées auprès d'opérateurs et fournisseurs de services M2M. Cet ensemble de données est alors rapproché de la définition établie par la GSMA, normalisé et analysé de manière à identifier des profils spécifiques d'adoption des services M2M. Enfin, ces profils sont appliqués à tous les opérateurs qui ont entrepris des déploiements commerciaux de services de communication entre machines, mais ne publient pas de chiffres sur les connexions M2M, afin de produire des statistiques au niveau national. Pour en savoir plus, voir www.gsmaintelligence.com. 


\section{1. ÉCONOMIE DU SAVOIR ET TRANSFORMATION NUMÉRIQUE}

\section{Science, innovation et révolution numérique}

\section{TIC de pointe}

Le développement et la maturation des technologies requièrent du temps, et leurs évolutions et profils d'adoption peuvent varier. Les technologies ouvrant la voie à des applications diverses peuvent, à un moment donné, voir leur développement s'accélérer. Les technologies de l'information et des communications (TIC) en sont un exemple probant. Les produits TIC, tels que les téléphones mobiles et les ordinateurs, sont connus pour leur complexité et leur modularité, leur obsolescence rapide et leur dépendance à l'égard d'une large gamme de technologies en constante évolution. Une nouvelle approche exploratoire des données permet de suivre de près l'émergence et le développement de différents domaines technologiques, et de détecter les évolutions fulgurantes. Au cours de la période 2012-15, cinq économies détenaient entre $69 \%$ et $98 \%$ des brevets sur 20 technologies liées aux TIC en pleine expansion. Le Japon et la Corée ont contribué à l'essor de l'ensemble des domaines TIC dont le développement s'est accéléré au cours de la période considérée ; ils représentaient à eux deux entre $21 \%$ et environ $70 \%$ des brevets pris dans ces domaines. Les États-Unis ont impulsé les progrès dans les domaines des protocoles de paiement (34 \% des dépôts de brevets), des dispositifs de transmission $(28 \%$ ) et du codage de signal vidéo numérique $(28 \%)$. La Chine faisait quant à elle partie des cinq premières économies à l'origine des progrès technologiques dans les domaines TIC les plus dynamiques, et notamment pour des inventions liées au contrôle et à la modulation de lumière (28 \%). En Europe, la Suède, l'Allemagne et la France se classaient également parmi les cinq premiers pays dans certains domaines TIC en forte accélération.

\section{Principaux acteurs des TIC émergentes, 2012-15}

Part des cinq économies ayant déposé le plus de brevets dans les 20 technologies affichant un développement accéléré à compter de 2010

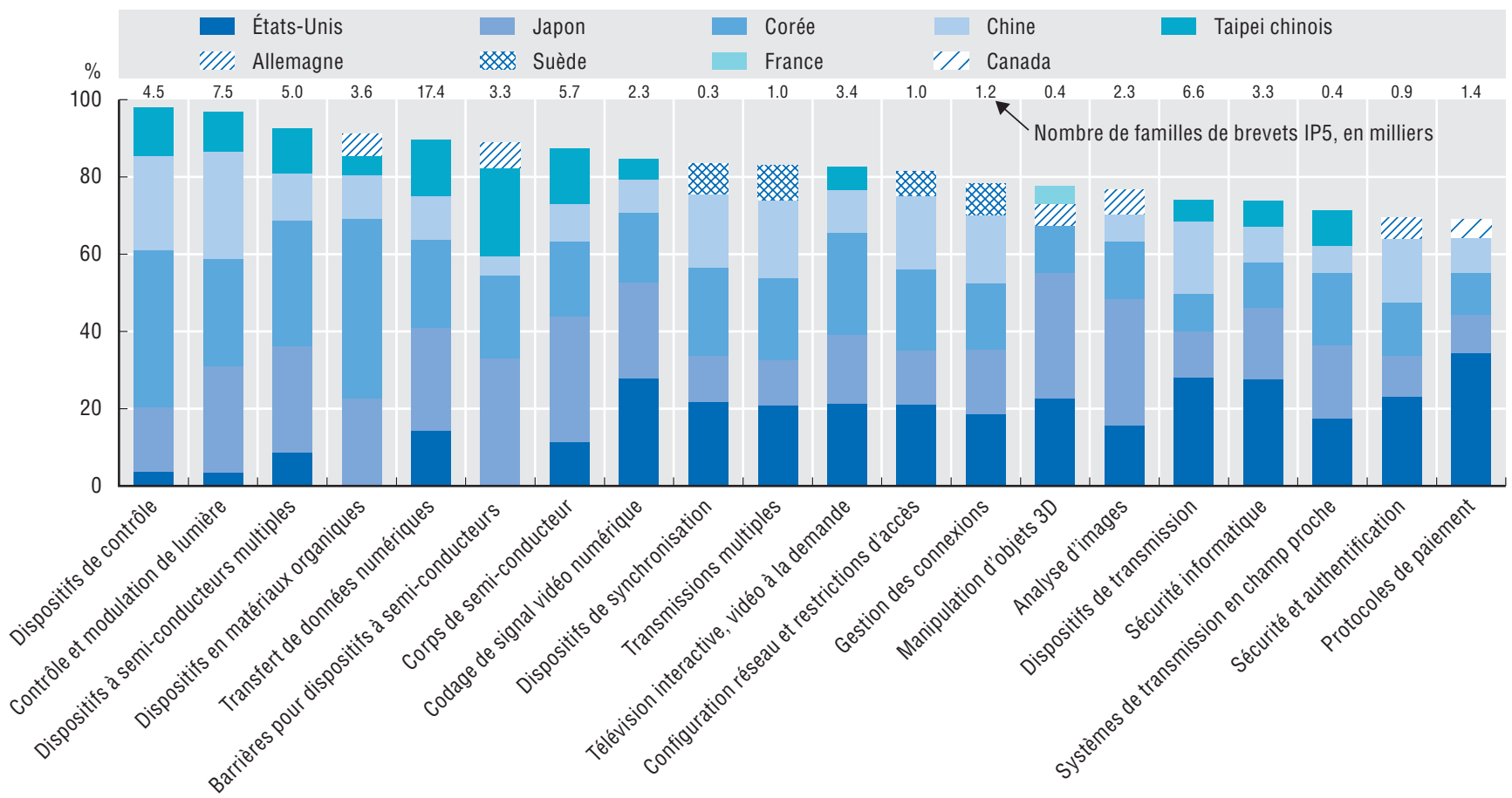

Source : OCDE, STI Microdata Lab : Base de données sur la propriété intellectuelle, http://oe.cd/ipstats, juin 2017. Davantage de données via StatLink. Voir notes de chapitre.

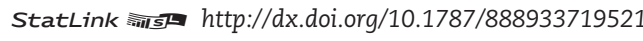

\section{Repérer les accélérations technologiques}

Dans la mesure où les brevets protègent des inventions et des technologies novatrices, les données de brevets peuvent apporter des éclairages sur un certain nombre de questions intéressant l'action des pouvoirs publics dans les domaines de l'innovation et du développement technologique. Une nouvelle approche en matière d'exploration des données, dénommée « DETECTS » (voir Dernis et al., 2016), met à profit les informations contenues dans les documents de brevets pour repérer les technologies dont le développement s'accélère brusquement, par rapport à des niveaux antérieurs et au développement d'autres technologies, tout en déterminant la chronologie de cette dynamique. On dit qu'un domaine technologique connaît une brusque accélération lorsque l'on observe une forte hausse du nombre de dépôts de brevets y afférents. DETECTS permet de suivre cette accélération en termes relatifs (c'est-à-dire en la comparant à des schémas de développement antérieurs dans le même domaine, et au rythme de développement observé dans d'autres domaines). Le suivi des domaines qui connaissent une forte accélération est essentiel pour l'action des pouvoirs publics, dans la mesure où ces évolutions tendent à s'inscrire dans les court et moyen termes. Qui plus est, les informations contenues dans les brevets sur les technologies elles-mêmes, ainsi que sur la localisation géographique des détenteurs et des inventeurs, permettent de repérer quelles économies conduisent ces développements technologiques, et mettent en lumière l'émergence de nouveaux domaines nés du croisement entre différentes technologies (TIC et technologies environnementales, par exemple). 


\section{TIC de pointe}

L'analyse des domaines liés aux TIC qui ont connu une brusque accélération au cours de la période 2000-14 permet de reconstituer la chronologie des évolutions technologiques sur ces 15 années, et d'observer l'accélération qui a marqué certains domaines TIC, ainsi que la durée de ces accélérations. Au début des années 2000, les activités ont particulièrement foisonné dans les domaines du traitement et de la préparation des données numériques, et de l'enregistrement optique, tandis qu'à la fin de la décennie, les accélérations concernaient surtout les dispositifs à semi-conducteurs et les communications sans fil. Depuis 2012, les inventions protégées dans les cinq grands offices de la propriété intellectuelle (IP5) liées aux transferts de données numériques ont affiché une accélération continue d'une intensité sans précédent : on a ainsi comptabilisé quelque 24000 familles de brevets IP5 pour la période 2012-14. À la fin de la période considérée, les domaines en plein essor (et dont l'accélération se poursuit) sont ceux liés aux dispositifs en matériaux organiques, à l'analyse d'images, à la gestion des connexions et aux protocoles de paiement. Par rapport au début de la période considérée, on note aujourd'hui un allongement de la durée des phases d'accélération et un nombre plus élevé d'inventions.

\section{Intensité et rapidité de développement des technologies liées aux TIC, 2000-14}

Intensité des accélérations (taille de la bulle) et durée

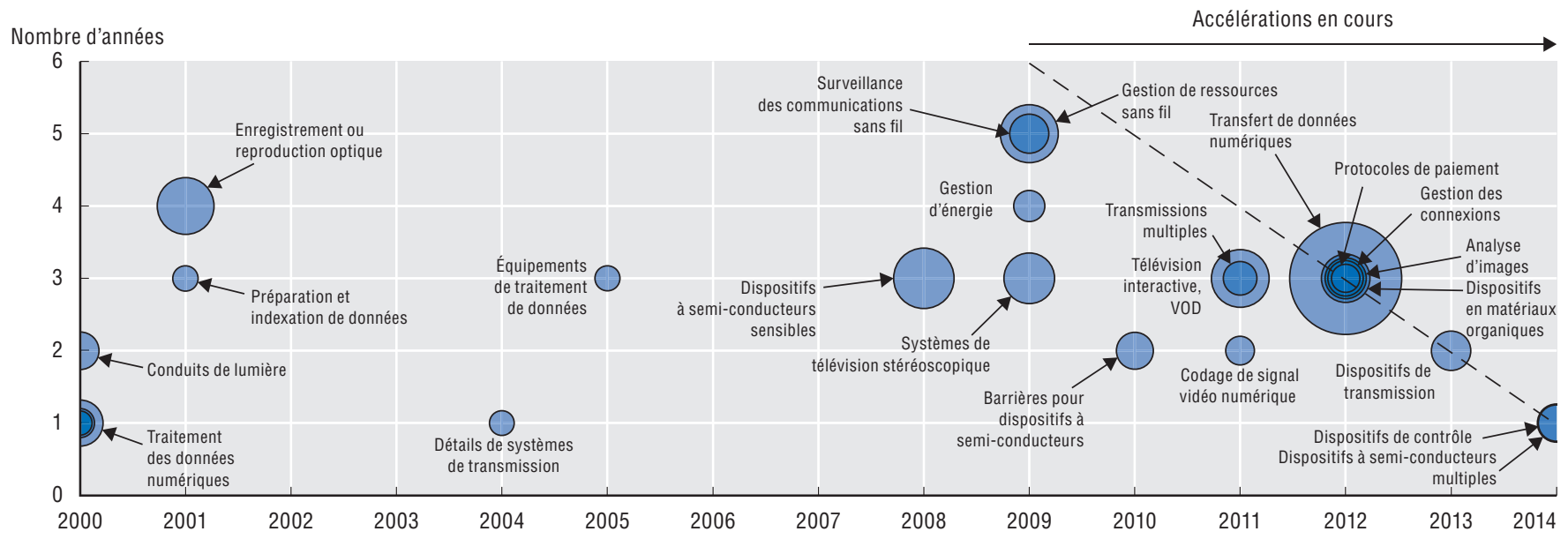

Source : OCDE, STI Microdata Lab : Base de données sur la propriété intellectuelle, http://oe.cd/ipstats, juillet 2017. Davantage de données via StatLink. Voir notes de chapitre.

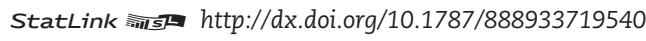

\section{Comment lire ce graphique}

La taille des bulles révèle l'intensité de l'accélération (à savoir le rythme auquel les technologies évoluent) ; différentes tonalités de couleur sont utilisées pour distinguer les technologies dont le développement s'accélère simultanément. Sur l'axe des abscisses sont représentées les années marquant le début de l'accélération du développement des technologies, et, sur l'axe des ordonnées, le nombre d'années pendant lesquelles le développement des technologies s'est poursuivi à un rythme soutenu avant de ralentir. Par exemple, le développement des technologies brevetées liées à l'enregistrement ou la reproduction optique (en haut à gauche du graphique) a connu une accélération fulgurante à partir de 2001 (axe des abscisses), qui s'est poursuivie pendant quatre ans (axe des ordonnées), jusqu'à fin 2004. Les bulles situées le long de la ligne diagonale en haut à droite du graphique représentent les accélérations technologiques en cours (c'est-à-dire les technologies qui sont toujours en phase de développement accéléré à la fin de la période considérée). Les technologies liées au transfert de données numériques, aux dispositifs en matériaux organiques et à l'analyse d'images comptent parmi les domaines TIC dont l'accélération a débuté en 2012. Si les développements dans ces domaines se caractérisent par un nombre variable de brevets - les technologies liées au transfert de données numériques arrivant en tête -, les activités d'invention dans tous ces domaines se poursuivaient à un rythme soutenu fin 2014. 


\title{
1. ÉCONOMIE DU SAVOIR ET TRANSFORMATION NUMÉRIQUE
}

\author{
1. Science, innovation et révolution numérique
}

\section{Intelligence artificielle}

L'intelligence artificielle (IA) désigne les machines simulant les fonctions cognitives humaines (apprentissage, compréhension, raisonnement ou interactions). Elle est à même de révolutionner la production et peut apporter des solutions susceptibles d'aider à affronter les défis mondiaux dans les domaines de la santé, des transports et de l'environnement. Le développement des technologies liées à l'IA, mesuré sur la base des inventions brevetées auprès des cinq grands offices de la propriété intellectuelle (IP5), a progressé en moyenne de $6 \%$ par an entre 2010 et 2015 , soit le double du taux de croissance annuel moyen observé pour les brevets déposés dans les autres domaines. En 2015, 18000 familles de brevets IP5 liées à l'IA ont été déposées dans le monde. Le Japon, la Corée et les États-Unis représentent à eux trois plus de 62 \% des brevets déposés dans ce domaine entre 2010 et 2015 contre $70 \%$ sur la période 2000-05. Toujours entre 2010 et 2015, le nombre de brevets liés à l'IA a augmenté par rapport à la période 2000-05 en Corée, en Chine et au Taipei chinois. Les économies de la zone UE28 ont contribué à hauteur de $12 \%$ des inventions liées à l'IA ayant donné lieu à des dépôts de brevets auprès des offices IP5 sur la période 2010-15, contre 19\% au cours de la décennie précédente. Les avancées technologiques dans le domaine de l'IA, à l'instar de l'apprentissage automatique, couplées aux technologies émergentes comme l'analytique des données massives et le cloud computing, renforcent l'impact potentiel de l'IA.

\section{Brevets portant sur des technologies liées à l'intelligence artificielle, 2000-15}

Nombre de familles de brevets IP5, taux de croissance annuels et principales économies dont relèvent les inventeurs
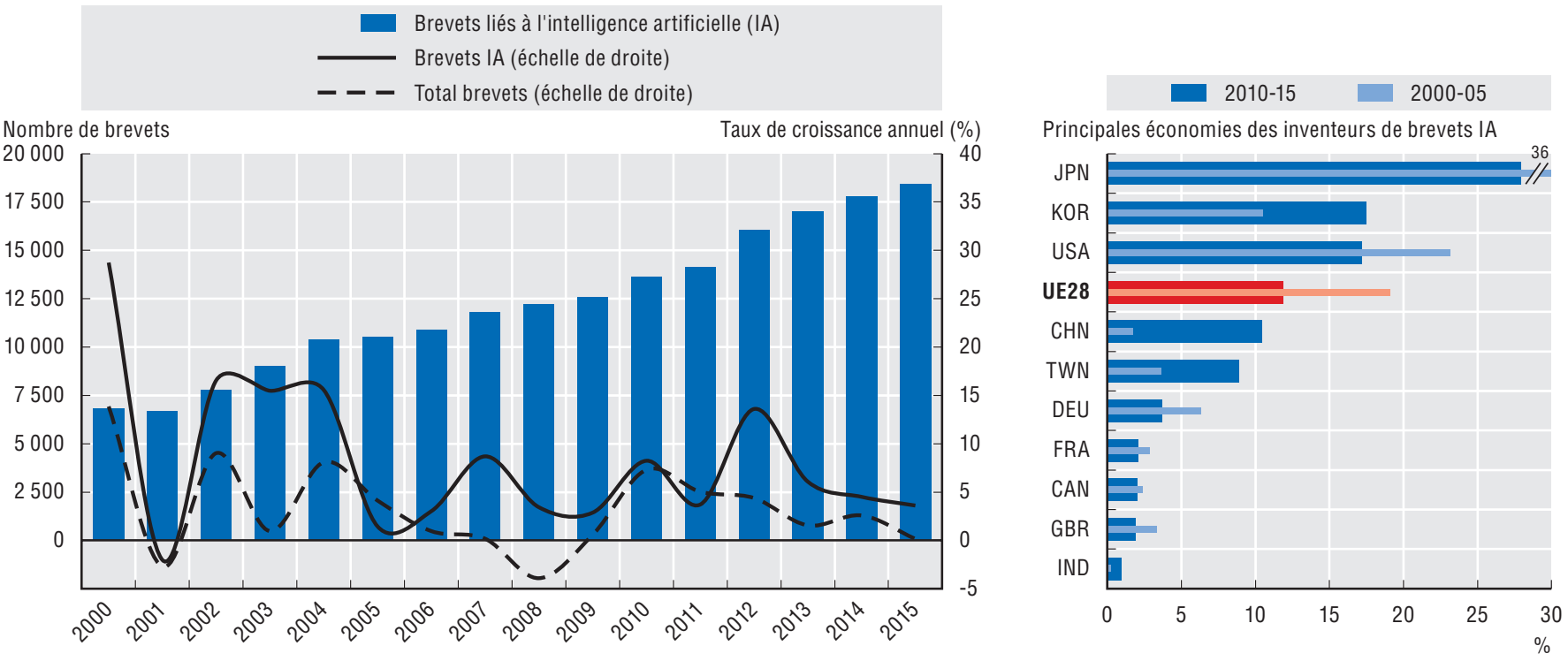

Source : OCDE, STI Microdata Lab : Base de données sur la propriété intellectuelle, http://oe.cd/ipstats, juin 2017. Davantage de données via StatLink. Voir notes de chapitre.

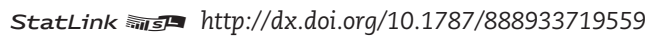

\section{Comment mesurer les technologies liées à l'intelligence artificielle?}

La mesure du développement des technologies liées à l'IA est délicate : les frontières entre l'IA et les autres technologies sont floues et en constante évolution. Les indicateurs présentés ici se fondent sur les domaines technologiques (identifiés par les codes de la Classification internationale des brevets, ou codes CIB) mentionnés dans les brevets pour recenser les inventions liées à l'IA. Sont considérées comme étant liées à l'IA toutes les inventions relevant des domaines ayant trait à l'interface homme-machine et à la cognition et la compréhension sémantique, décrits dans l'édition 2017 de la taxonomie de l'OCDE pour les brevets liés aux TIC (voir Inaba et Squicciarini, 2017).

Étant donné que les inventions brevetées peuvent être associées à plusieurs domaines technologiques, l'examen des " co-occurrences " des codes CIB dans les familles de brevets (à savoir les différents codes CIB énumérés dans un même brevet) permet d'étudier dans quelle mesure l'IA est associée à d'autres technologies. Les chiffres présentés ici mettent en lumière les technologies qui sont plus souvent associées à l'IA ; en se fondant sur la table de concordance CIB-technologie de l'OMPI (2013) et la taxonomie des brevets liés aux TIC. 
1. Science, innovation et révolution numérique

\section{Intelligence artificielle}

L'examen de l'ensemble des domaines technologiques dans lesquels des brevets liés à l'intelligence artificielle ont été déposés montre que l'IA est souvent associée à un certain nombre de technologies numériques utilisées aux fins de l'analytique des données massives - traitement et transfert de données numériques, ou encore applications pour le transport et la santé. Par exemple, une analyse plus approfondie des technologies médicales révèle que jusqu'à 30 \% des inventions utilisées à des fins de diagnostic médical (tests oculaires, examens médicaux généraux, etc.) intègrent des composants liés à l'IA.

8. Brevets portant sur les principales technologies intégrant des fonctions d'intelligence artificielle, 2000-05 et 2010-15 Nombre de familles de brevets IP5 liées à l'IA, par classe de brevets hors IA

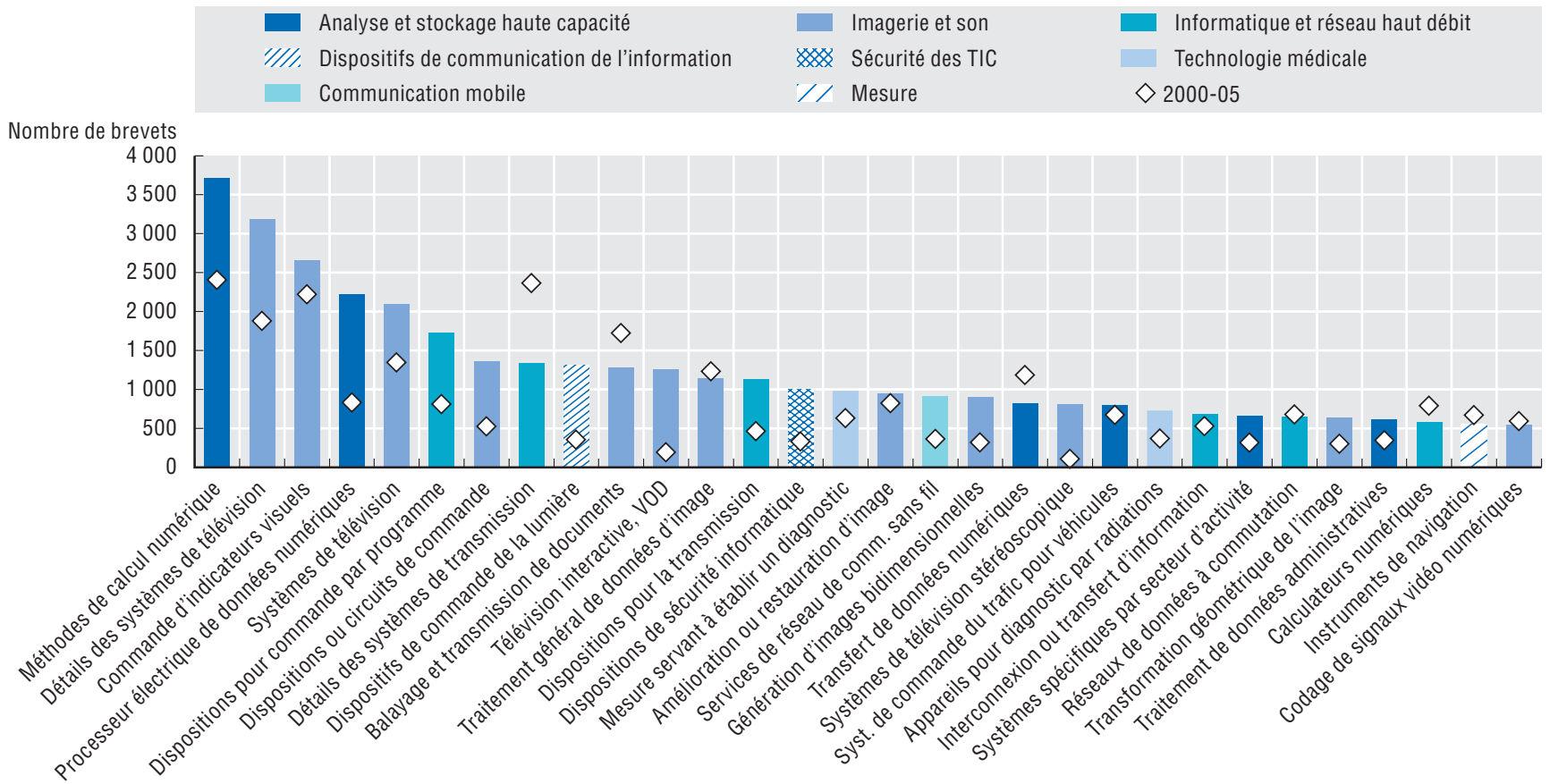

Source : OCDE, STI Microdata Lab : Base de données sur la propriété intellectuelle, http://oe.cd/ipstats, juin 2017. Davantage de données via StatLink. Voir notes de chapitre.

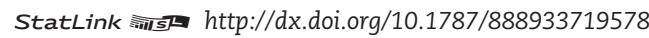

9. Dix principales technologies médicales couplées à l'intelligence artificielle, 2000-05 et 2010-15

Part des brevets liés à l’IA dans les familles de brevets IP5 portant sur des technologies médicales

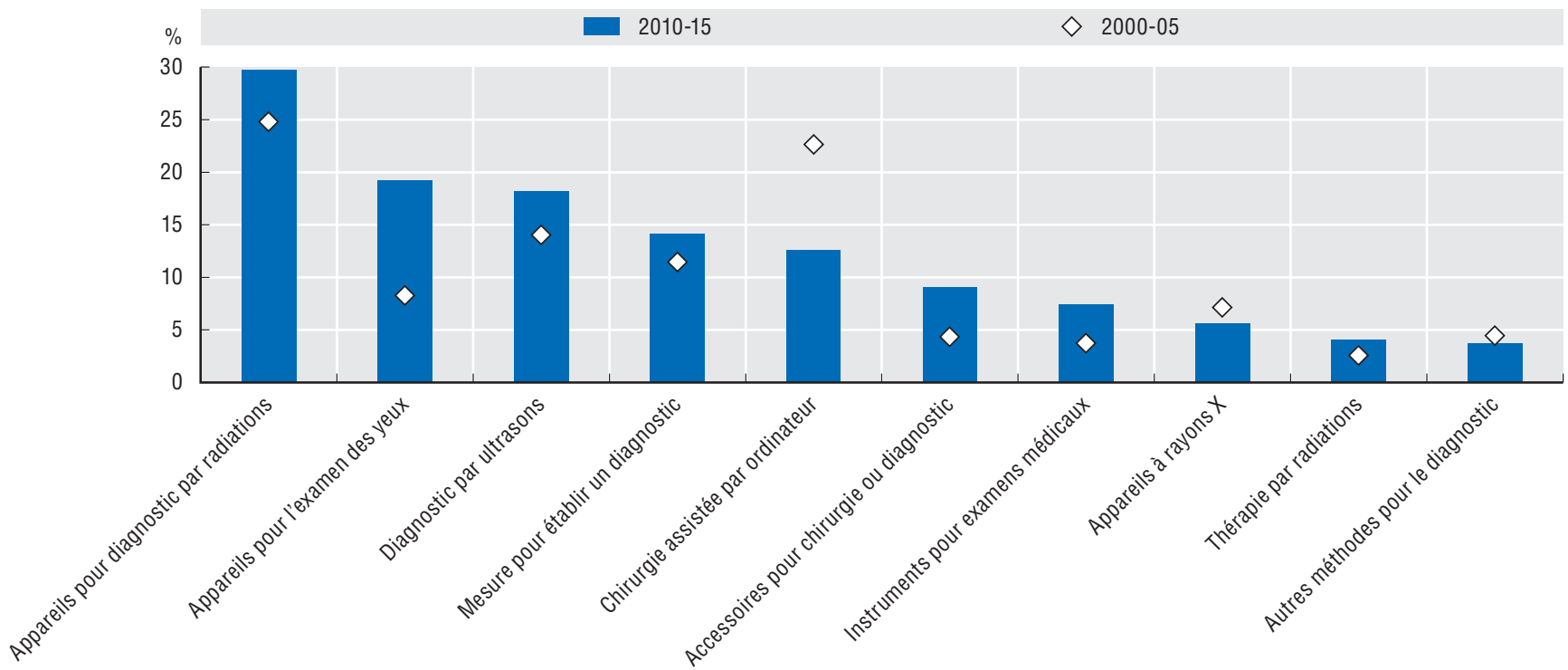

Source : OCDE, STI Microdata Lab : Base de données sur la propriété intellectuelle, http://oe.cd/ipstats, juin 2017. Davantage de données via StatLink. Voir notes de chapitre. 


\section{1. ÉCONOMIE DU SAVOIR ET TRANSFORMATION NUMÉRIQUE}

\section{Science, innovation et révolution numérique}

\section{Panorama de la science}

Premier acteur mondial de la R-D, les États-Unis ont consacré plus de 500 milliards USD aux dépenses intérieures de R-D en 2015. Arrive en deuxième position la Chine (avec 409 milliards USD à PPA), qui est passée devant la zone UE28 (386 milliards USD à PPA) en 2015. Israël et la Corée affichent le ratio le plus élevé de dépenses de R-D rapportées au PIB en raison d'une augmentation rapide de ces dépenses au cours des dernières années. Les économies partenaires de l'OCDE représentent quant à elles une part croissante de la R-D mondiale, mesurée par le nombre total de chercheurs et les dépenses de R-D. Les coûts de personnel, qui incluent ceux des chercheurs, sont, dans la plupart des économies, le principal poste de dépenses de R-D, ce qui explique l'étroite corrélation entre les mesures de R-D en pourcentage du PIB et le nombre de chercheurs exprimé en pourcentage de l'emploi total. Les écarts peuvent être dus à des différences au niveau des prix relatifs des intrants de la R-D (y compris la rémunération des chercheurs), du degré de spécialisation de la R-D dans chaque économie, et des dépenses de R-D en capital pour le développement des infrastructures de recherche à venir.

\section{La R-D dans la zone OCDE et les principales économies partenaires, 2015}

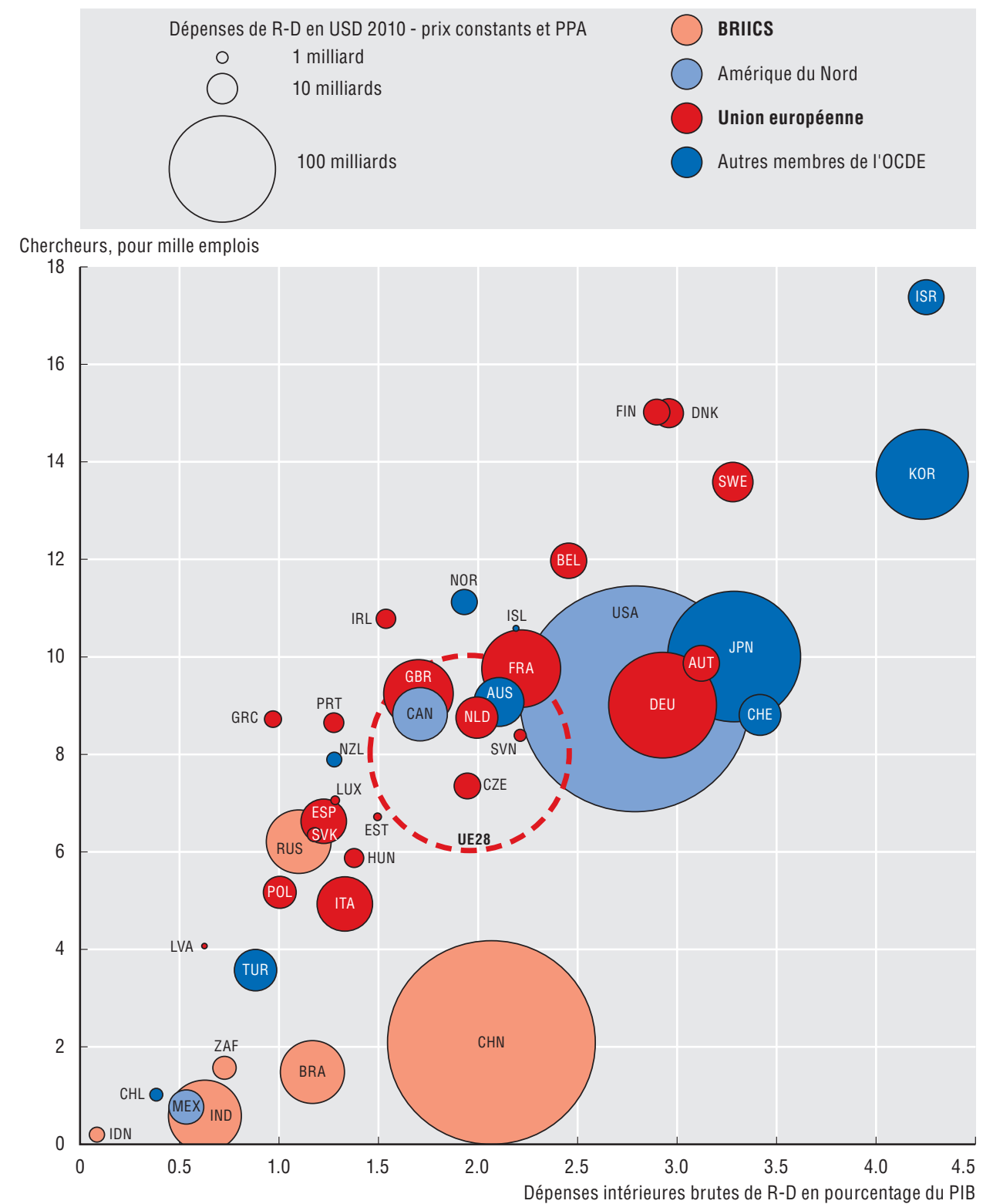

Note : En raison de divergences méthodologiques, les données relatives à certaines économies partenaires de l'OCDE peuvent ne pas être tout à fait comparables avec celles des autres pays.

Sources : OCDE, Base de données des Principaux indicateurs de la science et de la technologie, http://oe.cd/pist-fr, et Institut de statistique de l'UNESCO, Recherche et développement expérimental (complet), juillet 2017. Voir notes de chapitre. 
1. Science, innovation et révolution numérique

\section{Excellence scientifique}

Le volume mondial de la production scientifique, tel qu'indexé dans la base de données bibliométriques privée Scopus, a fortement augmenté au cours de la période 2005-16. Les indicateurs de l'« excellence scientifique » mettent en évidence l'évolution de la contribution des différents pays aux publications les plus citées. La Chine a enregistré une augmentation à la fois de son volume de publications scientifiques fréquemment citées et de sa part dans les $10 \%$ de publications les plus citées dans le monde. Passée de moins de 4 \% en 2005 à 14 \% en 2016 ; elle s'est ainsi hissée au deuxième rang mondial derrière les États-Unis. La part de l'Union européenne dans la production scientifique d'excellence est restée stable ; la zone est devenue la première puissance scientifique, devançant désormais les États-Unis. En revanche, comme le montre le deuxième graphique, le niveau d'excellence moyen de la recherche européenne reste à la traîne, à environ 12 \%. Un chiffre inférieur à ceux des États-Unis et du Royaume-Uni, qui continuent d'afficher les taux les plus élevés en termes de qualité de la recherche scientifique (14\%). La Fédération de Russie, partie d'un niveau relativement faible, a franchi la barre des $4 \%$ au cours de la période considérée.

\section{1. Économies en tête des volumes de publications scientifiques parmi les plus citées, 2005 et 2016} En pourcentage des $10 \%$ de publications les plus citées dans le monde

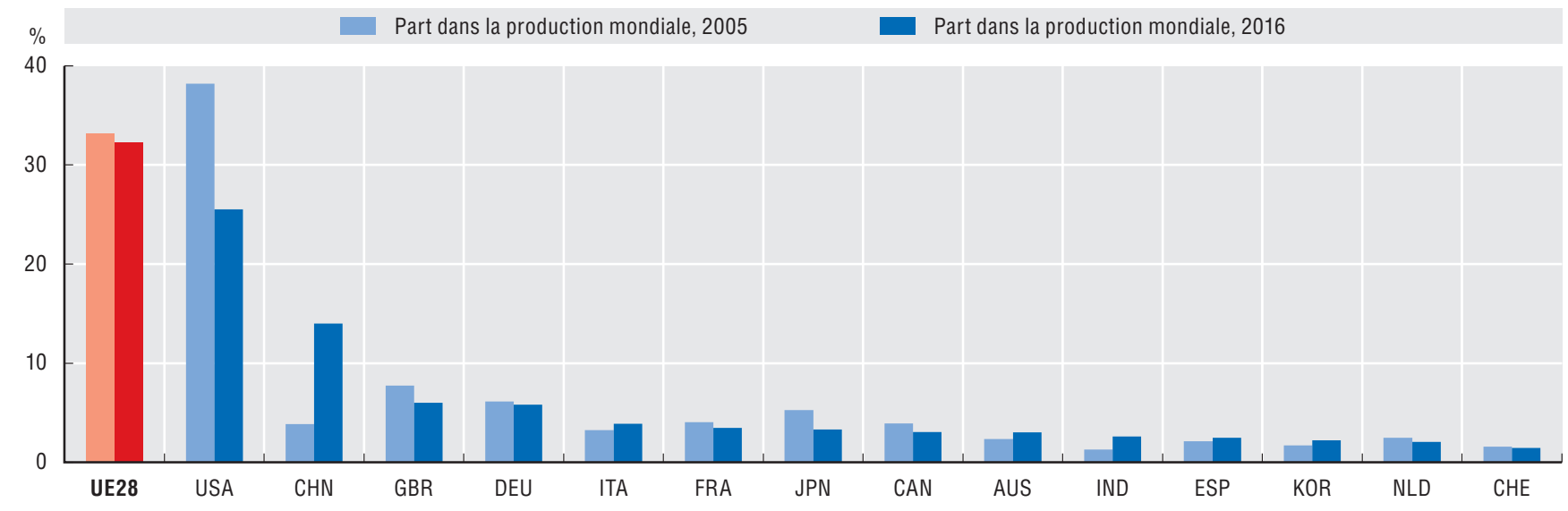

Source : Calculs de l'OCDE, d'après Scopus Custom Data, Elsevier, version 4.2017, juillet 2017. Davantage de données via StatLink. Voir notes de chapitre. StatLink न्ताs http://dx.doi.org/10.1787/888933719635

12. Évolution récente de l'excellence scientifique dans une sélection de pays, 2005-16

En pourcentage de la production scientifique du pays figurant parmi les $10 \%$ de publications les plus citées dans le monde

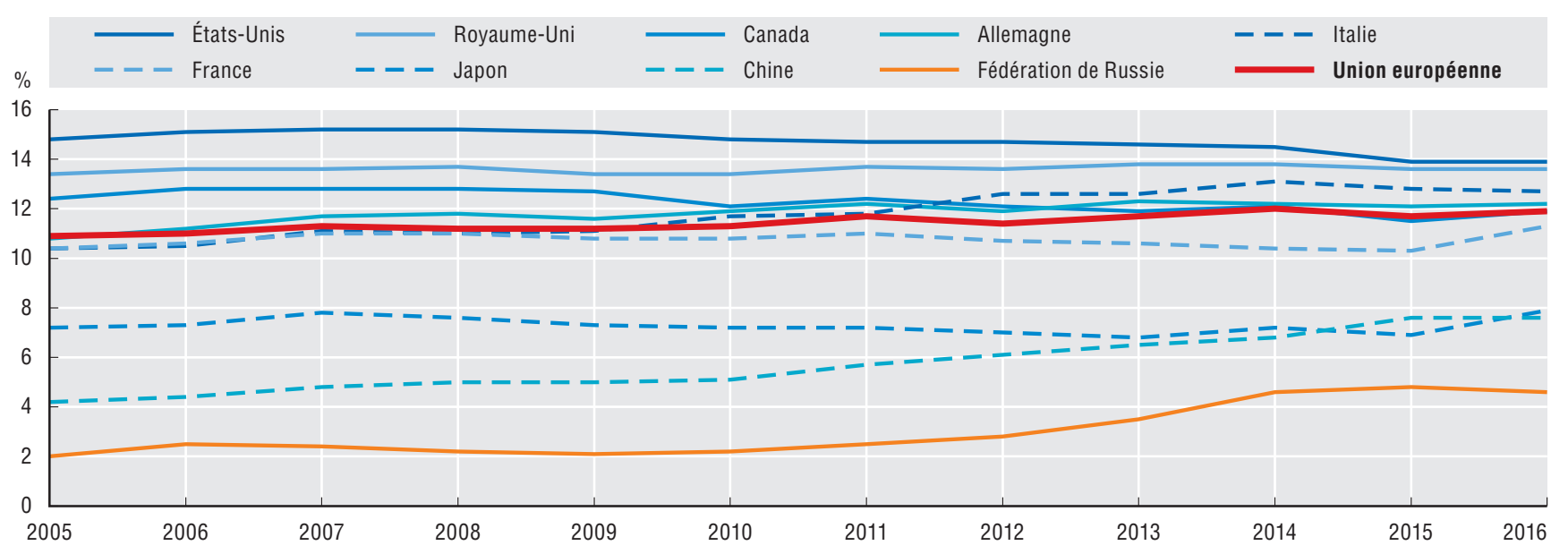

Source : Calculs de l'OCDE, d'après Scopus Custom Data, Elsevier, version 4.2017, juillet 2017. Voir notes de chapitre.

StatLink 完正四 http://dx.doi.org/10.1787/888933719654

\section{Comment lire ces graphiques}

Le graphique 11 illustre la part de chaque pays ou zone considéré(e) dans les $10 \%$ de documents les plus cités dans le monde, selon leur type (articles, évaluations et actes de conférences) et leur année de publication. Par exemple, plus de $30 \%$ des documents les plus cités sont publiés par des auteurs basés dans l'UE. Le graphique 12 représente quant à lui le pourcentage de documents produits dans chaque pays figurant parmi les 10 \% de publications les plus citées. Pour la zone UE, le taux avoisine les 12 \%. Le Scimago Journal Rank, qui mesure l'influence d'une revue d'après le nombre de citations, a été utilisé pour établir un classement des documents présentant un nombre de citations identique. Dans la mesure où les documents les plus récents comptent moins de citations, cet ajustement influe davantage sur les valeurs des dernières années - il en va de même pour les domaines dans lesquels les citations sont plus tardives. 


\section{1. ÉCONOMIE DU SAVOIR ET TRANSFORMATION NUMÉRIQUE}

\section{Science, innovation et révolution numérique}

\section{Tendances de la R-D}

Les dépenses intérieures brutes de R-D (DIRD) ont augmenté, dans la zone OCDE, de $2.3 \%$ en termes réels entre 2014 et 2015 , pour atteindre 1140 milliards USD. Cette hausse a contribué à consolider la reprise des dépenses de R-D amorcée au lendemain de la crise mondiale et financière de 2008-09. Depuis 2013, la part des DIRD dans le PIB dans la zone OCDE est restée stable, à 2.4 \% du PIB. La croissance récemment observée a été principalement tirée par les entreprises, qui représentent environ $70 \%$ de l'ensemble de la R-D. Les activités de R-D des institutions privées sans but lucratif (dont font partie la plupart des organismes caritatifs) ont également fortement progressé au cours de la période 2013-15, bien qu'elles ne représentent qu'une part réduite de l'ensemble de la R-D (2.4\%). La R-D de l'État a connu un léger rebond ; en revanche, le rythme de croissance des activités de R-D des établissements d'enseignement supérieur (deuxième secteur d'exécution de la R-D) a, quant à lui, ralenti. Parmi les pays entrant dans le champ des Principaux indicateurs de la science et de la technologie de l'OCDE (http://oe.cd/pist-fr), Israël et la Corée affichent la plus forte intensité de R-D - la Corée connaît d'ailleurs une évolution rapide depuis 2002, essentiellement due à la croissance de la R-D des entreprises. Même tendance en Chine, où la part des DIRD dans le PIB a dépassé celle de la zone UE28 en 2012 et continue de se rapprocher du niveau de la zone OCDE (2.4\%) - elle atteignait $2.07 \%$ en 2015. Le secteur de l'enseignement supérieur est un acteur essentiel de la R-D dans la plupart des pays, notamment pour ce qui est de la recherche fondamentale. Néanmoins, en Chine, la R-D des établissements d'enseignement supérieur représente seulement $7 \%$ des DIRD, soit un taux très inférieur à ceux de la zone OCDE et de l'UE28 (18\% et $23 \%$ respectivement).

\section{Dépenses de R-D par secteur d'exécution, zone OCDE, 1995-2015}

Indice en prix constants (USD à PPA, $1995=100)$ et part de la DIRD en 2015

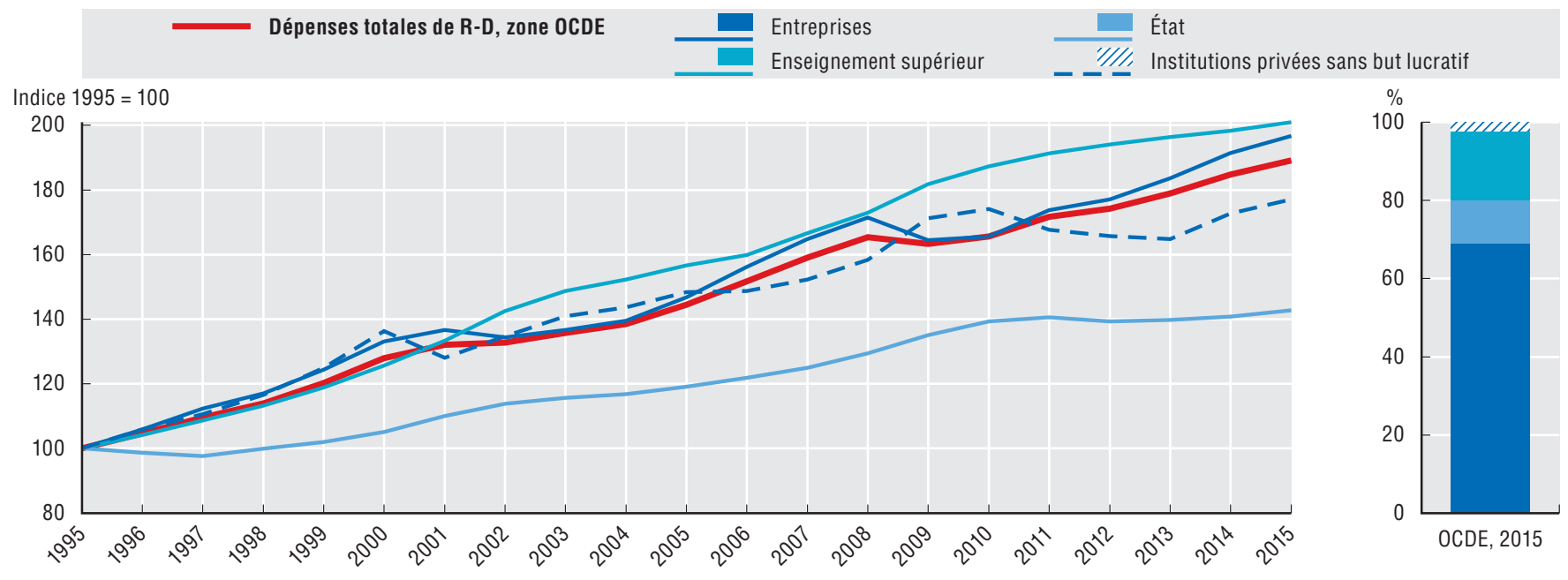

Source : OCDE, Base de données des Principaux indicateurs de la science et de la technologie, http://oe.cd/pist-fr, juillet 2017. Voir notes de chapitre. StatLink intsle $h$ ttp://dx.doi.org/10.1787/888933719673

\section{4. Évolution des activités de R-D dans la zone OCDE et une sélection d'économies, 1995-2015}

En pourcentage du PIB

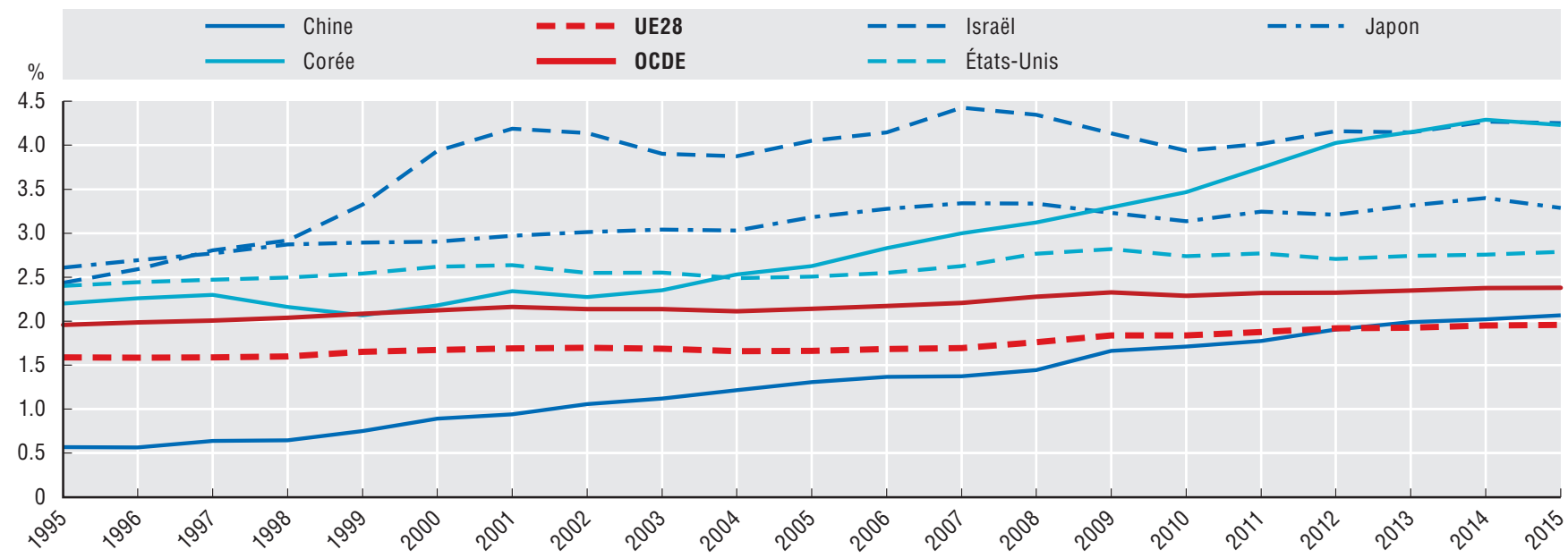

Source : OCDE, Base de données des Principaux indicateurs de la science et de la technologie, http://oe.cd/pist-fr, juillet 2017. Voir notes de chapitre.

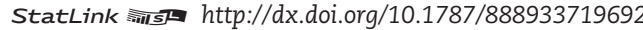




\section{Tendances de la R-D}

À l'instar des autres types d'investissement, les dépenses de R-D et d'innovation suivent l'évolution du cycle économique - elles affichent en effet une corrélation positive avec les résultats économiques. La R-D financée par les entreprises est particulièrement sensible aux conditions d'accès au financement et à la demande globale. L'effondrement, en 2008-09, du PIB et de la R-D des entreprises a été partiellement compensé par la progression de la R-D financée par l'État. À partir de 2010, la R-D financée par les entreprises s'est redressée, tandis que le financement public direct de la R-D a décliné - essentiellement du fait de politiques d'assainissement budgétaire. Depuis 1985, les trois composantes de la R-D ont évolué différemment : la recherche appliquée et le développement expérimental, qui représentent la majorité des dépenses de R-D, ont plus que doublé en termes réels. Ils s'élevaient respectivement à $21 \%$ et $62 \%$ de la DIRD de la zone OCDE en 2015, et représentaient, ensemble, $95 \%$ des dépenses de R-D en Chine. Quant à la recherche fondamentale (17\%), elle a presque quadruplé au cours de la même période, sous l'effet de la croissance soutenue de la R-D dans le secteur de l'enseignement supérieur. Ces tendances générales masquent toutefois des différences considérables selon les secteurs et les pays. Par exemple, la croissance relative de la recherche fondamentale menée dans les entreprises joue également un rôle dans certains pays comme les États-Unis, où elle a progressé de 3 \% à 5 \% de la DIRD entre 2005 et 2015.

\section{5. Évolution conjoncturelle des dépenses de R-D, par source de financement, zone OCDE, 1995-2016} Indice en prix constants (USD à PPA, $1995=100$ ) et part de la DIRD en 2015
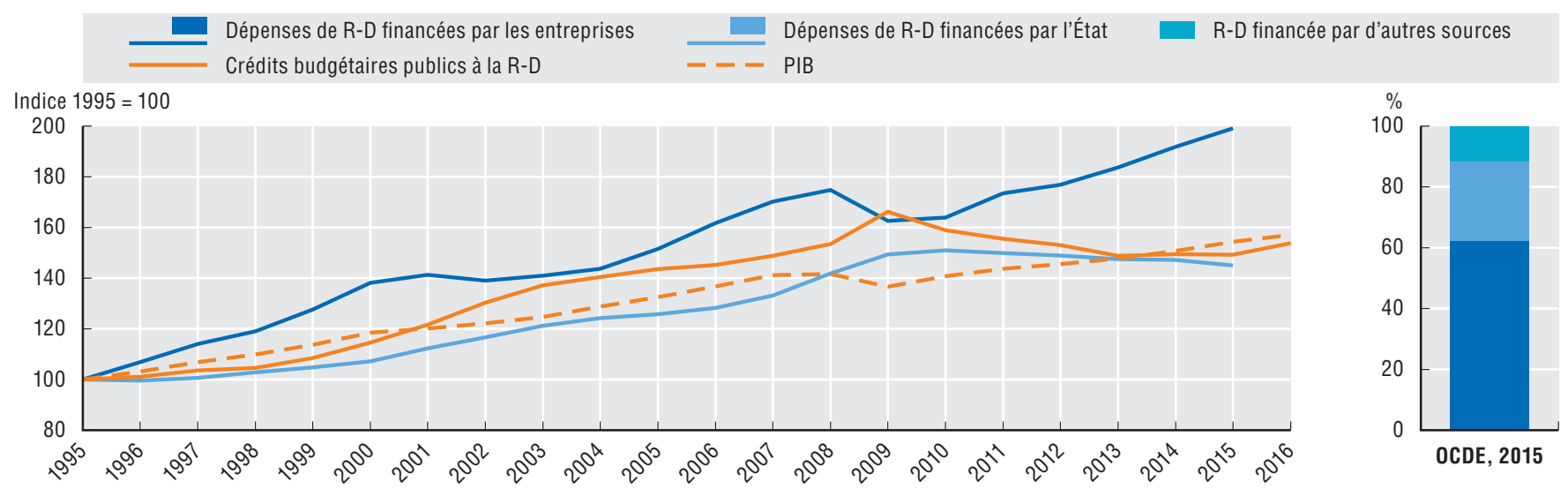

Source : OCDE, Base de données des Principaux indicateurs de la science et de la technologie, http://oe.cd/pist-fr, juillet 2017. Voir notes de chapitre.

StatLink -ilsts $h$ ttp://dx.doi.org/10.1787/888933719711

\section{6. Évolution de la recherche fondamentale, de la recherche appliquée et du développement expérimental dans la zone OCDE, 1985-2015}

Indice en prix constants (USD à PPA, 1985 = 100) et part de la DIRD en 2015

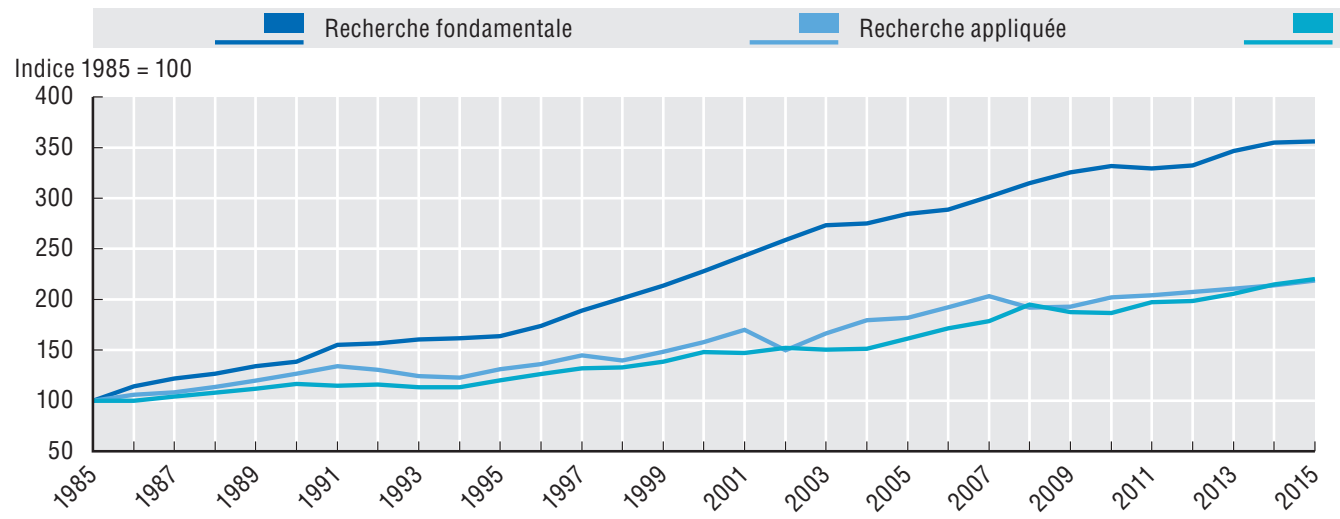

Développement expérimental

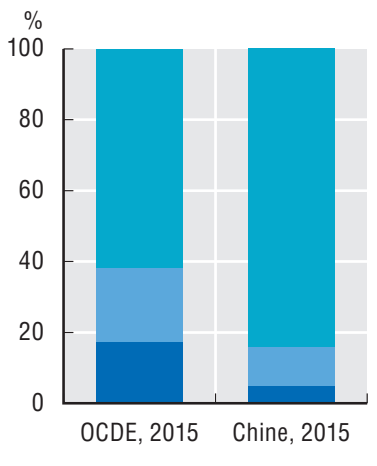

Note : L'indice a été estimé par enchaînement des taux de croissance en glissement annuel, calculés pour un ensemble variable de pays pour lesquels on dispose de données équilibrées pour des années consécutives, sans rupture de série.

Source: Calculs de l'OCDE, d'après les bases de données des Principaux indicateurs de la science et de la technologie, http://oe.cd/pist-fr, et des Statistiques de la recherche et développement, http://oe.cd/srd-fr, juin 2017. Voir notes de chapitre.

StatLink त्ञाIs http://dx.doi.org/10.1787/888933719730

\section{Mesure de la R-D et de ses composantes}

On mesure la R-D en additionnant l'ensemble des dépenses liées à l'exécution des activités de R-D, comme défini dans le Manuel de Frascati (OCDE, 2015a). La R-D comprend la recherche fondamentale (travaux entrepris en vue d'acquérir de nouvelles connaissances, sans viser une application particulière), la recherche appliquée (travaux entrepris en vue d'acquérir des connaissances nouvelles dans un but pratique déterminé) et le développement expérimental (développement de produits ou de procédés nouveaux). Dans certains pays et secteurs, il est difficile d'opérer une distinction entre ces différentes composantes, ce qui pose des problèmes en termes de couverture des données. Selon les incitations financières, en particulier les décisions et les priorités de financement des États, les répondants aux enquêtes peuvent être amenés à classer les projets de R-D du côté tantôt de la recherche fondamentale, tantôt de la recherche appliquée, ce qui a un impact sur la mesure de la spécialisation sectorielle dans les différents types de R-D. 


\section{1. ÉCONOMIE DU SAVOIR ET TRANSFORMATION NUMÉRIQUE}

\section{Science, innovation et révolution numérique}

\section{Concentration de la R-D des entreprises}

Les activités de R-D présentent une forte concentration : un nombre limité d'acteurs exécutent une large part de la R-D des entreprises (DIRDE) dans les pays. Un constat que vient corroborer une nouvelle analyse des activités de R-D, menée au niveau des entreprises, dans un certain nombre de pays de l'OCDE. Elle révèle en effet que les 50 premiers exécutants de la R-D représentent 40 \% de la DIRDE au Canada et aux États-Unis, 55 \% en Allemagne et au Japon, et 70 \% au Danemark et en Nouvelle-Zélande. L'élargissement du champ d'analyse aux 100 principaux acteurs de la R-D souligne une hausse relativement modérée de la part cumulée de la DIRDE qui leur est imputable. Ces chiffres doivent toutefois être examinés en gardant à l'esprit la taille du pays et le nombre total d'entreprises menant des activités de R-D. En Nouvelle-Zélande, par exemple, les 50 principaux exécutants de la R-D représentent $4 \%$ de l'ensemble des entreprises déployant des efforts dans ce domaine, tandis qu'en France ou en Allemagne, elles représentent une fraction bien plus ténue. L'examen de la concentration de la R-D des entreprises a des incidences directes sur l'affectation et le ciblage potentiel des aides publiques, généralement dirigées vers les grands exécutants.

\section{Concentration de la R-D des entreprises : 50 et 100 principaux exécutants, 2014}

En pourcentage des dépenses intérieures de R-D des entreprises et du nombre total d'acteurs de la R-D

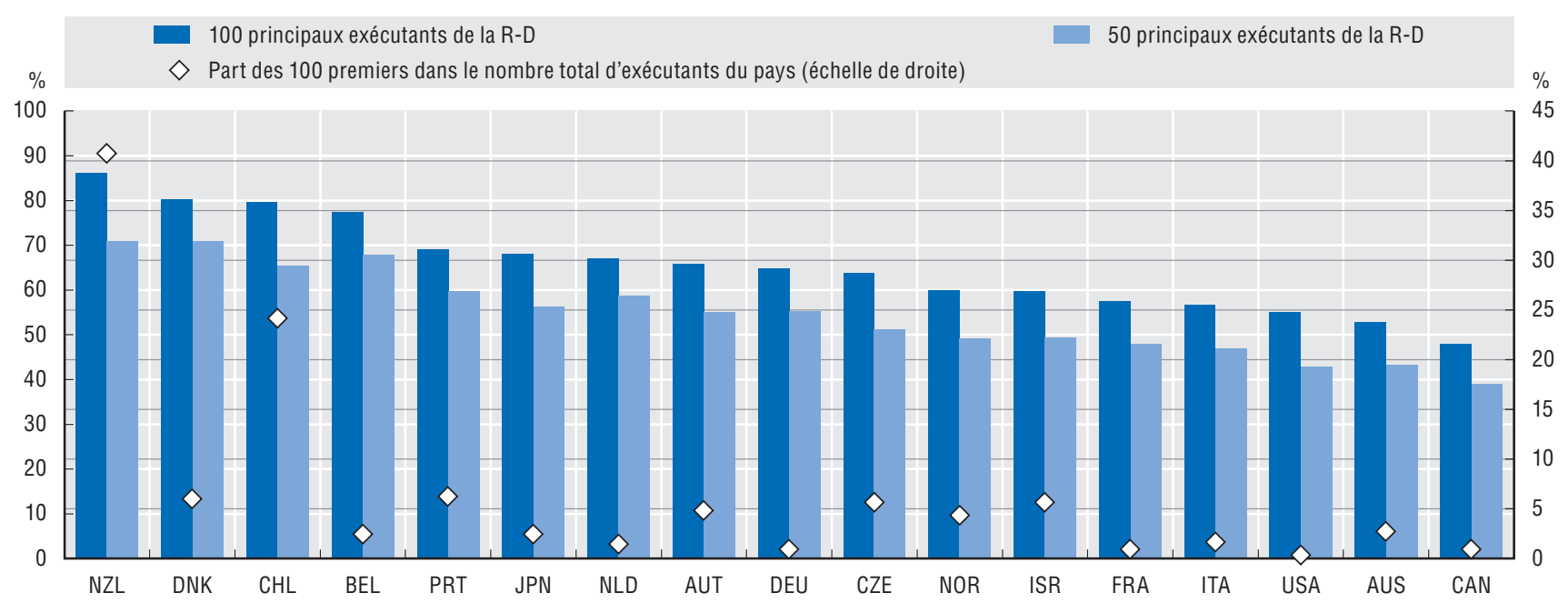

Source : OCDE, d'après les résultats préliminaires du projet microBeRD de l'OCDE, http://oe.cd/microberd, juillet 2017. Voir notes de chapitre.

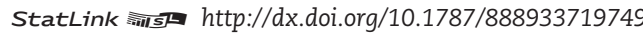

\section{Projet de micro-données de l'OCDE sur l'impact et les incidences du soutien public à la R-D des entreprises : microBeRD}

L'OCDE a lancé le projet microBeRD pour analyser l'ampleur et l'impact du soutien public à la R-D des entreprises au niveau microéconomique. Le projet vise à faciliter la reproductibilité des politiques publiques en examinant l'extrême hétérogénéité des conditions d'obtention et d'utilisation des aides publiques - aux niveaux tant infranationaux qu'internationaux. Pour ce faire, il s'appuie sur une approche coordonnée et distribuée de l'analyse des micro-données dans les différents pays et territoires, en collaboration avec des experts nationaux ayant accès aux micro-données sur la R-D et les aides publiques. La plupart de ces experts travaillent déjà de concert avec l'OCDE dans le cadre d'activités menées de longue date pour définir des normes de mesure de la R-D et développer des indicateurs agrégés comparables à l'échelle internationale.

L'utilisation d'un code commun adaptable permet de mener une analyse multi pays cohérente de l'hétérogénéité de l'utilisation et des incidences du soutien public à la R-D selon les entreprises. Cette approche garantit en outre la confidentialité des données (puisque seules des données agrégées anonymisées sont communiquées à l'OCDE). Elle présente enfin l'avantage de couvrir des questions qui ne sauraient être examinées par le biais d'analyses menées au sein des pays ou à partir des seules sources de données publiques.

L'établissement d'une série d'indicateurs dérivés des micro-données sur la R-D vise à éclairer l'analyse des marchés et des leviers de l'action publique susceptibles d'influer sur l'exécution de la R-D, ainsi que leurs impacts. Les indicateurs de la concentration des activités de R-D dans les pays de l'OCDE peuvent permettre de comprendre le rôle de la concurrence, par exemple, en établissant des comparaisons avec d'autres mesures de la concentration économique aux niveaux sectoriel ou national. Par ailleurs, la concentration réelle des efforts de R-D, comparée aux mesures de la concentration du soutien public à la R-D fondées sur les micro-données, permet d'observer l'existence d'éventuels biais et d'examiner la cohérence avec les critères que les pays déclarent employer pour l'affectation des aides. Si la forte concentration des activités de R-D ne fait aucun doute, on ne dispose que de peu de données probantes, comparables à l'échelle internationale, sur le degré de concentration de la R-D au sein des pays de l'OCDE. L'objectif du projet microBeRD est de remédier à ce manque.

Pour en savoir plus sur le projet microBeRD, voir http://oe.cd/microberd. 
1. Science, innovation et révolution numérique

\section{Concentration de la R-D des entreprises}

Si la majeure partie des activités de R-D est exécutée au sein des grandes entreprises dans la plupart des pays considérés, les petites et moyennes entreprises continuent de représenter une part notable de la DIRDE, avec des taux allant de $21 \%$ en Belgique à $56 \%$ en Norvège. Dans chaque classe de taille, la R-D est principalement exécutée par des entreprises établies depuis cinq ans ou plus. À l'exception de la République tchèque et de l'Italie, la majeure partie des activités de R-D menées par les entreprises les plus jeunes (affichant moins de cinq ans d'ancienneté) sont avant tout exécutées dans des petites structures (comptant entre 10 et 49 salariés), plutôt que dans des entreprises de taille moyenne (50 à 249 salariés) ou grande (250 salariés ou plus). Les pays affichant la proportion la plus élevée de R-D menée par de jeunes entreprises sont Israël (9.3\%), la Norvège (8.6 \%) et la République tchèque (7.6 \%).

Des écarts significatifs sont observés selon les pays dans le recours aux sources de financement externe de la R-D selon la taille et l'ancienneté des entreprises. En Belgique et en Norvège, par exemple, les sources externes de financement représentent en moyenne au moins $15 \%$ des dépenses de R-D dans chaque catégorie de taille et d'ancienneté, alors qu'elles comptent pour moins de 7 \% en République tchèque et en Israël. Dans l'ensemble, les acteurs de petite taille tendent à faire davantage appel au financement externe. Le financement public joue un rôle particulièrement important pour les petites entreprises ; en revanche, son importance relative varie selon les pays et la situation des petites entreprises les plus jeunes et les plus anciennes. Les fonds provenant de l'étranger jouent un rôle plus important pour les entreprises de taille moyenne et grande.

\section{Activités de R-D par taille et ancienneté des entreprises, 2014}

En pourcentage des dépenses de R-D des entreprises du pays considéré

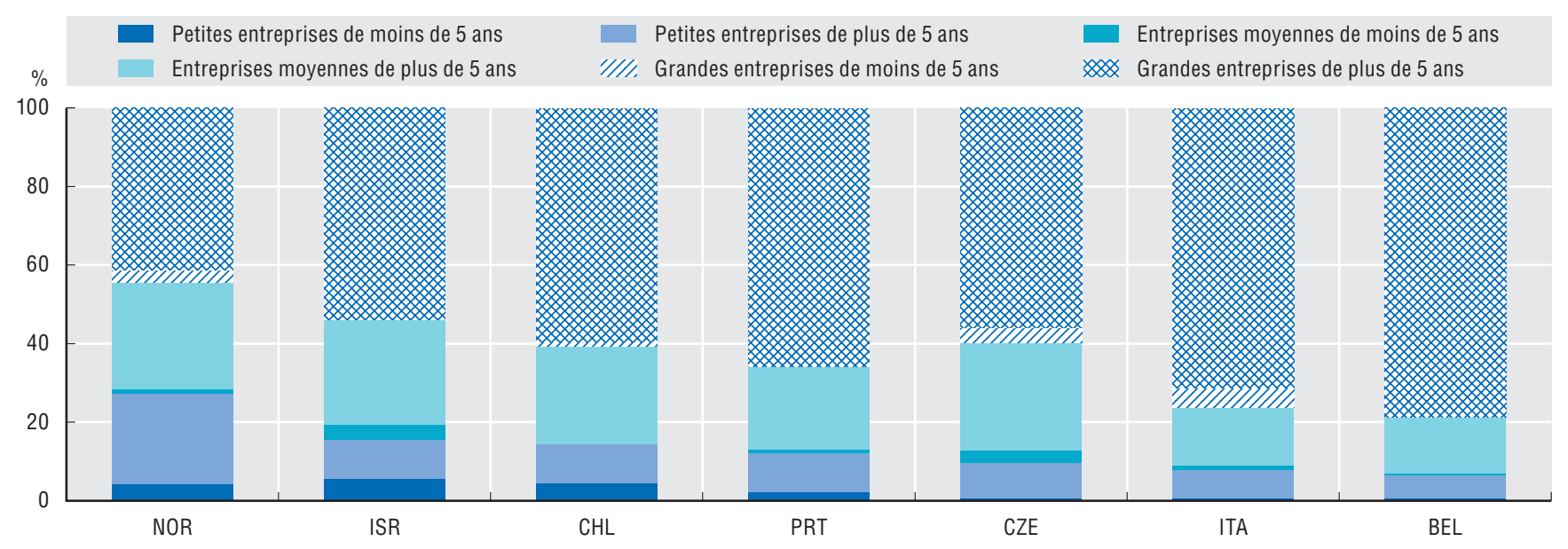

Source : OCDE, d'après les résultats préliminaires du projet microBeRD de l'OCDE, http://oe.cd/microberd, juillet 2017. Voir notes de chapitre.

StatLink त्राडs http://dx.doi.org/10.1787/888933719768

19. Sources externes de financement de la R-D par taille et ancienneté des entreprises, 2014 En pourcentage des dépenses de R-D intra-muros, moyenne pondérée

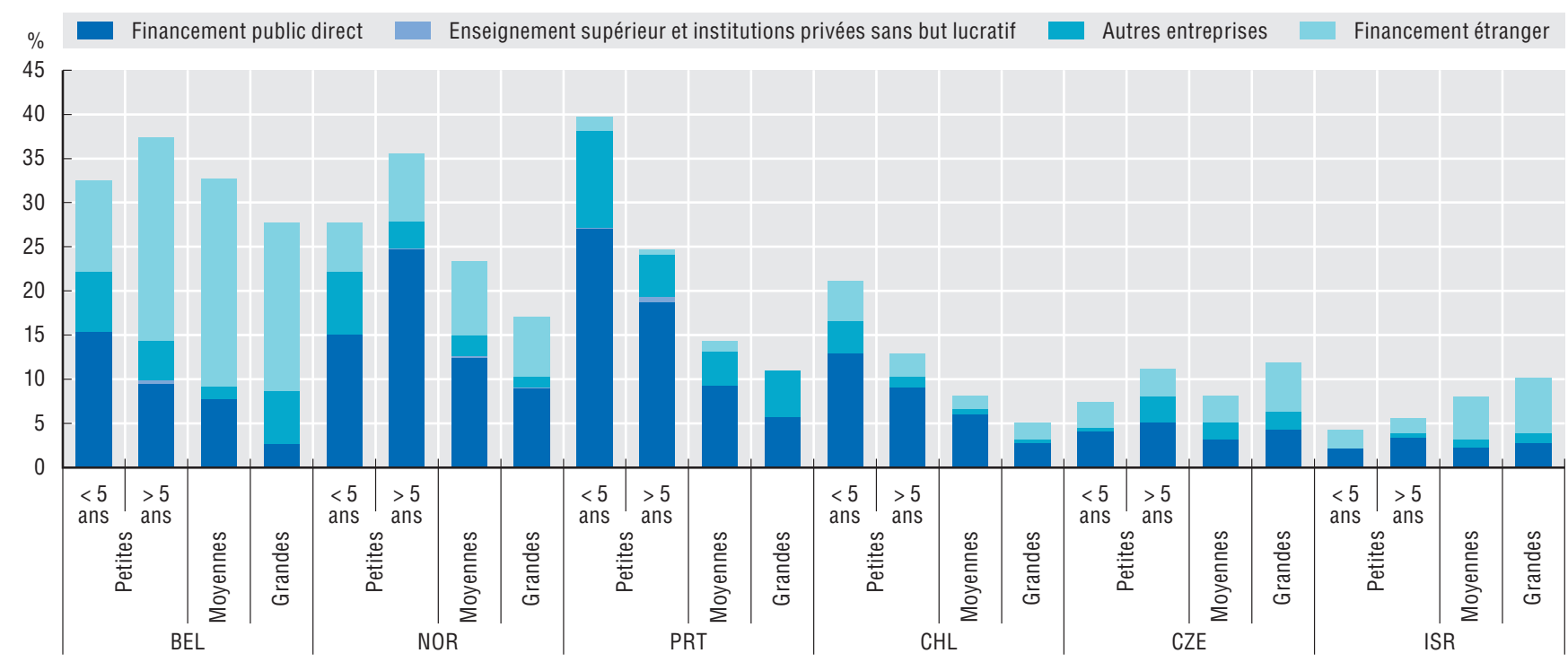

Source : OCDE, d'après les résultats préliminaires du projet microBeRD de l'OCDE, http://oe.cd/microberd, juillet 2017. Voir notes de chapitre. 


\section{1. ÉCONOMIE DU SAVOIR ET TRANSFORMATION NUMÉRIQUE}

\section{Science, innovation et révolution numérique}

\section{Principaux acteurs privés de la $R-D$}

Les acteurs qui investissent le plus dans la R-D sont des entreprises situées à la frontière technologique contribuant à un volume important des investissements et de la production en lien avec l'innovation. Leurs sièges sont concentrés dans un nombre restreint d'économies, notamment aux États-Unis, au Japon et en Chine. En moyenne, chacune des 2000 entreprises les plus actives en R-D possède des filiales dans 21 économies et exerce des activités dans 9 secteurs différents. Leurs dépenses de R-D, ainsi que leur production en matière d'innovation (mesurée d'après les brevets, marques, dessins et modèles déposés) apparaissent fortement concentrées. En 2014, le décile supérieur de ces investisseurs (à savoir les 200 premières entreprises et leurs filiales) représentaient environ 70 \% des dépenses de R-D, 60 \% des familles de brevets IP5 (inventions brevetées auprès des cinq grands offices de la propriété intellectuelle), $53 \%$ des dessins ou modèles déposés, et $38 \%$ des marques. La dynamique propre à certains secteurs, la complexité des produits et les stratégies de différenciation sur les marchés sont autant de facteurs qui peuvent expliquer les divergences d'utilisation des différents types de propriété intellectuelle selon les entreprises. Les principaux investisseurs en R-D jouent un rôle majeur dans le développement des technologies numériques. Ils détiennent respectivement environ $75 \%$ et $55 \%$ des brevets et des dessins ou modèles liés aux TIC déposés à l'échelle mondiale, et, en moyenne, 21 \% de leurs filiales opèrent dans les secteurs des TIC. Les brevets protégeant des inventions liées aux TIC représentent $44 \%$ du portefeuille total de brevets déposés par les principaux acteurs de R-D du secteur des TIC. En revanche, la part des brevets liés aux TIC détenus par des entreprises de secteurs autres que les TIC varie sensiblement; elle atteint par exemple $70 \%$ ou plus dans le cas des entreprises des secteurs « Finances et assurances » et "Services administratifs et de soutien ».

\section{Dépenses de R-D et portefeuille de PI des entreprises les plus actives en R-D, 2014}

Parts cumulées parmi les 2000 entreprises les plus actives en R-D

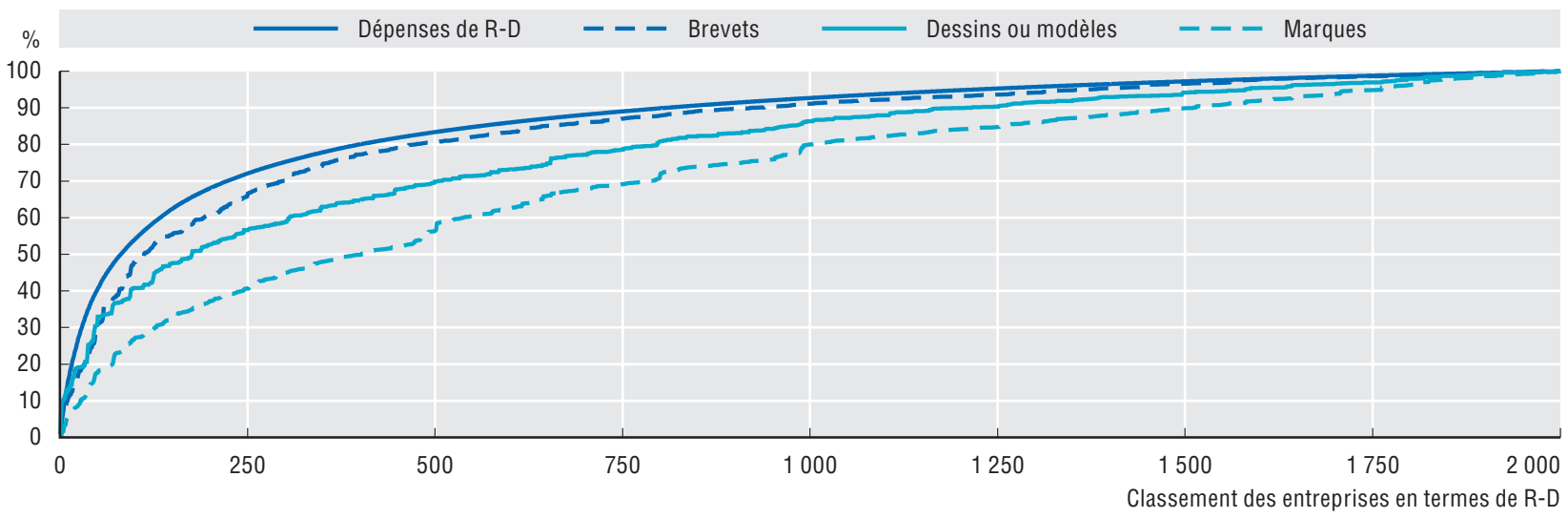

Source : Calculs de l'OCDE d'après CCR-OCDE, Base de données COR\&DIP@ v. 1, juillet 2017. Voir notes de chapitre.

StatLink +illst $h$ ttp://dx.doi.org/10.1787/888933719806

21. Portefeuille de brevets détenus par les entreprises les plus actives en R-D, par secteur, 2012-14 Nombre total des brevets et brevets liés aux TIC, familles de brevets IP5

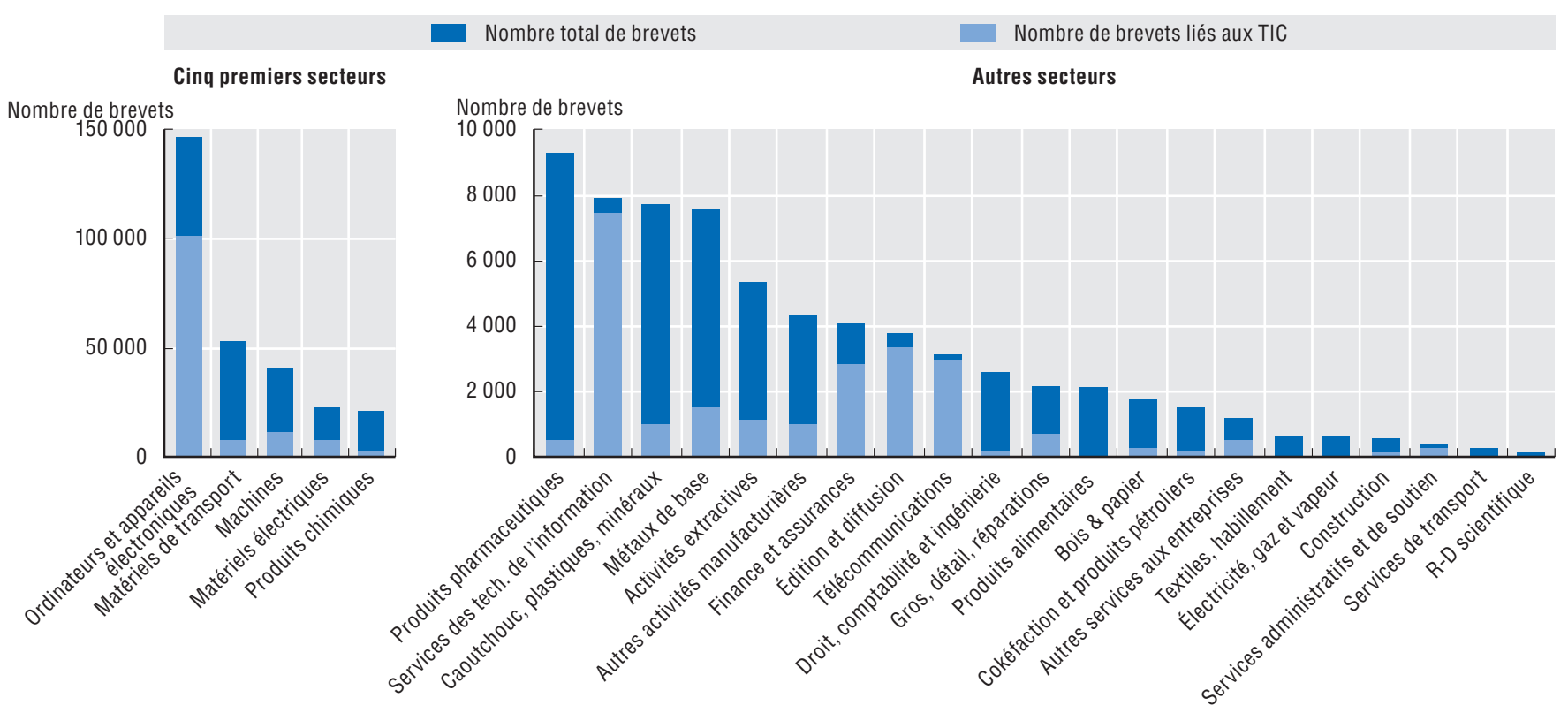

Source : Calculs de l'OCDE d'après CCR-OCDE, Base de données COR\&DIP@ v. 1, juillet 2017. Voir notes de chapitre. 


\section{Principaux acteurs privés de la R-D}

Les entreprises les plus actives en R-D dans le secteur « Ordinateurs et appareils électroniques » sont, de loin, celles qui comptent le plus d'actifs de propriété intellectuelle (DPI) ; elles représentent environ un tiers du nombre total de brevets déposés par les principaux acteurs investissant dans la R-D. Les secteurs " Matériels de transport ", " Machines " et " Produits chimiques " tendent eux aussi à déposer un nombre élevé de brevets. Les entreprises ne recourent pas dans les mêmes proportions aux différents actifs de propriété intellectuelle. Parmi les acteurs des TIC, ceux qui investissent massivement dans la R-D, à l'instar de Samsung ou de Sony, recourent autant aux brevets qu'aux dessins ou modèles; d'autres misent davantage sur les développements technologiques (comme Fujitsu et Toshiba), ou, au contraire, sur les dessins ou modèles (tel est le cas de Microsoft et d'Apple).

\section{Principaux acteurs de la R-D détenant des actifs de propriété intellectuelle, 2012-14}

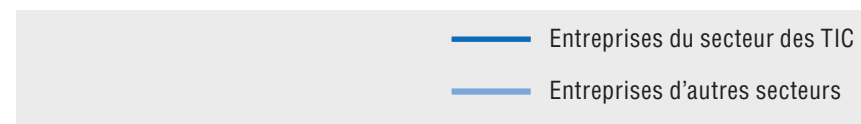

Familles de brevets IP 5

Dessins ou modèles
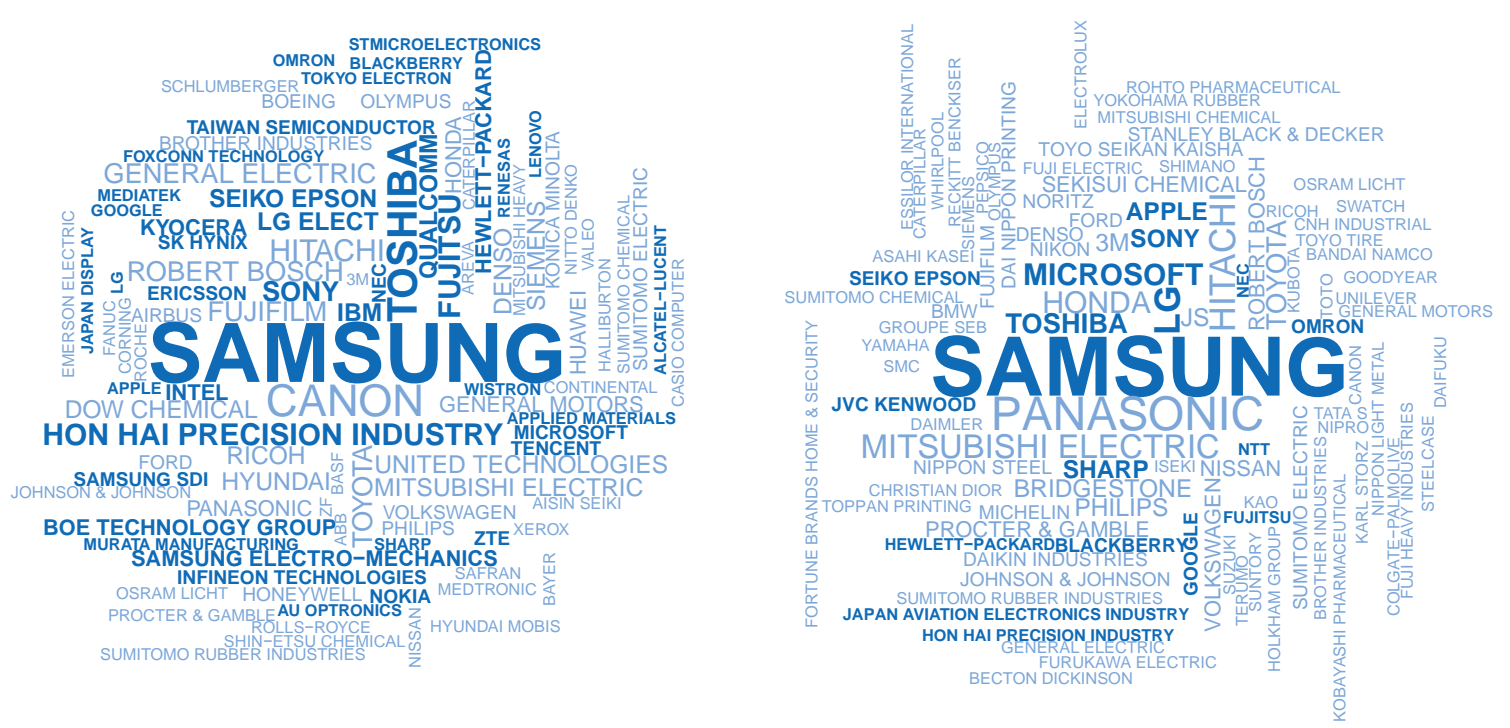

Source : Calculs de l'OCDE d'après CCR-OCDE, Base de données COR\&DIP@ v. 1, juillet 2017.Voir notes de chapitre.

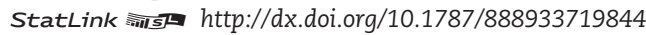

\section{Comment lire les nuages de noms}

Les deux nuages de noms d'entreprises ont été élaborés à partir d'informations relatives à la répartition des portefeuilles de brevets et de dessins ou modèles des entreprises les plus actives en R-D. La taille des caractères reflète la taille relative des portefeuilles de brevets et de dessins ou modèles des différentes entreprises qui composent l'échantillon étudié. Le nom des entreprises qui investissent le plus dans la R-D au sein du secteur des TIC apparaît en gras et en bleu foncé, tandis que le nom de celles relevant d'autres secteurs apparaît en bleu clair. La position et l'orientation (horizontale ou verticale) des noms n'ont pas de signification particulière, l'objectif étant simplement de garantir une lisibilité optimale.

\section{Qui sont les principaux acteurs de la R-D au niveau mondial ?}

Les entreprises qui investissent dans la R-D à l'échelle mondiale sont soit des sociétés mères d'une ou de plusieurs filiale(s), soit des entités indépendantes. Dans le premier cas, les dépenses de R-D prises en compte pour l'établissement du classement sont celles qui apparaissent dans les comptes consolidés ; elles intègrent donc les dépenses des entreprises affiliées. Quelque 82 \% des entreprises qui figurent parmi les principaux acteurs de la R-D en 2014 étaient déjà présentes dans le classement établi en 2012 (voir Dernis et al., 2015). Toutefois, les deux classements révèlent des différences notables : en 2014, l'échantillon dénombre moins d'entreprises du secteur "Ordinateurs et appareils électroniques ", et un plus grand nombre d'entreprises pharmaceutiques. Dans l'échantillon étudié, la plupart des détenteurs de brevets sont des entreprises installées en Asie. De fait, sur les 50 principaux détenteurs de familles de brevets IP5, 30 avaient leur siège en Asie, dont 19 au Japon et 6 en Corée. Les principaux investisseurs dans la R-D dont le siège est situé dans l'Union européenne, aux États-Unis et au Japon sont spécialisés dans un éventail relativement large de technologies. Les entreprises implantées dans l'UE ou aux États-Unis sont souvent tournées vers des technologies jouant un rôle fondamental dans la recherche de solutions face aux grands défis sociétaux liés à la santé ou à l'environnement. En revanche, celles qui ont leur siège en Chine et en Corée sont presque exclusivement spécialisées dans les technologies liées aux TIC. Plus de la moitié des grands acteurs de la R-D disposent d'un portefeuille complet de propriété intellectuelle (brevets, marques et dessins ou modèles). Toutefois, les stratégies de dépôt de propriété intellectuelle varient selon le marché ciblé et le secteur dans lequel évoluent les entreprises. Pour plus d'informations sur ces entreprises et leurs activités liées aux brevets, dessins ou modèles, et marques, voir Daiko et al. (2017). 


\section{1. ÉCONOMIE DU SAVOIR ET TRANSFORMATION NUMÉRIQUE}

\section{Science, innovation et révolution numérique}

\section{Technologies à la frontière mondiale}

Les entreprises les plus actives en R-D à l'échelle mondiale conduisent le développement de nombreuses technologies émergentes. Ce phénomène apparaît clairement lorsque l'on examine les domaines technologiques dans lesquels ces entreprises ont intensifié leurs activités d'invention au cours de la période 2012-14, ainsi que leur contribution au développement global de ces domaines. Les entreprises les plus actives en R-D ont accéléré leurs activités d'invention dans des domaines tels que les moteurs, les systèmes de conduite automatisée, et les technologies de l'information et des communications (TIC) liées à la connectivité, à la transmission et au transfert de données numériques. Dans la plupart de ces domaines, les principaux investisseurs détiennent $80 \%$ ou plus des portefeuilles de brevets liés à ces technologies déposés dans le monde.

\section{Classement des 20 premières technologies émergentes développées par les principaux} acteurs de la R-D, 2012-14

Part des brevets détenus par les 2000 entreprises les plus actives en R-D, en pourcentage du total des familles de brevets IP5 dans le domaine concerné

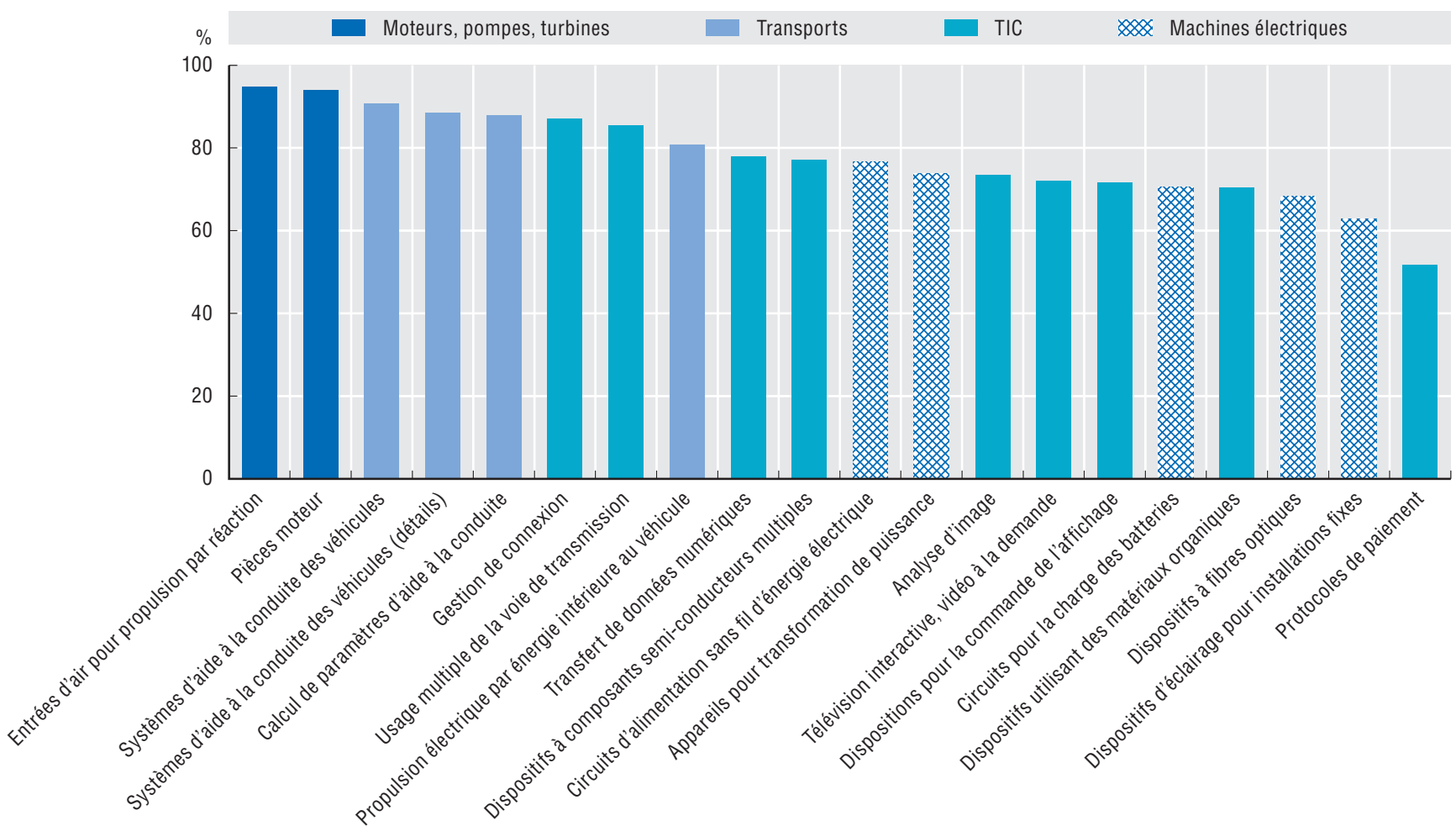

Sources: Calculs de l'OCDE d'après CCR-OCDE, Base de données COR\&DIP@ v. 1, et OCDE, STI Microdata Lab : Base de données sur la propriété intellectuelle, http://oe.cd/ipstats, juillet 2017. Voir notes de chapitre.

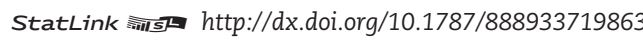

\section{Quelles sont les technologies phares développées par les entreprises les plus actives en R-D ?}

Les activités de recherche et développement menées par les entreprises les plus actives R-D débouchent sur le développement de nouvelles technologies. La méthodologie " DETECTS " (pour plus d'informations, voir Dernis et al., 2016) a été appliquée au portefeuille des 2000 entreprises les plus actives en R-D afin d'identifier les domaines technologiques dont le développement s'accélère brusquement, par rapport à d'autres technologies. On parle d'“ accélération » lorsque l'on observe une hausse soudaine et persistante du nombre de brevets déposés dans un domaine donné, par rapport aux dépôts de brevets comptabilisés dans les autres domaines ; l'analyse s'appuie sur les groupes de la Classification internationale des brevets (CIB). Les technologies émergentes présentées, identifiées par les codes CIB, sont celles qui affichent une accélération rapide et continue du nombre de dépôts de brevet depuis le début des années 2010. L'intelligence artificielle correspond aux catégories Interface homme-machine et Cognition et compréhension sémantique de la taxonomie des brevets liés aux TIC, décrite dans Inaba et Squicciarini (2017). 


\section{Principaux acteurs de l'intelligence artificielle}

Les 2000 entreprises les plus actives en R-D détiennent 75 \% des familles de brevets IP5 liées à l'intelligence artificielle (IA). Il ne s'agit pas nécessairement d'entreprises internationales du secteur des TIC : des entreprises de tous les secteurs contribuent en effet aux progrès de l'IA, bien qu'à des degrés divers. Outre le secteur "Ordinateurs et appareils électroniques ", qui représente $64 \%$ du portefeuille de brevets liés à l'IA déposés par les principaux acteurs de la R-D, les entreprises des secteurs « Matériels de transport » et « Machines » se sont également démarquées par le dynamisme de leurs activités d'invention dans le domaine de l'IA au cours de la période considérée (avec, en moyenne, près de 1000 brevets déposés par an). Le développement des technologies liées à l'IA se caractérise par une concentration relativement forte. Les acteurs de la R-D installés au Japon, en Corée, au Taipei chinois et en Chine représentent environ 70 \% de l'ensemble des inventions liées à l'IA imputables aux 2000 entreprises qui investissent le plus dans la R-D et à leurs filiales; la part des entreprises basées aux États-Unis s'élève quant à elle à 18 \%.

\section{Brevets liés à l'intelligence artificielle déposés par les 2000 entreprises les plus actives en $R-D$, par secteur, 2012-14 \\ Nombre de familles de brevets IP5, 20 principaux secteurs}
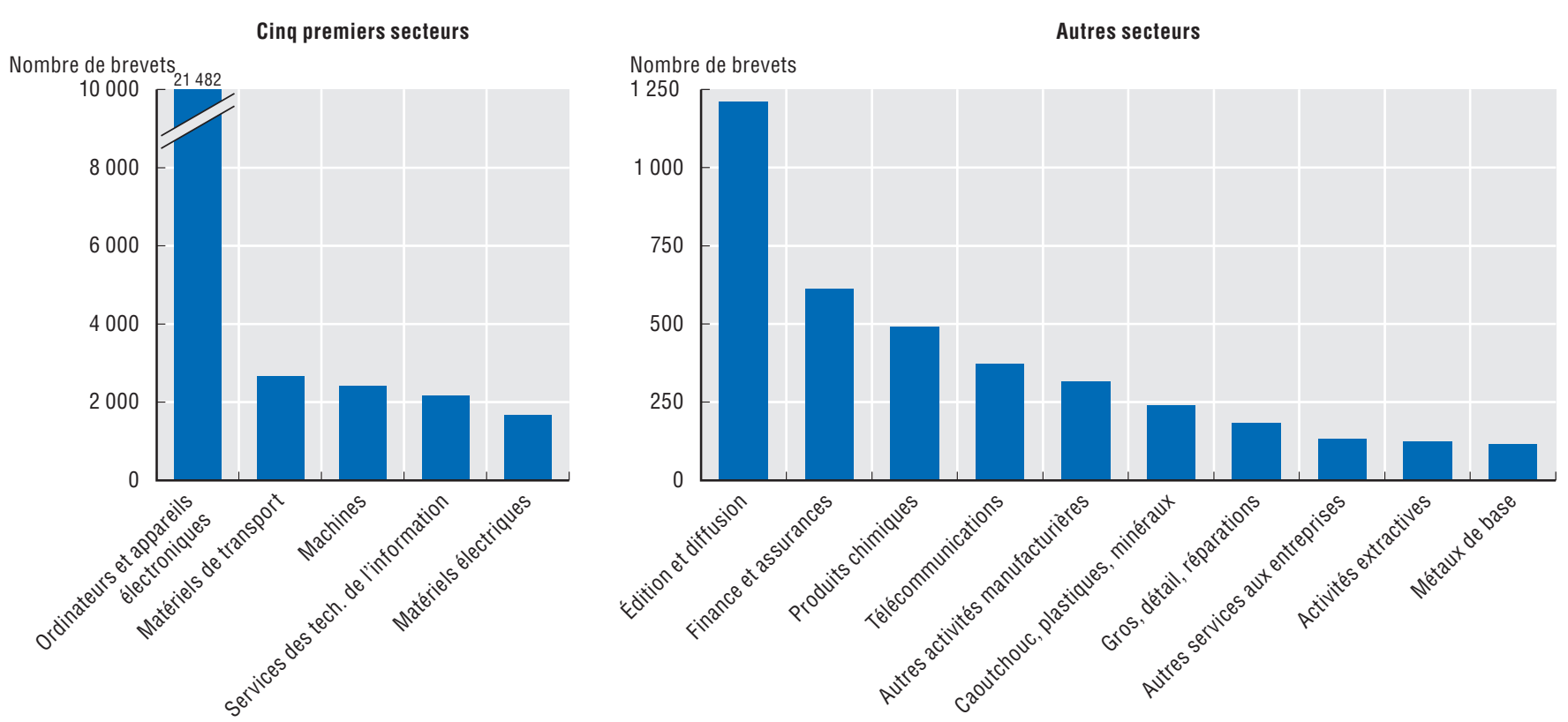

Sources : Calculs de l'OCDE d'après CCR-OCDE, Base de données COR\&DIP@ v. 1, et OCDE, STI Microdata Lab : Base de données sur la propriété intellectuelle, http://oe.cd/ipstats, juillet 2017. Voir notes de chapitre.

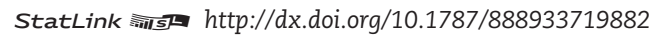

\section{Brevets liés à l’intelligence artificielle déposés par les entreprises les plus actives en R-D,} selon l'implantation du siège, 2012-14

Part des économies dans le nombre total de familles de brevets IP5 liés à l'IA détenues par les 2000 entreprises les plus actives en R-D
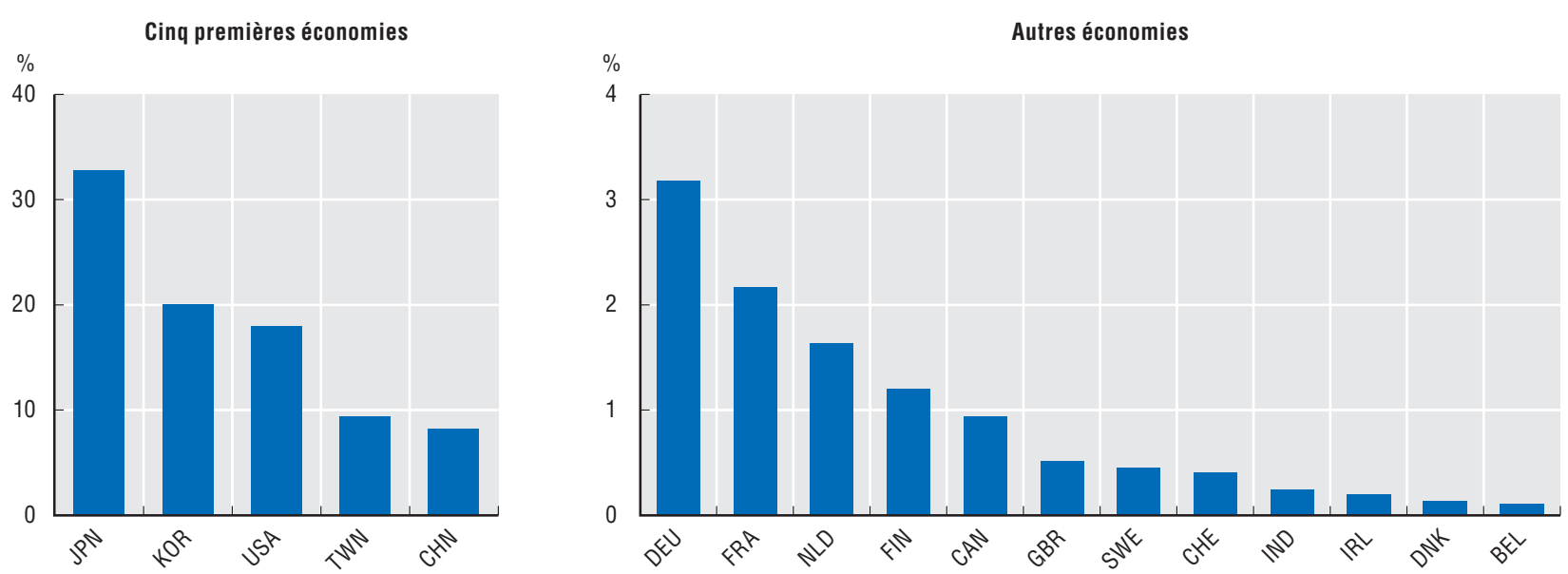

Sources : Calculs de l'OCDE d'après CCR-OCDE, Base de données COR\&DIP@ v. 1, et OCDE, STI Microdata Lab : Base de données sur la propriété intellectuelle, $h$ ttp://oe.cd/ipstats, juillet 2017. Davantage de données via StatLink. Voir notes de chapitre. 


\section{1. ÉCONOMIE DU SAVOIR ET TRANSFORMATION NUMÉRIQUE}

\section{Science, innovation et révolution numérique}

\section{Recherche dans le domaine de l'apprentissage automatique}

Depuis des décennies, la recherche dans le domaine de l'intelligence artificielle (IA) vise à permettre aux machines de simuler les fonctions cognitives humaines. Les progrès en termes de puissance de calcul et de conception des systèmes ont projeté l'IA sur le devant de la scène, avec des résultats de plus en plus semblables aux comportements humains. C'est grâce à ces avancées que l'ordinateur Deep Blue d'IBM a pu battre le champion du monde d'échecs, Garry Kasparov, en 1997, et que les machines sont désormais capables d'opérer une distinction entre des objets et du texte dans des images et des vidéos. La mise au point de techniques d'apprentissage automatique a joué un rôle déterminant dans ces évolutions. L'objectif est de développer des algorithmes informatiques qui apprennent de manière autonome à partir de données et informations disponibles. Exploitant la puissance des sources de données massives, les algorithmes sont aujourd'hui capables de traiter de problèmes plus complexes que seuls les humains pouvaient jusque-là prendre en charge. Une analyse bibliométrique expérimentale fait apparaître un accroissement notable des publications scientifiques liées à l'apprentissage automatique, en particulier au cours de la période 201415. Les États-Unis sont en tête des efforts de recherche dans ce domaine, que l'on observe le nombre total de publications ou le nombre de publications parmi les plus citées. Il convient par ailleurs de souligner la progression rapide de l'Inde, qui s'est hissée au troisième rang en termes de publication d'articles scientifiques sur l'apprentissage automatique, derrière la Chine, et pointe en quatrième position derrière le Royaume-Uni si l'on tient compte de la qualité des publications.

26. Évolution des publications scientifiques liées à l'apprentissage automatique, 2003-16

Économies présentant le nombre le plus élevé d'articles consacrés à ce sujet, comptages fractionnaires

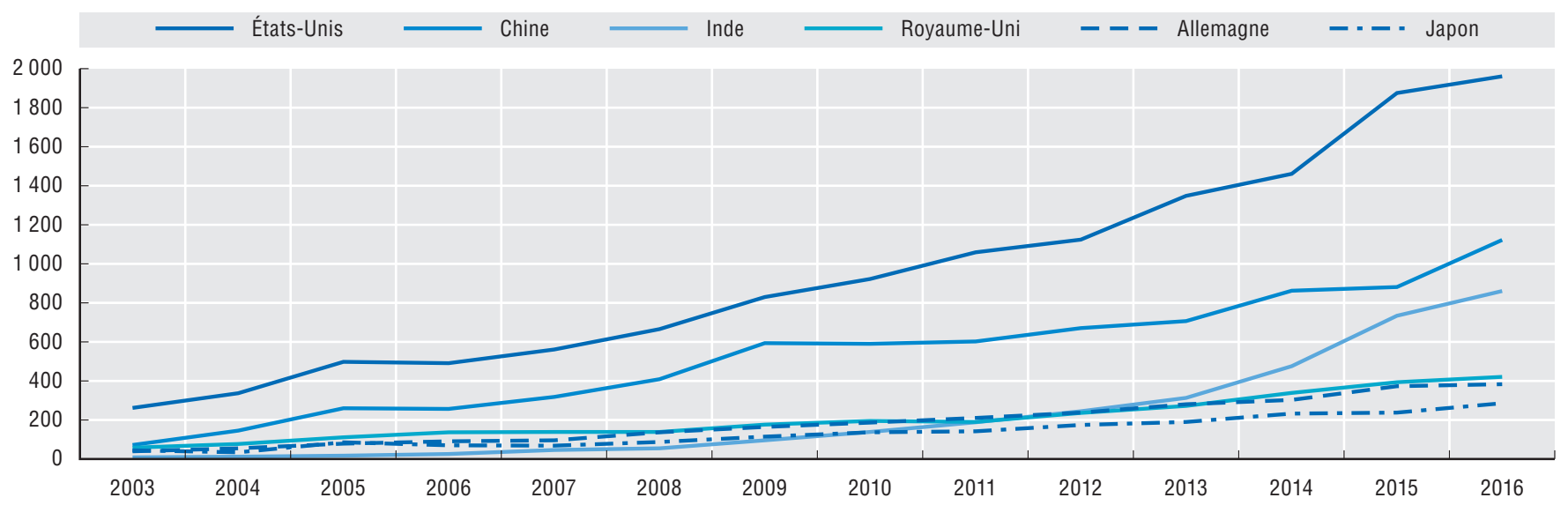

Source : Calculs de l'OCDE d'après Scopus Custom Data, Elsevier, version 4.2017, juillet 2017. Davantage de données via StatLink. Voir notes de chapitre.

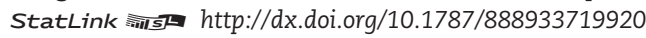

\section{Articles scientifiques sur l'apprentissage automatique parmi les publications les plus citées, 2006 et 2016}

Économies présentant le nombre le plus élevé d'articles consacrés à l'apprentissage automatique parmi les $10 \%$ de publications les plus citées, comptages fractionnaires

2016

Dix premiers pays

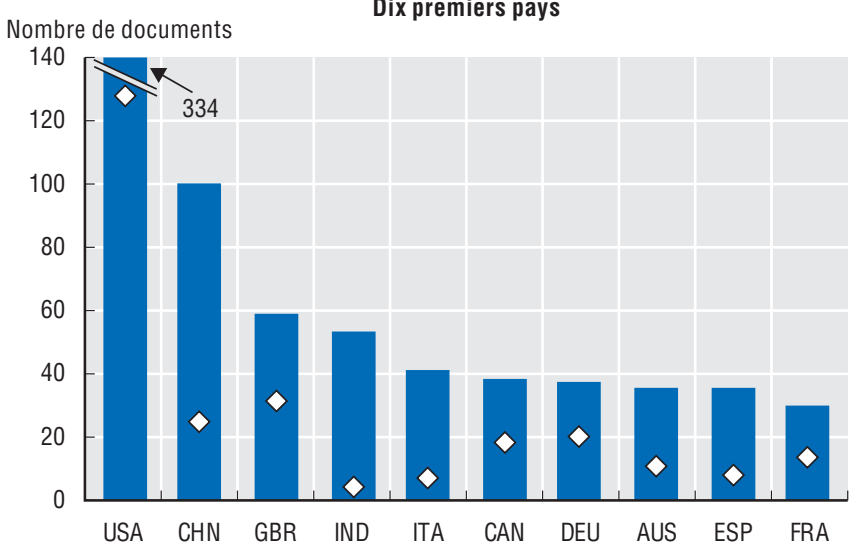

$\diamond 2006$

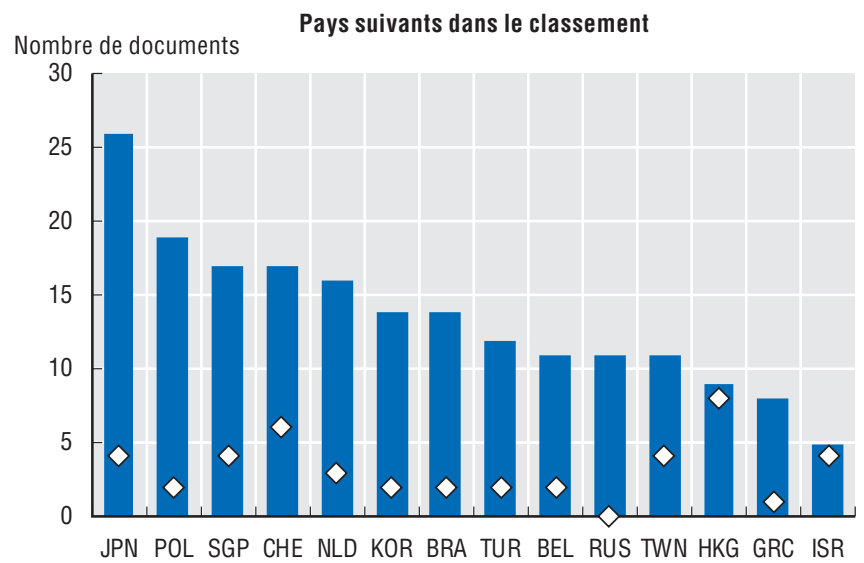

Sources : Calculs de l'OCDE, d'après Scopus Custom Data, Elsevier, version 4.2017 ; et valeurs SJR (Scimago Journal Rank) de 2015 des titres de la liste de revues Scopus (consulté en juin 2017), juillet 2017. Voir notes de chapitre. 


\section{Quelles publications scientifiques ont été identifiées comme étant liées à l'apprentissage automatique ?}

Pour procéder aux estimations expérimentales, une recherche a été menée sur l'élément de texte " *machine learn* » dans les résumés, titres et mots-clés des articles publiés (en anglais) jusqu'en 2016 et indexés dans la base Scopus. La fiabilité de la méthode dépend de l'exhaustivité de l'indexation des résumés, d'où un biais en faveur des revues anglophones. Dans le cadre d'une enquête réalisée par Kalantari et al. (2017), 142 experts des données massives ont répondu à la question suivante : "quels mots-clés utiliseriez-vous pour effectuer une recherche sur les données massives ? "; " machine learning " (apprentissage automatique) a été cité comme pertinent dans $29 \%$ des cas. Parmi la liste de mots-clés ainsi identifiée, " machine learning " a été trouvé dans $40 \%$ des 11000 documents recensés dans la base Web of Science, qui couvre la période 1980-2015; il s'agit donc du mot-clé le plus fréquent, devant « Data centre » (Centre de données), « Big data » (Données massives) et « Data warehouse » (Entrepôt de données). 


\section{1. ÉCONOMIE DU SAVOIR ET TRANSFORMATION NUMÉRIQUE}

\section{Croissance, emploi et transformation numerique}

\section{Robotisation de la production}

Les progrès en matière de données massives, d'impression 3D, de communication entre machines et de robots transforment la production. Des données comparables et représentatives portant sur le déploiement des technologies liées aux robots industriels, révèlent qu'en 2015, la Corée et le Japon étaient les champions de l'intensité robotique (mesurée par le nombre de robots industriels rapporté à la valeur ajoutée du secteur manufacturier). De fait, l'intensité robotique de ces pays est environ trois fois plus élevée que la moyenne de l'OCDE. Certaines économies d'Europe de l'Est utilisent également de manière intensive la robotique industrielle ; cette tendance pourrait refléter leur degré de spécialisation au sein des chaînes de valeur manufacturières et leur rôle éventuel en tant que fournisseurs des grandes entreprises multinationales. Depuis 2005, l'intensité robotique en Hongrie, en République slovaque, en République tchèque et en Slovénie a augmenté de trois à six fois, affichant un taux de croissance nettement supérieur au taux moyen observé pour les pays de l'OCDE ou ceux de la zone UE28 (respectivement de $29 \%$ et $54 \%$ ). L'intensité robotique a aussi progressé dans les BRICS, à un niveau relativement faible par rapport à celui des pays de l'OCDE. La Chine, notamment, a vu sa part passer de $23 \%$ à 88 \% du niveau observé aux États-Unis. Ces chiffres doivent toutefois être interprétés avec prudence, dans la mesure où les indicateurs se fondent sur le nombre de robots opérationnels dans une économie à un moment donné et ne reflètent ni l'évolution de l'efficacité, ni celle de la qualité des robots dans le temps.

\section{8. Économies et BRICS les plus robotisés, 2005 et 2015}

Parc de robots industriels rapporté à la valeur ajoutée du secteur manufacturier, en millions USD, valeurs courantes

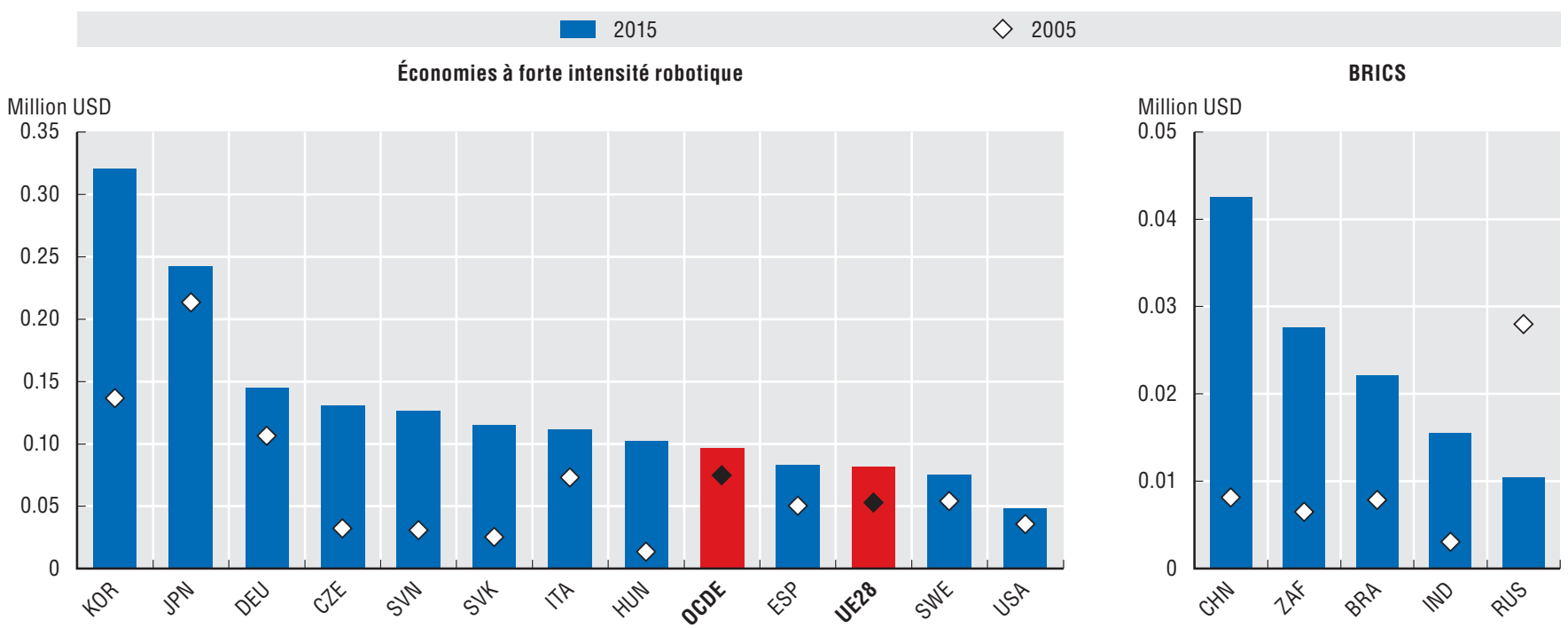

Sources : Calculs de l'OCDE d'après les données de la Fédération internationale de la robotique, et Banque mondiale, Indicateurs du développement dans le monde (base de données), septembre 2017. Davantage de données via StatLink. Voir notes de chapitre.

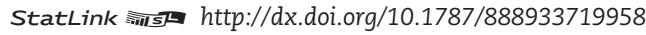

\section{Qu'est-ce qu'un robot industriel ?}

L'ISO 8373:2012 définit le robot industriel comme suit : « manipulateur multi-application reprogrammable commandé automatiquement, programmable sur trois axes ou plus, qui peut être fixé sur place ou mobile, destiné à être utilisé dans des applications d'automatisation industrielle ». La Fédération internationale de la robotique (IFR) collecte des informations sur le nombre de robots industriels livrés par la quasi-totalité des fournisseurs de robots à l'échelle mondiale. Le parc robotique présenté ci-dessus a été évalué en additionnant la valeur du stock la première année, publiée par l'IFR, et les ventes de robots au cours des années suivantes, en tenant compte d'un taux d'amortissement annuel de $10 \%$. Ces chiffres ne reflètent donc pas la progression de la valeur des robots, ni leur capacité d'exécution des tâches (pour les robots, il n'existe pas de calcul équivalent à celui de la puissance des moteurs des véhicules automobiles, mesurée en " cheval »). En outre, seules sont considérées les activités de fabrication, d'extraction, de construction et de distribution d'électricité, de gaz et d'eau ; de fait, les données de l'IFR, dont dispose l'OCDE, ne tiennent pas compte des robots utilisés dans les activités de services autres que celles du secteur de la R-D. 


\section{Robotisation de la production}

Outre leur rôle de catalyseur de croissance, les innovations technologiques peuvent induire des bouleversements profonds dans de nombreux domaines, dont la productivité, l'emploi et le bien-être. D'aucuns craignent qu'avoir massivement recours aux robots n'occasionne d'importantes pertes d'emplois, notamment lorsque l'on fait appel à des robots industriels conçus pour mener à bien des tâches traditionnellement exécutées par des hommes. Des études récentes révèlent que les robots contribuent à améliorer la productivité, bien que leur impact sur l'emploi et les salaires soit ambigu. En effet, le graphique ci-dessous montre que faire usage de la robotique peut optimiser l'utilisation d'autres technologies, car s'ils n'entrent pas dans la catégorie des outils TIC, les robots dépendent néanmoins des TIC (e.g. les logiciels). Les travailleurs devront donc disposer de compétences en TIC pour les faire fonctionner. La corrélation entre l'utilisation des TIC au travail et l'intensité robotique s'avère positive, bien que modérée, et dénote une complémentarité des investissements dans le capital technologique et le capital humain ; investissements nécessaires pour mettre en œuvre les procédés de mutations industrielles. Dans la mesure où ces données ont trait à l'utilisation des robots dans la production, les économies dont le secteur manufacturier est relativement développé affichent, en toute logique, une intensité robotique moyenne plus élevée. Certaines, à l'instar du Japon, de la Corée, de l'Allemagne et des États-Unis, présentent une intensité supérieure à la moyenne à la fois en matière de densité robotique et d'utilisation des TIC. À l'inverse, celles où les services sont relativement plus prépondérants (telles le Royaume-Uni, l'Irlande et les Pays-Bas) se caractérisent par une intensité en TIC supérieure à la moyenne, mais une densité robotique inférieure à la moyenne.

29. Intensité robotique et intensité des tâches liées aux TIC dans les emplois manufacturiers, 2012 ou 2015 Corrélation entre la densité robotique et l'intensité moyenne des tâches liées aux TIC

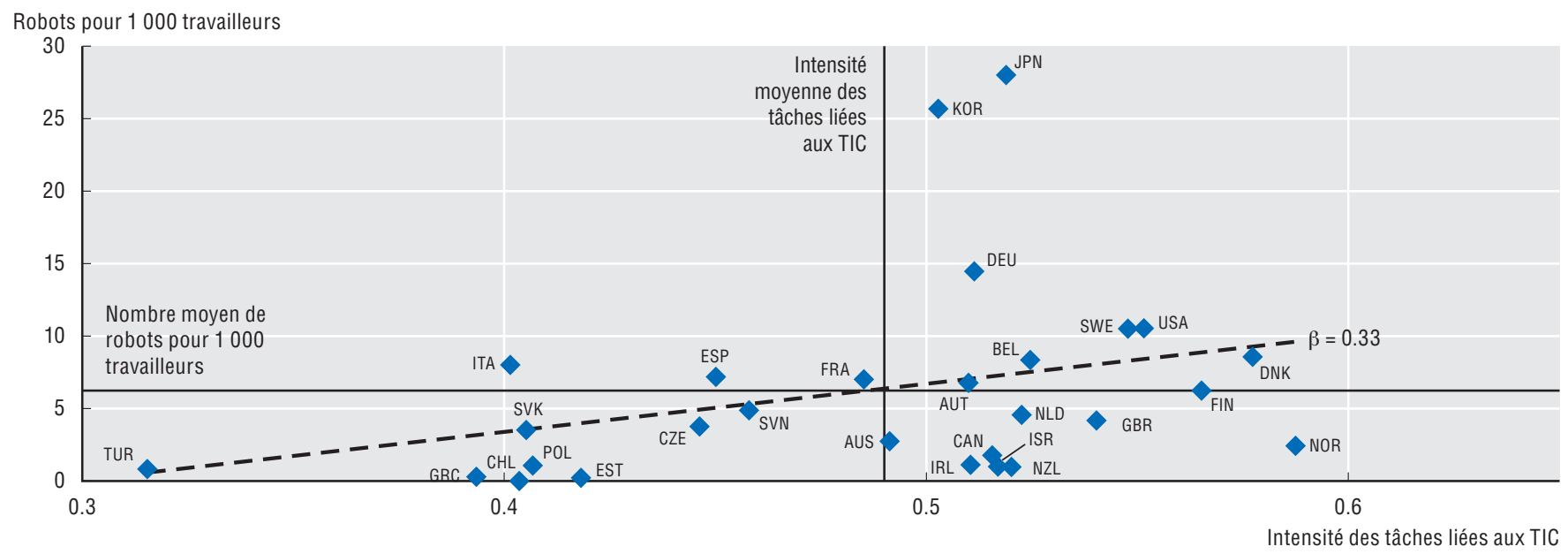

Source: Calculs de l'OCDE d'après la Base de données du Programme pour l'évaluation internationale des compétences des adultes (PIAAC) et la Fédération internationale de la robotique, septembre 2017. Voir notes de chapitre.

StatLink तiाls http://dx.doi.org/10.1787/888933719977

\section{Comment mesurer la composante TIC ?}

Récemment, une étude de l'OCDE a permis d'établir un indicateur de l'intensité d'utilisation des TIC selon les professions (Grundke et al., 2017), en s'appuyant sur les informations du Programme pour l'évaluation internationale des compétences des adultes (PIAAC). Cette approche, comparée aux études précédentes, permet de distinguer les compétences des travailleurs des tâches qu'ils exécutent dans le cadre de leur travail. L'indicateur reflète l'étendue des caractéristiques des tâches, allant de la simple consultation de l'internet à l'utilisation des logiciels Word ou Excel, ou d'un langage de programmation. Certains pays, présentés dans le graphique 28, n'apparaissent pas dans le graphique 29 car ils ne figurent pas dans la base de données du PIAAC ; l'intensité des tâches liées aux TIC n'est donc pas mesurée pour ces pays. 


\section{1. ÉCONOMIE DU SAVOIR ET TRANSFORMATION NUMÉRIQUE}

\section{Croissance, emploi et transformation numerique}

\section{Maturité des secteurs à l'égard du numérique}

Les technologies numériques, devenues omniprésentes, transforment en profondeur les économies et les sociétés. La digitalisation bouleverse, d'innombrables façons, les activités de production et de services, d'où la difficulté de donner une définition exhaustive de ce phénomène multidimensionnel. Les travaux récemment menés par l'OCDE visent à évaluer le contenu numérique des secteurs, en examinant la montée en puissance du digital sous différents angles : le volet technologique (investissements TIC matériels et immatériels, achats de biens et de services TIC intermédiaires, utilisation de robots), le capital humain nécessaire pour intégrer la technologie à la production (intensité de spécialistes des TIC), et les changements qu'il induit dans la façon dont les entreprises interagissent avec le marché (ventes sur l'internet). Si la transformation numérique touche progressivement tous les secteurs de l'économie, ses modalités et son ampleur ne sont en revanche pas uniformes, et certains secteurs se démarquent dans différents domaines. Ainsi, le secteur " Activités informatiques et services d'information " se classe dans le trio de tête pour tous les indicateurs pour lesquels les données sont disponibles, à l'exception des ventes sur l'internet. En revanche, son équivalent manufacturier, soit le secteur " Fabrication d'ordinateurs, d'articles électroniques et optiques ", ne se distingue pas des autres secteurs.

\section{Dispersion des secteurs pour chaque dimension du numérique étudiée, 2013-15}

Moyennes des valeurs des pays et des années confondus, normalisées entre les secteurs

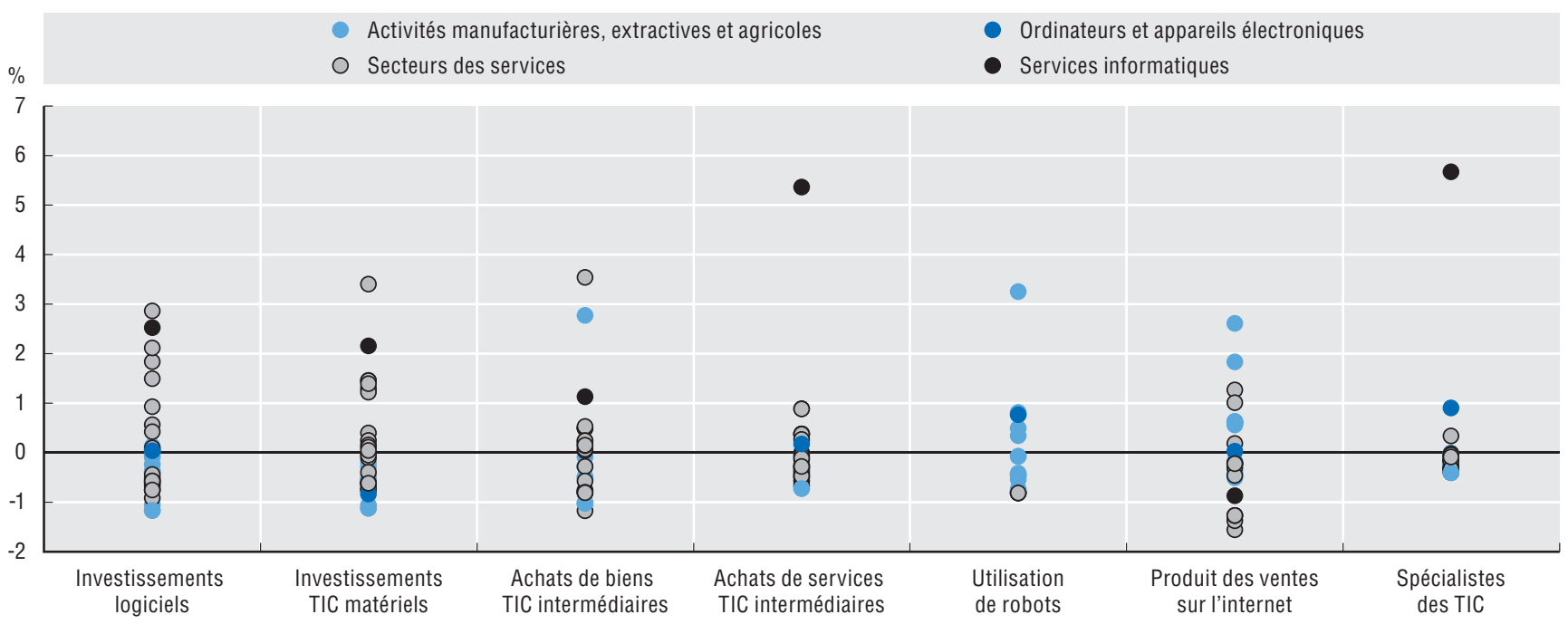

Source : Calculs de l'OCDE d'après les données des Comptes nationaux annuels, de la Base de données STAN, des TIES, du PIAAC, de la Fédération internationale de la robotique, de la Banque mondiale, de la Base de données Économie et société numériques d'Eurostat, des enquêtes nationales sur la population active, de l'enquête US CPS, d'INTAN-Invest et d'autres sources nationales. Voir notes de chapitre.

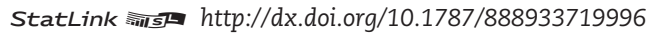

\section{Comment lire ce graphique}

Chaque point représente un secteur. Pour chaque indicateur, la valeur sectorielle est obtenue en calculant la moyenne des valeurs pour toutes les années et tous les pays considérés. Sur le graphique, les valeurs représentées sont normalisées par secteurs, de sorte que le secteur moyen correspond à la valeur zéro. Les points bleus correspondent aux secteurs manufacturiers, et les gris, aux secteurs des services. Les points bleu foncé et gris foncé représentent respectivement les industries manufacturières TIC et le secteur des services TIC.

\section{Mesurer la transformation numérique dans les secteurs : une approche multidimensionnelle}

Dans le cadre de travaux récents, l'OCDE (Calvino et al., à paraître) a mis au point une taxonomie des secteurs selon leur contenu numérique, en tenant compte d'une multiplicité de dimensions. Tous les indicateurs sont exprimés en intensités sectorielles. Les investissements des types "logiciels » et " TIC matériels " correspondent au rapport entre, d'une part, la formation brute de capital fixe (FBCF) dans les logiciels et les TIC et, d'autre part, la FBCF totale, toutes deux exprimées en volumes. Les achats de biens et de services intermédiaires correspondent au rapport entre les biens ou services TIC intermédiaires achetés par le secteur et la production du secteur acquéreur, exprimés en termes réels. Les valeurs sur l'utilisation des robots correspondent au quotient du parc de robots et de l'emploi dans le secteur. Le produit des ventes sur l'internet reflète la part du chiffre d'affaires issue des ventes en ligne. La catégorie "Spécialistes des TIC » reflète la part de ces professionnels dans l'emploi total. La taxonomie s'appuie sur les informations relatives aux pays suivants : Australie, Autriche, Danemark, États-Unis, Finlande, France, Italie, Japon, Norvège, Pays-Bas, Royaume-Uni et Suède, pour lesquels les valeurs sont disponibles pour l'ensemble des indicateurs, des secteurs et des années considérés ; font exception les indicateurs " Utilisation de robots » et « Produit des ventes sur l'internet » qui n'intègrent pas certains secteurs. 


\section{Maturité des secteurs à l'égard du numérique}

Les secteurs génèrent, adoptent et utilisent les technologies à des rythmes divers, et font appel à différents types de compétences dans des proportions inégales. Ce constat vaut pour la vaste palette de technologies et de compétences qui façonnent la transformation numérique des économies et des sociétés. L'analyse de l'OCDE révèle que certains secteurs d'activité n'ont pas franchi le cap du numérique au même rythme que d'autres, et ce, quel que soit le type d'indicateurs utilisé pour mesurer la mutation à l'œuvre. L'agriculture, les activités extractives et l'immobilier figurent dans le quartile inférieur de l'intensité numérique pour tous les indicateurs disponibles. À l'inverse, les télécommunications et les services informatiques arrivent systématiquement en tête du peloton. Les secteurs qui figurent au milieu du classement général affichent généralement une forte hétérogénéité, ce qui tend à montrer qu'ils sont engagés dans cette transition numérique à des degrés divers, selon les aspects considérés.

\section{Taxonomie des secteurs par quartile d'intensité numérique, 2013-15}

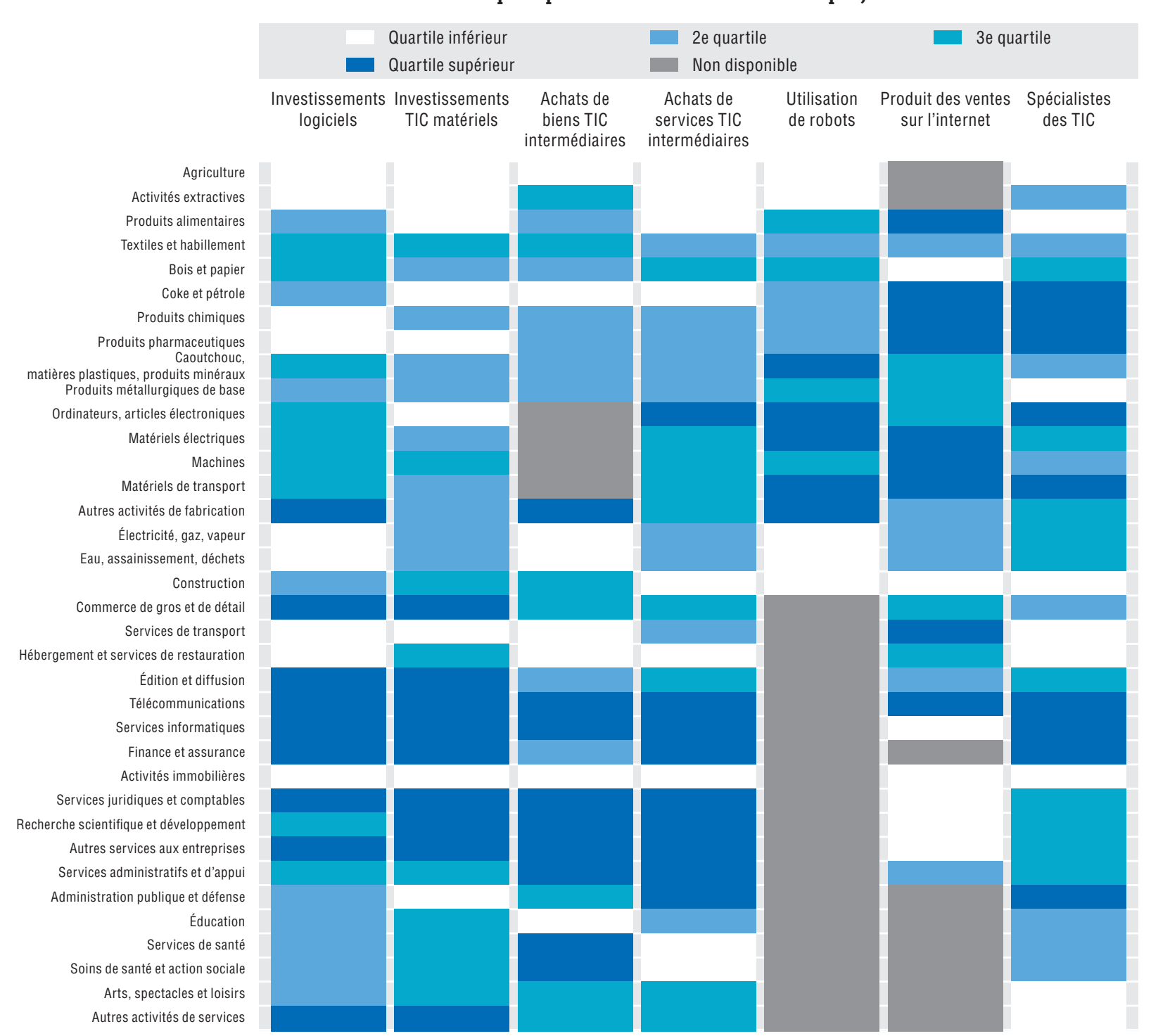

Source : Calculs de l'OCDE d'après les données des Comptes nationaux annuels, de la Base de données STAN, des TIES, du PIAAC, de la Fédération internationale de la robotique, de la Banque mondiale, de la Base de données Économie et société numériques d'Eurostat, des enquêtes nationales sur la population active, de l'enquête US CPS, d'INTAN-Invest et d'autres sources nationales. Davantage de données via StatLink. Voir notes de chapitre.

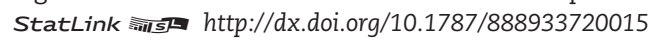

\section{Comment lire ce graphique}

Pour chaque indicateur, les secteurs sont classés selon leur valeur, qui correspond à la moyenne des valeurs des pays et des années. Les secteurs présentant la plus forte intensité numérique (quartile supérieur) apparaissent en bleu foncé, tandis que ceux qui affichent la plus faible intensité apparaissent en blanc. On ne dispose pas de données relatives à l'utilisation des robots pour les services autres que la distribution d'électricité, de gaz et de vapeur, et la construction (soit tous les services au-delà de la division 43 de la CITI rév. 4); pour ce qui est des ventes sur l'internet, on ne dispose pas de données pour l'agriculture (divisions 01-03), les activités extractives (05-09), les activités financières (64-66) et tous les secteurs au-delà de la division 84 de la CITI. Les achats de biens TIC intermédiaires, dans les secteurs liés à la fabrication de machines, ne sont pas pris en considération, afin d'éviter toute erreur de mesure. 


\section{1. ÉCONOMIE DU SAVOIR ET TRANSFORMATION NUMÉRIQUE}

\section{Croissance, emploi et transformation numerique}

\section{Quelles compétences pour le monde numérique de demain?}

Le numérique transforme les emplois et les marchés du travail dans les pays de l'OCDE et au-delà. Sous l'effet de l'automatisation, certains postes disparaissent, quand d'autres voient évoluer leur nature et les tâches qui leur sont inhérentes ; enfin, l'avènement de technologies comme l'intelligence artificielle (IA), l'internet des objets (IdO) ou l'analytique des données massives débouchera sur de nouveaux métiers. Les salariés des secteurs les plus exposés à la transformation numérique aujourd'hui, sont dotés de plus fortes compétences cognitives, non cognitives et sociales. À mesure que la transformation numérique progresse vers d'autres pans de l'économie jusqu'alors plutôt épargnés, la main-d'œuvre devra disposer de solides compétences cognitives et sociales en adéquation avec les besoins futurs.

32. Niveaux de compétences dans les secteurs à plus ou moins forte intensité numérique, 2012 ou 2015 Moyenne des différents pays

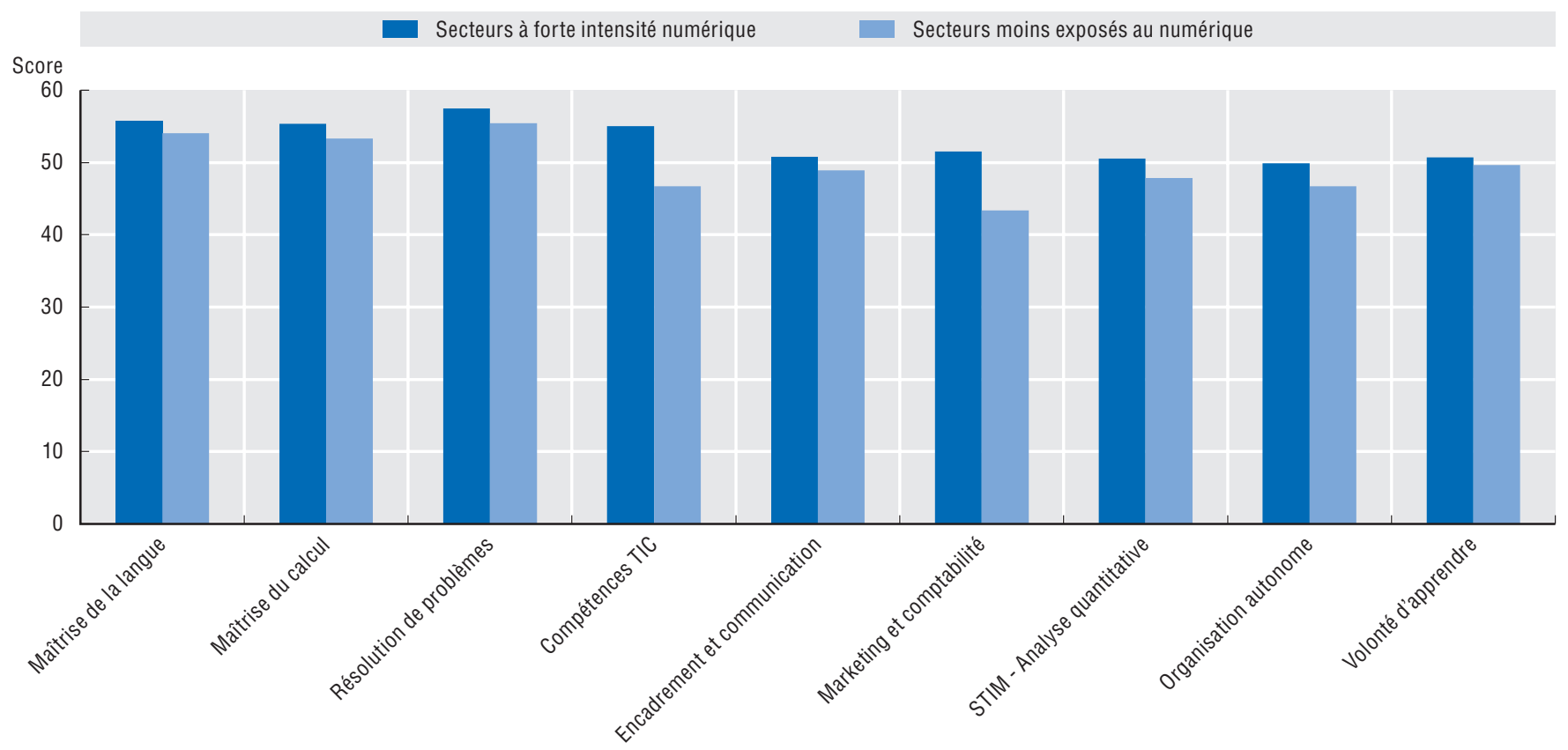

Source : Calculs de l'OCDE d'après la Base de données du Programme pour l'évaluation internationale des compétences des adultes (PIAAC), juin 2017. Voir notes de chapitre.

StatLink न्ताइ http://dx.doi.org/10.1787/888933720034

\section{Cartographier les compétences et identifier les secteurs à forte intensité numérique}

Cette analyse couvre les compétences cognitives et non cognitives, c'est-à-dire les compétences qui, en règle générale, ne sont acquises que partiellement à l'école et ont trait à l'attitude et à la personnalité des individus. La mesure des compétences non cognitives et des compétences sociales se fonde sur les informations relatives aux tâches que les travailleurs exécutent dans le cadre de leurs fonctions, tirées du Programme de l'OCDE pour l'évaluation internationale des compétences des adultes (PIAAC). L'exercice de cartographie a permis d'identifier six groupes de compétences fonctionnelles liées aux performances professionnelles et aux résultats économiques, à savoir : les compétences liées aux technologies de l'information et des communications (TIC) ; les compétences en sciences, technologies, ingénierie et mathématiques (STIM) et en analyse quantitative ; les compétences non cognitives telles que l'encadrement, la communication et l'organisation autonome; et les compétences socio-émotionnelles comme la volonté d'apprendre et la résolution créative de problèmes (voir Grundke et al., 2017).

Les secteurs à forte intensité numérique, et ceux qui y sont moins exposés, ont été identifiés en procédant à une analyse comparative sous différents angles : le rapport entre la formation brute de capital fixe (FBCF) dans les TIC et les logiciels, et la FBCF totale; le rapport entre les biens ou services TIC intermédiaires achetés par le secteur et la production du secteur acquéreur ; la densité robotique ; la part du chiffre d'affaires du secteur issue des ventes sur l'internet; et la part des spécialistes des TiC dans l'emploi total, par secteur (pour en savoir plus, voir Calvino et al., à paraître). 


\section{1. ÉCONOMIE DU SAVOIR ET TRANSFORMATION NUMÉRIQUE}

2. Groissance, emploi et transformation numerique

\section{Quelles compétences pour le monde numérique de demain?}

Pour identifier les compétences nécessaires à la transformation digitale, il faut pouvoir appréhender quelles qualifications font défaut (et/ou sont particulièrement demandées) et appellent une majoration salariale dans les secteurs plus ou moins exposés au numérique.

Un certain nombre de compétences ont une incidence notable sur la performance des entreprises. De fait, les secteurs à forte intensité numérique ont de meilleurs rendements du marché du travail que les autres secteurs. Par ailleurs, certaines compétences semblent jouer un rôle particulièrement important dans les activités à forte intensité numérique, en particulier celles ayant trait à l'analyse quantitative, à l'utilisation des TIC, à la maîtrise du calcul, au domaine des STIM, ainsi qu'à l'organisation autonome, aux qualités d'encadrement, et à la communication. Ce phénomène s'expliquerait par le fait que les personnes exerçant dans ces domaines travaillent de façon plus indépendante et décentralisée (e.g. en télétravail), exécutent une proportion relativement plus élevée de tâches non routinières, ou sont confrontées à des environnements en constante évolution, dans lesquels les compétences techniques, alliées aux qualités de communication et d'organisation, gagnent en puissance.

\section{Rendements additionnels des compétences sur le marché du travail dans les secteurs à forte intensité numérique,} 2012 ou 2015

Évolution (en pourcentage) de la rémunération horaire pour une augmentation d'un écart type des compétences

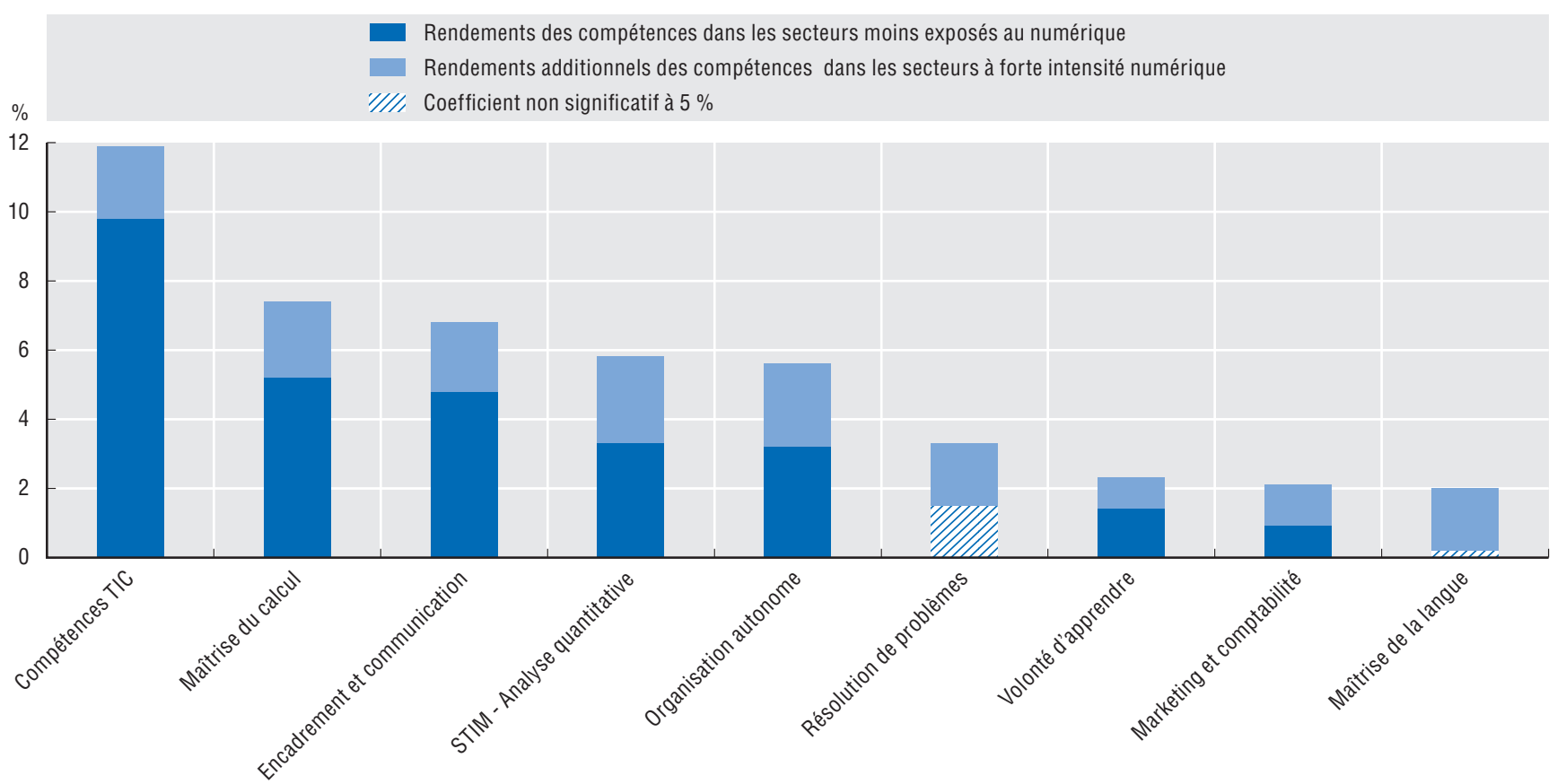

Source : Calculs de l'OCDE d'après la Base de données du Programme pour l'évaluation internationale des compétences des adultes (PIAAC), juin 2017. Voir notes de chapitre. 


\section{1. ÉCONOMIE DU SAVOIR ET TRANSFORMATION NUMÉRIQUE}

\section{Croissance, emploi et transformation numerique}

\section{Emploi : des gagnants et des perdants}

Entre 2010 et 2015, l'emploi total a progressé de 4.9 \% dans la zone OCDE (soit un gain net d'environ 27 millions d'emplois). Cette hausse s'observe principalement dans les pays hors UE - un gain net de 12.9 millions de postes est enregistré dans la seule zone de l'ALENA, contre seulement 3.6 millions dans l'Union européenne. La plupart des créations nettes d'emplois se concentrent dans les secteurs des services des pays de l'OCDE (en hausse de 24.8 millions) ; à cela s'ajoutent 2 millions de postes dans les activités manufacturières. En 2016, l'emploi dans l'Union européenne a solidement progressé pour la troisième année consécutive, portant à 6.4 millions le nombre de créations nettes pour la période 2010-16, dont une hausse notable de 3.9 millions d'emplois concerne les Activités professionnelles, scientifiques et techniques, et les Autres services aux entreprises. Derrière ces chiffres se cachent toutefois d'importantes disparités. De fait, l'Allemagne et le Royaume-Uni affichent tous deux des gains nets de quelque 2.5 millions d'emplois, tandis que l'Espagne, la Grèce et le Portugal peinent à retrouver leurs niveaux d'avant la crise et subissent une perte nette collective s'élevant à 1.5 million de postes au cours de la période considérée.

\section{Géographie des pertes et des créations d'emplois, 2010-16}

Contribution relative à l'évolution de l'emploi total, par grand secteur d'activité économique
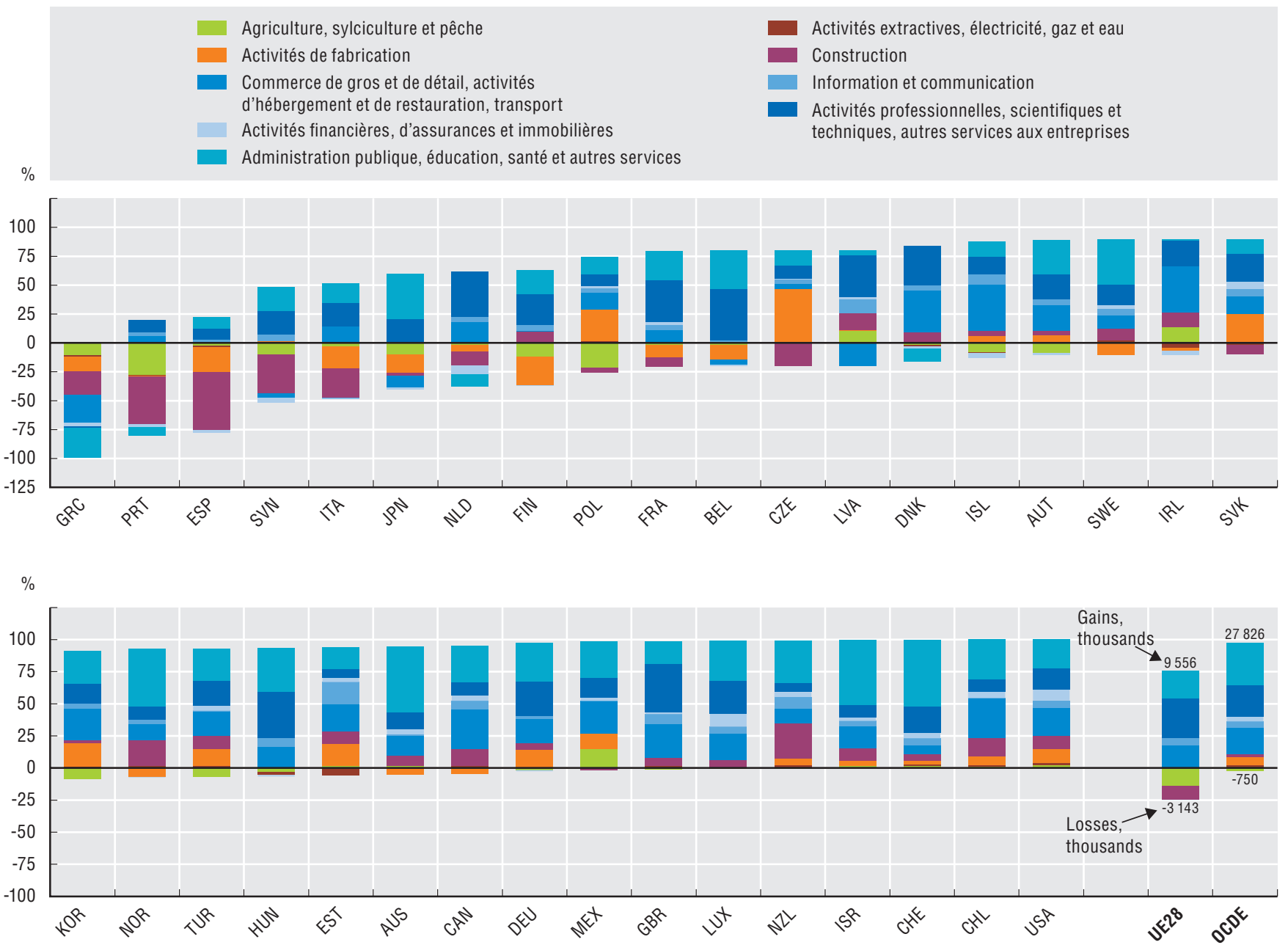

Note : Les données pour la Corée, Israël, le Japon, le Mexique, la Nouvelle-Zélande et l'agrégat de la zone OCDE se rapportent à la période 2010-15.

Source : Calculs de l'OCDE d'après la Base de données des Comptes nationaux annuels, http://www.oecd.org/fr/std/cn, la Base de données pour l'analyse structurelle (STAN), http://oe.cd/stan-fr, et des sources nationales, septembre 2017. Voir notes de chapitre.

StatLink 제패 http://dx.doi.org/10.1787/888933720072

\section{Comment lire ces graphiques}

On peut " normaliser » les variations de l'emploi par activité économique afin de mettre en évidence la contribution relative de chacune d'elles à la variation totale de l'emploi entre deux périodes, dans chaque pays. Pour ce faire, les variations sectorielles sont exprimées, pour chaque pays, en pourcentage de la somme des variations absolues. Les groupes d'activité sont définis suivant les divisions de la CITI rév. 4. Les créations et les pertes d'emplois correspondent respectivement à la somme des secteurs dans lesquels les variations sont positives et à la somme des secteurs présentant des variations négatives. Le recours à une ventilation plus fine des activités (en se fondant par exemple sur les codes de divisions à deux chiffres de la CITI rév. 4) donnerait lieu à des estimations différentes du nombre total de créations et de pertes d'emplois ; la variation nette totale resterait toutefois inchangée. 


\section{1. ÉCONOMIE DU SAVOIR ET TRANSFORMATION NUMÉRIQUE}

2. Croissance, emploi et transformation numerique

\section{Emploi : des gagnants et des perdants}

Les secteurs de l'information sont réputés être d'importants leviers de croissance dans la zone OCDE, bien qu'ils ne représentent que $5.5 \%$ de l'emploi du secteur des entreprises de cette zone. Entre 1997 et 2015, l'emploi lié aux activités de l'information a progressé de 18 \% à l'échelle de la zone, soit une croissance plus rapide que celle de l'emploi dans le secteur des entreprises (13\%) au cours de la même période. Ce taux est toutefois sujet à une volatilité conjoncturelle relativement importante depuis 1997. À titre d'exemple, après la crise financière, il a enregistré, dans la zone OCDE, un recul de $4.2 \%$ entre 2008 et 2010, conduisant à la perte de 800000 emplois. Les États-Unis, après avoir atteint environ $35 \%$ avant 2001, représentent aujourd'hui quelque $30 \%$ de l'emploi de l'OCDE dans les secteurs de l'information, et demeurent un moteur puissant de l'évolution de la main-d'œuvre dans ces secteurs. Les services d'information dominent en matière de créations de postes, tandis que sur la dernière décennie, le secteur manufacturier des TIC a enregistré, des pertes dans bon nombre de pays de l'OCDE, y compris aux États-Unis.

\section{Croissance de l'emploi dans les secteurs de l'information, OCDE, 1997-2015}

Variation annuelle en pourcentage et en milliers d'individus

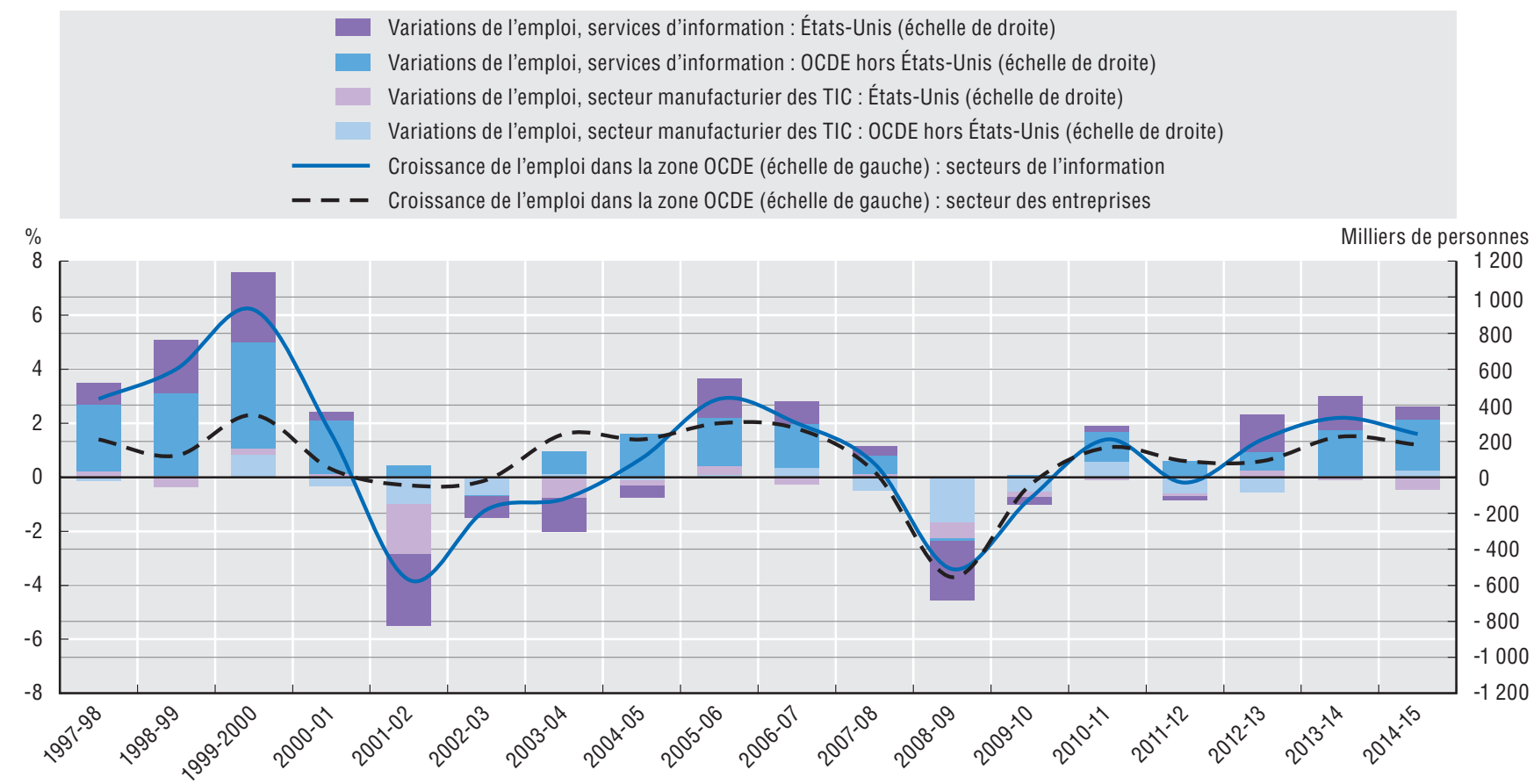

Source : Calculs de l'OCDE d'après la Base de données des Comptes nationaux annuels, www.oecd.org/fr/std/cn, la Base de données pour l'analyse structurelle (STAN), http://oe.cd/stan-fr, et des sources nationales, juin 2017. Davantage de données via StatLink. Voir notes de chapitre.

StatLink 젶ㄴ $h$ ttp://dx.doi.org/10.1787/888933720091

\section{Définition des secteurs de l'information}

Les « secteurs de l'information » sont définis d'après la classification CITI rév. 4 ; ils englobent la fabrication d'équipements TIC (division 26 : Fabrication d'ordinateurs, d'articles électroniques et optiques), les services d'information (divisions 58 à 60 : Activités d'édition, audiovisuel et activités de diffusion), ainsi que la division 61 (Télécommunications) et les divisions 62 et 63 (Activités informatiques et autres services d'information). Cette définition couvre donc à la fois le secteur des TIC et le secteur des contenus et médias, comme défini dans OCDE (2011). Le secteur des entreprises englobe pour sa part les divisions 05 à 66 et 69 à 82 de la CITI rév. 4, soit l'ensemble de l'économie à l'exception des divisions suivantes: Agriculture, sylviculture et pêche (divisions 01 à 03), Activités immobilières (68), Administration publique (84), Éducation (85), Santé et activités d'action sociale (86 à 88) et Arts, spectacles et loisirs, Activités de réparation d'articles ménagers et Autres activités de services personnels (90 à 99).

Les données sur l'emploi proviennent essentiellement des comptes nationaux et sont exprimées en nombre de personnes, sauf pour le Canada, le Japon et le Mexique, lesquels utilisent le nombre de postes comme unité de mesure. Il conviendra donc de faire attention lorsque l'on comparera l'évolution de l'emploi structurel dans ces trois pays à la situation d'autres pays. 


\section{1. ÉCONOMIE DU SAVOIR ET TRANSFORMATION NUMÉRIQUE}

\section{Croissance, emploi et transformation numerique}

\section{L'emploi des uns dépend de la demande de produits des autres}

Le renforcement de l'intégration économique et politique à l'échelle mondiale rend l'emploi national ou régional de plus en plus tributaire de la demande d'autres pays ou régions. Les Tableaux internationaux des entrées-sorties (TIES) de l'OCDE permettent de décomposer les variations annuelles de l'emploi dans la zone OCDE, afin de rendre compte des fluctuations de la demande finale de biens et de services dans différents pays et régions. Par exemple, entre 2009 et 2013, l'emploi dans le secteur des entreprises de l'OCDE a globalement augmenté de 9.2 millions, mais ce résultat n'est qu'apparence car il dissimule la perte de quelque 10 millions de postes, imputable à une réduction de la demande dans l’Union européenne, largement compensée par la création d'environ 19.2 millions d'emplois destinés à satisfaire la demande émanant des économies hors UE. En 2014, la demande des pays de l'UE avait suffisamment progressé pour induire un effet positif sur les emplois du secteur des entreprises de la zone OCDE. Au cours des dernières années, la fluctuation de la demande dans les économies partenaires a exercé une influence croissante sur l'évolution de l'emploi dans les pays de l'OCDE.

\section{Origine de la demande soutenant les emplois dans le secteur des entreprises, zone OCDE, 1995-2014}

En millions d'individus, variations annuelles par région d'origine de la demande

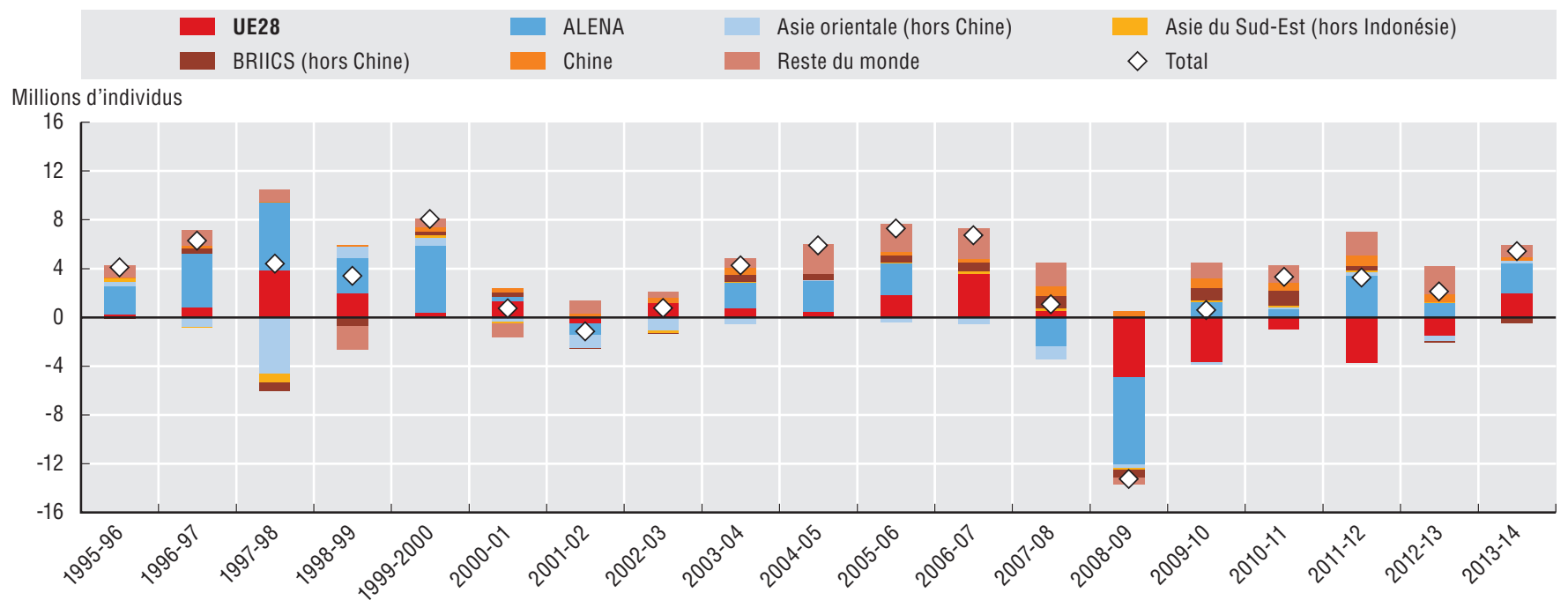

Source : Calculs de l'OCDE d'après les Tableaux internationaux des entrées-sorties (TIES), la Base de données pour l'analyse structurelle (STAN), la Base de données des Comptes nationaux annuels, la Base de données sur le contenu en emploi des échanges, et des sources nationales, juin 2017. Voir notes de chapitre.

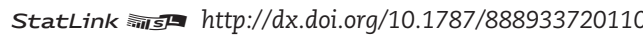

37. Origine de la demande soutenant les emplois dans les secteurs de l'information, zone OCDE, 1995-2014 En millions d'individus, variations annuelles par région d'origine de la demande

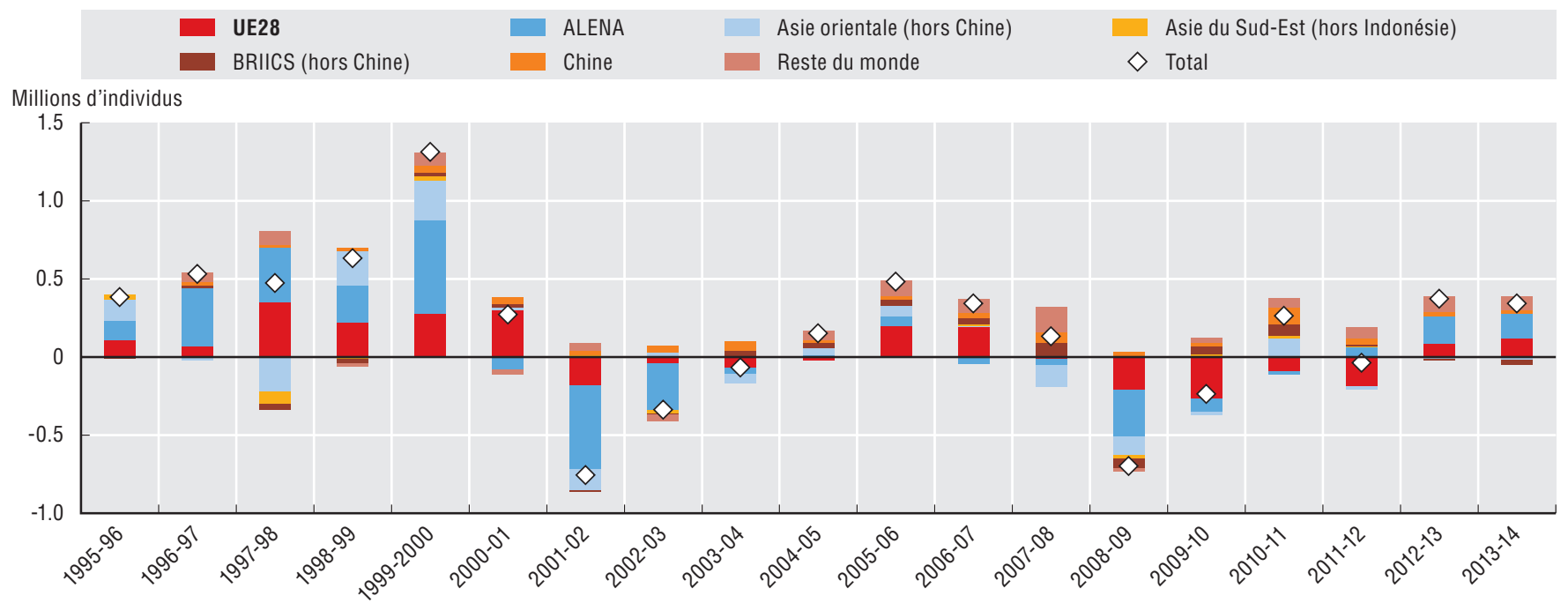

Source : Calculs de l'OCDE d'après les Tableaux internationaux des entrées-sorties (TIES), la Base de données pour l'analyse structurelle (STAN), la Base de données des Comptes nationaux annuels, la Base de données sur le contenu en emploi des échanges, et des sources nationales, juin 2017. Voir notes de chapitre. 


\section{L'emploi des uns dépend de la demande de produits des autres}

L'intégration croissante aux chaînes de valeur mondiales n'est pas sans conséquences sur la demande de compétences dans les pays. On estime qu'en 2014, aux États-Unis, les travailleurs très qualifiés représentaient 38 \% des quelque 13 millions d'employés du secteur des entreprises dont les activités productives étaient destinées à satisfaire la demande finale extérieure. Même constat dans les 22 pays de l'UE membres de l'OCDE, où les professions hautement qualifiées représentaient $36 \%$ des 54 millions de salariés travaillant pour répondre à la demande extérieure ; demande qui, en majorité, émanait d'autres pays de la zone. Toutefois on constate des disparités selon les pays de l'UE, avec des taux variant d'environ $25 \%$ en Grèce et en République slovaque, à plus de $40 \%$ dans les pays où le secteur des services occupe une place prépondérante, à l'instar du Luxembourg (56 \%), du Royaume-Uni (47 \%), de la Suède (46 \%), de la Finlande (43\%) et de la France (43\%). Dans les autres pays de l'OCDE, la part des emplois hautement qualifiés soutenus par la demande extérieure allait d'environ $21 \%$ en Australie et en Corée à $40 \%$ au Canada. Ces écarts reflètent les disparités observées quant aux compétences requises pour produire à des fins de consommation intérieure et d'exportation, aux profils de compétences de la main-d'œuvre des sociétés étrangères et nationales, et à la composition structurelle de la demande finale intérieure et extérieure.

\section{Emplois du secteur des entreprises soutenus par la demande finale extérieure, par intensité de qualification, 2014} En pourcentage de l'emploi total du secteur des entreprises

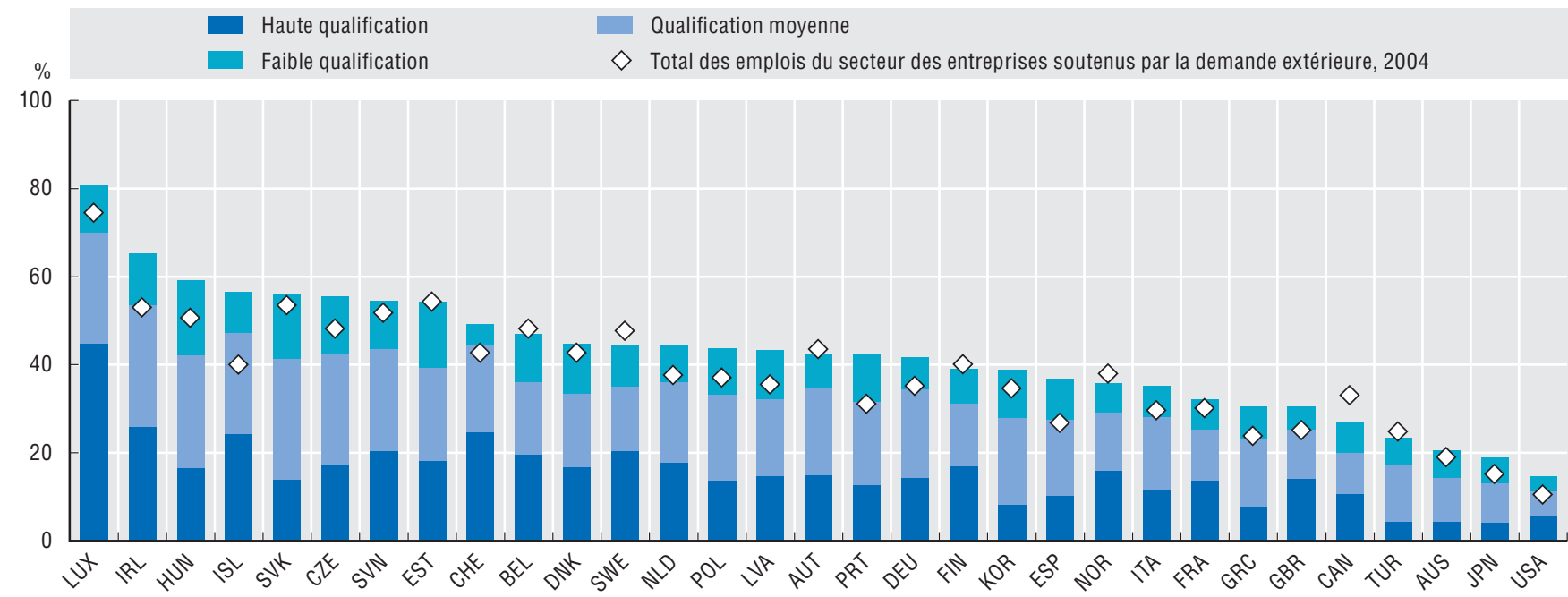

Note : Les estimations des emplois soutenus par la demande finale extérieure sont tirées des Tableaux internationaux d'entrées-sorties (TIES) de l'OCDE pour 2004 ; celles concernant 2014 sont des projections ou actualisations préliminaires. Cet indicateur expérimental décompose l'emploi total soutenu par la demande finale extérieure en trois catégories d'intensité de qualification, définies selon les grands groupes de la Classification internationale type des professions 2008 (CITP-08) : professions très qualifiées (grands groupes 1 à 3 de la CITP-08), moyennement qualifiées (groupes 4 à 7) et peu qualifiées (groupes 8 et 9).

Source : Calculs de l'OCDE d'après les Tableaux internationaux des entrées-sorties (TIES) de l'OCDE, la Base de données des Comptes nationaux annuels, les bases de données pour l'analyse structurelle (STAN) et sur le Contenu en emploi des échanges, la Base de données mondiale des entrées-sorties, les enquêtes européennes sur les forces de travail, les enquêtes nationales sur la population active et d'autres sources nationales, juin 2017. Voir notes de chapitre.

StatLink inils $h$ ttp://dx.doi.org/10.1787/888933720148

\section{Mesurer l'emploi soutenu par la demande finale extérieure}

Les biens et services acquis par les individus comprennent des intrants produits sur le territoire national ou importés de différents pays du monde. Or les flux de biens et de services circulant au sein des chaînes de production mondiales ne transparaissent pas toujours dans les statistiques traditionnelles sur les échanges internationaux, ni dans les tableaux nationaux des entrées-sorties, ou des ressources et des emplois, lesquels décrivent les flux intersectoriels (ou entre groupes de produits) des biens et services intermédiaires que les pays utilisent pour produire et satisfaire leur demande intérieure et extérieure. S'appuyant sur ces sources de données, et bien d'autres, les Tableaux internationaux des entrées-sorties (TIES) de l'OCDE fournissent des estimations des flux de biens et de services circulant entre 63 économies, ventilés selon 34 activités économiques fondées sur la CITI rév. 3 (dont 16 secteurs manufacturiers et 14 secteurs de services) et couvrant la période 1995-2011. Dans cette analyse, le secteur des TIC, défini conformément à la CITI rév. 3, englobe la fabrication d'ordinateurs, d'articles électroniques et optiques (divisions 30, 32 et 33), les postes et télécommunications (division 64) et les activités informatiques et activités rattachées (division 72).

Les TIES sont notamment utilisés pour bâtir un ensemble d'indicateurs sur les échanges en valeur ajoutée (TiVA) permettant d'identifier l'origine (tant locale qu'étrangère) de la valeur ajoutée des exportations des pays et de la demande finale. Ils permettent également d'estimer le contenu en emplois (autrement dit les emplois soutenus par) de la demande finale extérieure, à l'instar des estimations du contenu en valeur ajoutée locale de la demande finale extérieure. Les indicateurs expérimentaux sur l'emploi reposent néanmoins sur plusieurs grandes hypothèses. On suppose notamment qu'au sein de chaque secteur, la productivité de la main-d'œuvre des entreprises exportatrices est la même que celle des entreprises productrices de biens et de services destinés à un usage exclusivement national, et que, pour une production donnée, la part d'importations dans la production est la même pour l'ensemble des entreprises, qu'elles soient exportatrices ou non. Il s'avère néanmoins que les entreprises exportatrices enregistrent une productivité du travail plus élevée et ont davantage recours aux importations pour produire. Des efforts complémentaires devront être déployés pour tenir compte de l'hétérogénéité des entreprises dans le cadre des TIES et réduire les éventuelles surestimations découlant des hypothèses actuelles. 


\section{1. ÉCONOMIE DU SAVOIR ET TRANSFORMATION NUMÉRIQUE}

\section{Croissance, emploi et transformation numerique}

\section{Évolution de la nature des emplois}

L'innovation et les nouvelles technologies - en particulier les technologies de l'information et des communications (TIC) -, alliées à l'évolution de l'organisation de la production des entreprises, à l'échelle tant locale qu'internationale, transforment les emplois et les profils de compétences de la main-d'œuvre. Les économies à forte intensité d'utilisation des TIC sont aussi celles qui présentent une part d'emplois non routiniers plus importante. Ces derniers se caractérisent par l'exécution de tâches relativement complexes, difficiles à codifier ou à séquencer (comme la programmation ou la prise de décisions). On retrouve ce type de postes à la fois dans les services et dans le secteur de la fabrication. Si, en apparence, les emplois dans les services ont davantage recours aux TIC pour l'exécution de tâches, la corrélation positive entre la composante non répétitive des tâches et leur intensité en TIC est généralement plus marquée dans le secteur manufacturier. La structure organisationnelle des entreprises, l'adoption des technologies, leur participation aux chaînes de valeur mondiales, ou le degré d'automatisation, de délocalisation et de relocalisation des emplois manufacturiers routiniers sont autant de facteurs qui contribuent à ces phénomènes.

\section{Part de l'emploi non répétitif et de l'intensité des tâches liées aux TIC, 2012 ou 2015}

Corrélation avec les valeurs sectorielles moyennes dans chacun des grands secteurs

\section{Secteurs des services marchands}
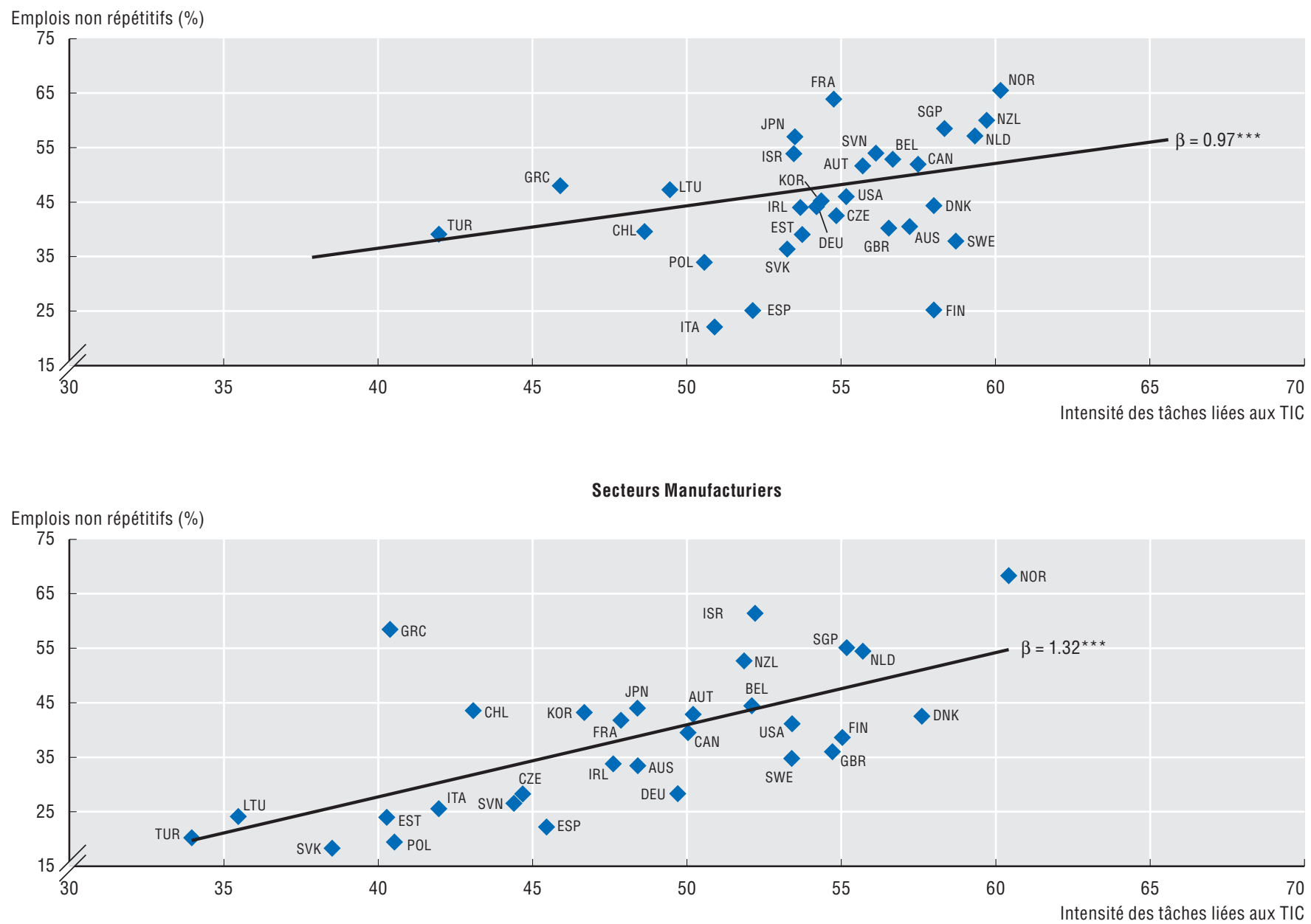

Source : Calculs de l'OCDE d'après la Base de données du Programme pour l'évaluation internationale des compétences des adultes (PIAAC), juin 2017. Voir notes de chapitre.

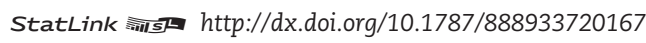

\section{Contenu des emplois en tâches répétitives et en tâches liées aux TIC}

L'OCDE a dernièrement mis au point des indicateurs fondés sur les composantes des emplois, afin de mesurer l'" intensité routinière " (Marcolin et al., 2016) et l'« intensité des tâches liées aux TIC » des professions (Grundke et al., 2017). Les deux ensembles d'indicateurs s'appuient sur des informations extraites du Programme pour l'évaluation internationale des compétences des adultes (PIAAC) de l'OCDE. L'« intensité routinière » des emplois reflète le degré d'autonomie dont disposent les travailleurs pour planifier et organiser leurs activités et leur temps, ainsi que la liberté dont ils jouissent pour décider de l'exécution et de l'ordonnancement de leurs tâches. L'« intensité des tâches liées aux TIC ", quant à elle, révèle la palette des tâches exécutées, allant de la simple consultation de l'internet à l'utilisation des logiciels Word ou Excel, ou d'un langage de programmation. Ces approches fondées sur les composantes des emplois présentent un avantage par rapport aux études précédentes : elles permettent d'opérer une distinction entre les tâches que les salariés exécutent au travail et les compétences dont ils sont dotés. 


\section{Formation en entreprise}

Les travailleurs qui exécutent des tâches non répétitives ou des tâches à forte intensité de TIC présentent généralement un niveau de qualification relativement élevé. La formation en entreprise est un moyen de motiver et de récompenser les travailleurs, tout en alignant leurs compétences sur les besoins des entreprises. Elle peut en outre contribuer à réduire les inégalités et doter les travailleurs peu qualifiés des compétences nécessaires pour affronter la transformation numérique. Pourtant, les faits montrent que la formation a surtout servi à renforcer les compétences des personnes disposant déjà d'un niveau de qualification moyen à élevé. En moyenne, dans les pays examinés, entre 30 \% (pour la Fédération de Russie et la Grèce) et 76 \% (aux Pays-Bas, au Danemark et en Finlande) des travailleurs ont bénéficié de formations proposées par leur employeur. Exception faite de la Turquie, moins d'un quart des travailleurs ayant bénéficié d'une formation sont peu qualifiés ; en revanche, entre un quart (en Autriche) et les trois quarts (dans la Fédération de Russie) des plus qualifiés ont suivi une formation.

\section{Travailleurs ayant bénéficié d'une formation en entreprise, par niveau de qualification, 2012 ou 2015}

En pourcentage du nombre total d'actifs occupés

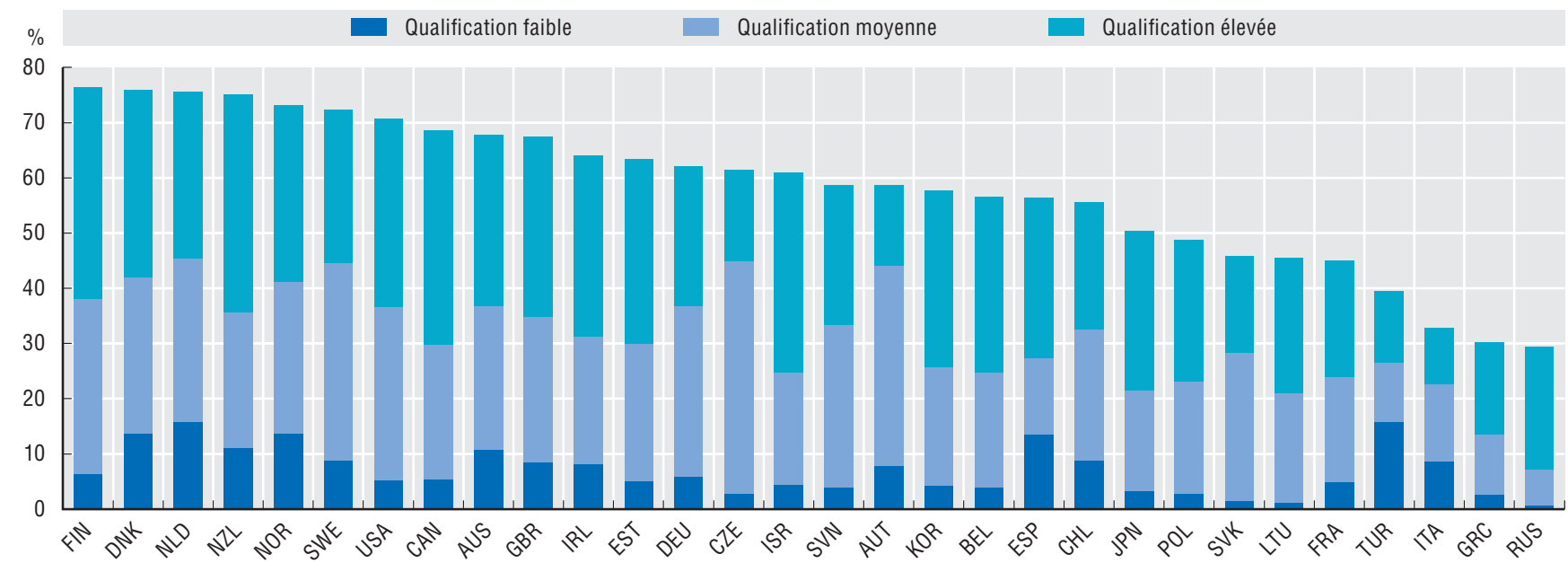

Source: Calculs de l'OCDE, d'après la Base de données du Programme pour l'évaluation internationale des compétences des adultes (PIAAC), juin 2017. Voir notes de chapitre.

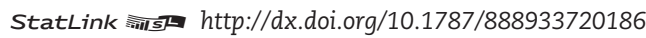

\section{Comment mesurer la formation}

La formation en entreprise permet de doter les individus des compétences dont ils ont besoin pour mener à bien leur mission et pour passer d'un emploi à un autre, des atouts particulièrement essentiels dans un environnement marqué par l'évolution rapide de la technologie. En l'absence de méthodologies établies à l'échelle internationale pour mesurer les investissements des entreprises en matière de formation, l'OCDE (Squicciarini et al., 2015) a mis au point une nouvelle approche pour estimer le recours à différents types de formations. On distingue ainsi la formation formelle, dispensée dans un cadre organisé, extérieur à l'environnement professionnel, sanctionnée par un diplôme délivré par un établissement d'enseignement, de la formation en cours d'emploi, qui peut être dispensée au sein ou en dehors de l'entreprise mais ne donne généralement pas lieu à l'obtention d'un diplôme officiel. Les chiffres concernant la formation reflètent le nombre d'employés qui, dans le cadre de l'évaluation internationale des compétences des adultes (PIAAC), ont déclaré avoir suivi une formation au moins une fois dans l'année, qu'ils appartiennent au secteur public ou au secteur privé. Une pondération est appliquée pour que ces valeurs soient représentatives sur le plan national. Les chiffres relatifs à la fréquence des formations peuvent masquer des différences quant à la durée de la période de formation, selon les individus et les pays. 


\section{1. ÉCONOMIE DU SAVOIR ET TRANSFORMATION NUMÉRIQUE}

\section{Croissance, emploi et transformation numerique}

\section{Les femmes au travail}

La rapidité, l'échelle et la portée de la transformation numérique remodèlent tous les aspects de la vie des individus, y compris sur le plan professionnel. Les modalités de travail nouvelles et atypiques peuvent certes ouvrir la voie à davantage de flexibilité, mais au détriment de la qualité de l'emploi. La manière dont les connaissances sont générées et partagées peut contribuer à lever un certain nombre de barrières culturelles ou institutionnelles, mais également à en créer de nouvelles. Dans un monde de plus en plus incertain, établir des prévisions relève de la gageure. D'où l'importance de développer et de renforcer les compétences, afin d'aider les travailleurs à affronter le changement numérique et en tirer le meilleur parti.

La rémunération des femmes reste souvent très inférieure à celle des hommes, même si l'on tient compte des caractéristiques personnelles et professionnelles. Les compétences n'expliquent qu'en partie les écarts de salaire entre les hommes et les femmes dans les différents pays. Par exemple, les hommes tendent à être dotés d'un niveau relativement plus élevé de compétences STIM, lesquelles sont valorisées sur le marché du travail. Les écarts se resserrent lorsque l'on tient compte des compétences, sans toutefois disparaître totalement, révélant ainsi d'autres sources d'inégalités salariales qui vont des choix organisationnels d'affectation des responsabilités de projet, à la titularisation des employés, en passant par la discrimination.

Les compétences TIC sont l'un des facteurs qui expliquent les inégalités salariales entre les hommes et les femmes. Les estimations indiquent que, toutes choses égales par ailleurs, le rendement de la composante TIC est plus élevé pour les femmes que pour les hommes. La formation des femmes et l'acquisition de compétences TIC complémentaires pourraient donc ouvrir la voie à de meilleures rémunérations et à une réduction des écarts salariaux entre les sexes.

\section{1. Écarts salariaux hommes-femmes, par pays, 2012 ou 2015}

Différences de salaire horaire, en pourcentage (avec et sans prise en compte des différents types de compétences)

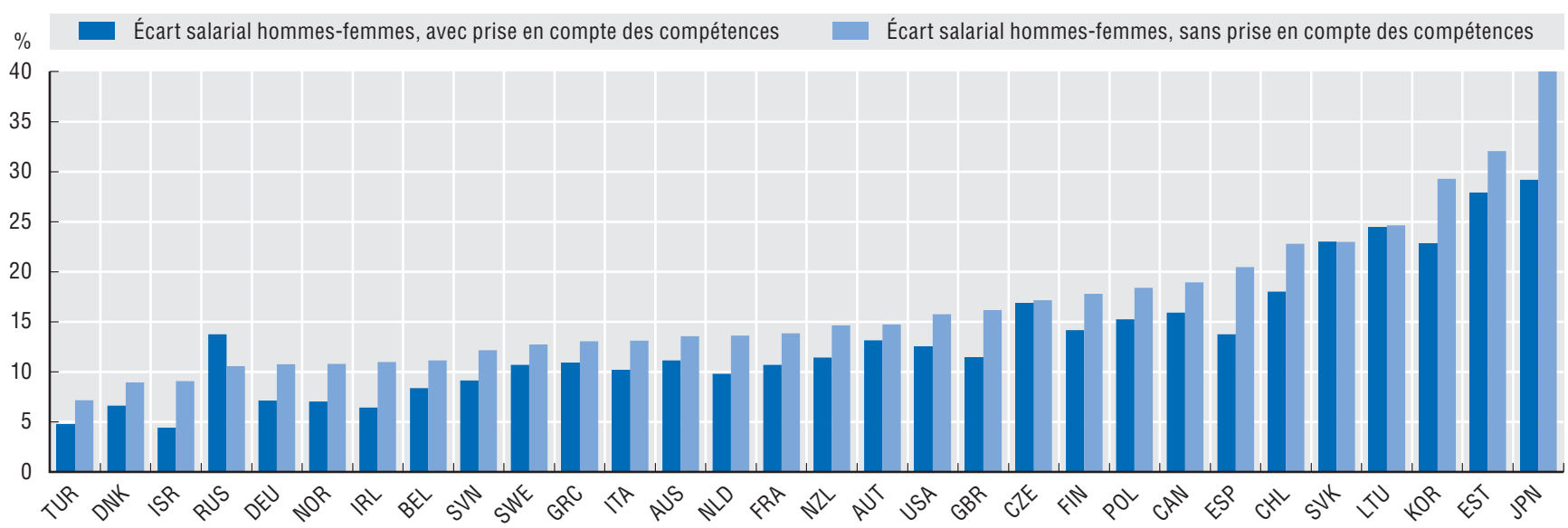

Source: Calculs de l'OCDE d'après la Base de données du Programme pour l'évaluation internationale des compétences des adultes (PIAAC), septembre 2017. Voir notes de chapitre.

42. Rendement de la composante TIC sur le marché du travail, par sexe, 2012 ou 2015

Pourcentage de variation du salaire horaire pour une augmentation de 10 \% de l'intensité des tâches liées aux TIC (par rapport à la moyenne nationale, par sexe)

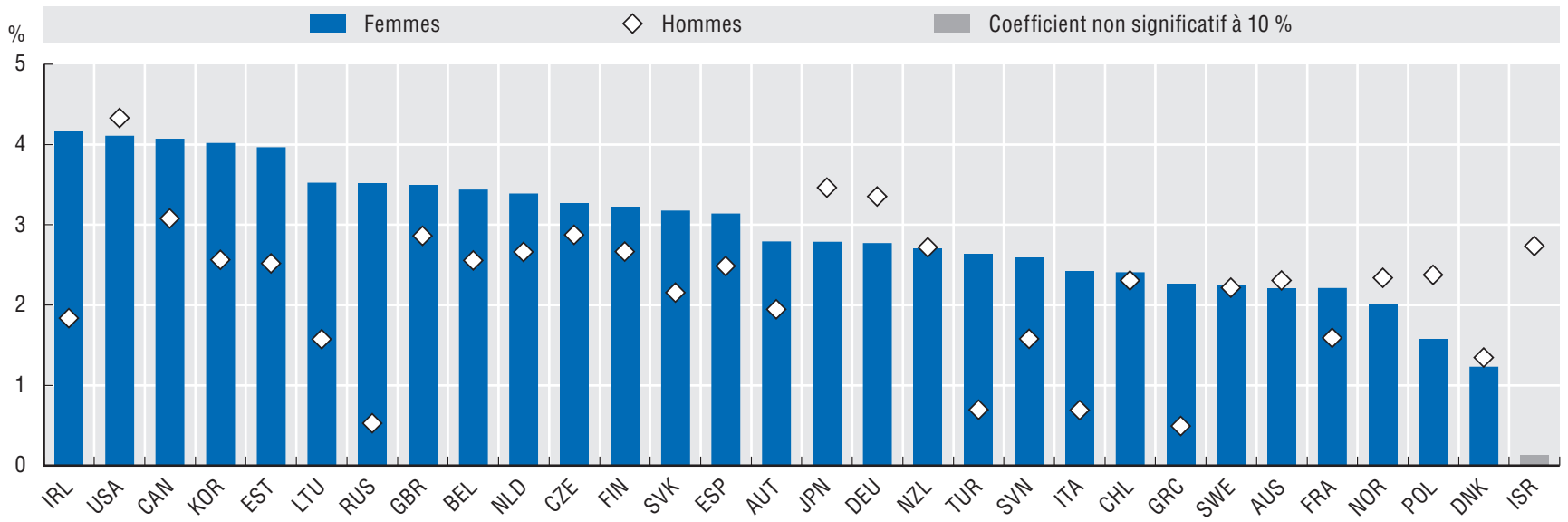

Source: Calculs de l'OCDE d'après la Base de données du Programme pour l'évaluation internationale des compétences des adultes (PIAAC), septembre 2017. Voir notes de chapitre. 


\section{Les femmes au travail}

La formation joue un rôle essentiel dans l'amélioration des compétences de la main-d'œuvre et augmente par là même la base de capital humain d'une économie. Des travaux de recherche de l'OCDE ont montré que les femmes sont proportionnellement plus nombreuses à suivre des formations en cours d'emploi ; en revanche, elles sont sensiblement moins nombreuses que les hommes à le faire pendant les heures de travail. Peuvent toutefois entrer en ligne de compte des facteurs tels que la propension plus ou moins élevée à travailler à temps partiel ou à développer ses compétences, les choix des employeurs quant aux personnes à former, et les rendements que les entreprises attendent de la formation.

43. Employés ayant suivi des formations en cours d'emploi, par sexe, 2012 ou 2015 En pourcentage du nombre total d'employés du même sexe, dans l'économie considérée

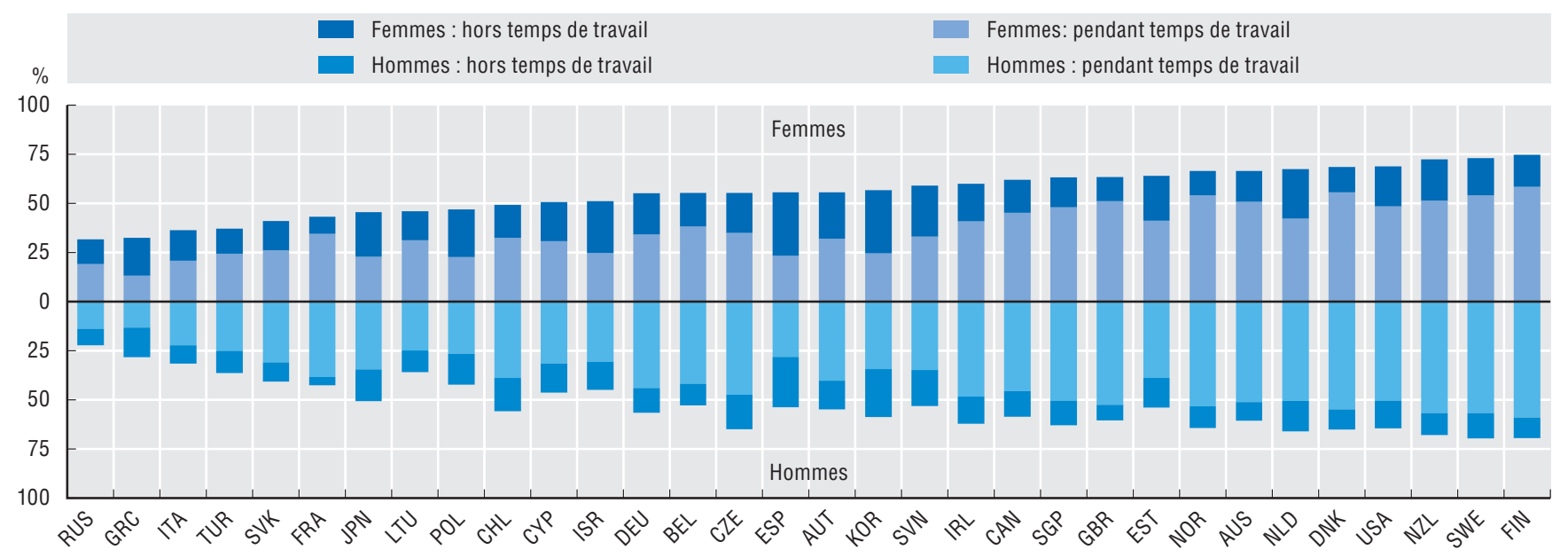

Source: Calculs de l'OCDE d'après la Base de données du Programme pour l'évaluation internationale des compétences des adultes (PIAAC), septembre 2017. Voir notes de chapitre.

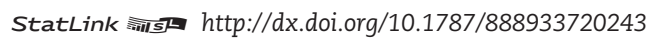

\section{Investir dans les compétences pour réduire les écarts salariaux entre hommes et femmes}

En l'absence de mesures convenues à l'échelle internationale pour évaluer l'investissement dans la formation, l'OCDE propose de recourir à une méthodologie expérimentale pour estimer l'investissement dans différents types de formations. La formation formelle est une formation dispensée dans un cadre organisé, extérieur à l'environnement professionnel, et sanctionnée par un diplôme. La formation en cours d'emploi peut quant à elle être dispensée au sein ou en dehors de l'entreprise, mais ne débouche généralement pas sur l'obtention d'un diplôme officiel (Squicciarini et al., 2015). Pour évaluer quelles compétences sont davantage valorisées sur le marché du travail, l'OCDE (Grundke et al., à paraître) estime le rendement des compétences ; pour ce faire, elle analyse la corrélation entre la rémunération du travail, sous la forme des salaires, et les compétences dont disposent les travailleurs. L'évaluation du rendement des compétences par sexe peut aider à repérer quels types de formation sont davantage susceptibles de contribuer à réduire l'écart de salaire associé. Les estimations s'appuient sur des indicateurs de compétences cognitives comme la maîtrise de la langue et du calcul, sur les compétences mises en évidence par une analyse des tâches menées par les individus dans le cadre de leur travail (pour de plus amples informations, voir Grundke et al., 2017), ainsi que sur les données du PIAAC. L'indicateur de la composante TIC tient compte de tâches allant de la simple consultation de l'internet à l'utilisation des logiciels Word ou Excel, ou d'un langage de programmation. 


\section{1. ÉCONOMIE DU SAVOIR ET TRANSFORMATION NUMÉRIQUE}

\section{Croissance, emploi et transformation numerique}

\section{Productivité sectorielle}

Comprendre les moteurs de la croissance de la productivité dans l'économie totale nécessite de connaître chaque contribution sectorielle. Dans les années qui ont précédé la crise économique (2001-07), un grand nombre de pays de l'OCDE ont vu leur productivité croître presque entièrement du fait de la hausse de la productivité dans le secteur manufacturier et de l'augmentation de la part des services aux entreprises dans l'activité globale. La plupart des pays de l'OCDE, pour lesquels les données sont disponibles, affichaient une croissance de la productivité du travail en baisse après le déclenchement de la crise financière en 2008, et ce dans tous les secteurs. L'Estonie, la Finlande, la Grèce, la Lettonie, la République slovaque, la République tchèque, le Royaume-Uni et la Slovénie ont connu un net ralentissement (de plus de $2 \%$ ) de la croissance moyenne de leur productivité entre 2009 et 2015 par rapport à la période 2001-07, en particulier avec un recul notable de la croissance de leur productivité manufacturière. Toutefois, certains pays - comme l'Australie, l'Espagne, l'Irlande, Israël, l'Italie et la Pologne - ont dans le même temps enregistré des gains modestes.

\section{Décomposition sectorielle de la croissance de la productivité du travail, 2001-07 et 2009-15}

Contributions du secteur des entreprises non agricoles à la variation annuelle moyenne en pourcentage

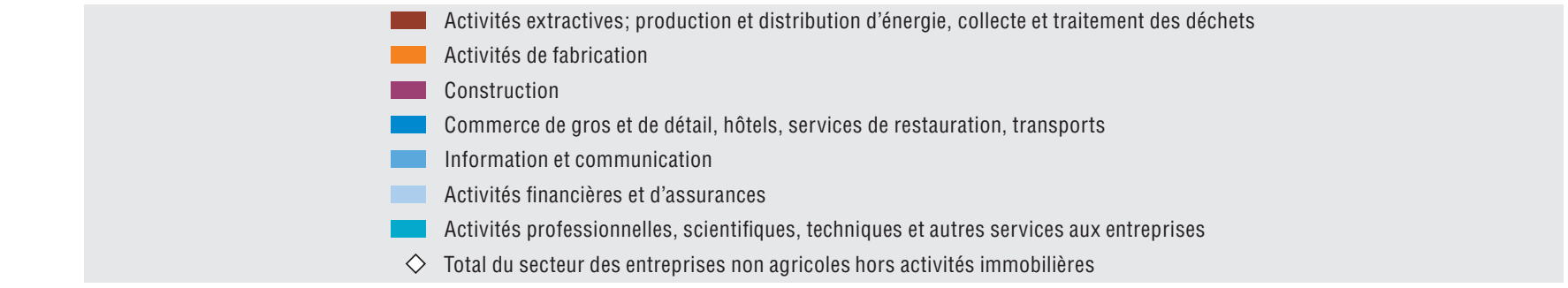

Percentage points
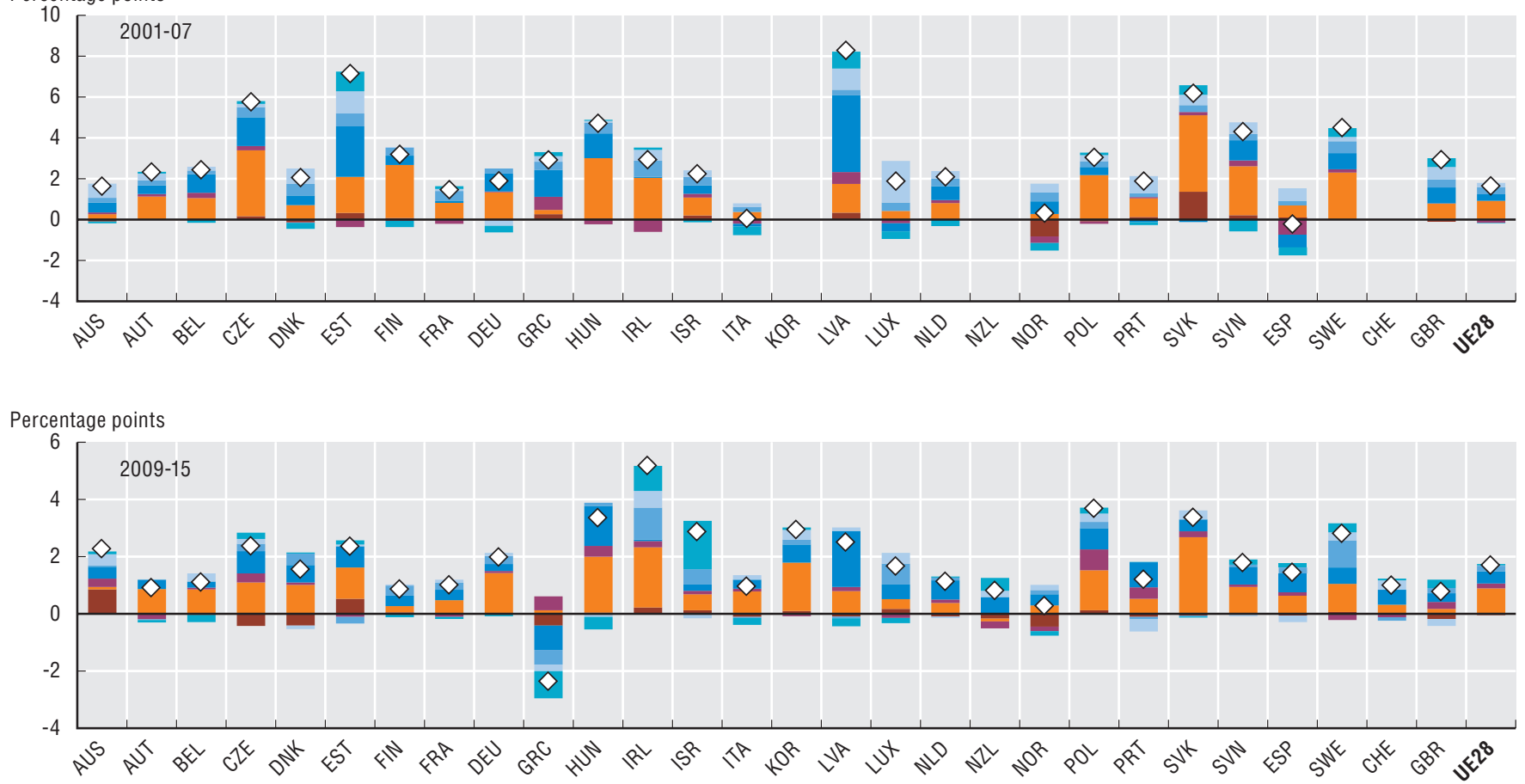

Source : OCDE, Base de données sur la productivité, http://www.oecd.org/fr/std/stats-productivite, septembre 2017. Voir notes de chapitre.

StatLink नiाडs http://dx.doi.org/10.1787/888933720262

\section{Mesurer la productivité du travail par secteur}

On entend par " croissance de la productivité du travail » le taux de croissance en valeur ajoutée réelle par heure travaillée. Les écarts de croissance observés pour la productivité du travail de différents secteurs peuvent être liés, par exemple, à l'intensité d'utilisation du capital (y compris le capital intellectuel) et de la main-d'œuvre qualifiée dans la production, à l'ampleur de l'innovation de produit et de procédé, au degré d'uniformisation des produits, à l'importance des économies d'échelle et à la participation des secteurs aux chaînes de valeur mondiales. Les problèmes liés à la mesure de la valeur ajoutée réelle peuvent influer sur l'aptitude à comparer la croissance de la productivité entre secteurs et entre pays. Par exemple, la plupart des pays postulent l'absence de variation de la productivité du travail dans les activités des administrations publiques, raison pour laquelle ce secteur n'apparaît pas ici. Les activités immobilières sont également exclues, car leur production correspond principalement à la valeur attribuée aux services d'hébergement que les propriétaires fournissent et consomment. En outre, le travail à temps partiel et l'activité indépendante occupent une grande place dans des secteurs comme la construction et certains services (par exemple, hôtellerie et restauration), ce qui peut nuire à la qualité des estimations du nombre réel d'heures travaillées. Pour un examen plus approfondi des questions liées à la mesure de la productivité, voir OCDE (2017a). 


\section{Productivité sectorielle}

Les secteurs de l'information, pour la plupart des pays de l'OCDE, ont une contribution à la croissance totale de la productivité du travail relativement faible. Ils affichent néanmoins une productivité du travail bien supérieure à la moyenne, du fait de leur relative intensité de capital fixe (actifs corporels) et intellectuel. En 2015, dans la zone OCDE, la productivité du travail dans les secteurs de l'information dépassait de 60 \% en moyenne celle des autres branches du secteur des entreprises. L'Irlande arrivait en tête, sous l'effet particulier de la forte croissance de la productivité des services des TIC et, en partie, de l'implantation de multinationales américaines ayant généré une forte valeur ajoutée dans ce secteur, avec relativement peu de salariés.

La productivité du travail reflète les changements dans l'utilisation et l'efficacité tant du capital fixe que du capital intellectuel (actifs incorporels). Les estimations de la productivité totale des facteurs (PTF), du fait qu'elles incluent la contribution " évaluée " du capital au PIB (y compris achats de logiciels et dépenses de R-D considérés comme des investissements), rendent aussi compte de l'apport d'actifs incorporels " non évalués » tels que le capital organisationnel ou l'investissement dans des formations personnalisées. Dans l'ensemble, la PTF, tous secteurs confondus, a été notablement plus faible au cours des six années qui ont suivi la crise économique qu'au cours des deux périodes de six ans (1995-2001 et 2001-07) la précédant. Parmi les pays présentés, seuls le Danemark et le Japon ont enregistré une PTF plus élevée en 2009-15 qu'au cours des périodes 1995-2001 et 2001-07.

\section{Productivité du travail des secteurs de l'information, 2015}

Rapportée à la productivité du travail agrégée des autres branches du secteur des entreprises non agricoles

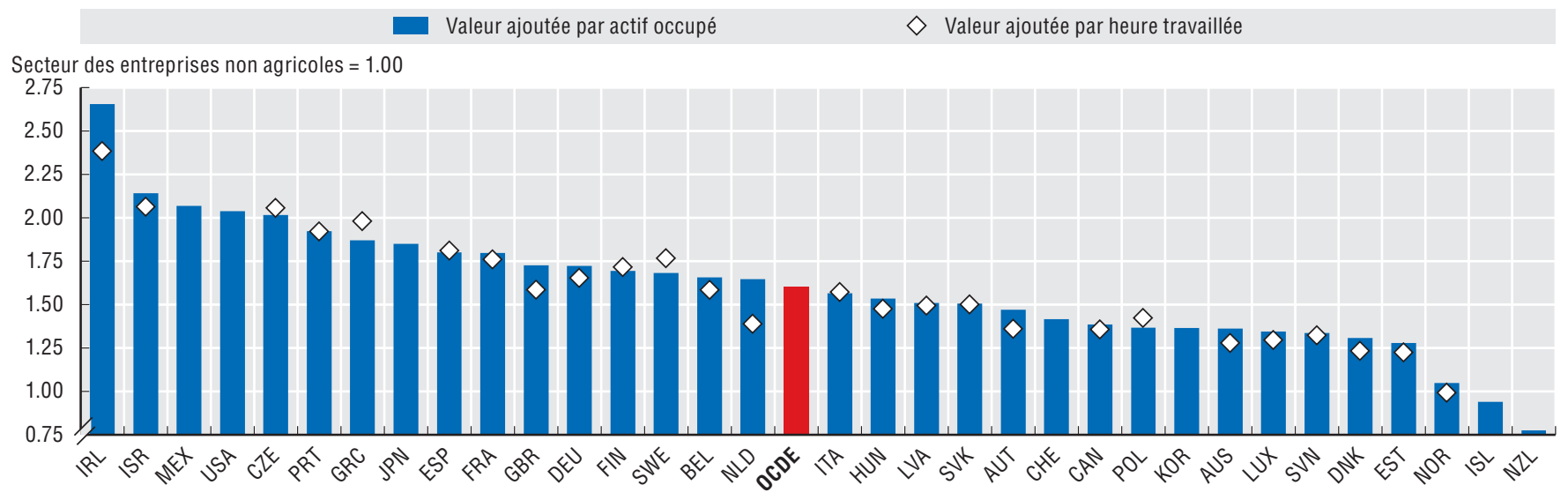

Note : La mesure privilégiée de la productivité du travail est la valeur ajoutée par heure travaillée. Toutefois, les données sectorielles détaillées sur les heures travaillées ont parfois une disponibilité limitée, ce qui conduit à utiliser en remplacement la valeur ajoutée par actif occupé. Les différences entre ces deux mesures correspondent au nombre moyen d'heures travaillées par personne. Dans ce graphique, une valeur ajoutée par actif occupé relative plus élevée indique que les secteurs de l'information ont un nombre supérieur d'heures travaillées par personne. Les secteurs de l'information sont définis conformément à la CITI rév. 4 : Fabrication d'ordinateurs, d'articles électroniques et optiques (division 26), Activités d'édition, audiovisuel et activités de diffusion (58 à 60), Télécommunications (61) et Activités informatiques et autres services d'information (62 et 63).

Source : OCDE, Base de données pour l'analyse structurelle (STAN), http://oe.cd/stan, et Base de données des Comptes nationaux annuels, www.oecd.org/ $\mathrm{fr} / \mathrm{std} / \mathrm{cn}$, septembre 2017. Voir notes de chapitre.

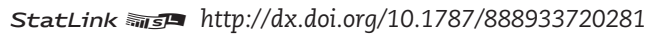

\section{Croissance de la productivité totale des facteurs, 1995-2015}

Ensemble de l'économie, variation annuelle moyenne en pourcentage

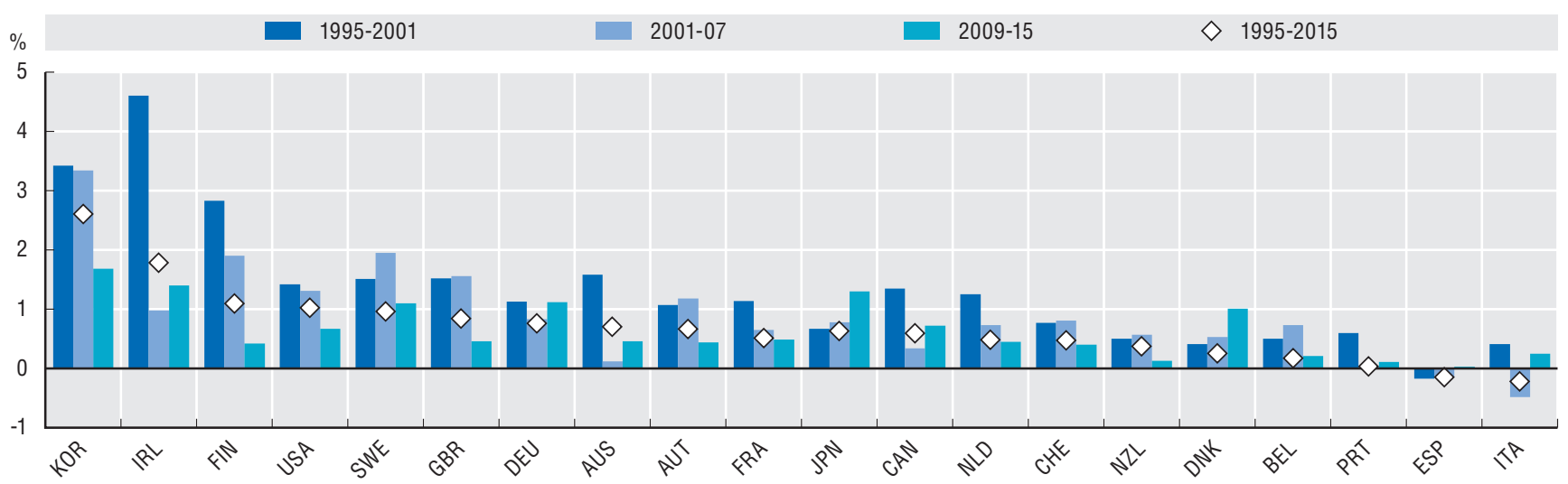

Source : Calculs de l'OCDE, d'après Base de données de l'OCDE sur la productivité, http://www.oecd.org/fr/std/stats-productivite, septembre 2017. Davantage de données via StatLink. Voir notes de chapitre. 


\section{1. ÉCONOMIE DU SAVOIR ET TRANSFORMATION NUMÉRIQUE}

\section{Croissance, emploi et transformation numerique}

\section{Empreinte élargie des TIC}

Mesurer la valeur ajoutée des secteurs des technologies de l'information et de la communication (TIC) ne donne qu'une vision partielle de l'importance des TIC dans l'économie d'un pays. En effet, la production du secteur des TIC d'une économie se retrouve non seulement dans ses produits finals mais aussi (via les produits intermédiaires) dans une large gamme d'autres biens et services destinés à satisfaire la demande finale (investissements des entreprises, consommation des ménages et des administrations publiques), tant intérieure qu'étrangère. Réciproquement, la production des secteurs d'activités non TIC d'un pays est présente dans de nombreux produits TIC consommés dans le monde entier par l'intermédiaire d'interconnexions locales et de la participation aux chaînes de valeur mondiales. La demande mondiale de biens et services TIC, constituée des investissements et des échanges internationaux, peut stimuler les activités non TIC de nombreux secteurs nationaux en amont. Par conséquent, une ébauche de la définition de l'empreinte élargie des TIC consiste à combiner la valeur ajoutée du secteur des TIC de chaque pays avec celle de ses secteurs non TIC, laquelle est contenue dans la demande mondiale de biens et services TIC.

En 2011, les États-Unis, le Japon et la Chine confondus totalisaient quelque 45 \% de l'empreinte élargie des TIC dans le monde. La part de l'Union européenne dans son ensemble était de $23 \%$, un pourcentage légèrement supérieur à celui des États-Unis. Pour estimer l'importance de l'économie "numérique », il convient de ne pas négliger la valeur ajoutée qui est produite par les secteurs non TIC de l'économie afin de répondre à la demande mondiale de produits TIC finals. Dans les pays de l'OCDE, le poids de la valeur ajoutée non TIC représente entre $19 \%$ et 34 \% de l'empreinte élargie des TIC ; ce chiffre atteint 41 \% en Chine.

\section{Empreinte élargie des TIC de la valeur ajoutée locale, 2011}

Milliards USD et part mondiale en pourcentage

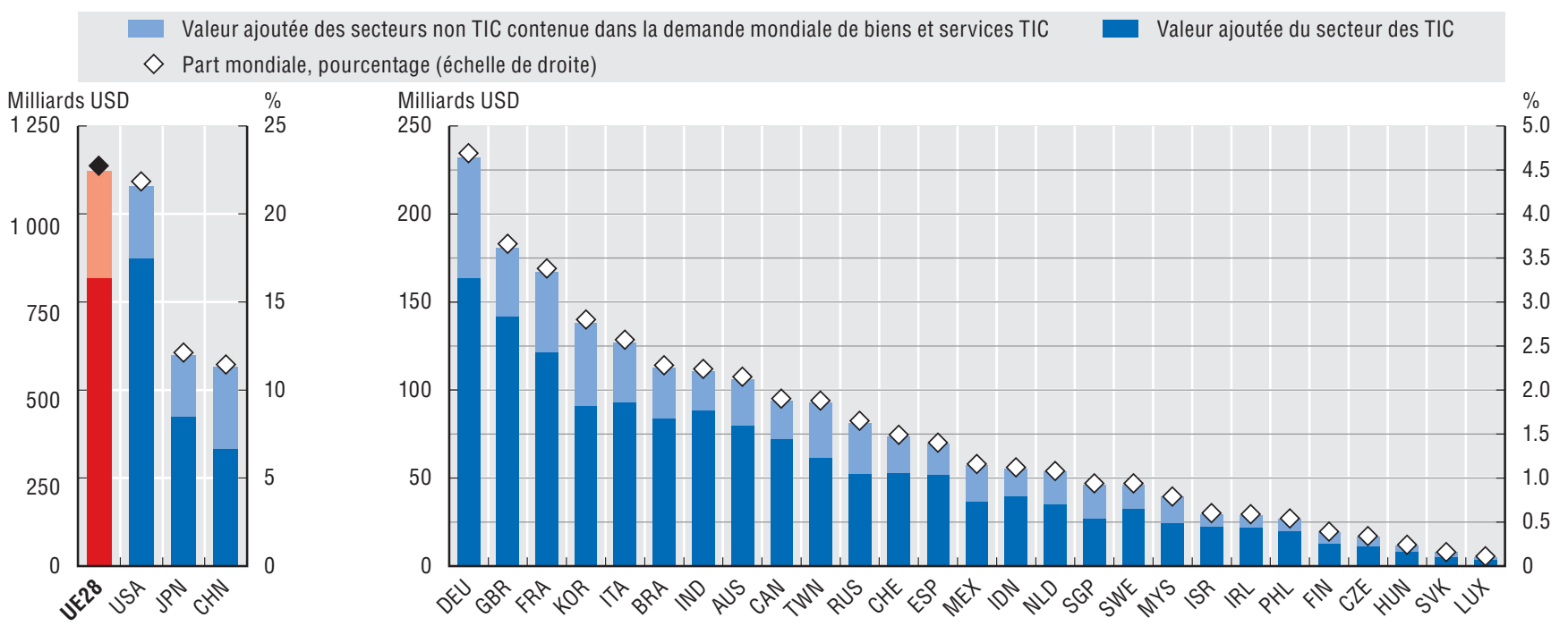

Source : OCDE, Tableaux internationaux des entrées-sorties (TIES), http://oe.cd/icio, et Base de données sur les échanges en valeur ajoutée (TiVA), http://oe.cd/tiva-fr, juillet 2017. Davantage de données via StatLink. Voir notes de chapitre.

StatLink त्ताड़ http://dx.doi.org/10.1787/888933720319

\section{TIC et origine de la valeur ajoutée}

Dans cette analyse, le secteur des TIC est établi à partir de la CITI rév. 3 et recouvre la fabrication d'ordinateurs, d'articles électroniques et optiques (divisions 30, 32 et 33), les postes et télécommunications (division 64) et les activités informatiques et activités rattachées (division 72). Cette définition, du fait des données disponibles, représente une approximation de la définition plus détaillée, et conforme à la CITI rév. 3, donnée dans OCDE (2011).

Si la valeur ajoutée du secteur des TIC figure généralement dans les statistiques des comptes nationaux, les indicateurs TiVA sont néanmoins nécessaires pour identifier l'origine (tant géographique que sectorielle) de la valeur ajoutée incorporée aux biens et services TIC finals. On utilise en particulier l'indicateur " origine du contenu en valeur ajoutée de la demande finale ", établi à partir de la base de données des TIES de l'OCDE. Les calculs fournissent des estimations des flux internationaux et intersectoriels de biens et services intermédiaires et finals, à partir desquels sont élaborés des indicateurs à même de renseigner sur la participation des pays à l'économie mondiale. En effet, de telles informations n'apparaissent ni dans les statistiques officielles conventionnelles sur les échanges « bruts » de produits, ni dans les tableaux nationaux des entrées-sorties ou ceux des ressources et des emplois. 


\section{Empreinte élargie des TIC}

Le concept d'empreinte élargie des TIC apparaît d'autant plus important si on s'intéresse au poids de la valeur ajoutée locale liée aux TIC dans la valeur ajoutée de l'ensemble de l'économie (ou PIB). En 2011, les parts des économies d'Asie de l'Est et du Sud-Est étaient parmi les plus élevées. La valeur ajoutée liée aux TIC représentait 20 \% du PIB dans le Taipei chinois et 18 \% à Singapour, deux économies particulièrement tributaires de la fabrication de biens TIC. En Corée, en Irlande, en Israël, au Japon et en Suisse, cette part dépassait $10 \%$, néanmoins, comme dans la plupart des autres pays de l'OCDE hormis la Corée, la principale contribution observée provenait des activités de services TIC. L'empreinte TIC permet également de déterminer quelle valeur ajoutée attribuable aux TIC est produite à l'étranger par le biais de la demande intérieure : en combinant notamment deux composantes dérivées des importations, à savoir la valeur ajoutée par les secteurs des TIC étrangers contenue dans la demande finale intérieure de tous les biens et services avec la valeur ajoutée par les autres (i.e. non TIC) secteurs étrangers incorporée à la demande intérieure de biens et services TIC. En 2011, la valeur ajoutée étrangère liée aux TIC représentait $2.4 \%$ du PIB en moyenne dans la zone OCDE.

\section{Valeur ajoutée locale liée aux TIC, 2011}

En pourcentage du PIB

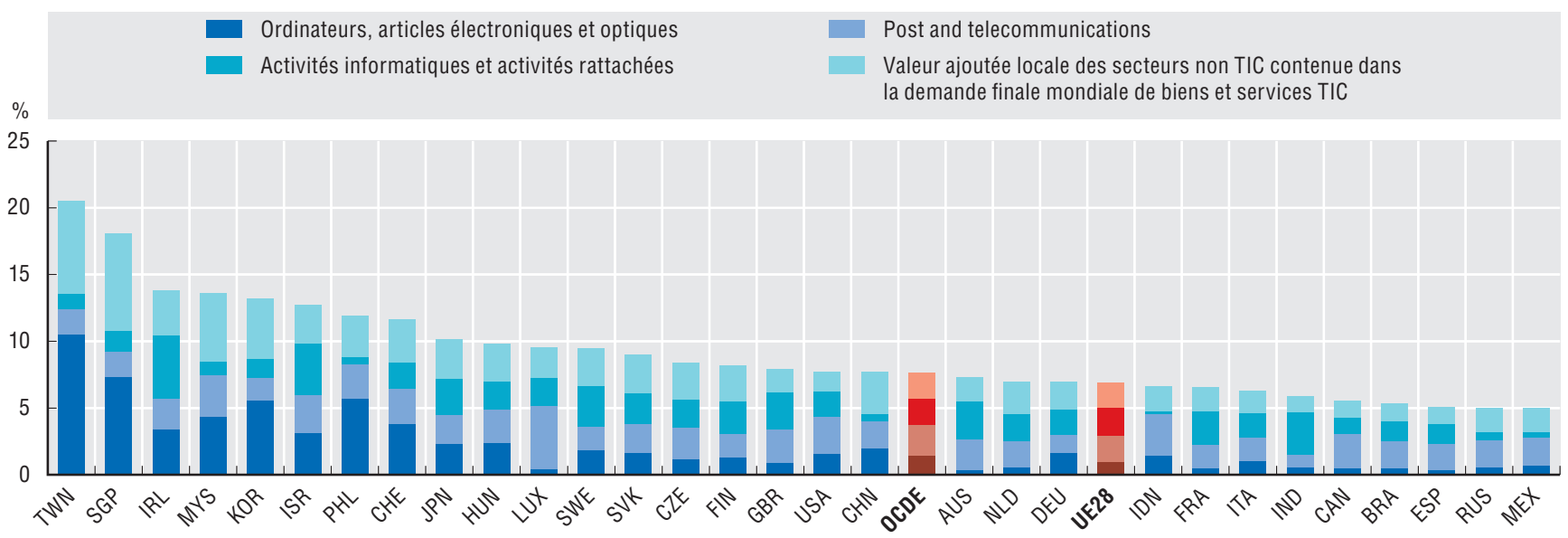

Source : OCDE, Tableaux internationaux des entrées-sorties (TIES), http://oe.cd/icio, et Base de données sur les échanges en valeur ajoutée (TiVA), http://oe.cd/tiva-fr, juillet 2017. Davantage de données via StatLink. Voir notes de chapitre.

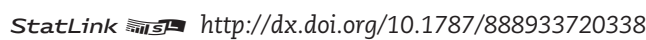

49. Valeur ajoutée étrangère liée aux TIC contenue dans la demande finale intérieure, 2011

En pourcentage du PIB

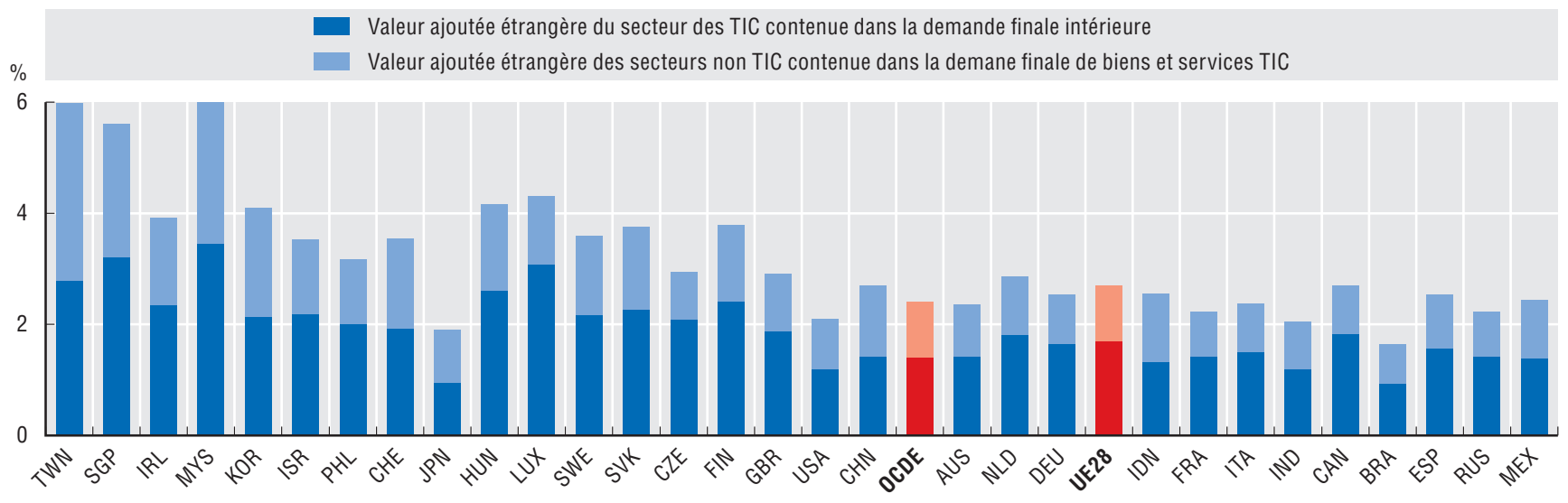

Source : OCDE, Tableaux internationaux des entrées-sorties (TIES), http://oe.cd/icio, et Base de données sur les échanges en valeur ajoutée (TiVA), http://oe.cd/tiva-fr, juillet 2017. Davantage de données via StatLink. Voir notes de chapitre.

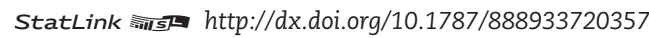

\section{Améliorer la mesure de l'empreinte des TIC}

Compte tenu des développements en cours concernant les TIES de l'OCDE, on pourrait approfondir le concept d'empreinte élargie des TIC et en améliorer sa mesure. Par exemple, utiliser une liste des secteurs d'activités fondée sur la CITI rév. 4 et, partant, une définition plus " fine » des secteurs des TIC et des TIES pour les années postérieures à 2011, permettrait d'obtenir des indicateurs plus récents. Estimer des matrices de flux de capitaux, pour l'heure absentes de l'infrastructure TIES, permettrait aussi d'inclure le contenu non TIC des investissements réalisés par les secteurs TIC, par exemple les machines et équipements utilisés pour fabriquer les pièces et composants TIC. Cette méthode élargirait le concept de l'empreinte TIC. Le contenu en TIC des biens d'équipement est, ici, déjà implicitement pris en compte dans l'analyse. 


\section{1. ÉCONOMIE DU SAVOIR ET TRANSFORMATION NUMÉRIQUE}

\section{Croissance, emploi et transformation numerique}

\section{Capital intellectuel}

Fabriquer de nouveaux produits encore meilleurs nécessite d'investir non seulement dans la recherche-développement (R-D), mais aussi dans des actifs complémentaires tels que des logiciels, des outils de conception, du capital humain et les capacités organisationnelles des entreprises - en résumé, du capital intellectuel. Selon de nouvelles estimations de l'OCDE (Haines et al., à paraître), le capital intellectuel et les actifs corporels étudiés pour la période 2000-14 représentaient en moyenne $6 \%$ et $14 \%$, respectivement, de la croissance de la productivité du travail. La contribution cumulée des logiciels et des équipements informatiques à la croissance de la productivité du travail s'échelonnait entre $2 \%$ (Luxembourg) et 18 \% (États-Unis), tandis que les capacités organisationnelles et la R-D atteignaient 8 \% (Belgique). Le capital intellectuel considéré semble également avoir contribué indirectement à la croissance de la productivité du travail : il existe en effet une relation positive entre le capital intellectuel et la productivité totale des facteurs (autrement dit, les économies où la croissance de la PTF est relativement plus forte enregistrent également une contribution plus élevée du capital intellectuel). Les facteurs qui pourraient expliquer ces tendances observées sont notamment la structure sectorielle des économies et la mesure dans laquelle les investissements dans le capital intellectuel génèrent des externalités de connaissances et des rendements d'échelle.

\section{Contribution des équipements informatiques et des actifs intellectuels à la croissance de la productivité du travail augmentée du capital intellectuel, 2000-14}

Estimations des contributions à la croissance, en pourcentage de la croissance de la productivité du travail, secteur marchand

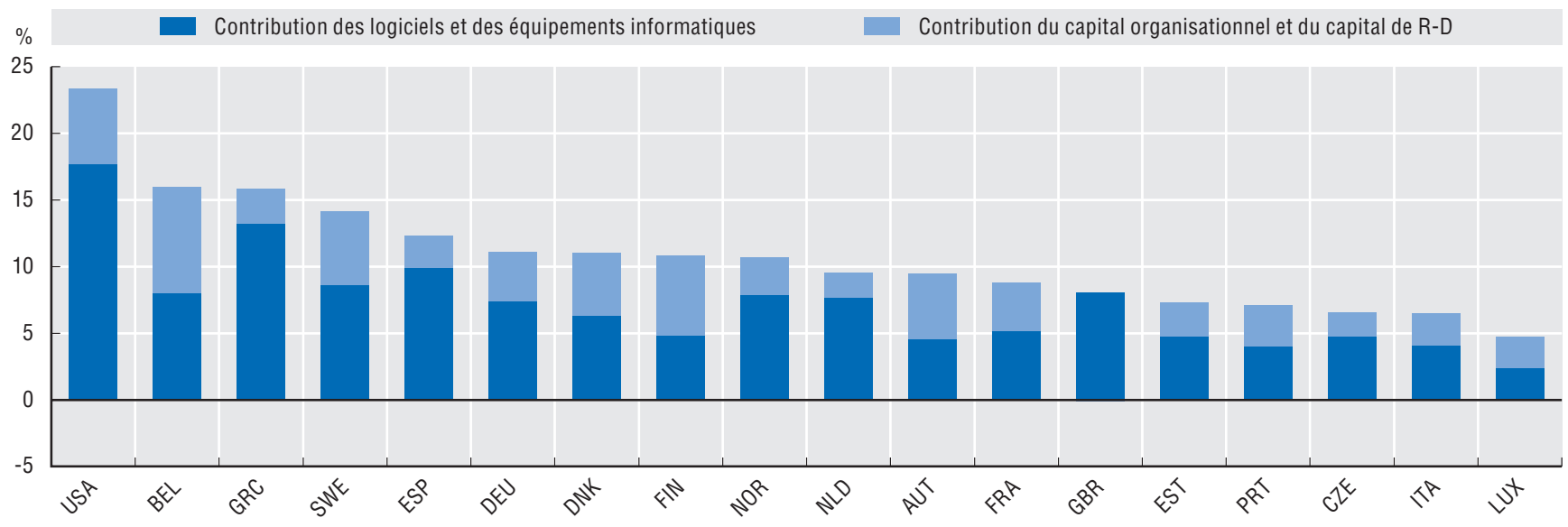

Source: Calculs de l'OCDE, d'après Base de données OCDE ANBERD, Base de données OCDE des Comptes nationaux annuels, Programme de l'OCDE pour l'évaluation internationale des compétences des adultes (PIAAC), Base de données EU-KLEMS, INTAN-Invest et comptes satellites du Bureau d'analyse économique (BEA, Bureau of Economic Analysis) des États-Unis, juin 2017. Voir notes de chapitre.

StatLink 需田 http://dx.doi.org/10.1787/888933720376

\section{Contribution du capital intellectuel et de la PTF à la croissance de la productivité du travail augmentée du capital intellectuel, 2000-14}

Estimations des contributions à la croissance, secteur des entreprises

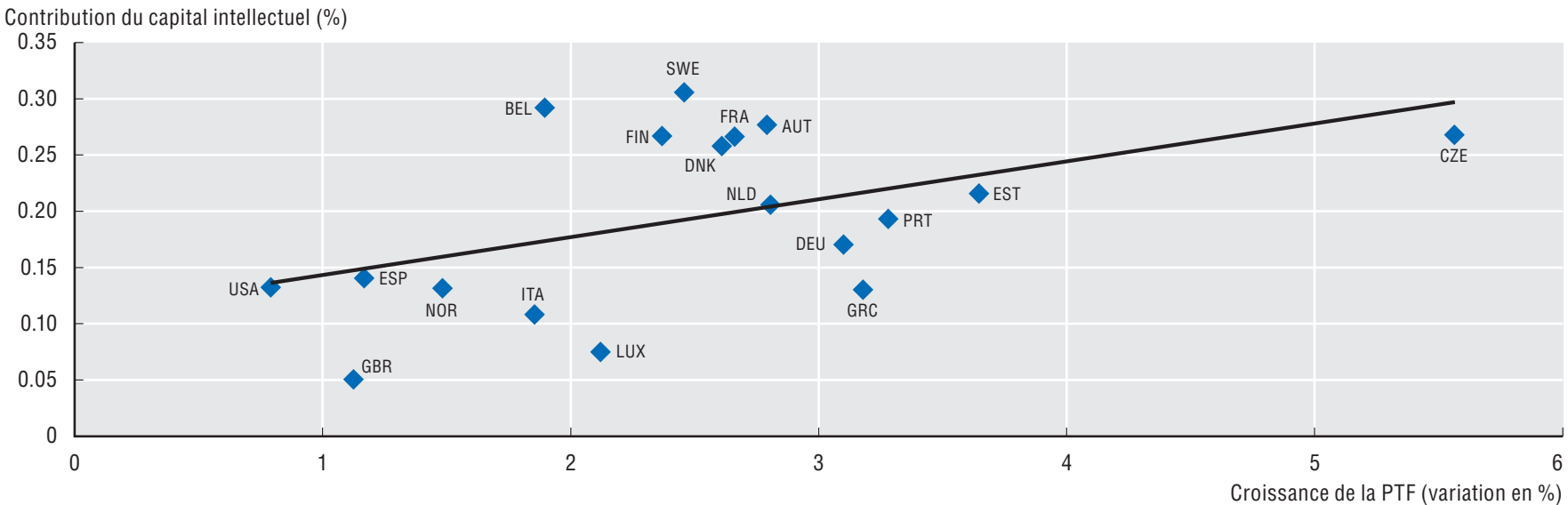

Source : Calculs de l'OCDE, d'après Base de données OCDE ANBERD, Base de données OCDE des Comptes nationaux annuels, Programme de l'OCDE pour l'évaluation internationale des compétences des adultes (PIAAC), Base de données EU-KLEMS, INTAN-Invest, www.intan-invest.net, comptes satellites du Bureau d'analyse économique (BEA, Bureau of Economic Analysis) des États-Unis et enquête PIAAC, juin 2017. Voir notes de chapitre. 


\section{Capital intellectuel}

Les estimations de la comptabilité de la croissance se concentrent généralement sur le seul secteur marchand, étant donné que mesurer la productivité dans le secteur non marchand peut être sujet à controverse. Cependant, de premières mesures du capital intellectuel dans le secteur non marchand, produites dans le contexte du réseau SPINTAN, montrent que les investissements dans les secteurs marchand et non marchand vont de pair dans les pays examinés. De ce fait, la contribution du capital intellectuel à la croissance économique pourrait être encore plus élevée que ne le suggère l'analyse fondée sur le secteur marchand, si les investissements publics dans le capital intellectuel devaient être pris en compte.

\section{Intensité en capital intellectuel des secteurs marchand et non marchand, 2015}

Corrélation des intensités, investissement rapporté à la valeur ajoutée brute

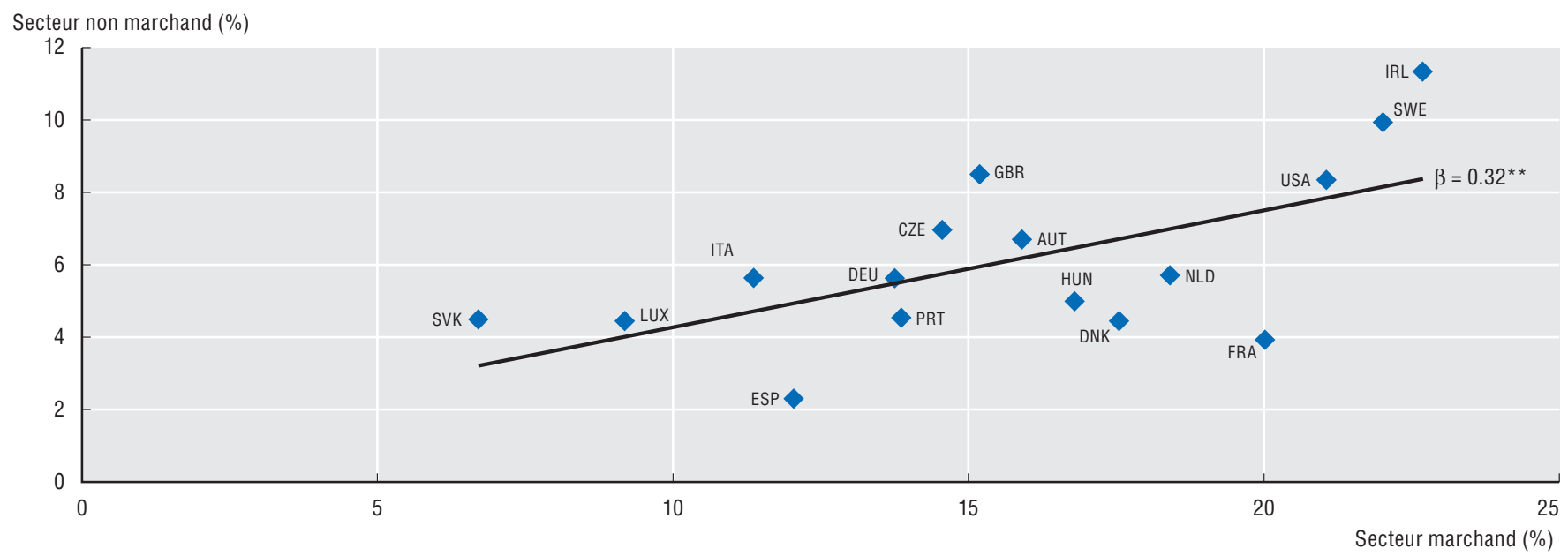

Source : Calculs de l'OCDE, d'après Base de données OCDE des Comptes nationaux annuels, www.oecd.org/std/ana, données INTAN-Invest, www.intaninvest.net, et données SPINTAN, www.spintan.net, mai 2017. Voir notes de chapitre.

\section{Qu'entend-on par « augmenté du capital intellectuel » et « comptabilité de la croissance " ?}

Le capital intellectuel, également appelé « actifs incorporels ", renvoie aux actifs qui, par nature, ne sont pas physiques. Leur valeur tient à leur contenu en termes de connaissances et à leur capacité à valoriser d'autres actifs. Corrado et al. (2009) répertorient dans le capital intellectuel des actifs tels que les logiciels et les bases de données, la R-D scientifique et non scientifique, les droits d'auteur, les dessins et modèles, la valeur de marque, la recherche marketing, les formations propres aux entreprises, et le savoir-faire organisationnel. Depuis quelques années, la communauté statistique internationale reconnaît les logiciels, la R-D, les spectacles, les originaux littéraires et artistiques et la prospection minière comme des actifs capitalisés et, à ce titre, les a comptabilisés dans le Système des comptes nationaux (SCN), révision 2008. En outre, des progrès méthodologiques ont récemment permis de proposer des moyens de mesurer d'autres actifs intellectuels, parmi lesquels les activités de conception, les marques, les formations propres aux entreprises et le capital organisationnel. Si des aspects tels que les prix et les taux d'amortissement de ces actifs ne font pas encore consensus, les méthodologies en question permettent néanmoins, à partir d'informations rapportées sous la forme de dépenses intermédiaires dans les comptes nationaux, d'estimer ce capital. Capitaliser ces actifs impose par conséquent d'ajuster les mesures de la valeur ajoutée et de la productivité du travail utilisées dans l'analyse de la comptabilité de la croissance : c'est ce que l'on entend ici par " augmenté du capital intellectuel ". On effectue en particulier un tel ajustement pour l'investissement dans le capital organisationnel, estimé sur la base de Le Mouel et al. (2016). Les estimations de l'investissement en capital intellectuel dans le secteur non marchand proviennent du réseau SPINTAN.

Selon la théorie économique classique, la croissance peut résulter d'une augmentation de la quantité de facteurs ou d'une augmentation de l'efficacité d'utilisation de ces facteurs dans la production. Sous réserve d'un certain nombre d'hypothèses, notamment celles de marchés parfaitement concurrentiels et de rendements d'échelle constants dans la production, la méthode de la comptabilité de la croissance fait la distinction entre la contribution de l'accumulation des facteurs et la contribution de la productivité totale des facteurs (PTF) au PIB ou à la croissance de la productivité du travail. On trouvera de plus amples informations sur la méthodologie et les solutions aux contraintes liées aux données dans Haines et al. (à paraître). 


\section{1. ÉCONOMIE DU SAVOIR ET TRANSFORMATION NUMÉRIQUE}

\section{Croissance, emploi et transformation numerique}

\section{Réseaux mondiaux de production des TIC}

Les réseaux mondiaux de production sont des tissus complexes de flux de connaissances, de biens et de services qui se combinent à diverses étapes de la production. Les entreprises et les industries positionnées au centre des chaînes de valeur mondiales (CVM) ont accès à une plus grande variété de ressources étrangères que celles de la périphérie. Les gains de productivité d'une entité résultent donc non seulement de sa participation aux CVM mais aussi de son positionnement au sein des CVM. Au cours des dernières décennies, la structure noyau/périphérie des CVM a profondément évolué, en particulier dans les secteurs des TIC. En 1995, les chaînes de valeur de la fabrication d'ordinateurs et d'articles électroniques étaient organisées autour d'une poignée de pôles centraux dans les économies à revenu élevé, notamment les États-Unis et le Japon. Puis, entre 1995 et 2011 , les centres traditionnels de production (cercles rouges sur le graphique) ont presque tous beaucoup perdu en importance. Au contraire, dans de nombreux pays d'Asie et d'Europe centrale et orientale, la centralité des activités a fortement augmenté, en particulier en Chine, et aussi en Corée, en Hongrie, en Malaisie et en République tchèque (cercles bleus sur le graphique). En 2011, la production mondiale d'articles informatiques et électroniques n'était plus centrée sur quelques économies à revenu élevé, et s'acheminait vers une distribution plus répartie.

53. Évolution de la centralité de la fabrication de matériel informatique dans les économies, 1995-2011

Centralité mesurée en centralité étrangère totale

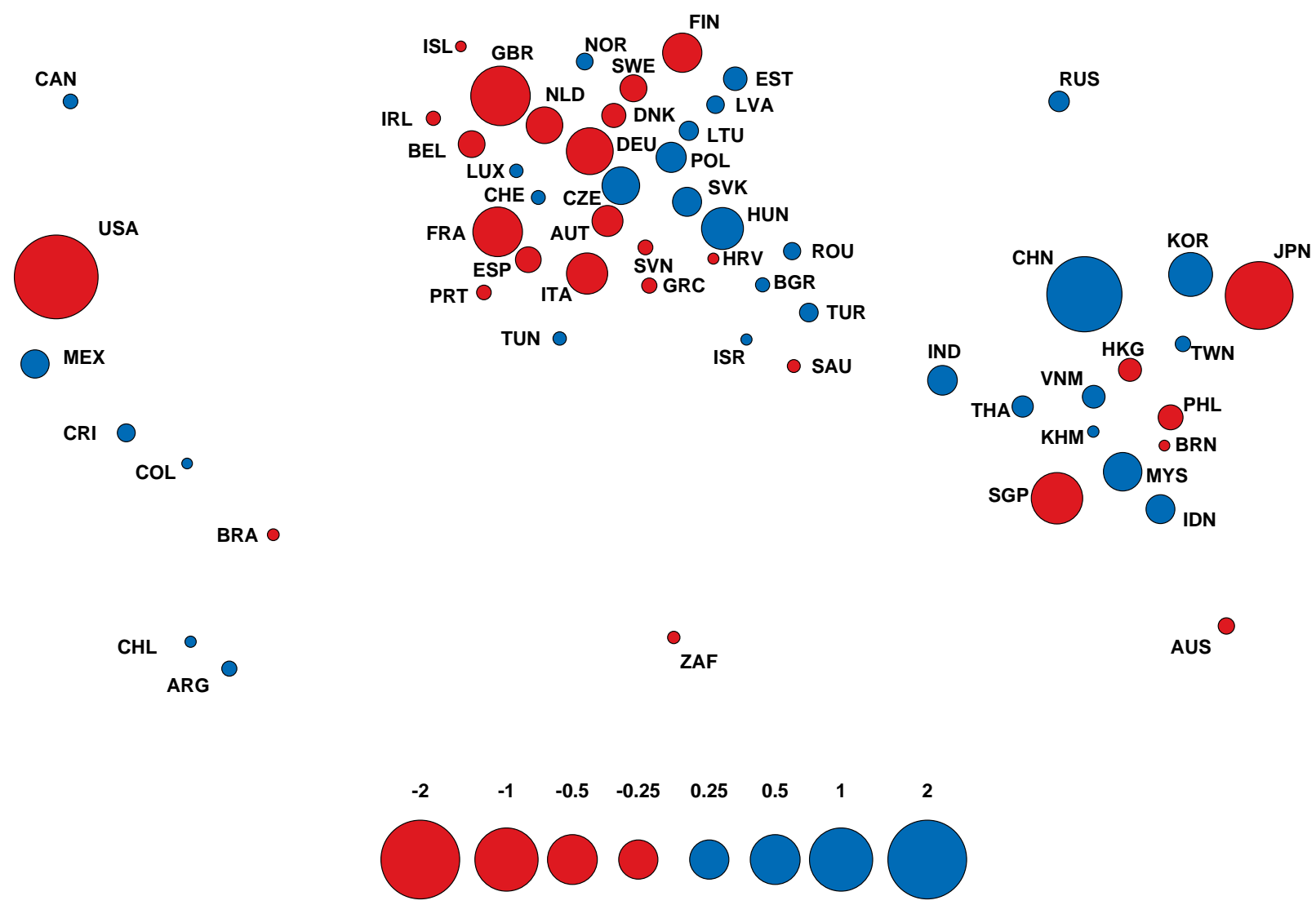

NZL

Source : OCDE, Tableaux internationaux des entrées-sorties (TIES), http://oe.cd/icio, juillet 2017. Davantage de données via StatLink. Voir notes de chapitre.

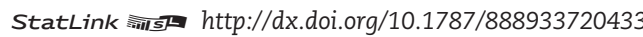

\section{Comment lire ces graphiques}

Les économies sont positionnées en fonction de leur situation géographique. La taille des cercles traduit l'ampleur des variations (en valeur absolue) de la centralité étrangère totale au cours de la période 1995-2001. À des fins de lisibilité, et comme indiqué dans la légende, on a représenté ces variations suivant une échelle logarithmique. Les cercles bleus indiquent une augmentation de la centralité et les cercles rouges une diminution. 


\section{Réseaux mondiaux de production des TIC}

L'évolution de la structure des réseaux mondiaux de production est liée à l'augmentation spectaculaire de l'importance des services informatiques. Encore limitée en 1995, la dépendance des réseaux mondiaux de production aux services informatiques a augmenté dans presque toutes les économies au cours de la période 1995-2011 (cercles bleus sur le graphique 54). Cette influence croissante des services informatiques est particulièrement visible dans les pays où la centralité des activités de fabrication d'ordinateurs et d'articles électroniques a le plus baissé, à savoir l'Allemagne, les États-Unis et le Royaume-Uni. Cependant, l'amplitude de cette évolution reste quelque peu inférieure à celle de la délocalisation de la fabrication d'ordinateurs et d'articles électroniques. Enfin, l'importance croissante des services informatiques, qui va de pair avec la restructuration des économies à revenu élevé, s'observe aussi dans un grand nombre d'économies telles que la Corée, l'Espagne et l'Irlande, et dans beaucoup d'économies émergentes, notamment la Chine, l'Inde et Singapour.

54. Évolution de la centralité des services informatiques dans les économies, 1995-2011

Centralité mesurée en centralité étrangère totale

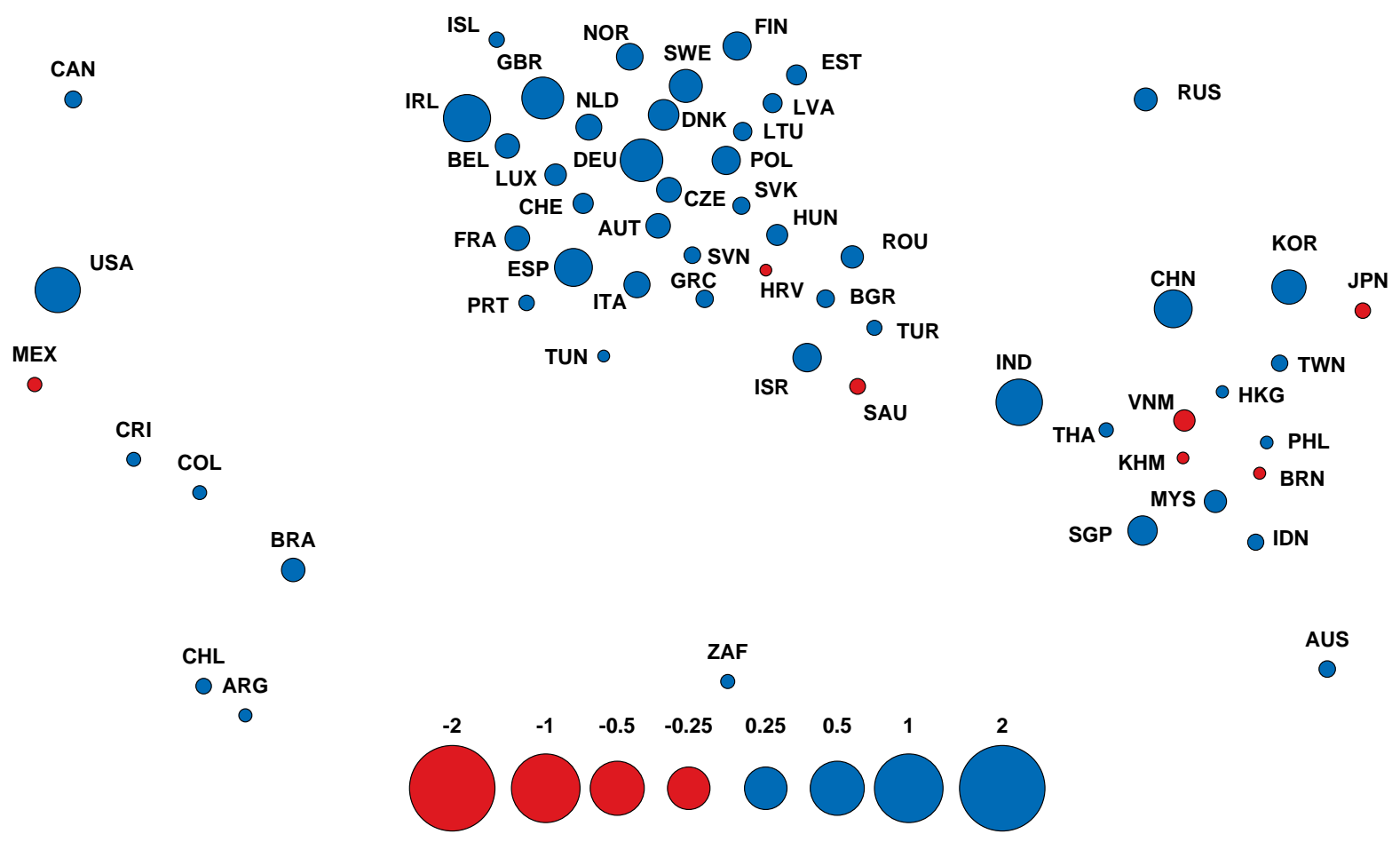

Source : OCDE, Tableaux internationaux des entrées-sorties (TIES), http://oe.cd/icio, juillet 2017. Davantage de données via StatLink. Voir notes de chapitre.

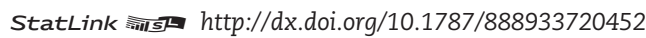

\section{Qu'entend-on par « centralité » et comment la calcule-t-on ?}

La centralité est une mesure de l'influence ou de la connectivité au sein du réseau mondial de production. Les secteurs centraux sont ceux qui sont très connectés (à la fois directement et indirectement) et influents dans ce réseau mondial de production. Inversement, les secteurs périphériques ont des liens ténus avec les autres secteurs et pays et, de ce fait, sont moins influents. Pour calculer cet indicateur, on applique la variante PageRank des centralités de vecteur propre de Bonacich-Katz au réseau des flux d'intrants entre les pays et les secteurs, détaillés dans les Tableaux internationaux des entrées-sorties (TIES) de l'OCDE, édition 2015. Pour chaque couple pays-secteur, on calcule la centralité de référence, ainsi que la somme pondérée des centralités des partenaires commerciaux, où les poids sont les parts des intrants. Ainsi, un pays ou un secteur est dit central s'il est très connecté à d'autres pays et secteurs, à la fois directement et indirectement, du fait d'échanges avec des partenaires commerciaux eux-mêmes très centraux. La centralité totale est la moyenne des centralités calculées avec les liaisons en aval (exportations d'intrants) et les liaisons en amont (importations d'intrants). On décompose la centralité selon son origine étrangère ou locale. La centralité étrangère est celle qui est due aux liaisons (directes et indirectes) avec les secteurs étrangers, tandis que la centralité locale est celle qui est due aux liaisons (directes et indirectes) avec les secteurs locaux. La fabrication de matériel informatique recouvre les divisions 30, 32 et 33 de la CITI rév. 3 : "fabrication d'ordinateurs, d'articles électroniques et optiques". Les services informatiques sont ceux de la division 72 de la CITI rév. 3 : "activités informatiques et activités rattachées ". Voir Criscuolo et Timmis (à paraître). 


\section{1. ÉCONOMIE DU SAVOIR ET TRANSFORMATION NUMÉRIQUE}

\section{Croissance, emploi et transformation numerique}

\section{Pôles TIC}

La centralité est une mesure de l'influence relative d'un pays-secteur vis-à-vis de tous les autres du réseau. Elle peut être étudiée à l'échelle du réseau mondial de production comme à l'échelon d'un réseau local. La position relative (centralité) d'un secteur tend à ne pas être la même au sein du réseau mondial de production et au sein des réseaux locaux. Pourtant, la réorganisation radicale des réseaux mondiaux de fabrication de matériel informatique a souvent été accompagnée d'évolutions largement semblables au niveau local. Bon nombre d'économies à revenu élevé, qui ont vu leur influence chuter dans les réseaux mondiaux de fabrication de matériel informatique (par exemple, les États-Unis, le Japon et le Royaume-Uni) ont presque toujours vu aussi l'influence de ce secteur reculer dans leurs réseaux locaux de production. Toutefois, la variation de la centralité locale est généralement de moindre ampleur que celle de la centralité étrangère. Au contraire, plusieurs économies d'Europe de l'Est et d'Asie ont vu la centralité de leurs activités de fabrication de matériel informatique augmenter dans le réseau mondial et dans leur réseau national (partie A). Mais la relation entre les deux indicateurs est ici plus faible : par exemple, dans le cas de l'Indonésie, la centralité locale a progressé beaucoup plus vite que la centralité étrangère, tandis qu'en Corée, l'évolution a été inverse.

De la même façon, les variations de la centralité des services informatiques au sein des réseaux mondiaux de production sont souvent reflétées dans les réseaux locaux de production. Ainsi, les activités de services informatiques des États-Unis, de l'Irlande, de l'Inde et de plusieurs autres économies sont devenues de plus en plus centrales dans la production mondiale, comme l'indique l'augmentation de la centralité étrangère correspondante (partie B). Dans beaucoup de ces économies, elles ont aussi gagné en influence dans les réseaux locaux de production, la variation locale étant parfois supérieure à la variation observée à l'échelle mondiale, par exemple au Danemark, en Inde et en République tchèque.

\section{Variations les plus fortes de la centralité étrangère et locale : fabrication et services informatiques, 1995-2011}

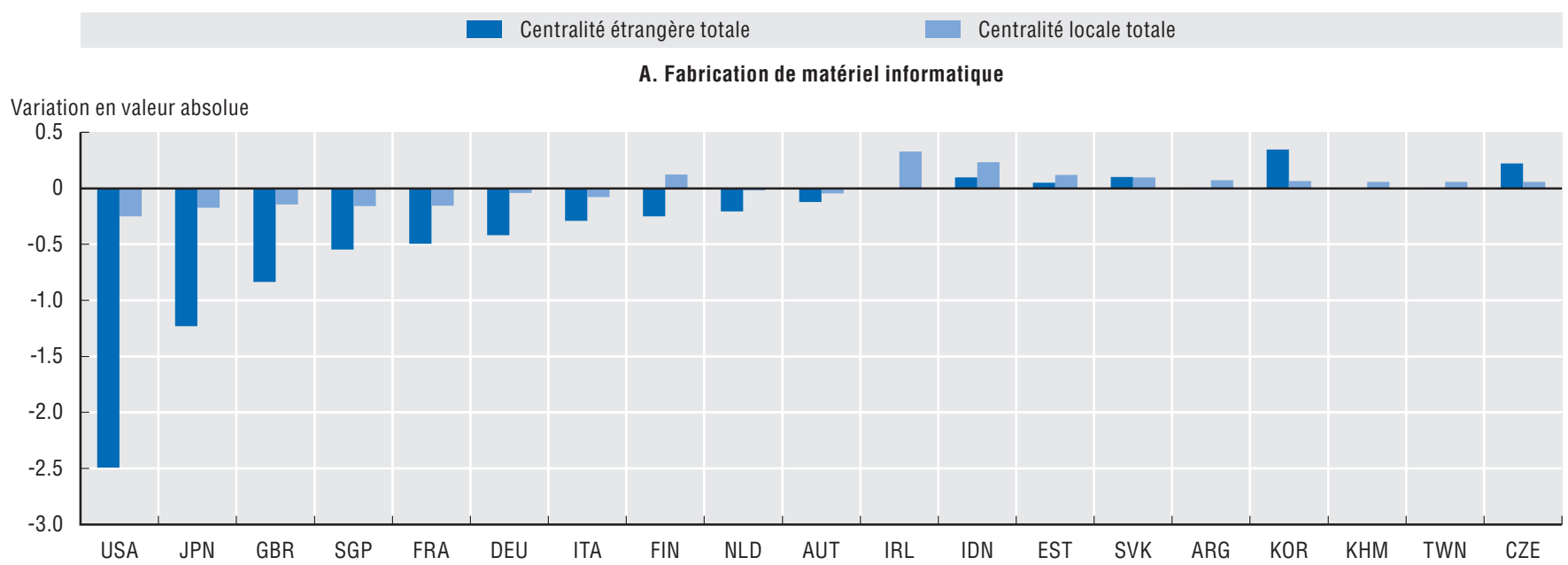

B. Services informatiques

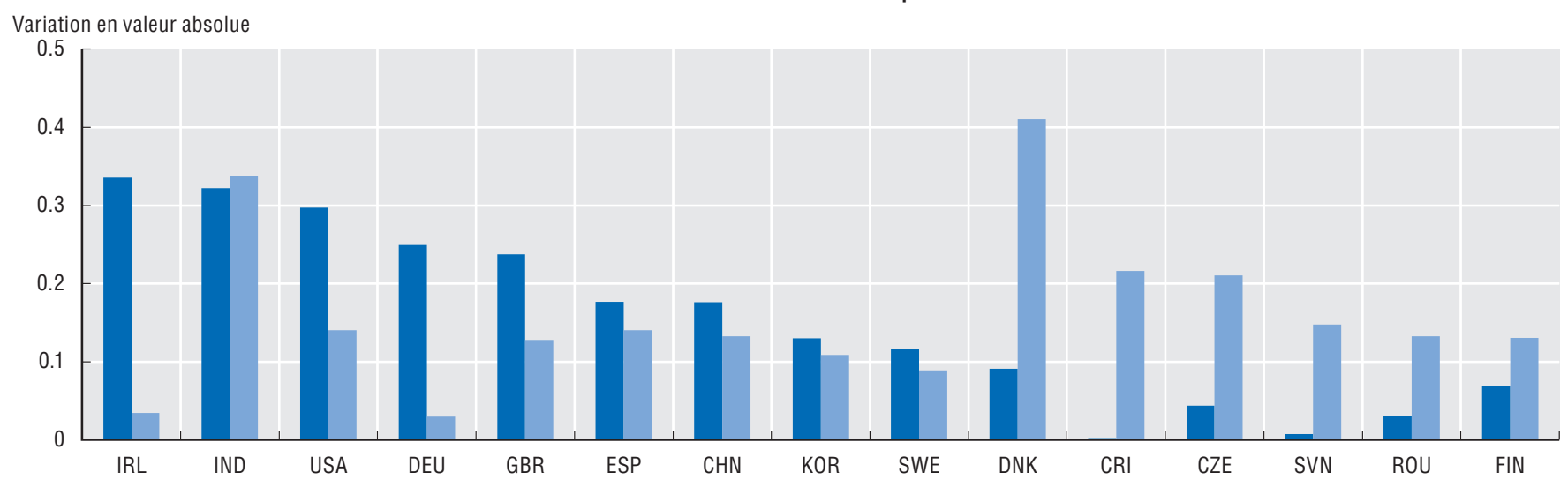

Source : OCDE, Tableaux internationaux des entrées-sorties (TIES), http://oe.cd/icio, juillet 2017. Davantage de données via StatLink. Voir notes de chapitre. StatLink 角西 http://dx.doi.org/10.1787/888933720471

\section{Comment lire ces graphiques}

Les barres représentent les variations (en valeur absolue) des centralités locale et étrangère totales au cours de la période 1995-2011. La partie A concerne les activités de fabrication de matériel informatique ("fabrication d'ordinateurs, d'articles électroniques et optiques ") et la partie B les activités de services informatiques (" activités informatiques et activités rattachées »). 


\section{1. ÉCONOMIE DU SAVOIR ET TRANSFORMATION NUMÉRIQUE}

2. Croissance, emploi et transformation numerique

\section{Pôles TIC}

Au cours de la période 1995-2011, la fabrication de matériel informatique s'est éloignée des quelques pôles à revenu élevé qui en concentraient les activités pour s'implanter dans les économies d'Asie, lesquelles sont alors devenues les nouveaux pôles centraux dans ce domaine. En 1995, la production mondiale était largement centrée sur les États-Unis et le Japon (graphique 56, partie en haut à gauche). En 2011, ces pôles centraux avaient perdu en importance et la production mondiale s'était déplacée vers l'est. Des dix pôles prédominants en 1995, seuls la Corée et le Taipei chinois avaient plus d'influence en 2011. En effet, la très large réorganisation de la production a conduit des économies émergentes relativement périphériques en 1995 à devenir des pôles centraux en 2011. Par exemple, la Chine (20en 1995) a remplacé les États-Unis à la tête des pays-secteurs les plus centraux en 2011.

En revanche, la centralité des services informatiques a presque partout augmenté. En 1995, les services informatiques n'avaient pas d'influence particulière dans la production mondiale (graphique 56, partie en bas à gauche). Même des pays centraux comme l'Allemagne et les États-Unis affichaient une centralité faible par rapport à celle de la fabrication de matériel informatique. Puis, entre 1995 et 2011 , les services informatiques ont gagné en importance relative à l'échelle mondiale, y compris dans bon nombre d'économies qui occupaient déjà les positions les plus centrales dans ce secteur au début de la période. Par exemple, les États-Unis, l'Allemagne et le Royaume-Uni, trois des quatre pays les plus centraux pour les services informatiques en 1995, l'étaient toujours en 2011. Toutefois, la période a également vu émerger de nouveaux pôles centraux, notamment l'Inde et l'Irlande.

\section{Les dix pôles informatiques les plus centraux, 1995 et 2011}

Centralité mesurée en centralité étrangère totale
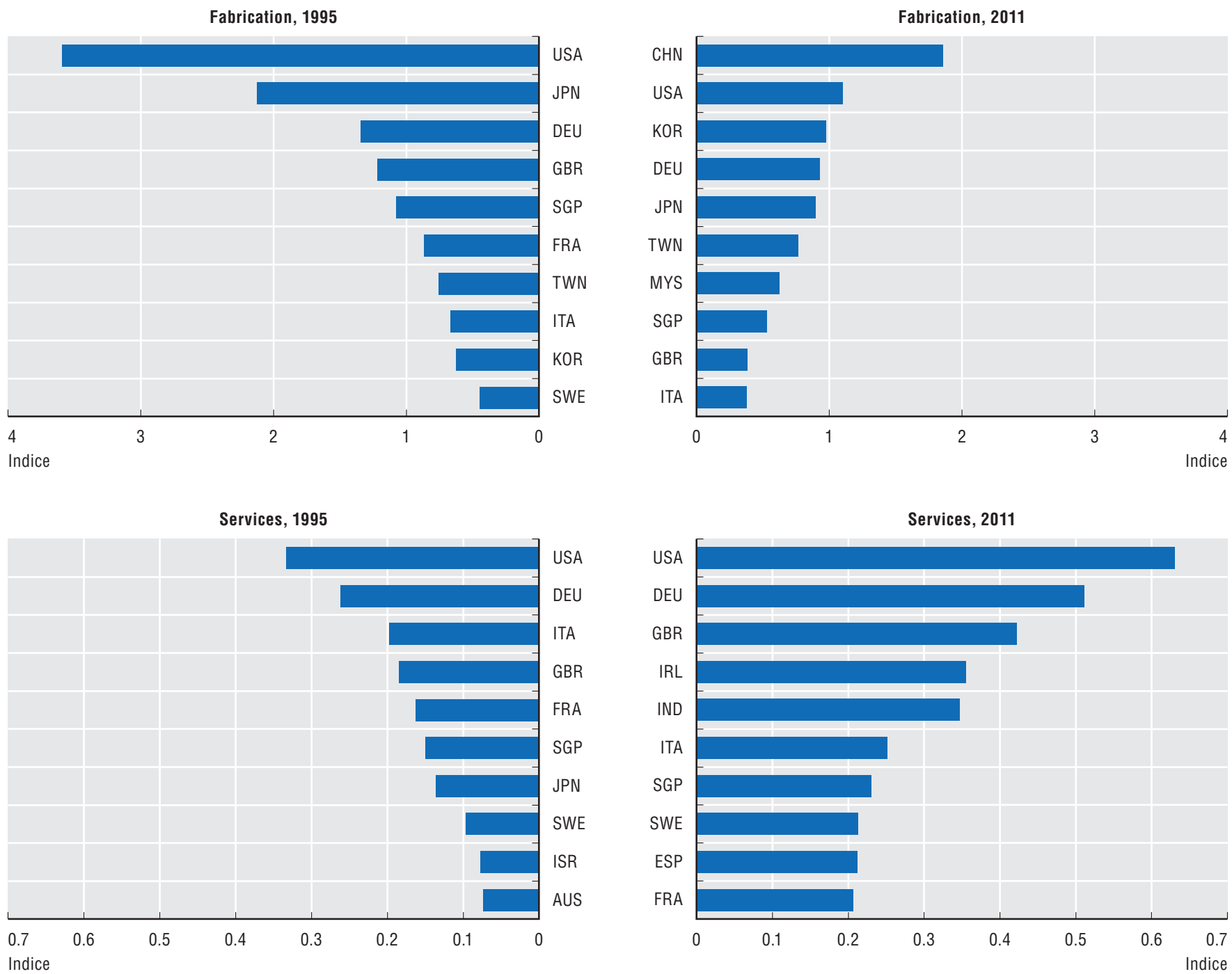

Source : OCDE, Tableaux internationaux des entrées-sorties (TIES), http://oe.cd/icio, juillet 2017. Voir notes de chapitre.

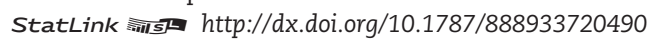

\section{Comment lire ces graphiques}

Les barres représentent la centralité étrangère totale des dix économies en tête de ce classement en 1995 (parties de gauche du graphique) et en 2011 (parties de droite du graphique). Les parties du haut concernent les activités de fabrication de matériel informatique (" fabrication d'ordinateurs, d'articles électroniques et optiques ») et les parties du bas les activités de services informatiques (« activités informatiques et activités rattachées »). 


\section{1. ÉCONOMIE DU SAVOIR ET TRANSFORMATION NUMÉRIQUE}

\section{Croissance, emploi et transformation numerique}

\section{Réduire la fracture numérique}

L'économie numérique d'aujourd'hui est caractérisée par l'existence d'une connectivité entre utilisateurs et entre terminaux, ainsi que par la convergence de pans précédemment distincts des écosystèmes de communication, comme les réseaux fixe et mobile, les données et la voix, ou encore les télécommunications et la diffusion. Dans les pays de l'OCDE et les économies émergentes, l'internet et les terminaux connectés occupent désormais une place déterminante dans la vie quotidienne de la majeure partie de la population. La part des internautes dans les pays de l'OCDE a augmenté en moyenne de 30 points de pourcentage au cours des dix dernières années (85\% en 2016 contre $56 \%$ en 2005), et plus que doublé dans les cas de la Grèce, du Mexique et de la Turquie. Comblant l'écart avec la zone OCDE, l'Afrique du Sud, le Brésil et la Chine sont aujourd'hui des pays où plus de $50 \%$ des 16 à 74 ans utilisent l'internet. Mais si certaines économies parviennent à saturation (le nombre d'internautes approche $100 \%$ ), d'autres ont encore une marge de rattrapage importante, en particulier les pays à faible revenu.

\section{7. Évolution de l'utilisation de l'internet, 2005-16}

\section{A. Pourcentage d'internautes chez les 16-74 ans, fourchette observée dans la zone OCDE}

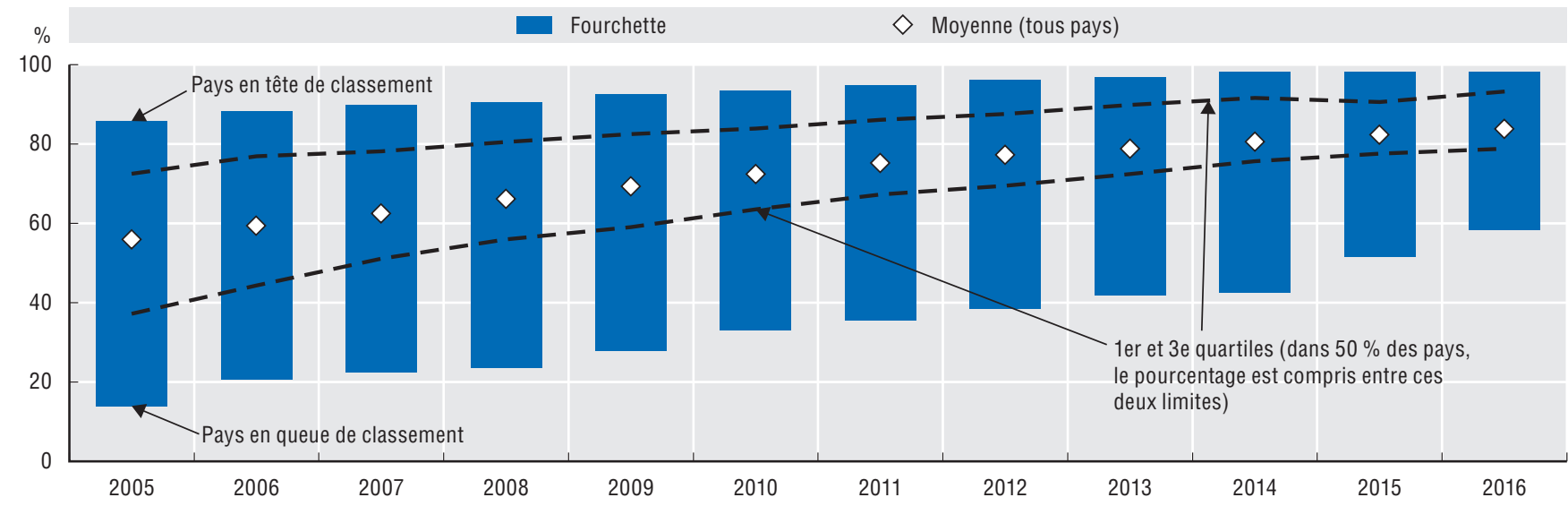

B. Variation du pourcentage d'internautes entre 2006 et 2016

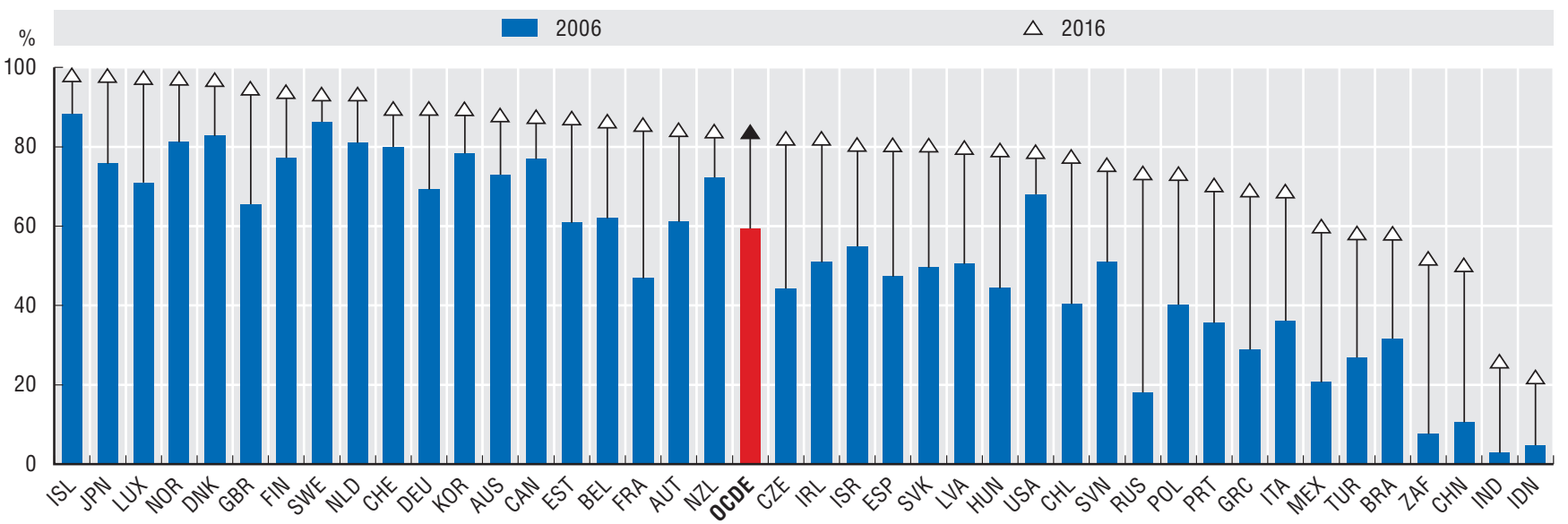

Source : Calculs de l'OCDE à partir de la Base de données sur l'accès et utilisation des TIC par les ménages et les individus, http://oe.cd/hhind ; UIT, Base de données Indicateurs des télécommunications/TIC dans le monde; et sources nationales, juin 2017. Voir notes de chapitre.

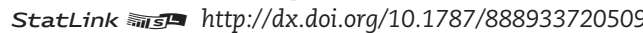

\section{Comment lire ces graphiques}

La partie A indique, pour chaque année comprise entre 2005 et 2016, l'écart entre le pays où les 16-74 ans utilisent le moins internet et le pays où ils l'utilisent le plus. En 2016, dans la zone OCDE, en moyenne 84 \% des personnes âgées de 16 à 74 ans utilisaient internet, les moyennes nationales allant de 58 \% (niveau le plus bas) à $98 \%$ (niveau le plus haut), et la moitié des pays affichant une moyenne comprise entre $79 \%$ (premier quartile) et $93 \%$ (troisième quartile). La partie B indique dans quelle mesure l'utilisation de l'internet chez les 16-74 ans a progressé entre 2006 et 2016 dans chaque pays. En Grèce, par exemple, la proportion est passée d'un tiers à 70 \% 


\section{1. ÉCONOMIE DU SAVOIR ET TRANSFORMATION NUMÉRIQUE}

2. Croissance, emploi et transformation numerique

\section{Réduire la fracture numérique}

Le fossé intergénérationnel observé chez les internautes se réduit régulièrement depuis 2005, une tendance qui devrait se poursuivre, à mesure que les technologies permettent de réduire le coût de l'accès en ligne et que les " natifs numériques " deviennent adultes. Cependant, il reste encore des écarts importants entre générations dans une majorité de pays de l'OCDE, ce qui soulève la question stratégique de l'inclusion numérique des personnes âgées. En 2016, dans la zone OCDE, le taux de pénétration de l'internet était légèrement supérieur à $60 \%$ chez les $55-74$ ans, alors qu'il dépassait $95 \%$ chez les $16-24$ ans. Le pourcentage d'internautes chez les séniors varie aussi très largement d'un pays à l'autre. En 2016, plus de $90 \%$ des $55-74$ ans déclaraient utiliser l'internet au Danemark, en Islande, au Luxembourg, en Norvège et en Suède, contre moins de $20 \%$ en Turquie.

\section{8. Évolution de l'utilisation de l'internet, par âge, 2005-16}
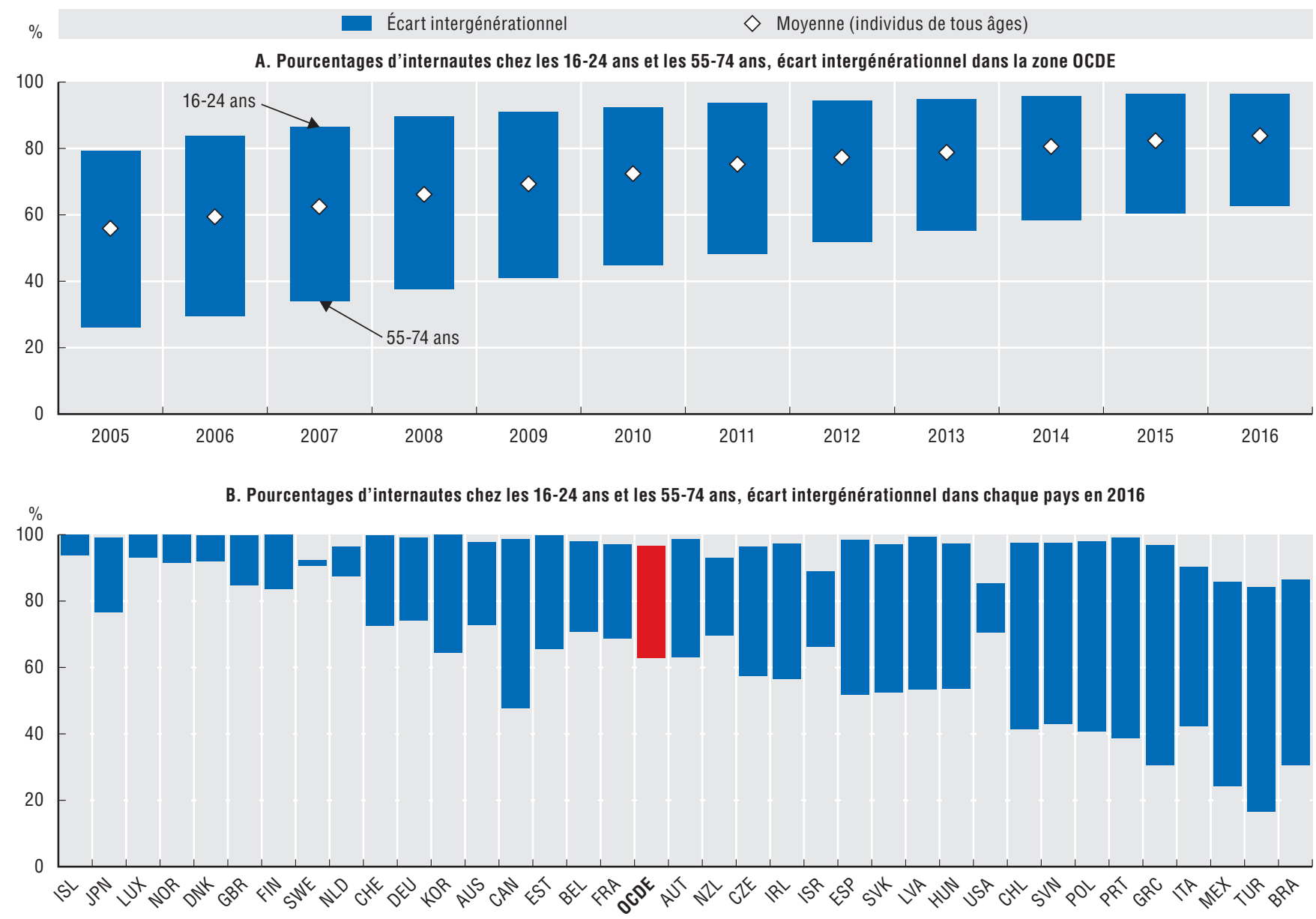

Source : Calculs de l'OCDE à partir de la Base de données sur l'accès et utilisation des TIC par les ménages et les individus, http://oe.cd/hhind, juin 2017. Davantage de données via StatLink. Voir notes de chapitre. 


\section{1. ÉCONOMIE DU SAVOIR ET TRANSFORMATION NUMÉRIQUE}

\section{Agir maintenant au service de l'innovation}

\section{Place aux femmes dans la science et l'innovation}

L'égalité entre les femmes et les hommes est l'un des objectifs des politiques de recherche et d'innovation de nombreux pays et organisations. Il s'agit de garantir aux femmes et aux hommes les mêmes possibilités de participation et opportunités, de l'instruction obligatoire jusqu'aux carrières de recherche où les femmes doivent avoir les mêmes opportunités que les hommes, des postes de début de carrière jusqu'aux postes décisionnels importants. L'objectif est également d'intégrer la dimension hommesfemmes dans le contenu de la recherche, en tenant compte des caractéristiques biologiques, sociales et culturelles des hommes et des femmes. Dans la zone OCDE, environ 30 \% des titulaires d'un diplôme de sciences naturelles, d'ingénierie ou de TIC sont des femmes, ce qui témoigne d'une sous-représentation considérable. De plus, la part des femmes dans la population des auteurs scientifiques correspondants, proche de $22 \%$, est très inférieure au pourcentage de femmes diplômées dans l'enseignement supérieur et au niveau doctoral. Ce pourcentage est même encore plus faible dans les sous-groupes d'auteurs ayant des responsabilités décisionnelles, c'est-à-dire les auteurs qui ont des revenus élevés, une activité rémunérée de recension, une activité éditoriale, ou qui se consacrent entièrement à la recherche. Un point pourrait s'avérer préoccupant : la relative sous-représentation des femmes dans certaines activités extérieures de plus en plus valorisées dans les exercices d'évaluation de la recherche publique, telles que la citation de de travaux de recherche dans des rapports des médias ou dans des documents techniques tels que les brevets.

\section{Diplômées de l'enseignement supérieur en sciences naturelles, ingénierie et TIC, 2015}

En pourcentage de la population diplômée de l'enseignement supérieur en sciences naturelles, ingénierie et TIC

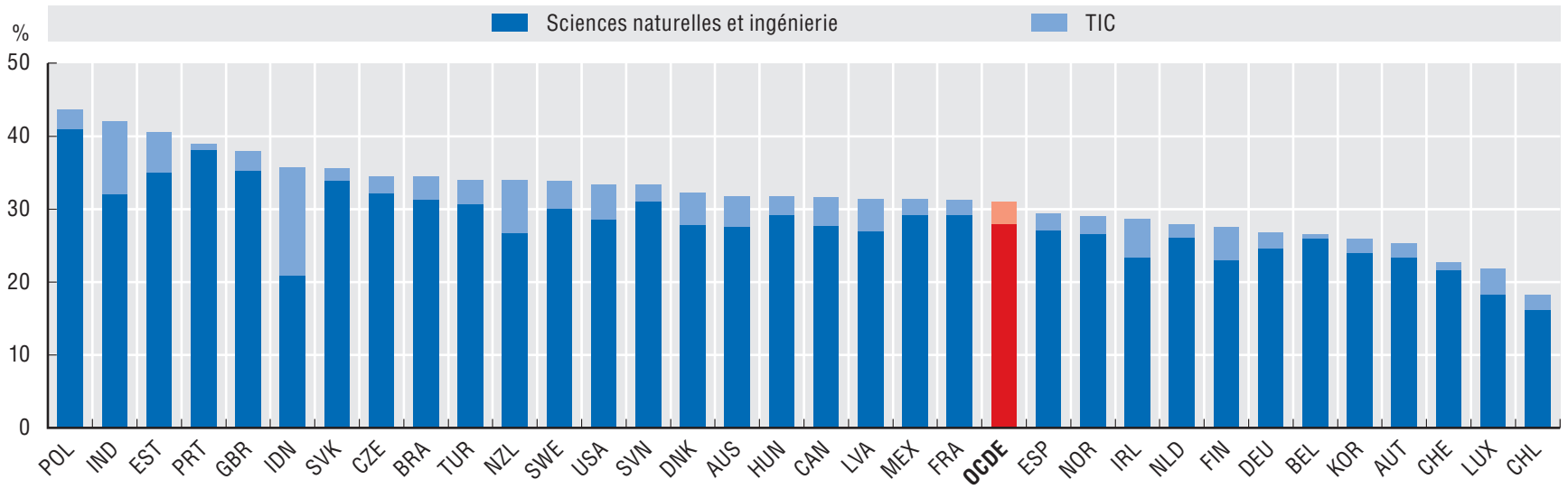

Source : Calculs de l'OCDE, d'après OCDE, Statistiques et bases de données sur l'éducation, septembre 2017. Davantage de données via StatLink. Voir notes de chapitre.

60. Les femmes dans la science, $\mathbf{2 0 1 5}$

Part des femmes dans les catégories suivantes d'auteurs scientifiques correspondants

Ensemble des auteurs correspondants

Responsabilités scientifiques Activité de recension Activité rémunérée de recension Membre du comité éditorial Membre rémunéré du comité éditoria

Pertinence / impact de la production scientifique Cité dans une revue à comité de lecture

Cité dans un document technique (brevet) Cité dans un rapport gouvernemental/stratégique Cité dans le protocole d'un praticien (médecine) Cité dans les médias

Cité dans le cadre d'une action en justice

Temps pour la recherche (travailleurs à temps plein): Recherche à temps plein Recherche à temps partie

Revenus : Revenus annuels élevés > 105000 USD

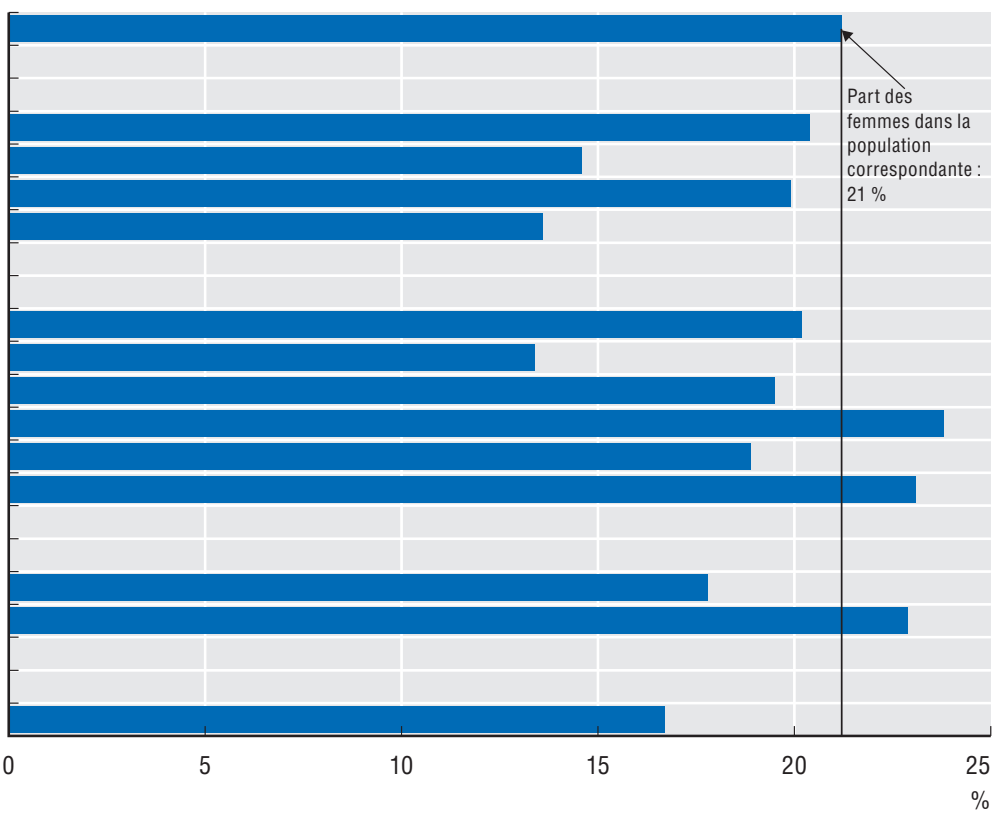

Source : Analyse de l'OCDE, d'après OCDE (2016), « International Survey of Scientific Authors: Public use microdata for 2015 pilot study - ISSA1 », OCDE, Direction de la science, de la technologie et de l'innovation, http://oe.cd/issa, juin 2017. Voir notes de chapitre. 


\section{Place aux femmes dans la science et l'innovation}

La contribution des femmes au développement de nouvelles technologies, mesurée en pourcentage des brevets ayant au moins une inventrice, s'échelonne d'environ $4 \%$ en moyenne en Autriche à plus de $15 \%$ en moyenne au Portugal. Entre 2012 et 2015 , des femmes ont figuré parmi les inventeurs de $10 \%$ des brevets aux États-Unis, et de $7 \%$ des brevets au Japon. La part des femmes dans la population des inventeurs varie également beaucoup d'un domaine technologique à un autre. Dans la plupart des pays, la contribution des femmes aux inventions brevetées est la plus élevée dans le secteur pharmaceutique (jusqu'à $42 \%$ en Espagne) et les biotechnologies ( $49 \%$ en Pologne), et la plus faible dans le génie civil et les télécommunications (0.3\% en Autriche et en République tchèque). Les facteurs qui peuvent expliquer les tendances observées sont notamment les différences de spécialisation technologique et sectorielle des pays, la proportion de diplômées dans les disciplines scientifiques, et le degré de participation des femmes au marché du travail.

\section{Contribution des femmes aux inventions brevetées, 2012-15}

En pourcentage du nombre de familles de brevets IP5 par technologie et par pays de résidence des inventeurs

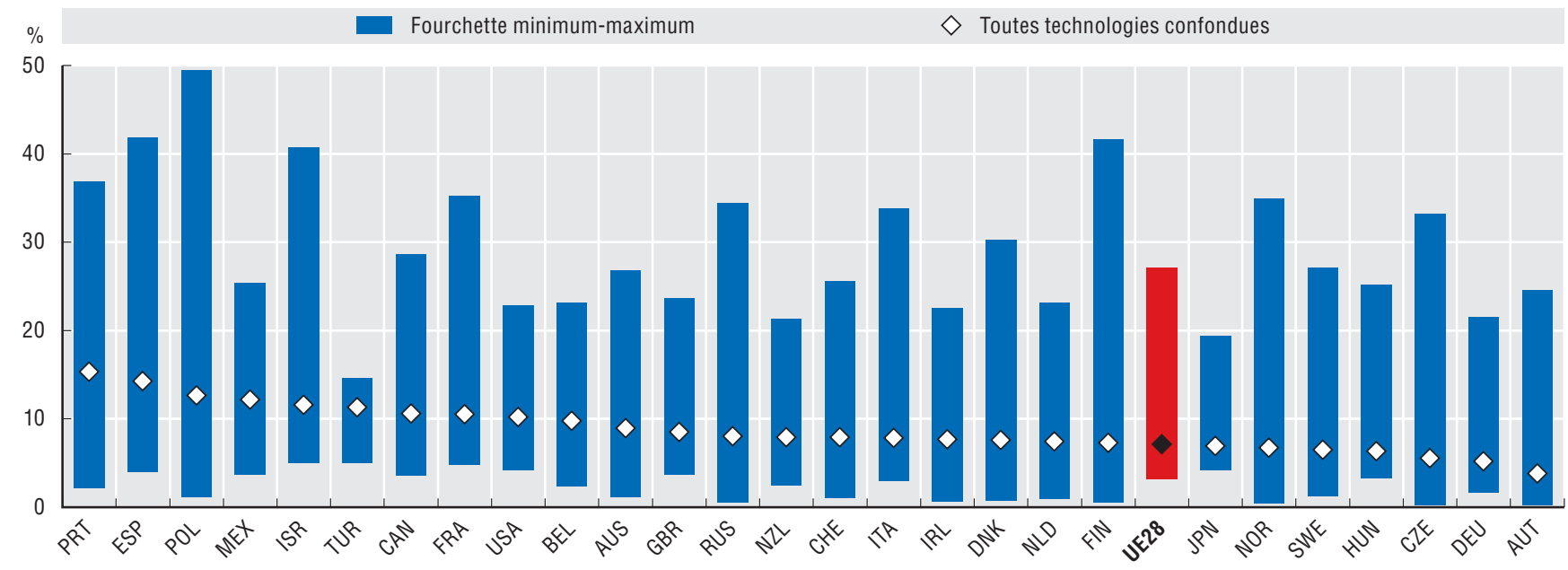

Source : OCDE, STI Microdata Lab : Base de données sur la propriété intellectuelle, http://oe.cd/ipstats, juin 2017. Davantage de données via StatLink. Voir notes de chapitre.

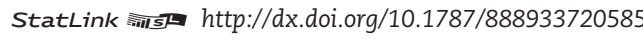

\section{Comment recenser les inventrices}

Pour recenser les inventrices, on utilise des dictionnaires de genre des noms, spécifiques pour chaque pays, que l'on croise avec les noms des inventeurs et inventrices cités dans les brevets (pour de plus amples informations, voir Lax Martínez, Raffo et Saito, 2016). Les statistiques sont disponibles uniquement pour les économies où plus de $80 \%$ des prénoms peuvent être clairement attribués à l'un ou l'autre des sexes. Comme il est difficile de faire la distinction entre les noms d'inventeurs et d'inventrices dans les économies d'Asie, il n’a pas été possible de calculer cet indicateur pour des économies comme la Chine et la Corée. Les données se rapportent aux familles IP5 par date de dépôt du brevet, et sont groupées en fonction du lieu de résidence et du sexe de la personne à l'origine de l'invention, avec des comptages fractionnaires. Les brevets sont affectés aux domaines technologiques sur la base de leurs codes dans la Classification internationale des brevets (CIB), suivant la concordance établie par l'OMPI (2013).

\section{Comment mesurer la représentation des femmes dans la science}

On estime la représentation des femmes dans la science sur la base des informations rapportées par les autrices elles-mêmes dans une enquête pilote conduite par l'OCDE auprès des autrices et des auteurs scientifiques en juillet 2015 (http://oe.cd/issa). L'échantillon est tiré des documents publiés en 2011 et indexés dans la base Scopus. On s'intéresse aux personnes désignées dans les documents comme " auteurs correspondants ». Les domaines couverts dans cette étude sont les suivants : Arts et lettres, Commerce, Génie chimique, Immunologie et microbiologie, Science des matériaux, Neurosciences, Physique et Astronomie. Les moyennes pondérées tiennent compte de la structure de l'échantillon et des schémas de non-réponse par domaine, pays d'affiliation et statut de la revue. Dans le graphique, on peut évaluer la représentation des femmes, en valeur absolue ou relative, selon différentes caractéristiques liées à la recherche scientifique en comparant les chiffres obtenus à la valeur de référence de $50 \%$ ou à la proportion de femmes dans la population considérée. Les fichiers à usage public contenant les micro-données de l'enquête pilote ISSA de 2015 peuvent être téléchargés à des fins de recherche depuis le site du projet. 


\section{1. ÉCONOMIE DU SAVOIR ET TRANSFORMATION NUMÉRIQUE}

\section{Agir maintenant au service de l'innovation}

\section{Financer la recherche à long terme plus risquée}

La recherche-développement (R-D) est un moteur essentiel de la performance économique à long terme. Les pouvoirs publics jouent un rôle important en soutenant les investissements dans ce domaine, en particulier lorsque le secteur privé est réticent à engager des dépenses étant donné l'échelle des investissements requis, la non-exclusivité des résultats exploitables et le degré d'incertitude ou de risque qui entoure le projet.

Au cours de la période 2007-16, le total des investissements publics a baissé tant en pourcentage des dépenses publiques qu'en pourcentage du PIB dans presque tous les pays de l'OCDE (OCDE, 2017b). Il en résulte que les budgets publics de R-D se sont aplanis ou ont décliné dans de nombreux pays de l'OCDE et économies du G20. Si le niveau des budgets publics de R-D dans l'UE28 est resté globalement constant entre 2008 et 2015, ceux de la France et de l'Italie ont reculé en termes réels de plus de $20 \%$, et ceux de l'Espagne et de la Lettonie de plus de $30 \%$. Au contraire, les budgets de R-D des États-Unis et du Royaume-Uni ont commencé à repartir à la hausse après avoir baissé entre 2009 et 2012/13. Pendant ce temps, d'autres pays ont augmenté leur budget de R-D, le plus notablement la Turquie (progression de près de $80 \%$ ) et la Corée (progression de $50 \%$ ).

Dans certains cas, la baisse des budgets publics de R-D peut s'expliquer en partie par une réorientation des ressources vers d'autres instruments de soutien à l'innovation, par exemple des incitations fiscales à la R-D. Cependant, la modification des trains de mesures en faveur de la R-D peut avoir un impact sur le financement de la recherche à long terme plus risquée, car les incitations fiscales à la R-D visent généralement les entreprises, qui tendent à moins s'engager dans la recherche fondamentale que les autres secteurs.

62. Budgets publics destinés à la R-D dans une sélection d'économies, 2008-16 Indice en prix constants (USD à PPA $2008=100)$
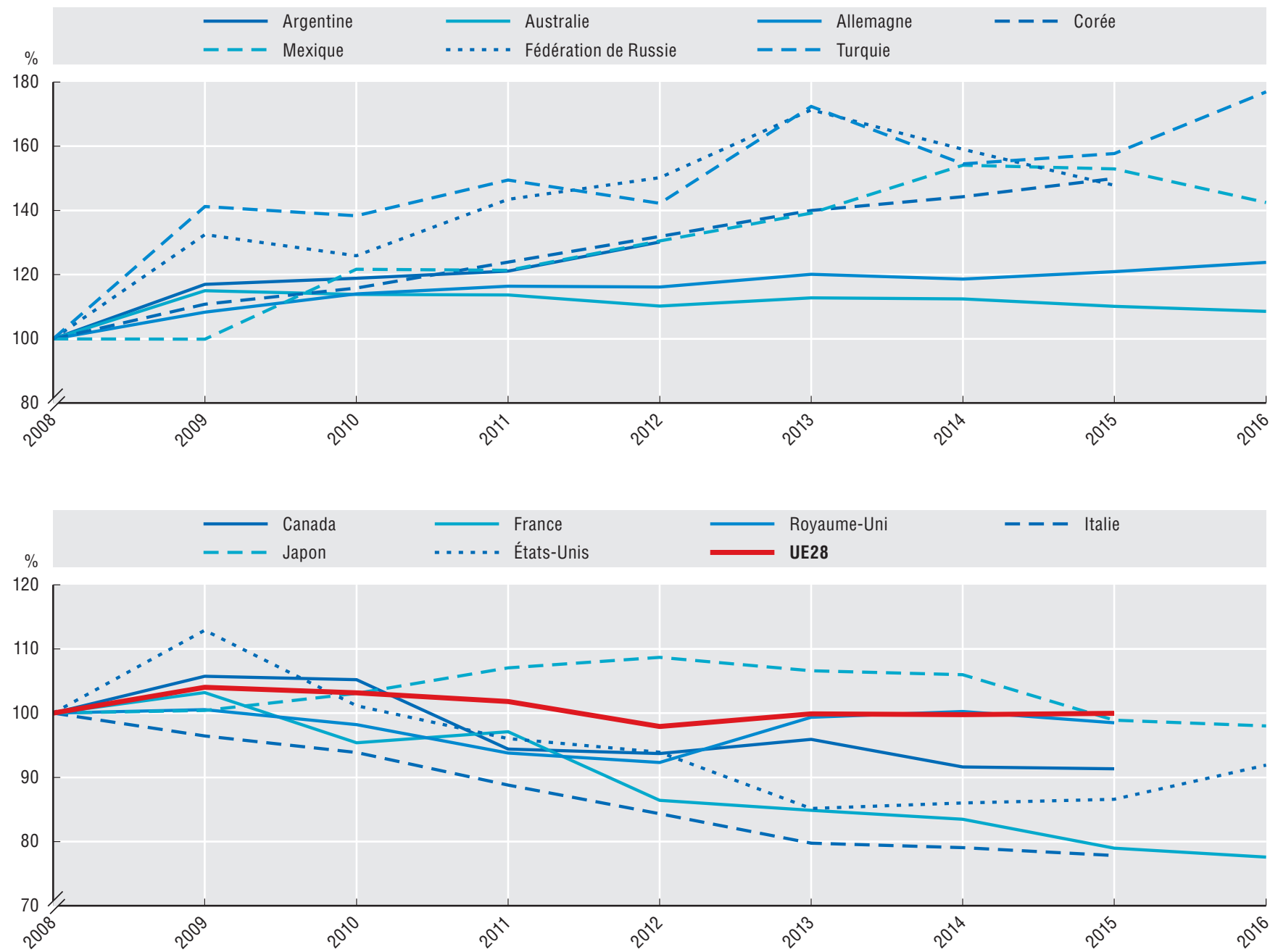

Source : OCDE, Base de données sur les Statistiques de la recherche et développement, http://oe.cd/srd-fr, et Eurostat, Statistiques de la recherche et développement, juillet 2017. Davantage de données via StatLink. Voir notes de chapitre. 


\section{Financer la recherche à long terme plus risquée}

Les États financent des domaines de recherche très divers, mais augmentent aussi leur soutien aux activités de recherche à long terme plus risquées liées aux défis sociétaux tels que le changement climatique, l'alimentation de la population mondiale toujours plus nombreuse, et les problèmes de santé tels que la démence. En 2016, le budget public de R-D des États-Unis a atteint 149 milliards USD - soit plus que les neuf pays suivants combinés ; $60 \%$ de cette somme était destinée à la R-D relative à la défense et à l'espace. Le Royaume-Uni et la Turquie sont les seuls autres pays de l'OCDE à consacrer $20 \%$ ou plus de leur budget public de R-D à la défense et à l'espace. Les pourcentages applicables à la Chine, à Israël et la Fédération de Russie sont indisponibles.

Le progrès général des connaissances est le principal objectif socio-économique de beaucoup de pays parce qu'il inclut tous les crédits budgétaires consacrés à la R-D mais non rattachables à un objectif spécifique, ainsi que toute la R-D financée sur les subventions générales des ministères de l'Éducation. D’après les données plus détaillées disponibles pour un sous-ensemble de 15 pays européens, les sciences naturelles sont le principal récipiendaire de ces fonds pour la R-D.

De nombreux pays allouent une part appréciable de leurs financements à l'agriculture, l'environnement et l'exploration du milieu terrestre, dans la perspective de relever les grands défis sociétaux que sont l'alimentation durable de la population mondiale en augmentation et l'atténuation du changement climatique. La production industrielle, la technologie, l'énergie et les infrastructures recueillent également une proportion importante des financements. Au contraire, la part consacrée aux structures et relations sociales qui comprend la R-D liée à l'amélioration de l'offre éducative, est généralement relativement faible.

Dans la zone OCDE, les crédits budgétaires de R-D liés à la défense et à l'espace ont reculé en termes réels, de $32 \%$ en 2006 à $24 \%$ en 2016. Le gros de cette baisse a concerné le secteur de la défense, notamment en France (-80 \%) et en Suède (-77 \%). Seules la Corée et la Pologne ont augmenté leurs budgets de R-D dédiés à la défense. Au cours de la même période, les crédits budgétaires imputés au progrès général des connaissances ont été portés de $28 \%$ à $32 \%$, ceux des autres domaines restant relativement stables.

\section{Crédits budgétaires publics de R-D, par objectif socio-économique, 2016}

En pourcentage du total des CBPRD

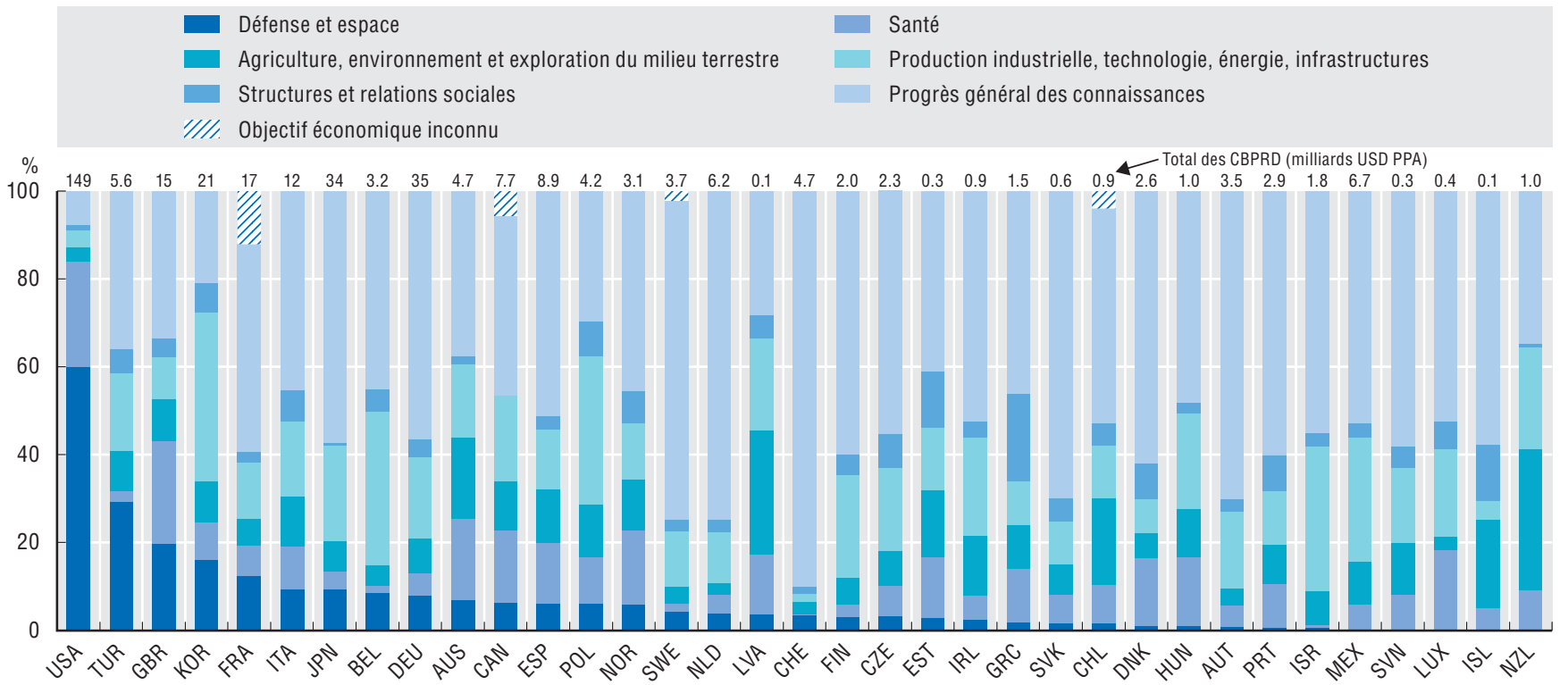

Source : OCDE, Base de données sur les Statistiques de la recherche et développement, http://oe.cd/srd-fr ; et Eurostat, Statistiques de la recherche et développement, juillet 2017. Davantage de données via StatLink. Voir notes de chapitre.

StatLink *iाls http://dx.doi.org/10.1787/888933720623

\section{Comment mesurer l'aide publique directe à la R-D}

Les données relatives aux " crédits budgétaires publics de R-D " (CBPRD) recouvrent l'ensemble des crédits budgétaires financés par des recettes publiques (par exemple des recettes fiscales) inscrites au budget. Autrement dit, elles incluent les crédits alloués dans le cadre de la procédure budgétaire, mais excluent les unités extrabudgétaires et le financement de la R-D par des entreprises publiques. Globalement plus récentes que les données d'enquête sur la R-D, elles peuvent néanmoins ne pas suivre précisément la définition de la R-D du Manuel de Frascati de l'OCDE du fait du niveau de détail limité dans les systèmes budgétaires. De même, il peut être difficile de toutes les imputer à des objectifs socio-économiques précis. Dans certains cas, le financement d'un projet à long terme peut être alloué à la R-D en une seule année. Enfin, dans certaines économies du G20 (par exemple, la Chine et la Fédération de Russie), il n'est pas possible de distinguer l'aide budgétaire à la R-D des autres investissements publics dans les STI. Pour obtenir davantage de données, voir http://oe.cd/rds. 


\section{1. ÉCONOMIE DU SAVOIR ET TRANSFORMATION NUMÉRIQUE}

\section{Agir maintenant au service de l'innovation}

\section{Un défi mondial : soigner la démence}

La démence est, comme beaucoup de maladies neurodégénératives, une maladie débilitante pour laquelle il n'existe à l'heure actuelle aucun remède. Les coûts humains et financiers qu'elle engendre sont une préoccupation croissante, en particulier du fait du vieillissement de la population mondiale. La recherche scientifique étant un pilier des efforts entrepris pour relever ce défi mondial, l'OCDE a appelé à agir pour rééquilibrer les risques et les bénéfices de la recherche afin d'encourager une approche plus large qui aide à cibler la maladie à un stade précoce (OCDE, 2015b). Une analyse expérimentale des résumés de publications scientifiques des vingt dernières années montre que le nombre de travaux de recherche consacrés à la démence dans les pays les plus actifs en la matière a régulièrement augmenté jusqu'en 2012. Après cette date, la tendance haussière a ralenti dans la plupart de ces pays, y compris aux États-Unis où, selon les estimations, $1.2 \%$ des crédits budgétaires publics de recherche avaient été imputés aux maladies neurodégénératives en 2012 (OCDE, 2015b). L'indexation incomplète des publications des dernières années, courante dans les bases de données bibliométriques mais qui devrait n'avoir impacté que les chiffres de 2015 et 2016 , ne suffit pas à expliquer cette évolution. Cela étant, de grands progrès ont été enregistrés en Chine, où le nombre de travaux de recherche était faible à l'origine.

\section{Recherche scientifique sur la démence et les maladies neurodégénératives dans une sélection de pays, 1996-2016}

Nombre total de documents relatifs à la démence dans la base de données Scopus, comptages fractionnaires

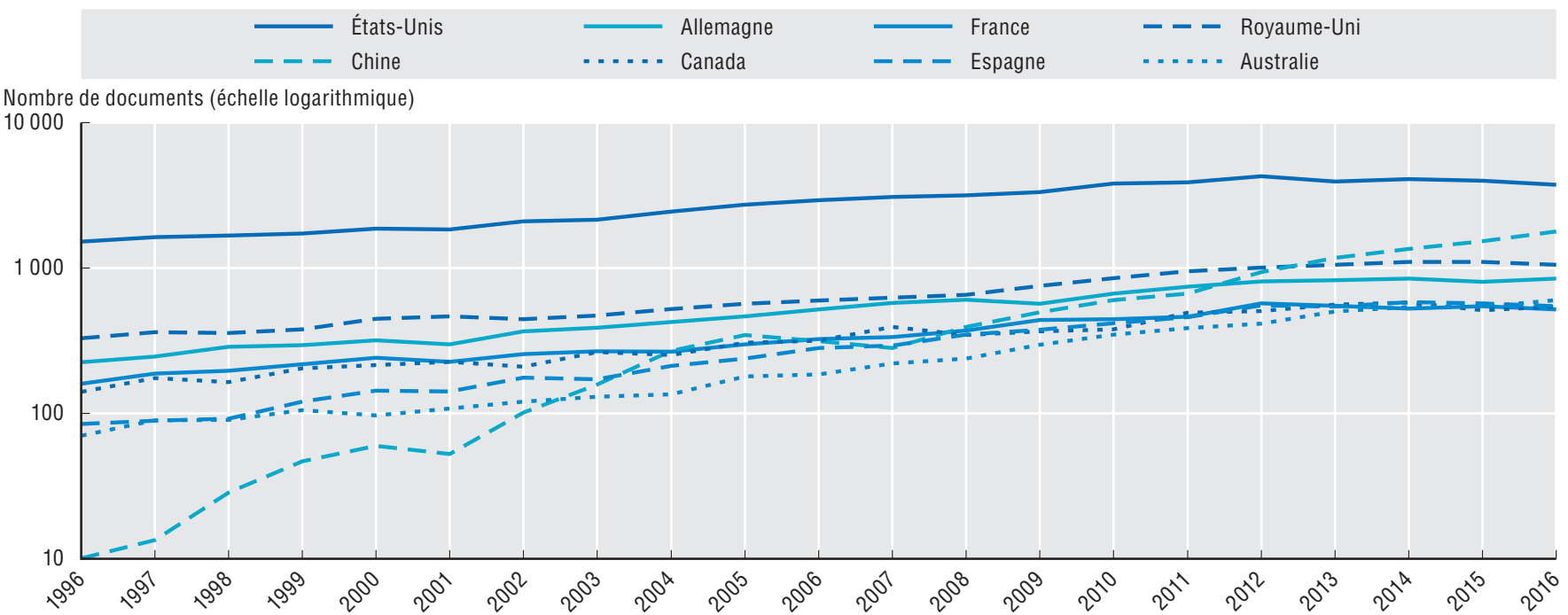

Source : Calculs de l'OCDE, d'après Scopus Custom Data, Elsevier, version 4.2017, juillet 2017. Davantage de données via StatLink. Voir notes de chapitre.

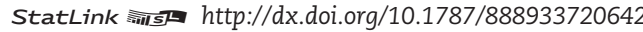

\section{Comment recenser les documents relatifs à la démence}

Ces estimations expérimentales se fondent sur des recherches des descripteurs «neurodegenerat* ", "dementia » et « Alzheimer » dans les résumés des articles publiés (en anglais) entre 1996 et 2016 et indexés dans la base de données Scopus. Les maladies neurodégénératives incluent la maladie d'Alzheimer et la démence associée, la maladie de Huntington, la maladie de Parkinson et d'autres maladies des motoneurones. Même s'il existe un risque de retenir des documents non strictement pertinents (faux positifs), la difficulté principale est de réussir à identifier tous les documents pertinents avec les mots-clés choisis en limitant autant que possible l'incidence des faux négatifs sur le processus de sélection. Cette difficulté est accrue dans le cas de la recherche fondamentale sur le cerveau, quand le résumé ne fait allusion à aucune application spécifique. De plus, la précision de la méthode dépend de l'exhaustivité de l'indexation des résumés, ce qui entraîne un biais en faveur des revues anglophones. 


\section{Un défi mondial : soigner la démence}

En recensant les champs disciplinaires des revues qui publient des articles sur la démence, on constate que les recherches dans ce domaine bénéficient de la contribution d'un large éventail de disciplines, qui vont des neurosciences et de la pharmacologie à la psychologie et aux soins infirmiers. Comme le montre également une récente analyse de l'OCDE relative aux crédits budgétaires publics pour la recherche sur les maladies neurodégénératives (OCDE, 2015b), les efforts de recherche (représentés par la production de publications) sont importants dans les disciplines visant à mieux comprendre les principes scientifiques fondamentaux à l'origine de la maladie (par exemple, neurosciences et biochimie). Les disciplines de la médecine (le nombre le plus important), des soins infirmiers et de la psychologie, font aussi référence à des travaux sur la recherche clinique et les soins de santé. Enfin, l'augmentation rapide du poids de l'informatique témoigne du rôle crucial que les données massives peuvent jouer dans l'analyse de la complexité de la démence en nous permettant de mieux cerner les facteurs de réduction des risques, ainsi que les soins et les traitements possibles.

\section{Disciplines contribuant à la recherche scientifique sur la démence et les maladies} neurodégénératives, 1996-2016

Nombre total de documents relatifs à la démence dans la base de données Scopus, par code ASJC, comptages fractionnaires

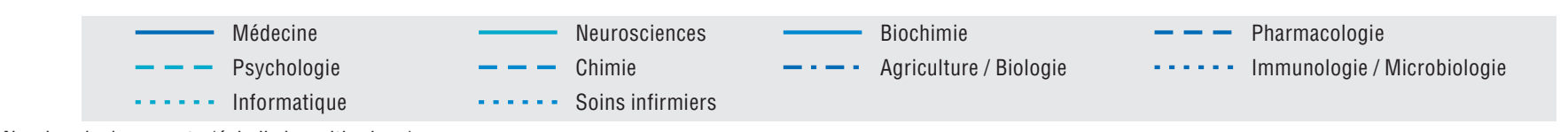

Nombre de documents (échelle logarithmique)

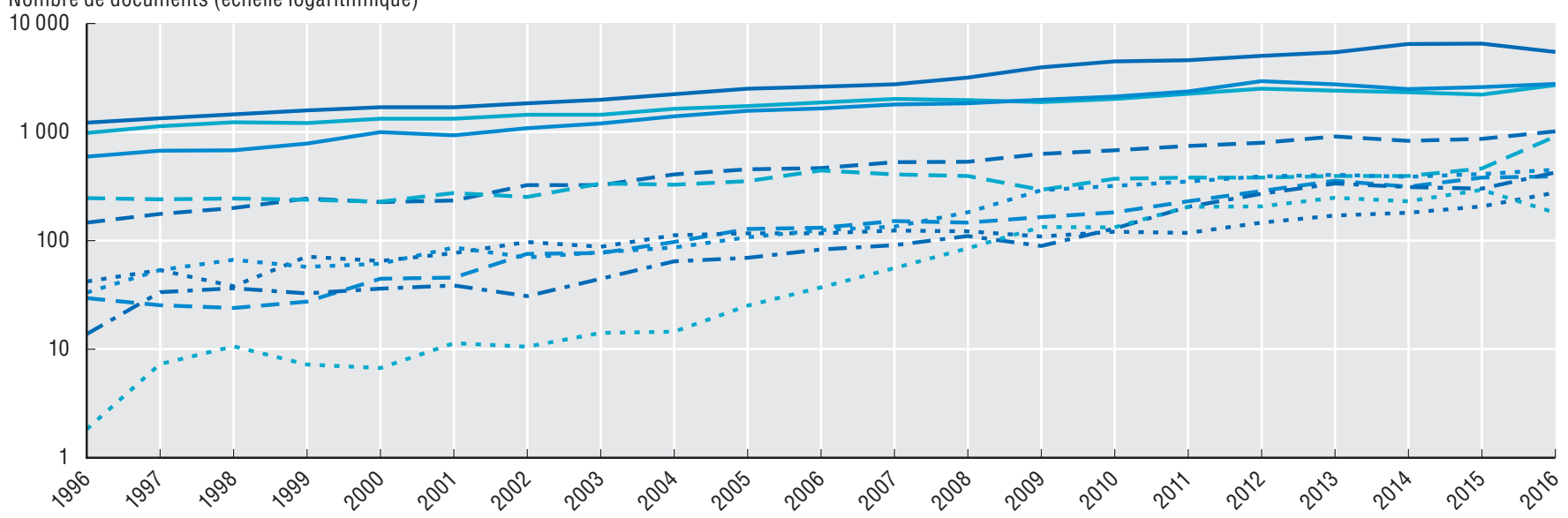

Source : Calculs de l'OCDE, d'après Scopus Custom Data, Elsevier, version 4.2017, juillet 2017. Davantage de données via StatLink. Voir notes de chapitre. StatLink -iाsta http://dx.doi.org/10.1787/888933720661

\section{Comment recenser les champs disciplinaires}

Pour recenser les disciplines, on utilise la classification d'Elsevier qui affecte à chaque revue de la base Scopus un ou plusieurs sujets au moyen des codes ASJC (All Science Journal Classification). Cette classification comprend 27 domaines principaux et 334 sujets définis plus étroitement. On répartit les publications entre les domaines principaux en effectuant des comptages fractionnaires, et on affecte les documents publiés dans des revues généralistes en proportion des différents domaines de fond, en fonction de la distribution des codes ASJC des documents qui les citent et des documents qu'ils citent. De plus amples travaux sont nécessaires pour mieux comprendre l'ampleur et la nature de l'interdisciplinarité dans ce domaine. La preuve de cette interdisciplinarité est apportée notamment par des documents très cités sur l'application du graphène (un produit des nanotechnologies) à la détection précoce de la démence. 


\section{1. ÉCONOMIE DU SAVOIR ET TRANSFORMATION NUMÉRIQUE}

\section{Agir maintenant au service de l'innovation}

\section{Ouvrir l'accès à la science}

L'accès aux articles de recherche joue un rôle important dans la diffusion des connaissances scientifiques. Les moyens dont nous disposons désormais grâce au numérique facilitent le partage de ces connaissances et donc leur utilisation à des fins de recherche et d'innovation. La promotion du libre accès aux publications est pertinente pour favoriser la science ouverte (c'est-àdire, les efforts déployés pour offrir à la communauté scientifique en particulier, et à la société en général, un accès plus large, au format numérique, aux résultats de la recherche financée sur fonds publics). D'après les résultats fournis par un nouvel indicateur expérimental fondé sur des requêtes en ligne de documents un an environ après leur publication, $60 \%$ à $80 \%$ des contenus parus en 2016 sont uniquement disponibles, un an après, moyennant un abonnement ou une redevance. La publication en libre accès dans une revue (voie dorée) est particulièrement visible au Brésil et dans beaucoup d'autres économies d'Amérique latine. L'auto-archivage en libre accès dans un référentiel (voie verte) prédomine pour les auteurs basés au Royaume-Uni. Aujourd'hui, il semble qu'environ $5 \%$ des auteurs paient une redevance pour donner libre accès aux articles qu'ils publient dans des revues traditionnelles à abonnement (voie dorée hybride).

\section{Documents scientifiques publiés en libre accès, 2017}

En pourcentage d'un échantillon aléatoire de 100000 documents publiés en 2016

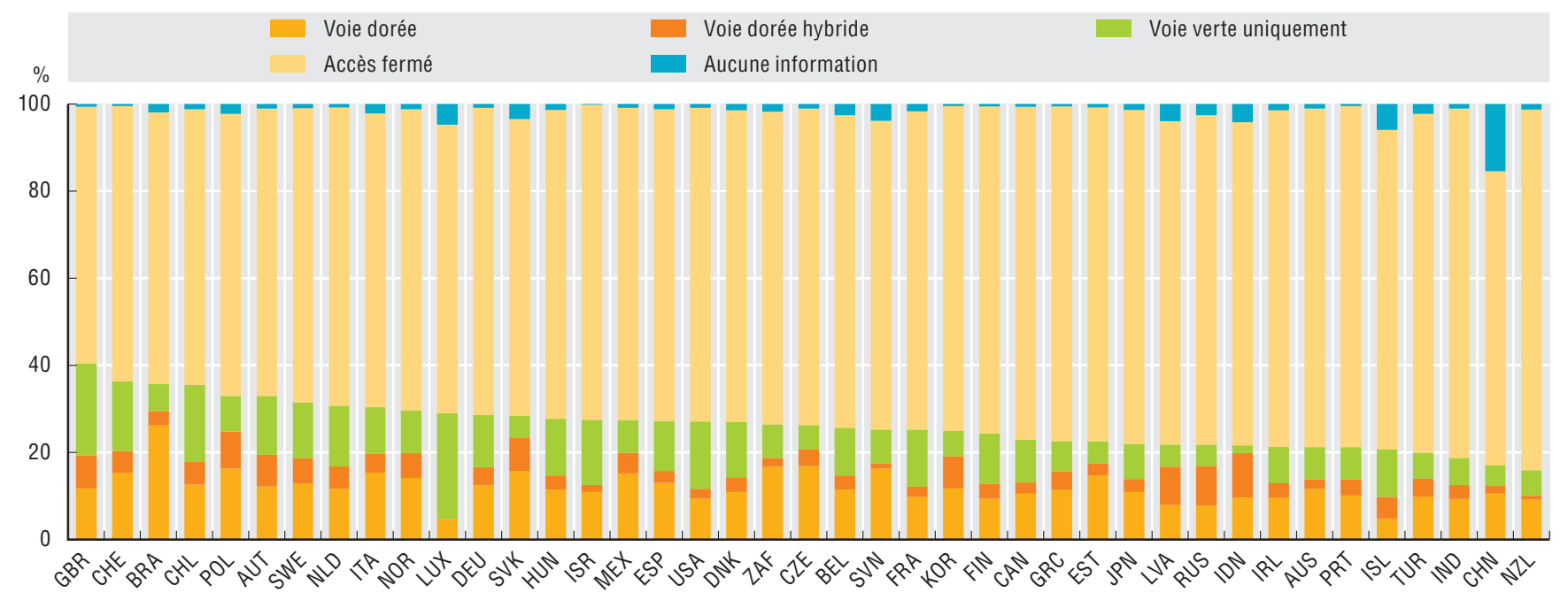

Source : Calculs de l'OCDE, d'après Scopus Custom Data, Elsevier, version 4.2017, et routine wrapper roaDOI pour l'API oaDOI, https://oaDOI.org, juillet 2017. Davantage de données via StatLink. Voir notes de chapitre.

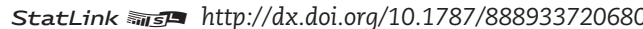

\section{Dénombrer les documents en libre accès à l'aide d'outils en ligne}

L'édition précédente de cette publication dénombrait les documents scientifiques en libre accès sur la base des résultats d'une enquête en ligne conduite par l'OCDE auprès des auteurs scientifiques du monde entier (http://oe.cd/issa). Dans cette édition, on utilise un indicateur obtenu en appliquant une requête informatique à un échantillon aléatoire (non stratifié) de 100000 documents citables (articles, recensions et actes de conférences) publiés en 2016 et indexés dans la base de données Scopus avec un DOI valide associé (plus de 90 \% des cas). La requête en question est une routine de type "wrapper ", codée en langage R, conçue pour interroger l'interface de programmation d'application (API) oaDOI développée par l'organisation ImpactStory, dont les outils en libre accès disponibles en ligne visent à aider les chercheurs à partager leurs recherches et à en mesurer l'impact. L'évaluation, conduite en juin 2017, a consisté à déterminer le statut des documents de l'échantillon. L'API oaDOI renvoie des informations sur les possibilités d'obtenir gratuitement des copies légales des documents selon différents mécanismes :

La voie dorée indique que l'éditeur du document est listé dans le Dictionnaire des revues en libre accès, c'est-à-dire qu'il donne gratuitement et librement accès à ses contenus.

La voie dorée hybride indique que l'éditeur du document, qui facture généralement un abonnement à ses lecteurs, propose ce document précis en libre accès, normalement parce que l'auteur ou ceux qui le financent ont payé les frais de publication de l'article, et donc obtenu un droit de parution en libre accès (sur le modèle des revues qui appliquent l'approche de la voie dorée).

La voie verte indique que des versions légales du document existent dans des référentiels ou d'autres types d'archives, sans qu'aucune autre version ne soit accessible via la voie dorée ou la voie dorée hybride. Le nombre de documents de cette catégorie peut être sous-estimé si l'API oaDOI n'arrive pas à recenser toutes les copies ou versions presque identiques légalement disponibles.

Quand le DOI du document ne peut pas être mis en correspondance avec un moyen quelconque d'accès à l'information, il est affecté à la catégorie « aucune information " (statut non disponible), une catégorie particulièrement importante (supérieure à $15 \%$ ) en Chine. Quand le DOI peut être mis en correspondance avec des sources mais que l'API oaDOI indique qu'aucune version légale en libre accès n'est disponible, il est affecté à la catégorie « accès fermé ». C'est le cas, notamment, des documents sous embargo. 


\section{Ouvrir l'accès à la science}

Évaluer dans quelle mesure les publications en libre accès sont plus citées que les autres peut aider les responsables de l'élaboration des politiques à estimer les coûts et les avantages sociaux des mécanismes de financement alternatif des publications scientifiques. Il a donc été entrepris de mesurer ce que l'on considère comme l'avantage du libre accès en termes de citation. Des résultats bibliométriques fondés sur le dernier ensemble de données disponibles confirment ceux d'une précédente enquête, qui présentaient une image contrastée (OCDE, 2015c ; Boselli et Galindo-Rueda, 2016) car toutes les formes de libre accès ne semblent pas conférer un avantage. En effet, si le libre accès est globalement associé à des taux de citation plus élevés dans le cas des documents recensés par les grands index de citation, la tendance n'est pas la même pour les documents publiés dans les revues en libre accès qui, plus récentes en moyenne, sont donc d'autant moins citées qu'on remonte dans le temps. La publication dans un référentiel (voie verte) confère elle systématiquement un avantage en termes de citation. Enfin, dans beaucoup de pays, les taux de citation sont plus élevés pour les documents publiés dans des revues à abonnement mais gratuitement accessibles en ligne car leur auteur a versé la redevance requise à l'éditeur (voie dorée hybride).

\section{Documents scientifiques les plus cités, par modalité d'accès, 2017}

En pourcentage des $10 \%$ de publications les plus citées en 2016

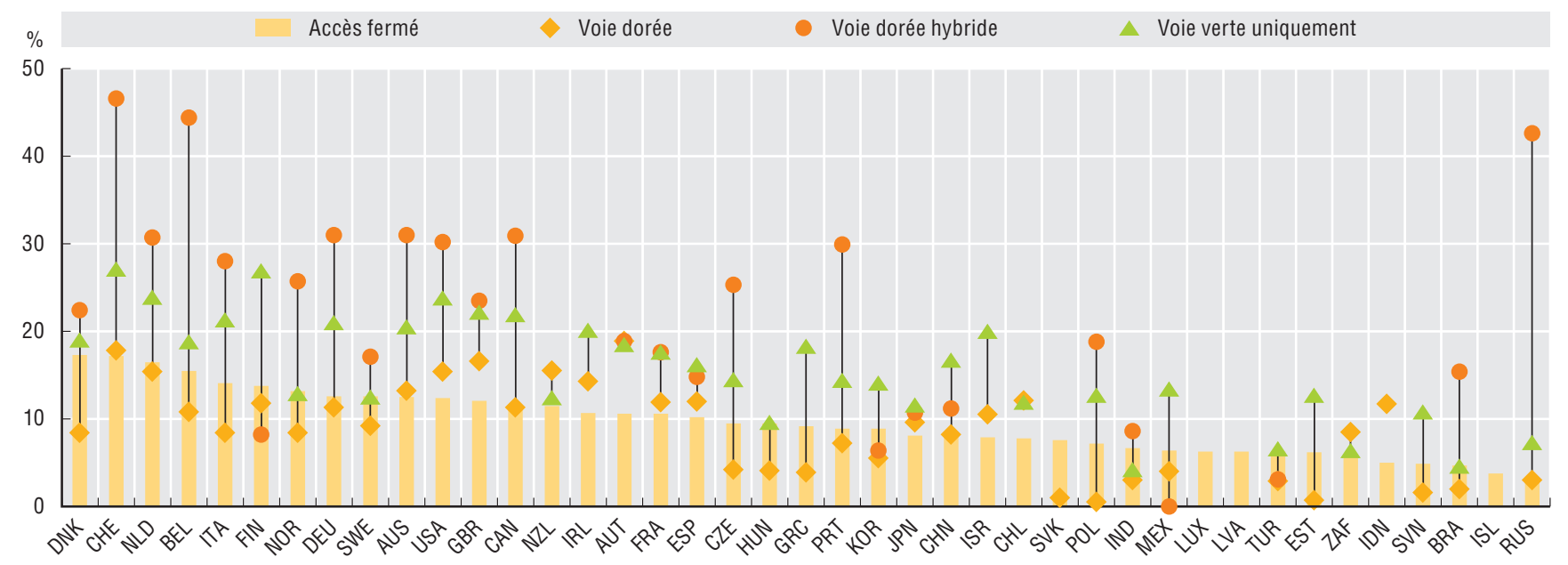

Source : Calculs de l'OCDE, d'après Scopus Custom Data, Elsevier, version 4.2017, et routine wrapper roaDOI pour l'interface oaDOI, https://oaDOI.org, juillet 2017. Davantage de données via StatLink. Voir notes de chapitre.

\section{L'impact du libre accès au-delà des citations par les pairs}

Les taux de citation sont des mesures imparfaites de l'impact scientifique, même si l'on normalise les résultats par domaine, cohorte et type de document. En particulier, et c'est l'une de leurs principales limitations, ils ne rendent pas compte de la pertinence et de l'utilité des activités de recherche auprès d'une plus large communauté d'utilisateurs potentiels de ces connaissances scientifiques, moins susceptibles de publier dans des revues spécialisées à comité de lecture (par exemple, médecins généralistes, inventeurs dans le secteur commercial, agents publics, etc.). L'étude ISSA1 de l'OCDE, conduite sur la base d'informations transmises par les auteurs eux-mêmes, montre que les documents en libre accès sont plus susceptibles d'être cités dans des contextes d'utilisation plus divers, par exemple dans des rapports de l'administration publique, des brevets, des affaires juridiques, des protocoles de soins, ou encore des documents de travail non soumis à des comité de lecture. Étant donné la place croissante du numérique dans nombre de ces contextes, on tend à utiliser davantage d'indicateurs fondés sur des données de l'internet pour étudier les formes plus larges d'impact des publications. Cependant, ces indicateurs ont pour inconvénient possible de risquer de mesurer la " popularité ", donc de modifier les comportements si l'on s'en sert pour conduire des évaluations des recherches (http://oe.cd/blue-sky). L'analyse de la relation entre les différentes formes de libre accès aux documents scientifiques et l'impact des recherches semble mettre en lumière un découplage possible entre l'assurance de la qualité et la diffusion de l'information, deux rôles traditionnellement joués par les revues académiques. 


\section{1. ÉCONOMIE DU SAVOIR ET TRANSFORMATION NUMÉRIQUE}

\section{Agir maintenant au service de l'innovation}

\section{Promouvoir la collaboration et la mobilité internationales}

La collaboration entre acteurs d'un même pays ou de pays différents est une caractéristique universelle des activités de recherche et d'innovation et un moteur clé des échanges de connaissances. Pour mieux cerner le phénomène, on peut étudier les affiliations des co-auteurs de publications scientifiques et des co-inventeurs d'inventions brevetées. On observe ainsi que dans tous les pays BRIICS (cercles blancs) et de l'OCDE (cercles bleus), sauf en Inde et en Indonésie, le taux de publications conjointes internationales est supérieur à celui des co-inventions internationales. Si des économies scientifiquement et technologiquement avancées comme la Corée, les États-Unis et le Japon participent autant que les autres à des co-autorats internationaux, elles se démarquent par des niveaux différents de co-inventions, les inventeurs asiatiques étant en outre relativement moins engagés dans des activités de collaboration internationale. Les petites économies ouvertes tendent à enregistrer des taux de collaboration plus élevés. Des facteurs tels que la spécialisation scientifique et technologique, les opportunités de collaboration et la proximité géographique et institutionnelle peuvent contribuer à expliquer ces différences.

68. Collaboration internationale en science et innovation, 2005-16

Publications et inventions conjointes en pourcentage des publications scientifiques et familles de brevets IP5

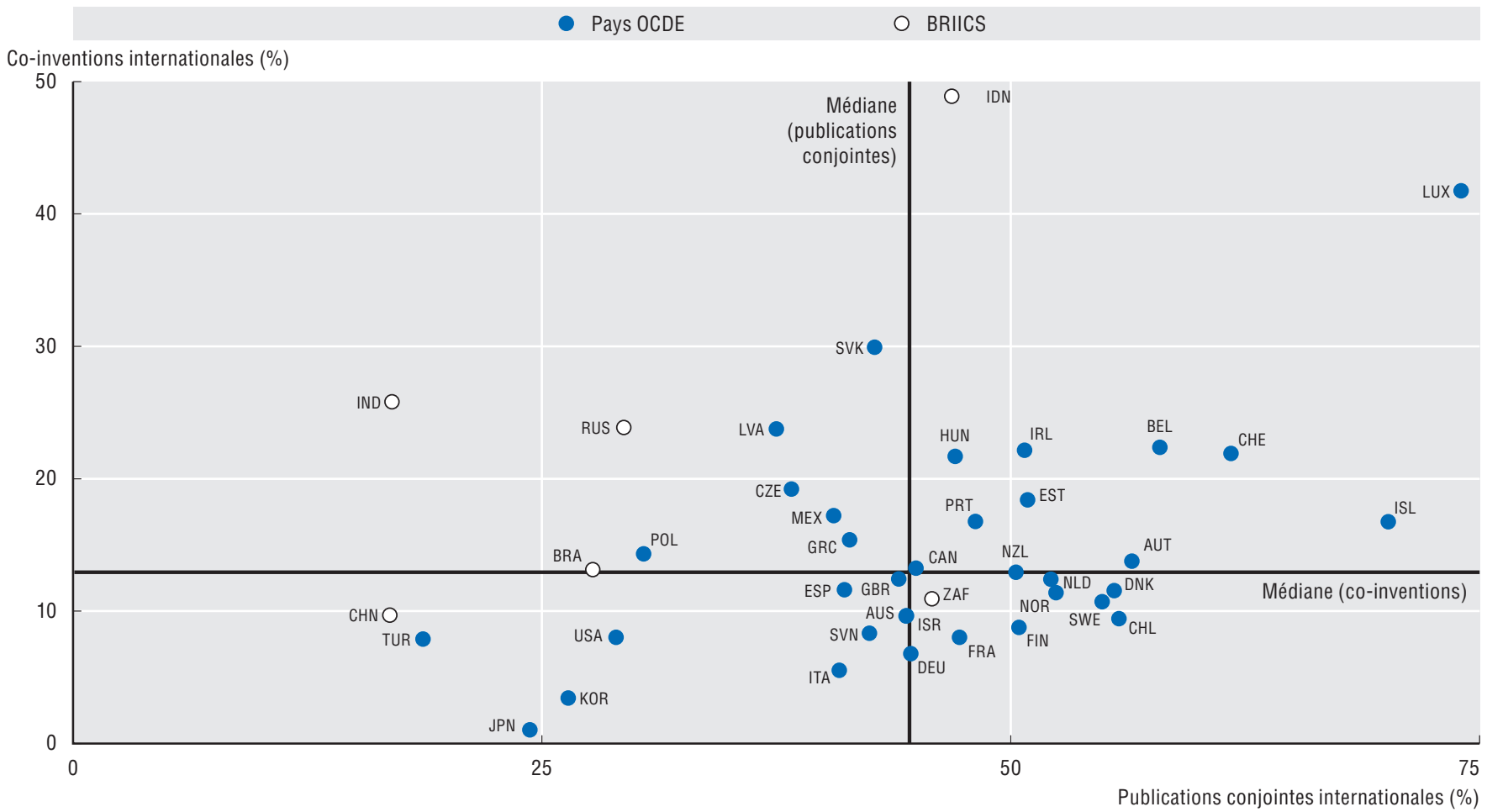

Source : Calculs de l'OCDE, d'après Scopus Custom Data, Elsevier, version 4.2017, et OCDE, STI Microdata Lab : Base de données sur la propriété intellectuelle, $h$ ttp://oe.cd/ipstats, juillet 2017. Davantage de données via StatLink. Voir notes de chapitre.

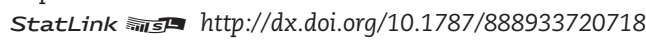

\section{Comment interpréter les indicateurs}

Les co-inventions internationales sont mesurées en calculant la part, dans le nombre total de brevets d'un pays donné, des demandes de brevets impliquant au moins un co-inventeur installé dans une économie différente. Les données se rapportent aux familles de brevets IP5 (inventions brevetées auprès des cinq grands offices de la propriété intellectuelle) déposées entre 2005 et 2015, par première date de dépôt et lieu de résidence de l'inventeur, avec comptages simples.

Le co-autorat international de publications scientifiques est mesuré en calculant la part, dans le nombre total d'articles produits par les établissements nationaux, des articles émanant d'au moins un auteur affilié à un établissement étranger (d'une économie ou d'un pays différent). À des fins de comparabilité avec les données sur les co-inventions, on utilise également le comptage simple. Les estimations présentées ici sont donc supérieures à celles que contient le chapitre 3, qui reposent sur des comptages fractionnaires. 


\section{Promouvoir la collaboration et la mobilité internationales}

Les indicateurs de la mobilité scientifique internationale, établis sur la base des changements d'affiliation des auteurs, révèlent que la mobilité est surtout caractérisée par une circulation des chercheurs (renouvellement des " cerveaux ") plutôt que par des déplacements unidirectionnels (gains ou pertes de " cerveaux "). Cependant, l'analyse des flux nets d'entrée et de sortie des auteurs scientifiques au cours du temps peut donner beaucoup d'informations sur la réaction de la communauté scientifique aux événements ou politiques nationales en lien avec le financement de la recherche scientifique, le soutien à la mobilité scientifique internationale et les mesures visant à attirer les plus qualifiés. Les données sur les flux nets enregistrés depuis 2001 montrent que la Suisse, les États-Unis et l'Australie attirent le plus de scientifiques, juste devant la Chine qui, après avoir affiché des niveaux élevés de sorties nettes, est désormais celle des grandes économies qui enregistre le plus d'entrées nettes. Aux États-Unis, la balance positive diminue depuis quelques années. Le Japon et l'Inde accumulent les sorties nettes, tandis que la Fédération de Russie attire davantage d'auteurs scientifiques depuis 2014. L'UE28, devenue bénéficiaire nette pendant une courte période en 2008-09, a enregistré un déficit très important après 2011. Sur l'ensemble des 15 dernières années, le nombre d'auteurs scientifiques qui en sont partis est supérieur de près de 36000 à celui des auteurs scientifiques qui s'y sont installés, ce qu'explique en partie la mobilité de retour des scientifiques qui étaient venus y faire leurs études avant de devenir des auteurs publiés. Au sein même des grands pays de l'UE, il existe des différences notables : le Royaume-Uni a attiré des scientifiques en 2014-16 tandis que l'Espagne a rejoint l'Italie à la tête du classement des économies très productrices de contenus scientifiques qui voient partir davantage de scientifiques qu'elles n'en accueillent.

69. Flux internationaux nets d'auteurs scientifiques dans une sélection d'économies, 2002-16 Différence entre les entrées et les sorties annuelles, en pourcentage des flux totaux

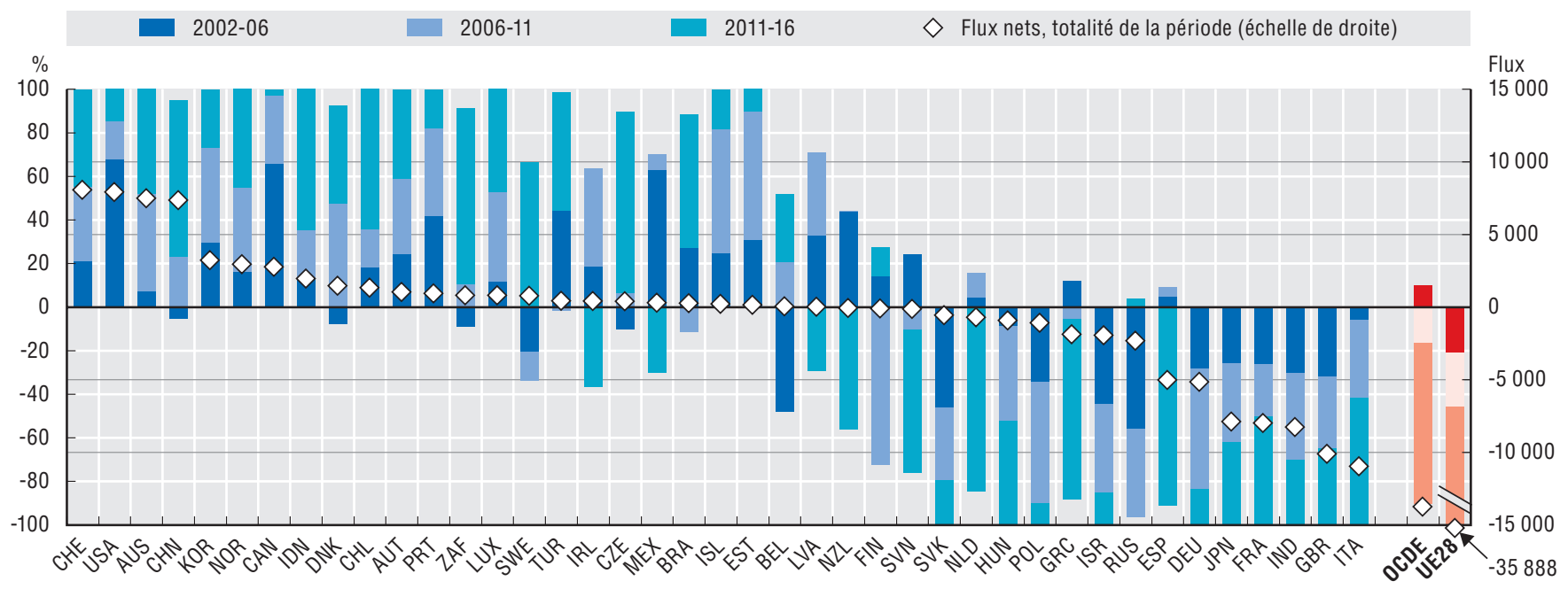

Source : Calculs de l'OCDE, d'après Scopus Custom Data, Elsevier, version 4.2017, juillet 2017. Davantage de données via StatLink. Voir notes de chapitre. StatLink aills $h$ ttp://dx.doi.org/10.1787/888933720737

\section{Comment lire ce graphique}

Le graphique décompose le flux global net des auteurs scientifiques dans les pays de l'OCDE et les économies BRIICS entre 2001 et 2016 , en termes relatifs. Il permet de mettre en évidence la chronologie et l'intensité des différentes phases d'entrées et de sorties nettes du point de vue d'un pays donné. Ainsi, l'Allemagne et l'Espagne ont enregistré une perte nette cumulée de même ordre au cours de la période (valeur représentée par un losange sur l'axe de droite). Toutefois, en Espagne, cette évolution est le résultat d'une phase de sorties nettes modérées dans les années 2000 puis d'un important flux net de sorties d'auteurs scientifiques après la crise. Au contraire, la tendance observée en Allemagne est relativement plus stable, et son déficit a baissé ces dernières années.

Établir un suivi des changements d'affiliation des scientifiques à partir des registres mondiaux des publications fournit une source complémentaire d'informations détaillées, mais ces informations sont limitées aux auteurs qui publient régulièrement : dans le cas contraire, les affiliations ne peuvent être détectées et datées avec suffisamment de précision. De plus, il n'est possible de calculer la mobilité que pour les auteurs d'au moins deux publications. Ces indicateurs sous-estiment en outre vraisemblablement les flux par pays et par domaine qui impliquent une mobilité vers un secteur ou des établissements dans lesquels la publication dans des revues spécialisées n'est pas la norme. Enfin, la mesure de la mobilité peut être difficile à distinguer de celle de la collaboration dans le cas des auteurs affiliés à plusieurs établissements dans différents pays. Dans ce cas, on a procédé à des comptages fractionnaires. 


\section{1. ÉCONOMIE DU SAVOIR ET TRANSFORMATION NUMÉRIQUE}

\section{Agir maintenant au service de l'innovation}

\section{Soutenir l'innovation des entreprises}

Le soutien public à la R-D des entreprises vise à encourager celles-ci à investir dans des connaissances pouvant déboucher sur des innovations à même de transformer les marchés et les activités et, partant, de bénéficier à toute la société. Pour inciter les entreprises à s'engager dans la R-D, les pouvoirs publics peuvent avoir recours à divers dispositifs. En 2017, 30 des 35 pays de l'OCDE ont proposé des allègements fiscaux en plus de l'aide directe traditionnellement apportée sous forme notamment de subventions et de contrats, alors qu'ils n'étaient que 16 à le faire en 2000. Les grandes entreprises, où se concentrent la majeure partie des activités de R-D, tendent à être les principales bénéficiaires des aides directes et fiscales accordées pour soutenir les dépenses intérieures de R-D des entreprises (DIRDE). Une nouvelle analyse de l'OCDE apporte des éclairages sur la répartition des aides en fonction de la taille des entreprises dans un certain nombre de pays. La part des aides fiscales à la R-D qui revient aux PME varie de $5 \%$ en Hongrie à $71 \%$ en Lettonie et $79 \%$ en Norvège (petites entreprises uniquement). Si le soutien direct relève le plus souvent de mesures discrétionnaires, la part de l'aide fiscale destinée aux PME tend à être plus étroitement corrélée à la part de celles-ci dans les DIRDE, ce qui tend à prouver que les incitations fiscales sont généralement, dans la panoplie des mesures de soutien public direct à la R-D, un instrument stimulé par la demande. Il est intéressant de noter que l'Autriche, le Canada, la France, la Norvège, les Pays-Bas et le Royaume-Uni allouent aux PME une part plus importante des aides fiscales que des aides directes. Tous ces pays ont mis en place des crédits d'impôt qui ciblent en priorité les plus petites entreprises actives en R-D : celles-ci peuvent ainsi bénéficier des crédits même lorsque l'impôt qu'elles doivent est inférieur au montant total alloué, la différence leur étant versée en totalité ou en partie par l'administration.

\section{Aides directes et fiscales à la R-D des PME, 2015}

En pourcentage de l'aide publique aux DIRDE, dans chaque catégorie

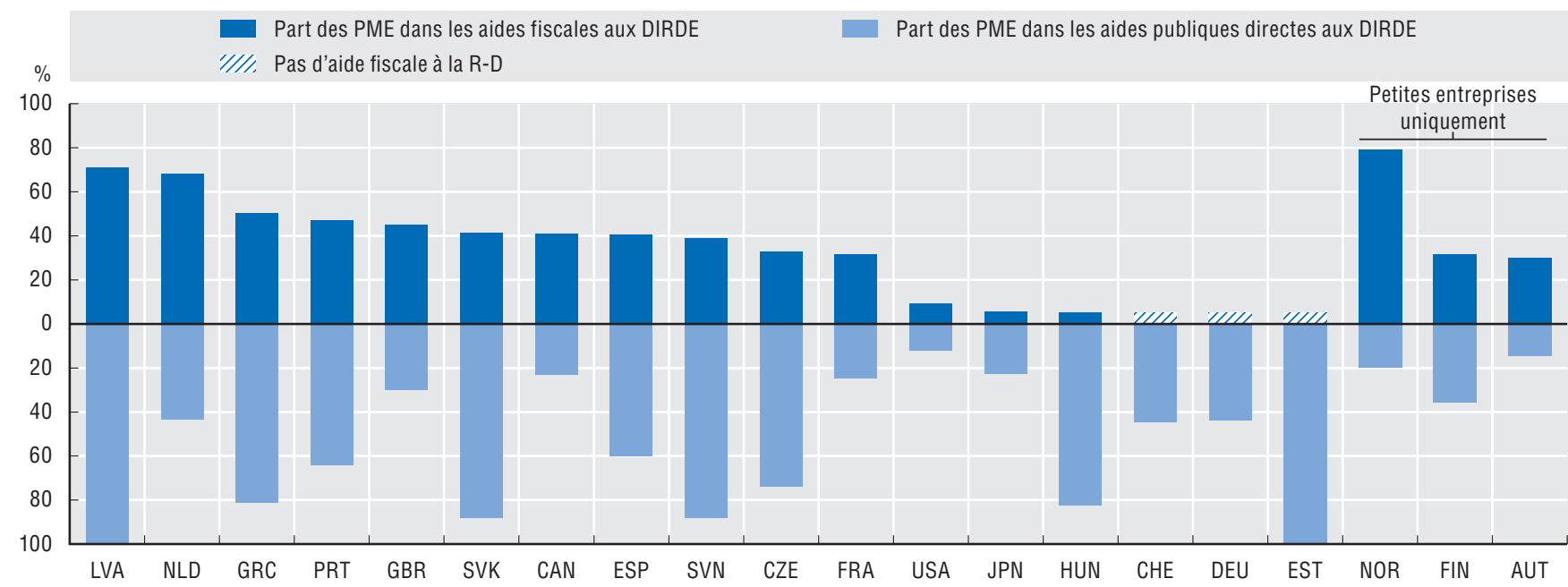

Source : OCDE, Indicateurs des incitations fiscales en faveur de la R-D, http://oe.cd/rdtax, juillet 2017. Voir notes de chapitre.

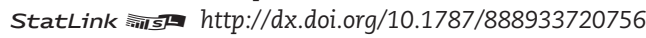

\section{Comment mesurer les aides fiscales à la R-D}

Les estimations établies par l'OCDE du coût des allègements fiscaux en faveur de la R-D sont associées aux données sur le financement direct de la R-D, telles que communiquées par les entreprises dans le cadre d'enquêtes sur la R-D, afin d'obtenir un tableau plus complet des initiatives publiques à l'appui de la R-D des entreprises. On peut alors avoir un suivi de ces initiatives au fil du temps. Le recueil de données de l'OCDE sur les incitations fiscales à la R-D (qui en est à sa cinquième édition) s'efforce de mettre en lumière et d'analyser les différences subtiles au niveau du traitement fiscal de la R-D, les systèmes fiscaux de référence pertinents et les approches en matière de métrique. Des experts nationaux des indicateurs de la science et de la technologie ont travaillé en collaboration avec des administrations fiscales et des administrations chargées des finances publiques afin de fournir des données aussi comparables à l'échelle internationale et à jour que possible. Dans la mesure du possible, le coût estimé des dispositions accordant un traitement préférentiel aux dépenses de R-D des entreprises est rapporté à une référence commune (déductibilité totale des dépenses courantes de R-D). Ces estimations rendent compte de la somme du manque à gagner fiscal - calculé selon le principe de la comptabilité d'exercice - et des remboursements d'impôts, le cas échéant. La dernière édition du Manuel de Frascati de l'OCDE inclut un nouveau chapitre consacré à la mesure des incitations fiscales à la R-D (OCDE, 2015e), voir http://oe.cd/frascati.

L'indicateur spécifique présenté ici est expérimental. La comparabilité internationale des données peut être limitée (par exemple, parce que les définitions des PME ne sont pas les mêmes aux fins de la comptabilisation de la R-D des entreprises et des allègements fiscaux en faveur de la R-D). Pour les DIRDE et les DIRDE financées par l'État, les PME s'entendent généralement des entreprises de 1 à 249 salariés (c'est-à-dire, à l'exclusion des entreprises de zéro salarié), sauf mention contraire. Mais un certain nombre de pays appliquent des critères supplémentaires pour définir les PME. Pour de plus amples informations sur les définitions des PME, voir http://oe.cd/sb2017_notes_rdtax. 


\section{1. ÉCONOMIE DU SAVOIR ET TRANSFORMATION NUMÉRIQUE}

3. Agir maintenant au service de l'innovation

\section{Soutenir l'innovation des entreprises}

L'intensité de la R-D du secteur des entreprises affiche une corrélation positive (0.3) avec le niveau de financement public de ce secteur de R-D dans presque tous les pays, quelques exceptions étant à noter. L'Allemagne et la Corée présentent des intensités de R-D relativement élevées dans les entreprises par rapport à leur niveau de soutien public, alors que la France, la Hongrie et la Fédération de Russie présentent des taux de soutien supérieurs à ceux de pays dont le rapport entre la R-D des entreprises et le PIB est pourtant similaire. Un indicateur complémentaire compare l'évolution du soutien public à la R-D et de l'intensité de la R-D du secteur des entreprises. Entre 2006 et 2015, les pays dans lesquels le soutien public a connu la plus forte progression ont aussi connu une croissance plus élevée de l'intensité de R-D. En Chine et en Corée, la croissance de l'intensité de la R-D a été supérieure à celle que laissait prévoir la variation du soutien public. Celle-ci semble être à l'origine d'environ $17 \%$ de la variation observée de l'intensité de la R-D des entreprises au cours de la période 2006-15. Une analyse supplémentaire montre que cette variation expliquée est due, pour deux tiers, à l'évolution des aides directes et, pour le tiers restant, à l'évolution des aides fiscales.

71. Intensité de la R-D des entreprises et soutien public à la R-D des entreprises, 2015

En pourcentage du PIB

Volume de l'aide fiscale à la R-D des entreprises, en millions USD à prix courants et à PPA, 2015

- Aucune incitation $\times$ Aucune donnée disponible $\quad 200$ millions USD $\bigcirc 1500$ millions USD

6000 millions USD

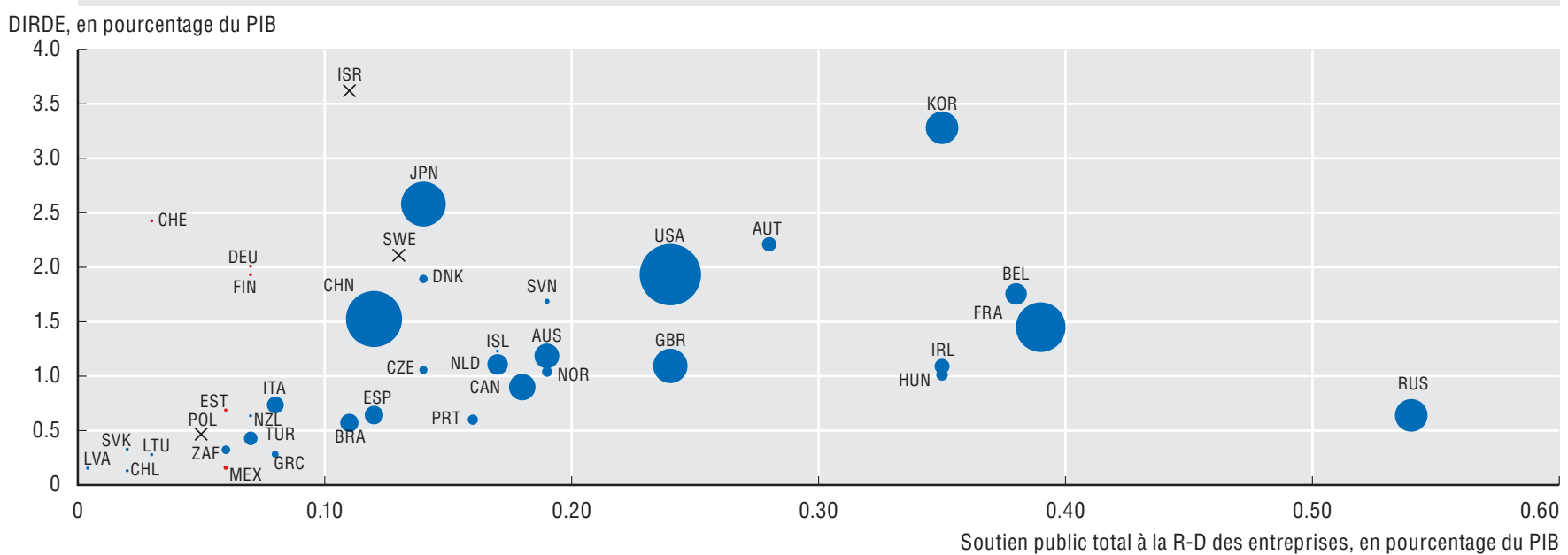

Source : OCDE, Indicateurs des incitations fiscales en faveur de la R-D, http://oe.cd/rdtax, juillet 2017. Davantage de données via StatLink. Voir notes de chapitre. StatLink inist http://dx.doi.org/10.1787/888933720775

72. Évolution du soutien public à la R-D des entreprises et des DIRDE, 2006-15

Variation absolue annualisée des valeurs exprimées en pourcentage du PIB

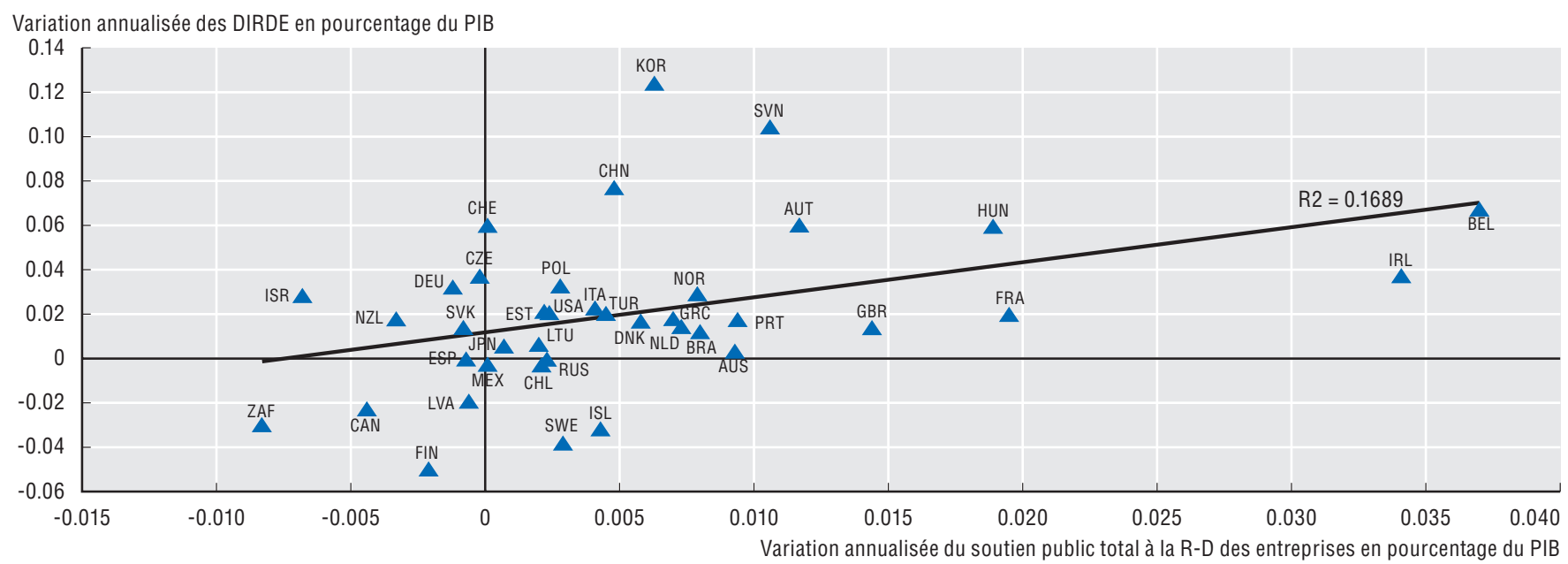

Source : OCDE, Indicateurs des incitations fiscales en faveur de la R-D, http://oe.cd/rdtax, juillet 2017. Davantage de données via StatLink. Voir notes de chapitre.

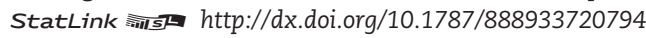




\section{1. ÉCONOMIE DU SAVOIR ET TRANSFORMATION NUMÉRIQUE}

\section{Agir maintenant au service de l'innovation}

\section{Financer l'entrepreneuriat et l'innovation}

Les petites entreprises nouvelles et innovantes peuvent financer leurs activités par l'emprunt ou par des fonds propres. Dans ce deuxième cas, le capital-risque et le capital apporté par des investisseurs providentiels ( business angels ") constituent des sources importantes, en particulier pour les jeunes entreprises axées sur les technologies. Les données disponibles au niveau des entreprises montrent qu'en 2016, les investissements en capital-risque se sont concentrés principalement sur le secteur des TIC, en particulier dans des pays tels que l'Espagne, l'Estonie et la République slovaque. En Europe, des données de 2015 montrent que plus d'un tiers des contrats passés avec des investisseurs providentiels concernaient le secteur des TIC, tandis qu'aux États-Unis, des données de 2016 indiquent que 45 \% de tous les accords portaient sur le logiciel, l'internet ou la téléphonie mobile.

\section{Investissement en capital-risque dans une sélection de pays, par secteur, 2016}

En pourcentage du total des investissements en capital-risque

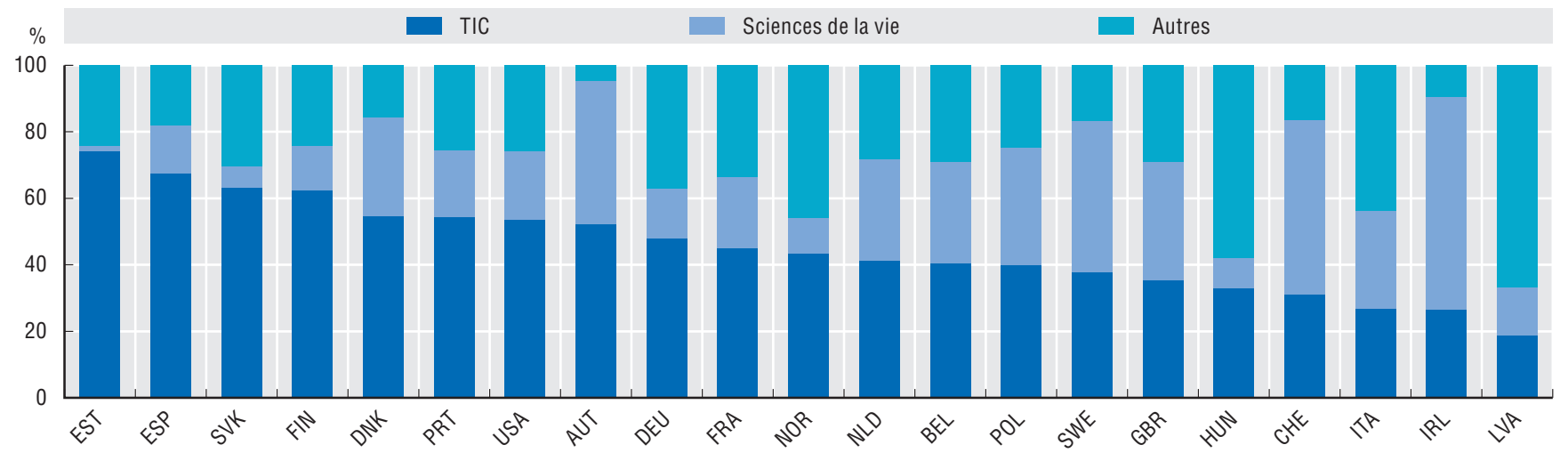

Source : OCDE, d'après OCDE, Base de données sur le financement de l'entrepreneuriat, septembre 2017. Voir notes de chapitre.

StatLink 完正四 http://dx.doi.org/10.1787/888933720813

74. Accords conclus avec des investisseurs providentiels par secteur, Europe, 2015 et États-Unis, 2016

En pourcentage du total des accords conclus avec des investisseurs providentiels

Europe, 2015

\begin{tabular}{|c|c|c|}
\hline TIC & $\square$ & Mobile \\
\hline Industries créatives & 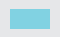 & Biotech et sciences de la vie \\
\hline Technologies médicales / soins de santé & & Fabrication \\
\hline Énergie & $1 / 1 /$, & Autres \\
\hline
\end{tabular}

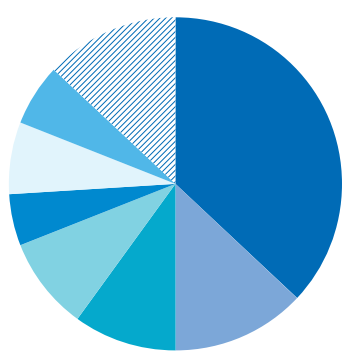

États-Unis, 2016

$\begin{array}{ll}\text { Logiciel } & \text { Internet et mobile } \\ \text { Produits et services grand public } & \text { Produits et services destinés } \\ \text { Soins de santé } & \text { aux entreprises } \\ \text { III/, Autres } & \end{array}$

Source : Calculs de l'OCDE, d'après ARI (Angel Resource Institute) et réseaux interrogés par EBAN (European Trade Association for Business Angels, Seed Funds and other Early Stage Market Players), septembre 2017. Voir notes de chapitre.

\section{Mesurer le capital-risque et le capital apporté par des investisseurs providentiels}

Les données sur le capital-risque proviennent principalement d'associations de capital-risque nationales ou régionales et de fournisseurs de données du secteur privé. Il n'existe pas de définition type au niveau international du capital-risque ou de la ventilation des investissements de capital-risque par stade de développement et par secteur d'activités. La base de données de l'OCDE sur le financement de l'entrepreneuriat agrège des données originales pour correspondre à la classification OCDE du capital-risque par stade de développement et par groupe de secteurs d'activités. Le renforcement de la coopération entre fournisseurs nationaux/régionaux de données sur le capital-risque devrait améliorer la disponibilité des données et fournir des informations comparables à l'échelle internationale sur l'investissement de capitalrisque dans les différents secteurs et les différents pays. Les données sur le capital apporté par des investisseurs providentiels proviennent des enquêtes conduites par les associations d'investisseurs providentiels auprès des réseaux et des groupes de tels investisseurs. Elles sont difficiles à évaluer du fait de la discrétion de ces financements (ce qui conduit à parler de " marché invisible ") et des différences entre pays au niveau de ce que l'on choisit de définir comme un investisseur providentiel. En outre, les données recueillies dans le cadre d'enquêtes sont généralement sujettes à des incohérences, d'abord parce que le nombre de répondants n'est pas le même d'une année à l'autre, ensuite parce que la couverture du marché est incomplète (OCDE, à paraître). 


\section{Financer l'entrepreneuriat et l'innovation}

Les indicateurs expérimentaux fondés sur les données détaillées de la source Crunchbase ${ }^{\odot}$ révèlent que la majorité des financements sur fonds propres destinés à des start-ups axées sur le numérique se retrouve dans les secteurs des applis, des données et de l'analytique de données. Entre 2011 et 2016, la part de ces secteurs dans l'ensemble des financements sur fonds propres d'entreprises axées sur le numérique s'est élevée à $80 \%$ en Estonie et en Lettonie, et à $70 \%$ en Chine.

75. Start-ups axées sur le numérique ayant attiré des fonds propres dans les économies de l’OCDE et BRIICS, 2011-16 Entreprises de cinq ans d'ancienneté ou moins

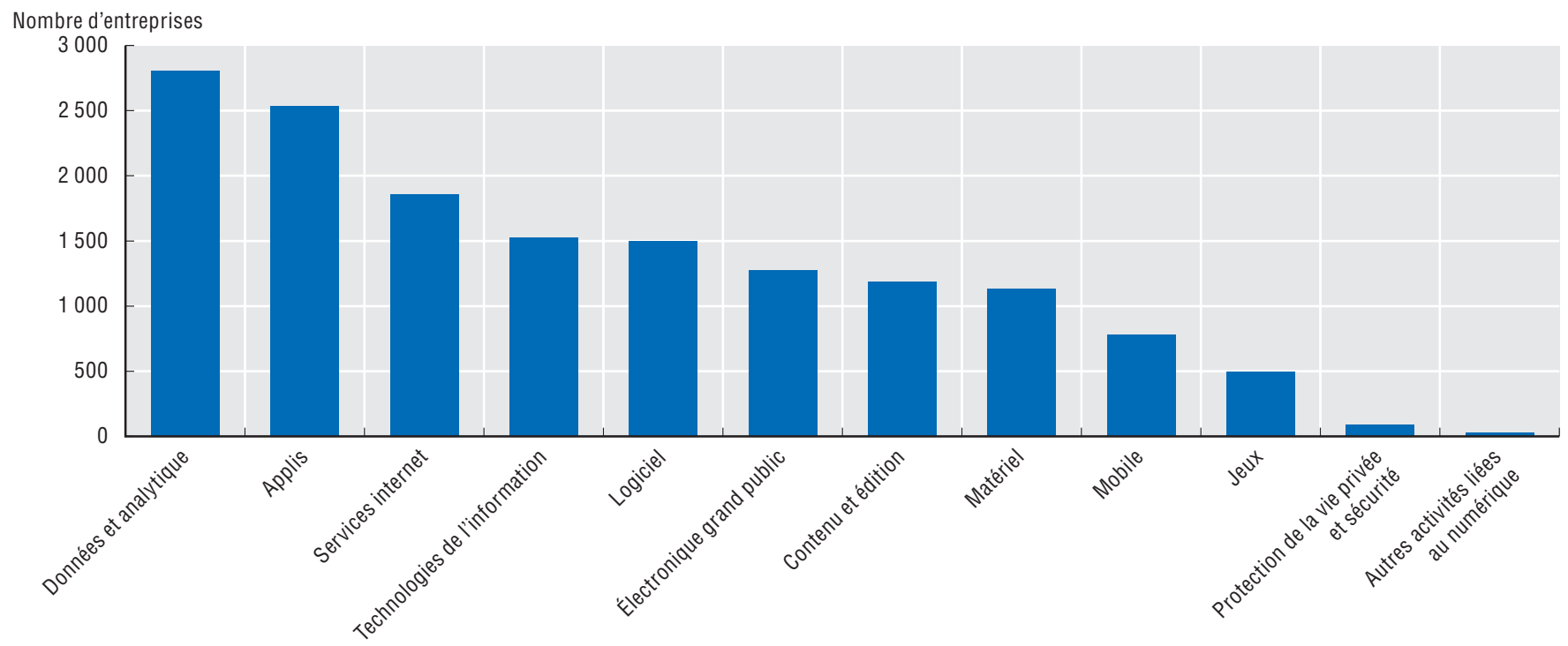

Source : Données de Crunchbase ${ }^{\odot}$, www.crunchbase.com, septembre 2017, comme indiqué dans Breschi, Lassebie et Menon (à paraître). Voir notes de chapitre. StatLink त्ताइा http://dx.doi.org/10.1787/888933720851

76. Activités liées au numérique ayant attiré le plus de financements sur fonds propres, 2011-16 En pourcentage du total des financements sur fonds propres d'activités liées au numérique

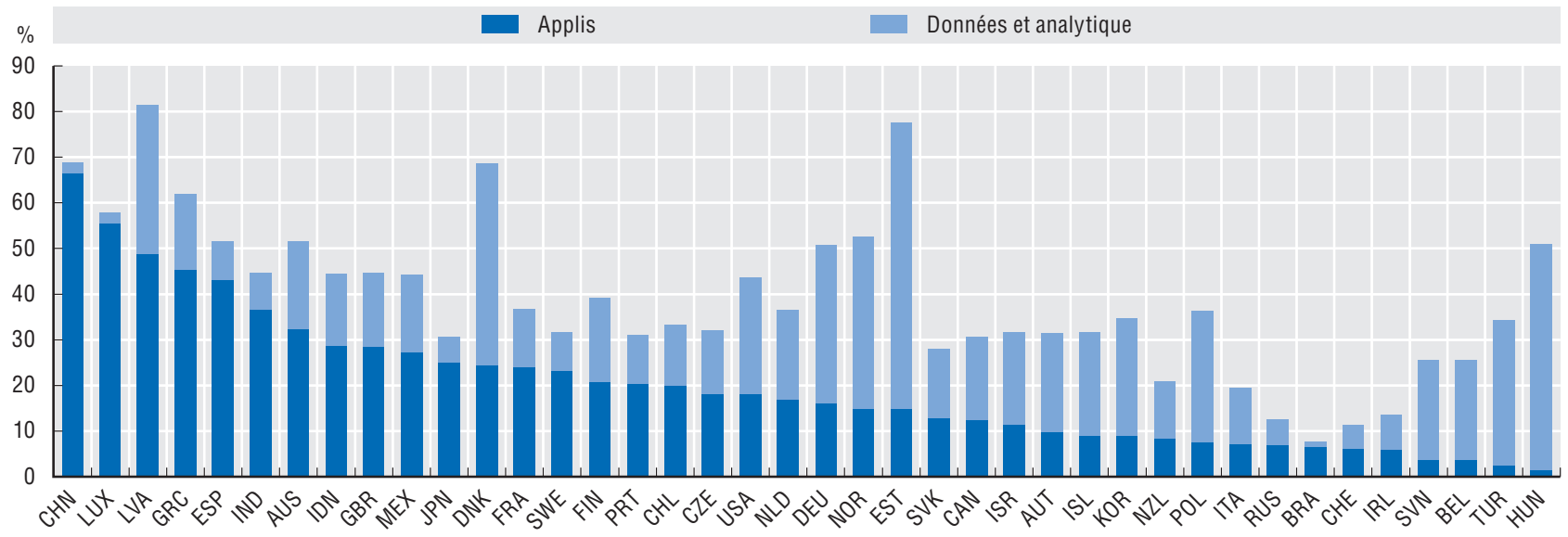

Source : Données de Crunchbase ${ }^{\odot}$, www.crunchbase.com, septembre 2017, comme indiqué dans Breschi, Lassebie et Menon (à paraître). Voir notes de chapitre.

StatLink 제패 http://dx.doi.org/10.1787/888933720870

\section{Ce que nous apprend Crunchbase ${ }^{\odot}$}

Le site Crunchbase ${ }^{\odot}$ fournit des informations sur l'activité et le financement des start-ups dans les différents pays du monde. Les variables disponibles sont notamment la taille de l'entreprise, son emplacement (ville et région), le rôle des acteurs concernés (firme, groupe, investisseur, université), le statut (en activité, acquisition, introduction en bourse, ou fermé), sa date de création et les dates auxquelles l'enregistrement a été créé et mis à jour. Pour de plus amples informations, voir Dalle, Denbesten et Menon (à paraître). Dans les graphiques présentés ici, l'échantillon est limité aux entreprises créées après 2010 (donc qui avaient cinq ans d'ancienneté ou moins en 2016) et qui ont attiré des financements sur fonds propres au cours de la période 2011-16. Le financement sur fonds propres inclut le capital-risque et d'autres financements à risque tels que les capitaux apportés par des investisseurs providentiels ou le financement par emprunt. Les activités liées au numérique sont répertoriées sur la base d'une correspondance entre les secteurs listés dans la base de données et les codes de la CITI rév. 4. Il convient de considérer les résultats rapportés ici comme une première exploration des données de Crunchbase ${ }^{\circledR}$ aux fins d'une analyse statistique et économique. 


\section{1. ÉCONOMIE DU SAVOIR ET TRANSFORMATION NUMÉRIQUE}

\section{Agir maintenant au service de l'innovation}

\section{Promouvoir l'excellence scientifique}

Quelle est l'efficacité des différents mécanismes de financement de la recherche scientifique ? Un nouvel indicateur expérimental, fondé sur les remerciements que les auteurs scientifiques adressent à des financeurs dans leurs articles publiés, permet d'appréhender la mesure dans laquelle ces chercheurs réussissent à pérenniser des financements directs pour leur recherche. Cette méthode fournit une vision ascendante approximative, du point de vue des chercheurs, de l'amplitude des financements d'activités spécifiques (sans doute en concurrence les unes avec les autres pour ces financements) en lien avec la production scientifique. Les auteurs qui ne citent aucun financeur en particulier sont plus susceptibles de dépendre de ressources institutionnelles pour leurs travaux et leurs propres salaires. Les auteurs basés en Chine, en Corée et aux États-Unis - des économies où le financement par projet est très courant - sont les plus à même de remercier des sources de financement dans leurs articles. Au contraire, les auteurs basés en France et en Italie affichent certains des taux de remerciements de financeurs les plus bas, ce qui témoigne de l'importance relativement plus élevée des financements institutionnels dans ces pays. Une comparaison des profils de citation au sein des différents pays montre que les remerciements pour financement sont associés à un taux d'excellence beaucoup plus élevé, mesuré comme la proportion de chaque catégorie qui figure parmi les $10 \%$ de documents les plus cités dans leur domaine et dans leur cohorte. Cette observation peut laisser entendre que les financeurs sélectionnent les activités à même d'avoir le plus d'impact, ou être le reflet des avantages dont bénéficient les chercheurs qui obtiennent des financements supplémentaires. Évaluer l'efficacité relative d'autres mécanismes d'allocation des ressources nécessite de plus amples informations sur les montants des financements et les mécanismes de sélection appliqués au niveau micro-économique. Il faudrait pour cela une infrastructure de données plus fine pour compléter le panorama descendant fourni par les statistiques sur les budgets publics de R-D.

\section{Publications scientifiques avec remerciements pour financement direct, 2016}

En pourcentage de tous les documents citables

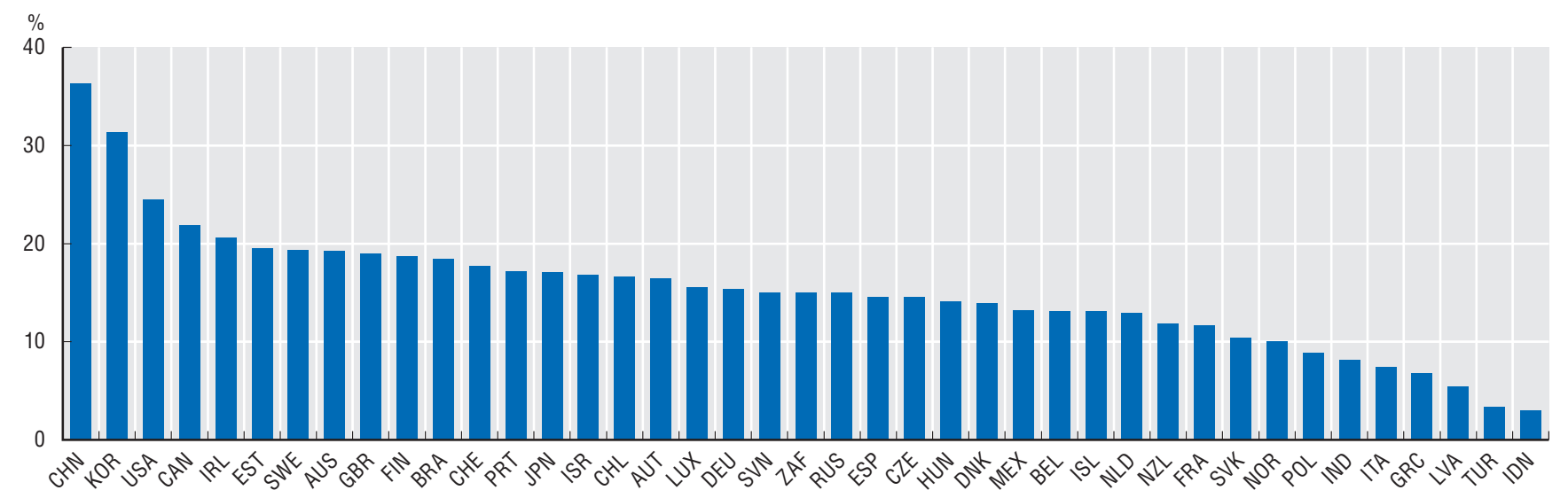

Source : Calculs de l'OCDE, d'après Scopus Custom Data, Elsevier, version 4.2017, juillet 2017. Davantage de données via StatLink. Voir notes de chapitre.

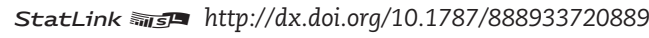


78. Impact de citation des publications scientifiques avec ou sans remerciements pour financement direct, 2016 En pourcentage des $10 \%$ de documents qui figurent en tête de classement dans chaque catégorie

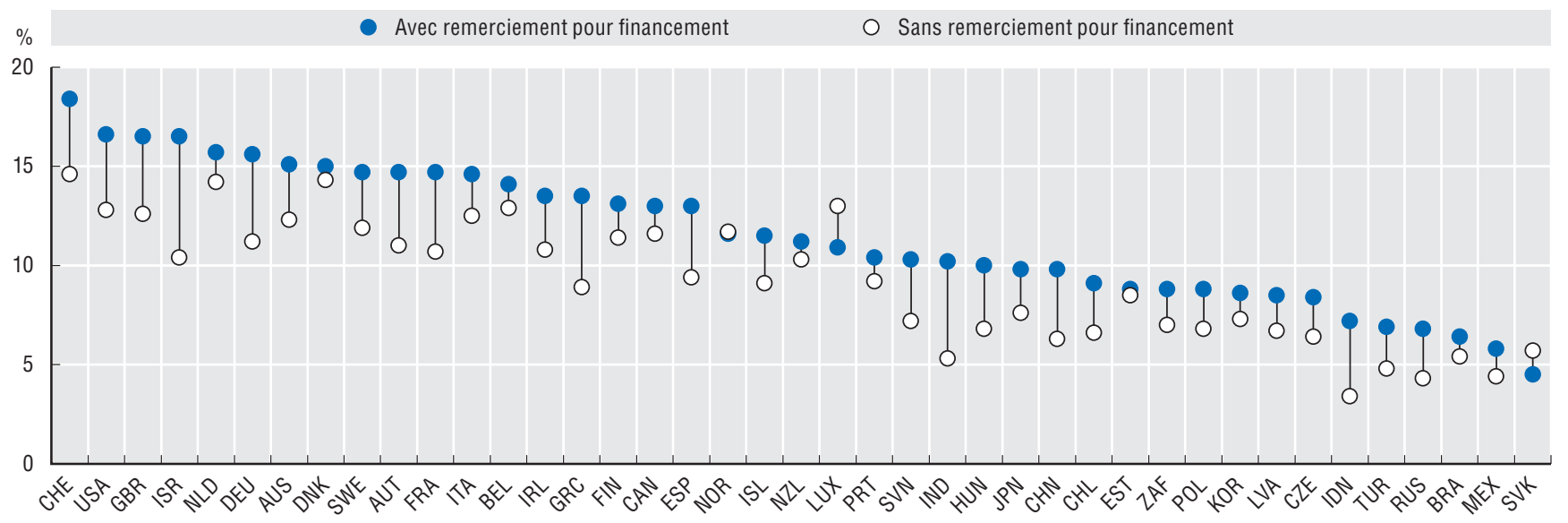

Source : Calculs de l'OCDE, d'après Scopus Custom Data, Elsevier, version 4.2017, et valeurs SJR (Scimago Journal Rank) de 2015 des titres de la liste de revues Scopus (consulté en juin 2017), juillet 2017. Davantage de données via StatLink. Voir notes de chapitre. 


\section{1. ÉCONOMIE DU SAVOIR ET TRANSFORMATION NUMÉRIQUE}

\section{Notes et Références}

\section{Chypre}

La note suivante est incluse à la demande de la Turquie :

"Les informations figurant dans ce document qui font référence à “Chypre” concernent la partie méridionale de l'île. Il n'y a pas d'autorité unique représentant à la fois les Chypriotes turcs et grecs sur l'île. La Turquie reconnaît la République Turque de Chypre Nord (RTCN). Jusqu'à ce qu'une solution durable et équitable soit trouvée dans le cadre des Nations Unies, la Turquie maintiendra sa position sur la "question chypriote". "

La note suivante est ajoutée à la demande de tous les États de l'Union européenne membres de l'OCDE et de l'Union européenne :

"La République de Chypre est reconnue par tous les membres des Nations Unies sauf la Turquie. Les informations figurant dans ce document concernent la zone sous le contrôle effectif du gouvernement de la République de Chypre. »

\section{Israël}

"Les données statistiques concernant Israël sont fournies par et sous la responsabilité des autorités israéliennes ou d'un tiers compétents. L'utilisation de ces données par l'OCDE est sans préjudice du statut des hauteurs du Golan, de JérusalemEst et des colonies de peuplement israéliennes en Cisjordanie aux termes du droit international. »

" Il est à noter que les données statistiques sur les brevets et marques israéliens sont fournies par les offices des brevets et des marques des pays concernés. "

\section{Pénétration du haut débit mobile, OCDE, G20 et BRIICS, 2016}

Pour l'Afrique du Sud, l'Arabie saoudite, l'Argentine, le Brésil, la Chine, la Fédération de Russie, l'Inde et l'Indonésie, les données sont issues de la base des Indicateurs des télécommunications/TIC dans le monde de l'UIT, consultée en juillet 2017.

Pour Israël, les données proviennent de GSMA Intelligence.

Pour les États-Unis et la Suisse, les données sont des estimations.

\section{Pénétration des cartes SIM M2M, OCDE, Monde et pays du G20, juin 2017}

Les données pour 2017 se rapportent au deuxième trimestre.

Pour disposer de données comparables, obtenues à l'aide d'une méthodologie unique, les données de l'ensemble des économies, y compris des pays de l'OCDE, proviennent de GSMA Intelligence (www.gsmaintelligence.com, consulté en septembre 2017). La GSMA s'appuie sur la définition suivante pour recenser les connexions par carte SIM M2M : ensemble des cartes SIM uniques enregistrées sur un réseau mobile à la fin de la période considérée, permettant la transmission de données mobiles entre au moins deux machines. N'entrent pas dans le champ de cette définition les équipements informatiques relevant de l'électronique grand public comme les liseuses, les smartphones, les dongles et les tablettes.

\section{Pays comptant le plus de connexions par carte SIM M2M, juin 2017}

Les données se rapportent au deuxième trimestre de 2017.

Pour disposer de données comparables, obtenues à l'aide d'une méthodologie unique, les données de l'ensemble des économies, y compris des pays de l'OCDE, proviennent de GSMA Intelligence (www.gsmaintelligence.com, consulté en septembre 2017). La GSMA s'appuie sur la définition suivante pour recenser les connexions par carte SIM M2M : ensemble des cartes SIM uniques enregistrées sur un réseau mobile à la fin de la période considérée, permettant la transmission de données mobiles entre au moins deux machines. N'entrent pas dans le champ de cette définition les équipements informatiques relevant de l'électronique grand public comme les liseuses, les smartphones, les dongles et les tablettes.

\section{Principaux acteurs des TIC émergentes, 2012-15}

Les données se rapportent aux familles de brevets IP5, par date de dépôt et lieu de résidence des déposants (comptages fractionnaires). Les " accélérations » correspondent aux périodes caractérisées par une hausse soudaine et persistante du nombre de brevets déposés, sur la base des classes de la Classification internationale des brevets (CIB). Les accélérations les plus fortes sont mises en évidence par comparaison avec l'évolution des dépôts de brevets relevant des autres classes de la CIB. L'intensité d'une accélération correspond au degré relatif de l'augmentation du nombre de dépôts observée. Seules les classes de la CIB présentant une évolution positive à compter de 2010 sont prises en compte. Les données pour 2014 et 2015 sont incomplètes. Les classes de la CIB sont décrites à l'adresse http://web2.wipo.int/classifications/ipc/ipcpub.

\section{Intensité et rapidité de développement des technologies liées aux TIC, 2000-14}

Les " accélérations " correspondent aux périodes caractérisées par une hausse soudaine et persistante du nombre de dépôts de brevets portant sur des technologies liées aux TIC. Les accélérations les plus fortes sont mises en évidence par comparaison avec l'évolution des dépôts de brevets liés aux autres technologies. L'intensité d'une accélération correspond au degré relatif de l'augmentation du nombre de dépôts observée. Les données se rapportent aux familles de brevets IP5, par date de dépôt (comptages fractionnaires). Les brevets liés aux TIC sont recensés à l'aide des codes de la CIB, présentés dans Inaba et Squicciarini (2017). Seules les 25 principales classes de brevets liés aux TIC présentant une évolution positive à compter de 2000 sont prises en compte. Les classes de la CIB sont décrites à l'adresse http://web2.wipo.int/classifications/ipc/ipcpub. 


\section{Brevets portant sur des technologies liées à l'intelligence artificielle, 2000-15}

Les données portent sur le nombre de familles de brevets IP5 ayant trait à l'intelligence artificielle (IA), par date de dépôt et pays de l'inventeur (comptages fractionnaires). L'intelligence artificielle correspond aux catégories Interface homme-machine et Cognition et compréhension sémantique de la taxonomie des brevets liés aux TIC, décrite dans Inaba et Squicciarini (2017). Les chiffres de 2014 et 2015 sont estimés à partir des données disponibles pour ces années.

\section{Brevets portant sur les principales technologies intégrant des fonctions d'intelligence artificielle, 2000-05 et 2010-15}

Les données se rapportent au nombre de familles de brevets IP5 ayant trait à l'intelligence artificielle (IA), par date de dépôt et d'après les codes de la Classification internationale des brevets (CIB) figurant dans les documents de brevets ne portant pas sur l'IA (comptages fractionnaires). L'intelligence artificielle correspond aux catégories Interface homme-machine et Cognition et compréhension sémantique de la taxonomie des brevets liés aux TIC, décrite dans Inaba et Squicciarini (2017). Les données pour 2014 et 2015 sont incomplètes.

\section{Dix principales technologies médicales couplées à l'intelligence artificielle, 2000-05 et 2010-15}

Les données portent sur le nombre de familles de brevets IP5 ayant trait aux technologies médicales et à l'intelligence artificielle (IA), par date de dépôt et d'après les codes de la Classification internationale des brevets (CIB) figurant dans les documents de brevets non liés à l'IA (comptages fractionnaires). Les brevets sont associés aux technologies médicales sur la base des codes de la CIB, selon la table de concordance établie par l'OMPI (2013). L'intelligence artificielle correspond aux catégories Interface hommemachine et Cognition et compréhension sémantique de la taxonomie des brevets liés aux TIC, décrite dans Inaba et Squicciarini (2017). Les données pour 2014 et 2015 sont incomplètes.

\section{La R-D dans la zone OCDE et les principales économies partenaires, 2015}

Pour le Brésil, l'Inde et l'Indonésie, les données sont fournies par l'Institut de statistique de l'UNESCO.

Les données relatives au nombre de chercheurs sont exprimées en équivalent temps plein.

Pour l'Afrique du Sud, les données se rapportent à 2013.

Pour l'Australie, elles se rapportent à 2013, 2010 et 2013.

Pour le Brésil, elles se rapportent à 2014, 2010 et 2014.

Pour le Canada et le Mexique, les données se rapportent à 2015, 2013 et 2015.

Pour les États-Unis, les données sur les chercheurs ont été estimées d'après des données contemporaines sur les chercheurs travaillant dans le secteur des entreprises, et des données passées sur les chercheurs travaillant dans les autres secteurs.

Pour la France, elles se rapportent à 2015, 2014 et 2015.

Pour l'Indonésie, elles se rapportent à 2013, 2009 et 2013.

Pour l'Irlande, elles se rapportent à 2014, 2015 et 2014.

Pour Israël, les données se rapportent à 2015, 2012 et 2015 ; la R-D du secteur de la défense est partiellement exclue des estimations disponibles.

\section{1. Économies en tête des volumes de publications scientifiques parmi les plus citées, 2005 et 2016}

Les "publications les plus citées " correspondent aux $10 \%$ de documents les plus cités, les valeurs étant normalisées par domaine scientifique et par type de document (articles, recensions et actes de conférences). Les valeurs SJR (Scimago Journal Rank) sont utilisées en complément pour hiérarchiser les publications ayant obtenu le même nombre de citations au sein d'une catégorie. Cette mesure est un indicateur indirect de l'excellence de la recherche. Les estimations sont fondées sur des comptages fractionnaires des documents publiés par des auteurs affiliés aux établissements de chaque économie.

\section{2. Évolution récente de l'excellence scientifique dans une sélection de pays, 2005-16}

Les « publications les plus citées » correspondent aux $10 \%$ de documents les plus cités, les valeurs étant normalisées par domaine scientifique et par type de document (articles, recensions et actes de conférences). Les valeurs SJR (Scimago Journal Rank) sont utilisées en complément pour hiérarchiser les publications ayant obtenu le même nombre de citations au sein d'une catégorie. Cette mesure est un indicateur indirect de l'excellence de la recherche. Les estimations sont fondées sur des comptages fractionnaires des documents publiés par des auteurs affiliés aux établissements de chaque économie.

\section{Dépenses de R-D par secteur d'exécution, zone OCDE, 1995-2015}

Ces statistiques se fondent sur la base de données des Principaux indicateurs de la science et de la technologie de l'OCDE (http:// oe.cd/msti-fr). Pour en savoir plus sur les données, en particulier sur les ruptures de séries et autres problèmes analogues, se référer à cette source. 


\section{1. ÉCONOMIE DU SAVOIR ET TRANSFORMATION NUMÉRIQUE}

\section{Notes et Références}

\section{4. Évolution des activités de R-D dans la zone OCDE et une sélection d'économies, 1995-2015}

Pour les États-Unis, à l'exception des DIRDET, qui couvrent les dépenses en capital allouées à la R-D, les chiffres portent sur les dépenses courantes, mais intègrent une composante d'amortissement, d'où une possible variation par rapport au niveau réel des dépenses en capital.

Les estimations établies par l'OCDE pour la zone UE28 peuvent différer légèrement de celles publiées par Eurostat. Dans la présente publication, les estimations nationales sont agrégées en utilisant les taux en USD à parité de pouvoir d'achat (PPA), alors qu'Eurostat utilise les taux de change en EUR. Ainsi, pour l'UE28, la mesure de l'intensité de la DIRD par rapport au PIB correspond à la moyenne des intensités des pays de l'UE, pondérée par la part du PIB national dans celui de l'UE, exprimée en USD à PPA, et non pas à la part des dépenses dans le PIB, exprimée en EUR.

Ces statistiques se fondent sur la base de données des Principaux indicateurs de la science et de la technologie de l'OCDE (http:// oe.cd/msti-fr). Pour en savoir plus sur les données, en particulier sur les ruptures de séries et autres problèmes analogues, se référer à cette source.

\section{5. Évolution conjoncturelle des dépenses de R-D, par source de financement, zone OCDE, 1995-2016}

Les dépenses de R-D financées par les entreprises et l'État sont des composantes des dépenses intérieures brutes de R-D (DIRD), c'est-à-dire des dépenses intra-muros destinées à la R-D exécutée sur le territoire national. Les sources de financement sont habituellement identifiées par les unités qui mènent des travaux de R-D.

Les données sur les crédits budgétaires publics sont souvent plus récentes mais ne coïncident pas toujours avec le montant de la R-D financée par l'État qui est déclaré par les exécutants de la R-D, du fait, notamment, des écarts entre dépenses programmées et décaissements effectifs.

Ces statistiques se fondent sur la base de données des Principaux indicateurs de la science et de la technologie de l'OCDE (http:// oe.cd/msti-fr). Pour en savoir plus sur les données, en particulier sur les ruptures de séries et autres problèmes analogues, se référer à cette source.

\section{6. Évolution de la recherche fondamentale, de la recherche appliquée et du développement expérimental dans la zone OCDE, 1985-2015}

En raison de l'absence de ventilations de la DIRD par type de R-D (recherche fondamentale, recherche appliquée et développement expérimental) et de l'existence de ruptures de séries, l'évolution à long terme a été estimée par enchaînement des taux de croissance en glissement annuel. Ces taux sont calculés chaque année à partir d'un ensemble variable de pays pour lesquels on dispose de données équilibrées pour des années consécutives, sans rupture de série. Cette série sur les tendances donne une indication du volume des dépenses allouées à la recherche fondamentale, à la recherche appliquée et au développement expérimental, et repose sur des données de la DIRD en USD à PPA (prix constants 2010). Certains pays de l'OCDE sont entièrement absents des calculs car ils ne ventilent pas leurs données par type de R-D. De plus amples détails sur les calculs sont disponibles sur demande.

Pour la Chine, la ventilation de la DIRD par type de R-D a été estimée sur la base de la somme des dépenses courantes et des dépenses en capital. Pour l'OCDE, une estimation pondérée de la DIRD a été calculée à partir d'un ensemble de 14 pays pour lesquels on disposait de données par type de R-D en 2015. Les données utilisées pour chaque pays correspondent à la somme des dépenses courantes et des dépenses en capital, sauf pour le Chili, les États-Unis et la Norvège, pour lesquels seules les dépenses courantes sont incluses dans les estimations communiquées à l'OCDE.

\section{Concentration de la R-D des entreprises : 50 et 100 principaux exécutants, 2014}

Cet indicateur est expérimental. La comparabilité des données à l'échelle internationale peut être limitée. Pour en savoir plus sur le projet microBeRD de l'OCDE, voir http://oe.cd/microberd.

Les chiffres peuvent différer ou sembler différer des statistiques officielles de la R-D, les méthodologies adoptées aux fins de l'analyse des micro-données n'étant pas les mêmes. Il convient de garder à l'esprit que les estimations présentées sont expérimentales et ne sont pas destinées à se substituer aux statistiques officielles existantes.

Les données pour l'Allemagne, l'Autriche, la Belgique, la France et l'Italie se rapportent à 2013. Pour le Portugal, les données se rapportent à 2012 .

L'unité statistique d'analyse est l'entreprise, sauf pour Israël, où les chiffres sont calculés au niveau des établissements.

L'analyse porte sur les entreprises de 10 salariés ou plus, à l'exception du Japon, où elle couvre les entreprises de 50 salariés ou plus. L'analyse couvre les secteurs 05 à 72 (codes à deux chiffres de la CITI rév. 4), à l'exception des secteurs 45, 47, 55-56 et 68-69, sauf pour le Canada et les États-Unis.

Les données pour le Canada et les États-Unis ont été calculées par les deux pays conformément à leurs propres procédures.

\section{Activités de R-D par taille et ancienneté des entreprises, 2014}

Cet indicateur est expérimental. La comparabilité des données à l'échelle internationale peut être limitée. Pour en savoir plus sur le projet microBeRD de l'OCDE, voir http://oe.cd/microberd.

Les chiffres peuvent différer ou sembler différer des statistiques officielles de la R-D, les méthodologies adoptées aux fins de l'analyse des micro-données n'étant pas les mêmes. Il convient de garder à l'esprit que les estimations présentées sont expérimentales et ne sont pas destinées à se substituer aux statistiques officielles existantes. 
Pour la Belgique et l'Italie, les données se rapportent à 2013. Pour le Portugal, elles se rapportent à 2012.

L'unité statistique d'analyse est l'entreprise, sauf pour Israël, où les chiffres sont calculés au niveau des établissements.

L'analyse porte sur les entreprises de 10 salariés ou plus. Les petites entreprises comptent entre 10 et 49 salariés, les entreprises de taille moyenne, entre 50 et 249 salariés, et les grandes entreprises, 250 salariés ou plus. La distinction entre les jeunes entreprises et les entreprises dites " anciennes " se fait à cinq ans d'ancienneté.

L'analyse couvre les secteurs 05 à 72 (codes à deux chiffres de la CITI rév. 4), à l'exception des secteurs 45, 47, 55-56 et 68-69.

\section{Sources externes de financement de la R-D par taille et ancienneté des entreprises, 2014}

Cet indicateur est expérimental. La comparabilité des données à l'échelle internationale peut être limitée. Pour en savoir plus sur le projet microBeRD de l'OCDE, voir http://oe.cd/microberd.

Les chiffres peuvent différer ou sembler différer des statistiques officielles de la R-D, les méthodologies adoptées aux fins de l'analyse des micro-données n'étant pas les mêmes. Il convient de garder à l'esprit que les estimations présentées sont expérimentales et ne sont pas destinées à se substituer aux statistiques officielles existantes.

Pour la Belgique, les données se rapportent à 2011. Pour le Portugal, elles se rapportent à 2012.

L'unité statistique d'analyse est l'entreprise, sauf pour Israël, où les chiffres sont calculés au niveau des établissements.

L'analyse porte sur les entreprises de 10 salariés ou plus. Les petites entreprises comptent entre 10 et 49 salariés, les entreprises de taille moyenne, entre 50 et 249 salariés, et les grandes entreprises, 250 salariés ou plus. La distinction entre les jeunes entreprises et les entreprises dites « anciennes " se fait à cinq ans d'ancienneté.

L'analyse couvre les secteurs 05 à 72 (codes à deux chiffres de la CITI rév. 4), à l'exception des secteurs 45, 47, 55-56 et 68-69.

\section{Dépenses de R-D et portefeuille de PI des entreprises qui investissent le plus dans la R-D, 2014}

Les données renvoient aux sociétés figurant parmi les 2000 entreprises qui investissent le plus dans la R-D, classées d'après les montants des dépenses de R-D.

Le portefeuille de PI renvoie au nombre de brevets, marques, et dessins ou modèles déposés au cours de la période 2012-14, et détenus par les entreprises qui investissent le plus dans la R-D (comptages fractionnaires). Sont comptabilisés ici : les familles de brevets IP5 ; les marques déposées auprès de l'EUIPO, du JPO et de l'USPTO ; les dessins ou modèles déposés auprès de l'EUIPO et du JPO, et les brevets liés à des dessins ou modèles, déposés auprès de l'USPTO.

\section{Portefeuille de brevets détenus par les principaux acteurs privés de la R-D, par secteur,} 2012-14

Les données se rapportent aux familles de brevets IP5, par date de dépôt, détenues par les entreprises qui investissent le plus dans la R-D (comptages fractionnaires). Les brevets liés aux TIC sont identifiés à l'aide des codes CIB recensés dans la taxonomie présentée dans Inaba et Squicciarini (2017). Les données pour 2014 sont partielles.

\section{Principaux acteurs de la R-D détenant des actifs de propriété intellectuelle, 2012-14}

Les données reflètent la part du portefeuille de brevets (ou dessins et modèles) des entreprises dans le nombre total de brevets (ou dessins et modèles) déposés par les 2000 entreprises qui ont investi le plus dans la R-D au cours de la période 2012-14.

Les données relatives aux brevets correspondent aux familles de brevets IP5 ; celles sur les dessins ou modèles englobent les dépôts effectués auprès de l'EUIPO et du JPO, ainsi que les brevets liés à des dessins ou modèles, déposés auprès de l'USPTO.

Les secteurs d'activité sont définis conformément à la CITI rév. 4. Le secteur des TIC englobe les secteurs ayant trait à la fabrication d'équipements TIC (classes 2610, 2620, 2630, 2640 et 2680), au commerce d'équipements TIC (classes 4651 et 4652 ), aux services TIC (classe 5820), aux télécommunications (division 61), à la programmation informatique (division 62), au traitement des données (groupe 631), et à la réparation d'ordinateurs et de matériel de communication (groupe 951).

\section{Classement des 20 premières technologies émergentes développées par les principaux acteurs de la R-D, 2012-14}

Les données reflètent la part des familles de brevets IP5 détenues par les 2000 entreprises qui investissent le plus dans la R-D, dans l'ensemble des familles de brevets IP5, par date de dépôt et classe de la Classification internationale des brevets (CIB). Les 20 premières technologies émergentes correspondent aux classes de la CIB qui, au sein du portefeuille de brevets des entreprises qui investissent le plus dans la R-D depuis 2010, affichent une accélération. Les " accélérations " correspondent à des périodes caractérisées par une hausse soudaine et persistante du nombre de dépôts de brevets, par classe de la CIB. Les accélérations les plus fortes sont mises en évidence par comparaison avec l'évolution des dépôts de brevets relevant de l'ensemble des classes de la CIB, dans le portefeuille des entreprises qui investissent le plus dans la R-D. L'intensité d'une accélération correspond au degré relatif de l'augmentation du nombre de dépôts observée. Les données pour 2014 sont partielles.

Les technologies recensées dans le graphique sont fondées sur la table de concordance CIB-Technologie de l'OMPI (2013) et la taxonomie des brevets liés aux TIC.

Les classes de la CIB sont décrites à l'adresse http://web2.wipo.int/classifications/ipc/ipcpub. 


\section{Brevets liés à l'intelligence artificielle déposés par des sociétés figurant parmi les 2000 entreprises les plus actives en R-D, par secteur, 2012-14}

Les données se rapportent aux familles de brevets IP5 liées à l'intelligence artificielle (IA), détenues par des sociétés figurant parmi les 2000 entreprises qui investissent le plus dans la R-D et déposées au cours de la période 2012-14. Sont considérées comme liées à l'IA les familles de brevets IP5 relevant des catégories Interface homme-machine et Cognition et compréhension sémantique de la taxonomie des brevets liés aux TIC, exposée dans Inaba et Squicciarini (2017). Les données pour 2014 sont partielles.

Les secteurs d'activité sont définis conformément à la CITI rév. 4.

\section{Brevets liés à l’intelligence artificielle déposés par les entreprises les plus actives en R-D, selon l'implantation du siège, 2012-14}

Les données se rapportent aux familles de brevets IP5 liées à l'intelligence artificielle (IA), détenues par des sociétés figurant parmi les 2000 entreprises qui investissent le plus dans la R-D et déposées au cours de la période 2012-14, par pays d'implantation du siège. Sont considérées comme liées à l'IA les familles de brevets IP5 relevant des catégories Interface homme-machine et Cognition et compréhension sémantique de la taxonomie des brevets liés aux TIC, exposée dans Inaba et Squicciarini (2017). Les données pour 2014 sont partielles.

\section{6. Évolution des publications scientifiques liées à l’apprentissage automatique, 2003-16 Cet indicateur est expérimental.}

Les estimations sont fondées sur des comptages fractionnaires des documents publiés, par auteur affilié à des établissements dans chaque économie.

Pour procéder aux estimations, une recherche a été lancée sur l'élément de texte " *machine learn* " dans les résumés, titres et mots-clés des articles publiés (en anglais) entre 2003 et 2016 et indexés dans la base Scopus.

\section{Articles scientifiques sur l'apprentissage automatique parmi les publications les plus citées, 2006 et 2016}

Cet indicateur est expérimental.

Il recense le nombre d'articles traitant de l'apprentissage automatique figurant parmi les publications les plus citées, publiés dans chaque pays ou économie. Sont pris en considération les $10 \%$ de publications les plus citées, les valeurs étant normalisées par domaine scientifique et type de document (articles, recensions et actes de conférences). Les valeurs SJR (Scimago Journal Rank) sont utilisées en complément pour hiérarchiser les publications ayant obtenu le même nombre de citations au sein d'une catégorie. Cette mesure est un indicateur de l'excellence de la recherche. Les estimations sont fondées sur des comptages fractionnaires des documents publiés, par auteur affilié à des établissements dans chaque économie.

Ces estimations ont été établies à partir d’une recherche sur l'élément de texte " *machine learn* " dans les résumés, titres et mots-clés des articles publiés (en anglais) entre 2006 et 2016 et indexés dans la base Scopus.

\section{8. Économies et BRICS les plus robotisés, 2005 et 2015}

L'utilisation de robots, mesurée à partir des informations collectées par la Fédération internationale de la robotique (IFR), reflète le nombre de robots achetés dans un pays ou un secteur donné. Le parc robotique a été évalué en additionnant la valeur du stock la première année, publiée par l'IFR, et les achats de robots au cours des années suivantes, en tenant compte d'un taux d'amortissement annuel de $10 \%$. Sont pris en considération les secteurs liés aux activités de fabrication, d'extraction et de distribution d'électricité, de gaz et de vapeur. En l'absence de données pour les pays suivants, les valeurs de 2014 et 2015 ont été obtenues par extrapolation : Australie, Chili, Estonie, Finlande, Grèce, Irlande, Islande, Lettonie, Lituanie, Norvège, Nouvelle-Zélande et Slovénie. Faute de données, la moyenne de l'OCDE n'intègre pas le Canada, Israël, le Luxembourg ni le Mexique. La moyenne de l'UE28 ne couvre pas Chypre ni le Luxembourg.

\section{Intensité robotique et intensité des tâches liées aux TIC dans les emplois manufacturiers, 2012 ou 2015}

L'utilisation de robots, mesurée à partir des informations collectées par la Fédération internationale de la robotique (IFR), reflète le nombre de robots achetés dans un pays ou un secteur donné. Le parc robotique a été évalué en additionnant la valeur du stock la première année, publiée par l'IFR, et les achats de robots au cours des années suivantes, en tenant compte d'un taux d'amortissement annuel de $10 \%$. L'échantillon couvre uniquement les secteurs de la fabrication et de la distribution d'électricité, de gaz et de vapeur. L'indicateur de l'intensité en TIC des emplois repose sur une analyse factorielle exploratoire de pointe. Il donne un aperçu de l'utilisation des TIC pour effectuer des tâches dans le cadre des emplois examinés, et s'appuie sur les réponses apportées à 11 questions de l'évaluation internationale des compétences des adultes (PIAAC) de l'OCDE ; ces tâches peuvent aller de la simple consultation de l'internet, à l'utilisation des logiciels Word ou Excel, ou d'un langage de programmation. Pour en savoir plus sur la méthodologie utilisée, voir Grundke et al. (2017).

Les données issues de la première phase du PIAAC pour les 23 pays suivants se rapportent à l'année 2012 : Allemagne, Australie, Autriche, Belgique (Flandre), Canada, Corée, Danemark, Espagne, Estonie, États-Unis, Fédération de Russie (sauf Moscou), Finlande, France, Irlande, Italie, Japon, Norvège, Pays-Bas, Pologne, République slovaque, République tchèque, Royaume-Uni (Angleterre et Irlande du Nord) et Suède. Les données afférentes aux autres pays se rapportent à 2015 et proviennent de la deuxième phase d'enquête du PIAAC concernant l'intensité des tâches liées aux TIC. 


\section{Dispersion des secteurs pour chaque dimension du numérique étudiée, 2013-15}

Tous les indicateurs sous-jacents sont exprimés sous la forme d'intensités sectorielles. Pour chacun d'entre eux, les valeurs sectorielles correspondent à la moyenne des valeurs des pays et des années considérés. Les valeurs obtenues sont ensuite normalisées par rapport à la moyenne, de sorte que, pour la série correspondant à chaque indicateur, la moyenne ait la valeur zéro, et l'écart type, la valeur 1.

La taxonomie est fondée sur les informations relatives aux pays suivants: Australie, Autriche, Danemark, États-Unis, Finlande, France, Italie, Japon, Norvège, Pays-Bas, Royaume-Uni et Suède, pour lesquels on dispose de valeurs pour l'ensemble des indicateurs, dans tous les secteurs et pour toutes les années considérés ; font exception les indicateurs " Utilisation de robots " et « Produit des ventes sur l'internet ", qui n'intègrent pas certains secteurs.

Les « investissements logiciels » correspondent au rapport entre les volumes de FBCF dans les logiciels et les volumes totaux de FBCF. Il en va de même pour les « investissements TIC matériels ". Les données qui entrent dans la composition de ces indicateurs sont tirées de la base de données des Comptes nationaux annuels de l'OCDE et d'INTAN-Invest. Les volumes sont obtenus à partir des valeurs à prix courants, ajustées à l'aide des déflateurs propres aux pays, dérivés des données d'INTAN-Invest (pour les logiciels) et des comptes nationaux (pour les actifs TIC matériels).

Les données relatives aux achats de biens TIC intermédiaires correspondent au rapport entre, d'une part, les achats de biens intermédiaires par le secteur observé, auprès du secteur produisant les équipements TIC (" Fabrication d'ordinateurs, d'articles électroniques et optiques ", ou secteurs 30, 32, 33 de la CITI rév. 3) et, d'autre part, la production du secteur acquéreur ; les données proviennent dans les deux cas de la base de données des tableaux internationaux d'entrées-sorties de l'OCDE et des tableaux nationaux des entrées-sorties. Pour ce qui est des achats de services TIC, on a procédé de même, mais en prenant cette fois les achats des différents secteurs auprès du secteur produisant les services TIC ("Activités informatiques et activités rattachées ", ou secteur 72 de la CITI rév. 3). Les achats de biens ou de services TIC sont corrigés des prix de la production des secteurs proposant les biens ou les services TIC d'un pays donné, tandis que les valeurs de la production sectorielle sont corrigées des prix de la production du secteur dans le pays observé. Les déflateurs sont issus de la base de données pour l'analyse structurelle (STAN) ou de la base de données des Comptes nationaux de l'OCDE. Les valeurs des achats de biens TIC par les secteurs liés aux activités de fabrication de machines (secteurs 29 à 35 de la CITI rév. 3) sont, par défaut, remplacées par des valeurs manquantes.

Les valeurs relatives aux achats de robots correspondent au nombre de robots achetés dans un pays ou un secteur donné, collecté par la Fédération internationale de la robotique (IFR). Les données sur l'utilisation des robots reflètent le rapport entre le parc de robots achetés dans un secteur et le nombre d'employés dudit secteur. Le parc robotique a été évalué en additionnant la valeur du stock la première année, publiée par l'IFR, et les achats de robots au cours des années suivantes, en tenant compte d'un taux d'amortissement annuel de $10 \%$. L'ensemble de données couvre l'agriculture, les activités extractives, la production manufacturière, la construction et la distribution d'électricité, de gaz et de vapeur (ainsi que le secteur de la R-D, qui n'entre pas dans le cadre de la présente analyse).

Le produit des ventes sur l'internet mesure la part du chiffre d'affaires du secteur étudié issue du commerce électronique ; les données sont tirées de la base de données Économie et société numériques d'Eurostat. Elles concernent uniquement les pays européens et la structure de l'échantillon ne couvre pas les secteurs suivants de la CITI rév. 4 : divisions 01 à 09 (agriculture et activités extractives), 64 à 66 (activités financières et d'assurances), et 84 et suivantes (administration publique, activités d'action sociale et de services personnels).

La catégorie "Spécialistes des TIC » reflète le rapport entre le nombre d'individus exerçant des fonctions de spécialiste des TIC dans le secteur étudié et l'emploi total du secteur. Les professions assimilées aux spécialistes des TIC aux fins de la présente analyse sont exposées dans Calvino et al. (à paraître). Elles correspondent aux codes CITP-08 suivants : 251 (Concepteurs et analystes de logiciels et de multimédia), 252 (Spécialistes des bases de données et des réseaux d'ordinateurs), 133 (Directeurs et cadres de direction, technologies de l'information et des communications) et 351 (Techniciens, opérations et soutien aux utilisateurs des technologies de l'information et des communications). Les données sur l'emploi par profession et par secteur sont issues des enquêtes sur la population active australienne, canadienne, européenne et japonaise, de la Current Population Survey des États-Unis, du recensement de la population active au Japon, et de la Korean Labour and Income Panel Study (KIPS), pour la Corée.

Pour en savoir plus sur les principes qui ont guidé le calcul des indicateurs, ainsi que sur toute opération de nettoyage, d'interpolation ou d'extrapolation réalisée sur les séries de données, voir Calvino et al. (à paraître) : A Taxonomy of Digital Sectors.

\section{Taxonomie des secteurs par quartile d'intensité numérique, 2013-15}

Tous les indicateurs sous-jacents sont exprimés sous la forme d'intensités sectorielles. Pour chacun d'entre eux, les valeurs sectorielles correspondent à la moyenne des valeurs des pays et des années considérés. Les valeurs obtenues sont ensuite normalisées par rapport à la moyenne, de sorte que, pour la série correspondant à chaque indicateur, la moyenne ait la valeur zéro, et l'écart type, la valeur 1. La couleur des cellules du tableau correspond au quartile dans lequel se situe le secteur dans la ventilation sectorielle. Les valeurs utilisées pour la constitution des quartiles par indicateur sont exposées en bas du tableau.

La taxonomie est fondée sur les informations relatives aux pays suivants: Australie, Autriche, Danemark, États-Unis, Finlande, France, Italie, Japon, Norvège, Pays-Bas, Royaume-Uni et Suède, pour lesquels on dispose de valeurs pour l'ensemble des indicateurs, dans tous les secteurs et pour toutes les années considérés; font exception les indicateurs " Utilisation de robots " et « Produit des ventes sur l'internet », qui n'intègrent pas certains secteurs.

Les « investissements logiciels " correspondent au rapport entre les volumes de FBCF dans les logiciels et les volumes totaux de FBCF. Il en va de même pour les « investissements TIC matériels ". Les données qui entrent dans la composition de ces indicateurs sont tirées de la base de données des Comptes nationaux annuels de l'OCDE et d'INTAN-Invest. Les volumes sont obtenus à 


\section{1. ÉCONOMIE DU SAVOIR ET TRANSFORMATION NUMÉRIQUE}

Notes et Références

partir des valeurs à prix courants, ajustées à l'aide des déflateurs propres aux pays, dérivés des données d'INTAN-Invest (pour les logiciels) et des comptes nationaux (pour les actifs TIC matériels).

Les données relatives aux achats de biens TIC intermédiaires correspondent au rapport entre, d'une part, les achats de biens intermédiaires par le secteur observé, auprès du secteur produisant les équipements TIC ("Fabrication d'ordinateurs, d'articles électroniques et optiques ", ou secteurs 30, 32, 33 de la CITI rév. 3) et, d'autre part, la production du secteur acquéreur ; les données proviennent dans les deux cas de la base de données des tableaux internationaux d'entrées-sorties de l'OCDE et des tableaux nationaux des entrées-sorties. Pour ce qui est des achats de services TIC, on a procédé de même, mais en prenant cette fois les achats des différents secteurs auprès du secteur produisant les services TIC (" Activités informatiques et activités rattachées ", ou secteur 72 de la CITI rév. 3). Les achats de biens ou de services TIC sont corrigés des prix de la production des secteurs proposant les biens ou les services TIC d'un pays donné, tandis que les valeurs de la production sectorielle sont corrigées des prix de la production du secteur dans le pays observé. Les déflateurs sont issus de la base de données pour l'analyse structurelle (STAN) ou de la base de données des Comptes nationaux de l'OCDE. Les valeurs des achats de biens TIC par les secteurs liés aux activités de fabrication de machines (secteurs 29 à 35 de la CITI rév. 3) sont, par défaut, remplacées par des valeurs manquantes.

Les valeurs relatives aux achats de robots correspondent au nombre de robots achetés dans un pays ou un secteur donné, collecté par la Fédération internationale de la robotique (IFR). Les données sur l'utilisation des robots reflètent le rapport entre le parc de robots achetés dans un secteur et le nombre d'employés dudit secteur. Le parc robotique a été évalué en additionnant la valeur du stock la première année, publiée par l'IFR, et les achats de robots au cours des années suivantes, en tenant compte d'un taux d'amortissement annuel de $10 \%$. L'ensemble de données couvre l'agriculture, les activités extractives, la production manufacturière, la construction et la distribution d'électricité, de gaz et de vapeur (ainsi que le secteur de la R-D, qui n'entre pas dans le cadre de la présente analyse).

Le produit des ventes sur l'internet mesure la part du chiffre d'affaires du secteur étudié issue du commerce électronique ; les données sont tirées de la base de données Économie et société numériques d'Eurostat. Elles concernent uniquement les pays européens et la structure de l'échantillon ne couvre pas les secteurs suivants de la CITI rév. 4 : divisions 01 à 09 (agriculture et activités extractives), 64 à 66 (activités financières et d'assurances), et 84 et plus (administration publique, activités d'action sociale et de services personnels).

La catégorie "Spécialistes des TIC » reflète le rapport entre le nombre d'individus exerçant des fonctions de spécialiste des TIC dans le secteur étudié, et l'emploi total du secteur. Les professions assimilées aux spécialistes des TIC aux fins de la présente analyse sont exposées dans Calvino et al. (à paraître). Elles correspondent aux codes CITP-08 suivants : 251 (Concepteurs et analystes de logiciels et de multimédia), 252 (Spécialistes des bases de données et des réseaux d'ordinateurs), 133 (Directeurs et cadres de direction, technologies de l'information et des communications) et 351 (Techniciens, opérations et soutien aux utilisateurs des technologies de l'information et des communications). Les données sur l'emploi par profession et par secteur sont issues des enquêtes sur la population active australienne, canadienne, européenne et japonaise, de la Current Population Survey des États-Unis, du recensement de la population active au Japon, et de la Korean Labour and Income Panel Study (KIPS), pour la Corée.

Pour en savoir plus sur les principes qui ont guidé le calcul des indicateurs, ainsi que sur toute opération de nettoyage, d'interpolation ou d'extrapolation réalisée sur les séries de données, voir Calvino et al. (à paraître) : A Taxonomy of Digital Sectors.

\section{Niveaux de compétences dans des secteurs plus ou moins exposés au numérique, 2012 ou 2015}

Les différences au niveau des compétences moyennes entre les secteurs à plus ou moins forte intensité numérique sont significatives à $5 \%$.

Les indicateurs de compétences au niveau individuel sont fondés sur les données du Programme pour l'évaluation internationale des compétences des adultes (PIAAC) de l'OCDE. La maîtrise de la langue, la maîtrise du calcul et la résolution de problèmes en environnement à forte composante technologique font partie des compétences cognitives mesurées dans le cadre des tests d'évaluation. Les autres indicateurs de compétences sont établis à l'aide de données sur la fréquence des tâches exécutées par les individus dans le cadre de leur travail et en menant une analyse factorielle de pointe. Pour en savoir plus sur la méthodologie utilisée, voir Grundke et al. (2017). Tous les indicateurs de compétences sont exprimés sur une échelle de 0 à 100. Les moyennes sont calculées pour les secteurs exposés ou non aux technologies numériques dans l'ensemble des 31 pays ayant participé au PIAAC, chacun d'entre eux ayant le même poids.

Une taxonomie des secteurs à forte intensité numérique est proposée dans Calvino et al. (à paraître). Le caractère multidimensionnel de la transformation numérique est évalué en examinant la place des composantes suivantes dans les secteurs : investissements TIC matériels et immatériels, achats de biens et de services TIC, utilisation de robots, produit des ventes sur l'internet et emploi de spécialistes des TIC. Les secteurs se situant au-delà du secteur médian au regard de la combinaison de ces indicateurs sont considérés comme présentant une forte intensité numérique.

L'échantillon couvre 31 pays (phases 1 et 2 du PIAAC). Les données issues de la première phase du PIAAC pour les 23 pays ci-après se rapportent à l'année 2012 : Allemagne, Australie, Autriche, Belgique (Flandre), Canada, Corée, Danemark, Espagne, Estonie, États-Unis, Fédération de Russie (sauf Moscou), Finlande, France, Irlande, Italie, Japon, Norvège, Pays-Bas, Pologne, République slovaque, République tchèque, Royaume-Uni (Angleterre et Irlande du Nord) et Suède. Les données afférentes aux huit autres pays, issues de la deuxième phase de la première vague d'enquête, se rapportent à 2015 : Chili, Grèce, Israël, Lituanie, NouvelleZélande, Singapour, Slovénie et Turquie. 


\section{Rendements additionnels des compétences sur le marché du travail dans les secteurs à forte intensité numérique, 2012 ou 2015}

Les barres zébrées indiquent que le coefficient n'est pas significatif à $5 \%$.

Les indicateurs de compétences au niveau individuel sont fondés sur les données du Programme pour l'évaluation internationale des compétences des adultes (PIAAC) de l'OCDE. La maîtrise de la langue, la maîtrise du calcul et la résolution de problèmes en environnement à forte composante technologique font partie des compétences cognitives mesurées dans le cadre des tests d'évaluation. Les autres indicateurs de compétences sont établis à l'aide de données sur la fréquence des tâches exécutées par les individus dans le cadre de leur travail et en menant une analyse factorielle de pointe. Pour en savoir plus sur la méthodologie utilisée, voir Grundke et al. (2017).

Les rendements des compétences sur le marché du travail sont obtenus par des régressions des salaires selon la méthode des moindres carrés ordinaires (équations de Mincer) à partir des données de l'évaluation internationale des compétences des adultes (PIAAC) de l'OCDE. Les estimations s'appuient sur le logarithme des salaires horaires en tant que variable dépendante et intègrent diverses variables de contrôle liées aux individus (notamment l'âge, les années d'étude, le sexe et les compétences cognitives que sont la maîtrise de la langue et du calcul), ainsi que les variables nominales pays, secteur et profession. Les coefficients sont obtenus en estimant la spécification pour l'échantillon de 31 pays.

Une taxonomie des secteurs à forte intensité numérique est proposée dans Calvino et al. (à paraître). Le caractère multidimensionnel de la transformation numérique est évalué en examinant la place des composantes suivantes dans les secteurs : investissements TIC matériels et immatériels, achats de biens et de services TIC, utilisation de robots, produit des ventes sur l'internet et emploi de spécialistes des TIC. Les secteurs se situant au-delà du secteur médian au regard de la combinaison de ces indicateurs sont considérés comme présentant une forte intensité numérique.

L'échantillon couvre 31 pays (phases 1 et 2 du PIAAC). Les données issues de la première phase du PIAAC pour les 23 pays ci-après se rapportent à 2012 : Allemagne, Australie, Autriche, Belgique (Flandre), Canada, Corée, Danemark, Espagne, Estonie, États-Unis, Fédération de Russie (sauf Moscou), Finlande, France, Irlande, Italie, Japon, Norvège, Pays-Bas, Pologne, République slovaque, République tchèque, Royaume-Uni (Angleterre et Irlande du Nord) et Suède. Les données afférentes aux huit autres pays, issues de la deuxième phase de la première vague d'enquête, se rapportent à 2015 : Chili, Grèce, Israël*, Lituanie, Nouvelle-Zélande, Singapour, Slovénie et Turquie.

\section{Géographie des pertes et des créations d'emplois, 2010-16}

Les données pour la Corée, Israël, le Japon, le Mexique, la Nouvelle-Zélande et l'agrégat de la zone OCDE se rapportent à la période 2010-15.

Pour mettre en évidence leur contribution relative à la variation totale de l'emploi entre deux périodes dans chaque pays, on peut «normaliser » les variations de l'emploi par activité économique. À cette fin, les variations sectorielles sont exprimées, pour chaque pays, en pourcentage de la somme des variations absolues. Les groupes d'activités sont définis suivant les divisions de la CITI rév. 4.

Les activités sectorielles agrégées sont définies sur la base de la CITI rév. 4 : Agriculture, sylviculture et pêche (divisions 01-03) ; Activités extractives, production et distribution d'électricité, de gaz, de vapeur, de climatisation et d'eau (05-09 et 35-39) ; Activités de fabrication (10-33) ; Construction (41-43) ; Commerce de gros et de détail, Activités d'hébergement et de restauration, transport (45-56) ; Information et communication (58-63) ; Activités financières et d'assurances (64-68) ; Activités professionnelles, scientifiques et techniques, Autres services aux entreprises (69-82) ; et Administration publique, éducation, santé et autres services (84-99).

Les créations et les pertes sont exprimées en milliers d'emplois et correspondent respectivement à la somme des secteurs dans lesquels les variations sont positives et à la somme des secteurs présentant des variations négatives. Le recours à une ventilation plus fine (en se fondant par exemple sur les codes à deux chiffres de la CITI rév. 4) donnerait lieu à des estimations différentes du nombre total de créations et de pertes d'emplois.

Les données sur l'emploi proviennent essentiellement des comptes nationaux et sont exprimées en nombre de personnes, sauf pour le Canada, le Japon et le Mexique, où l'unité de mesure est le nombre de postes.

\section{Croissance de l'emploi dans les secteurs de l'information, OCDE, 1997-2015}

Les « secteurs de l'information " sont définis d'après la CITI rév. 4 ; ils, couvrent les activités de fabrication d'équipements TIC, à savoir la division 26 (Fabrication d'ordinateurs, d'articles électroniques et optiques), les services d'information, soit les divisions 58 à 60 (Activités d'édition, audiovisuel et activités de diffusion), ainsi que la division 61 (Télécommunications) et les divisions 62 et 63 (Activités informatiques et autres services d'information).

Le secteur des entreprises englobe pour sa part les divisions 05 à 66, et 69 à 82 de la CITI rév. 4, soit l'ensemble de l'économie à l'exception des divisions suivantes : Agriculture, sylviculture et pêche (divisions 01 à 03), Activités immobilières (68), Administration publique (84), Éducation (85), Santé et activités d'action sociale (86 à 88), et Arts, spectacles et loisirs, Activités de réparation d'articles ménagers et Autres activités de services personnels (90 à 99).

\section{Origine de la demande soutenant les emplois dans le secteur des entreprises, zone OCDE, 1995-2014}

Le secteur des entreprises correspond aux divisions 10 à 74 de la CITI rév. 3, soit l'ensemble des activités économiques hors Agriculture, sylviculture et pêche (divisions 01 à 05), Administration publique (75), Éducation (80), Santé (85) et Autres activités de services collectifs, sociaux et personnels (90 à 95). 


\section{1. ÉCONOMIE DU SAVOIR ET TRANSFORMATION NUMÉRIQUE}

Notes et Références

La zone UE28 couvre les 28 pays membres de l'Union européenne ; l'Asie du Sud-Est (hors Indonésie) comprend Brunei Darussalam, le Cambodge, la Malaisie, les Philippines, Singapour, la Thaillande et le Viet Nam ; l'Asie orientale englobe la Corée, le Japon, Hong Kong (Chine) et le Taipei chinois ; la zone ALENA comprend le Canada, les États-Unis et le Mexique ; et les BRIICS (hors Chine) rassemblent le Brésil, la Fédération de Russie, l’Inde, l'Indonésie et l'Afrique du Sud.

\section{Origine de la demande soutenant les emplois dans les secteurs de l'information, zone OCDE, 1995-2014}

Les secteurs de l'information renvoient aux divisions 30, 32, 33, 64 et 72 de la CITI rév. 3.

La zone UE28 couvre les 28 pays membres de l'Union européenne ; l'Asie du Sud-Est (hors Indonésie) comprend Brunei Darussalam, le Cambodge, la Malaisie, les Philippines, Singapour, la Thailande et le Viet Nam ; l'Asie orientale englobe la Corée, le Japon, Hong Kong (Chine) et le Taipei chinois ; la zone ALENA comprend le Canada, les États-Unis et le Mexique ; et les BRIICS (hors Chine) rassemblent le Brésil, la Fédération de Russie, l'Inde, l’Indonésie et l'Afrique du Sud.

\section{Emplois du secteur des entreprises soutenus par la demande finale extérieure, par intensité de qualification, 2014}

Le secteur des entreprises correspond aux divisions 10 à 74 de la CITI rév. 3, soit l'ensemble des activités économiques hors Agriculture, sylviculture et pêche (divisions 01 à 05), Administration publique (75), Éducation (80), Santé (85) et Autres activités de services collectifs, sociaux et personnels (90 à 95).

Les trois catégories d'intensité de qualification sont définies selon les grands groupes de la Classification internationale type des professions 2008 (CITP-08) : professions très qualifiées (grands groupes 1 à 3 de la CITP-08), moyennement qualifiées (groupes 4 à 7) et peu qualifiées (groupes 8 et 9).

Pour les États-Unis, les données concernant les activités professionnelles proviennent de l'Enquête sur la population active. Le papier d'Eckardt and Squicciarini (2015) décrit la table utilisée pour convertir les données de la classification SOC et celles de recensement selon la classification des professions CITP-08.

Les données pour l’Australie se rapportent à 2011, celles pour la Corée à 2012.

\section{Part de l'emploi non répétitif et de l'intensité des tâches liées aux TIC, 2012 ou 2015}

L'indicateur relatif à la composante TIC des emplois s'appuie sur une analyse factorielle exploratoire de pointe et rend compte de l'utilisation de ces technologies en milieu professionnel. Il repose sur les réponses à 11 questions de l'évaluation internationale des compétences des adultes (PIAAC) de l'OCDE ; les tâches considérées vont de la simple consultation de l'internet, à l'utilisation des logiciels Word ou Excel, ou d'un langage de programmation. Pour en savoir plus sur la méthodologie utilisée, voir Grundke et al. (2017). Les intensités sont exprimées sur une échelle de 0 à 100 (l'échelle allait, à l'origine, de 0 à 1).

La part de l'emploi non routinier correspond à la part, dans l'emploi total d'un secteur, des professions (codes à trois chiffres) affichant une forte proportion de tâches non répétitives. La classification des professions selon la proportion de tâches répétitives s'appuie sur la méthodologie décrite dans Marcolin et al. (2015). Les professions à forte proportion de tâches répétitives affichent des valeurs supérieures à la médiane en termes d'intensité de tâches répétitives exécutées dans le cadre du travail ; les professions dites non routinières se situent en deçà de la médiane.

Les différences observées dans les lignes de tendances des grands secteurs doivent être prises avec prudence, dans la mesure où le test de Wald ne permet pas de rejeter l'hypothèse d'une égalité des corrélations dans les secteurs des services marchands et des industries manufacturières.

Les points représentent des moyennes simples des valeurs dans les secteurs manufacturiers et les services marchands. Les industries manufacturières couvrent les activités extractives ; les produits alimentaires et boissons ; les textiles, l'habillement et le cuir ; le bois, le papier et l'édition ; les métaux de base et usinés ; les produits chimiques, le caoutchouc, les matières plastiques et autres produits minéraux non métalliques ; la fabrication de machines et de matériel, n.c.a. ; la fabrication d'ordinateurs, d'articles électroniques et optiques ; la fabrication de matériels de transport ; et les autres activités de fabrication, n.c.a. Les services marchands couvrent la distribution d'électricité, de gaz, de vapeur et d'eau, la construction, le commerce, les activités de réparation, les activités d'hébergement ; les services de transport et de télécommunication ; les activités financières ; et les services aux entreprises.

Les données issues de la première phase du PIAAC pour les 22 pays suivants se rapportent à 2012 : Allemagne, Australie, Autriche, Belgique (Flandre), Canada, Corée, Danemark, Espagne, Estonie, États-Unis, Finlande, France, Irlande, Italie, Japon, Norvège, Pays-Bas, Pologne, République slovaque, République tchèque, Royaume-Uni (Angleterre et Irlande du Nord) et Suède. Les données concernant les autres pays se rapportent à 2015 et sont issues de la deuxième phase de la première vague d'enquête.

\section{Travailleurs ayant bénéficié d'une formation en entreprise, par niveau de qualification, 2012 ou 2015}

Le pourcentage de travailleurs avant suivi une formation est obtenu en calculant le rapport entre, d'une part, le nombre total d'employés dotés d'un niveau de qualification donné et ayant suivi au moins une formation au cours de l'année considérée et, d'autre part, l'emploi total dans l'économie. Sont prises en considération les formations formelles, les formations en cours d'emploi, ou les deux, telles que définies dans Squicciarini et al. (2015). Les individus peu qualifiés sont les personnes qui n'ont pas reçu un enseignement structuré ou ont atteint les niveaux 1 à 3C de la classification CITE 1997 (si le niveau 3C a été d'une 
durée inférieure à deux ans). Les individus présentant une qualification moyenne sont ceux qui ont atteint un niveau 3C (d'une durée supérieure à deux ans) à 4. Les individus hautement qualifiés ont dépassé le niveau 4 de la CITE 1997. Les valeurs ont été pondérées afin d'être représentatives de la population des pays.

Les données issues de la première phase du PIAAC pour les 23 pays ci-après se rapportent à 2012 : Allemagne, Australie, Autriche, Belgique (Flandre), Canada, Corée, Danemark, Espagne, Estonie, États-Unis, Fédération de Russie (sauf Moscou), Finlande, France, Irlande, Italie, Japon, Norvège, Pays-Bas, Pologne, République slovaque, République tchèque, Royaume-Uni (Angleterre et Irlande du Nord) et Suède. Les données concernant les autres pays se rapportent à 2015 et sont issues de la deuxième phase de la première vague d'enquête.

\section{1. Écarts salariaux hommes-femmes par pays, 2012 ou 2015}

Les estimations quant aux écarts de salaire entre hommes et femmes sont fondées sur des régressions des salaires selon la méthode des moindres carrés ordinaires (équations de Mincer), à partir des données de l'évaluation internationale des compétences des adultes (PIAAC) de l'OCDE. Elles s'appuient sur le logarithme des salaires horaires en tant que variable dépendante et intègrent diverses variables de contrôle liées aux individus (notamment l'âge, les années d'étude, le sexe et des mesures de compétences détaillées dans Grundke et al., 2017), ainsi que des variables nominales propres au secteur concerné.

Les données issues de la première phase du PIAAC pour les 23 pays ci-après se rapportent à l'année 2012 : Allemagne, Australie, Autriche, Belgique (Flandre), Canada, Corée, Danemark, Espagne, Estonie, États-Unis, Fédération de Russie (sauf Moscou), Finlande, France, Irlande, Italie, Japon, Norvège, Pays-Bas, Pologne, République slovaque, République tchèque, Royaume-Uni (Angleterre et Irlande du Nord) et Suède. Les données concernant les autres pays se rapportent à 2015 et sont issues de la deuxième phase de la première vague d'enquête.

\section{Rendement de la composante TIC sur le marché du travail, par sexe, 2012 ou 2015}

L'indicateur de la composante TIC des emplois repose sur une analyse factorielle exploratoire de pointe. Il donne un aperçu des tâches liées aux TIC effectuées dans le cadre des emplois étudiés, à la lumière des réponses à 11 questions de l'évaluation internationale des compétences des adultes (PIAAC) de l'OCDE ; ces tâches vont de la simple consultation de l'internet, à l'utilisation des logiciels Word ou Excel, ou d'un langage de programmation. Pour en savoir plus sur la méthodologie utilisée, voir Grundke et al. (2017).

Les rendements de la composante TIC sur le marché du travail sont obtenus en opérant des régressions des salaires selon la méthode des moindres carrés ordinaires (équations de Mincer), à partir de données de l'évaluation internationale des compétences des adultes (PIAAC) de l'OCDE. Les estimations s'appuient sur le logarithme des salaires horaires en tant que variable dépendante, et intègrent diverses variables de contrôle ayant trait aux individus (notamment l'âge, les années d'étude, le sexe et d'autres mesures de compétences détaillées dans Grundke et al., 2017), ainsi que des variables nominales propres au secteur concerné. Les coefficients pour les travailleurs masculins et féminins sont obtenus en estimant la spécification respective pour chaque sous-échantillon. La composante TIC moyenne par pays utilisée pour calculer le pourcentage d'évolution des salaires pour une augmentation de $10 \%$ de la composante TIC correspond aux moyennes nationales respectives observées pour les hommes et les femmes.

Les données issues de la première phase du PIAAC pour les 23 pays ci-après se rapportent à l'année 2012 : Allemagne, Australie, Autriche, Belgique (Flandre), Canada, Corée, Danemark, Espagne, Estonie, États-Unis, Fédération de Russie (sauf Moscou), Finlande, France, Irlande, Italie, Japon, Norvège, Pays-Bas, Pologne, République slovaque, République tchèque, Royaume-Uni (Angleterre et Irlande du Nord) et Suède. Les données concernant les autres pays se rapportent à 2015 et sont issues de la deuxième phase de la première vague d'enquête.

\section{Employés ayant suivi des formations en cours d'emploi, par sexe, 2012 ou 2015}

La proportion de femmes et d'hommes ayant suivi des formations en cours d'emploi ne tient pas compte des individus n'ayant pas précisé si la formation a eu lieu pendant les heures de travail ou en dehors du temps de travail (environ $4 \%$ de l'échantillon global). Le nombre d'hommes et de femmes ayant suivi des formations pendant les heures de travail correspond au nombre de salariés ayant indiqué que la formation avait eu lieu exclusivement ou principalement sur leur temps de travail. La proportion est obtenue en calculant le rapport entre ce chiffre et le nombre total d'employés du sexe considéré, dans chacune des économies. Les données issues de la première phase du PIAAC pour les 23 pays ci-après se rapportent à 2012 : Allemagne, Australie, Autriche, Belgique (Flandre), Canada, Corée, Danemark, Espagne, Estonie, États-Unis, Fédération de Russie (sauf Moscou), Finlande, France, Irlande, Italie, Japon, Norvège, Pays-Bas, Pologne, République slovaque, République tchèque, Royaume-Uni (Angleterre et Irlande du Nord) et Suède. Les données concernant les autres pays se rapportent à 2015 et sont issues de la deuxième phase de la première vague d'enquête.

\section{Décomposition sectorielle de la croissance de la productivité du travail, 2001-07 et 2009-15}

Pour l'Irlande et la Nouvelle-Zélande, la période la plus récente est 2009-14. Pour la Suisse, la période la plus récente est 2010-15, et les activités de fabrication incluent les activités extractives, la production et distribution d'énergie, et la collecte et traitement des déchets.

On entend par croissance de la productivité du travail la variation annuelle de la valeur ajoutée brute (en termes de volume) par heure travaillée.

Les regroupements d'activités sont établis à partir de la CITI rév. 4 : Activités extractives ; distribution d'électricité, de gaz, de vapeur, d'air conditionné ; distribution d'eau, traitement et gestion des déchets (divisions 05 à 09 et 35 à 39 ); Activités de fabrication (10 à 33); Construction (41 à 43); Commerce de gros et de détail, hôtels, services de restauration, transports (45 à 56) ; Information et 


\section{1. ÉCONOMIE DU SAVOIR ET TRANSFORMATION NUMÉRIQUE}

Notes et Références

communication (58 à 63) ; Activités financières et d'assurances (64 à 66) ; Activités professionnelles, scientifiques et techniques et autres services aux entreprises (69 à 82). Le secteur des entreprises non agricoles recouvre les divisions 05 à 66 et 69 à 82 . Les activités immobilières (68) sont exclues car la valeur ajoutée de ce secteur comprend une imputation correspondant aux services d'hébergement que les propriétaires fournissent et consomment.

\section{Productivité du travail des secteurs de l'information, 2015}

La productivité du travail est la valeur ajoutée en prix courants par heure travaillée et par actif occupé.

Les secteurs de l'information sont définis conformément à la CITI rév. 4 : Fabrication d'ordinateurs, d'articles électroniques et optiques (division 26), Activités d'édition, audiovisuel et activités de diffusion (58 à 60), Télécommunications (61) et Activités informatiques et autres services d'information (62 et 63).

Le secteur des entreprises non agricoles recouvre les divisions 05 à 66 et 69 à 82 de la CITI rév. 4. Les activités immobilières (division 68) sont exclues car la valeur ajoutée de ce secteur inclut une imputation pour les services d'hébergement que les propriétaires fournissent et consomment.

Les estimations portant sur la Corée, Israël, la Lettonie et le Luxembourg n'incluent pas la fabrication d'ordinateurs, d'articles électroniques et optiques (division 26).

Les données de l'Allemagne, l'Espagne, l'Irlande, la Nouvelle-Zélande, la Pologne, le Portugal, la Suède et la Suisse se rapportent à 2014 ; celles du Canada et de la Corée se rapportent à 2013 ; celles de l'Australie et de la Nouvelle-Zélande se rapportent à l'année fiscale 2014/15.

La moyenne de la zone OCDE est une moyenne non pondérée de la valeur ajoutée par actif occupé dans les pays indiqués.

\section{Croissance de la productivité totale des facteurs, 1995-2015}

Pour l’Espagne, l’Irlande et le Portugal, les données se rapportent à la période 1995-2014.

\section{Contribution élargie des TIC en valeur ajoutée locale, 2011}

Dans cette analyse, le secteur des technologies de l'information et des communications (TIC) est défini conformément à la CITI rév. 3 et recouvre la fabrication d'ordinateurs, d'articles électroniques et optiques (divisions 30, 32 et 33), les postes et télécommunications (division 64) et les activités informatiques et activités rattachées (division 72). La base de données sous-jacente des TIES étant construite à partir de statistiques du Système des comptes nationaux (SCN) de 1993, les chiffres sur la valeur ajoutée des TIC, présentés ici, pourraient ne pas correspondre aux statistiques sur la valeur ajoutée calculées à partir de la CITI rév. 4 et du SCN de 2008 .

\section{Valeur ajoutée locale liée aux TIC, 2011}

Le secteur des technologies de l'information et des communications (TIC) est défini conformément à la CITI rév. 3 et recouvre la fabrication d'ordinateurs, d'articles électroniques et optiques (divisions 30, 32 et 33), les postes et télécommunications (division 64) et les activités informatiques et activités rattachées (division 72).

La valeur ajoutée du secteur des TIC national se retrouve dans une large gamme de biens et services finals destinés à répondre à la demande finale tant intérieure qu'étrangère. De même, la valeur ajoutée des autres secteurs nationaux (non TIC) peut se retrouver dans des biens et services TIC finals consommés à l'étranger.

\section{Valeur ajoutée étrangère liée aux TIC dans la demande finale intérieure, 2011}

Le secteur des technologies de l'information et des communications (TIC) est défini conformément à la CITI rév. 3 et recouvre la fabrication d'ordinateurs, d'articles électroniques et optiques (divisions 30, 32 et 33), les postes et télécommunications (division 64) et les activités informatiques et activités rattachées (division 72).

La valeur ajoutée des secteurs des TIC étrangers peut se retrouver dans une large gamme de biens et services finals destinés à satisfaire la demande intérieure. De même, la valeur ajoutée des autres secteurs étrangers (non TIC) peut se retrouver dans des biens et services finals consommés localement.

\section{Contribution des équipements informatiques et des actifs intellectuels à la croissance de la productivité du travail augmentée du capital intellectuel, 2000-14}

Le graphique montre les contributions du capital intellectuel et des actifs corporels des TIC à la croissance de la productivité du travail en pourcentage de la croissance de la productivité du travail elle-même entre 2000 et 2014 . On calcule ces contributions à l'aide d'une méthode de comptabilité de la croissance non paramétrique standard pour l'ensemble de la période considérée, en supposant des rendements d'échelle constants et des marchés pleinement concurrentiels, une technologie de production log-linéaire et des élasticités de production égales à des parts des facteurs. Le capital intellectuel comprend les logiciels, la R-D et le capital organisationnel (d'après Le Mouel et al., 2016). Les données sur les investissements dans les logiciels, la R-D et les équipements TIC proviennent de la base de données de l'OCDE sur les Comptes nationaux, sauf dans le cas des États-Unis pour lesquels les chiffres sur les investissements dans la R-D proviennent des comptes satellites du Bureau d'analyse économique (BEA, Bureau of Economic Analysis). Toutes les données sous-jacentes sont exprimées en termes réels. Les estimations des stocks de capital sont obtenues en appliquant la méthode de l'inventaire permanent aux données sur les investissements, avec 1993 comme année de départ. Certains points de données sont interpolés ou extrapolés le cas échéant.

L'échantillon couvre les secteurs marchands uniquement (c'est-à-dire les divisions 01 à 82, sauf 68, et 90 à 96 de la CITI rév. 4). 


\section{Contribution du capital intellectuel et de la PTF à la croissance de la productivité du travail augmentée du capital intellectuel, 2000-14}

Le graphique montre les contributions du capital intellectuel et de la productivité totale des facteurs (PTF) à la croissance de la productivité du travail en pourcentage de la croissance de la productivité du travail elle-même entre 2000 et 2014 . On calcule ces contributions à l'aide d'une méthode de comptabilité de la croissance non paramétrique standard pour l'ensemble de la période considérée, en supposant des rendements d'échelle constants et des marchés pleinement concurrentiels, une technologie de production log-linéaire et des élasticités de production égales à des parts des facteurs. Le capital intellectuel comprend les logiciels, la R-D et le capital organisationnel (d'après Le Mouel et al., 2016). Les données sur les investissements dans les logiciels et la R-D proviennent de la base de données de l'OCDE sur les Comptes nationaux, sauf dans le cas des États-Unis pour lesquels les chiffres sur les investissements dans la R-D proviennent des comptes satellites du Bureau d'analyse économique (BEA, Bureau of Economic Analysis).

Toutes les données sous-jacentes sont exprimées en termes réels. Les estimations des stocks de capital sont obtenues en appliquant la méthode de l'inventaire permanent aux données sur les investissements, avec 1993 comme année de départ. Certains points de données sont interpolés ou extrapolés le cas échéant.

L'échantillon couvre les secteurs marchands uniquement (c'est-à-dire les divisions 01 à 82, sauf 68 , et 90 à 96 de la CITI rév. 4).

\section{Intensité en capital intellectuel des secteurs marchand et non marchand, 2015}

Le secteur marchand recouvre les divisions 01 à 82, sauf 68, et 90 à 96 de la CITI rév. 4. Le secteur non marchand se réfère à la définition proposée par SPINTAN et couvre les établissements publics et les entités sans but lucratif appartenant aux divisions 72 et 84 à 88 de la CITI rév. 4.

Les intensités sont définies comme l'investissement rapporté à la valeur ajoutée brute telle qu'elle figure dans la base de données sur les Comptes nationaux de l'OCDE. Pour le secteur non marchand, les données sur les investissements dans le capital intellectuel ont été fournies par SPINTAN et extrapolées, lorsque nécessaire, à l'aide du taux de croissance moyen de ces investissements dans l'ensemble des pays, tel qu'il est donné par la même source. Les données relatives aux investissements dans les autres actifs intellectuels, hors champ des comptes nationaux, ont été fournies par INTAN-Invest et extrapolées, lorsque nécessaire, à l'aide du taux de croissance de la formation brute de capital fixe associée à la propriété intellectuelle, tiré de la base de données sur les Comptes nationaux de l'OCDE. Les chiffres des investissements et de la valeur ajoutée sont exprimés en prix courants.

\section{3. Évolution de la centralité de la fabrication de matériel informatique dans les économies, 1995-2011}

La fabrication de matériel informatique recouvre les divisions 30, 32 et 33 de la CITI rév. 3 : fabrication d'ordinateurs, d'articles électroniques et optiques.

La position des économies sur le graphique correspond à leur situation géographique. La taille des cercles traduit l'ampleur des variations (en valeur absolue) de la centralité étrangère totale au cours de la période 1995-2001. À des fins de lisibilité, on a représenté ces variations sur une échelle logarithmique. Les cercles bleus indiquent une augmentation de la centralité et les cercles rouges une diminution.

Les indicateurs de la centralité sont calculés à partir des données de l'édition 2015 de la base de l'OCDE sur les Tableaux internationaux des entrées-sorties, qui fournissent des données sur les flux d'intrants de 61 pays et 34 secteurs d'activités de 1995 à 2011. Pour chaque couple pays-secteur, on calcule la centralité de référence, ainsi que la somme pondérée des centralités des partenaires commerciaux, où les poids sont les parts des intrants. La centralité totale est la moyenne des centralités calculées avec les liaisons en aval (exportations d'intrants) et les liaisons en amont (importations d'intrants). On décompose la centralité selon son origine étrangère ou locale. La centralité étrangère est celle qui est due aux liaisons (directes et indirectes) avec les secteurs étrangers, tandis que la centralité locale représente la composante due aux liaisons (directes et indirectes) avec les secteurs locaux.

\section{4. Évolution de la centralité des services informatiques dans les économies, 1995-2011}

Les services informatiques sont ceux de la division 72 de la CITI rév. 3 : activités informatiques et activités rattachées.

La position des économies sur le graphique correspond à leur situation géographique. La taille des cercles traduit l'ampleur des variations (en valeur absolue) de la centralité étrangère totale au cours de la période 1995-2001. À des fins de lisibilité, on a représenté ces variations sur une échelle logarithmique. Les cercles bleus indiquent une augmentation de la centralité et les cercles rouges une diminution.

Les indicateurs de la centralité sont calculés à partir des données de l'édition 2015 de la base de l'OCDE sur les Tableaux internationaux des entrées-sorties, qui fournissent des données sur les flux d'intrants de 61 pays et 34 secteurs d'activités de 1995 à 2011. Pour chaque couple pays-secteur, on calcule la centralité de référence, ainsi que la somme pondérée des centralités des partenaires commerciaux, où les poids sont les parts des intrants. La centralité totale est la moyenne des centralités calculées avec les liaisons en aval (exportations d'intrants) et les liaisons en amont (importations d'intrants). On décompose la centralité selon son origine étrangère ou locale. La centralité étrangère est celle qui est due aux liaisons (directes et indirectes) avec les secteurs étrangers, tandis que la centralité locale représente la composante due aux liaisons (directes et indirectes) avec les secteurs locaux.

\section{Variations les plus fortes de la centralité étrangère et locale : fabrication et services informatiques,} 1995-2011

La fabrication de matériel informatique recouvre les divisions 30, 32 et 33 de la CITI rév. 3 : fabrication d'ordinateurs, d'articles électroniques et optiques. 


\title{
1. ÉCONOMIE DU SAVOIR ET TRANSFORMATION NUMÉRIQUE
}

\author{
Notes et Références
}

Les services informatiques sont ceux de la division 72 de la CITI rév. 3 : activités informatiques et activités rattachées.

Les indicateurs de la centralité sont calculés à partir des données de l'édition 2015 de la base de l'OCDE sur les Tableaux internationaux des entrées-sorties, qui fournissent des données sur les flux d'intrants de 61 pays et 34 secteurs d'activités de 1995 à 2011. Pour chaque couple pays-secteur, on calcule la centralité de référence, ainsi que la somme pondérée des centralités des partenaires commerciaux, où les poids sont les parts des intrants. La centralité totale est la moyenne des centralités calculées avec les liaisons en aval (exportations d'intrants) et les liaisons en amont (importations d'intrants). On décompose la centralité selon son origine étrangère ou locale. La centralité étrangère est celle qui est due aux liaisons (directes et indirectes) avec les secteurs étrangers, tandis que la centralité locale représente la composante due aux liaisons (directes et indirectes) avec les secteurs locaux.

\section{Les dix pôles informatiques les plus centraux, 1995 et 2011}

Les indicateurs de la centralité sont calculés à partir des données de l'édition 2015 de la base de l'OCDE sur les Tableaux internationaux des entrées-sorties, qui fournissent des données sur les flux d'intrants de 61 pays et 34 secteurs d'activités de 1995 à 2011. Pour chaque couple pays-secteur, on calcule centralité de référence, ainsi que la somme pondérée des centralités des partenaires commerciaux, où les poids sont les parts des intrants. La centralité totale est la moyenne des centralités calculées avec les liaisons en aval (exportations d'intrants) et les liaisons en amont (importations d'intrants). On décompose la centralité selon son origine étrangère ou locale. La centralité étrangère est celle qui est due aux liaisons (directes et indirectes) avec les secteurs étrangers, tandis que la centralité locale représente la composante due aux liaisons (directes et indirectes) avec les secteurs locaux.

\section{7. Évolution de l'utilisation de l'internet, 2005-16}

Notes relatives à la partie A :

Les données sont fondées sur des estimations de l'OCDE.

Notes relatives à la partie $B$ :

Sauf indication contraire, la période de référence prise en compte pour recenser les internautes est de 3 mois. Pour l'Australie, le Canada et le Japon, elle est de 12 mois. Pour les États-Unis, elle est de 6 mois en 2015 ; elle n'est pas précisée en 2006. Pour la Corée et la Nouvelle-Zélande, elle est de 12 mois en 2006. Pour l'Afrique du Sud, le Chili en 2009, la Chine, la Fédération de Russie, l'Inde et l'Indonésie, aucune période de référence n'est spécifiée.

Pour l'Australie, les données se rapportent aux exercices 2006/07 et 2014/15 clos le 30 juin.

Pour le Brésil, les données se rapportent à 2008 et 2015.

Pour le Canada, les données se rapportent à 2007 et 2012. En 2007, elles concernent les individus âgés de 16 ans ou plus, et non de 16 à 74 ans.

Pour le Chili, les données se rapportent à 2009 et 2015.

Pour l'Afrique du Sud, la Chine, la Fédération de Russie, l'Inde et l'Indonésie, les données proviennent de la base des Indicateurs des télécommunications/TIC dans le monde de l'UIT, et se rapportent à 2015 au lieu de 2016.

Pour l'Islande et la Suisse, les données se rapportent à 2014 au lieu de 2016.

Pour l'Indonésie, les données concernent les individus âgés de 5 ans ou plus.

Pour Israël, les données se rapportent à 2015 au lieu de 2016, et concernent les individus âgés de 20 ans ou plus, et non de 16 à 74 ans. Pour le Japon, les données se rapportent à 2015 au lieu de 2016, et concernent les individus âgés de 15 à 69 ans.

Pour la Corée, les données se rapportent à 2015 au lieu de 2016.

Pour la Nouvelle-Zélande, les données se rapportent à 2012 au lieu de 2016.

Pour la Turquie, les données se rapportent à 2007 au lieu de 2006.

Pour les États-Unis, les données se rapportent à 2007 et 2015.

\section{8. Évolution de l'utilisation de l'internet, par âge, 2005-16}

Notes relatives à la partie $\mathrm{A}$ :

Les données sont fondées sur des estimations de l'OCDE.

Notes relatives à la partie $B$ :

Sauf indication contraire, la période de référence prise en compte pour recenser les internautes est de 12 mois et les données relatives à l'ensemble des individus se rapportent aux 16-74 ans. Pour les États Unis, aucune période de référence n'est spécifiée.

Pour l'Australie, les données se rapportent à l'exercice 2014/15 et la période de référence est de 3 mois.

Pour le Brésil, le Chili, la Colombie, la Corée, les États-Unis, Israël et le Japon, les données se rapportent à 2015.

Pour le Canada, les données se rapportent à 2012 et concernent les individus âgés de 65 ans ou plus, et non de 55 à 64 ans.

Pour l'Islande et la Suisse, les données se rapportent à 2014.

Pour Israël, les données concernent les individus âgés de 20 ans ou plus, et non de 16 à 74 ans, et les individus âgés de 20 à 24 ans, et non de 16 à 24 ans. 
Pour le Japon, les données concernent les individus âgés de 15 à 69 ans, et non de 16 à 74 ans, et les individus âgés de 60 à 69 ans, et non de 55 à 74 ans. Les données relatives aux 60-69 ans proviennent de l'enquête de 2015 sur les habitudes des consommateurs, conduite par le ministère des Affaires intérieures et des Communications.

Pour la Nouvelle-Zélande, les données se rapportent à 2012.

\section{Diplômées de l'enseignement supérieur en sciences naturelles, ingénierie et TIC, 2015}

L'enseignement supérieur correspond aux niveaux 5 à 8 de la CITE 2011.

Le domaine d'études « Technologies de l'information et de la communication » (TIC) renvoie aux Domaines d'études et de formation de la CITE (CITE-F 2013).

L'agrégat OCDE correspond à la moyenne non pondérée des pays pour lesquels des données sont disponibles.

\section{Les femmes dans la science, 2015}

Cet indicateur, expérimental, a été obtenu à partir d'un échantillon aléatoire stratifié d'auteurs scientifiques.

Les échantillons proviennent de publications parues en 2011 et indexées dans la base de données Scopus. Parmi les domaines examinés figurent : Arts et lettres, Commerce, Génie chimique, Immunologie et microbiologie, Science des matériaux, Neurosciences, et Physique et astronomie.

Les estimations pondérées tiennent compte de la structure de l'échantillon et des schémas de non-réponse par domaine, pays d'affiliation et statut de la revue.

\section{Contribution des femmes aux inventions brevetées, 2012-15}

La part des brevets à l'origine desquels on trouve des femmes est calculée comme le nombre de brevets comptant des inventrices dans un pays donné divisé par le nombre total de brevets déposés dans ce pays. Les données se rapportent aux familles IP5 par date de dépôt du brevet, et sont groupées en fonction du lieu de résidence et du sexe de la personne à l'origine de l'invention, avec des comptages fractionnaires. Pour recenser les inventrices, on utilise des dictionnaires de genre des noms (dictionnaires de prénoms par pays), suivant la méthodologie décrite par Lax Martínez, Raffo et Saito (2016). Les brevets sont affectés aux domaines technologiques sur la base de leurs codes dans la Classification internationale des brevets (CIB), suivant la concordance établie par l'OMPI (2013). Ne sont inclus que les pays pour lesquels on dénombre, pour 2012-15, plus de 100 familles de brevets au total, au moins 25 familles de brevets dans chaque technologie représentée et plus de $80 \%$ de noms qu'on peut répartir entre inventrices et inventeurs. Les chiffres fournis pour 2014 et 2015 sont estimés sur la base des données disponibles pour ces années.

\section{Budgets publics destinés à la R-D dans une sélection d'économies, 2008-16}

Ces statistiques se fondent sur les bases de données de l'OCDE sur la R-D, dont la Base de données des Statistiques de la recherchedéveloppement (http://oe.cd/rds) et la Base de données des Principaux indicateurs de la science et de la technologie (http://oe.cd/ msti). Se reporter à ces sources pour davantage de renseignements sur les données, en particulier sur les ruptures de séries et autres problèmes analogues.

Pour l'Australie, le Canada, la Corée, les États-Unis et le Japon, seuls les crédits budgétaires de R-D alloués par le gouvernement central ou fédéral sont inclus.

\section{Crédits budgétaires publics de R-D, par objectif socio-économique, 2016}

Ces statistiques se fondent sur les bases de données de l'OCDE sur la R-D, dont la Base de données des Statistiques de la recherchedéveloppement (http://oe.cd/srd-fr) et la Base de données des Principaux indicateurs de la science et de la technologie (http://oe.cd/ pist-fr). Se reporter à ces sources pour davantage de renseignements sur les données, en particulier sur les ruptures de séries et autres problèmes analogues.

Pour l'Australie, l'Autriche, le Canada, la Corée, les États-Unis, l'Islande et le Japon, seuls les crédits budgétaires de R-D alloués par le gouvernement central ou fédéral sont inclus.

\section{Recherche scientifique sur la démence et les maladies neurodégénératives dans une sélection de pays, 1996-2016 \\ Cet indicateur est expérimental.}

Ces estimations se fondent sur des recherches des descripteurs " neurodegenerat* ", " dementia " et " Alzheimer " dans les résumés des articles publiés (en anglais) entre 1996 et 2016 et indexés dans la base de données Scopus.

Les comptages effectués au niveau des pays sont fractionnaires.

\section{Disciplines contribuant à la recherche scientifique sur la démence et les maladies neurodégénératives, 1996-2016}

Cet indicateur est expérimental.

Ces estimations se fondent sur des recherches des descripteurs " neurodegenerat* ", "dementia " et " Alzheimer " dans les résumés des articles publiés (en anglais) entre 1996 et 2016 et indexés dans la base de données Scopus.

Les comptages effectués au niveau des champs disciplinaires sont fractionnaires. 


\section{1. ÉCONOMIE DU SAVOIR ET TRANSFORMATION NUMÉRIQUE}

\section{Documents scientifiques publiés en libre accès, 2017}

Cet indicateur est expérimental.

Il s'appuie sur une requête automatisée appliquée à un échantillon aléatoire (non stratifié) de 100000 documents citables (articles, recensions et actes de conférences) publiés en 2016 et indexés dans la base de données Scopus avec un DOI valide associé (plus de 90 \% des cas). La requête en question est une routine de type "wrapper ", codée en langage R, conçue pour interroger l'interface de programmation d'application (API) oaDOI développée par l'organisation ImpactStory, dont les outils en libre accès disponibles en ligne visent à aider les chercheurs à partager leurs recherches et à en mesurer l'impact. L'évaluation a consisté à déterminer le type d'accès aux documents de l'échantillon. L'API oaDOI renvoie des informations sur les possibilités d'obtenir des copies légales des documents selon différents mécanismes.

La « voie dorée » indique que l'éditeur du document est listé dans le Dictionnaire des revues en libre accès, c'est-à-dire qu'il donne gratuitement accès à ses contenus. La "voie dorée hybride " indique que l'éditeur du document, qui facture généralement un abonnement à ses lecteurs, propose ce document précis en libre accès, normalement parce que l'auteur ou ceux qui le financent ont payé les frais de publication de l'article, et donc obtenu un droit de parution en libre accès (sur le modèle des revues qui appliquent l'approche de la voie dorée). La "voie verte " indique que des versions légales du document existent dans des référentiels ou d'autres types d'archives, sans qu'aucune autre version ne soit accessible via la voie dorée ou la voie dorée hybride. Quand le DOI du document ne peut pas être mis en correspondance avec un moyen quelconque d'accès à l'information, il est affecté à la catégorie " aucune information " (statut non disponible). Quand le DOI peut être mis en correspondance avec des sources mais que l'API indique qu'aucune version légale en libre accès n'est disponible, il est affecté à la catégorie " accès fermé ".

Le nombre de documents en libre accès peut être sous-estimé car le DOI ne peut pas toujours être mis en correspondance avec toutes les versions légales en libre accès et car il peut exister des versions non conformes au droit d'auteur. L'indicateur indique le type possible d'accès aux documents tels qu'ils apparaissent entre six et dix-huit mois après leur publication. Les documents sous embargo temporaire appartiendront ensuite à la catégorie " accès fermé " mais pourraient être disponibles en accès libre à une étape ultérieure.

\section{Documents scientifiques les plus cités, par modalité d'accès, 2017}

Cet indicateur est expérimental.

Il s'appuie sur une requête automatisée appliquée à un échantillon aléatoire (non stratifié) de 100000 document citables (articles, recensions et actes de conférences) publiés en 2016 et indexés dans la base de données Scopus avec un DOI valide associé (plus de $90 \%$ des cas). La requête en question est une routine de type "wrapper ", codée en langage R, conçue pour interroger l'interface de programmation d'application (API) oaDOI développée par l'organisation ImpactStory, dont les outils en libre accès disponibles en ligne visent à aider les chercheurs à partager leurs recherches et à en mesurer l'impact. L'évaluation a consisté à déterminer le type d'accès aux documents de l'échantillon. L'API oaDOI renvoie des informations sur les possibilités d'obtenir des copies légales des documents selon différents mécanismes.

La « voie dorée » indique que l'éditeur du document est listé dans le Dictionnaire des revues en libre accès, c'est-à-dire qu'il donne gratuitement accès à ses contenus. La « voie dorée hybride » indique que l'éditeur du document, qui facture généralement un abonnement à ses lecteurs, propose ce document précis en libre accès, normalement parce que l'auteur ou ceux qui le financent ont payé les frais de publication de l'article, et donc obtenu un droit de parution en libre accès (sur le modèle des revues qui appliquent l'approche de la voie dorée). La "voie verte » indique que des versions légales du document existent dans des référentiels ou d'autres types d'archives, sans qu'aucune autre version ne soit accessible via la voie dorée ou la voie dorée hybride. Quand le DOI du document ne peut pas être mis en correspondance avec un moyen quelconque d'accès à l'information, il est affecté à la catégorie " aucune information " (statut non disponible). Quand le DOI peut être mis en correspondance avec des sources mais que l'API indique qu'aucune version légale en libre accès n'est disponible, il est affecté à la catégorie " accès fermé ".

Le nombre de documents en libre accès peut être sous-estimé car le DOI ne peut pas toujours être mis en correspondance avec toutes les versions légales en libre accès et car il peut exister des versions non conformes au droit d'auteur. L'indicateur indique le type possible d'accès aux documents tels qu'ils apparaissent entre six et dix-huit mois après leur publication. Les documents sous embargo temporaire appartiendront ensuite à la catégorie " accès fermé " mais pourraient être disponibles en accès libre à une étape ultérieure.

Les documents très cités sont les $10 \%$ de publications les plus citées, les valeurs étant normalisées par domaine scientifique et par type de document (articles, recensions et actes de conférences). Les valeurs SJR (Scimago Journal Rank) sont utilisées en complément pour hiérarchiser les publications ayant obtenu le même nombre de citations au sein d'une catégorie. Cette mesure est un indicateur de l'excellence de la recherche. Les estimations sont fondées sur des comptages fractionnaires des documents publiés, par auteur affilié à des établissements dans chaque économie.

\section{Collaboration internationale en science et innovation, 2005-16}

Le pourcentage de co-inventions internationales est égal au rapport entre le nombre de familles de brevets IP5 dont des inventeurs se trouvent dans au moins deux économies et le nombre total de familles de brevets IP5 dont des inventeurs se trouvent dans l'économie de référence. Les données se rapportent aux familles de brevets IP5 déposées pendant la période 2005-15, classées par lieu de résidence de l'inventeur. Seules les économies totalisant plus de 100 familles de brevets au cours de cette période sont incluses. On applique la méthode du comptage simple.

Le co-autorat international de publications scientifiques est défini au niveau institutionnel. On considère qu'un document scientifique est le fruit d'une collaboration internationale si la liste des affiliations communiquée par le ou les auteurs comprend 
des établissements de différents pays ou économies. À des fins de comparabilité avec les données sur les co-inventions, on utilise également dans ce cas le comptage simple. Les estimations présentées ici sont donc supérieures à celles que contient le chapitre 3 , qui reposent sur des comptages fractionnaires.

\section{Flux internationaux nets d'auteurs scientifiques dans une sélection d'économies, 2002-16}

Cet indicateur est expérimental.

Les estimations reposent sur les différences entre les entrées et les sorties implicites d'auteurs scientifiques pour l'économie de référence, telles qu'indiquées par le changement de la principale affiliation d'un auteur donné ayant publié un article référencé dans la base Scopus, au cours de la durée pendant laquelle cet auteur a eu des articles référencés. Le graphique décompose les flux nets enregistrés année après année au cours de la période, dans une sélection de pays. Une entrée est comptabilisée pour une année t et une économie e si un auteur auparavant affilié à une autre économie est observé à l'année t comme étant pour la première fois affilié à un établissement de l'économie e. De même, une sortie est enregistrée lorsqu'un auteur affilié à l'économie e au cours de l'année précédente est observé à l'année t comme étant affilié à une économie différente. Si un auteur est affilié à plusieurs économies, on applique un comptage fractionnaire. Si, au cours d'une année donnée, un auteur a produit plusieurs publications, la dernière d'entre elles est utilisée comme référence, les autres sont ignorées.

La date réelle de la mobilité est indéterminée, car il peut s'écouler plus d'une année entre les publications. Par conséquent, la chronologie que sous-entend le graphique peut être en décalage par rapport au moment où la mobilité est survenue. La chronologie sera plus fiable pour les auteurs plus prolifiques. Les estimations relatives aux premières années ne sont pas communiquées car les flux de mobilité ne peuvent être calculés que lorsqu'une deuxième publication au nom d'un même auteur est répertoriée dans la base de données. De même, l'indexation incomplète de tous les auteurs au cours de la période 2000-03 peut entraîner une sous-estimation des flux totaux et, par conséquent, bien que dans une moindre mesure, des flux nets estimés.

\section{Aides directes et fiscales à la R-D des PME, 2015}

Cet indicateur est expérimental. La comparabilité internationale des données peut être limitée (par exemple, parce que les définitions des PME ne sont pas les mêmes aux fins de la comptabilisation de la R-D des entreprises et des allègements fiscaux en faveur de la R-D). Pour les DIRDE et les DIRDE financées par l'État, les PME s'entendent généralement des entreprises de 1 à 249 salariés (c'est-àdire, à l'exclusion des entreprises de zéro salarié), sauf mention contraire. Mais un certain nombre de pays appliquent des critères supplémentaires pour définir les PME. Par exemple, l'indépendance est un critère pertinent actuellement utilisé par quelques pays (le Canada et le Royaume-Uni, notamment) pour rendre compte des DIRDE financées par l'État et des aides fiscales à la R-D par taille d'entreprise. La comparabilité internationale en est d'autant plus limitée. Pour de plus amples informations sur les définitions des PME, voir les notes par pays.

Pour de plus amples informations sur les incitations fiscales à la R-D, voir http://oe.cd/rdtax. Pour consulter les notes générales et les notes par pays applicables à cet indicateur sur les incitations fiscales à la R-D, voir http://oe.cd/sb2017_notes_rdtax.

\section{Intensité de la R-D des entreprises et soutien public à la R-D des entreprises, 2015}

Pour de plus amples informations sur les incitations fiscales à la R-D, voir http://oe.cd/rdtax. Pour consulter les notes générales et les notes par pays applicables à cet indicateur sur les incitations fiscales à la R-D, voir http://oe.cd/sb2017_notes_rdtax.

\section{2. Évolution du soutien public à la R-D des entreprises et des DIRDE, 2006-15}

Pour de plus amples informations sur les incitations fiscales à la R-D, voir http://oe.cd/rdtax. Pour consulter les notes générales et les notes par pays applicables à cet indicateur sur les incitations fiscales à la R-D, voir http://oe.cd/sb2017_notes_rdtax.

\section{Investissement en capital-risque dans une sélection de pays, par secteur, 2016}

Pour les États-Unis, les données incluent également les investissements de capital-risque effectués par d'autres investisseurs engagés aux côtés de sociétés de capital-risque, mais excluent les accords d'investissements financés à 100 \% par des firmes et/ ou des investisseurs providentiels.

Les fournisseurs des données sont Invest Europe pour les pays européens et NVCA pour les États-Unis.

Les TIC désignent « les communications » et " l'informatique et l'électronique grand public » pour les pays européens et « les technologies de l'information » pour les États-Unis.

La catégorie « autres » recouvre l'agriculture, les produits et services destinés aux entreprises, les produits chimiques et matériaux, la construction, les produits et services grand public, l'énergie et l'environnement, les activités financières et d'assurance, l'immobilier et les transports pour les pays européens ; elle recouvre l'énergie, les ressources et matériaux, le commerce d'entreprise à consommateur (B2C), le commerce interentreprises (B2B) et les services financiers pour les États-Unis.

\section{Accords conclus avec des investisseurs providentiels par secteur, Europe, 2015 et États-Unis, 2016}

Un investisseur providentiel est un investisseur privé qui, généralement, propose des ressources financières et une expertise commerciale à une entreprise en échange d'une part dans le capital de cette entreprise. Certains investisseurs providentiels se regroupent dans le cadre de consortiums ou de réseaux afin de prendre part à des projets plus importants en partageant les risques. Un groupe d'investisseurs providentiels réunit des individus ayant décidé d'unir leurs forces pour évaluer et investir dans des entreprises. Ces individus mettent en commun leur capital et peuvent ainsi réaliser de plus gros investissements. 


\section{1. ÉCONOMIE DU SAVOIR ET TRANSFORMATION NUMÉRIQUE}

Notes et Références

Un réseau d'investisseurs providentiels est une organisation conçue pour faciliter la mise en relation des entrepreneurs avec les investisseurs providentiels.

Les données se rapportent aux réseaux et aux groupes auprès desquels les associations d'investisseurs providentiels ont effectué leur enquête.

L'Europe inclut les pays suivants : Andorre, Allemagne, Autriche, Belgique, Bosnie-Herzégovine, Bulgarie, Croatie, Chypre, Danemark, Espagne, Estonie, Ex-République yougoslave de Macédoine, Fédération de Russie, Finlande, France, Grèce, Hongrie, Irlande, Italie, Kosovo, Lettonie, Lituanie, Luxembourg, Malte, Norvège, Pays-Bas, Pologne, Portugal, Serbie, République slovaque, République tchèque, Royaume-Uni, Slovénie, Suède, Suisse, Turquie et Ukraine.

Pour les États-Unis, les données se rapportent à la moyenne simple des régions suivantes : Nord-Ouest, Californie, Sud-Ouest et Texas, Grandes Plaines, Grands Lacs, Sud-Est, zone atlantique intermédiaire, New York et Nord-Est.

\section{Start-ups axées sur le numérique ayant attiré des fonds propres dans les économies de l'OCDE et BRIICS, 2011-16}

L'échantillon est limité aux entreprises créées après 2010 (donc qui avaient cinq ans d'ancienneté ou moins en 2016) et qui ont attiré des financements sur fonds propres au cours de la période 2011-16.

Le financement sur fonds propres inclut le capital-risque et d'autres formes de financements à risque tels que les capitaux apportés par des investisseurs providentiels ou le financement par emprunt.

Les activités liées au numérique sont répertoriées par l’OCDE sur la base d'une correspondance entre les secteurs listés dans la base de données et les codes de la CITI rév. 4.

La catégorie " autres activités liées au numérique " comprend la navigation et la cartographie, les paiements, la messagerie et les télécommunications, et les plateformes.

\section{Activités liées au numérique ayant attiré le plus de financements sur fonds propres, 2011-16}

L'échantillon est limité aux entreprises créées après 2010 (donc qui avaient cinq ans d'ancienneté ou moins en 2016) et qui ont attiré des financements sur fonds propres au cours de la période 2011-16.

Le financement sur fonds propres inclut le capital-risque et d'autres formes de financements à risque tels que les capitaux apportés par des investisseurs providentiels ou le financement par emprunt.

Les activités liées au numérique sont répertoriées par l'OCDE sur la base d'une correspondance entre les secteurs listés dans la base de données et les codes de la CITI rév. 4.

\section{Publications scientifiques avec remerciements à des financeurs directs, 2016}

Cet indicateur est expérimental.

Il est construit pour les documents scientifiques citables (articles, recensions et actes de conférences) publiés en 2016 et indexés dans la base de données Scopus. Il indique s'il existe un enregistrement de l'auteur remerciant une ou plusieurs organisation(s) de leur financement. Il est un indicateur de substitution de la mesure dans laquelle les scientifiques doivent obtenir des financements directs pour leurs activités de recherche, sous réserve que ces financements doivent faire l'objet d'un remerciement dans les publications produites concernant ces activités.

\section{Impact de citation des publications scientifiques avec remerciements à des financeurs directs Cet indicateur est expérimental.}

Il est construit pour les documents scientifiques citables (articles, recensions et actes de conférences) indexés dans la base de données Scopus. Il indique s'il existe un enregistrement de l'auteur remerciant une ou plusieurs organisation(s) de leur financement. La proportion dans les documents les plus cités par pays et par type de document, selon que le document contient ou non un remerciement pour financement, est calculée grâce à des indicateurs d'impact normalisés par type de document et par discipline qui classent les documents de chaque groupe en fonction du nombre de citations effectives et, de pair avec les citations, en fonction du prestige de la revue conformément à la valeur SJR (Scimago Journal Rank) de 2015. Les documents sont affectés aux $10 \%$ supérieurs de leur catégorie et agrégés à l'aide de comptages fractionnaires par discipline et par pays. Compte tenu du court délai de citation possible (un an après la publication), les résultats dépendent très fortement du classement de la revue elle-même.

\section{Références}

Boselli, B. et F. Galindo-Rueda (2016), « Drivers and Implications of Scientific Open Access Publishing: Findings from a Pilot OECD International Survey of Scientific Authors ", OECD Science, Technology and Industry Policy Papers, $\mathrm{n}^{\circ} 33$, Éditions OCDE, Paris, http:// dx.doi.org/10.1787/5jlr2z70k0bx-en.

Breschi, S., J. Lassebie et C. Menon (à paraître), A micro-data analysis of innovative start-ups and venture-capital investments across countries. Calvino et al. (à paraître), A Taxonomy of Digital Sectors.

Corrado, C., C. Hulten et D. Sichel (2009), "Intangible capital and US economic growth », Review of Income and Wealth, vol. 55/3, pp. 661-685. 
Criscuolo, C. et J. Timmis (à paraître), « GVCs and centrality: Mapping key hubs, spokes and the periphery », OECD Productivity Working Papers.

Daiko, T. et al. (2017), World Corporate Top R\&D Investors: Industrial Property Strategies in the Digital Economy, rapport conjoint du JRC et de l'OCDE, Office des publications de l'Union européenne, Luxembourg.

Dalle, J. M., M. Denbesten et C. Menon (à paraître), Using Crunchbase for academic and managerial research.

Dernis, H. et al. (2015), World Corporate Top R\&D Investors: Innovation and IP Bundles, rapport conjoint du JRC et de l'OCDE, Office des publications de l'Union européenne, Luxembourg.

Dernis, H., M. Squicciarini et R. de Pinho (2016), « Detecting the emergence of technologies and the evolution and co-development trajectories in science (DETECTS): A 'burst' analysis-based approach », Journal of Technology Transfer, vol. 41/5, pp. 930-960.

Fédération internationale de la robotique (2016), World Robotics: Industrial Robots, Frankfurt am Main, Allemagne.

Grundke, R. et al. (à paraître), Which skills for the digital era? A returns to skills analysis.

Grundke, R. et al. (2017), "Skills and global value chains: A characterisation », Documents de travail de l’OCDE sur la science, la technologie et l'industrie, $n^{\circ}$ 2017/05, Éditions OCDE, Paris, http://dx.doi.org/10.1787/cdb5de9b-en.

Haines, W., L. Marcolin et M. Squicciarini (à paraître), Intangibles and productivity growth: a new assessment.

Inaba, T. et M. Squicciarini (2017), «ICT: A new taxonomy based on the international patent classification ", Documents de travail de l'OCDE sur la science, la technologie et l'industrie, n² 2017/01, Éditions OCDE, Paris, http://dx.doi.org/10.1787/ab16c396-en.

Kalantari, A. et al. (2017), « A bibliometric approach to tracking big data research trends », Journal of Big Data, vol. 4/30, pp. 1-18, https:// doi.org/10.1186/s40537-017-0088-1.

Lax Martínez, G., J. Raffo et K. Saito (2016), « Déterminer la part des inventeurs et des inventrices dans le contexte du PCT », Document de recherche économique $n^{\circ} 33$, Organisation mondiale de la propriété intellectuelle (OMPI), Genève, www.wipo.int/publications/fr/ details.jsp?id=4125.

Le Mouel, M., L. Marcolin et M. Squicciarini (2016), « Investment in organisational capital: Methodology and panel estimates », SPINTAN Working Papers, $n^{\circ} 21$, Commission européenne, Bruxelles.

Marcolin, L., S. Miroudot et M. Squicciarini (2016), "The routine content of occupations: New cross-country measures based on PIAAC ", Documents de travail de l'OCDE sur la politique commerciale, $n^{\circ} 188$, Éditions OCDE, Paris.

OCDE (2018), Financing SMEs and Entrepreneurs 2018: An OECD Scoreboard, Éditions OCDE, Paris,http://dx.doi.org/10.1787/fin_sme_ ent-2018-en.

OCDE (2017a), OECD Compendium of Productivity Indicators 2017, Éditions OCDE, Paris, http://dx.doi.org/10.1787/pdtuy-2017-en.

OCDE (2017b), Panorama des administrations publiques 2017, Éditions OCDE, Paris, http://dx.doi.org/10.1787/gov_glance-2017-fr.

OCDE (2015a), Manuel de Frascati 2015 : Lignes directrices pour le recueil et la communication des données sur la recherche et le développement expérimental, Mesurer les activités scientifiques, technologiques et d'innovation, Éditions OCDE, Paris, http://dx.doi. org/10.1787/9789264257252-fr.

OCDE (2015b), Addressing Dementia: The OECD Response, Éditions OCDE, Paris, http://dx.doi.org/10.1787/9789264231726-en.

OCDE (2015c), « Making Open Science a Reality », OECD Science, Technology and Industry Policy Papers, $\mathrm{n}^{\circ} 25$, Éditions OCDE, Paris, http:// dx.doi.org/10.1787/5jrs2f963zs1-en.

OCDE (2011), OECD Guide to Measuring the Information Society 2011, Éditions OCDE, Paris, http://dx.doi.org/10.1787/9789264113541-en.

OMPI (2013), Table de concordance CIB, Organisation mondiale de la propriété intellectuelle, Genève, www.wipo.int/ipstats/fr/statistics/ technology_concordance.html (consulté le $1^{\mathrm{er}}$ juin 2017).

Squicciarini, M., L. Marcolin et P. Horvát (2015), « Estimating cross-country investment in training: An experimental methodology using PIAAC data ", Documents de travail de l'OCDE sur la science, la technologie et l'industrie, n²015/09, Éditions OCDE, Paris, http:// dx.doi.org/10.1787/5jrs3sftp8nw-en. 



\section{LE SAVOIR, LES TALENTS ET LES COMPÉTENCES}

1. Investir dans le savoir

2. Enseignement supérieur et recherche fondamentale

3. Scientifiques et ingénieurs

4. Chercheurs

5. Compétences dans le monde numérique

6. Rendements des compétences en TIC

7. Capital intellectuel

Notes et références 


\section{Dépenses consacrées à l'enseignement supérieur et à l'enseignement professionnel, 2014}

En pourcentage du PIB

Enseignement supérieur Enseignement professionnel

Dépenses consacrées à l'enseignement professionnel indisponibles

$\diamond$ Enseignement supérieur, 2005

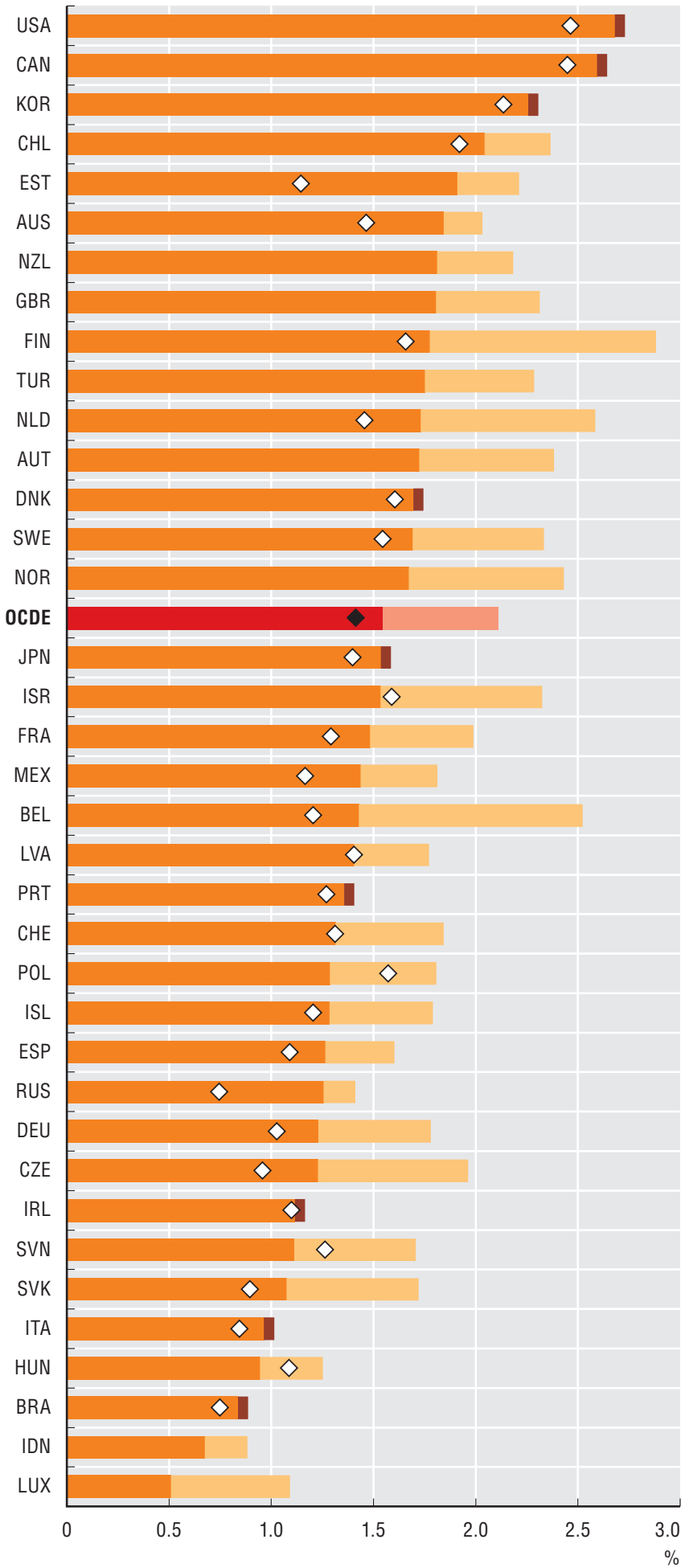

Source : OCDE, d'après OCDE (2017), Regards sur l'éducation 2017 : Les indicateurs de l'OCDE, Éditions OCDE, Paris. Voir notes de chapitre.

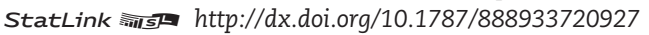

\section{Le saviez-vous ?}

$\mathrm{Au}$ cours des dix dernières années, les pays de l'OCDE ont accru leurs dépenses d'enseignement supérieur d'environ $9 \%$ et leurs dépenses de R-D de $11 \%$.

L'investissement dans le capital humain est indispensable à l'innovation, au progrès technologique et à la croissance à long terme. Il peut prendre la forme de dépenses consacrées à l'éducation - notamment à l'enseignement supérieur et à la formation professionnelle - de dépenses de recherchedéveloppement (R-D) et d'investissements dans les technologies génériques, parmi lesquelles se rangent les technologies de l'information et des communications (TIC).

En l'espace d'une décennie, la quasi-totalité des pays de l'OCDE ont intensifié leurs investissements dans l'enseignement supérieur, jusqu'à atteindre une moyenne de 1.5 \% du PIB en 2014. Les dépenses dédiées à la formation professionnelle augmentent d'un tiers celles consacrées, en moyenne, à l'enseignement post-secondaire. C'est l'Estonie qui a le plus grossi ses dépenses d'enseignement supérieur (67\%), suivie par six autres pays de l'OCDE, dont la République tchèque, l'Australie et le Mexique (avec une progression d'environ $20 \%$, sinon davantage).

Les dépenses de R-D ont elles aussi été orientées à la hausse dans pratiquement tous les pays, à commencer par la République slovaque (avec une augmentation de 140 \%), entre 2005 et 2015. Cette année-là, elles représentaient de $4.25 \%$ du PIB, en Israël, à $0.38 \%$, au Chili. La manière dont ces dépenses se répartissent entre " recherche » et " développement expérimental » est par ailleurs très hétérogène : en 2015 toujours, la « recherche » comptait pour moins du quart de leur total en Israël, alors qu'elle en drainait plus de $70 \%$ ailleurs, comme en Lettonie et aux Pays-Bas.

De 2005 à 2015, les investissements dans les actifs TIC sont demeurés stables dans l'OCDE, à $2.3 \%$ du PIB en moyenne. Cette constance trouve partiellement son explication dans le remplacement des investissements en capital par l'achat de services TIC, avec la généralisation des services de cloud computing, et le déclin rapide du prix des équipements informatiques. En 2015, la part des investissements TIC dévolue aux logiciels et bases de données était comprise entre $40 \%$ environ en Estonie, en Lettonie et en Norvège et $80 \%$, voire plus, en France et aux Pays-Bas.

\section{Définitions}

Les dépenses d'enseignement mesurent les dépenses que les États, les entreprises et les particuliers consacrent aux établissements d'enseignement.

La formation professionnelle s'entend des programmes du second cycle du secondaire conçus pour donner aux élèves qui les suivent une qualification professionnelle pertinente sur le marché du travail permettant l'exercice immédiat de professions spécifiques.

Les dépenses intérieures brutes de R-D (DIRD) sont la somme des dépenses afférentes aux moyens mis en œuvre dans le cadre d'activités de R-D. La recherche désigne les travaux originaux dont la finalité est l'acquisition de nouveaux savoirs ; le développement expérimental s'appuie sur elle pour définir des produits ou procédés nouveaux ou améliorés.

L'investissement dans les TIC s'entend de la formation brute de capital fixe (FBCF) en " équipements TIC » et en "logiciels et bases de données ", telles que ces catégories d'actifs sont définies dans le Système de comptabilité nationale de 2008 (SCN 2008). 


\section{Dépenses intérieures brutes de R-D, par type d'activité, 2015}

En pourcentage du PIB

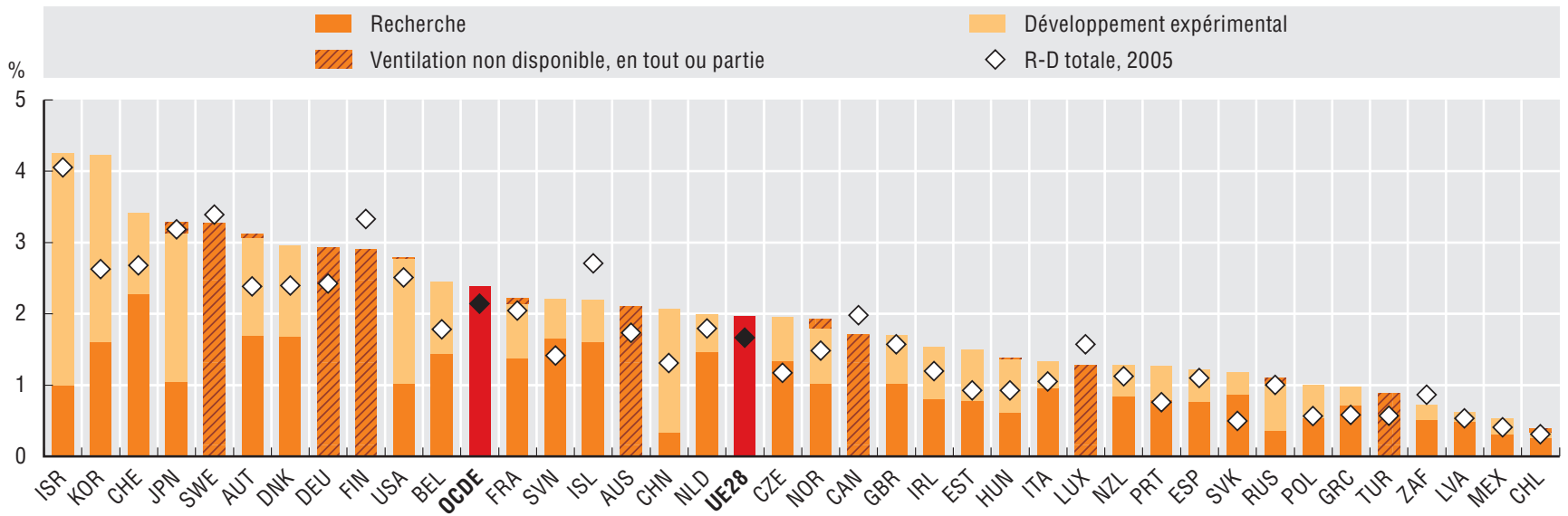

Source : OCDE, Base de données des Principaux indicateurs de la science et de la technologie, http://oe.cd/pist-fr, juillet 2017, et Base de données sur les Statistiques de la recherche et développement, http://oe.cd/srd-fr, juin 2017. Davantage de données via StatLink. Voir notes de chapitre.

\section{Investissement en TIC, par type d'actifs, 2015}

En pourcentage du PIB

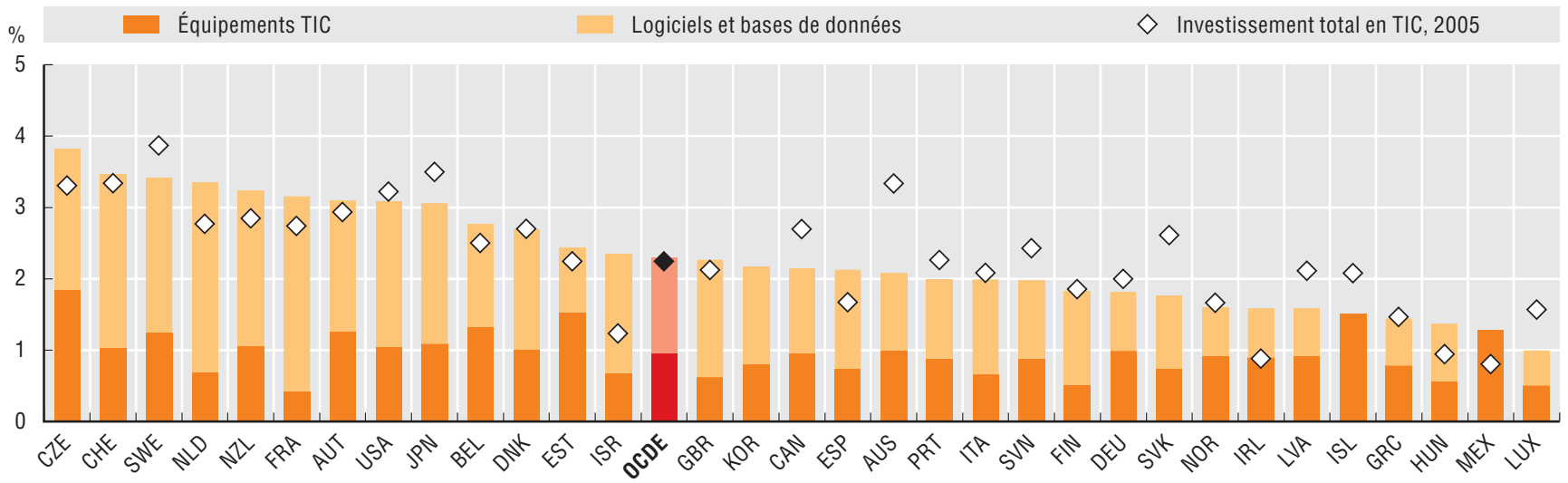

Source : OCDE, Base de données des Comptes nationaux annuels, http://www.oecd.org/fr/std/cn/, Eurostat et sources nationales, juillet 2017. Davantage de données via StatLink. Voir notes de chapitre.

\section{Mesurabilité}

Plusieurs facteurs influent sur les dépenses consacrées à l'enseignement, comme la composition démographique, les taux d'inscription, la nature de la demande de compétences et la rémunération des enseignants. Les données relatives aux dépenses de R-D proviennent pour l'essentiel d'enquêtes auprès des structures menant des activités de R-D. Ces dépenses sont définies comme étant celles liées à la recherche (fondamentale ou appliquée) ou au développement expérimental ; leur inventaire est parfois difficile à dresser - en particulier dans le cas des dépenses en capital consacrées à la R-D ou dans certains secteurs (notamment celles de l'enseignement supérieur) - si bien que leur ventilation peut s'avérer impossible, en tout ou partie. Si la mesure de l'investissement matériel (aux prix courants) dans les actifs TIC, comme les équipements informatiques et de télécommunication, est une pratique relativement bien établie, mesurer l'investissement dans les logiciels et bases de données s'avère autrement complexe. On constate ainsi des différences significatives de méthode dans le cas des logiciels (en particulier des logiciels pour compte propre). S'agissant des bases de données, le SCN 2008 recommande de ne comptabiliser que leurs coûts de maintenance physique et de construction, et non le rendement potentiel de leur contenu (voir Ahmad et Schreyer, 2016). 


\section{LE SAVOIR, LES TALENTS ET LES COMPÉTENCES}

\section{Enseignement supérieur et recherche fondamentale}

\section{Dépenses de R-D de l'enseignement supérieur, 2015}

En pourcentage du PIB

DIRDES
Part financée sur les fonds généraux des universités (FGU)
\[ \text { Estimation de la composante FGU indisponible } \]
$\diamond$ DIRDES, 2005

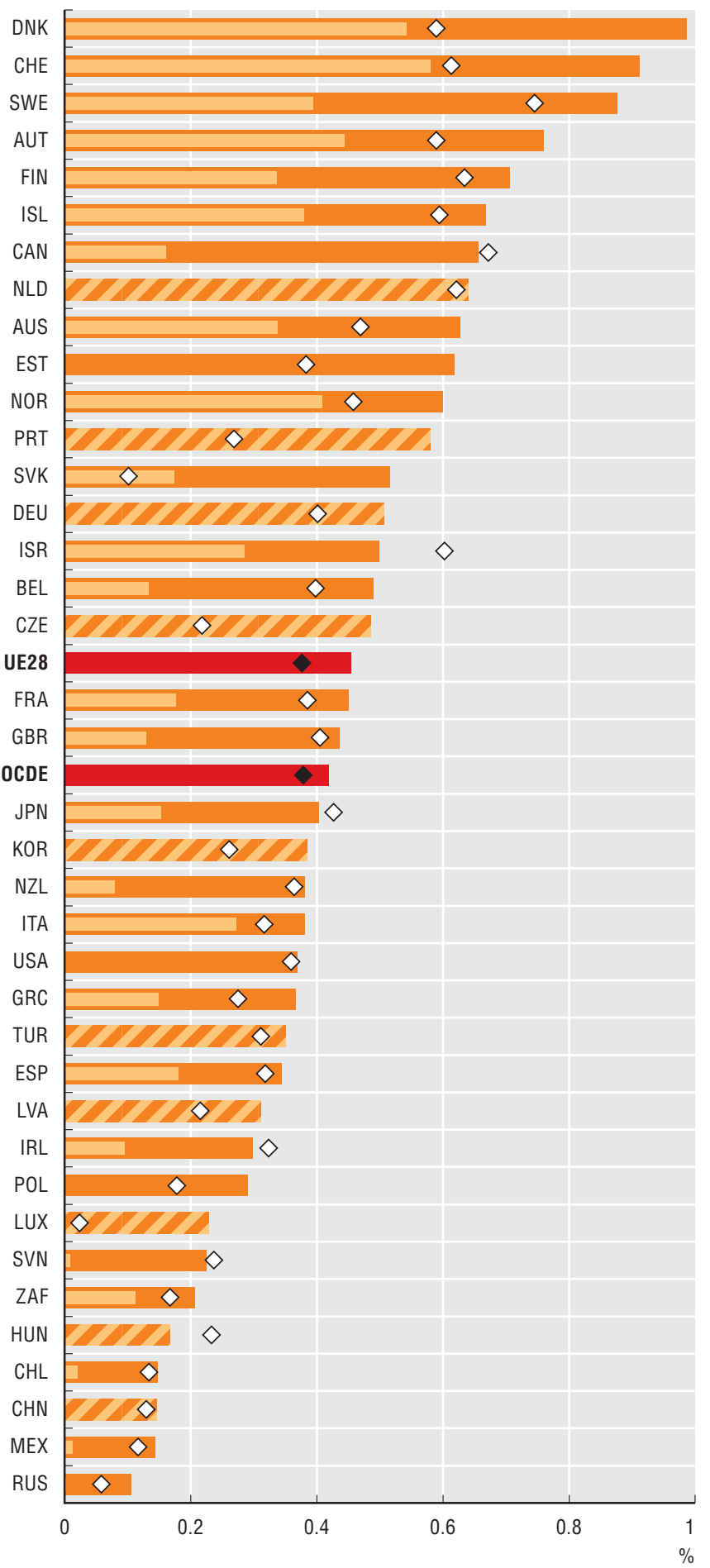

Source : OCDE, Base de données des Principaux indicateurs de la science et de la technologie, http://oe.cd/pist-fr, juillet 2017. Davantage de données via StatLink. Voir notes de chapitre.

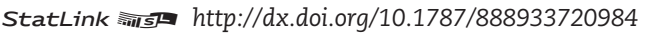

\section{Le saviez-vous ?}

En moyenne, un tiers de la R-D du secteur de l'enseignement supérieur dans les pays de l'OCDE est financée par l'intermédiaire de la dotation générale que les universités reçoivent des pouvoirs publics.

Les pays de l'OCDE ont consacré $0.4 \%$ de leur PIB aux dépenses intérieures de R-D de l'enseignement supérieur (DIRDES) en 2015. Toutefois, au Danemark, en Suisse et en Suède, ces dépenses représentaient plus du double de cette moyenne. Par rapport à 2005, les DIRDES, exprimées en proportion du PIB, avaient progressé dans la plupart des pays mais accusaient un net recul en Hongrie et en Israël.

Les établissements d'enseignement supérieur (EES) et les organismes de recherche publics concentrent, en moyenne, plus des trois-quarts de la recherche fondamentale. La proportion est cependant moindre en Corée, avec $43 \%$, contre $56 \%$ pour le secteur des entreprises. D'une manière générale, la recherche fondamentale est exécutée pour l'essentiel par les EES, même si le secteur de l'État arrive en tête en République tchèque (53\%), en Hongrie (44\%), en Fédération de Russie (76\%) et au Mexique (52\%), tandis qu'en Chine, c'est plus de $40 \%$ de cette même recherche fondamentale qu'il faut mettre à son actif. Le secteur de l'État finance en moyenne 70 \% des DIRDES, souvent par l'intermédiaire des fonds généraux des universités que les EES utilisent à discrétion pour la R-D ou d'autres activités. Les EES peuvent aussi utiliser leurs propres ressources (par exemple les recettes au titre des frais de scolarité) ou des fonds reçus d'autres établissements avec lesquels ils collaborent pour financer la R-D ; la pratique est courante en Turquie (46\% des DIRDES) et au Japon (44\%). En République slovaque, $56 \%$ des DIRDES sont financées de l'étranger, principalement par l'UE. Le financement par les entreprises des activités de R-D des EES est un marqueur de la collaboration que les deux secteurs entretiennent en ce domaine. Il atteint les plus hauts niveaux, en proportion, en Chine et en Fédération de Russie, où les entreprises (y compris les entreprises d'État) prennent en charge environ $30 \%$ des DIRDES quand la moyenne OCDE s'établit à $6 \%$. Les entreprises peuvent aussi contribuer de manière indirecte au financement de ces dépenses via le paiement de redevances pour l'utilisation des installations de R-D des EES, l'achat de résultats de R-D ou l'investissement dans des entreprises dérivées.

\section{Définitions}

Le secteur de l'enseignement supérieur comprend l'ensemble des universités, établissements d'enseignement post-secondaire et autres établissements proposant des programmes d'enseignement supérieur formel indépendamment de leur source de financement ou de leur statut juridique, ainsi que l'ensemble des instituts et centres de recherche, stations d'expérimentation et centres de soins dont les activités de R-D relèvent du contrôle direct d'établissements d'enseignement supérieur.

Les fonds généraux des universités (FGU) d'origine publique correspondent à la part des ressources financières destinées à la R-D qui sont issues de la dotation globale allouée aux universités, à l'appui de leurs activités de recherche et d'enseignement, par le ministère de l'éducation de l'administration centrale (fédérale) ou par les autorités régionales (d’États fédérés) ou locales (municipales) compétentes.

La recherche fondamentale est une composante de la R-D - avec la recherche appliquée et le développement expérimental consistant en des travaux de recherche expérimentaux ou théoriques entrepris en vue d'acquérir de nouvelles connaissances sur les fondements des phénomènes et des faits observables, sans envisager une application ou une utilisation particulière. 
Recherche fondamentale dans les secteurs de l'enseignement supérieur et de l'État, 2015

En pourcentage des dépenses intérieures de recherche fondamentale

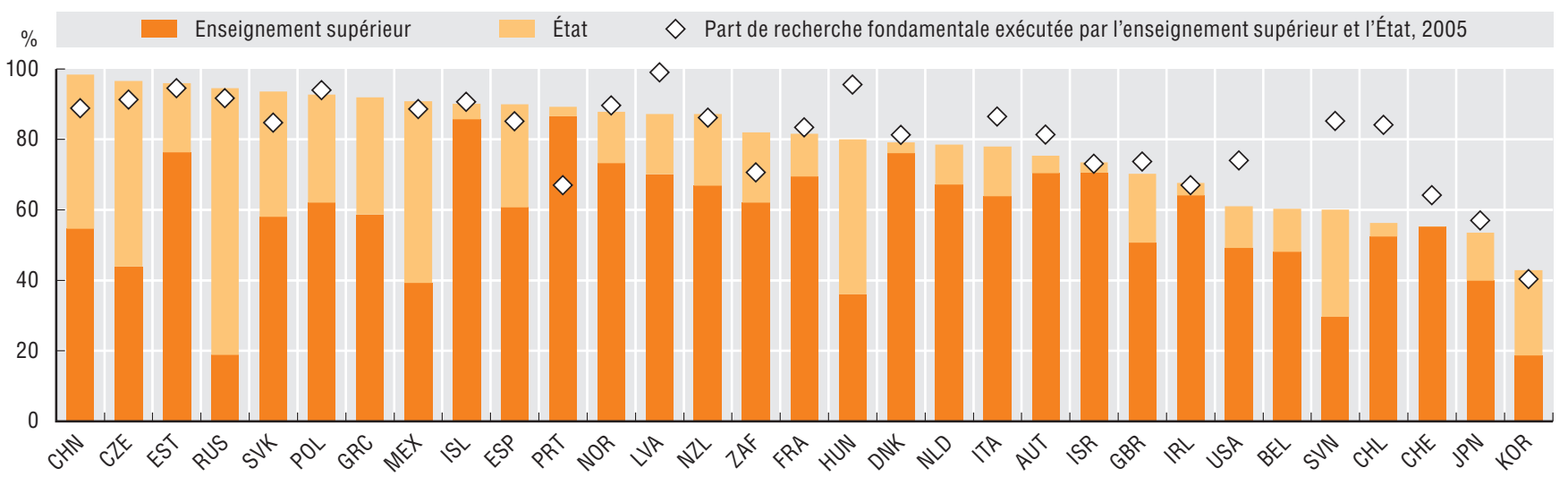

Source : OCDE, Base de données sur les Statistiques de la recherche et développement, http://oe.cd/srd-fr, juin 2017. Davantage de données via StatLink. Voir notes de chapitre.

StatLink 完节四 http://dx.doi.org/10.1787/888933721003

Financement de la R-D dans l'enseignement supérieur, 2015

En pourcentage des dépenses de R-D de l'enseignement supérieur

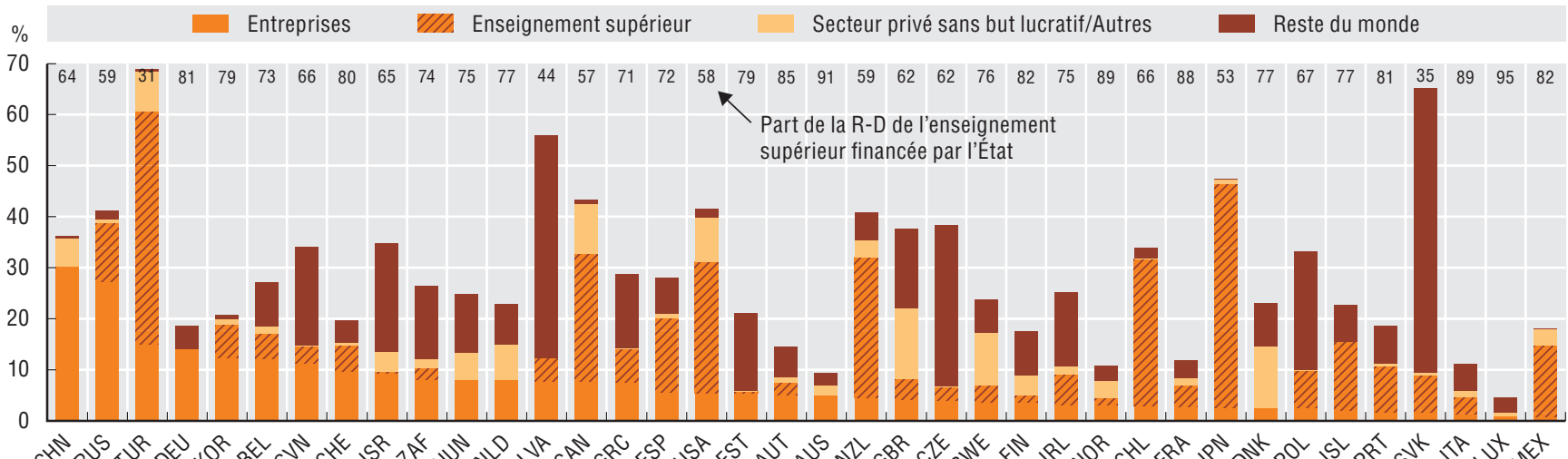

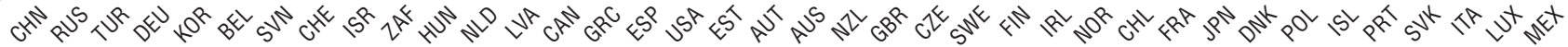

Source : OCDE, Base de données sur les Statistiques de la recherche et développement, http://oe.cd/srd-fr, juin 2017. Davantage de données via StatLink. Voir notes de chapitre.

StatLink 尚ISD http://dx.doi.org/10.1787/888933721022

\section{Mesurabilité}

Le secteur de l'enseignement supérieur fait l'objet d'un traitement à part dans les statistiques sur la R-D, en raison du rôle important des universités et des établissements assimilés dans l'exécution de la R-D et la formation des chercheurs dans le cadre des doctorats et autres diplômes de recherche. La mesure des DIRDES s'appuie sur des enquêtes spécifiques auprès des institutions de la plupart des pays de l'OCDE, souvent complétées par des sources de données administratives.

Il peut s'avérer difficile d'obtenir des mesures comparables en ce qui concerne les fonds que l'enseignement supérieur reçoit de différentes sources du fait de la grande diversité des dispositifs de financement de la R-D en place dans les pays, qui déterminent la nature des données sur lesquelles les EES peuvent s'appuyer pour rendre compte des activités qu'ils mènent. Les fonds publics généraux des universités sont présentés comme une forme d'aide publique à la R-D bien qu'ils puissent être considérés comme des ressources internes des universités, sachant que celles-ci sont relativement libres de décider si elles les emploieront ou non pour financer des activités de R-D. 


\section{Diplômés de l'enseignement supérieur en sciences naturelles, ingénierie et TIC, 2005 et 2015}

En pourcentage de l'ensemble des diplômés de l'enseignement supérieur

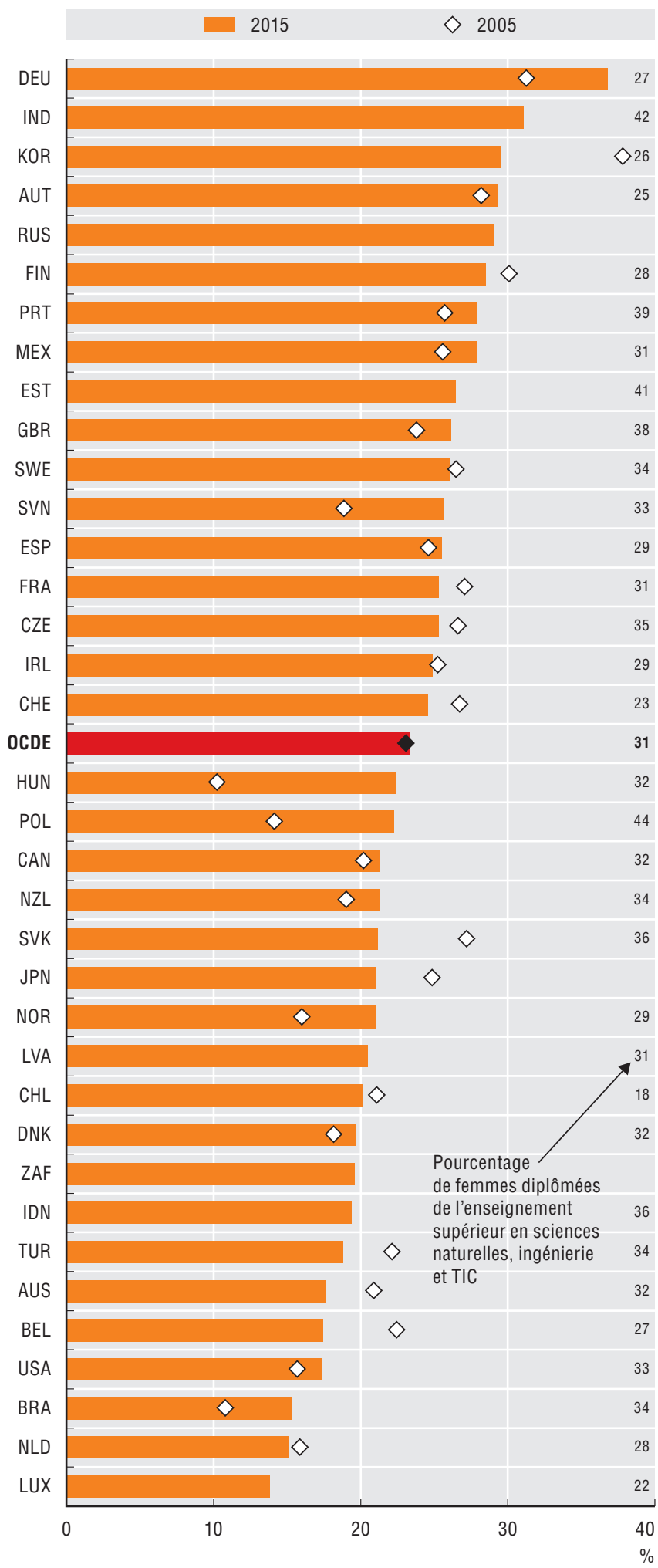

Source : OCDE, d'après OCDE (2017), Regards sur l'éducation 2017 : Les indicateurs de l'OCDE et OCDE (2007), Regards sur l'éducation 2007 : Les indicateurs de l'OCDE, Éditions OCDE, Paris. Davantage de données via StatLink. Voir notes de chapitre.

StatLink 青正 http://dx.doi.org/10.1787/888933721041

\section{Le saviez-vous ?}

L'Inde compte près de 600000 diplômés de l'enseignement supérieur dans le domaine des TIC, soit pratiquement cinq fois plus que les États-Unis.

L'enseignement supérieur s'est développé dans le monde entier pour soutenir l'offre de travailleurs ayant un niveau d'instruction élevé et répondre à la demande croissante de compétences cognitives. Les décideurs s'intéressent particulièrement à l'offre de scientifiques, d'ingénieurs et d'experts en TIC, directement associés au progrès technologique et à la transformation numérique en cours. En 2015, les diplômés en sciences naturelles, en ingénierie et en TIC représentaient $23 \%$ des diplômés de l'enseignement supérieur de la zone OCDE. En dépit des pénuries ressenties, cette proportion est restée plutôt constante au cours de la décennie écoulée dans toute la zone. Les femmes ne comptaient toutefois, en moyenne, que pour $31 \%$ de l'effectif en 2015 , le rapport allant de $18 \%$ au Chili à $44 \%$ en Pologne. L'Inde et l'Indonésie affichaient la plus forte proportion de diplômés de l'enseignement supérieur issus d'une filière associée aux TIC. De surcroît, en plus d'être le plus important contributeur au stock mondial de diplômés en TIC, avec près de 585000 individus en 2015, l'Inde est aussi le pays qui se rapproche le plus de la parité.

Les compétences de chercheur revêtent également une grande importance aux yeux des décideurs et leur acquisition dans le cadre de programmes doctoraux fait souvent l'objet de subventions publiques. L'effectif de titulaires de doctorat, rapporté à la population, varie sensiblement d'un pays à l'autre. La Suisse arrive en tête pour le nombre de ces diplômés exprimé en proportion de la population en âge de travailler, ce qu'elle doit en partie à la présence relativement forte d'étrangers parmi eux. En 2016, dans plus d'un tiers des pays pour lesquels des données sont disponibles, les titulaires de doctorat représentaient un peu plus de $1 \%$ de la population s'inscrivait dans une progression régulière. En moyenne, 40 \% d'entre eux étaient des femmes.

\section{Définitions}

Les filières sciences naturelles, ingénierie et TIC correspondent aux domaines suivants de la classification CITE-2013 : 05 - Sciences naturelles, mathématiques et statistiques ; 06 - Technologies de l'information et de la communication ; et 07 - Ingénierie, industries de transformation et construction.

Les diplômés de l'enseignement supérieur sont les étudiants ayant obtenu un diplôme de niveau 5 à 8 selon la CITE-2011. Les titulaires de doctorat sont les individus ayant obtenu un diplôme de niveau 8 selon la CITE-2011, soit un diplôme de chercheur hautement qualifié, dont la préparation s'achève normalement par la remise et la soutenance d'un travail écrit de fond, d'une qualité suffisante pour en permettre la publication, étayé par des recherches originales. 


\section{Diplômés de l'enseignement supérieur en technologies de l'information et de la communication, selon le sexe, 2015}

En pourcentage de l'ensemble des diplômés de l'enseignement supérieur

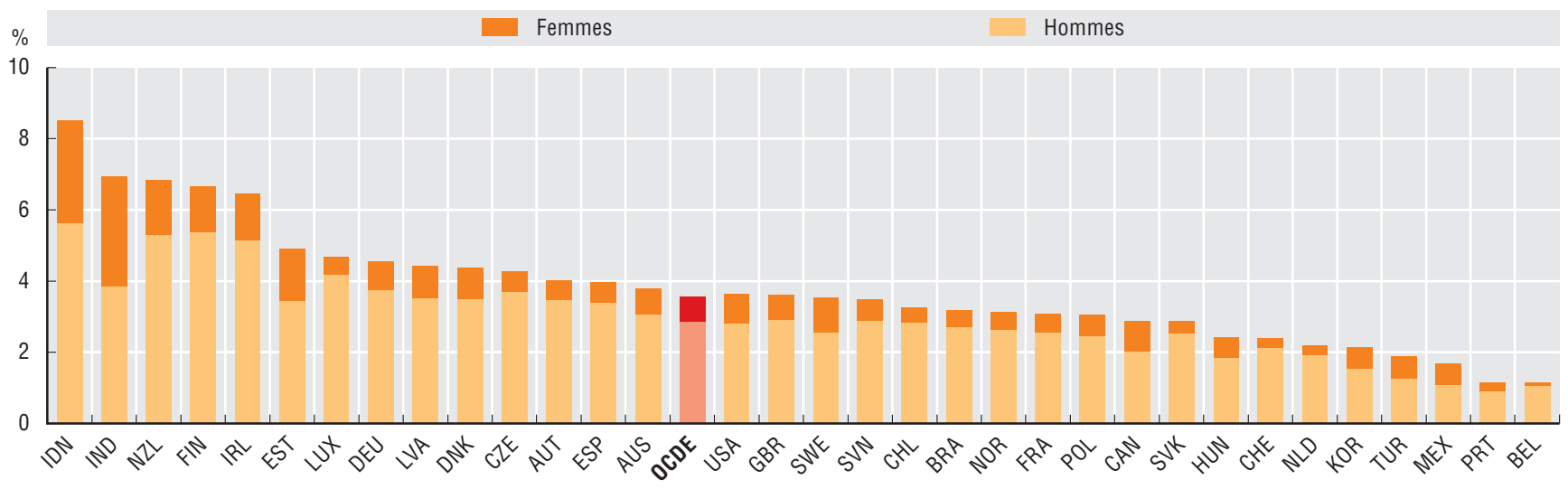

Source : Calculs de l'OCDE d'après OCDE, Statistiques et base de données sur l'éducation, septembre 2017. Davantage de données via StatLink. Voir notes de chapitre.

Titulaires de doctorat dans la population en âge de travailler, 2016

Pour mille individus âgés de 25 à 64 ans

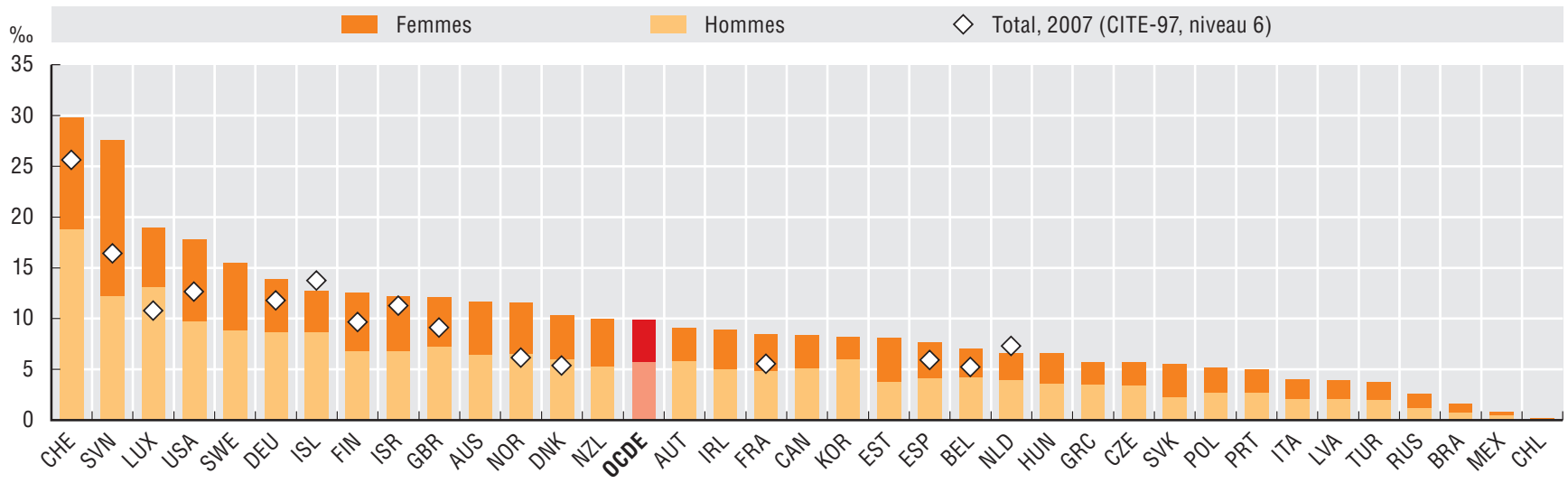

Source : Calculs de l'OCDE à partir de la collecte de données de l'OCDE sur les carrières des titulaires de doctorat 2017, http://oe.cd/ctd-fr, OCDE (2017), Regards sur l'éducation 2017 : les indicateurs de l'OCDE et OCDE (2009), Regards sur l'éducation 2009 : les indicateurs de l'OCDE, Éditions OCDE, Paris. Davantage de données via StatLink. Voir notes de chapitre.

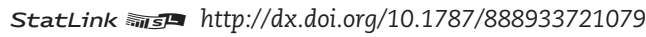

\section{Mesurabilité}

Les indicateurs relatifs aux diplômés par domaine d'études sont calculés à partir de données annuelles collectées conjointement par l'UNESCO-UIS, l'OCDE et Eurostat. L'exercice de collecte de données vise à produire des informations comparables internationalement sur des aspects clés des systèmes éducatifs dans plus de 60 pays du monde (http://www. oecd.org/fr/education/base-de-donnees.htm). L'adoption, dans ce cadre, de la Classification internationale type de l'éducation telle que révisée en 2011 (CITE-11) et de la Classification des domaines d'études et de formation de 2013 a une incidence sur les comparaisons avec les séries de données antérieures. Il s'agit toutefois d'un problème mineur au niveau d'agrégation retenu, où les équivalences sont plutôt évidentes.

Obtenir des données statistiquement représentatives au sujet du rôle des titulaires de doctorat dans la population tient de la gageure compte tenu du nombre relativement faible d'individus appartenant à ce groupe. Un projet horizontal est en cours au sein de l'OCDE afin d'encourager les pays à rendre compte, de façon régulière et systématique, des caractéristiques clés de ce groupe en expansion rapide, mobile à l'échelle internationale et doté de compétences uniques en recherche qui peuvent être fort utiles au-delà du milieu universitaire (http://oe.cd/ctd-fr). 


\section{LE SAVOIR, LES TALENTS ET LES COMPÉTENCES}

\section{Chercheurs}

Personnel de R-D, 2015

Pour mille emplois

VII/. Données " Autre personnel de R-D » non disponibles

$\diamond$ Chercheurs, 2005

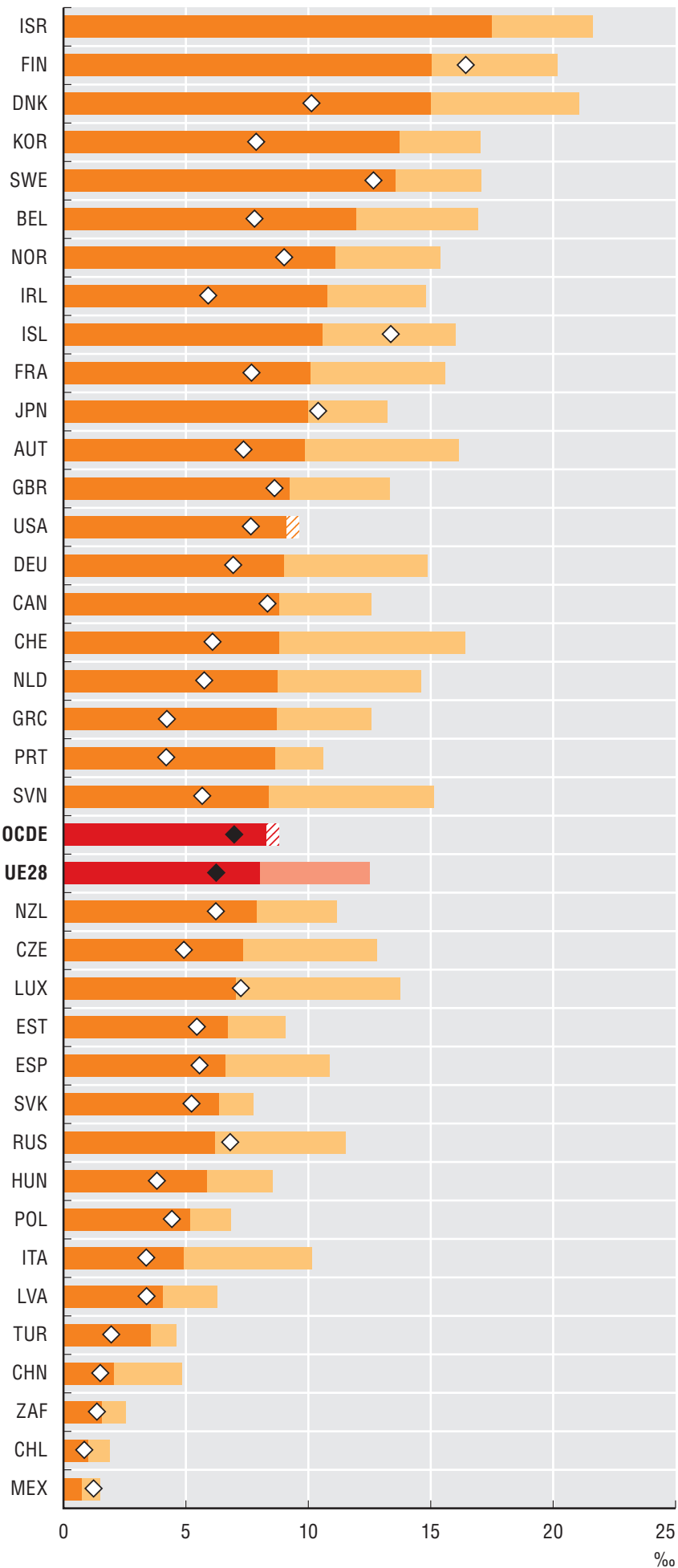

Source : OCDE, Base de données des Principaux indicateurs de la science et de la technologie, http://oe.cd/pist-fr, juillet 2017. Davantage de données via StatLink. Voir notes de chapitre.

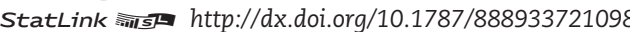

\section{Le saviez-vous ?}

En Lituanie, il y a autant d'hommes que de femmes chez les chercheurs tandis qu'en Suède les secondes sont plus actives que les premiers dans la recherche en entreprise.

Les chercheurs, et plus généralement le personnel de R-D, apportent un concours indispensable aux activités de R-D. Si l'on mesure en équivalent temps plein (ETP), plus de $2 \%$ des personnes pourvues d'un emploi au Danemark, en Finlande et en Israël travaillent dans le domaine - soit pratiquement deux fois la moyenne des autres pays (1.1\%). La Norvège et l'Irlande arrivent en bonne position malgré des dépenses de R-D inférieures à la moyenne OCDE. Que ce soit en termes absolus ou relatifs, les effectifs de chercheurs ont pratiquement partout progressé entre 2005 et 2015, même si leur poids dans le total du personnel de R-D est très variable puisqu'il est supérieur à $80 \%$ en Israël, en Corée, en République slovaque, en Suède et au Portugal, contre $49 \%$ en Italie et $43 \%$ en Chine.

Le secteur des entreprises et celui de l'enseignement supérieur sont les principaux employeurs de chercheurs. Les entreprises arrivent en tête - avec plus de $70 \%$ des chercheurs aux États-Unis, en Corée, en Israël et au Japon. Entre 2005 et 2015, la proportion de chercheurs employés par elles a fait un bond considérable en Hongrie, en Pologne et en Turquie, alors qu'elle s'est contractée en Afrique du Sud, en Grèce, au Luxembourg et au Mexique.

Les femmes comptent pour plus de $40 \%$ des chercheurs en Afrique du Sud, en Estonie, en Lettonie, au Portugal et en République slovaque, quand elles ne représentent pas $25 \%$ de l'effectif en Allemagne, en Autriche et en République tchèque. Ce sont souvent les établissements d'enseignement supérieur qui les emploient en majorité. Au Luxembourg, la création en 2003 de la première et seule université publique du pays dédiée à la recherche, a contribué à faire croître la proportion de femmes parmi les chercheurs, qui avait ainsi gagné 10 points de pourcentage en 2015.

\section{Définitions}

Le personnel de recherche-développement réunit toutes les personnes employées directement pour des activités de R-D et comprend aussi bien les chercheurs que les techniciens et le personnel auxiliaire.

Les chercheurs s'entendent des spécialistes travaillant à la conception ou à la création de nouveaux savoirs. Ils mènent des travaux de recherche en vue d'améliorer ou de mettre au point des concepts, théories, modèles, techniques, instruments, logiciels ou modes opératoires et sont, en règle générale, directement associés à la gestion des projets de R-D.

Le personnel de R-D est comptabilisé en équivalent temps plein, soit le nombre d'heures de travail réellement consacrées à la R-D au cours d'une période de référence donnée (habituellement une année civile) rapporté au nombre total d'heures qu'une personne ou un groupe de personnes est censé travailler au cours de la même période. Ainsi une personne travaillant à mi-temps à des activités de R-D pendant une année donnée compte pour 0.5 personne-année en ETP de R-D. Cela donne une mesure plus juste du volume de ressources humaines consacrées à la R-D dans un pays que les effectifs ou le nombre de postes. À des fins de comparaisons internationales, les chiffres afférents au personnel de R-D sont normalisés par rapport à ceux de l'emploi total figurant dans les statistiques de l'OCDE sur les comptes nationaux. 
Chercheurs, par secteur d'emploi, 2015

En pourcentage de l'effectif total des chercheurs, équivalents temps plein

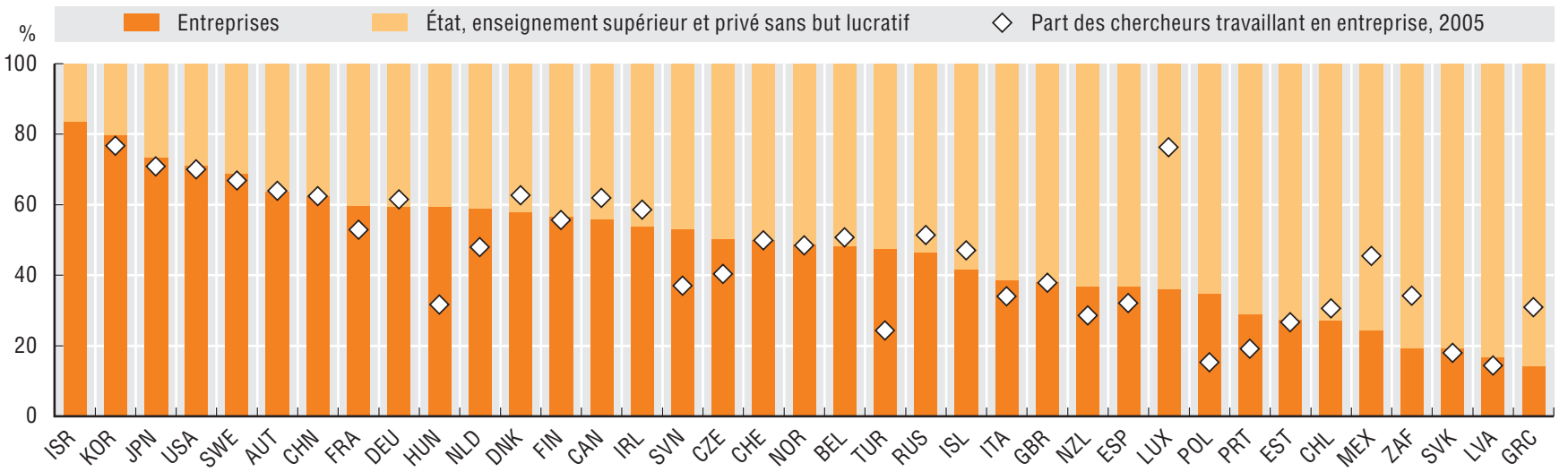

Source : OCDE, Base de données sur les Statistiques de la recherche et développement, http://oe.cd/srd-fr, juin 2017. Davantage de données via StatLink. Voir notes de chapitre.

StatLink :

Femmes chercheurs, 2015

En pourcentage de l'effectif total des chercheurs, équivalents temps plein

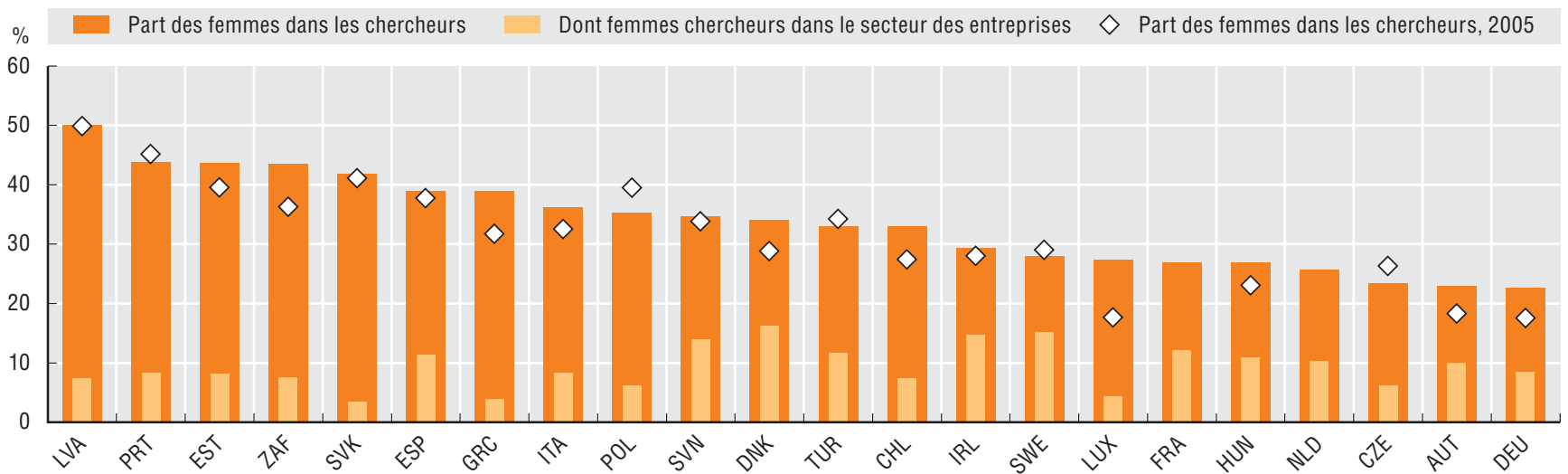

Source: OCDE, Base de données sur les Statistiques de la recherche et développement, http://oe.cd/srd-fr, juin 2017. Davantage de données via StatLink. Voir notes de chapitre.

StatLink त्ताइ http://dx.doi.org/10.1787/888933721136

\section{Mesurabilité}

Il s'avère parfois difficile de réunir des renseignements détaillés (par ex. : sexe, tranche d'âge, qualifications, origine géographique, etc.) sur les personnes qui exécutent des activités de R-D. Le Manuel de Frascati 2015 (http://oe.cd/frascati) préconise diverses ventilations en ce qui concerne les personnes occupées à des activités de R-D car les exécutants sont mieux à même de fournir ces renseignements au sujet de leurs employés directs. Certains pays toutefois prennent aussi en considération des acteurs externes - comme les consultants intervenant sur site ou le personnel non rémunéré ou bénévole dans leurs données sur le personnel de R-D. Plusieurs pays ces dernières années ont fait entrer de nouveaux groupes - tels les étudiants en doctorat bénéficiaires de subventions ou l'ensemble des enseignants des universités titulaires d'un poste permanent - dans la catégorie des chercheurs. Les estimations elles non plus ne sont pas toujours établies selon les mêmes règles ; ainsi, les méthodes de calcul des ETP peuvent varier non seulement d'un pays à l'autre, mais encore entre les secteurs d'un même pays, du fait de l'utilisation de données issues de sources différentes pour y mesurer la R-D. L'estimation des ETP est particulièrement délicate dans le secteur de l'enseignement supérieur, de nombreux chercheurs ayant en effet d'autres activités - enseignement, gestion administrative - dont certaines se situent parfois à la frontière de la R-D. Ces disparités entre les pays affectent la comparabilité des résultats à l'échelle internationale. 


\section{LE SAVOIR, LES TALENTS ET LES COMPÉTENCES}

\section{Compétences dans le monde numérique}

\section{Élèves forts et élèves faibles en sciences et mathématiques, enquête PISA, 2015}

En pourcentage des élèves de 15 ans

Élèves forts en sciences et mathématiques

Élèves faibles en sciences et mathématiques

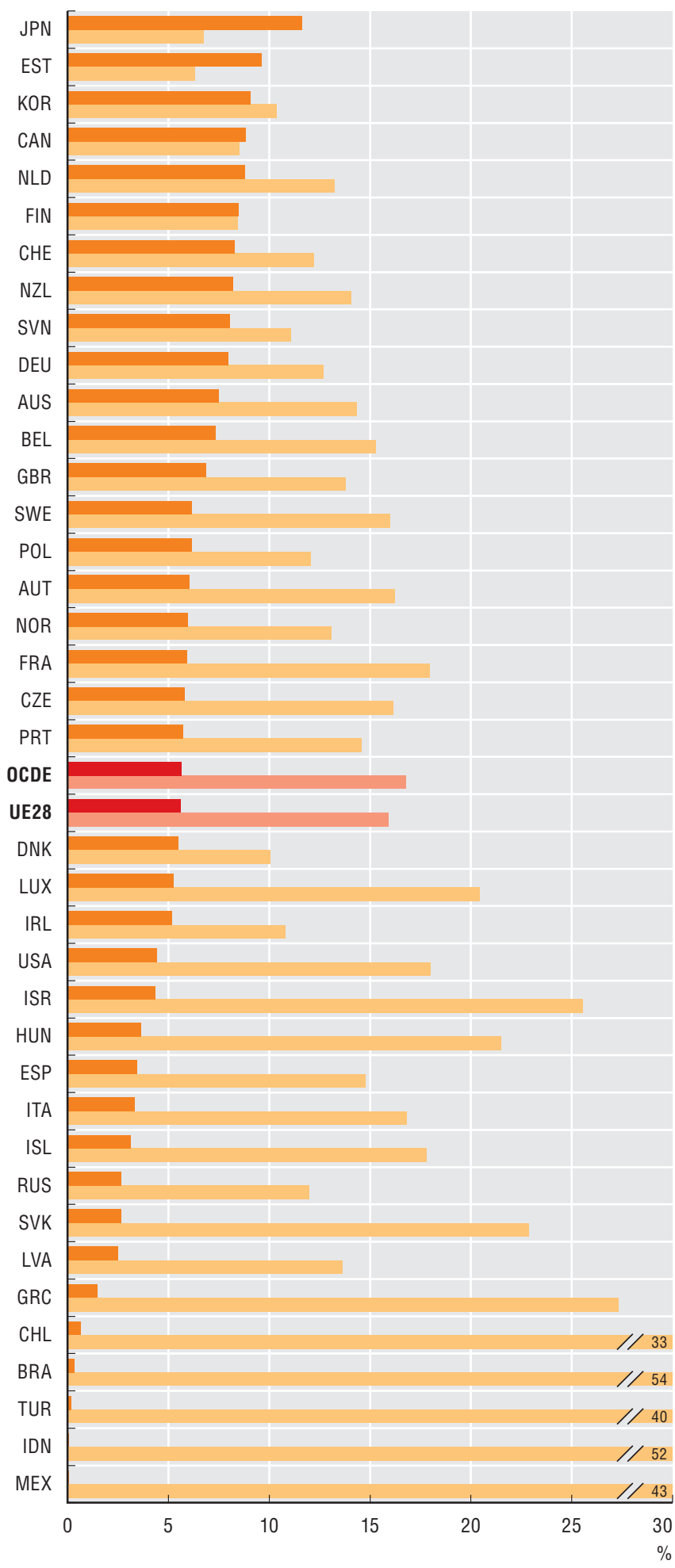

Source : Calculs de l'OCDE, d'après OCDE, Base de données de l'enquête du Programme international pour le suivi des acquis des élèves (PISA) 2015, juillet 2017. Davantage de données via StatLink. Voir notes de chapitre.

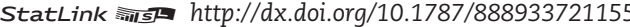

\section{Le saviez-vous ?}

La proportion d'individus présentant une bonne aptitude à la résolution de problèmes en environnement à forte composante technologique est trois à quatre fois plus élevée chez les jeunes actifs, hommes et femmes, que chez leurs aînés en fin de carrière.

De solides compétences cognitives, jointes à la capacité de résoudre des problèmes et d'apprendre et penser de manière créative, sont essentielles pour s'adapter aux transformations rapides, de grande ampleur et de vaste portée, induites par le numérique.

En moyenne, $6 \%$ des élèves de 15 ans dans la zone OCDE sont très performants en sciences et en mathématiques, si l'on en croit les résultats de l'enquête réalisée 2015 au titre du Programme de l'OCDE pour l'évaluation internationale des acquis des élèves (PISA). Deux tiers de ces jeunes démontrent également de solides capacités en lecture. À l'inverse, environ $17 \%$ sont à la peine dans au moins deux de ces trois domaines. Le Canada, l'Estonie, la Finlande et le Japon sont les seuls pays où la proportion d'élèves très performants est au moins aussi élevée que celle d'élèves peu performants (de $8.5 \%$ à $11.6 \%$ des élèves obtiennent de bons résultats tandis que $6.3 \%$ à $8.5 \%$ se situent en bas de l'échelle de notation).

L'instruction reçue et les compétences acquises dans le jeune âge influent en règle générale sur le niveau de performance professionnelle. Les différences observées, dans les pays couverts par le Programme pour l'évaluation internationale des compétences des adultes (PIAAC), entre les actifs âgés de 25 à 34 ans et ceux âgés de 55 à 65 ans tendent à mettre en évidence l'action positive de l'éducation sur les compétences. Partout, les jeunes travailleurs se montrent plus aptes à résoudre des problèmes que ne le sont leurs aînés. Les différences entre générations sont souvent plus marquées chez les femmes que chez les hommes. Les jeunes femmes relèvent d'ailleurs très sensiblement la moyenne dans les pays où $30 \%$ seulement des travailleurs, sinon moins, s'avèrent tout à fait, ou passablement, capables de résoudre des problèmes dans un environnement à forte composante technologique. C'est le cas, par exemple, en Slovénie, en Grèce et en Turquie, où $47 \%, 30 \%$ et $24 \%$ d'entre elles, respectivement, démontrent de bonnes aptitudes à cet égard, contre $34 \%, 19 \%$ et $15 \%$ des hommes de la même génération.

En ce qui concerne la disposition à l'apprentissage et la créativité, les différences liées à l'âge et au sexe sont plus marquées entre les pays qu'au sein des pays, ce qui souligne l'influence de facteurs tels que la culture et les conventions sociales dans la formation de la personnalité.

\section{Définitions}

Les élèves très performants sont, parmi les élèves âgés de 15 ou 16 ans, ceux qui se sont classés en haut de l'échelle de compétences (soit aux Niveaux 5 et 6), les élèves peu performants n'ont pas atteint le Niveau 2 lors de l'évaluation PISA 2015.

La résolution de problèmes en environnement à forte composante technologique désigne des épreuves auxquelles on a soumis des travailleurs âgés de 25 à 34 ans et de 55 à 65 ans démontrant un niveau de compétences intermédiaire ou élevé (c'est-à-dire des individus atteignant le Niveau 2 ou 3 à l'évaluation PIAAC) qui consistaient à résoudre des problèmes liés à l'utilisation des technologies de l'information et des communications. L'indicateur disposition à l'apprentissage et créativité correspond à des traits de personnalité et est construit à partir de six questions du PIAAC. 
Résolution de problèmes en environnement à forte composante technologique, 2012 ou 2015

Pourcentage d'actifs obtenant un résultat moyen ou élevé, selon le sexe, individus âgés de 25 à 34 ans et de 55 à 65 ans

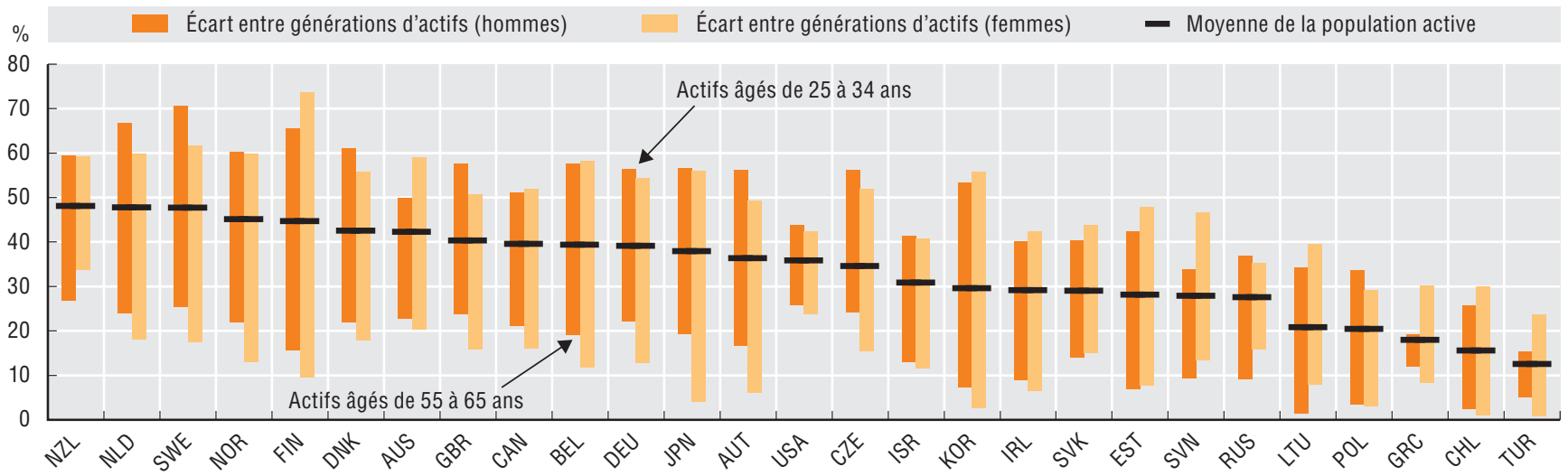

Source : Calculs de l'OCDE, d'après la base de données du Programme international pour l'évaluation des compétences des adultes (PIAAC), juin 2017. Voir notes de chapitre.

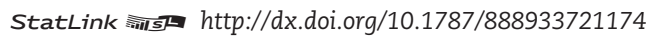

Disposition à l'apprentissage et créativité, 2012 ou 2015

Résultats moyens selon le sexe, actifs âgés de 25 à 34 ans et de 55 à 65 ans

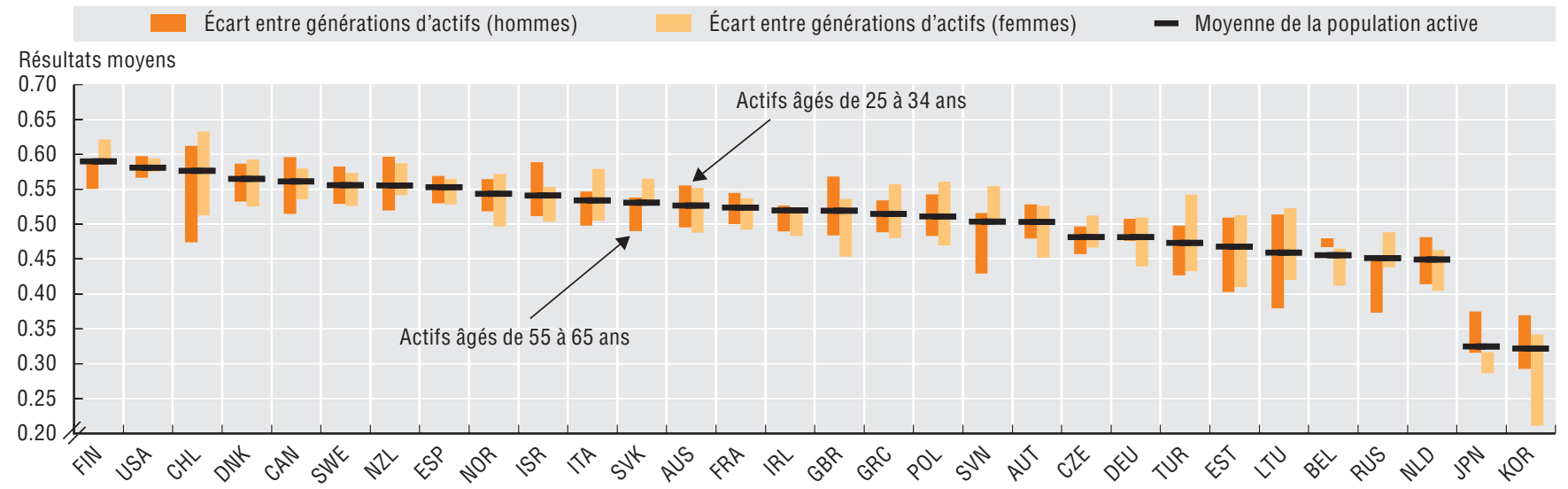

Source : Calculs de l'OCDE, d'après base de données du Programme international pour l'évaluation des compétences des adultes (PIAAC), juin 2017. Voir notes de chapitre.

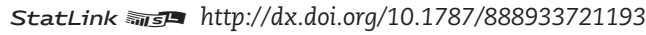

\section{Mesurabilité}

L'âge des participants au Programme pour l'évaluation internationale des acquis des élèves (PISA) de l'OCDE est compris entre 15 ans et trois mois et 16 ans et deux mois. Ces élèves doivent être scolarisés, depuis six années au moins, sans considération du type d'établissement fréquenté, du programme suivi ou du régime d'enseignement (temps plein ou temps partiel). Les données PISA les plus récentes dont on dispose ont été réunies au cours de l'année scolaire 2015 : plus d'un demi-million d'élèves, représentant 28 millions de leurs contemporains dans 72 pays et économies différents, ont passé un examen international d'une durée de 2 heures. Les chiffres concernant la résolution de problèmes en environnement à forte composante technologique sont ceux d'un sous-ensemble de pays participant au PIAAC, l'Espagne, la France et l'Italie n'ayant pas pris part à ce volet de l'évaluation. L'indicateur relatif à la disposition à l'apprentissage et la créativité a été développé à partir d'une analyse factorielle exploratoire de pointe. Il repose sur six questions en rapport avec l'ouverture à la nouveauté et la pensée créative, comme " Met en œuvre des idées nouvelles dans la vie réelle " ou " Aime apprendre de nouvelles choses ». La méthodologie détaillée est exposée par Grundke et al. (2017). 


\section{LE SAVOIR, LES TALENTS ET LES COMPÉTENCES}

\section{Rendements des compétences en TIC}

Composante TIC des emplois, 2012 ou 2015

Écart interquartile, médiane et moyenne
25th percentile-75th percentile
$\diamond$ Moyenne
I Médiane

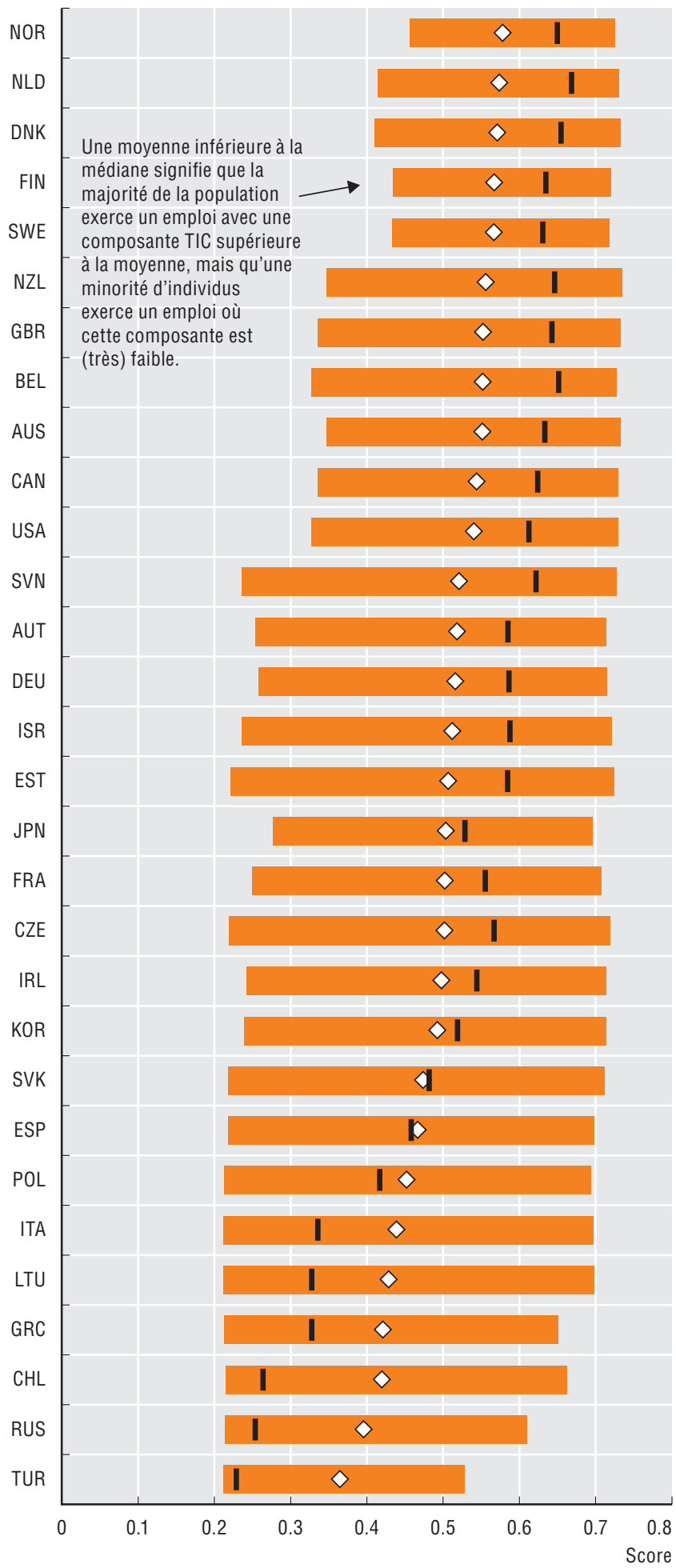

Source: Calculs de l'OCDE, d'après la base de données du Programme international pour l'évaluation des compétences des adultes (PIAAC), juin 2017. Voir notes de chapitre.

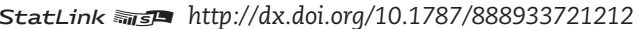

\section{Le saviez-vous ?}

Les actifs qui occupent un emploi dont la composante TIC est supérieure de $10 \%$ à la moyenne perçoivent un salaire horaire jusqu'à $4 \%$ plus élevé.

La transformation numérique change les modes de vie et de production et modifie la configuration des lieux de travail et la nature même des emplois. Dans le cadre de leur activité, les individus, de plus en plus, doivent exécuter des tâches qui exigent de savoir utiliser les technologies de l'information et des communications (TIC). Leur rémunération est en rapport avec l'éventail de compétences dont ils sont pourvus, notamment dans le domaine des TIC, celles-ci ayant une incidence bénéfique sur leur performance professionnelle et sur les résultats de l'entreprise.

Un nouvel indicateur, bâti à partir d'informations sur les tâches accomplies au travail, montre que la composante TIC, quel que soit le type d'emploi, diffère largement d'un pays à un autre. Les travailleurs des pays nordiques et des Pays-Bas exercent ainsi des activités dans lesquelles cette composante est, dans l'ensemble, plus développée qu'ailleurs.

Les compétences en TIC sont particulièrement recherchées et toutes choses étant égales par ailleurs (notamment l'éducation et les compétences des autres actifs) - plus un emploi les sollicitera, plus la rémunération horaire accordée sera élevée. Si l'on en croit les estimations, l'avantage offert par les emplois à forte composante TIC serait très variable selon les pays. Ainsi, en Corée et aux États-Unis, quand cette composante est supérieure de $10 \%$ à la moyenne nationale, le salaire horaire est augmenté de plus de $3.5 \%$. Le bénéfice est en revanche relativement moindre en Israël et en Turquie (voisin de $1 \%$ seulement). Le rendement de ces emplois dépend de bien des facteurs, au nombre desquels la situation qui prévaut dans le pays sur le plan de l'offre et de la demande de compétences connexes et la structure salariale.

Cependant les compétences en TIC ne suffisent pas à elles seules pour tirer son épingle du jeu dans l'économie numérique. L'analyse donne à penser que d'autres compétences encore sont nécessaires, en particulier en gestion et communication, et que ce domaine est très complémentaire au précédent. Le surcroît de rémunération conféré à ceux que leur emploi met au contact de ces deux domaines en vaut confirmation. À titre d'exemple, une progression de $10 \%$ de la composante gestion et communication par rapport à la moyenne nationale se traduit par des salaires horaires plus élevés de $0.6 \%$ (en Belgique) à $2.1 \%$ (en Allemagne), et ajoute encore, en moyenne, $0.3 \%$ au salaire horaire des emplois à forte composante TIC. Au Royaume-Uni, le bonus additionnel de $1.4 \%$ lié aux TIC double le rendement des tâches de gestion et communication sur le marché du travail. La Fédération de Russie semble être le seul pays de l'échantillon à faire exception, puisque les compétences en gestion et communication n'y apportent aucun avantage salarial supplémentaire lorsqu'elles sont utilisées en association avec les compétences en TIC.

\section{Définitions}

Les emplois à forte composante TIC s'entendent des emplois dont la composante TIC est supérieure de $10 \%$ à la moyenne nationale. Les rendements moyens des tâches de gestion et communication sont calculés par rapport à la moyenne du pays, toutes les autres variables (notamment celles ayant trait à l'instruction et aux compétences) étant par ailleurs constantes. 
Rendements de la composante TIC sur le marché du travail, 2012 ou 2015

Pourcentage de variation du salaire horaire pour une augmentation de 10 \% (par rapport à la moyenne nationale) de la composante TIC des emplois

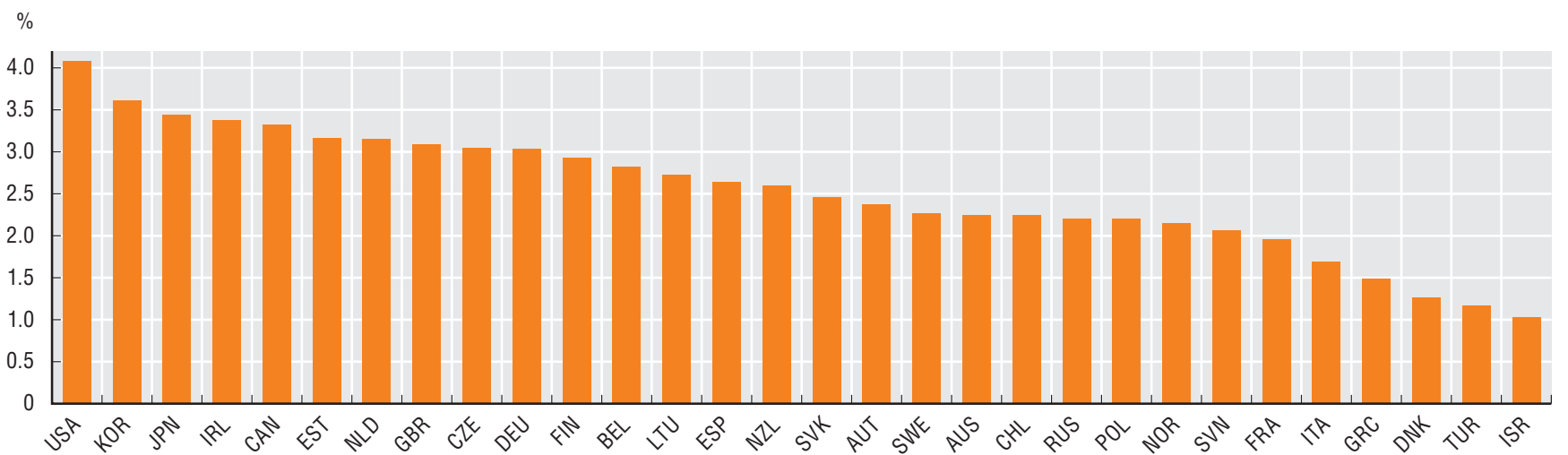

Source : Calculs de l'OCDE, d'après la base de données du Programme international pour l'évaluation des compétences des adultes (PIAAC), juin 2017. Voir notes de chapitre.

\section{Rendements de la composante gestion et communication des emplois : la prime TIC, 2012 ou 2015}

Pourcentage de variation du salaire horaire pour une augmentation de $10 \%$

(par rapport à la moyenne nationale) de la composante gestion et communication des emplois

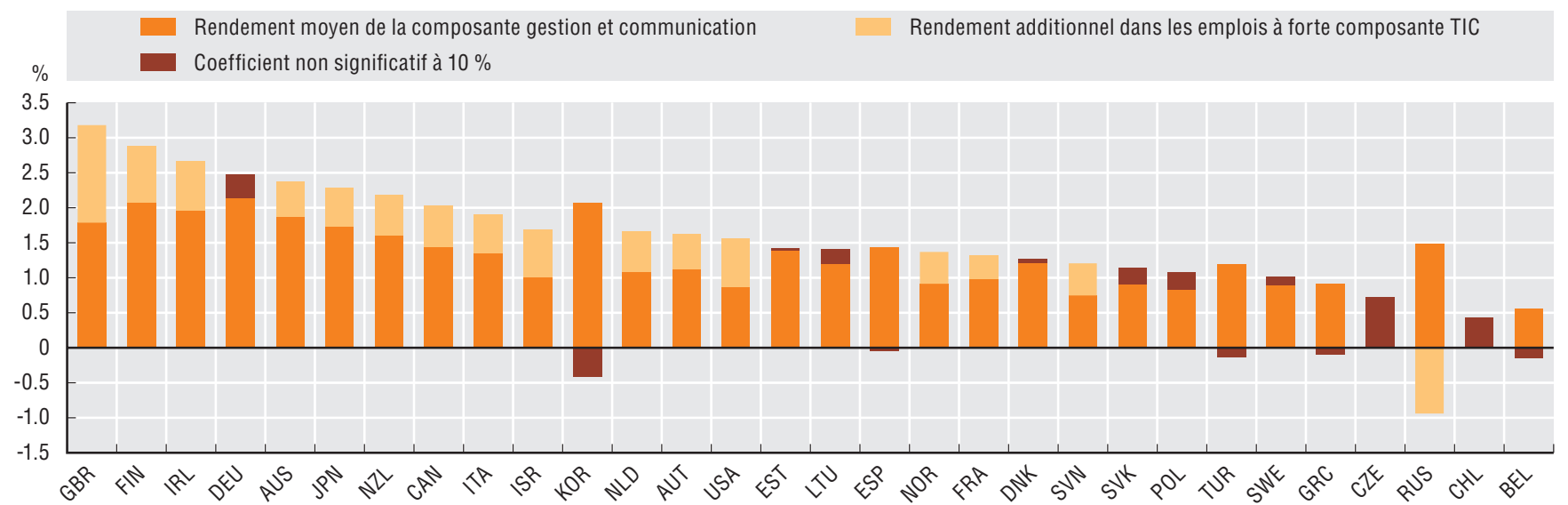

Source : Calculs de l'OCDE, d'après la base de données du Programme international pour l'évaluation des compétences des adultes (PIAAC), juin 2017. Voir notes de chapitre.

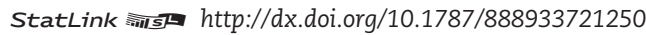

\section{Mesurabilité}

Les indicateurs relatifs aux composantes TIC et gestion et communication des emplois sont construits à partir d'une analyse factorielle exploratoire de pointe et expriment la mesure dans laquelle les individus accomplissent des tâches relevant de ces domaines dans le cadre de leur activité professionnelle. L'indicateur concernant les TIC repose sur les réponses à 11 questions de l'Évaluation sur les compétences des adultes (PIAAC), réalisée par l'OCDE, qui vont de la simple utilisation de l'internet, à celle des logiciels Word ou Excel, ou à l'emploi d'un langage de programmation. L'indicateur concernant la gestion et la communication est fondé quant à lui sur cinq questions en rapport avec les activités de négociation ou de planification des tâches d'autres personnes, ou encore avec la fourniture de conseils ou d'instructions à des tiers. On trouvera une description détaillée de la méthodologie suivie chez Grundke et al. (2017). Les rendements offerts sur le marché du travail en fonction du contenu des emplois sont calculés par régression des salaires, selon la méthode des moindres carrés ordinaires (équations de Mincer), à partir de données du PIAAC. Les estimations s'appuient sur le logarithme des salaires horaires en tant que variable liée et sur diverses variables de contrôle ayant trait aux individus (dont l'âge, la durée de formation initiale, le sexe et les autres mesures de compétences) ainsi que sur des variables auxiliaires du secteur pertinent prises en tant que variables explicatives. Voir Grundke et al. (à paraître) pour de plus amples détails. 


\section{Travailleurs suivant une formation, selon le type de formation, 2012 ou 2015}

En pourcentage du nombre total de personnes occupant un emploi

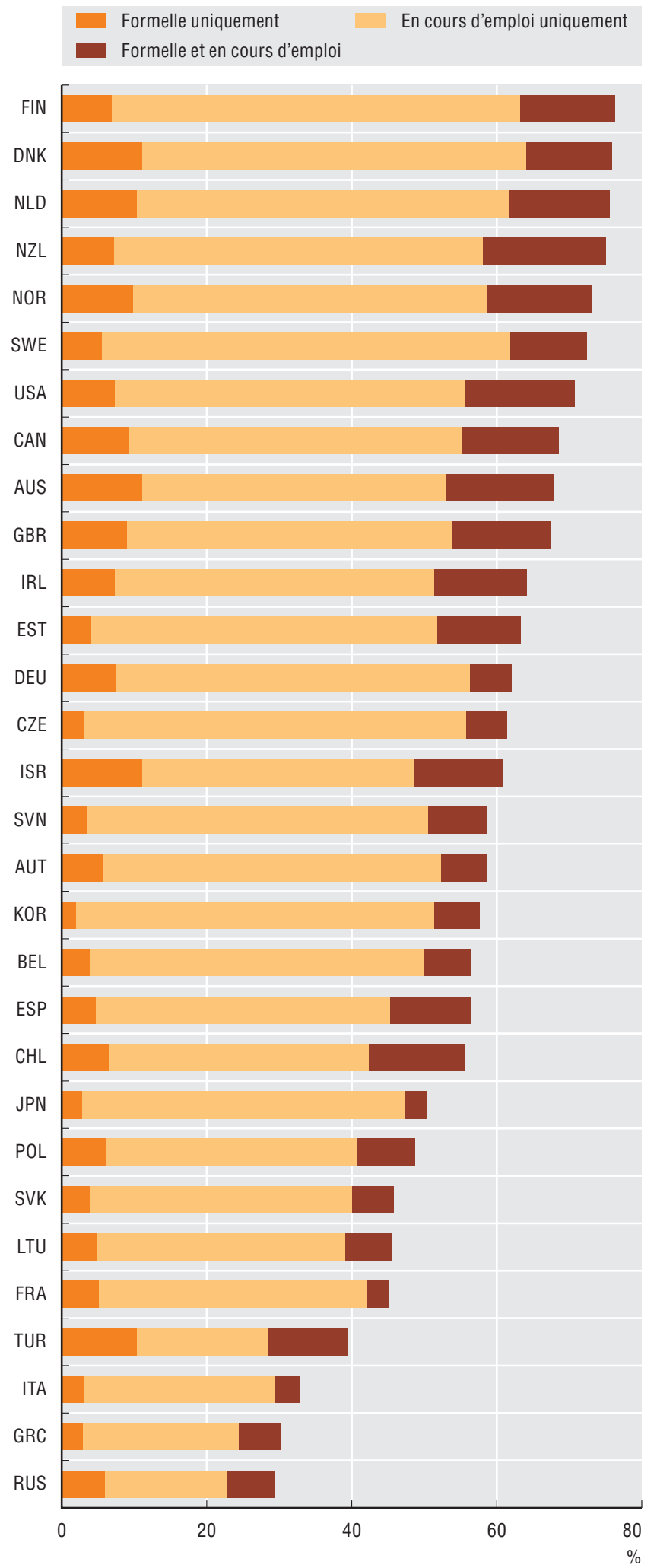

Source : Calculs de l'OCDE, d'après la base de données du Programme international pour l'évaluation des compétences des adultes (PIAAC), juin 2017. Voir notes de chapitre.

StatLink ainsth http://dx.doi.org/10.1787/888933721269

\section{Le saviez-vous ?}

Dans de nombreux pays de l'OCDE, les entreprises investissent davantage dans les actifs incorporels que dans les machines et le matériel - plus de deux fois plus en Irlande et en Norvège.

Le capital intellectuel désigne des actifs qui, par nature, sont dépourvus de substance physique (on parle d'actifs " incorporels "). Ces actifs ont trait pour la plupart à l'investissement dans le capital humain et portent sur la création, la codification et l'utilisation de la connaissance. Entrent dans le capital intellectuel le capital organisationnel, la recherche et l'innovation, les logiciels et les bases de données. Certains de ces éléments, à commencer par la R-D et les logiciels, sont désormais pris en considération dans le Système de comptabilité nationale (SCN, tel que révisé en 2008) et équivalent en moyenne à plus d'un tiers de l'investissement total que les entreprises consacrent aux machines et au matériel.

En 2015, en moyenne pour l'ensemble des pays étudiés, l'investissement total consenti par le secteur des entreprises dans le capital intellectuel - en tenant compte de l'investissement dans le capital organisationnel et la formation, lorsque l'on disposait d'estimations - était d'importance comparable à l'investissement dans les actifs corporels et représentait $15 \%$ de la valeur ajoutée brute. C'est en France, en Irlande, en Suède et aux États-Unis que cette proportion était la plus élevée (excédant les $20 \%$ ).

L'investissement du secteur non marchand dans le capital intellectuel, qui vient compléter celui du secteur des entreprises, connaît lui aussi d'amples variations puisqu'il représente de $2 \%$ (en Espagne) à $11 \%$ (en Irlande) de la valeur ajoutée brute du secteur. Les niveaux les plus élevés sont atteints par l'Irlande, la Suède, les États-Unis et le Royaume-Uni. En comparaison, l'intensité de capital intellectuel du secteur marchand demeure entre 1.5 et 5 fois plus importante, et progresse à un rythme plus soutenu (40\% contre $11 \%$ en moyenne depuis 2000).

Investir dans la formation permet de doter les individus des compétences nécessaires pour accomplir les tâches qui leur incombent et pour passer d'un emploi à un autre, surtout s'ils opèrent dans un domaine où la technologie évolue rapidement. Les estimations de l'OCDE tendent à indiquer que si près de $60 \%$ des travailleurs, en moyenne, bénéficient d'une formation formelle ou en cours d'emploi, la probabilité de suivre une telle formation est très variable : allant de $30 \%$ environ (en Fédération de Russie) à plus de $75 \%$ (en Finlande). Les formations ont principalement lieu en cours d'emploi (dans $72 \%$ des cas en moyenne), pour une meilleure adéquation aux besoins de l'entreprise.

\section{Définitions}

Une formation formelle est une formation dispensée dans un cadre organisé, extérieur à l'environnement professionnel, et sanctionnée par un diplôme. Une formation en cours d'emploi est dispensée au sein ou en dehors de l'entreprise mais ne débouche pas, en règle générale, sur l'obtention d'un diplôme officiel. Les actifs intellectuels entrant dans le SCN comprennent la $\mathrm{R}-\mathrm{D}$, les logiciels, la prospection minière et les œuvres artistiques originales. L'investissement dans les autres actifs intellectuels fait l'objet d'estimations fondées sur les données d'INTAN-Invest et concerne les marques, les dessins et modèles, les nouveaux produits financiers, le capital organisationnel et la formation en entreprise. La valeur ajoutée brute se calcule comme la valeur de la production diminuée de celle de la consommation intermédiaire. La formation brute de capital fixe (FBCF) hors logement est constituée de l'investissement dans les actifs corporels à l'exclusion du logement. 
Investissement des entreprises en capital fixe et en capital intellectuel, 2015

En pourcentage de la valeur ajoutée brute du secteur des entreprises

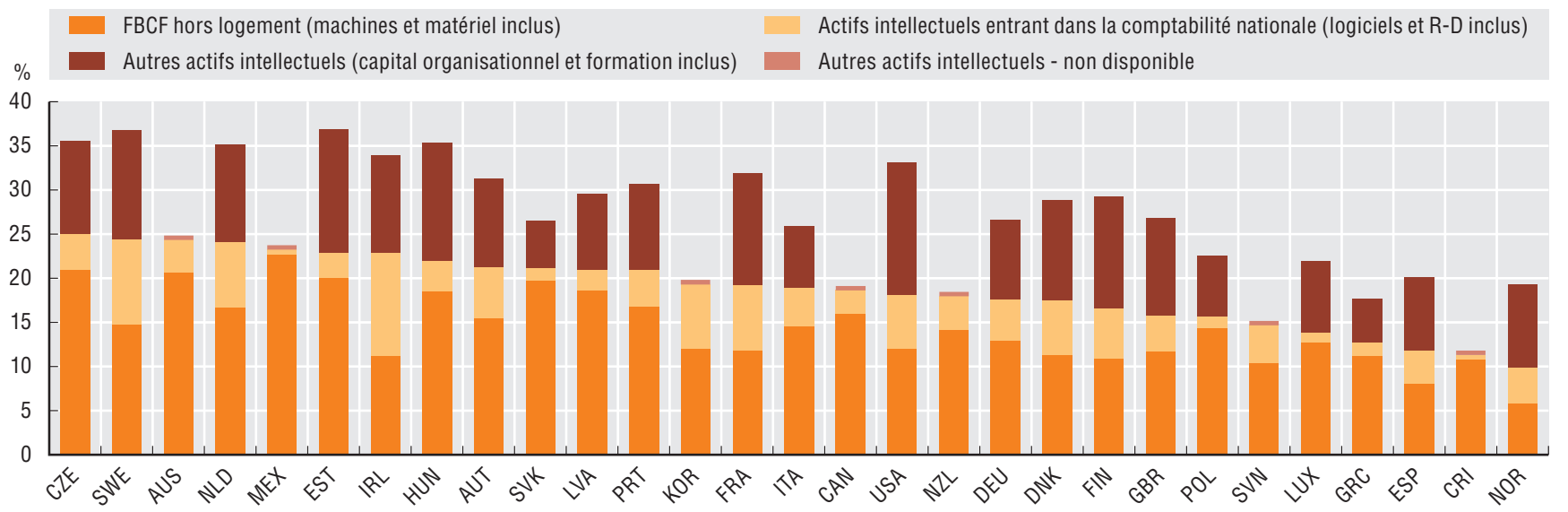

Source : Calculs de l'OCDE, d'après la Base de données des Comptes nationaux des pays de l'OCDE ; des données d'INTAN-Invest (http://www.intan-invest.net) ; et des données du Bureau d'analyse économique (BEA, Bureau of Economic Analysis) des États-Unis, mai 2017. Voir notes de chapitre.

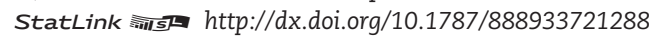

Investissement en capital intellectuel des secteurs marchand et non marchand, 2000 et 2015

En pourcentage de la valeur ajoutée brute du secteur

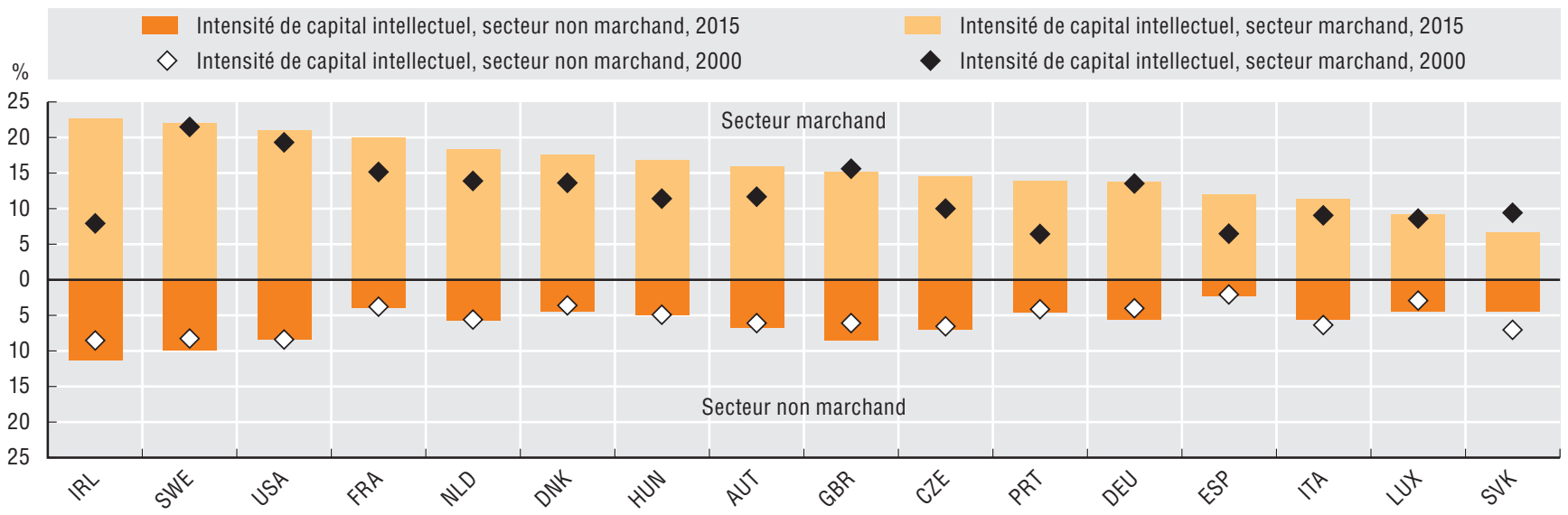

Source : Calculs de l'OCDE, d'après la Base de données des Comptes nationaux des pays de l'OCDE ; des données d'INTAN-Invest (http://www.intan-invest.net) ; et des données de SPINTAN (http://www.spintan.net), mai 2017. Voir notes de chapitre.

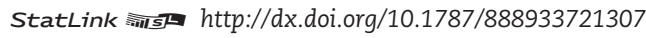

\section{Mesurabilité}

Les chiffres concernant la formation sont dérivés du nombre d'employés qui, dans le cadre de l'Évaluation des compétences des adultes (PIAAC) ont déclaré avoir suivi une formation d'un certain type au moins une fois dans l'année, qu'ils appartiennent au secteur public ou au secteur privé. Une pondération est appliquée pour que ces chiffres soient représentatifs au plan national. La fréquence des formations est susceptible de masquer des différences en ce qui concerne leur durée. L'investissement dans les actifs intellectuels entrant dans la comptabilité nationale est repris de la Base de données des Comptes nationaux. Les autres actifs intellectuels sont une estimation, établie à partir des données d'INTAN-Invest, que l'on a extrapolée en se fondant sur le taux de croissance de l'investissement dans les actifs intellectuels entrant dans la comptabilité nationale. Dans la définition retenue ici, les investissements du secteur marchand s'entendent des investissements réalisés par les entreprises qui relèvent des divisions 1 à 82 de la CITI rév. 4, exception faite des divisions 68 et 72 . Les investissements du secteur non marchand sont tels que définis par SPINTAN et couvrent les établissements publics et les entités sans but lucratif appartenant aux divisions 72 et 84 à 93 de la CITI rév. 4. Les intensités de capital sont obtenues en calculant le quotient de l'investissement par la valeur ajoutée brute, telle qu'elle figure dans la Base de données des Comptes nationaux, dans les divisions correspondantes. Le dénominateur n'est pas corrigé : i) du caractère institutionnel des agents économiques, contrairement aux données de l'investissement figurant au numérateur, ni ii) de la capitalisation des actifs hors SCN. 


\section{LE SAVOIR, LES TALENTS ET LES COMPÉTENCES}

\section{Notes et Références}

\section{Chypre}

La note suivante est incluse à la demande de la Turquie :

« Les informations figurant dans ce document qui font référence à “Chypre” concernent la partie méridionale de l'île. Il n'y a pas d'autorité unique représentant à la fois les Chypriotes turcs et grecs sur l'île. La Turquie reconnaît la République Turque de Chypre Nord (RTCN). Jusqu'à ce qu'une solution durable et équitable soit trouvée dans le cadre des Nations Unies, la Turquie maintiendra sa position sur la "question chypriote". "

La note suivante est ajoutée à la demande de tous les États de l'Union européenne membres de l’OCDE et de l'Union européenne :

"La République de Chypre est reconnue par tous les membres des Nations Unies sauf la Turquie. Les informations figurant dans ce document concernent la zone sous le contrôle effectif du gouvernement de la République de Chypre. »

\section{Israël}

"Les données statistiques concernant Israël sont fournies par et sous la responsabilité des autorités israéliennes ou d'un tiers compétents. L'utilisation de ces données par l'OCDE est sans préjudice du statut des hauteurs du Golan, de JérusalemEst et des colonies de peuplement israéliennes en Cisjordanie aux termes du droit international. »

" Il est à noter que les données statistiques sur les brevets et marques israéliens sont fournies par les offices des brevets et des marques des pays concernés. "

\subsection{Investir dans le savoir}

\section{Dépenses consacrées à l'enseignement supérieur et à l'enseignement professionnel, 2014}

Pour le Chili et l'Indonésie, les données se rapportent à 2015.

Pour le Danemark et la Pologne, les informations sur l'enseignement professionnel portent sur des programmes du premier et du second cycle du secondaire.

Pour la République slovaque, les données concernant l'enseignement supérieur correspondent aux dépenses des seuls établissements publics.

Pour la Suisse, les données correspondent aux dépenses publiques uniquement.

\section{Dépenses intérieures brutes de R-D, par type d'activité, 2015}

Les données afférentes aux DIRD totales (tous types de R-D confondus), se rapportent à 2005 et 2015. Lorsque la ventilation par type de R-D pour l'année 2015 n'est pas directement disponible, elle est estimée à partir de l'année la plus récente pour laquelle on dispose de données ventilées, à savoir : 2014 pour la France, l'Italie, les Pays-Bas, la Pologne, le Portugal, le Royaume-Uni et la Slovénie ; 2013 pour l'Autriche, la Belgique, le Danemark, la Grèce, l'Irlande et Israël.

La ventilation par type de R-D s'appuie en règle générale sur l'ensemble des DIRD, y compris les dépenses en capital. Toutefois, pour le Chili, la Fédération de Russie et la Norvège, la part correspondant à chaque type d'activité est calculée sur la base des estimations des dépenses courantes de R-D, les dépenses en capital étant comptabilisées dans la catégorie "Ventilation non disponible, en tout ou partie ». Pour les États-Unis à l'exception des DIRDET, qui couvrent les dépenses en capital dédiées à la R-D, les chiffres donnés (aussi bien pour la R-D totale que par type d'activité) portent sur les dépenses courantes mais intègrent une composante d'amortissement qui peut différer du niveau réel des dépenses en capital (indiqué dans la catégorie "Ventilation non disponible, en tout ou partie ").

Ces statistiques se fondent sur les Statistiques de la recherche et développement (http://oe.cd/srd-fr) et les Principaux indicateurs de la science et de la technologie (http://oe.cd/pist-fr) de l'OCDE. Se reporter à ces sources pour davantage de renseignements sur les données, en particulier sur les ruptures de séries et autres problèmes analogues.

Pour l'Afrique du Sud, les données relatives aux DIRD totales se rapportent à 2013.

Pour l'Australie, les données relatives aux DIRD totales se rapportent à 2004 et 2013.

Pour le Chili, les données relatives aux DIRD totales se rapportent à 2007 et 2015.

Pour le Chili, les chiffres officiels du PIB utilisés pour normaliser les niveaux relatifs de R-D sont calculés conformément au Système de comptabilité nationale (SCN) de 1993 et non au SCN de 2008, utilisé dans tous les autres cas.

Pour l'Irlande, les données relatives aux DIRD totales se rapportent à 2014.

Pour Israël, la R-D du secteur de la défense est partiellement exclue des estimations disponibles.

Pour la Suisse, les données relatives aux DIRD totales se rapportent à 2004 et 2015.

\section{Investissement en TIC, par type d'actifs, 2015}

L'investissement désigne la Formation brute de capital fixe (FBCF) au sens du Système de comptabilité nationale de 2008 (SCN 2008 ). Les données pour l'Islande correspondent à l'investissement du secteur des entreprises dans les machines de bureau et les ordinateurs. 
Les données pour la Corée ont été calculées par l'OCDE à partir des tableaux détaillés des entrées et sorties nationaux fournis par la Banque de Corée et des Comptes nationaux annuels des pays de l'OCDE.

\subsection{Enseignement supérieur et recherche fondamentale}

\section{Dépenses de R-D de l'enseignement supérieur, 2015}

Les fonds généraux des universités (FGU) d'origine publique correspondent à la part des dotations institutionnelles générales faites par les pouvoirs publics au secteur de l'enseignement supérieur qui est employée par celui-ci à des fins de R-D. Pour l'Estonie, les États-Unis et la Pologne, aucune dotation correspondant à la description des FGU n'est déclarée. On ne dispose pas d'estimations pour l'Allemagne, la Chine, la Corée, la Hongrie, la Lettonie, le Luxembourg, les Pays-Bas, le Portugal, la République tchèque et la Turquie. Les chiffres des FGU se rapportent à la même année de référence que ceux des DIRDES ou, à défaut, sont calculés sur la base des chiffres de l'année la plus récente disponible. Tel est le cas pour la Belgique (2013), la France (2014), Israël (2013), l'Italie (2014), la Nouvelle-Zélande (2013) et la Suède (2013).

Ces statistiques se fondent sur la Base de données des Principaux indicateurs de la science et de la technologie de l'OCDE (http://oe.cd/ pist-fr). Se reporter à cette source pour davantage de renseignements sur les données, en particulier sur les ruptures de séries et autres problèmes analogues.

Pour l'Afrique du Sud, les données se rapportent à 2013.

Pour l'Australie, les données se rapportent à 2004 et 2014.

Pour le Chili, les données se rapportent à 2007 et 2015.

Pour le Chili, les chiffres officiels du PIB utilisés pour normaliser les niveaux relatifs de R-D sont calculés conformément au Système de comptabilité nationale (SCN) de 1993 et non au SCN de 2008, utilisé dans tous les autres cas.

Pour la Corée et Israël, la R-D dans les sciences sociales et humaines n'est pas incluse dans les estimations de 2005.

Pour les États-Unis, les chiffres portent sur les dépenses courantes, mais intègrent une composante d'amortissement qui peut différer du niveau réel des dépenses en capital.

Pour la Suisse, les données se rapportent à 2004 et 2015.

\section{Recherche fondamentale dans les secteurs de l'enseignement supérieur et de l'État, 2015}

Les données correspondent à la somme des dépenses courantes et des dépenses en capital, sauf pour le Chili, les États-Unis, la Fédération de Russie et la Norvège, pour lesquels seules les dépenses courantes sont prises en compte dans les estimations communiquées à l'OCDE.

Ces statistiques se fondent sur les bases de données de l'OCDE sur la R-D, dont la Base de données des Statistiques de la recherche et développement (http://oe.cd/srd-fr) et la Base de données des Principaux indicateurs de la science et de la technologie (http://oe.cd/pist-fr). Se reporter à ces sources pour davantage de renseignements sur les données, en particulier sur les ruptures de séries et autres problèmes analogues.

Pour l'Afrique du Sud, l'Autriche, la Belgique, le Danemark, la Grèce, l'Irlande et Israël, les données se rapportent à 2013.

Pour la France, l'Italie, les Pays-Bas, le Portugal, le Royaume-Uni et la Slovénie, les données se rapportent à 2014.

L'année de référence utilisée comme base de comparaison pour l'évaluation de la part de la recherche fondamentale exécutée par les secteurs de l'enseignement supérieur et de l'État dans les DIRD est 2005, sauf pour l'Autriche et la Suisse (2004), le Chili, le Danemark et le Royaume-Uni (2007) et le Mexique (2003).

Pour la France, l'enquête auprès des administrations publiques a fait l'objet en 2010 de changements méthodologiques : la méthode de mesure des ressources consacrées à la R-D par les ministères et certains organismes publics a été modifiée, ce qui s'est traduit par une meilleure caractérisation de leurs activités de financement et une révision à la baisse, de l'ordre de 900 millions EUR, des DIRDET.

Pour Israël, la R-D du secteur de la défense est partiellement exclue des estimations disponibles.

Pour les Pays-Bas, une partie des dépenses allouées au développement expérimental dans le secteur de l'enseignement supérieur sont rattachées à la recherche fondamentale. Par ailleurs, les dépenses du secteur privé sans but lucratif (PSBL) sont comptabilisées avec celles du secteur de l'État.

Pour la Pologne, la part de la recherche fondamentale des secteurs de l'enseignement supérieur et de l'État dans les DIRD en 2005 est calculée à partir des seules dépenses courantes, tandis que les données pour 2014 portent également sur les dépenses en capital.

Pour l'Espagne, la part de la recherche fondamentale exécutée par les secteurs de l'enseignement supérieur et de l'État dans les DIRD en 2005 est calculée à partir des seules dépenses courantes, tandis que les données pour 2015 portent également sur les dépenses en capital.

Pour la Suisse, le secteur de l'État correspond uniquement aux administrations fédérales ou centrales.

Pour le Royaume-Uni, la méthode de décomposition des DIRDET en fonction du type d'activité de R-D a été améliorée en 2010, ce qui a entraîné une rupture de série.

Pour les États-Unis, les chiffres portent sur les dépenses courantes, mais intègrent une composante d'amortissement qui peut différer du niveau réel des dépenses en capital ; les DIRDET font exception, puisqu'elles intègrent à la place les dépenses en capital. 


\section{LE SAVOIR, LES TALENTS ET LES COMPÉTENCES}

\section{Notes et Références}

\section{Financement de la R-D dans l'enseignement supérieur, 2015}

Ces statistiques se fondent sur les bases de données de l'OCDE sur la R-D, dont la Base de données des Statistiques de la recherche et développement (http://oe.cd/srd-fr) et la Base de données des Principaux indicateurs de la science et de la technologie (http://oe.cd/pist-fr). Se reporter à ces sources pour davantage de renseignements sur les données, en particulier sur les ruptures de séries et autres problèmes analogues.

Pour tous les pays à l'exception de la Chine, la catégorie « Secteur privé sans but lucratif/Autres n.c.a. » est exclusivement composée des fonds provenant d'institutions privées sans but lucratif étrangères.

Les fonds généraux des universités (FGU) que les pouvoirs publics versent à des établissements universitaires à des fins de R-D sont comptabilisés dans le secteur de l'État d'où ils proviennent.

Pour l'Afrique du Sud, la Belgique, Israël, le Luxembourg et la Suède, les données se rapportent à 2013.

Pour l'Allemagne, la France, l'Italie et le Portugal, les données se rapportent à 2014.

Pour l'Allemagne, les fonds de l'enseignement supérieur et du secteur privé sans but lucratif sont rattachés aux financements directs de l'État.

Pour l'Australie, les données se rapportent à 2014.

Pour l'Australie, les Australian competitive grants (ACG) - dispositifs fédéraux et autres - sont identifiés séparément et inclus respectivement dans les financements directs de l'État et ceux du secteur privé sans but lucratif.

Pour l'Autriche, les données se rapportent à 2013.

Pour la Chine, les sources de financement des dépenses se décomposent comme suit : l'État, les entreprises, les fonds étrangers et " autres ». Ces catégories diffèrent légèrement de celles énoncées dans le Manuel de Frascati. Les fonds ne provenant pas de sources spécifiques apparaissent sous " autre secteur (intérieur) ». Entrent notamment dans cette catégorie les fonds levés auprès des circuits non bancaires, en particulier ceux des établissements de recherche indépendants (IRI, anciennement GRI) et du secteur de l'enseignement supérieur, ainsi que les reliquats de financements publics d'années ou de subventions passées. Les montants correspondants sont comptabilisés sous « Secteur privé sans but lucratif/Autres n.c.a. » dans le présent graphique.

Pour le Danemark, les fonds de l'enseignement supérieur sont rattachés aux financements de l'État.

Pour Israël, la R-D du secteur de la défense est partiellement exclue des estimations disponibles.

Dans l'enquête du Luxembourg, les données de R-D par source de financement se décomposent comme suit : groupe d'entreprises, ministère de l'Économie, entreprises partenaires de projets de R-D, Commission européenne, organisations internationales, autres sources étrangères (autres gouvernements nationaux, enseignement supérieur, autres).

\subsection{Scientifiques et ingénieurs}

\section{Diplômés de l'enseignement supérieur en sciences naturelles, ingénierie et TIC, 2005 et 2015}

L'enseignement supérieur correspond aux niveaux 5 à 8 de la CITE-2011.

Les domaines d'études renvoient aux Domaines d'études et de formation de la CITE (CITE-F 2013).

Pour le Japon, les données relatives aux technologies de l'information et de la communication sont comptabilisées dans les autres domaines.

Pour les Pays-Bas, les données excluent les titulaires de doctorat.

Pour l'Afrique du Sud, les données se rapportent à 2014.

Diplômés de l'enseignement supérieur en technologies de l'information et de la communication, selon le sexe, 2015

L'enseignement supérieur correspond aux niveaux 5 à 8 de la CITE-2011.

Le domaine d'études « Technologies de l'information et de la communication " renvoie aux Domaines d'études et de formation de la CITE (CITE-F 2013).

L'agrégat OCDE correspond à la moyenne non pondérée des pays pour lesquels des données sont disponibles.

\section{Titulaires de doctorat dans la population en âge de travailler, 2016}

La comparabilité internationale peut être limitée.

Sources (Population en âge de travailler) :

Nations Unies, Département des affaires économiques et sociales, Division de la population (2017), World Population Prospects: The 2017 Revision. Données personnalisées acquises depuis le site web.

Sources (Titulaires de doctorat) :

Pour l'Allemagne, le Brésil, le Canada, le Chili, la Corée, l'Estonie, la Norvège, le Portugal, la République tchèque et le RoyaumeUni : campagne de collecte de données de l'OCDE sur les carrières des titulaires de doctorats, 2017.

Pour les autres pays : OCDE (2017).

Les données pour 2007 ont été calculées à partir de OCDE (2009). 
Les données relatives aux niveaux d'études en 2016 et 2007 se fondent sur deux versions différentes de la CITE (CITE-2011 et CITE-97, respectivement) et n'ont pas fait l'objet d'une harmonisation. Même si les " titulaires de doctorats " sont définis de manière globalement identique dans les deux classifications, les comparaisons dans le temps sont à interpréter avec précaution. Pour le Brésil, les données concernant les titulaires de doctorat couvrent une période allant de 1996 à 2014.

Pour la Canada, les données se rapportent à 2011 et n'incluent pas les non-résidents ou les résidents étrangers, les personnes résidant en logement collectif, institutionnel ou non, les citoyens canadiens résidant à l'étranger et les membres à temps plein des Forces armées canadiennes stationnées à l'étranger. Les ressortissants étrangers sont en partie comptabilisés.

Pour le Chili, les données se rapportent à 2015.

Pour la Corée, les données se rapportent à 2012 et incluent les ressortissants étrangers sans qu'il soit toutefois possible de procéder à une ventilation par nationalité aux différents niveaux d'études.

Pour la Fédération de Russie, la couverture des données se rapportant aux diplômés sans emploi ou inactif, aux ressortissants étrangers et aux non-résidents est limitée.

Pour la République tchèque, les données correspondent à une moyenne mobile calculée sur la période 2014-16.

\subsection{Chercheurs}

\section{Personnel de R-D, 2015}

Ces statistiques se fondent sur la Base de données des Principaux indicateurs de la science et de la technologie de l'OCDE (http://oe.cd/ pist-fr). Se reporter à cette source pour davantage de renseignements sur les données, en particulier sur les ruptures de séries et autres problèmes analogues.

Pour l'Autriche, l'Espagne, la Grèce et la Lettonie, le personnel de R-D inclut le personnel interne ainsi que certains intervenants de l'extérieur; des doubles comptes sont possibles si plusieurs répondants comptabilisent les mêmes individus dans leur personnel de R-D. Pour l'Afrique du Sud, le Canada et le Mexique, les données se rapportent à 2013.

Pour la Chine, l'Irlande et la Turquie, aucune enquête sur la R-D n’a été menée auprès du secteur privé sans but lucratif (PSBL), ses activités en la matière étant tenues pour négligeables.

Pour la Chine et Israël, la part militaire de la R-D du secteur de la défense n'est pas prise en compte.

Pour la Chine, les données sur les chercheurs rassemblées dans le cadre d'enquêtes antérieures à 2009 l'ont été sur la base du concept de « scientifique et ingénieur » défini par l'UNESCO, et celles réunies depuis sur la base du concept de « chercheur » au sens du Manuel de Frascati, d'où une rupture de série entre 2008 et 2009.

Pour le Chili, les données se rapportent à 2007 et 2015.

Pour la Corée, les données sur le personnel de R-D en 2005 excluent la R-D dans les sciences sociales et humaines.

Pour les États-Unis, la proportion de chercheurs dans le personnel de R-D hors secteur des entreprises est une estimation à partir de données historiques.

Pour la Grèce, un changement d'ordre méthodologique a été introduit en 2011 avec l'extension du secteur de l'État et de l'enseignement supérieur de manière à y inclure les hôpitaux publics, l'ensemble des institutions relevant du ministère de la Culture, tous les établissements d'enseignement technologique et les établissements d'enseignement post-secondaire, ce qui s'est traduit par une augmentation du nombre de chercheurs.

Pour l'Irlande, un changement d'ordre méthodologique a été introduit en 2014 avec l'inclusion des doctorants dans le secteur de l'enseignement supérieur, ce qui a considérablement accru les effectifs chercheurs.

Pour l'Islande et l'année de référence 2013, la méthode de collecte des données a été modifiée, donnant lieu à des ruptures de séries. Les principales différences tiennent à la refonte du questionnaire, l'utilisation des registres des entreprises, l'obligation légale faite à celles-ci de répondre à l'enquête, la définition, dans le questionnaire, de certaines notions essentielles en matière de R-D, et diverses modifications dans la répartition des établissements entre les secteurs des entreprises ou de l'État.

Pour Israël, les données se rapportent à 2014 et les proportions sont des estimations fondées sur les données disponibles pour cette année-là.

Pour le Luxembourg, un changement d'ordre méthodologique, en 2012, a permis de mieux isoler la R-D dans les activités logicielles et, par conséquent, entraîné une diminution de l'effectif de chercheurs.

Pour la Norvège, dans le secteur des entreprises, les données se rapportent aux diplômés universitaires et non aux chercheurs.

Pour les Pays-Bas, un changement d'ordre méthodologique est intervenu en 2012 avec la modification, dans les enquêtes nationales, des données relatives au personnel selon la fonction exercée, entraînant une augmentation substantielle de l'effectif de chercheurs.

Pour le Portugal, le personnel de R-D a augmenté en 2008, sous l'effet principalement d'améliorations d'ordre méthodologique dans les différents secteurs institutionnels (État, enseignement supérieur et institutions privées sans but lucratif) : les réponses consignées dans chaque formulaire d'enquête ont été confrontées au contenu d'autres bases de données internes ce qui a abouti, entre autres, à la prise en compte de l'ensemble des enseignants des universités titulaires d'un poste permanent et de tous les chercheurs financés par le ministère de la Science, de la Technologie et de l'Enseignement supérieur en 2008.

Pour la Slovénie, la méthodologie employée a changé en 2011, d'où une meilleure analyse des non-réponses et l'utilisation de nouvelles sources administratives pour mieux identifier les exécutants de R-D, ce qui s'est traduit par une forte hausse de l'effectif de chercheurs. 


\section{LE SAVOIR, LES TALENTS ET LES COMPÉTENCES}

Pour la Suède, les données de 2005 du secteur des entreprises se rapportent aux diplômés universitaires et non aux chercheurs. Un changement d'ordre méthodologique a été introduit en 2013 : dans le secteur des entreprises, le secteur PSBL et le secteur de l'État, il a été demandé aux unités déclarantes de classer leur personnel en deux catégories, " chercheurs " et " autre personnel ", et non plus trois. "Autre personnel " regroupe les catégories « techniciens et personnel équivalent " et " autre personnel de soutien ». Une partie du personnel, à compter de l'année de référence 2013, a été transférée de la catégorie des « techniciens » à celle des " chercheurs".

Pour la Suisse, les données se rapportent à 2004 et 2015, et le personnel de R-D du secteur PSBL n'entre pas dans le total.

\section{Chercheurs, par secteur d'emploi, 2015}

Ces statistiques se fondent sur la Base de données des Statistiques de la recherche et développement (http://oe.cd/srd-fr). Se reporter à cette source pour davantage de renseignements sur les données, en particulier sur les ruptures de séries et autres problèmes analogues.

Pour l'Afrique du Sud, le Canada et le Mexique, les données se rapportent à 2005 et 2013.

Pour l'Autriche, l'Espagne, la Grèce et la Lettonie, le personnel de R-D inclut le personnel interne ainsi que certains intervenants de l'extérieur; des doubles comptes sont possibles si plusieurs répondants comptabilisent les mêmes individus dans leur personnel de R-D.

Pour la Canada, la R-D dans les sciences sociales et humaines n'est pas prise en considération en ce qui concerne le secteur des entreprises et le secteur PSBL.

Pour le Chili, les données se rapportent à 2007 et 2015.

Pour la Chine, l'Irlande et la Turquie, aucune enquête sur la R-D n'a été menée auprès du secteur privé sans but lucratif (PSBL), ses activités en la matière étant tenues pour négligeables.

Pour la Chine et Israël, la part militaire de la R-D du secteur de la défense n'est pas prise en compte.

Pour la Chine, les données sur les chercheurs rassemblées dans le cadre d'enquêtes antérieures à 2009 l'ont été sur la base du concept de " scientifique et ingénieur » défini par l'UNESCO, et celles réunies depuis sur la base du concept de " chercheur » au sens du Manuel de Frascati, d'où une rupture de série entre 2008 et 2009.

Pour la Corée, les données sur le personnel de R-D en 2005 excluent la R-D dans les sciences sociales et humaines.

Pour les États-Unis, les données se rapportent à 2008 et 2015 et la proportion de chercheurs dans le personnel de R-D hors secteur des entreprises est une estimation à partir de données historiques.

Pour la Grèce, un changement d'ordre méthodologique a été introduit en 2011 avec l'extension du secteur de l'État et de l'enseignement supérieur de manière à y inclure les hôpitaux publics, l'ensemble des institutions relevant du ministère de la Culture, tous les établissements d'enseignement technologique et les établissements d'enseignement post-secondaire, ce qui s'est traduit par une augmentation du nombre de chercheurs.

Pour l'Irlande, un changement d'ordre méthodologique a été introduit en 2013 avec l'inclusion des doctorants dans le secteur de l'enseignement supérieur, ce qui a considérablement accru les effectifs de chercheurs.

Pour l'Islande et l'année de référence 2013, la méthode de collecte des données a été modifiée, donnant lieu à des ruptures de séries. Les principales différences tiennent à la refonte du questionnaire, l'utilisation des registres des entreprises, l'obligation légale faite à celles-ci de répondre à l'enquête, la définition, dans le questionnaire, de certaines notions essentielles en matière de R-D, et diverses modifications dans la répartition des établissements entre les secteurs des entreprises ou de l'État.

Pour Israël, les données se rapportent à 2014 et les proportions sont des estimations fondées sur les données disponibles pour cette année-là.

Pour la Lettonie, le secteur PSBL est comptabilisé avec le secteur des entreprises.

Pour le Luxembourg, un changement d'ordre méthodologique, en 2012, a permis de mieux isoler la R-D dans les activités logicielles et, par conséquent, entraîné une diminution de l'effectif de chercheurs.

Pour la Nouvelle-Zélande, le secteur PSBL est comptabilisé avec le secteur des entreprises.

Pour la Norvège, dans le secteur des entreprises, les données se rapportent aux diplômés universitaires et non aux chercheurs.

Pour les Pays-Bas, un changement d'ordre méthodologique est intervenu en 2012 avec la modification, dans les enquêtes nationales, des données relatives au personnel selon la fonction exercée, entraînant une augmentation substantielle de l'effectif de chercheurs. Pour le Portugal, le personnel de R-D a augmenté en 2008, sous l'effet principalement d'améliorations d'ordre méthodologique dans les différents secteurs institutionnels (État, enseignement supérieur et institutions privées sans but lucratif) : les réponses consignées dans chaque formulaire d'enquête ont été confrontées au contenu d'autres bases de données internes ce qui a abouti, entre autres, à la prise en compte de l'ensemble des enseignants des universités titulaires d'un poste permanent et de tous les chercheurs financés par le ministère de la Science, de la Technologie et de l'Enseignement supérieur en 2008.

Pour la Slovénie, la méthodologie employée a changé en 2011, d'où une meilleure analyse des non-réponses et l'utilisation de nouvelles sources administratives pour mieux identifier les exécutants de R-D, ce qui s'est traduit par une forte hausse de l'effectif de chercheurs.

Pour la Suède, les données de 2005 du secteur des entreprises se rapportent aux diplômés universitaires et non aux chercheurs. Un changement d'ordre méthodologique a été introduit en 2013 : dans le secteur des entreprises, le secteur PSBL et le secteur de l'État, il a été demandé aux unités déclarantes de classer leur personnel en deux catégories, " chercheurs » et " autre personnel ", et non plus trois. " Autre personnel » regroupe les catégories" techniciens et personnel équivalent » et " autre personnel de 
soutien ». Une partie du personnel, à compter de l'année de référence 2013, a été transférée de la catégorie des « techniciens » à celle des " chercheurs".

Pour la Suisse, le personnel de R-D du secteur PSBL n'entre pas dans le total.

\section{Femmes chercheurs, 2015}

Ces statistiques se fondent sur la Base de données des Statistiques de la recherche et développement (http://oe.cd/srd-fr). Se reporter à cette source pour davantage de renseignements sur les données, en particulier sur les ruptures de séries et autres problèmes analogues.

Pour l'Afrique du Sud, le Danemark, la Grèce, l'Irlande, le Luxembourg et la Suède, les données se rapportent à 2013.

Pour l'Autriche, l'Espagne, la Grèce et la Lettonie, le personnel de R-D inclut le personnel interne ainsi que certains intervenants de l'extérieur; des doubles comptes sont possibles si ces individus sont déclarés par chacun de leurs employeurs directs.

Pour l'Autriche, les données se rapportent à 2004 et 2013.

Pour le Chili, les données se rapportent à 2007 et 2015.

Pour la Grèce, un changement d'ordre méthodologique a été introduit en 2011 avec l'extension du secteur de l'État et de l'enseignement supérieur de manière à y inclure les hôpitaux publics, l'ensemble des institutions relevant du ministère de la Culture, tous les établissements d'enseignement technologique et les établissements d'enseignement post-secondaire, ce qui s'est traduit par une augmentation du nombre de chercheurs.

Pour la Hongrie, les données se rapportent à 2006 et 2015.

Pour l'Irlande et la Turquie, aucune enquête sur la R-D n'a été menée auprès du secteur privé sans but lucratif (PSBL), ses activités en la matière étant tenues pour négligeables.

Pour l'Italie, les Pays-Bas, la Pologne, le Portugal et la Slovénie, les données se rapportent à 2014.

Pour la Lettonie, le secteur PSBL est comptabilisé avec le secteur des entreprises.

Pour le Luxembourg, un changement d'ordre méthodologique, en 2012, a permis de mieux isoler la R-D dans les activités logicielles et, par conséquent, entraîné une diminution de l'effectif de chercheurs.

Pour la Norvège, dans le secteur des entreprises, les données se rapportent aux diplômés universitaires et non aux chercheurs.

Pour le Portugal, le personnel de R-D a augmenté en 2008, sous l'effet principalement d'améliorations d'ordre méthodologique dans les différents secteurs institutionnels (État, enseignement supérieur et institutions privées sans but lucratif) : les réponses consignées dans chaque formulaire d'enquête ont été confrontées au contenu d'autres bases de données internes ce qui a abouti, entre autres, à la prise en compte de l'ensemble des enseignants des universités titulaires d'un poste permanent et de tous les chercheurs financés par le ministère de la Science, de la Technologie et de l'Enseignement supérieur en 2008.

Pour la Slovénie, la méthodologie employée a changé en 2011, d'où une meilleure analyse des non-réponses et l'utilisation de nouvelles sources administratives pour mieux identifier les exécutants de R-D, ce qui s'est traduit par une forte hausse de l'effectif de chercheurs.

Pour la Suède, les données de 2005 du secteur des entreprises se rapportent aux diplômés universitaires et non aux chercheurs. Un changement d'ordre méthodologique a été introduit en 2013 : dans le secteur des entreprises, le secteur PSBL et le secteur de l'État, il a été demandé aux unités déclarantes de classer leur personnel en deux catégories, " chercheurs " et " autre personnel ", et non plus trois. " Autre personnel " regroupe les catégories « techniciens et personnel équivalent " et " autre personnel de soutien ». Une partie du personnel, à compter de l'année de référence 2013, a été transférée de la catégorie des « techniciens » à celle des " chercheurs".

\subsection{Compétences dans le monde numérique}

\section{Élèves forts et élèves faibles en sciences et mathématiques, enquête PISA, 2015}

Les indicateurs correspondent à la part des élèves qui obtiennent des résultats élevés (faibles) en sciences et en mathématiques mais non en lecture et à celle des élèves qui obtiennent des résultats élevés (faibles) en sciences, en mathématiques et en lecture.

\section{Résolution de problèmes en environnement à forte composante technologique, 2012 ou 2015}

Les calculs sont fondés sur les résultats des épreuves de résolution de problèmes en environnement à forte composante technologique réalisées dans le cadre du Programme pour l'évaluation internationale des compétences des adultes (PIAAC).

Ont un niveau moyen ou élevé de compétences en résolution de problèmes en environnement à forte composante technologique les individus ayant atteint le niveau 2 ou 3 de l'échelle d'évaluation.

L'Italie, la France et l'Espagne n'ont pas pris part aux évaluations en ce qui concerne la résolution de problèmes en environnement à forte composante technologique.

Les données pour les 23 pays ci-après, qui sont issues de la première vague du PIAAC, se rapportent à l'année 2012 : Allemagne, Australie, Autriche, Belgique (Flandre), Canada, Corée, Danemark, Espagne, Estonie, États-Unis, Fédération de Russie (sauf Moscou), Finlande, France, Irlande, Italie, Japon, Norvège, Pays-Bas, Pologne, République slovaque, République tchèque, Royaume-Uni (Angleterre et Irlande du Nord) et Suède. Les données concernant les autres pays se rapportent à 2015 et sont issues de la deuxième vague du premier cycle de l'enquête. 


\section{Disposition à l'apprentissage et créativité, 2012 ou 2015}

L'indicateur relatif à la disposition à l'apprentissage et la créativité s'appuie sur une analyse factorielle exploratoire de pointe. Il repose sur six questions en rapport avec l'ouverture à la nouveauté et la pensée créative. La méthodologie détaillée est exposée par Grundke et al. (2017).

Les données pour les 23 pays ci-après, qui sont issues de la première vague du PIAAC, se rapportent à l'année 2012 : Allemagne, Australie, Autriche, Belgique (Flandre), Canada, Corée, Danemark, Espagne, Estonie, États-Unis, Fédération de Russie (sauf Moscou), Finlande, France, Irlande, Italie, Japon, Norvège, Pays-Bas, Pologne, République slovaque, République tchèque, Royaume-Uni (Angleterre et Irlande du Nord) et Suède. Les données concernant les autres pays se rapportent à 2015 et sont issues de la deuxième vague du premier cycle de l'enquête.

\subsection{Rendements des compétences en TIC}

\section{Composante TIC des emplois, 2012 ou 2015}

Une moyenne inférieure à la médiane signifie que la majorité de la population exerce un emploi avec une composante TIC supérieure à la moyenne, mais qu'une minorité d'individus exerce un emploi où cette composante est (très) faible.

L'indicateur relatif à la composante TIC des emplois s'appuie sur une analyse factorielle exploratoire de pointe et rend compte de l'utilisation de ces technologies en milieu professionnel. Il repose sur 11 questions de l'Évaluation des compétences des adultes (PIAAC) de l'OCDE, qui vont de la simple utilisation de l'internet, à celle des logiciels Word ou Excel, ou à l'emploi d'un langage de programmation. La méthodologie détaillée est exposée par Grundke et al. (2017).

Les données pour les 23 pays ci-après, qui sont issues de la première vague du PIAAC, se rapportent à l'année 2012 : Allemagne, Australie, Autriche, Belgique (Flandre), Canada, Corée, Danemark, Espagne, Estonie, États-Unis, Fédération de Russie (sauf Moscou), Finlande, France, Irlande, Italie, Japon, Norvège, Pays-Bas, Pologne, République slovaque, République tchèque, Royaume-Uni (Angleterre et Irlande du Nord) et Suède. Les données concernant les autres pays se rapportent à 2015 et sont issues de la deuxième vague du premier cycle de l'enquête.

\section{Rendements de la composante TIC sur le marché du travail, 2012 ou 2015}

L'indicateur relatif à la composante TIC des emplois s'appuie sur une analyse factorielle exploratoire de pointe et rend compte de l'utilisation de ces technologies en milieu professionnel. Il repose sur 11 questions de l'Évaluation des compétences des adultes (PIAAC) de l'OCDE, qui vont de la simple utilisation de l'internet, à celle des logiciels Word ou Excel, ou à l'emploi d'un langage de programmation. La méthodologie détaillée est exposée par Grundke et al. (2017).

Les rendements sur le marché du travail en fonction de l'importance de la composante TIC sont obtenus par des régressions des salaires selon la méthode des moindres carrés ordinaires (équations de Mincer) à partir de données de l'Enquête de l'OCDE sur les compétences des adultes (PIAAC). Les estimations s'appuient sur le logarithme des salaires horaires en tant que variable liée et sur diverses variables de contrôle ayant trait aux individus (dont l'âge, la durée de formation initiale, le sexe et les autres mesures de compétences, détaillées par Grundke et al., 2017) ainsi que sur des variables auxiliaires du secteur pertinent prises en tant que variables explicatives.

Les données pour les 23 pays ci-après, qui sont issues de la première vague du PIAAC, se rapportent à l'année 2012 : Allemagne, Australie, Autriche, Belgique (Flandre), Canada, Corée, Danemark, Espagne, Estonie, États-Unis, Fédération de Russie (sauf Moscou), Finlande, France, Irlande, Italie, Japon, Norvège, Pays-Bas, Pologne, République slovaque, République tchèque, Royaume-Uni (Angleterre et Irlande du Nord) et Suède. Les données concernant les autres pays se rapportent à 2015 et sont issues de la deuxième vague du premier cycle de l'enquête.

\section{Rendements de la composante gestion et communication des emplois : la prime TIC, 2012 ou 2015}

Les indicateurs relatifs à la place des tâches liées aux TIC et des tâches de gestion et communication dans l'exercice d'un emploi sont construits à partir d'une analyse factorielle exploratoire de pointe. Ces indicateurs rendent compte, respectivement, de l'utilisation des TIC et de l'exécution de tâches de gestion et de communication dans le cadre professionnel. L'indicateur concernant les TIC repose sur les réponses à 11 questions de l'Évaluation des compétences des adultes (PIAAC) de l'OCDE, qui vont de la simple utilisation de l'internet, à celle des logiciels Word ou Excel, ou à l'emploi d'un langage de programmation. L'indicateur concernant la gestion et la communication est fondé quant à lui sur cinq questions en rapport avec les activités de négociation ou de planification des tâches d'autres personnes, ou encore avec la fourniture de conseils ou d'instructions à des tiers. On trouvera une description détaillée de la méthodologie suivie chez Grundke et al. (2017).

Les rendements sur le marché du travail en fonction de l'intensité des tâches liées aux TIC sont obtenus par des régressions des salaires selon la méthode des moindres carrés ordinaires (équations de Mincer) à partir de données de l'Évaluation des compétences des adultes (PIAAC) de l'OCDE. Les estimations s'appuient sur le logarithme des salaires horaires en tant que variable liée et sur diverses variables de contrôle ayant trait aux individus (dont l'âge, la durée de formation initiale, le sexe et les autres mesures de compétences, détaillées par Grundke et al., 2017) ainsi que sur des variables auxiliaires du secteur pertinent prises en tant que variables explicatives.

Les données issues de la première vague du PIAAC pour les 23 pays ci-après se rapportent à l'année 2012 : Allemagne, Australie, Autriche, Belgique (Flandre), Canada, Corée, Danemark, Espagne, Estonie, États-Unis, Fédération de Russie (sauf Moscou), Finlande, France, Irlande, Italie, Japon, Norvège, Pays-Bas, Pologne, République slovaque, République tchèque, Royaume-Uni (Angleterre et 
Irlande du Nord) et Suède. Les données concernant les autres pays se rapportent à 2015 et sont issues de la deuxième vague du premier cycle de l'enquête.

\subsection{Capital intellectuel}

\section{Travailleurs suivant une formation, selon le type de formation, 2012 ou 2015}

Les pourcentages de travailleurs suivant une formation sont calculés comme le nombre total d'actifs occupés ayant reçu une formation au moins une fois par an, par type de formation (formelle, en cours d'emploi, mixte, comme dans Squicciarini et al., 2015), rapporté à l'emploi total dans l'économie. Ils sont repondérés pour être représentatifs de la population nationale.

Les données issues de la première vague du PIAAC pour les 23 pays ci-après se rapportent à l'année 2012 : Allemagne, Australie, Autriche, Belgique (Flandre), Canada, Corée, Danemark, Espagne, Estonie, États-Unis, Fédération de Russie (sauf Moscou), Finlande, France, Irlande, Italie, Japon, Norvège, Pays-Bas, Pologne, République slovaque, République tchèque, Royaume-Uni (Angleterre et Irlande du Nord) et Suède. Les données concernant les autres pays se rapportent à 2015 et sont issues de la deuxième vague d'enquête.

\section{Investissement des entreprises en capital fixe et en capital intellectuel, 2015}

Les données, en prix courants, concernent le secteur privé marchand suivant la définition d'INTAN-Invest (soit les divisions 01 à 82 de la CITI rév. 4, à l'exclusion des divisions 68 et 72).

Les intensités de capital sont définies comme l'investissement rapporté à la valeur ajoutée brute telle qu'elle figure dans la Base de données des Comptes nationaux des pays de l'OCDE. Les données sur la formation brute de capital fixe (FBCF) hors logement, qui se calcule comme la FBCF totale ôtée des investissements dans le logement et la propriété intellectuelle, proviennent de la même base de données. Il en va également ainsi pour les données sur les actifs intellectuels entrant dans la comptabilité nationale, qui correspondent à la FBCF associée à la propriété intellectuelle. Les investissements en R-D par secteur aux ÉtatsUnis sont connus par les données du Bureau d'analyse économique (Bureau of Economic Analysis). Les données relatives aux autres actifs intellectuels ont été fournies par INTAN-Invest et extrapolées, lorsque nécessaire, à l'aide du taux de croissance de la FBCF associée à la propriété intellectuelle tiré de la Base de données des comptes nationaux des pays de l'OCDE. La catégorie « Autres actifs intellectuel » comprend les dessins et modèles, les nouveaux produits financiers, les marques, la formation et le capital organisationnel.

\section{Investissement en capital intellectuel des secteurs marchand et non marchand, 2000 et 2015}

Le secteur marchand correspond à la définition donnée par INTAN-Invest (soit les divisions 01 à 82 de la CITI rév. 4, à l'exclusion des divisions 68 et 72). Le secteur non marchand se réfère à celle proposée par SPINTAN et couvre les établissements publics et les entités sans but lucratif appartenant aux divisions 72 et 84 à 93 de la CITI rév. 4.

Les intensités de capital sont définies comme l'investissement rapporté à la valeur ajoutée brute telle qu'elle figure dans la Base de données des Comptes nationaux des pays de l'OCDE. Pour le secteur non marchand, les données sur les investissements dans le capital intellectuel ont été fournies par SPINTAN et extrapolées, lorsque nécessaire, à l'aide du taux de croissance moyen de ces investissements dans l'ensemble des pays, tel qu'il est donné par la même source. Pour le secteur marchand, les investissements dans les actifs entrant dans la comptabilité nationale correspondent à la FBCF associée à la propriété intellectuelle d'après la Base de données des Comptes nationaux des pays de l'OCDE. Les données relatives aux investissements dans les autres actifs intellectuels, hors champ des comptes nationaux, ont été fournies par INTAN-Invest et extrapolées, lorsque nécessaire, à l'aide du taux de croissance de la FBCF associée à la propriété intellectuelle tiré de la Base de données des Comptes nationaux des pays de l'OCDE. Toutes les données sont en prix courants.

\section{Références}

Grundke, R. et al. (2017), "Skills and global value chains: A characterisation ", OECD Science, Technology and Industry Working Papers, $n^{\circ}$ 2017/05, Éditions OCDE, Paris, http://dx.doi.org/10.1787/cdb5de9b-en.

Grundke, R. et al. (à paraître), « Which skills for the digital era? A returns to skills analysis ».

OCDE (2009), Regards sur l'éducation 2009 : Les indicateurs de l'OCDE, Éditions OCDE, Paris, http://dx.doi.org/10.1787/eag-2009-fr.

OCDE (2015), Manuel de Frascati 2015 : Lignes directrices pour le recueil et la communication des données sur la recherche et le développement expérimental, Éditions OCDE, Paris, http://dx.doi.org/10.1787/9789264257252-fr.

OCDE (2017), Regards sur l'éducation 2017 : Les indicateurs de l'OCDE, Éditions OCDE, Paris, http://dx.doi.org/10.1787/eag-2017-fr.

Squicciarini, M., L. Marcolin et P. Horvát (2015), “ Estimating Cross-Country Investment in Training: An Experimental Methodology Using PIAAC Data ", OECD Science, Technology and Industry Working Papers, $n^{\circ}$ 2015/09, Éditions OCDE, Paris, http://dx.doi.org/10.1787/5jrs3sftp8nw-en. 


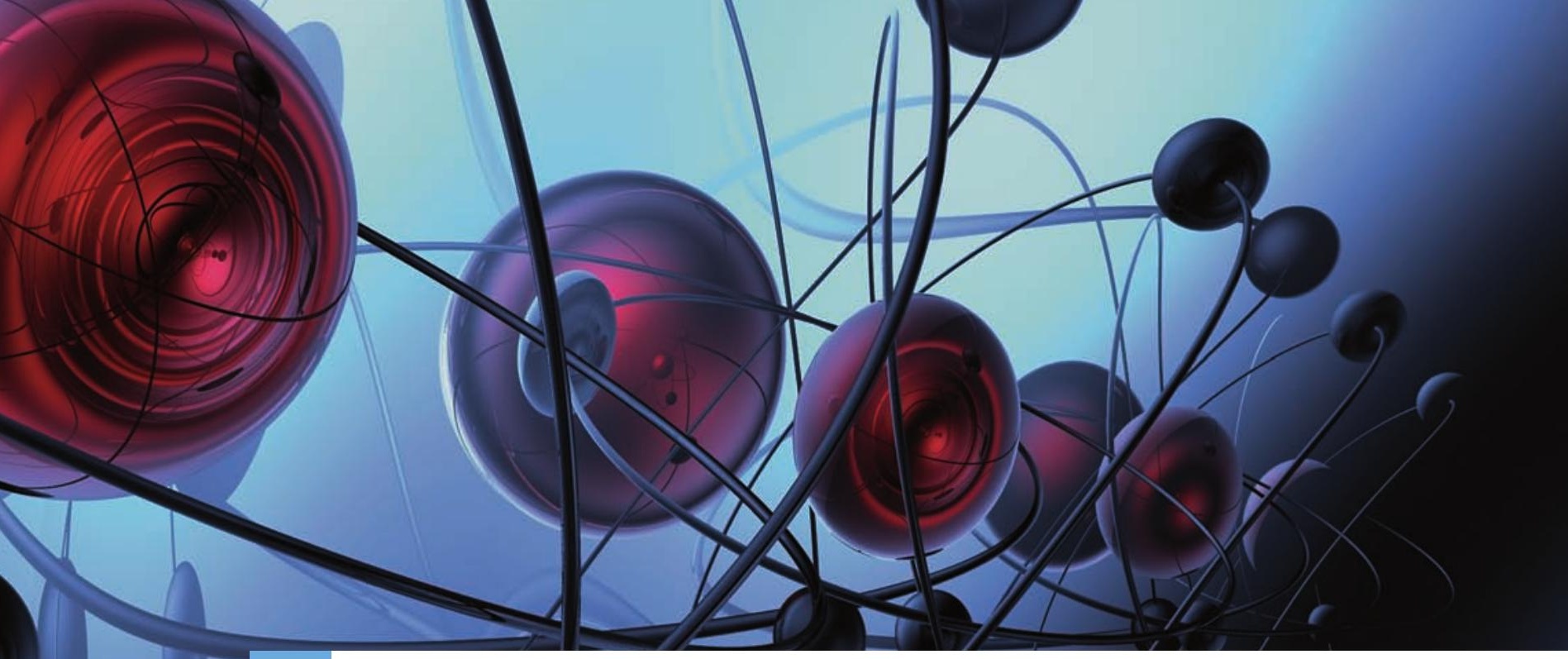

3. EXCELLENCE DE LA RECHERCHE ET COLLABORATION

1. Excellence de la recherche et spécialisation

2. Excellence de la collaboration scientifique

3. Mobilité internationale des personnes hautement qualifiées

4. Mobilité des scientifiques

5. Mondialisation de la R-D

6. Inventions internationales

7. Collaboration en matière d'innovation

Notes et références 


\section{EXCELLENCE DE LA RECHERCHE ET COLLABORATION}

\section{Fxcellence de la recherche et spécialisation}

\section{Quantité et qualité de la production scientifique, 2005 et 2015}

Nombre de publications et pourcentage parmi les $10 \%$ les plus citées au monde, comptages fractionnaires

Pourcentage de publications parmi les plus citées, 2015

$\diamond$ Pourcentage de publications parmi les plus citées, 2005

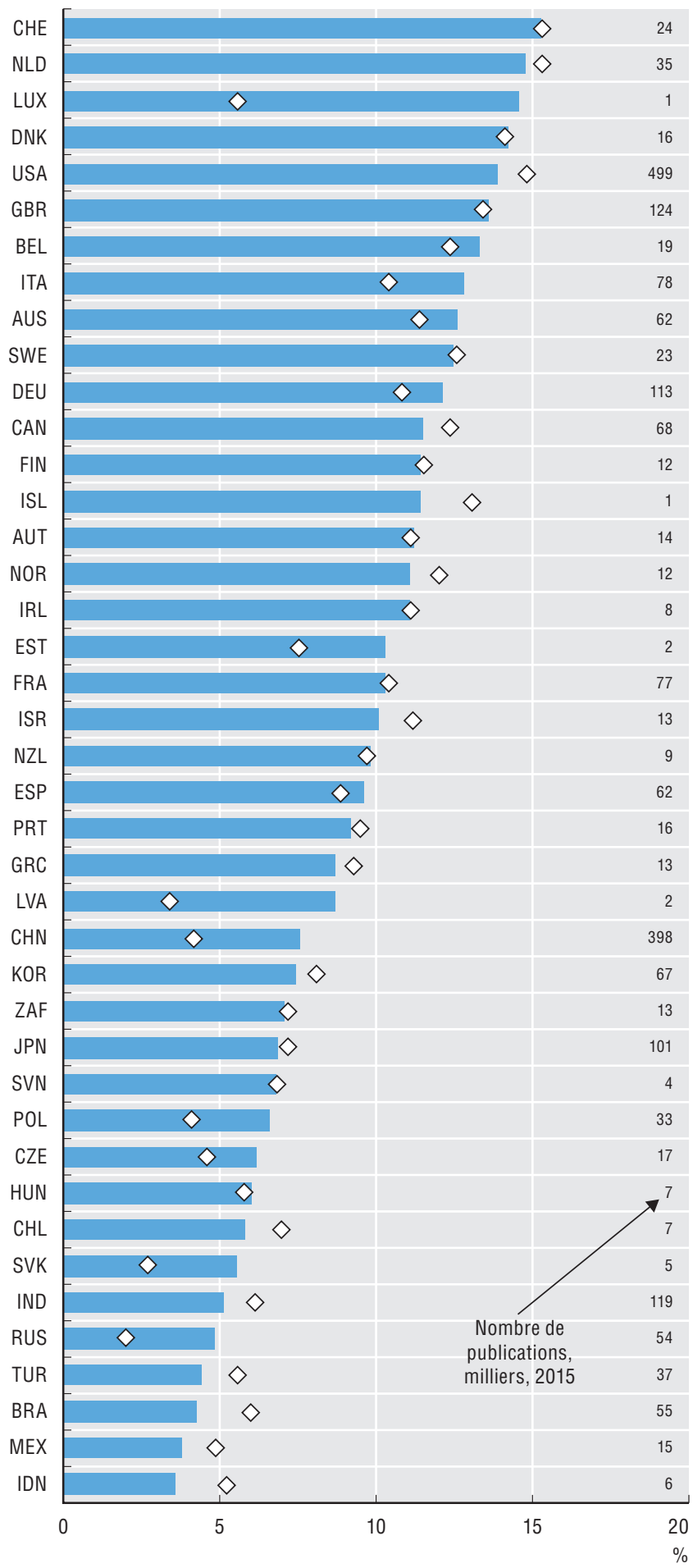

Source : Calculs de l'OCDE, d'après Scopus Custom Data, Elsevier, version 4.2017 et valeurs SJR (Scimago Journal Rank) de 2015 des titres de la liste de revues Scopus (consulté en juin 2017), juillet 2017. Davantage de données via StatLink. Voir notes de chapitre.

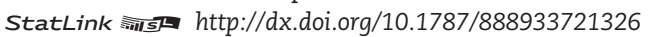

\section{Le saviez-vous ?}

La production scientifique est $20 \%$ à $30 \%$ plus élevée en Chine qu'aux États-Unis dans des disciplines comme l'informatique ou la science des matériaux, mais $70 \%$ plus faible en neurosciences.

L'indicateur reflétant les publications les plus citées offre une mesure de la production de la recherche scientifique « corrigée des variations de qualité ». En 2015, les États-Unis ont produit le plus de publications scientifiques, avec près d'un demimillion de documents. La Chine arrive au deuxième rang en nombre de publications les plus citées, avec presque autant de publications citées que l'Allemagne et le Royaume-Uni combinés. Pour ce qui est du pourcentage de publications scientifiques à fort impact de citation, par des auteurs affiliés à un établissement du pays, c'est en Suisse qu'il est le plus élevé puis, juste après, aux Pays-Bas.

Les pays se spécialisent dans des disciplines scientifiques différentes. Un indice de spécialisation met en évidence les domaines dans lesquels un pays donné contribue à une part relativement élevée de la production scientifique par rapport à la distribution mondiale de la production scientifique dans les différentes disciplines. La relation entre spécialisation et impact de citation est analysée dans quatre domaines : la biochimie, l'informatique, la science des matériaux et les neurosciences. Si une spécialisation accrue s'accompagne d'un plus fort impact de citation en biochimie, ce n'est pas le cas dans les autres disciplines. La plupart des pays ont un degré de spécialisation équivalent en biochimie, mais pas en neurosciences où l'on observe davantage d'hétérogénéité. L'Inde est un pays à la fois spécialisé et gros producteur de publications scientifiques dans le domaine de l'informatique. La Fédération de Russie est très spécialisée en science des matériaux. Les pays qui ont une production scientifique importante affichent des valeurs d'impact normalisé similaires d'une discipline à l'autre. Les plus petits pays s'illustrent dans des niches d'excellence dans certaines disciplines.

\section{Définitions}

La production scientifique est estimée sur la base du comptage des documents citables (articles, recensions et actes de conférences) indexés dans la base de données Scopus d'Elsevier, publiés par des auteurs affiliés aux établissements de chaque pays. Les documents sont comptabilisés de façon fractionnaire, en fonction du nombre d'auteurs et des affiliations respectives de chacun dans un pays donné.

L'indicateur de spécialisation est obtenu en divisant la part des documents d'une discipline dans la production scientifique d'un pays par la part des documents de cette discipline dans la production scientifique mondiale. Les économies dans lesquelles la distribution des disciplines est presque la même qu'au niveau mondial affichent des valeurs de spécialisation très proches de 1.

L'indicateur d'excellence scientifique (publications les plus citées) reflète le pourcentage de la production scientifique d'un pays qui figure dans le groupe des $10 \%$ de publications les plus citées dans les disciplines scientifiques correspondantes. Les valeurs SJR (Scimago Journal Rank) de 2015 sont utilisées en complément pour hiérarchiser les publications ayant obtenu le même nombre de citations au sein d'une classe. 
Spécialisation et impact de citation dans quelques disciplines scientifiques, 2015

En pourcentage de publications parmi les 10 \% les plus citées au monde, spécialisation relative, par discipline scientifique, comptages fractionnaires
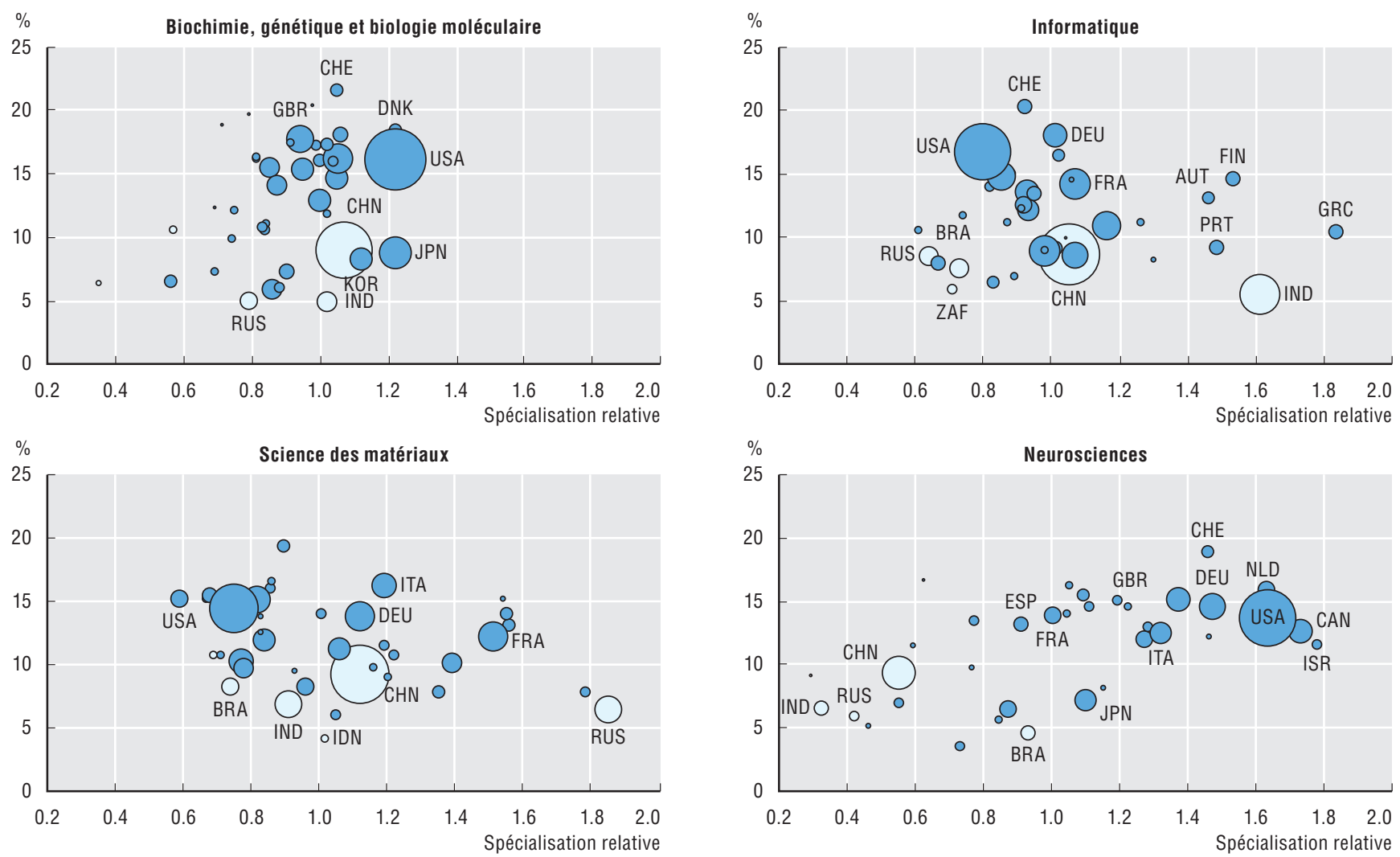

Source : Calculs de l'OCDE, d'après Scopus Custom Data, Elsevier, version 4.2017 et valeurs SJR (Scimago Journal Rank) de 2015 des titres de la liste de revues Scopus (consulté en juin 2017), juillet 2017. Davantage de données via StatLink. Voir notes de chapitre.

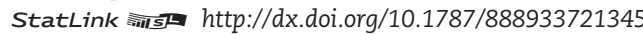

\section{Mesurabilité}

Les publications scientifiques fournissent une mesure de l'activité de production scientifique basée sur le nombre de documents publiés dans des revues à comité de lecture et indexés par les fournisseurs de données. Les normes de publication varient selon le domaine et le secteur (OCDE et CSIC, 2016), en fonction des mécanismes d'examen par les pairs ou de diffusion éventuellement appliqués ainsi que des différentes pratiques de divulgation propres aux établissements. L'indexation peut également présenter des biais d'ordre linguistique. On approxime l'excellence scientifique en mesurant la distribution de l'impact de citation normalisé par année de publication, type de document et champ(s) disciplinaire(s). Des chiffres élevés peuvent intégrer des autocitations, renvoyer à des articles ayant fait l'objet d'une rétractation, et ne pas rendre compte de la pertinence pour les publics qui ne publient pas d'articles.

Dans sa base de données Scopus, Elsevier utilise sa classification ASJC (All Science Journal Classification) pour répertorier chaque revue sous un ou plusieurs sujet(s). L'affectation à une discipline en fonction de celle de la revue est approximative, le classement d'une revue n'étant pas nécessairement le reflet exact du contenu thématique de chaque document qu'elle contient. Pour limiter autant que possible ce problème, les documents publiés dans des revues multidisciplinaires génériques ont été affectés sur une base fractionnaire aux codes ASJC trouvés aussi bien dans les articles qui citent que dans les articles cités. 


\section{EXCELLENCE DE LA RECHERCHE ET COLLABORATION}

\section{Fxcellence de la collaboration scientifique}

Collaboration scientifique internationale, 2015

En pourcentage des publications dont l'un des auteurs est affilié à un établissement du pays, comptages fractionnaires

Collaboration internationale conduite par un auteur affilié à un établissement du pays

Collaboration internationale conduite par un auteur affilié à un établissement étranger

$\diamond$ Pourcentage de collaboration internationale, 2005

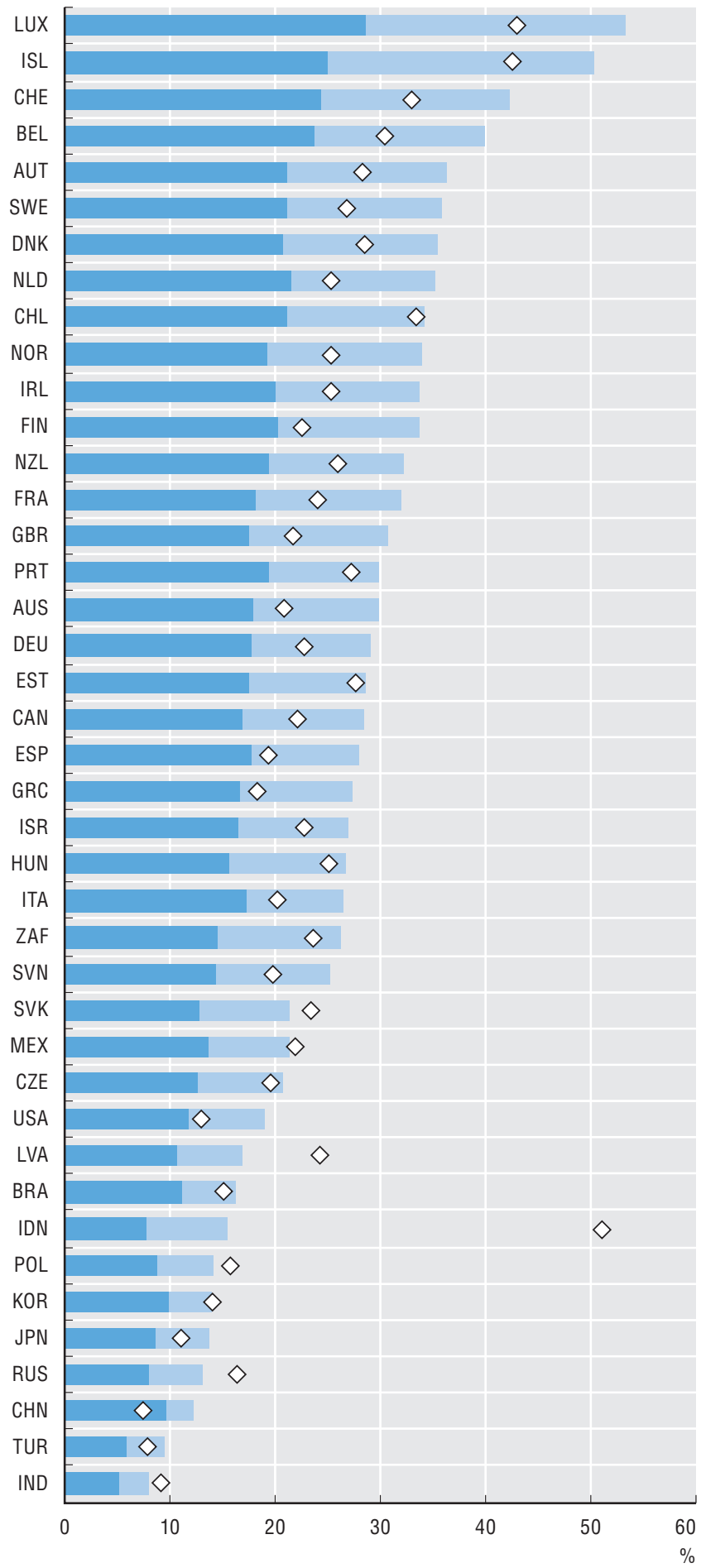

Source : Calculs de l'OCDE, d'après Scopus Custom Data, Elsevier, version 4.2017, juillet 2017. Davantage de données via StatLink. Voir notes de chapitre.

StatLink त्ताज http://dx.doi.org/10.1787/888933721364

\section{Le saviez-vous ?}

$\mathrm{Au}$ cours des dix dernières années, la collaboration scientifique internationale fondée sur la rédaction conjointe d'une publication par des auteurs de pays différents a augmenté dans 32 des 35 pays de l'OCDE.

Au cours de la période 2005-15, la collaboration internationale en matière de recherche scientifique s'est intensifiée dans toutes les régions du monde. Le pourcentage de collaboration de la Chine, certes très bas au départ, a presque doublé. En 2015, le Luxembourg, l'Islande, la Suisse et la Belgique affichaient les taux de collaboration internationale les plus élevés.

Les mesures de la collaboration en matière de recherche scientifique et de l'impact de citation (qui indique la qualité de la production scientifique) au niveau national sont positivement corrélées, en particulier pour les économies dont les niveaux de production scientifique sont plus faibles. Ces économies plus petites tentent de compenser les limitations liées à leur échelle en participant plus activement aux réseaux mondiaux. Une analyse conjointe de l'excellence et de l'affiliation de l'auteur principal apporte un éclairage supplémentaire sur l'origine des publications les plus citées dans un pays donné, puisque beaucoup d'entre elles sont le fruit de collaborations internationales, souvent conduites par des auteurs affiliés à des établissements étrangers. Les États-Unis comptent la plus grande part des publications les plus citées dont l'auteur principal est affilié à un établissement du pays ; suivent les Pays-Bas, la Suisse, le Danemark et le Royaume-Uni. Certains pays ont des taux d'excellence globaux élevés grâce à la contribution des articles collaboratifs dont les auteurs principaux sont affiliés à des établissements étrangers.

\section{Définitions}

On définit la collaboration internationale comme le nombre de publications dont un auteur au moins est affilié à l'économie de référence et dont un auteur au moins est affilié à un établissement d'un autre pays ou d'une autre économie, exprimé en pourcentage du nombre total de publications citables (articles, recensions et actes de conférences) dont un auteur au moins est affilié à l'économie de référence.

Il peut y avoir collaboration internationale quand l'auteur principal est affilié en premier lieu à un établissement dans l'économie de référence, mais aussi quand l'auteur principal est affilié en premier lieu à un établissement étranger. L'auteur principal est déterminé sur la base de l'identité de l'auteur correspondant désigné.

L'impact de citation normalisé est le rapport entre le nombre moyen de citations que reçoivent les documents publiés par des auteurs affiliés à un établissement dans l'économie de référence et le nombre moyen de citations à l'échelle mondiale, au cours de la même période, par type de document et discipline.

L'excellence scientifique est la proportion (en pourcentage) de la production scientifique d'une unité qui figure parmi le groupe mondial des $10 \%$ de publications les plus citées dans les disciplines scientifiques correspondantes. Cet indicateur peut être combiné aux informations sur l'affiliation de l'auteur correspondant - voir l'encadré Mesurabilité - afin de donner un aperçu plus fidèle du rôle de la collaboration internationale en tant que levier d'excellence scientifique. 
Impact de citation de la production scientifique et degré de collaboration internationale, 2012-16 Indice et pourcentage de tous les documents citables, comptages fractionnaires

Impact de citation normalisé
1.8

Source : Calculs de l'OCDE, d'après Scopus Custom Data, Elsevier, version 4.2017, juillet 2017. Davantage de données via StatLink. Voir notes de chapitre.

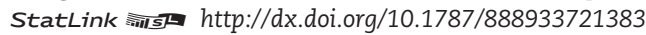

Production parmi les $10 \%$ de publications les plus citées et profils de collaboration internationale, 2015

Publications les plus citées dont l'auteur principal est affilié à un établissement du pays ou étranger, en pourcentage du total des publications, comptages fractionnaires

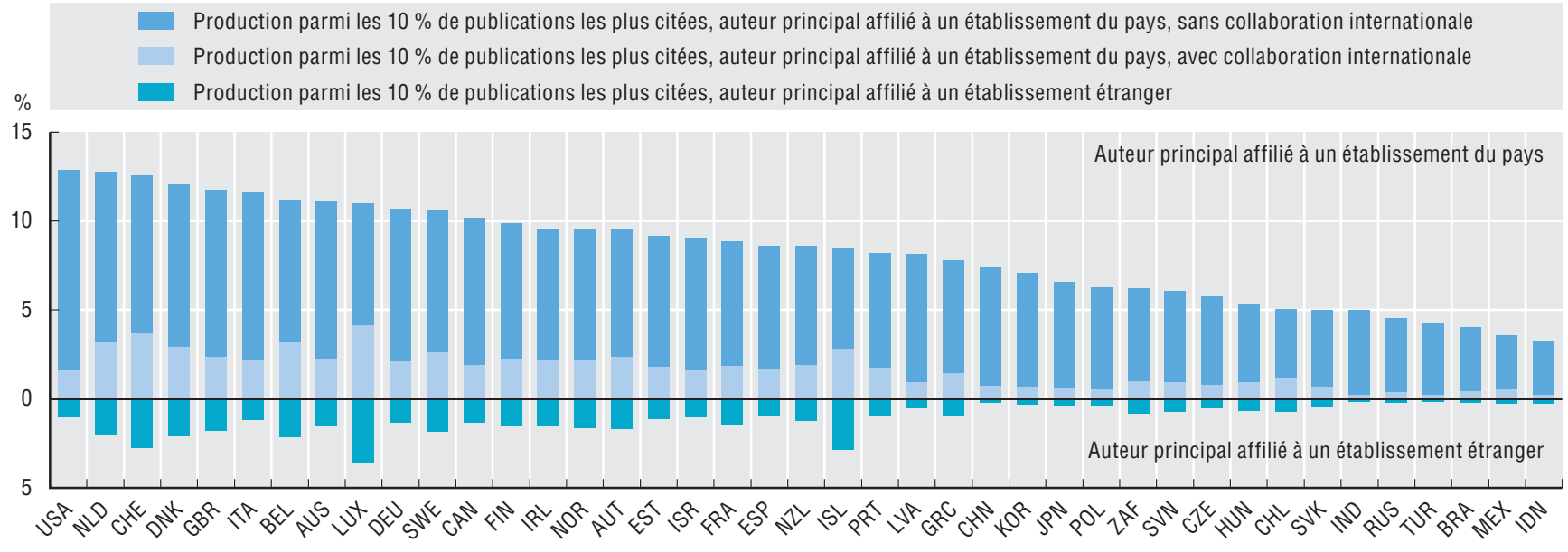

Source: Calculs de l'OCDE, d'après Scopus Custom Data, Elsevier, version 4.2017 et valeurs SJR (Scimago Journal Rank) de 2015 des titres de la liste de revues Scopus (consulté en juin 2017), juillet 2017. Davantage de données via StatLink. Voir notes de chapitre

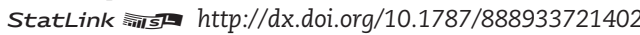

\section{Mesurabilité}

Les publications sont attribuées aux pays en fonction des établissements d'affiliation des auteurs. Les méthodes de comptage simple ou fractionnaire présentent des avantages et des limitations, le choix de la méthode ayant des effets potentiellement importants sur les chiffres mentionnés, en particulier les taux de collaboration. La méthode utilisée dans cette édition est celle du comptage fractionnaire, pour éviter les distorsions causées par le nombre croissant de documents associés à de très longues listes d'affiliation, qui peuvent conduire à surestimer les résultats.

En complément, on peut déterminer l'établissement d'affiliation de l'auteur principal (Moya-Anegón et al., 2013). L'attribution en fonction de l'auteur correspondant peut donner des informations sur le rôle d'un établissement ou d'un pays donné dans les activités de collaboration. De fait, elle montre la part de la production scientifique (en l'occurrence, les publications les plus citées) pour laquelle un auteur du pays en question est cité en tant qu'auteur correspondant. On peut alors analyser dans quelle proportion les documents attribués à un pays donné ont un auteur principal affilié à un établissement du pays, qui travaille ou non en collaboration avec le reste du monde, ou un auteur principal affilié à un établissement étranger. 


\section{EXCELLENCE DE LA RECHERCHE ET COLLABORATION}

\section{Mobilité internationale des personnes hautement qualifiées}

\section{Mobilité internationale des étudiants inscrits dans l'enseignement supérieur, 2015}

Totaux et répartition par domaine d'étude

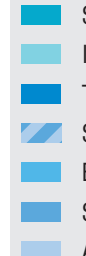

Sciences naturelles, mathématiques et statistiques Ingénierie, industries de transformation et construction

Technologies de l'information et de la communication

Santé et protection sociale

Éducation, arts et lettres

Sciences sociales, journalisme, commerce et droit

Agriculture, sylviculture, halieutique, sciences vétérinaires et services

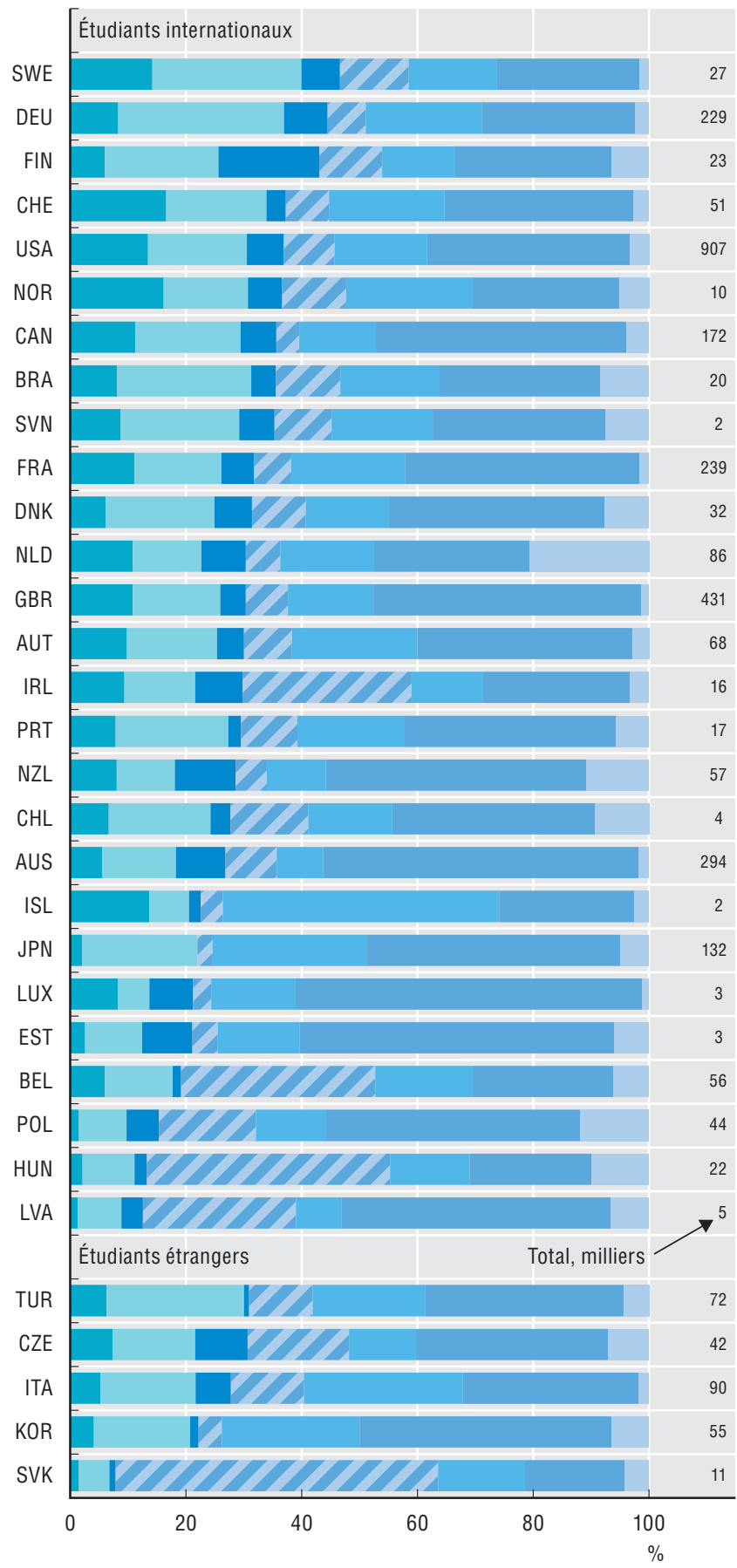

Source : OCDE, d'après OCDE (2017), Regards sur l'éducation 2017 : Les indicateurs de l'OCDE, Éditions OCDE, Paris. Voir notes de chapitre.

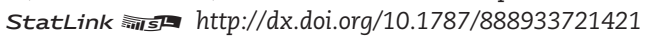

\section{Le saviez-vous?}

Aux États-Unis, les filières des sciences naturelles, de l'ingénierie et des technologies de l'information et des communications (TIC) totalisent moins de $25 \%$ des étudiants nationaux, mais près de $70 \%$ des doctorants mobiles à l'international.

La mobilité internationale des personnes ayant un niveau d'instruction élevé, à différents stades de leur développement personnel et de leur carrière professionnelle, constitue un moteur essentiel de circulation du savoir dans le monde. Les États-Unis attirent le plus grand nombre d'étudiants internationaux de l'enseignement supérieur (près d'un million). Ils sont suivis par le Royaume-Uni (près de 400 000). La répartition des étudiants internationaux entre les différentes filières est un indicateur des atouts de chaque pays perçus comme tels. Par exemple, le pourcentage d'étudiants internationaux est relativement élevé en Finlande dans le domaine des TIC, au Royaume-Uni en sciences sociales et en lettres, en Suisse dans le domaine des sciences naturelles et des mathématiques et en Allemagne dans celui de l'ingénierie.

Les doctorants internationaux de la zone OCDE sont généralement plus attirés par les sciences naturelles, l'ingénierie et les TIC que leurs homologues nationaux. Cette tendance est particulièrement marquée au Canada, aux ÉtatsUnis et en Suisse.

Le degré de mobilité internationale une fois les études supérieures achevées est également notable dans un certain nombre de cas. Dans plusieurs pays de l'OCDE, à l'instar du Canada et d'Israël, la part de la population en âge de travailler diplômée de l'enseignement supérieur est plus élevée parmi les personnes nées à l'étranger que parmi les natifs. Ces pays tendent à définir leurs politiques d'immigration de façon à attirer les personnes hautement qualifiées. À l'inverse, en Allemagne, aux États-Unis, en France, en Italie et au Japon, la population native affiche des taux de diplômés de l'enseignement supérieur plus élevés ou comparables.

\section{Définitions}

Les étudiants internationaux sont ceux qui partent à l'étranger dans l'intention expresse d'étudier. L'Institut de statistique de l'UNESCO (ISU), l'OCDE et Eurostat les définissent comme des étudiants qui ne sont pas résidents permanents du pays dans lequel ils étudient ou ceux dont la formation antérieure s'est déroulée dans un autre pays.

Les sciences naturelles, l'ingénierie et les TIC correspondent à des domaines spécifiques de la Classification internationale type de l'éducation (CITE-2013) : 05 sciences naturelles, mathématiques et statistiques ; 06 technologies de l'information et des communications ; 07 ingénierie, industries de transformation et construction.

L'enseignement supérieur correspond aux niveaux 5 à 8 de la CITE-2011. Les personnes dotées d'un niveau d'instruction élevé dans les populations immigrée et native sont diplômées de l'enseignement supérieur. Les titulaires de doctorat sont des personnes ayant obtenu une qualification avancée pour la recherche de niveau 8 de la CITE-2011. 
Doctorants internationaux et locaux dans les domaines des sciences naturelles, de l'ingénierie et des TIC, 2015 Part des doctorants en sciences naturelles, ingénierie et TIC dans le total des doctorants, selon que ces derniers étudient ou non à l'étranger

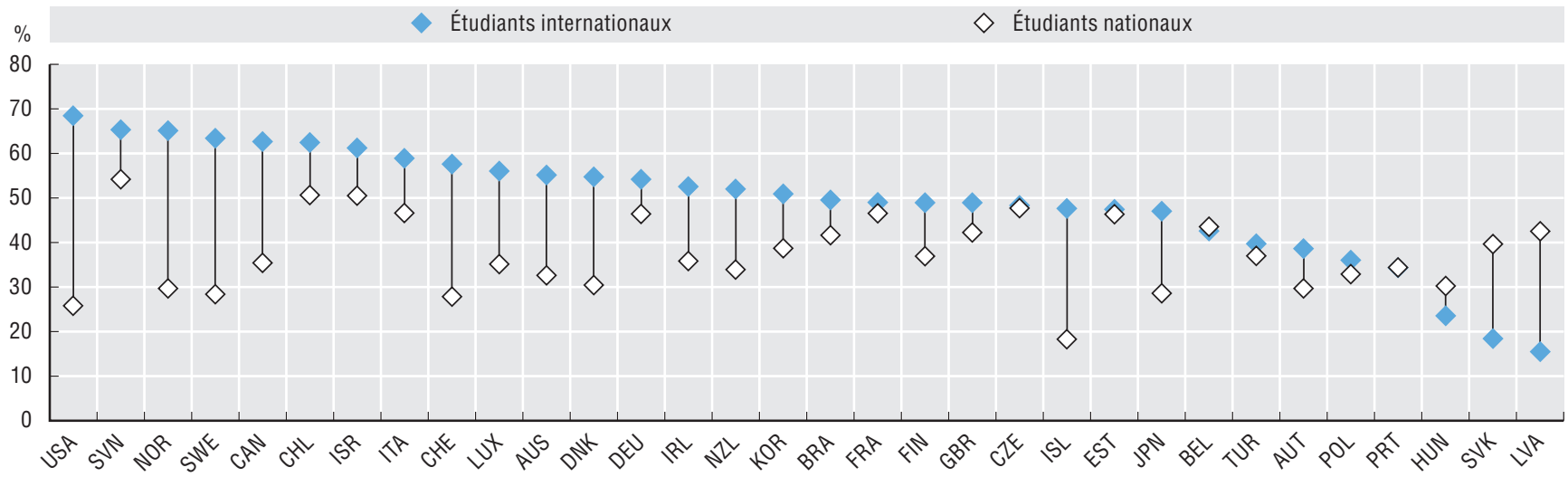

Source: Calculs de l'OCDE, d'après OCDE, Statistiques et bases de données sur l'éducation, septembre 2017. Voir notes de chapitre.

StatLink 需田 http://dx.doi.org/10.1787/888933721440

Personnes dotées d'un niveau d'instruction élevé dans la population en âge de travailler, par pays de naissance, 2015 En pourcentage du groupe correspondant, population âgée de 15 à 64 ans

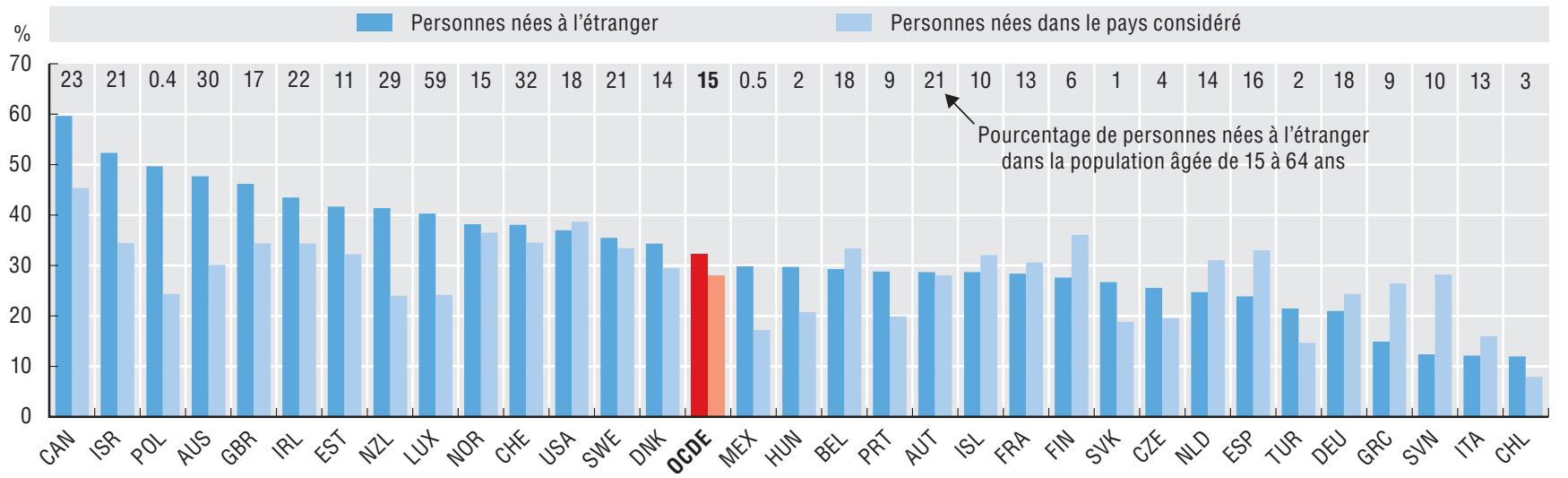

Source: Calculs de l'OCDE, d'après Eurostat, Enquête sur les forces de travail, et sources nationales, juillet 2017. Voir notes de chapitre.

\section{Mesurabilité}

La collecte de statistiques sur l'éducation par l'UNESCO-OCDE-Eurostat (UOE) constitue la principale source de données sur les inscriptions et les diplômes dans le supérieur, par pays d'origine et de destination. La notion d'étudiants internationaux est plus pertinente pour l'analyse de la mobilité des étudiants. En l'absence de données sur les étudiants internationaux, on utilise celles relatives aux étudiants étrangers afin d'obtenir un panorama plus complet.

La base de données sur les immigrés dans les pays de l'OCDE fournit les informations les plus complètes et comparables sur les immigrés qui vivent dans les pays de l'OCDE. Les données proviennent essentiellement des recensements démographiques et des registres de la population avec, en complément, des enquêtes sur la population active, moins précises pour les petites populations. Comme la plupart des recensements suivent un cycle décennal, on ne disposait pas de données suffisamment récentes pour offrir un panorama détaillé de la situation concernant les titulaires de doctorat. 


\section{EXCELLENCE DE LA RECHERCHE ET COLLABORATION}

\section{Mobilité des scientifiques}

\section{Flux bilatéraux internationaux d'auteurs scientifiques, 2006-16}

Grands flux bilatéraux, par première et dernière affiliation principale répertoriée
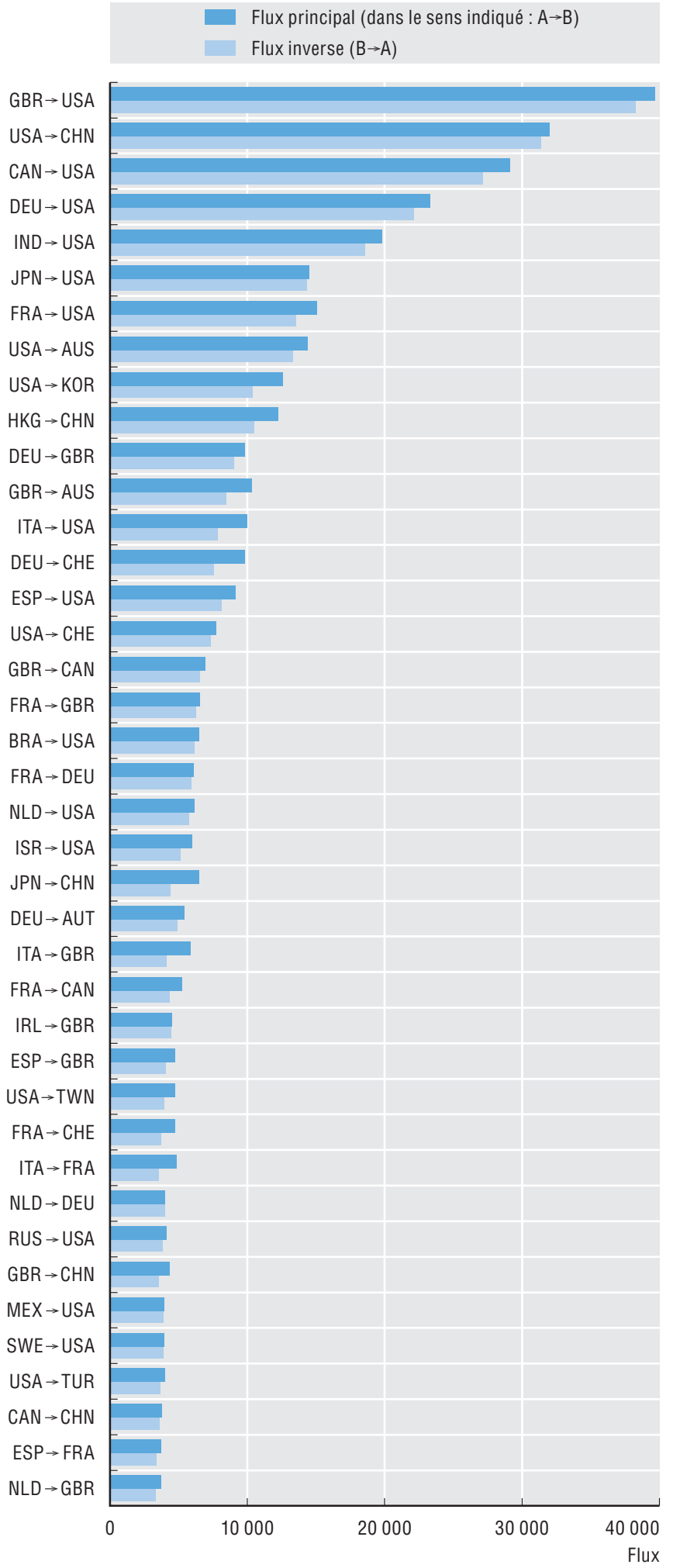

Source : Calculs de l'OCDE, d'après Scopus Custom Data, Elsevier, version 4.2017, juillet 2017. Davantage de données via StatLink. Voir notes de chapitre.

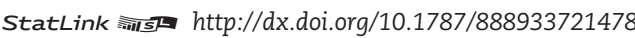

\section{Le saviez-vous?}

Les scientifiques qui entreprennent des recherches à l'étranger puis retournent dans l'économie dans laquelle ils ont publié pour la première fois contribuent à élever la qualité globale de la recherche de cette économie de $20 \%$ en moyenne.

La mobilité des scientifiques favorise la circulation des connaissances. L'une des solutions pour l'étudier consiste à observer les changements d'établissements d'affiliation mentionnés dans les publications parues dans les revues spécialisées. Cette approche montre que la circulation des cerveaux (renouvellement) est beaucoup plus importante que les gains ou les pertes (flux nets). Au cours de la période 200616 , les neuf plus grands flux bilatéraux internationaux de scientifiques ont fait intervenir les États-Unis. Sur les 40 premiers flux bilatéraux internationaux, 14 ont représenté un bénéfice net pour les États-Unis, 6 pour le Royaume-Uni et 5 pour la Chine.

En 2016, les auteurs établis au Luxembourg et en Suisse affichaient les taux de mobilité les plus élevés au sein de la zone OCDE. Dans l'économie médiane, $95 \%$ des scientifiques se trouvaient déjà là au moment de leur précédente publication. Les schémas de mobilité varient selon les économies ; par exemple, en Israël et en Italie, la majorité des entrants sont en réalité des réentrants, c'est-à-dire des chercheurs de retour dans leur pays de départ. Au contraire, en Suisse, la plupart des chercheurs affichant une mobilité internationale sont de nouveaux arrivants.

À quelques exceptions près, les scientifiques qui ne changent pas d'économie d'affiliation (résidents) sont davantage susceptibles de publier dans des revues de moindre notoriété. Les sortants tendent à être associés à des publications mieux cotées que leurs homologues résidents ou réentrants. C'est cependant la tendance inverse que l'on observe aux ÉtatsUnis, où les scores obtenus par les chercheurs entrants restent supérieurs à ceux des chercheurs qui sont restés dans le pays, ce qui montre une capacité durable du pays à attirer des scientifiques de haut niveau.

\section{Définitions}

Les auteurs scientifiques sont référencés dans la base de données Scopus des revues à comité de lecture, et associés à un identifiant d'auteur unique, attribué par Elsevier. La mobilité internationale est déterminée pour les auteurs ayant publié au moins deux articles au cours de la période de référence, sur la base des changements d'affiliation et de l'ordre de publication. Les résidents sont les scientifiques dont l'économie d'affiliation reste la même au cours de la période de référence. Les réentrants sont les auteurs qui sont de retour dans l'économie de leur première affiliation ; c'est ce qui les distingue des nouveaux arrivants. Enfin, les sortants sont évalués à partir de leur affiliation au début de la période de référence.

L'indicateur SJR (Scimago Journal Rank) mesure l'influence scientifique des revues spécialisées en tenant compte à la fois du nombre de citations dont elles ont bénéficié et de l'importance, ou de la notoriété, des revues dans lesquelles ces citations paraissent (González-Pereira et al., 2010). 
Mobilité internationale des auteurs scientifiques, 2016

En pourcentage des auteurs, par dernière affiliation principale répertoriée en 2016

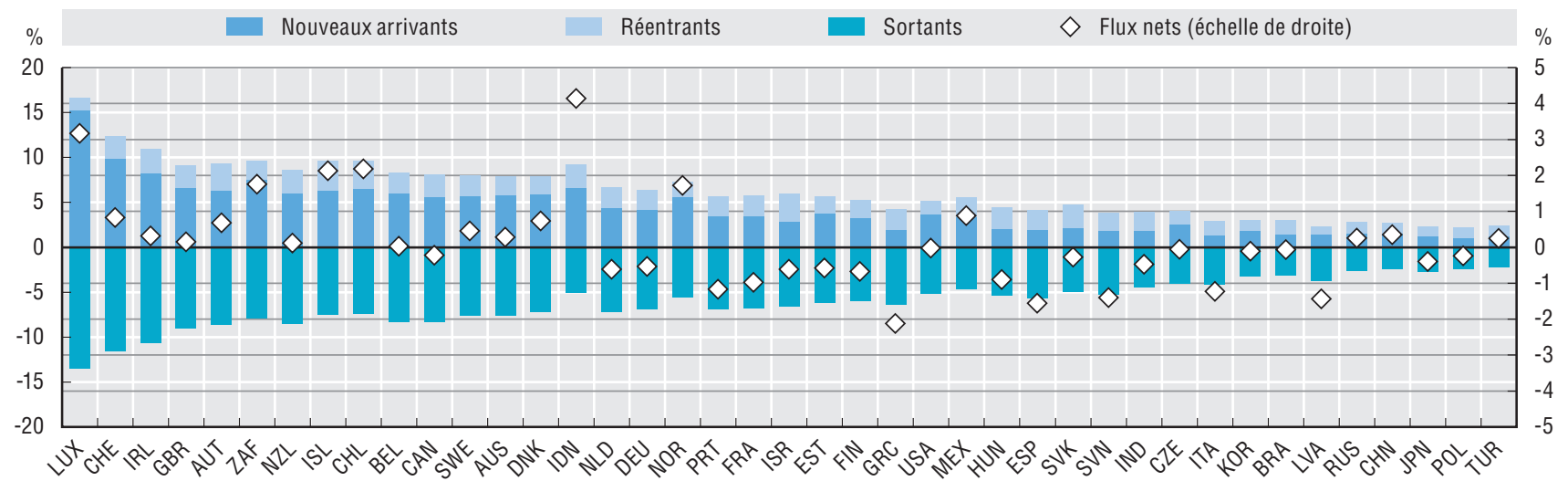

Source : Calculs de l'OCDE, d'après Scopus Custom Data, Elsevier, version 4.2017, juillet 2017. Davantage de données via StatLink. Voir notes de chapitre.

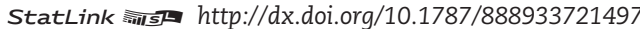

Impact attendu des citations des auteurs scientifiques, par profil de mobilité, 2016

Valeurs SJR (Scimago Journal Rank) moyennes pour 2015

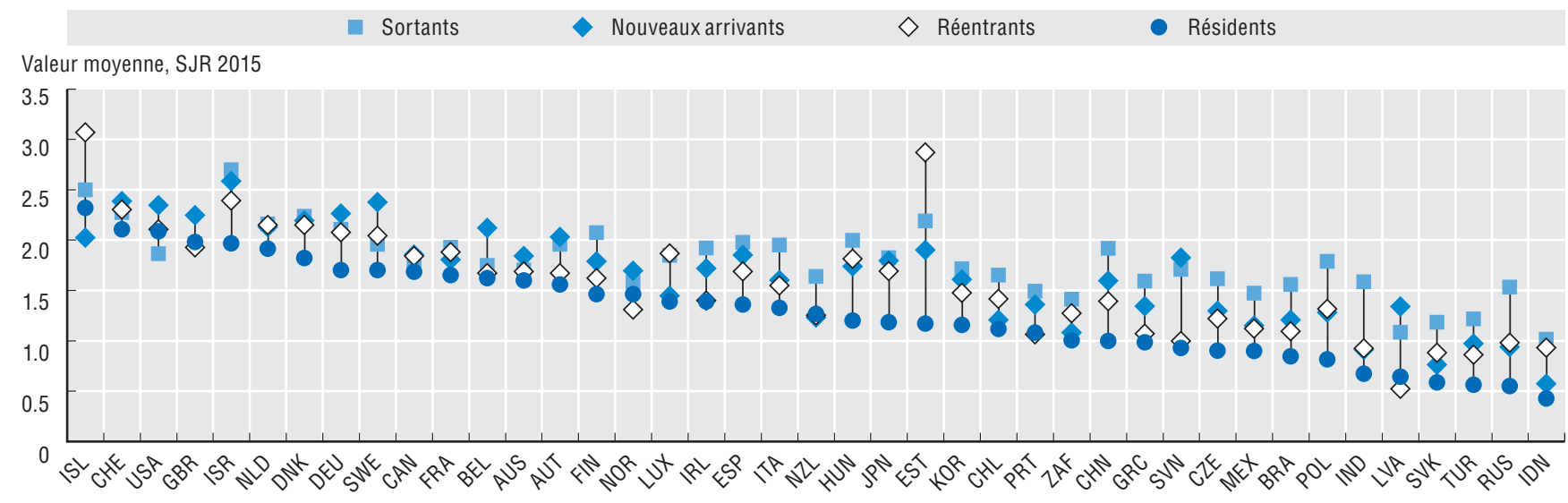

Source : Calculs de l'OCDE, d'après Scopus Custom Data, Elsevier, version 4.2017 et valeurs SJR (Scimago Journal Rank) de 2015 des titres de la liste de revues Scopus (consulté en juin 2017), juillet 2017. Davantage de données via StatLink. Voir notes de chapitre.

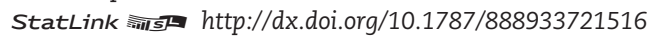

\section{Mesurabilité}

Élaborés à l'origine par Elsevier (2011), les indicateurs bibliométriques donnent des informations complémentaires sur la mobilité internationale des chercheurs ; ils sont toutefois expérimentaux et doivent, à ce titre, être interprétés avec prudence (Moed et al., 2013). Les données sur la mobilité sont moins précises voire manquantes pour les auteurs qui sont moins prolifiques, ou qui intègrent ou quittent des fonctions non universitaires. Il arrive que les affiliations à des établissements soient enregistrées après un certain délai et ne reflètent pas le lieu où la recherche a été menée. Dans cette édition, les auteurs qui ont plusieurs affiliations se voient associer une "économie principale " par document (celle-ci étant sélectionnée aléatoirement si toutes les économies ont le même poids). Plus important encore, une attribution non rigoureuse des identifiants aux auteurs peut fausser les estimations de la mobilité, qui seront sous-évaluées si plusieurs identifiants sont affectés à une même personne, ou surévaluées si les personnes possèdent des noms courants. L'initiative ORCID (Open Researcher and Contributor ID) promeut l'utilisation d'identifiants uniques pouvant être associés aux résultats de recherche des chercheurs. L'OCDE a réalisé et publié une analyse du réseau de mobilité internationale des scientifiques et de ses principaux moteurs (Appelt et al., 2015). 


\section{EXCELLENCE DE LA RECHERCHE ET COLLABORATION}

\section{Mondialisation de la R-D}

\section{R-D des entreprises financée par des fonds étrangers, par source de financement, 2015}

En pourcentage des dépenses intérieures de R-D des entreprises
Entreprises étrangères
Organisations internationales
Autres fonds du reste du monde
2. Ventilation non disponible
$\diamond$ Part des DIRDE financée par le reste du monde, 2005

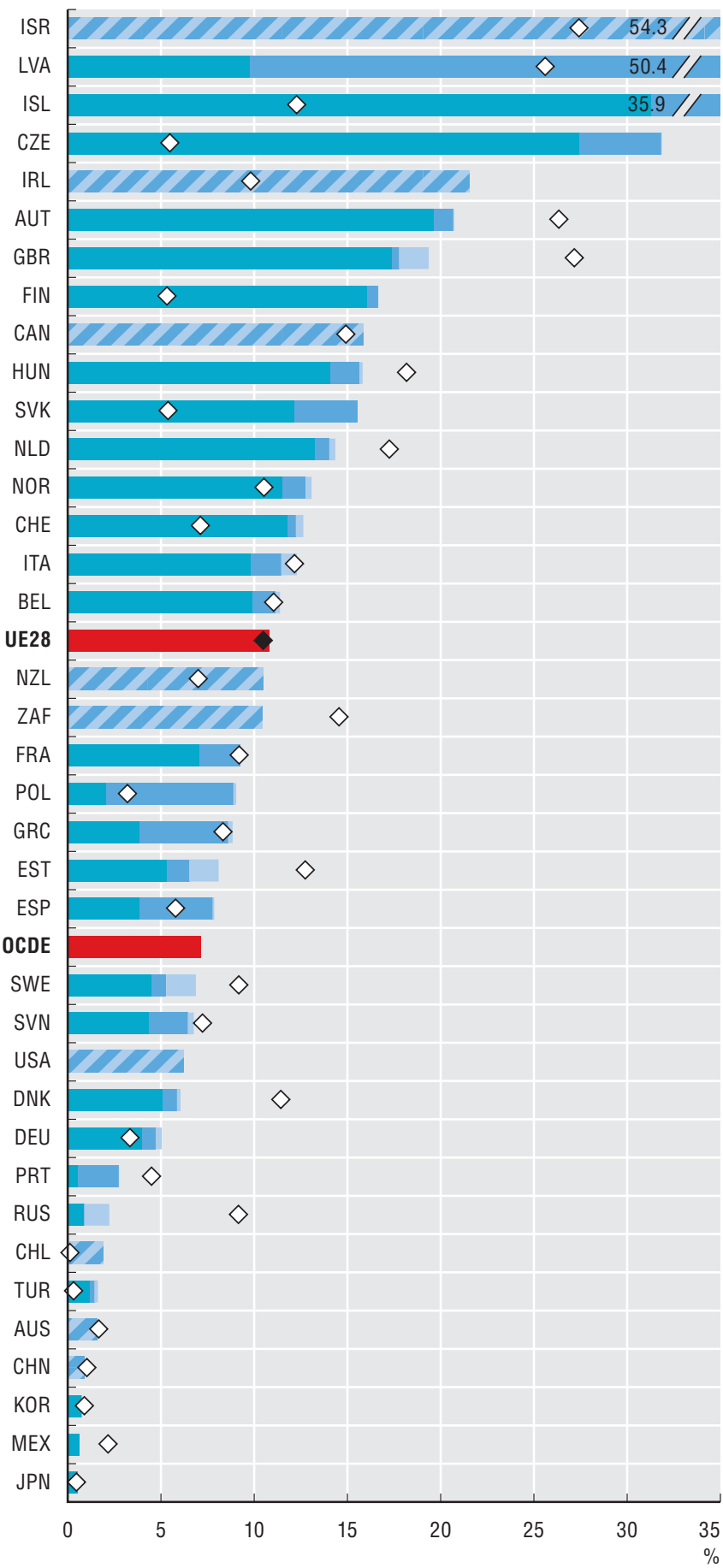

Source: OCDE, Base de données sur les statistiques de la recherche et développement, http://oe.cd/srd-fr, juin 2017. Davantage de données via StatLink. Voir notes de chapitre.

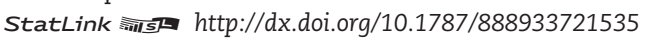

\section{Le saviez-vous ?}

Le Royaume-Uni est le premier bénéficiaire des financements de l'UE pour la R-D dans l'enseignement supérieur : il a reçu 615 millions EUR en 2014, c'est-à-dire $8 \%$ de la R-D dans l'enseignement supérieur.

Dans notre économie mondialisée actuelle, les entreprises financent leurs activités de R-D de diverses manières, et notamment à l'aide de leurs propres fonds (par exemple, bénéfices non distribués) et de sources nationales et internationales. La principale source internationale consiste en des paiements en provenance d'entreprises étrangères, dont celles qui entretiennent des relations de propriété ou de contrôle avec l'entreprise qui cherche à financer sa R-D. Les subventions et les contrats de recherche émanant d'organisations internationales sont une autre source internationale importante. Dans de nombreux pays, les fonds étrangers soutiennent une part considérable de la R-D des entreprises : plus de $20 \%$ en Autriche, en Irlande, en Islande et en République tchèque, et plus de $50 \%$ en Israël où une large part de la R-D des entreprises est menée par des entreprises affiliées sous contrôle étranger, et en Lettonie où près de $40 \%$ des DIRDE sont financées par l'UE.

Dans beaucoup de pays aussi, les entreprises affiliées sous contrôle étranger sont responsables d'une part importante de la R-D des entreprises : plus d'un cinquième dans la plupart des pays, et plus de la moitié en Autriche, en Belgique, en Irlande, en Israël, en République slovaque, en République tchèque et au Royaume-Uni. Cette tendance met en lumière la mondialisation de la R-D des entreprises et son importance dans les entreprises multinationales. Le Japon est le dernier du classement pour ce qui est des financements par des fonds étrangers et par des entreprises affiliées sous contrôle étranger, ce qui témoigne de son degré relativement faible d'intégration à l'environnement de R-D international.

Les organisations internationales, et notamment l'Union européenne, financent plus de $40 \%$ de la R-D des entreprises en Lettonie. Les fonds de la Commission européenne (CE) peuvent aussi être particulièrement importants pour la R-D menée par les établissements d'enseignement supérieur et les organismes publics de recherche. Les premiers bénéficiaires en sont l'Allemagne et le Royaume-Uni. Ces fonds sont plus déterminants au Royaume-Uni où ils soutiennent 7.4\% de la R-D de l'État et de l'enseignement supérieur contre seulement $3.9 \%$ en Allemagne - un pourcentage plus élevé que dans n'importe quel autre pays de l'Europe de l'Ouest, à l'exception de la Grèce ou de l'Irlande.

\section{Définitions}

Le reste du monde recouvre l'ensemble des institutions et personnes qui ne disposent pas, sur le territoire économique considéré, de site, de lieu de production ou de locaux dans lesquels ou depuis lesquels mener des activités économiques et des opérations économiques de grande envergure. Il regroupe toutes les unités (y compris parties d'entreprises, universités, organismes publics, organisations à but non lucratif, etc.) non résidentes dans le pays de référence, ainsi que les organisations internationales et entités supranationales, y compris les installations et activités qu'elles possèdent à l'intérieur du pays considéré.

Les entreprises affiliées sous contrôle étranger sont les entreprises du pays de référence détenues majoritairement par une société mère étrangère. 


\section{Dépenses de R-D engagées par des entreprises affiliées sous contrôle étranger, échantillon de pays, 2015 ou année disponible la plus récente}

En pourcentage des dépenses intérieures de R-D des entreprises

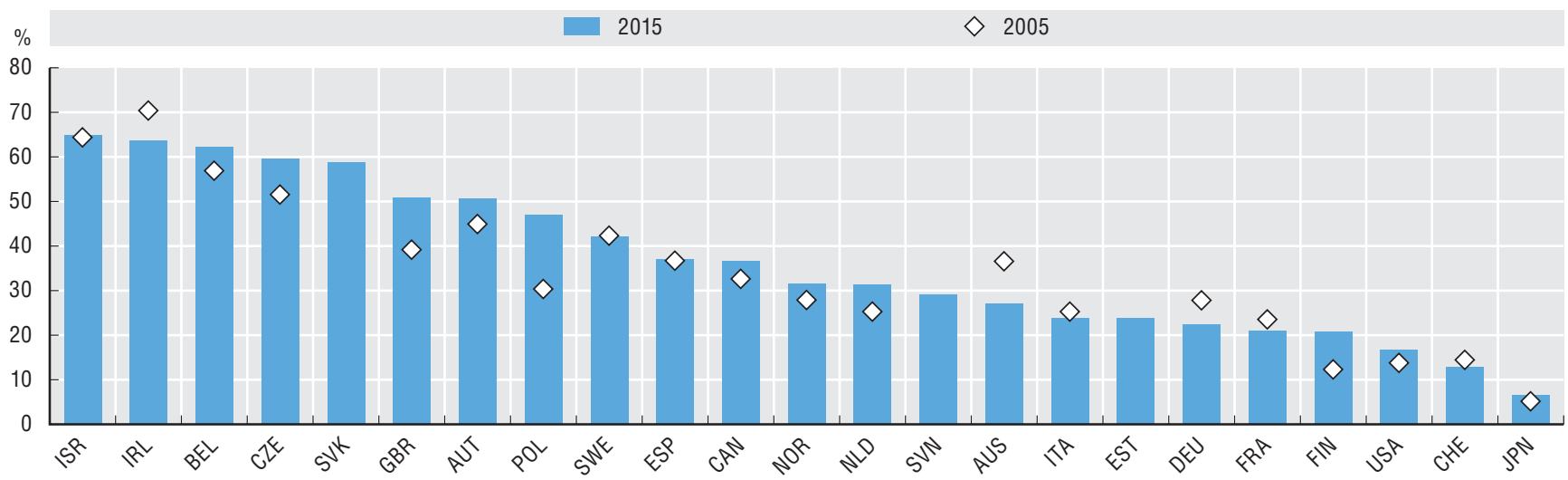

Source : OCDE, Base de données sur les activités des entreprises multinationales, http://oe.cd/amne ; Eurostat, Base de données FATS entrantes ; OCDE Base de données sur les statistiques de la recherche et développement, http://oe.cd/srd-fr, juin 2017. Davantage de données via StatLink. Voir notes de chapitre.

StatLink 尚Is] http://dx.doi.org/10.1787/888933721554

Financement de la R-D de l'État et de l'enseignement supérieur en Europe par la Commission européenne, 2015 Millions EUR PPP, prix de 2010

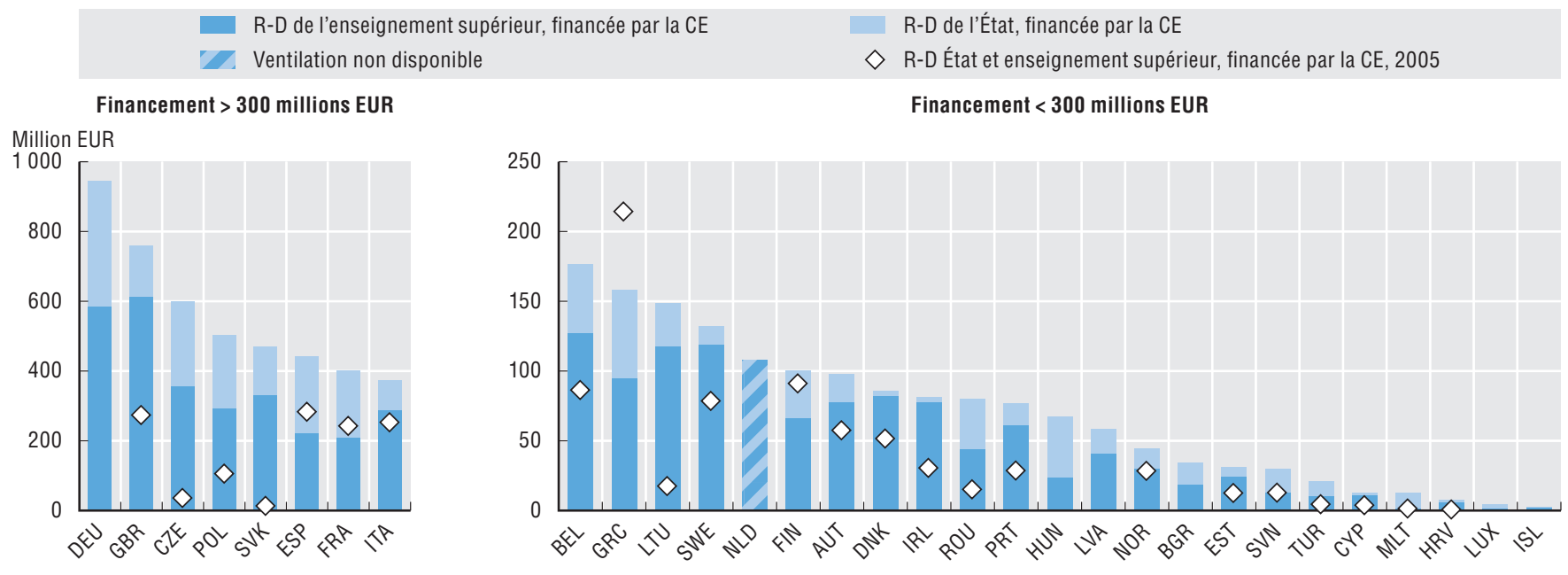

Source : OCDE, Base de données sur les statistiques de la recherche et développement, http://oe.cd/srd-fr ; Eurostat, statistiques de la recherchedéveloppement ; Eurostat, PPP pour agrégats SEC 2010, juillet 2017. Davantage de données via StatLink. Voir notes de chapitre.

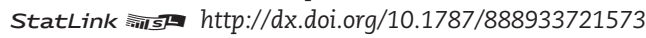

\section{Mesurabilité}

Du fait de l'internationalisation croissante de la R-D et des autres activités économiques, il devient difficile de déterminer précisément les flux financiers associés à la R-D qui circulent entre les entreprises, ainsi que la nature exacte de ces flux. Si les enquêtes sur la R-D permettent de recueillir des informations pertinentes, elles sont essentiellement axées sur les activités de R-D intra-muros nationales. Par conséquent, dans la plupart des pays, les informations sur les activités de R-D des multinationales à l'étranger sont rares, voire inexistantes. Sans compter que la collecte d'informations précises sur la valeur et la nature économique des flux transnationaux de R-D entre entreprises peut poser beaucoup de problèmes, dans la mesure où les pratiques des multinationales en la matière, y compris le financement et l'exploitation de la production intellectuelle qui en résulte, tendent à refléter des stratégies d'optimisation fiscale. La mesure de la mondialisation de la R-D au niveau des États et d'autres institutions hors entreprises n'en est qu'à ses débuts, et la comparabilité des données reste à ce jour limitée et concentrée dans un nombre restreint de pays. La dernière édition du Manuel de Frascati (OCDE, 2015) consacre un chapitre à la mesure des différents aspects de la mondialisation de la R-D (voir http://oe.cd/frascati). 


\section{EXCELLENCE DE LA RECHERCHE ET COLLABORATION}

\section{Inventions internationales}

\section{Co-inventions internationales dans le domaine des TIC, 2012-15}

En pourcentage du nombre total de familles de brevets IP5 dans les économies

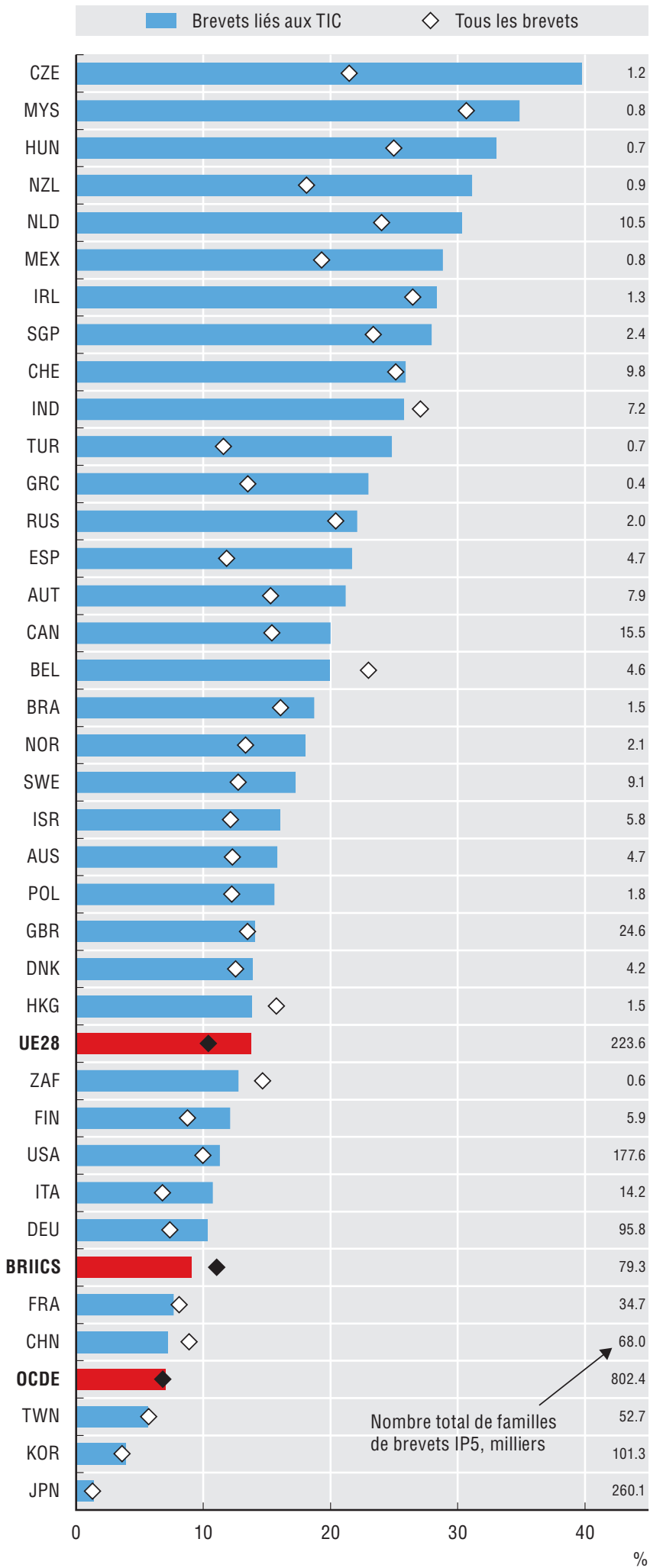

Source : OCDE, STI Microdata Lab : Base de données sur la propriété intellectuelle, http://oe.cd/ipstats, juin 2017. Davantage de données via StatLink. Voir notes de chapitre.

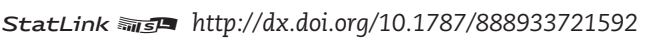

\section{Le saviez-vous ?}

Plus de $70 \%$ des brevets liés aux technologies de l'information et des communications (TIC) détenus par des entreprises des Bermudes, de la Barbade, des Îles Caïmans et des Îles Vierges britanniques sont inventés à l'étranger.

La diversité favorisant la créativité et l'innovation, les inventions naissent souvent de collaborations au sein d'une même économie ou entre acteurs de différentes économies. Les informations contenues dans les inventions brevetées, concernant le lieu de résidence des détenteurs et des inventeurs, aident à mettre en lumière les collaborations internationales en matière d'innovation. Elles montrent également la mesure dans laquelle les innovateurs accèdent aux connaissances dans d'autres économies pour trouver les compétences et les qualifications qui répondent le mieux à leurs besoins.

Sauf dans les technologies liées à la santé, la collaboration internationale entre inventeurs a augmenté dans tous les domaines technologiques et, en particulier, dans celui des TIC. Les co-inventions internationales sont plus courantes dans les TIC que dans d'autres domaines technologiques, et représentent un tiers ou plus de tous les brevets liés aux TIC pour des économies comme la République tchèque ou la Malaisie.

Qui plus est, à quelques exceptions près (la Chine, notamment), les inventions brevetées qui concernent les TIC reposent, en moyenne, sur des inventeurs basés dans un nombre d'économies relativement plus élevé. Il en ressort que les entreprises des TIC ont généralement accès aux connaissances développées dans un plus grand nombre d'économies, par rapport aux stratégies d'acquisition des savoirs appliquées dans d'autres domaines.

Il y a souvent découplage entre les inventeurs et les détenteurs d'inventions brevetées relatives aux TIC, une tendance plus marquée dans ce domaine technologique que dans les autres puisqu'elle concerne en moyenne $7.6 \%$ des brevets des TIC mais $6.4 \%$ des brevets toutes technologiques confondues. Dans la zone de l'OCDE, la part des inventions TIC étrangères que détiennent les économies varie entre $57 \%$ (Luxembourg) et $0.6 \%$ (Italie).

\section{Définitions}

Les familles de brevets IP5 correspondent aux brevets déposés auprès des cinq principaux offices de brevets (IP5). Les co-inventions internationales désignent les familles de brevets IP5 dont un coinventeur au moins est localisé à l'étranger. Les pourcentages sont calculés en divisant le nombre de co-inventions internationales par le nombre total de familles de brevets IP5, pour une économie donnée, dans le domaine technologique considéré. Le nombre d'économies de résidence des inventeurs est un indicateur fondé sur le nombre moyen d'économies dans lesquelles se situent les inventeurs des familles de brevets IP5 détenus par des résidents de l'économie en question. Les inventions étrangères détenues dans les économies correspondent à la part, dans le nombre total de familles de brevets IP5 détenues dans une économie, de celles qui sont détenues par un résident de l'économie alors qu'aucun des inventeurs n'en est luimême résident. Les brevets liés aux TIC sont recensés à l'aide des codes de la Classification internationale des brevets (CIB) (voir Inaba et Squicciarini, 2017) et alignés sur les définitions de l'OCDE du secteur des TIC (2017) et des produits TIC (2008). 
Nombre d'économies de résidence des inventeurs, par technologie, 2012-15

Moyennes, toutes technologies confondues, familles de brevets IP5 selon la résidence du détenteur du brevet

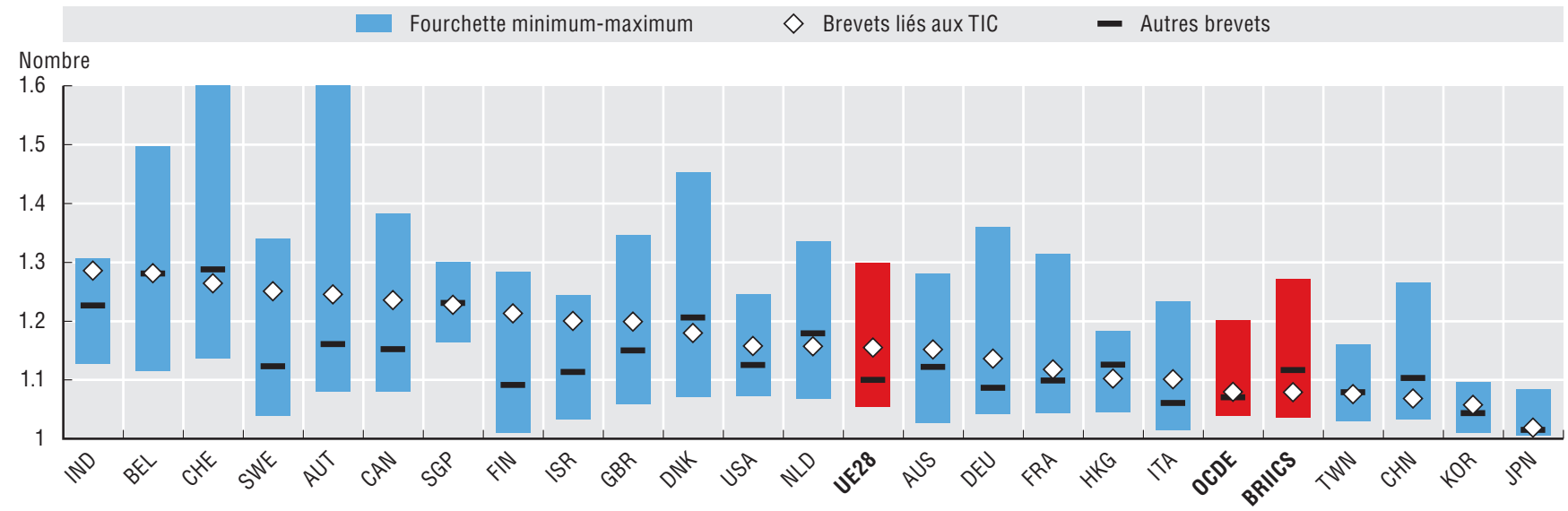

Source : OCDE, STI Micro-data Lab : Base de données sur la propriété intellectuelle, http://oe.cd/ipstats, juin 2017. Davantage de données via StatLink. Voir notes de chapitre.

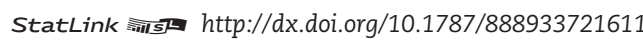

Inventions TIC étrangères détenues par les économies, 2012-15

En pourcentage du nombre total de familles de brevets IP5 dans les économies

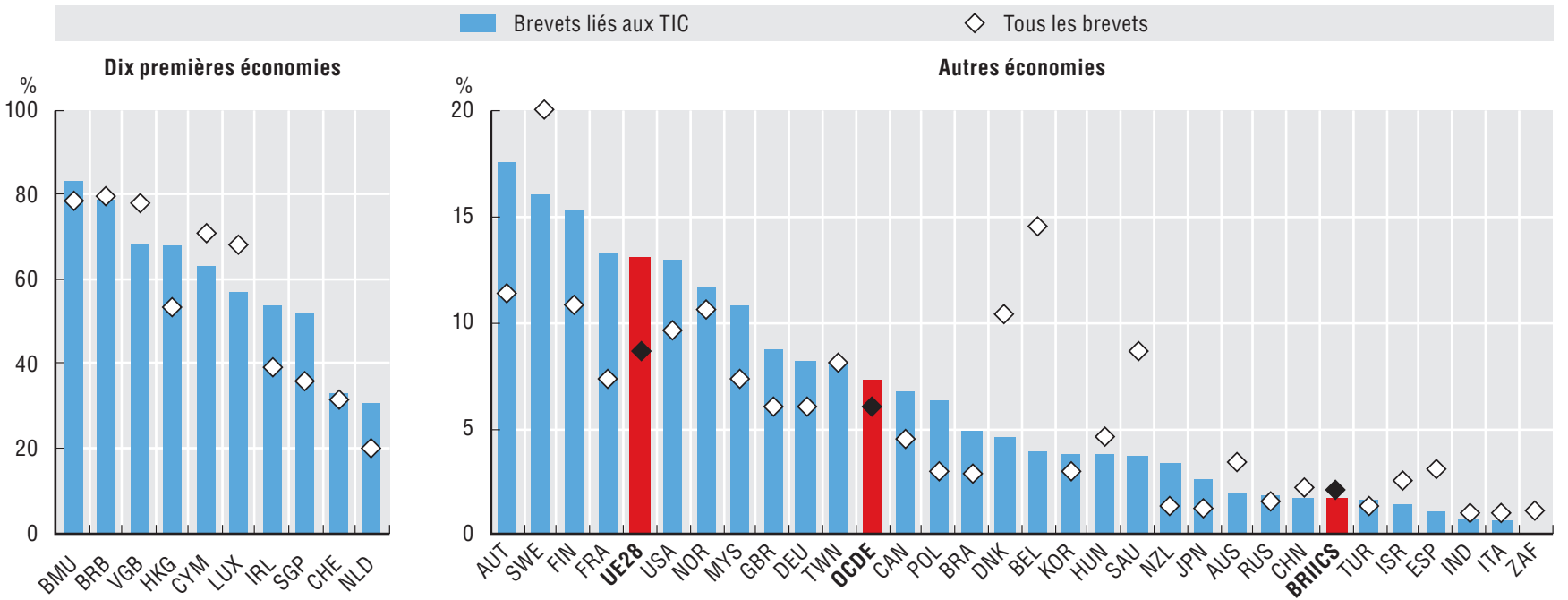

Source : OCDE, STI Micro-data Lab : Base de données sur la propriété intellectuelle, http://oe.cd/ipstats, juin 2017. Davantage de données via StatLink. Voir notes de chapitre.

StatLink :

\section{Mesurabilité}

Les collaborations peuvent revêtir différentes formes : co-inventions internationales par plusieurs entreprises, grandes ou petites, pôles de recherche conjointe mis en place par des établissements privés et publics (par exemple, entre des entreprises et des universités ou établissements publics de recherche), ou encore réseaux formels ou informels de scientifiques. Dans le cas des multinationales, la collaboration internationale témoigne souvent d'un processus suivant lequel les entreprises s'appuient sur des installations de recherche et d'innovation implantées dans plusieurs économies pour mobiliser des savoirs géographiquement dispersés et/ou développer des complémentarités avec des inventeurs étrangers. Le degré de collaboration entre des inventeurs à l'échelle internationale peut dépendre d'un large éventail de facteurs, notamment la structure de l'entreprise ou de l'établissement auxquels ils appartiennent, le domaine technologique de l'invention, et la proximité linguistique et culturelle. Les brevets liés aux TIC recouvrent 13 domaines définis selon les caractéristiques techniques et les fonctionnalités qu'ils permettent d'obtenir (par exemple, communication mobile, réseau à haut débit, informatique de haute puissance et analytique de données massives). Comme la plupart des inventions ne sont protégées que dans certaines économies, l'utilisation des données de différents offices de brevets peut conduire à des résultats différents. 


\section{EXCELLENCE DE LA RECHERCHE ET COLLABORATION}

\section{Collaboration en matière d'innovation}

Entreprises menant des activités d'innovation en collaboration avec des établissements d'enseignement supérieur ou de recherche, par taille, 2012-14

En pourcentage des entreprises actives dans l'innovation de produit et/ou de procédé, dans chaque catégorie de taille

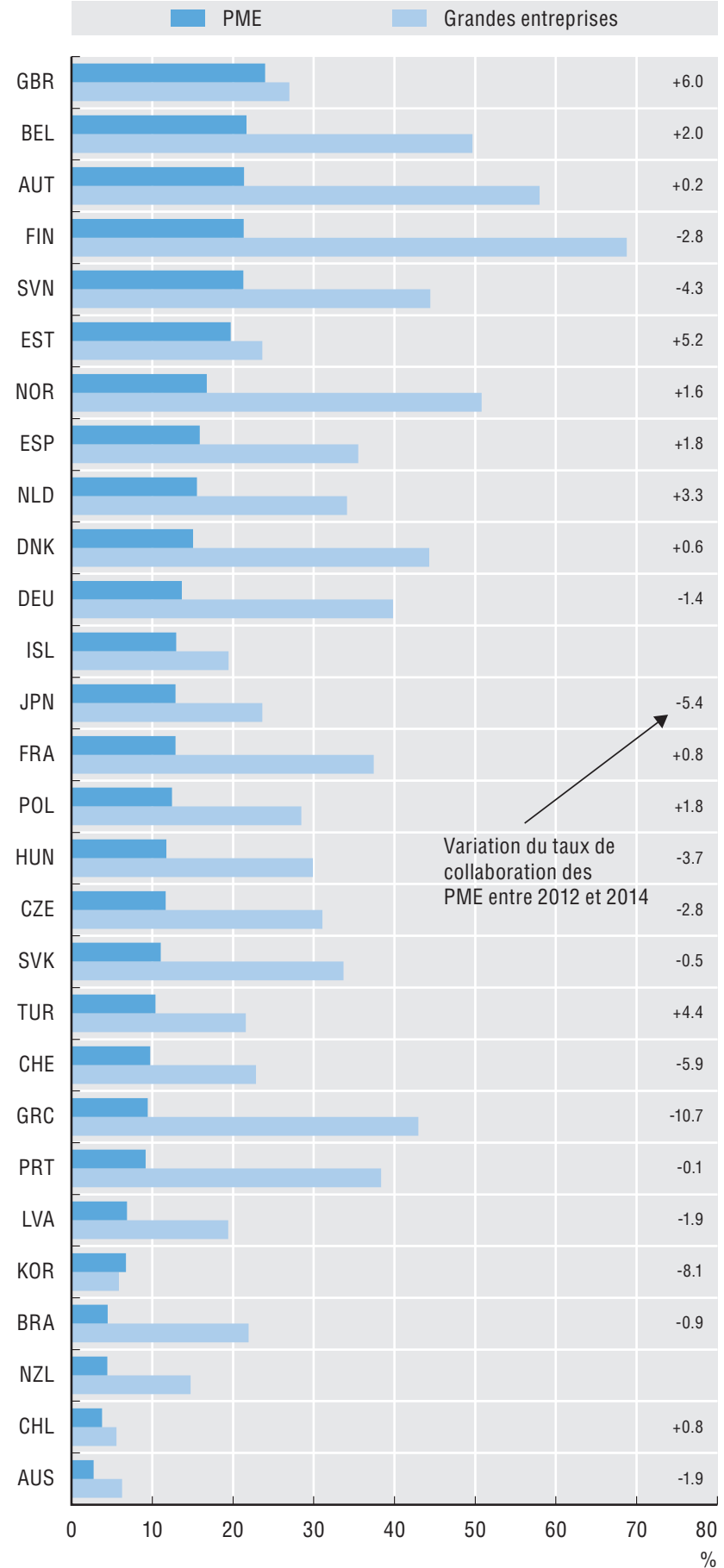

Note : La comparabilité internationale peut être limitée du fait des différences méthodologiques entre les enquêtes sur l'innovation et des profils de réponses propres à chaque pays. Les pays européens suivent le schéma harmonisé de l'Enquête communautaire sur l'innovation.

Source : OCDE, d'après OCDE, Enquête de 2017 sur les statistiques nationales de l'innovation et Eurostat, Enquête communautaire sur l'innovation (CIS-2014), http://oe.cd/inno-stats, juin 2017. Davantage de données via StatLink. Voir notes de chapitre.

StatLink तiाs] http://dx.doi.org/10.1787/888933721649

\section{Le saviez-vous ?}

En moyenne, seules $13 \%$ des petites et moyennes entreprises (PME) actives dans l'innovation mettent au point leurs innovations en collaboration avec des universités ou des établissements de recherche, contre $31 \%$ des grandes entreprises.

Les entreprises se spécialisent pour être plus compétitives ; la collaboration leur permet d'avoir recours à un plus grand vivier de ressources et de savoirs, et de partager les risques. Les profils de collaboration dépendent des caractéristiques des entreprises et de leurs objectifs en matière d'innovation. Par exemple, les formes d'innovation fondées sur la R-D peuvent nécessiter de faire appel à différents types de partenaires. Les travaux menés en collaboration avec des établissements d'enseignement supérieur ou des établissements publics de recherche constituent une source importante de transfert de connaissances pour les grandes entreprises. Dans la plupart des pays, celles-ci sont généralement deux à trois fois plus susceptibles d'y avoir recours que les PME.

La collaboration en matière d'innovation est plus fréquente avec les fournisseurs et les clients. Dans le cas des grandes entreprises, les fournisseurs jouent un rôle dominant compte tenu de l'intégration croissante des chaînes de valeur. Dans des pays comme l'Allemagne, la Corée, la Finlande et le Royaume-Uni, la collaboration avec les clients est au moins aussi importante, en particulier pour les PME actives dans l'innovation. Cette tendance pourrait être une indication de l'importance des utilisateurs en tant que moteurs de l'innovation.

Les partenaires étrangers peuvent aussi jouer un rôle majeur dans le processus d'innovation, compte tenu du rôle croissant des chaînes de valeur mondiales. Mais les taux de collaboration internationale varient sensiblement d'un pays à l'autre. Dans certaines petites économies ouvertes, les entreprises privilégient fortement la collaboration avec des partenaires étrangers, un phénomène qui pourrait être lié à des facteurs tels que la spécialisation sectorielle, des possibilités limitées de collaboration au niveau national et, parfois, à la proximité de pôles de savoir extérieurs. La taille des entreprises semble constituer un déterminant majeur de la collaboration internationale : quel que soit le taux global observé en la matière, les grandes entreprises sont bien plus susceptibles que les PME de choisir cette forme d'interaction.

\section{Définitions}

La collaboration en matière d'innovation implique une participation active à des projets d'innovation (c'est-à-dire visant à introduire sur le marché un produit ou un procédé nouveau ou sensiblement amélioré) menés conjointement avec d'autres organisations, mais exclut la sous-traitance pure et simple des activités correspondantes. Elle peut inclure la mise en œuvre conjointe d'innovations avec des clients et des fournisseurs, ainsi que des partenariats avec d'autres entreprises ou organisations.

La collaboration internationale en matière d'innovation suppose une participation transnationale active à des travaux d'innovation collaboratifs.

Le classement des entreprises selon leur taille est conforme aux recommandations du Manuel d'Oslo, et s'appuie, dans la majorité des pays, sur le nombre d'employés. Les PME s'entendent des entreprises de 10 à 249 salariés, à quelques exceptions près spécifiées dans les notes de chapitre. 
Entreprises menant des activités d'innovation en collaboration avec des fournisseurs ou des clients, par taille, 2012-14 En pourcentage des entreprises actives dans l'innovation de produit et/ou de procédé, dans chaque catégorie de taille

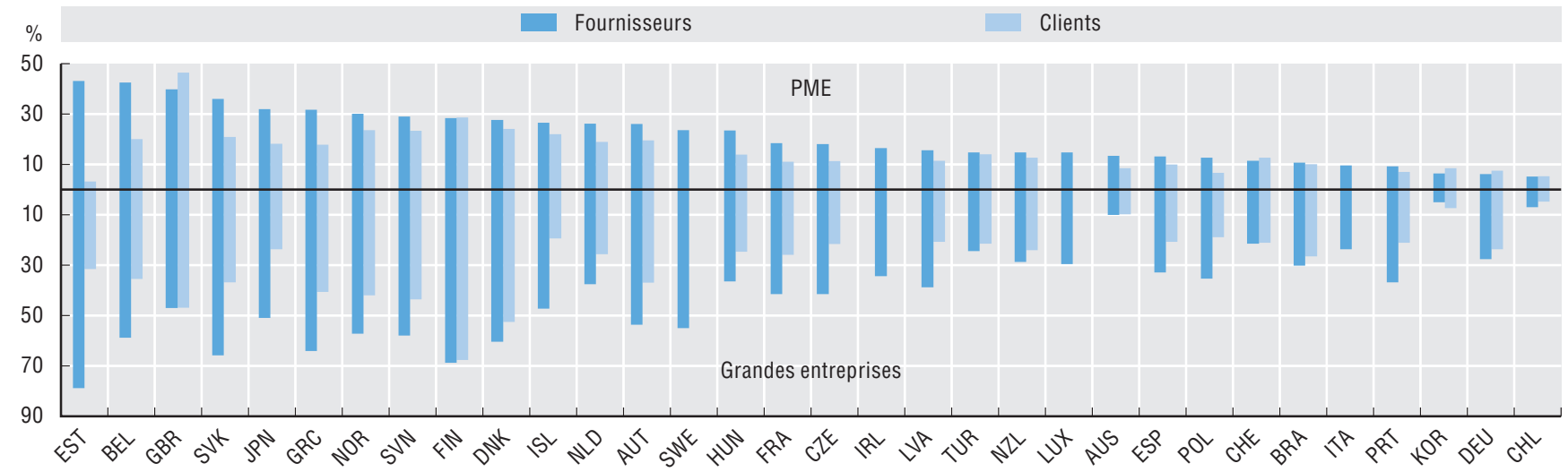

Note : La comparabilité internationale peut être limitée du fait des différences méthodologiques entre les enquêtes sur l'innovation et des profils de réponses propres à chaque pays. Les pays européens suivent le schéma harmonisé de l'Enquête communautaire sur l'innovation.

Source : OCDE, d'après OCDE, Enquête de 2017 sur les statistiques nationales de l'innovation et Eurostat, Enquête communautaire sur l'innovation (CIS-2014), http://oe.cd/inno-stats, juin 2017. Davantage de données via StatLink. Voir notes de chapitre.

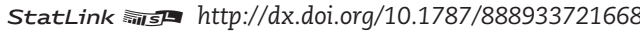

\section{Entreprises engagées dans des collaborations internationales à des fins d'innovation, par taille, 2012-14}

En pourcentage des entreprises actives dans l'innovation de produit et/ou de procédé, dans chaque catégorie de taille

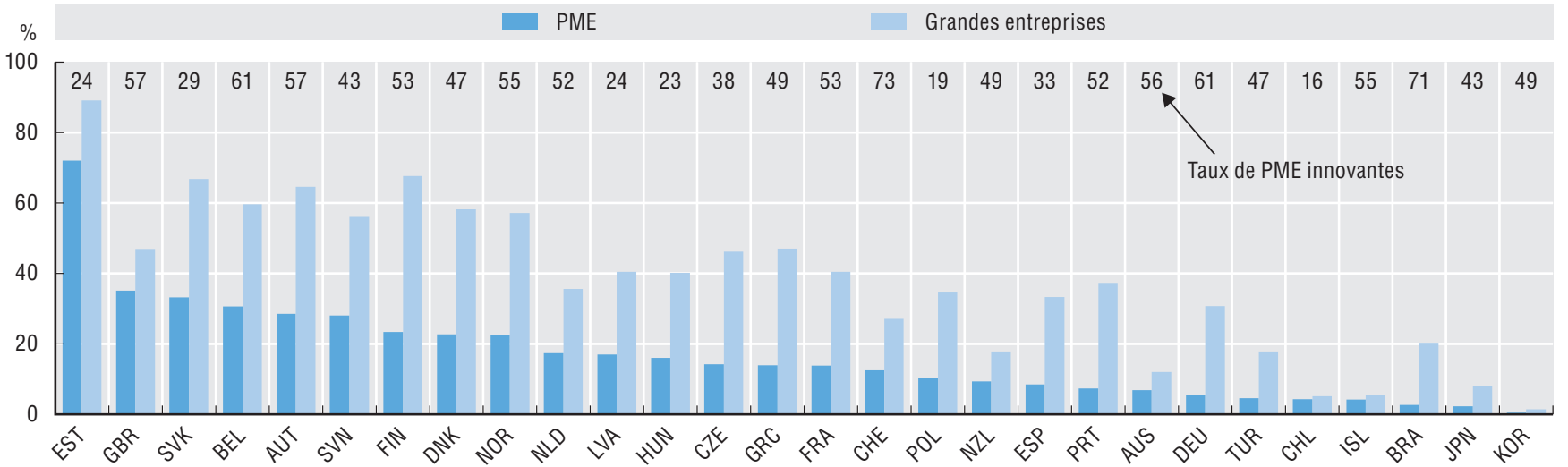

Note : La comparabilité internationale peut être limitée du fait des différences méthodologiques entre les enquêtes sur l'innovation et des profils de réponses propres à chaque pays. Les pays européens suivent le schéma harmonisé de l'Enquête communautaire sur l'innovation.

Source : OCDE, d'après OCDE, Enquête de 2017 sur les statistiques nationales de l'innovation et Eurostat, Enquête communautaire sur l'innovation (CIS-2014), http://oe.cd/inno-stats, juin 2017. Davantage de données via StatLink. Voir notes de chapitre.

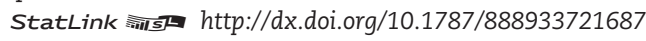

\section{Mesurabilité}

Dans les enquêtes qui suivent le schéma de l'Enquête communautaire sur l'innovation, les taux de collaboration se rapportent aux entreprises actives dans l'innovation de produit ou de procédé. Dans les autres cas, ils portent sur les entreprises pratiquant tous types d'innovation. Le concept de collaboration en matière d'innovation varie selon les modèles d'enquêtes, lorsque les groupes d'entreprises auxquelles la mesure s'applique diffèrent.

Par ailleurs, la structure et les caractéristiques des enquêtes peuvent influer sur les réponses des entreprises et, par conséquent, sur les résultats. Ainsi, les réponses aux questions sur les activités d'innovation et aux questions complémentaires sur la collaboration avec des tierces parties peuvent varier selon l'ordre des questions, le champ de l'enquête, ou la combinaison avec d'autres types d'enquêtes. Les problèmes de comparabilité qui en découlent sont examinés dans le cadre de la révision en cours du Manuel d'Oslo OCDE/Eurostat sur la mesure de l'innovation dans les entreprises (http://oe.cd/manueldoslo). 


\section{EXCELLENCE DE LA RECHERCHE ET COLLABORATION}

\section{Notes et Références}

\section{Chypre}

La note suivante est incluse à la demande de la Turquie :

"Les informations figurant dans ce document qui font référence à “Chypre” concernent la partie méridionale de lîle. Il n'y a pas d'autorité unique représentant à la fois les Chypriotes turcs et grecs sur l'île. La Turquie reconnaît la République Turque de Chypre Nord (RTCN). Jusqu'à ce qu'une solution durable et équitable soit trouvée dans le cadre des Nations Unies, la Turquie maintiendra sa position sur la "question chypriote". "

La note suivante est ajoutée à la demande de tous les États de l'Union européenne membres de l'OCDE et de l'Union européenne :

"La République de Chypre est reconnue par tous les membres des Nations Unies sauf la Turquie. Les informations figurant dans ce document concernent la zone sous le contrôle effectif du gouvernement de la République de Chypre. »

\section{Israël}

"Les données statistiques concernant Israël sont fournies par et sous la responsabilité des autorités israéliennes ou d'un tiers compétents. L'utilisation de ces données par l'OCDE est sans préjudice du statut des hauteurs du Golan, de JérusalemEst et des colonies de peuplement israéliennes en Cisjordanie aux termes du droit international. »

"Il est à noter que les données statistiques sur les brevets et marques israéliens sont fournies par les offices des brevets et des marques des pays concernés. ”

\subsection{Excellence de la recherche et spécialisation}

\section{Quantité et qualité de la production scientifique, 2005 et 2015}

Les "publications les plus citées " sont les $10 \%$ de publications les plus citées, les valeurs étant normalisées par domaine scientifique et par type de document (articles, recensions et actes de conférences). Les valeurs SJR (Scimago Journal Rank) sont utilisées en complément pour hiérarchiser les publications ayant obtenu le même nombre de citations au sein d'une catégorie. Cette mesure est un indicateur de substitution de l'excellence de la recherche. Les estimations sont fondées sur des comptages fractionnaires des documents publiés, par auteur affilié à des établissements dans chaque économie.

\section{Spécialisation et impact de citation dans quelques disciplines scientifiques, 2015}

"Les «publications les plus citées " sont les $10 \%$ de publications les plus citées, les valeurs étant normalisées par domaine scientifique et par type de document (articles, recensions et actes de conférences). Les valeurs SJR (Scimago Journal Rank) sont utilisées en complément pour hiérarchiser les publications ayant obtenu le même nombre de citations au sein d'une catégorie. Cette mesure est un indicateur de substitution de l'excellence de la recherche. Les estimations sont fondées sur des comptages fractionnaires des documents publiés, par auteur affilié à des établissements dans chaque économie. Les documents publiés dans des revues multidisciplinaires/génériques sont affectés sur une base fractionnaire aux codes ASJC des articles qui citent et des articles cités.

L'indicateur de spécialisation relative a été calculé en divisant la part d'un champ disciplinaire donné dans la production scientifique totale du pays par la part de ce champ disciplinaire dans la production scientifique mondiale. S'il est supérieur à 1, le pays est très spécialisé dans le champ disciplinaire en question, autrement dit la part de ce domaine dans la production du pays a une importance relative plus élevée que la part de ce domaine dans la production scientifique mondiale en général, selon les données collectées dans la base Scopus. Les chiffres ont été arrondis. Les cas où le nombre de documents d'une économie et d'un champ disciplinaire donnés est trop faible ont été supprimés.

\subsection{Excellence de la collaboration scientifique}

\section{Collaboration scientifique internationale, 2015}

On définit la collaboration internationale comme le nombre de publications dont un auteur au moins est affilié à l'économie de référence et dont un auteur au moins est affilié à un établissement d'un autre pays ou d'une autre économie, exprimé en pourcentage du nombre total de publications dont un auteur au moins est affilié à l'économie de référence. Ce nombre inclut une part relativement faible de documents attribués à un seul auteur affilié à plusieurs établissements dans différentes économies.

La collaboration internationale comprend les documents dont l'auteur principal est affilié en premier lieu à un établissement dans l'économie de référence et les documents dont l'auteur principal est affilié en premier lieu à un établissement étranger. L'auteur principal est déterminé dans la plupart des cas sur la base de l'identité de l'auteur correspondant désigné. Pour la part relativement réduite de documents pour lesquels on ne dispose pas de cette information, c'est le premier auteur mentionné qui est considéré comme l'auteur principal. 


\section{Impact de citation de la production scientifique et degré de collaboration internationale, 2012-16}

La production scientifique correspond au nombre total de documents citables (articles, recensions et actes de conférences) publiés dans des revues scientifiques indexées dans la base Scopus.

L'impact de citation normalisé est le rapport entre le nombre moyen de citations que reçoivent les documents publiés par des auteurs affiliés à un établissement dans l'économie de référence et le nombre moyen de citations à l'échelle mondiale, au cours de la même période, par type de document et discipline.

La normalisation des valeurs relatives aux citations est axée sur les documents (c'est-à-dire effectuée au niveau de chaque document). Si un document est publié dans une revue relevant de plusieurs disciplines, on calcule une moyenne sur l'ensemble de ces disciplines. Les valeurs indiquent la relation entre l'impact moyen d'une unité et la moyenne mondiale pour la discipline et le type de document considérés, à laquelle correspond la valeur 1 (par conséquent, un score de 0.8 signifie que l'unité se situe 20 \% en-dessous de la moyenne, tandis qu'un score de 1.3 signifie qu'elle se situe 30 \% au-dessus de la moyenne).

On définit la collaboration internationale comme le nombre de publications dont un auteur au moins est affilié à l'économie de référence et dont un auteur au moins est affilié à un établissement d'un autre pays ou d'une autre économie, exprimé en pourcentage du nombre total de publications dont un auteur au moins est affilié à l'économie de référence. Les articles émanant d'un auteur unique affilié à des établissements de plusieurs pays peuvent ainsi être comptabilisés au titre de la collaboration internationale entre les établissements.

Les chiffres pluriannuels sont des moyennes (ou des totaux) d'estimations annuelles.

\section{Production parmi les $10 \%$ de publications les plus citées et profils de collaboration internationale, 2015}

Ce graphique montre la distribution des publications les plus citées de chaque pays ou économie. Il s'agit des $10 \%$ de publications les plus citées, les valeurs étant normalisées par domaine scientifique et par type de document (articles, recensions et actes de conférences). Les valeurs SJR (Scimago Journal Rank) sont utilisées en complément pour hiérarchiser les publications ayant obtenu le même nombre de citations au sein d'une catégorie. Cette mesure est un indicateur de l'excellence de la recherche. Les estimations sont fondées sur des comptages fractionnaires des documents publiés, par auteur affilié à des établissements dans chaque économie.

Les publications les plus citées sont réparties entre plusieurs catégories, selon que leur auteur principal est affilié à un établissement du pays ou étranger (autrement dit, selon que l'auteur principal est affilié en premier lieu dans l'économie de référence ou à l'étranger). L'auteur principal est déterminé dans la plupart des cas sur la base de l'identité du premier auteur correspondant désigné. Pour la part relativement réduite de documents pour lesquels on ne dispose pas de cette information, c'est le premier auteur mentionné qui est considéré comme l'auteur principal. Les documents dont l'auteur principal est affilié à un établissement dans l'économie de référence peuvent ou non avoir fait l'objet d'une collaboration internationale, ce qui conduit à distinguer les collaborations internationales avec l'auteur principal affilié dans le pays, des documents dont tous les auteurs sont affiliés dans le pays.

\subsection{Mobilité internationale des personnes hautement qualifiées}

\section{Mobilité internationale des étudiants inscrits dans l'enseignement supérieur, 2015}

Pour la Corée, l'Italie, la République slovaque, la République tchèque et la Turquie, les données se rapportent aux étudiants étrangers. Les étudiants étrangers sont définis selon leur nationalité. Ces données ne peuvent être comparées à celles sur les étudiants internationaux. Elles sont donc présentées séparément dans le tableau et le graphique.

Le nombre total d'inscrits inclut l'ensemble des étudiants internationaux ou étrangers. La répartition par domaine d'étude est fondée sur le nombre d'étudiants dont le domaine d'étude est renseigné.

L'enseignement supérieur correspond aux niveaux 5 à 8 de la CITE-2011.

Les domaines d'étude mentionnés correspondent à des domaines d'étude et de formation de la classification CITE-F 2013.

Pour les États-Unis, la catégorie Santé et protection sociale inclut tous les programmes interdisciplinaires, y compris ceux sans composante spécifique Arts et lettres.

Pour le Japon, les données sur les technologies de l'information et des communications sont incluses dans d'autres domaines. Pour les Pays-Bas, le nombre total d'étudiants dans l'enseignement supérieur exclut le niveau doctoral.

Doctorants internationaux et nationaux dans les domaines des sciences naturelles, de l'ingénierie et des TIC, 2015

L'enseignement supérieur correspond aux niveaux 5 à 8 de la CITE-2011.

Les domaines d'étude mentionnés correspondent à des domaines d'étude et de formation de la classification CITE-F 2013.

Pour le Japon, les données sur les technologies de l'information et des communications sont incluses dans les autres domaines.

Personnes dotées d'un niveau d'instruction élevé dans la population en âge de travailler, par pays de naissance, 2015

Les personnes dotées d'un niveau d'instruction élevé sont définies comme étant celles dont le plus haut diplôme ou niveau de formation validée se situe au niveau 5 ou plus de la classification CITE-2011. 


\section{EXCELLENCE DE LA RECHERCHE ET COLLABORATION}

Notes et Références

Pour la Corée, les données se rapportent à 2013. Le statut d'immigré est défini sur la base de la nationalité, et non du pays de naissance. Pour les États-Unis, les données tiennent compte des individus de plus de 55 ans qui suivent un enseignement.

L'indicateur est calculé à partir des sources de données suivantes : Eurostat, Enquêtes sur les forces de travail (EFT-UE) ; Enquêtes sur la population active (Australie, Canada, Israël, Nouvelle-Zélande) ; Encuesta de Caracterización Socioeconómica Nacional (CASEN) (Chili) ; Enquête sur la population active étrangère (Corée) ; Encuesta Nacional de Ocupación y Empleo (ENOE) (Mexique) ; et Current Population Survey (CPS) (États-Unis).

\subsection{Mobilité des scientifiques}

\section{Flux bilatéraux internationaux d'auteurs scientifiques, 2006-16}

Les données sont fondées sur le pays de la principale affiliation des auteurs, pour au moins deux publications indexées dans la base de données Scopus au cours de la période 2006-16. Les comptages reflètent les nombres d'auteurs ayant changé d'affiliations entre leur première et leur dernière publication enregistrée au cours de la période considérée. Ils ne tiennent donc pas compte des flux vers et depuis les affiliations intermédiaires. Quand un auteur est affilié à plusieurs pays (ce qui concerne environ 2 \% des documents), on utilise pour cet auteur le pays d'affiliation qui revient le plus souvent.

\section{Mobilité internationale des auteurs scientifiques, 2016}

Les estimations sont fondées sur la comparaison entre la principale affiliation d'un auteur donné, doté d'un identifiant Scopus et ayant publié un article en 2016, et celle mentionnée dans la dernière publication disponible au cours d'une année antérieure. Seuls les auteurs d'au moins deux publications sont pris en compte. Un épisode de mobilité est observé en 2016 lorsqu'un auteur affilié à un établissement dans une économie donnée, sur la base de sa dernière publication en 2016, était auparavant affilié à un établissement dans une autre économie. Le statut est affecté aux auteurs en fonction de leur dernière affiliation en 2016. Le statut de " résident " est attribué à un auteur si son affiliation principale en 2016 et avant 2016 correspond à l'économie de référence. Le statut de « réentrant » lui est attribué si, lors de son dernier changement d'affiliation, il est arrivé dans la même économie de référence que celle de sa première publication enregistrée. Du point de vue de l'économie dans laquelle se trouve l'établissement d'affiliation précédent, l'auteur peut être enregistré comme "sortant " : il est comptabilisé en tant que tel dans les données présentées. Les données sont présentées triées en fonction de la part des sortants dans la somme étendue de tous les profils de mobilité du point de vue de l'économie de référence (résidents, réentrants, entrants et sortants).

L'indicateur correspond au ratio entre le nombre d'auteurs dans la catégorie pertinente et le nombre (absolu) d'auteurs recensés dans l'économie de référence en 2016, auquel s'ajoutent les flux sortants enregistrés pour cette même économie en 2016 . Il peut être ajusté afin de mettre en avant les profils d'auteurs du point de vue du pays final d'affiliation, comme le montrent les variables supplémentaires, la somme des pourcentages des nouveaux arrivants, des réentrants et des résidents étant égale à 100.

\section{Impact attendu des citations des auteurs scientifiques, par profil de mobilité, 2016}

Cet indicateur est expérimental.

Les estimations sont basées sur une comparaison des valeurs SJR (Scimago Journal Rank) de 2015 applicables aux documents publiés par des auteurs scientifiques, à partir du rang des revues dans lesquelles sont parus les articles en 2016, et de la mobilité des auteurs entre 2001 et 2016. Seuls les auteurs d'au moins deux publications sont pris en compte. Un épisode de mobilité est observé en 2016 lorsqu'un auteur affilié à un établissement dans une économie donnée, sur la base de sa dernière publication en 2016, était auparavant affilié à un établissement dans une autre économie. Le statut est affecté aux auteurs en fonction de leur dernière affiliation en 2016. Le statut de " résident » est attribué à un auteur si son affiliation principale en 2016 et avant 2016 correspond à l'économie de référence. Le statut de " réentrant " lui est attribué si, lors de son dernier changement d'affiliation, il est arrivé dans la même économie de référence que celle de sa première publication enregistrée. Du point de vue de l'économie dans laquelle se trouve l'établissement d'affiliation précédent, l'auteur peut être enregistré comme « sortant » et être comptabilisé en tant que tel dans les données présentées.

L'indicateur correspond à la valeur moyenne SJR 2015 obtenue pour les auteurs dans la catégorie et l'économie considérées.

\subsection{Mondialisation de la R-D}

\section{R-D des entreprises financée par des fonds étrangers, par source de financement, 2015}

En l'absence de ventilation par source de financement, c'est la part des DIRDE financée par le " reste du monde » (étranger) qui est présentée.

Ces statistiques sont fondées sur les données des bases de l'OCDE consacrées à la R-D, notamment les Statistiques de la recherche et développement (http://oe.cd/srd-fr) et les Principaux indicateurs de la science et de la technologie (http://oe.cd/pist-fr). Se reporter à ces sources pour davantage de renseignements sur les données, en particulier sur les ruptures de séries et autres problèmes analogues. Pour l'Afrique du Sud, l'Allemagne, l'Australie, la Belgique, le Danemark et la Suède, les données se rapportent à 2013.

Pour l'Autriche, les données se rapportent à 2004 et 2013. 
Pour le Danemark et l'Estonie, les DIRDE financées par les organisations internationales comprennent uniquement les fonds émanant de la Commission européenne.

Pour la France, Israël, l'Italie, les Pays-Bas, la Pologne, le Portugal, le Royaume-Uni, la Slovénie, la zone OCDE et la zone UE28, les données se rapportent à 2014.

Pour Israël, la R-D du secteur de la défense est partiellement exclue des estimations disponibles.

Pour le Mexique, seuls les fonds des entreprises étrangères sont disponibles.

Pour la Suisse, les données se rapportent à 2004 et 2015.

\section{Dépenses de R-D engagées par des entreprises affiliées sous contrôle étranger, échantillon de pays, 2015 ou année disponible la plus récente}

Pour l'Allemagne, l'Australie, l'Autriche, la Belgique, le Canada, l'Espagne, l'Estonie, la Finlande et la Pologne, les données se rapportent à 2013.

Pour l'Australie et Israël, les données de la période antérieure se rapportent à 2006.

Pour l'Autriche et les Pays-Bas, les données de la période antérieure se rapportent à 2004.

Pour l'Espagne, les Pays-Bas, la Pologne et la Slovénie, les données concernent uniquement les sections B à F de la CITI révision 4.

Pour l'Estonie et la Finlande, les données concernent uniquement les sections B à E de la CITI révision 4.

Pour les États-Unis, la France, l'Italie, le Japon et les Pays-Bas, les données se rapportent à 2014.

Pour Israël et la Slovénie, les données se rapportent à 2011.

Pour la Norvège, les données se rapportent à 2012.

Pour la Suisse, les données de la période antérieure se rapportent à 2008.

\section{Financement de la R-D de l'État et de l'enseignement supérieur en Europe par la Commission européenne, 2015}

Ces statistiques sont fondées sur les données des bases de l'OCDE consacrées à la R-D, notamment les Statistiques de la Recherche et développement (http://oe.cd/srd-fr) et les Principaux indicateurs de la science et de la technologie (http://oe.cd/pist-fr). Se reporter à ces sources pour davantage de renseignements sur les données, en particulier sur les ruptures de séries et autres problèmes analogues.

Il n'y a pas de ventilation disponible pour les Pays-Bas.

Pour l'Allemagne, la Bulgarie, la Croatie, Chypre, la France, l'Italie, la Lituanie, le Luxembourg, les Pays-Bas, la Pologne, le Portugal, la Roumanie et la Slovénie, les données se rapportent à 2014.

Pour l'Autriche, les données se rapportent à 2013 et 2006.

Pour la Belgique, le Danemark et la Suède, les données se rapportent à 2013.

Pour la République slovaque, les données de la période antérieure se rapportent à 2006.

Pour la République tchèque, les données de la période antérieure se rapportent à 2007.

\subsection{Inventions internationales}

\section{Co-inventions internationales dans le domaine des TIC, 2012-15}

Les co-inventions internationales sont mesurées comme la part des familles de brevets IP5 dont des inventeurs se trouvent dans au moins deux économies, rapportée au nombre total de familles de brevets IP5 dont des inventeurs se trouvent dans l'économie de référence. Les données se rapportent aux familles de brevets IP5, par date de dépôt, en fonction du lieu de résidence des inventeurs, et avec comptages fractionnaires. Les brevets liés aux TIC sont recensés à l'aide des codes de la Classification internationale des brevets (CIB) dans Inaba et Squicciarini (2017). Seules les économies totalisant plus de 100 familles au total et au moins 50 familles dans les TIC en 2012-15 sont incluses. Les chiffres de 2014 et 2015 sont estimés sur la base des données disponibles pour ces années.

\section{Nombre d'économies de résidence des inventeurs, par technologie, 2012-15}

Les données indiquent le nombre moyen d'économies des inventeurs des familles de brevets IP5, par domaine technologique et par date de dépôt, en fonction de l'économie de résidence du détenteur du brevet. Les familles de brevets sont affectées aux domaines technologiques sur la base de leurs codes dans la Classification internationale des brevets (CIB), suivant la concordance établie par l'OMPI (2013). Les familles de brevets IP5 liés aux TIC sont recensées à l'aide des codes de la Classification internationale des brevets (CIB) dans Inaba et Squicciarini (2017). Seules les économies totalisant plus de 100 familles par domaine technologique sont incluses. 


\section{EXCELLENCE DE LA RECHERCHE ET COLLABORATION}

\section{Inventions TIC étrangères détenues par les économies, 2012-15}

Les inventions étrangères détenues dans les économies correspondent à la part, dans le nombre total de familles de brevets IP5 détenues dans une économie, de celles qui sont détenues par un résident de l'économie alors qu'aucun des inventeurs n'en est lui-même résident. Les données se rapportent aux familles de brevets IP5, par date de dépôt, en fonction du lieu de résidence des inventeurs, et avec comptages fractionnaires. Les familles de brevets IP5 liés aux TIC sont recensées à l'aide des codes de la Classification internationale des brevets (CIB) dans Inaba et Squicciarini (2017). Seules les économies totalisant plus de 100 familles au total et au moins 50 familles dans les TIC en 2012-15 sont incluses. Les chiffres de 2014 et 2015 sont estimés sur la base des données disponibles pour ces années.

\subsection{Collaboration en matière d'innovation}

\section{Entreprises menant des activités d'innovation en collaboration avec des établissements d'enseignement supérieur ou de recherche, par taille, 2012-14}

La comparabilité internationale peut être limitée du fait des différences méthodologiques entre les enquêtes sur l'innovation et des profils de réponses propres à chaque pays. Les pays européens suivent le schéma harmonisé de l'Enquête communautaire sur l'innovation. Pour en savoir plus, voir www.oecd.org/sti/inno-stats.htm.

La taille d'une entreprise est déterminée sur la base du nombre de ses employés. Les PME ont de 10 à 249 salariés, les grandes entreprises ont 250 salariés ou plus.

Pour les pays qui suivent le schéma de l'Enquête communautaire sur l'innovation de 2014 d'Eurostat, les données sur la collaboration en matière d'innovation se rapportent aux entreprises actives dans l'innovation de produit ou de procédé (y compris activités d'innovation en cours ou abandonnées). La couverture sectorielle principale inclut les sections et divisions B, C, D, E, G46, H, J, K et M71-72-73 de la CITI révision 4. Seules les entreprises de 10 salariés ou plus sont prises en considération.

Pour l'Australie, les données sont tirées de la Business Characteristics Survey (BCS) et se rapportent à l'exercice 2014-15. Les données sur la collaboration en matière d'innovation avec des établissements d'enseignement supérieur ou des établissements de recherche sont calculées en incluant les entreprises ayant collaboré avec (f) des universités ou d'autres établissements d'enseignement supérieur, (g) d'autres établissements de recherche (toutes options) ou (g) des établissements publics. Les données se rapportent aux entreprises actives dans l'innovation de produit, de procédé, de commercialisation ou d'organisation (y compris activités d'innovation en cours ou abandonnées). Les entreprises actives dans l'innovation de commercialisation ou d'organisation sont moins susceptibles de collaborer avec d'autres établissements. Les secteurs d'activité et tailles d'entreprises pris en considération coïncident avec le champ de l'Enquête communautaire sur l'innovation.

Pour le Brésil, les données sont tirées de l'enquête nationale sur l'innovation de 2014 (PINTEC) et se rapportent à la période $2012-14$. Les données se rapportent aux entreprises actives dans l'innovation de produit ou de procédé (y compris activités d'innovation en cours ou abandonnées). Les secteurs d'activité retenus pour cette enquête ne coïncident pas avec le champ principal de l'Enquête communautaire sur l'innovation. La section E de la CITI révision 4 est hors étude et seules certaines activités de services sont couvertes (divisions et groupes 592, 61, 62, 631, 71 et 72).

Pour le Chili, les données sont tirées de la 9 e enquête nationale sur l'innovation, se rapportent aux années 2013-14, et concernent les entreprises actives dans l'innovation de produit, de procédé, de commercialisation ou d'organisation (sans identification des activités d'innovation en cours ou abandonnées). Les entreprises actives dans l'innovation de commercialisation ou d'organisation sont moins susceptibles de collaborer avec d'autres établissements. Cette enquête porte sur les entreprises réalisant un chiffre d'affaires annuel supérieur à 2400 UF, sans limite de taille. La couverture sectorielle est plus large pour le secteur industriel et s'étend, au-delà des activités qui forment le champ principal de l'Enquête communautaire sur l'innovation, aux sections A (Agriculture, chasse et sylviculture), B (Pêche) et F (Construction) de la CITI révision 3. Les activités de services couvertes correspondent aux sections $\mathrm{G}, \mathrm{I}$, J et $\mathrm{K}$ de la CITI révision 3.

Pour la Corée, les données sont tirées de la Korean Innovation Survey. Cette enquête est menée séparément pour les activités manufacturières et pour les activités de services, mais les données se rapportent dans les deux cas à la période 2013-15. Les données se rapportent aux entreprises actives dans l'innovation de produit ou de procédé (y compris activités d'innovation en cours ou abandonnées). Le champ sectoriel, plus restreint que celui de l'Enquête communautaire sur l'innovation en ce qui concerne le secteur industriel, se limite à la section C (Activités de fabrication) de la CITI révision 4. Toutes les activités de services sont visées par l'enquête, à l'exception de celles relevant de la section O (Administration publique et défense ; sécurité sociale obligatoire). Pour l'Estonie, les données de l'Enquête communautaire sur l'innovation de 2014 ont fait l'objet d'une révision méthodologique. Il y a donc eu une rupture de série par rapport aux précédentes éditions de l'Enquête communautaire sur l'innovation.

Pour le Japon, les données proviennent de la Japanese National Innovation Survey (J-NIS 2015), se rapportent aux exercices 2012$13,2013-14$ et 2014-15, et concernent les entreprises actives dans l'innovation de produit ou de procédé (y compris activités d'innovation en cours ou abandonnées). Les secteurs d'activité et tailles d'entreprises pris en considération coïncident avec le champ de l'Enquête communautaire sur l'innovation.

Pour la Nouvelle-Zélande, les données se rapportent aux exercices 2012-13 et 2013-14 et concernent les entreprises de six employés ou plus avec un chiffre d'affaires annuel tel que calculé aux fins de la taxe sur les produits et services (TPS) supérieur à 30000 NZD. Les données portent sur les entreprises ayant collaboré avec des universités, des instituts polytechniques ou des instituts de recherche de la Couronne, d'autres instituts de recherche ou des associations de recherche, par exemple Callaghan Innovation. Les 
données se rapportent aux entreprises actives dans l'innovation de produit, de procédé, de commercialisation ou d'organisation (y compris activités d'innovation en cours ou abandonnées).

Pour la Suisse, les données sont tirées de l'Enquête sur l'innovation dans l'économie suisse et se rapportent à la période 2012-14. Les données qui concernent la collaboration portent uniquement sur la collaboration en R-D.

\section{Entreprises menant des activités d'innovation en collaboration avec des fournisseurs ou des clients, par taille, 2012-14}

La comparabilité internationale peut être limitée du fait des différences méthodologiques entre les enquêtes sur l'innovation et des profils de réponses propres à chaque pays. Les pays européens suivent le schéma harmonisé de l'Enquête communautaire sur l'innovation. Pour en savoir plus, voir www.oecd.org/sti/inno-stats.htm.

La taille d'une entreprise est déterminée sur la base du nombre de ses employés. Les PME s'entendent des entreprises de 10 à 249 salariés, et les grandes entreprises des entreprises de 250 salariés ou plus.

Pour les pays qui suivent le schéma de l'Enquête communautaire sur l'innovation de 2014 d'Eurostat, les données sur la collaboration en matière d'innovation se rapportent aux entreprises actives dans l'innovation de produit ou de procédé (y compris activités d'innovation en cours ou abandonnées). La couverture sectorielle principale inclut les sections et divisions B, C, D, E, G46, H, J, K et M71-72-73 de la CITI révision 4. Seules les entreprises de 10 salariés ou plus sont prises en considération.

Pour l'Australie, les données sont tirées de la Business Characteristics Survey (BCS) et se rapportent à l'exercice 2014-15. Les données sur la collaboration en matière d'innovation avec des établissements d'enseignement supérieur ou des établissements de recherche sont calculées en incluant les entreprises ayant collaboré avec (f) des universités ou d'autres établissements d'enseignement supérieur, (g) d'autres établissements de recherche (toutes options) ou (g) des établissements publics. Les données se rapportent aux entreprises actives dans l'innovation de produit, de procédé, de commercialisation ou d'organisation (y compris activités d'innovation en cours ou abandonnées). Les entreprises actives dans l'innovation de commercialisation ou d'organisation sont moins susceptibles de collaborer avec d'autres établissements. Les secteurs d'activité et tailles d'entreprises pris en considération coïncident avec le champ de l'Enquête communautaire sur l'innovation.

Pour le Brésil, les données sont tirées de l'enquête nationale sur l'innovation de 2014 (PINTEC) et se rapportent à la période 2012-14. Les données se rapportent aux entreprises actives dans l'innovation de produit ou de procédé (y compris activités d'innovation en cours ou abandonnées). Les secteurs d'activité retenus pour cette enquête ne coïncident pas avec le champ principal de l'Enquête communautaire sur l'innovation. La section E de la CITI révision 4 est hors étude et seules certaines activités de services sont couvertes (divisions et groupes 592, 61, 62, 631, 71 et 72).

Pour le Chili, les données sont tirées de la 9e enquête nationale sur l'innovation, se rapportent aux années 2013-14, et concernent les entreprises actives dans l'innovation de produit, de procédé, de commercialisation ou d'organisation (sans identification des activités d'innovation en cours ou abandonnées). Les entreprises actives dans l'innovation de commercialisation ou d'organisation sont moins susceptibles de collaborer avec d'autres établissements. Cette enquête porte sur les entreprises réalisant un chiffre d'affaires annuel supérieur à 2400 UF, sans limite de taille. La couverture sectorielle est plus large pour le secteur industriel et s'étend, au-delà des activités qui forment le champ principal de l'Enquête communautaire sur l'innovation, aux sections A (Agriculture, chasse et sylviculture), B (Pêche) et F (Construction) de la CITI révision 3. Les activités de services couvertes correspondent aux sections G, I, J et $\mathrm{K}$ de la CITI révision 3).

Pour la Corée, les données sont tirées de la Korean Innovation Survey. Cette enquête est menée séparément pour les activités manufacturières et pour les activités de services, mais les données se rapportent dans les deux cas à la période 2013-15. Les données se rapportent aux entreprises actives dans l'innovation de produit ou de procédé (y compris activités d'innovation en cours ou abandonnées). Le champ sectoriel, plus restreint que celui de l'Enquête communautaire sur l'innovation en ce qui concerne le secteur industriel, se limite à la section C (Activités de fabrication) de la CITI révision 4. Toutes les activités de services sont visées par l'enquête, à l'exception de celles relevant de la section O (Administration publique et défense ; sécurité sociale obligatoire).

Pour l'Estonie, les données de l'Enquête communautaire sur l'innovation de 2014 ont fait l'objet d'un examen méthodologique. Il y a donc eu une rupture de série par rapport aux précédentes éditions de l'Enquête communautaire sur l'innovation.

Pour le Japon, les données proviennent de la Japanese National Innovation Survey (J-NIS 2015), se rapportent aux exercices 2012-13, 2013-14 et 2014-15, et concernent les entreprises actives dans l'innovation de produit ou de procédé (y compris activités d'innovation en cours ou abandonnées). Les secteurs d'activité et tailles d'entreprises pris en considération coïncident avec le champ de l'Enquête communautaire sur l'innovation.

Pour la Nouvelle-Zélande, les données se rapportent aux exercices 2012-13 et 2013-14 et concernent les entreprises de six employés ou plus avec un chiffre d'affaires annuel tel que calculé aux fins de la taxe sur les produits et services (TPS) supérieur à 30000 NZD. Les données sur la collaboration en matière d'innovation avec des établissements d'enseignement supérieur ou des établissements de recherche sont calculées en incluant les entreprises ayant collaboré avec des universités, des instituts polytechniques ou des instituts de recherche de la Couronne, d'autres instituts de recherche ou des associations de recherche, par exemple Callaghan Innovation. Les données se rapportent aux entreprises actives dans l'innovation de produit, de procédé, de commercialisation ou d'organisation (y compris activités d'innovation en cours ou abandonnées).

Pour la Suisse, les données sont tirées de l'Enquête sur l'innovation dans l'économie suisse et se rapportent à la période 2012-14. Les données qui concernent la collaboration portent uniquement sur la collaboration en R-D. 


\section{EXCELLENCE DE LA RECHERCHE ET COLLABORATION}

\section{Entreprises engagées dans des collaborations internationales à des fins d'innovation, par taille, 2012-14}

La comparabilité internationale peut être limitée du fait des différences méthodologiques entre les enquêtes sur l'innovation et des profils de réponses propres à chaque pays. Les pays européens suivent le schéma harmonisé de l'Enquête communautaire sur l'innovation. Pour en savoir plus, voir www.oecd.org/sti/inno-stats.htm.

La taille d'une entreprise est déterminée sur la base du nombre de ses employés. Les PME s'entendent des entreprises de 10 à 250 salariés, et les grandes entreprises des entreprises de plus de 250 salariés.

Pour les pays qui suivent le schéma de l'Enquête communautaire sur l'innovation de 2014 d'Eurostat, les données sur la collaboration en matière d'innovation se rapportent aux entreprises actives dans l'innovation de produit ou de procédé (y compris activités d'innovation en cours ou abandonnées). La couverture sectorielle principale inclut les sections et divisions B, C, D, E, G46, H, J, K et M71-72-73 de la CITI révision 4. Seules les entreprises de 10 salariés ou plus sont prises en considération.

Pour l'Australie, les données sont tirées de la Business Characteristics Survey (BCS) et se rapportent à l'exercice 2014-15. Les données se rapportent aux entreprises actives dans l'innovation de produit, de procédé, de commercialisation ou d'organisation (y compris activités d'innovation en cours ou abandonnées). Les entreprises actives dans l'innovation de commercialisation ou d'organisation sont moins susceptibles de collaborer avec d'autres établissements. Les secteurs d'activité et tailles d'entreprises pris en considération coïncident avec le champ de l'Enquête communautaire sur l'innovation.

Pour le Brésil, les données sont tirées de l'enquête nationale sur l'innovation de 2014 (PINTEC) et se rapportent à la période 2012-14. Les données se rapportent aux entreprises actives dans l'innovation de produit ou de procédé (y compris activités d'innovation en cours ou abandonnées). Les secteurs d'activité retenus pour cette enquête ne coïncident pas avec le champ principal de l'Enquête communautaire sur l'innovation. La section E de la CITI révision 4 est hors étude et seules certaines activités de services sont couvertes (divisions et groupes 592, 61, 62, 631, 71 et 72).

Pour le Chili, les données sont tirées de l'enquête nationale sur l'innovation de 2015, se rapportent aux années 2013-14, et concernent les entreprises actives dans l'innovation de produit, de procédé, de commercialisation ou d'organisation (sans identification des activités d'innovation en cours ou abandonnées). Les entreprises actives dans l'innovation de commercialisation ou d'organisation sont moins susceptibles de collaborer avec d'autres établissements. Cette enquête porte sur les entreprises réalisant un chiffre d'affaires annuel supérieur à 2400 UF, sans limite de taille. La couverture sectorielle est plus large pour le secteur industriel et s'étend, au-delà des activités qui forment le champ principal de l'Enquête communautaire sur l'innovation, aux sections A (Agriculture, chasse et sylviculture), B (Pêche) et $\mathrm{F}$ (Construction) de la CITI révision 3. Les activités de services couvertes correspondent aux sections G, I, J et K de la CITI révision 3.

Pour la Corée, les données sont tirées de la Korean Innovation Survey. Cette enquête est menée séparément pour les activités manufacturières et pour les activités de services, mais les données se rapportent dans les deux cas à la période 2011-13. Les données se rapportent aux entreprises actives dans l'innovation de produit ou de procédé (y compris activités d'innovation en cours ou abandonnées). Le champ sectoriel, plus restreint que celui de l'Enquête communautaire sur l'innovation en ce qui concerne le secteur industriel, se limite à la section C (Activités de fabrication) de la CITI révision 4. Toutes les activités de services sont visées par l'enquête, à l'exception de celles relevant de la section O (Administration publique et défense ; sécurité sociale obligatoire).

Pour l'Estonie, les données de l'Enquête communautaire sur l'innovation de 2014 ont fait l'objet d'un examen méthodologique. Il y a donc eu une rupture de série par rapport aux précédentes éditions de l'Enquête communautaire sur l'innovation.

Pour le Japon, les données proviennent de la Japanese National Innovation Survey (J-NIS 2015), se rapportent aux exercices 201213, 2013-14 et 2014-15, et concernent les entreprises actives dans l'innovation de produit ou de procédé (y compris activités d'innovation en cours ou abandonnées). Les secteurs d'activité et tailles d'entreprises pris en considération coïncident avec le champ de l'Enquête communautaire sur l'innovation.

Pour la Nouvelle-Zélande, les données se rapportent aux exercices 2012-13 et 2013-14 et concernent les entreprises de six employés ou plus avec un chiffre d'affaires annuel tel que calculé aux fins de la taxe sur les produits et services (TPS) supérieur à 30000 NZD. Les données se rapportent aux entreprises actives dans l'innovation de produit, de procédé, de commercialisation ou d'organisation (y compris activités d'innovation en cours ou abandonnées).

Pour la Suisse, les données sont tirées de l'Enquête sur l'innovation dans l'économie suisse et se rapportent à la période 2012-14. Les données qui concernent la collaboration portent uniquement sur la collaboration en R-D.

\section{Références}

Appelt, S., F. Galindo-Rueda, R. de Pinho et B. van Beuzekom (2015), « Which factors influence the international mobility of research scientists? ", Documents de travail de l'OCDE sur la science, la technologie et l'industrie, n 2015/02, Éditions OCDE, Paris, http://dx.doi. org/10.1787/5js1tmrr2233-en.

Elsevier (2011), International Comparative Performance of the UK Research Base: 2011, étude commanditée par le ministère britannique des Entreprises, de l'Innovation et des Compétences.

González-Pereira, B., V.P. Guerrero-Bote, et F. Moya-Anegón (2010), « A new approach to the metric of journals' scientific prestige: The SJR indicator ", Journal of Informetrics, vol. 4, n³, pp. 379-391. 
Inaba, T. et M. Squicciarini (2017), «ICT: A new taxonomy based on the international patent classification ", Documents de travail de l'OCDE sur la science, la technologie et l'industrie, n² 2017/01, Éditions OCDE, Paris, http://dx.doi.org/10.1787/ab16c396-en.

Moed, H., M. Aisati et A. Plume (2013), « Studying scientific migration in Scopus », Scientometrics, vol. 94, n 3, pp. $929-942$.

Moya-Anegón, F., V. P. Guerrero-Bote, L. Bornmann et H. Moed (2013), « The research guarantors of scientific papers and the output counting: A promising new approach », Scientometrics, vol. 97, pp. 421-434.

OCDE/Eurostat (2005), Manuel d'Oslo : Principes directeurs pour le recueil et l'interprétation des données sur l'innovation, 3e édition, La mesure des activités scientifiques et technologiques, Éditions OCDE, Paris, http://dx.doi.org/10.1787/9789264013124-fr.

OCDE (2015), Manuel de Frascati 2015 : Lignes directrices pour le recueil et la communication des données sur la recherche et le développement expérimental, Éditions OCDE, Paris, http://dx.doi.org/10.1787/9789264257252-fr.

OCDE et SCImago Research Group (CSIC) (2016), Compendium of Bibliometric Science Indicators, http://oe.cd/scientometrics. 

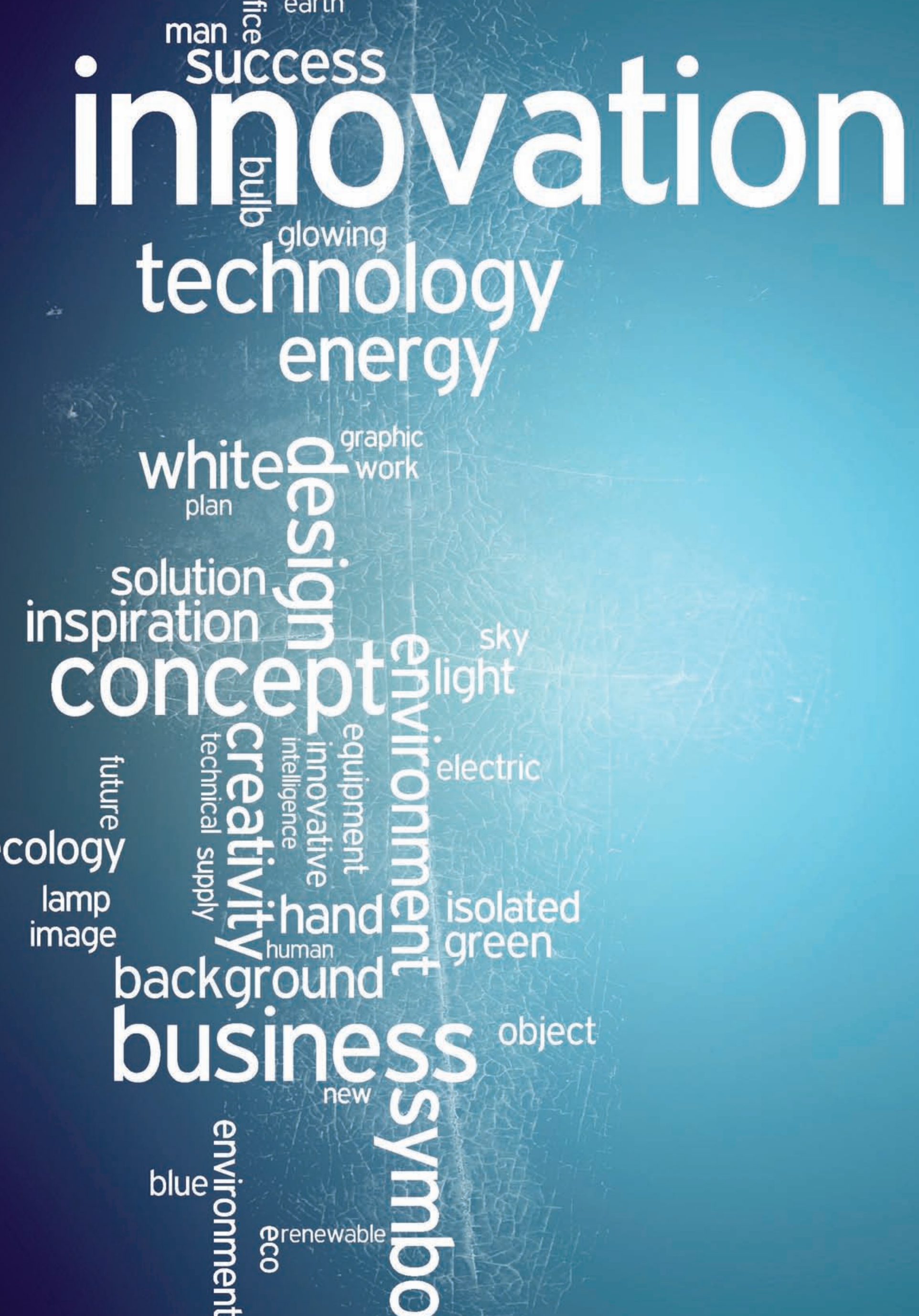


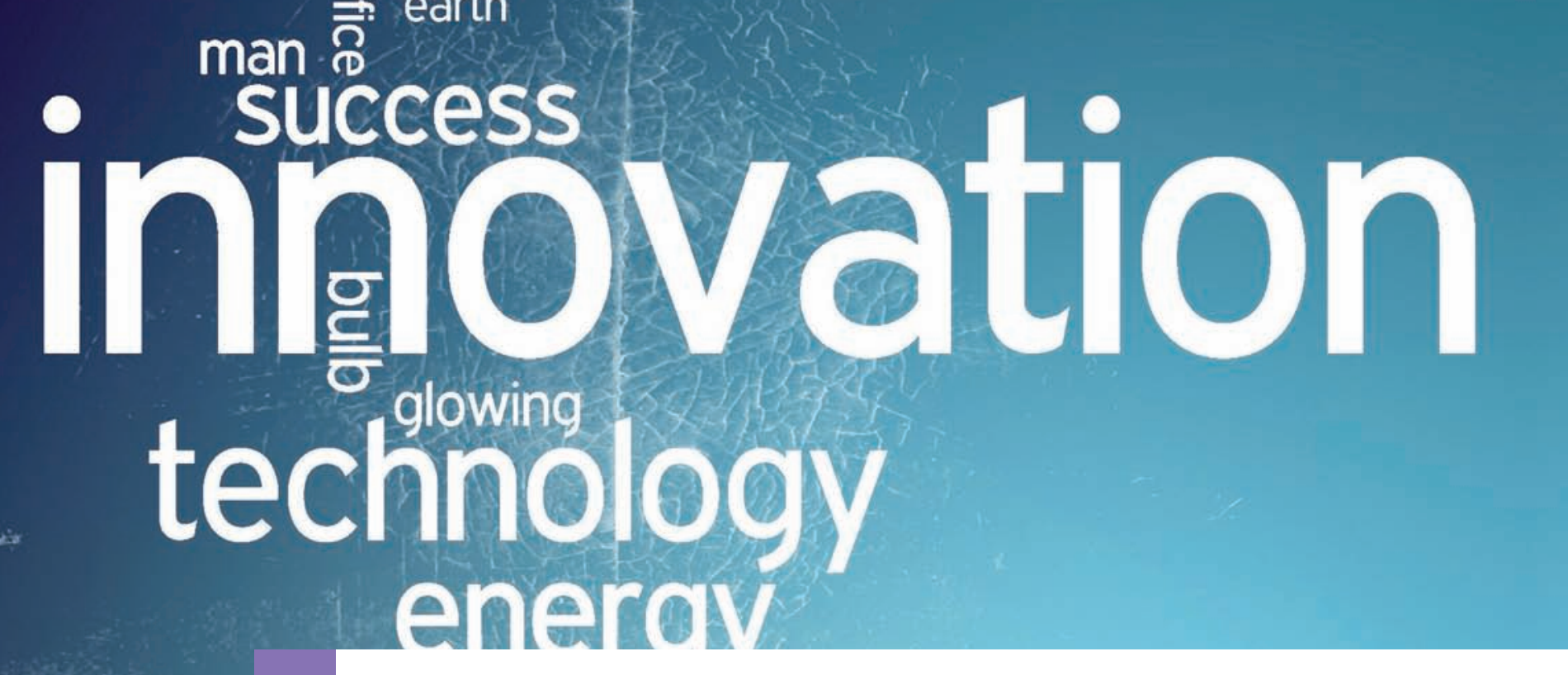

\section{L'INNOVATION DANS LES ENTREPRISES}

1. R-D des entreprises

2. Principaux acteurs de la R-D

3. Portefeuilles de PI

4. TIC et innovation

5. Panachage des modes d'innovation

6. Incitations fiscales en faveur de la R-D

7. Cadre de la politique économique et demande d'innovation Notes et références 


\section{R-D des entreprises, 2005 et $\mathbf{2 0 1 5}$}

En pourcentage des dépenses intérieures brutes de R-D

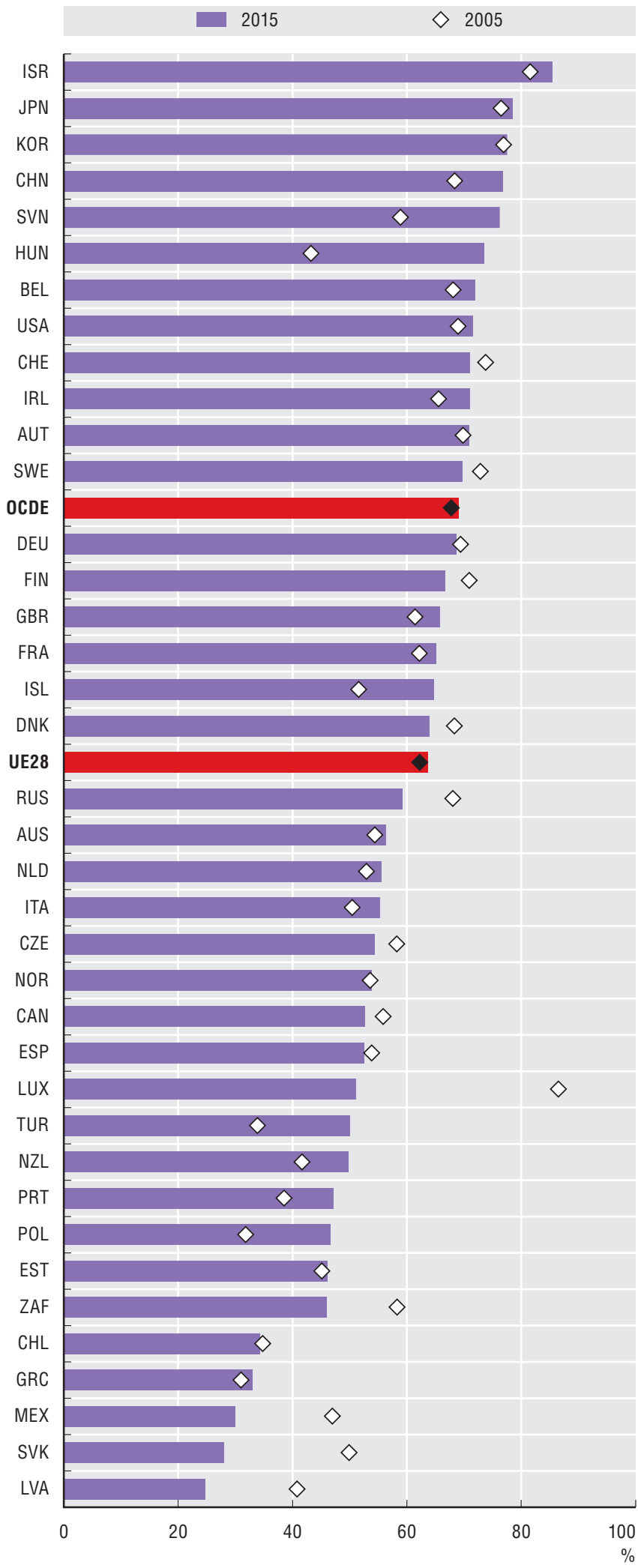

Source: OCDE, Base de données des Principaux indicateurs de la science et de la technologie, http://oe.cd/pist, juillet 2017. Davantage de données via StatLink. Voir notes de chapitre.

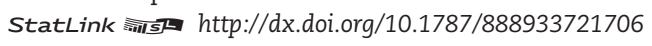

\section{Le saviez-vous?}

En moyenne, $35 \%$ de la R-D des entreprises est réalisée par des PME alors que celles-ci perçoivent près de $60 \%$ des crédits publics de la R-D destinés aux entreprises.

Principal exécutant de la R-D dans la plupart des économies, le secteur des entreprises est à l'origine de plus de $60 \%$ des dépenses de R-D réalisées dans la zone OCDE. Entre 2005 et 2015, la contribution des entreprises à la R-D est restée relativement stable dans la zone OCDE, au sein de l'Union européenne et aux États-Unis. En revanche, elle a bondi en Chine et en Turquie et a diminué en Afrique du Sud, en Fédération de Russie et au Mexique. Plusieurs États de l'UE ont par ailleurs connu un revirement de situation.

D'ordinaire, l'activité de R-D est l'apanage d'un petit nombre d'entreprises, en particulier de grandes dimensions. Dans certains pays, les petites et moyennes entreprises (PME) représentent une part importante des dépenses de R-D des entreprises (DIRDE). Cette part s'élève à $35 \%$ en moyenne, elle dépasse les deux tiers en Islande, en Lettonie et en NouvelleZélande et elle se situe sous la barre des $15 \%$ en Allemagne, aux États-Unis et au Japon. Le secteur des administrations publiques joue un rôle relativement secondaire dans l'exécution de la R-D, mais constitue une source importante de financement pour la $\mathrm{R}-\mathrm{D}$ de l'enseignement supérieur et des entreprises. Ce sont généralement les PME qui perçoivent la plus grande partie de ces fonds, $60 \%$ en moyenne, voire $100 \%$ en Estonie et en Lettonie. L'intensité de R-D varie d'un secteur et d'un pays à l'autre. Elle est particulièrement forte dans les branches d'activité liées aux technologies numériques, comme les équipements TIC et les services d'information. Ainsi, l'industrie des équipements TIC (fabrication d'ordinateurs, d'articles électroniques et optiques) est la plus intensive en R-D (24.7\%) juste derrière l'industrie pharmaceutique (25.1\%). Tous secteurs confondus, l'intensité moyenne de R-D s'élève à $5 \%$. De par les activités engagées dans des domaines comme le rendement énergétique des véhicules et l'automatisation, l'industrie automobile fait partie des 10 secteurs les plus intensifs dans la zone OCDE, avec $17 \%$ en Allemagne et $19 \%$ en Suède.

\section{Définitions}

Les dépenses intérieures brutes de R-D sont établies pour les secteurs exécutant des activités de R-D. Les dépenses intérieures de R-D des entreprises (DIRDE) couvrent les dépenses brutes de R-D engagées par l'ensemble des firmes, organisations et institutions (publiques et privées) dont l'activité première est la production marchande de biens ou de services (autres que d'enseignement supérieur) destinés à être vendus au public à un prix économiquement significatif, ainsi que par les institutions privées sans but lucratif servant principalement leurs intérêts.

La R-D des entreprises financée par l'État désigne les activités de R-D des entreprises commerciales que celles-ci imputent au financement public direct, c'est-à-dire les subventions et les paiements effectués au titre des marchés publics de R-D, à l'exclusion des incitations fiscales en faveur de la R-D, des avances remboursables et des prises de participation.

L'intensité de R-D d'une unité, d'une branche d'activité, d'un secteur ou d'un pays correspond à la part de ses dépenses de R-D dans sa valeur ajoutée.

Les petites et moyennes entreprises (PME) sont les entreprises dont le nombre de salariés est inférieur ou égal à 249. 
R-D des entreprises et aides publiques, par taille d'entreprise, 2015

Part des PME, en pourcentage du total correspondant

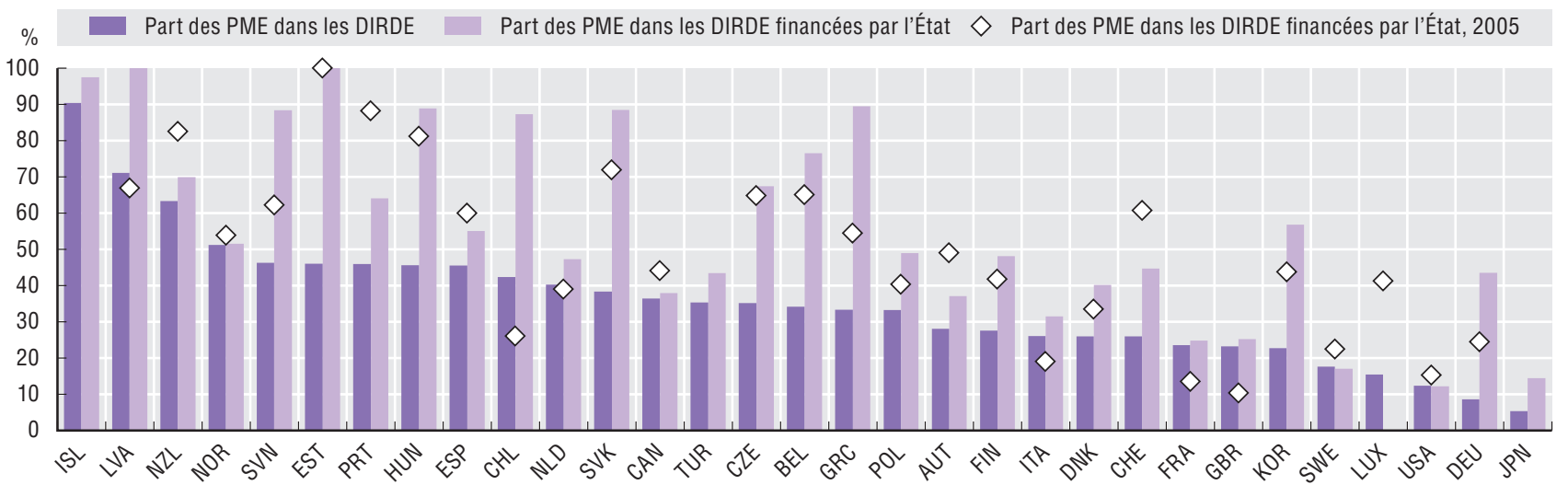

Source : OCDE, Base de données sur les Statistiques de la recherche et développement), http://oe.cd/srd-fr, juin 2017. Davantage de données via StatLink. Voir notes de chapitre.

Intensité de R-D par secteur, 2015

En pourcentage de la valeur ajoutée brute, échelle logarithmique

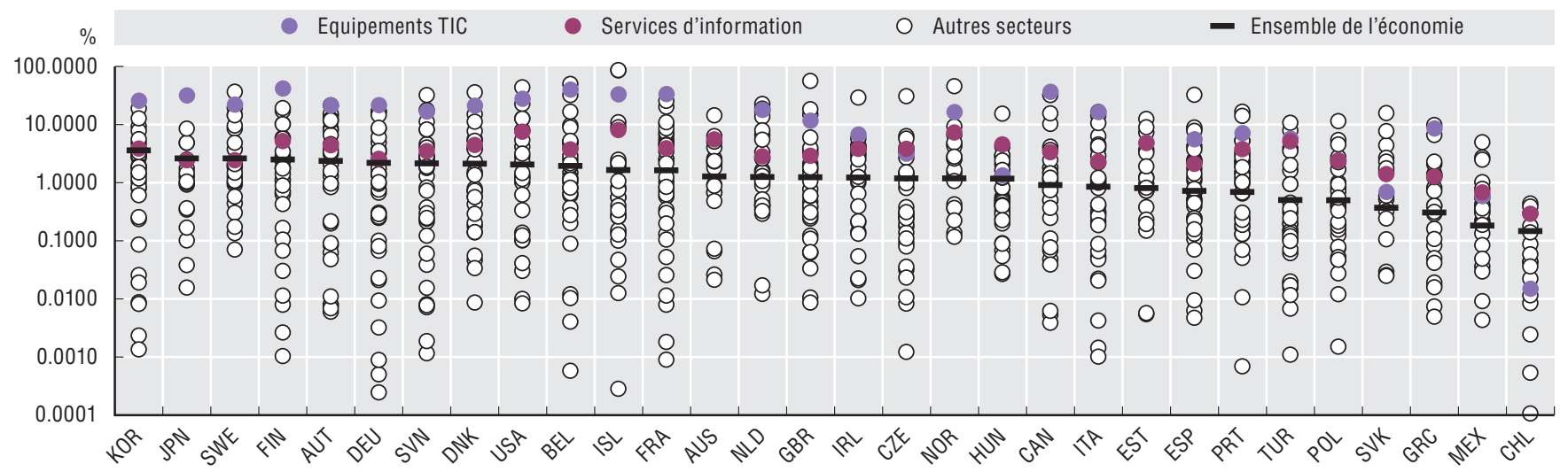

Source : Calculs de l'OCDE, d'après les bases de données ANBERD, http://oe.cd/anberd-fr, STAN, http://oe.cd/stan-fr, Comptes nationaux et Statistiques de la recherche et développement, http://oe.cd/srd-fr, juin 2017. Davantage de données via StatLink. Voir notes de chapitre.

StatLink त्ञाता http://dx.doi.org/10.1787/888933721744

\section{Mesurabilité}

Les DIRDE sont communément mesurées à partir d'enquêtes officielles sur le volume et la nature des dépenses de R-D des entreprises, qui concernent notamment l'origine des fonds employés. Ces enquêtes et toutes autres sources connexes, comme les registres des entreprises, fournissent également des informations utiles sur le contexte, telles que le nombre de salariés et la nature de la principale activité de production (c'est-à-dire la principale source de valeur ajoutée). Les DIRDE sont le premier critère sur la base duquel le classement sectoriel des activités de R-D est établi, tel que recommandé dans le Manuel de Frascati 2015 de l'OCDE (http://oe.cd/frascati-fr). Cependant, dans la pratique, plusieurs pays tiennent également compte d'un élément de redistribution fondé sur des renseignements plus détaillés quant au type de produits ou productions associés à l'activité de R-D (autrement dit, la R-D est ventilée par " orientation sectorielle »). Sont exclus les pays pour lesquels la décomposition de la R-D est limitée et pour lesquels il n'existe pas de données sur la valeur ajoutée par activité qui soient comparables et compilées selon le SCN.

Les données sur le financement public des DIRDE ne couvrent que les aides directes. Le soutien indirect (tel que le manque à gagner lié aux crédits d'impôt pour la R-D) est également important. Les données actuellement disponibles sur le soutien public alloué par taille d'entreprise ne permettent pas de distinguer les PME affiliées à un groupe de celles qui sont indépendantes. 


\section{Dépenses de R-D par brevet dans les entreprises les plus actives en $R-D$, selon l'implantation du siège, 2012-14 \\ En millions USD par famille de brevets IP5}

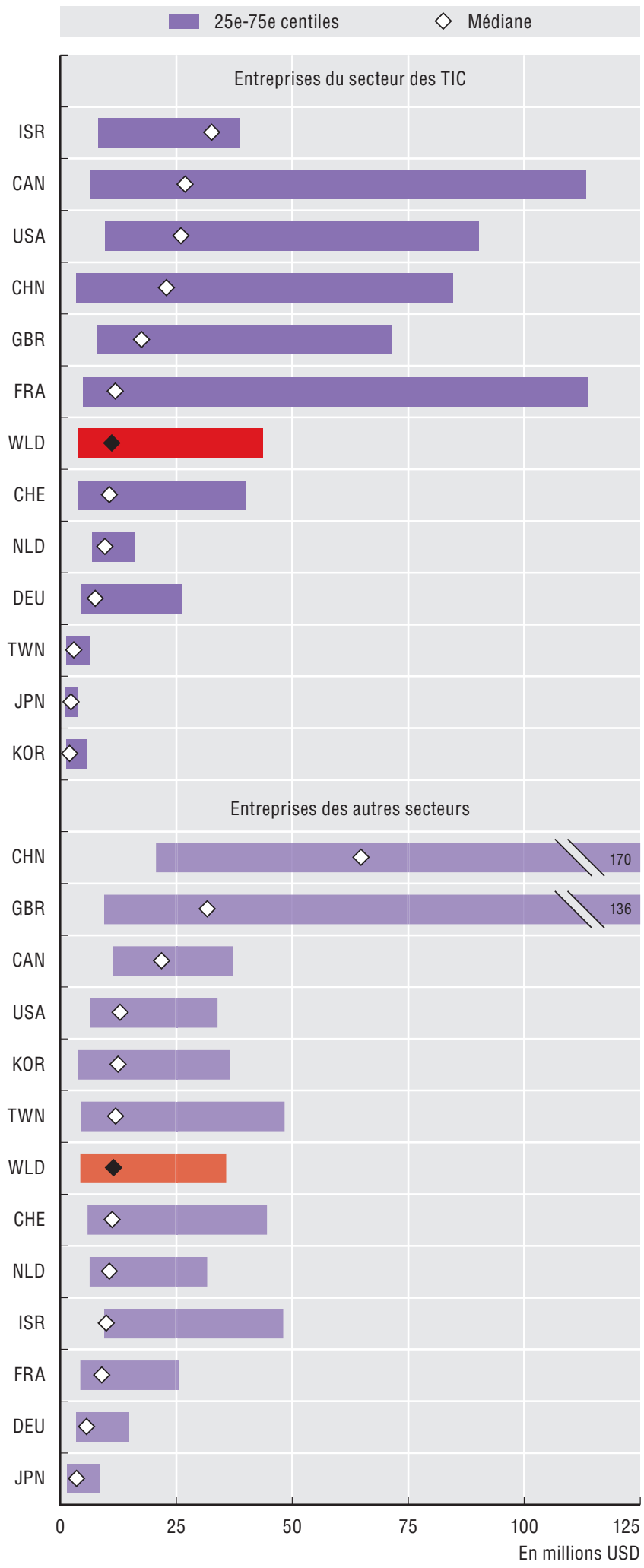

Source : Calculs de l'OCDE d'après CCR-OCDE, Base de données COR\&DIP@ v. 1, juin 2017. Davantage de données via StatLink. Voir notes de chapitre.

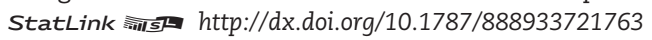

\section{Le saviez-vous?}

Les géants des TIC consacrent en moyenne plus de 11 millions USD à la R-D par famille de brevets IP5, montant atteignant 33 millions USD en Israël.

Les entreprises qui investissent le plus dans la recherche et développement (R-D) sont des acteurs mondiaux de premier plan à la frontière technologique. Elles détiennent environ $64 \%$ de l'ensemble des familles de brevets déposés auprès des cinq principaux offices de propriété intellectuelle au monde (IP5). Examiner dans quelle mesure ces grands acteurs investissent dans la R-D, diversifient leurs activités technologiques et sectorielles et s'appuient sur les marques pour rivaliser permet d'appréhender les tendances mondiales et le champ des futurs possibles.

Le montant médian investi par les entreprises des TIC les plus actives en R-D s'échelonne de 33 millions USD environ (Israël) à 1.9 million USD (Corée) par famille de brevets IP5. L'investissement médian par brevet réalisé dans les autres branches d'activité se situe entre 3.4 millions USD (Japon) et plus de 65 millions USD (Chine). Ces derniers chiffres révèlent des divergences de propension des entreprises à déposer des brevets et de composition sectorielle selon les économies.

Spécialisation technologique et spécialisation sectorielle ne vont manifestement pas de pair parmi les entreprises qui investissent le plus dans la R-D. Le niveau médian enregistré dans les quatre premiers domaines de spécialisation technologique oscille entre moins de $40 \%$ (Machines et Construction) et $85 \%$ ou plus (Télécommunications et R-D scientifique). À l'inverse, tous secteurs confondus, au moins la moitié des filiales des entreprises les plus actives en R-D interviennent dans quatre branches d'activité seulement. Cette proportion dépasse $80 \%$ dans le cas des Produits alimentaires. Le nombre médian d'économies dans lesquelles ces filiales sont implantées varie lui aussi. La plupart des principaux exécutants de la R-D comptent des filiales dans plus de 10 économies.

Les entreprises les plus actives R-D s'appuient à des degrés divers sur des marques commerciales. Dans les branches d'activité liées aux TIC, le montant médian des ventes nettes par marque déposée s'élève à 285 millions USD, contre près de 320 millions USD dans d'autres secteurs. Cet écart tient au niveau plus élevé de diversification sectorielle et économique des entreprises opérant dans d'autres secteurs que les TIC, ainsi qu'à leur positionnement en aval ou en amont des chaînes de valeur mondiales.

\section{Définitions}

Les indices de spécialisation sectorielle et technologique sont construits de la même manière que les ratios de concentration. L'indice de spécialisation sectorielle des principaux acteurs de la $\mathrm{R}-\mathrm{D}$ dans un secteur d'activité donné correspond au poids de leurs filiales dans les quatre secteurs prépondérants, rapporté au total de leurs filiales. L'indice de spécialisation technologique de ces entreprises correspond au poids de leurs brevets dans les quatre domaines technologiques où elles en déposent le plus, rapporté au total des brevets qu'elles ont déposés. 
Spécialisation sectorielle et technologique des entreprises les plus actives en R-D, 2012-14

Spécialisation au regard du ratio de concentration (4) aux niveaux sectoriel et technologique

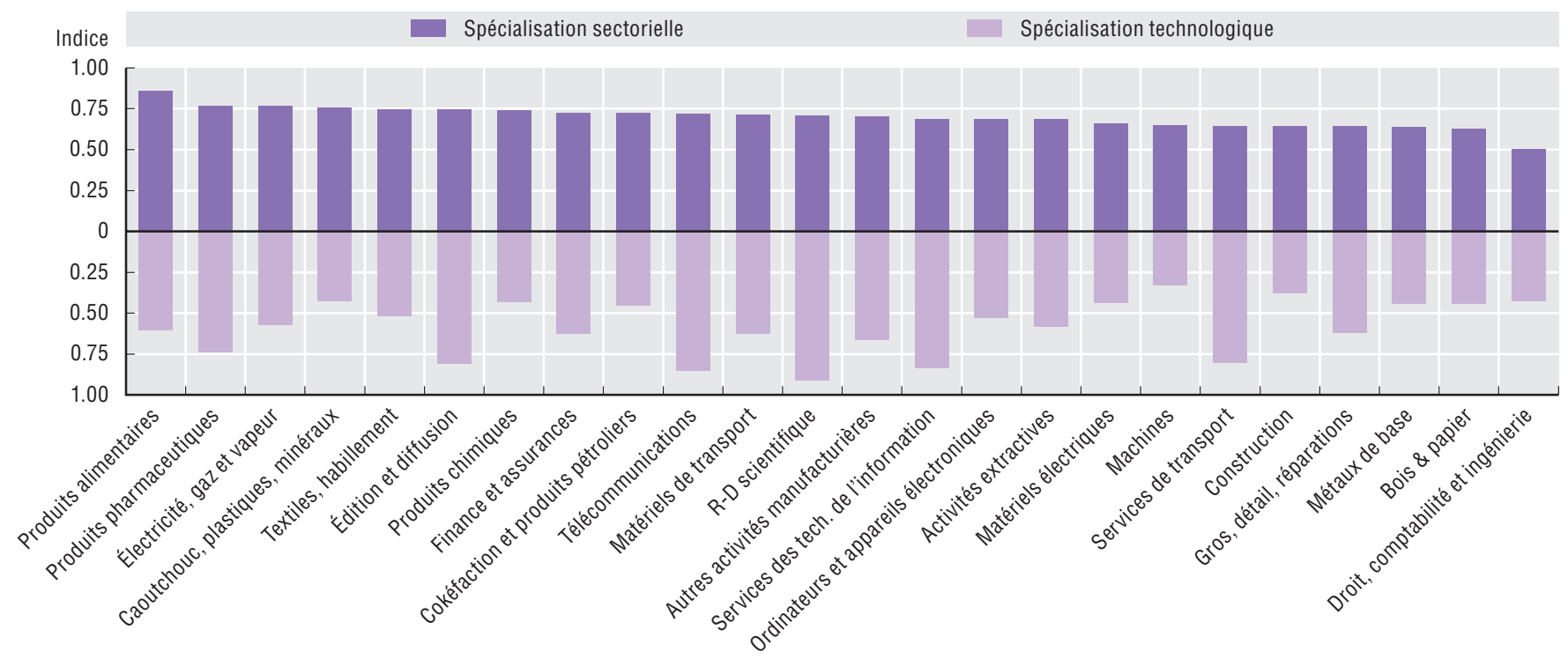

Source : Calculs de l'OCDE d'après CCR-OCDE, Base de données COR\&DIP@ v. 1, juin 2017. Davantage de données via StatLink. Voir notes de chapitre.

StatLink +intst $h$ ttp://dx.doi.org/10.1787/888933721782

Ventes nettes par dépôt de marques des entreprises des TIC les plus actives en R-D, selon l'implantation du siège, 2012-14 En millions USD par dépôt de marques auprès de l'EUIPO, du JPO et de l'USPTO

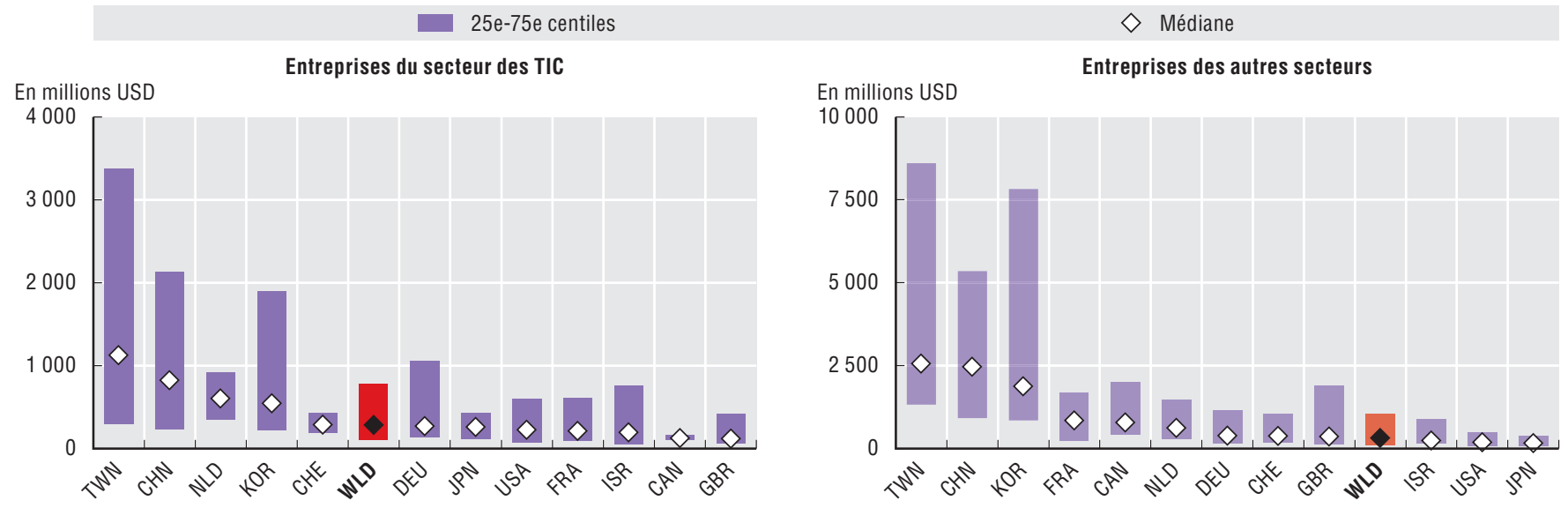

Source : Calculs de l'OCDE d'après CCR-OCDE, Base de données COR\&DIP@ v. 1, juin 2017. Davantage de données via StatLink. Voir notes de chapitre. StatLink intsta http://dx.doi.org/10.1787/888933721801

\section{Mesurabilité}

Les branches d'activité sont définies conformément à la CITI rév. 4. Le secteur des TIC comprend les Activités de fabrication dans le secteur des TIC (classes 2610, 2620, 2630, 2640 et 2680), les Activités commerciales du secteur des TIC (4651 et 4652 ), les Activités de services dans le secteur des TIC (5820), les Télécommunications (61), la Programmation informatique (62), les Activités de traitement de données (631) et la Réparation d'ordinateurs et de matériel de communication (951). Les données sur les brevets se rapportent aux familles de brevets déposés auprès des cinq principaux offices de propriété intellectuelle au monde, ou IP5 (www.fiveipoffices.org). Les données sur les marques correspondent aux nouvelles marque enregistrées auprès de l'Office de l'Union européenne pour la propriété intellectuelle (EUIPO), du Japan Patent Office (JPO) et de l'United States Patent and Trademark Office (USPTO). Les dépenses de R-D et les ventes nettes sont exprimées en millions USD. Les familles de brevets sont comptabilisées selon la date du premier dépôt, et les marques en fonction de la date de la demande d'enregistrement. Dans l'un et l'autre cas, les indicateurs reposent sur l'activité principale du siège de l'entreprise déposante et les comptages sont fractionnaires. Des divergences peuvent apparaître dans les chiffres suivant les types de familles de brevets, les autorités de la propriété intellectuelle et/ou la période considérée. Aucune comparaison temporelle n'est fournie car le classement et la structure des principaux exécutants de R-D sont susceptibles d'évoluer dans le temps. 
Brevets liés aux TIC, 2002-05 et 2012-15

En pourcentage du total des familles de brevets IP5 détenues dans les économies

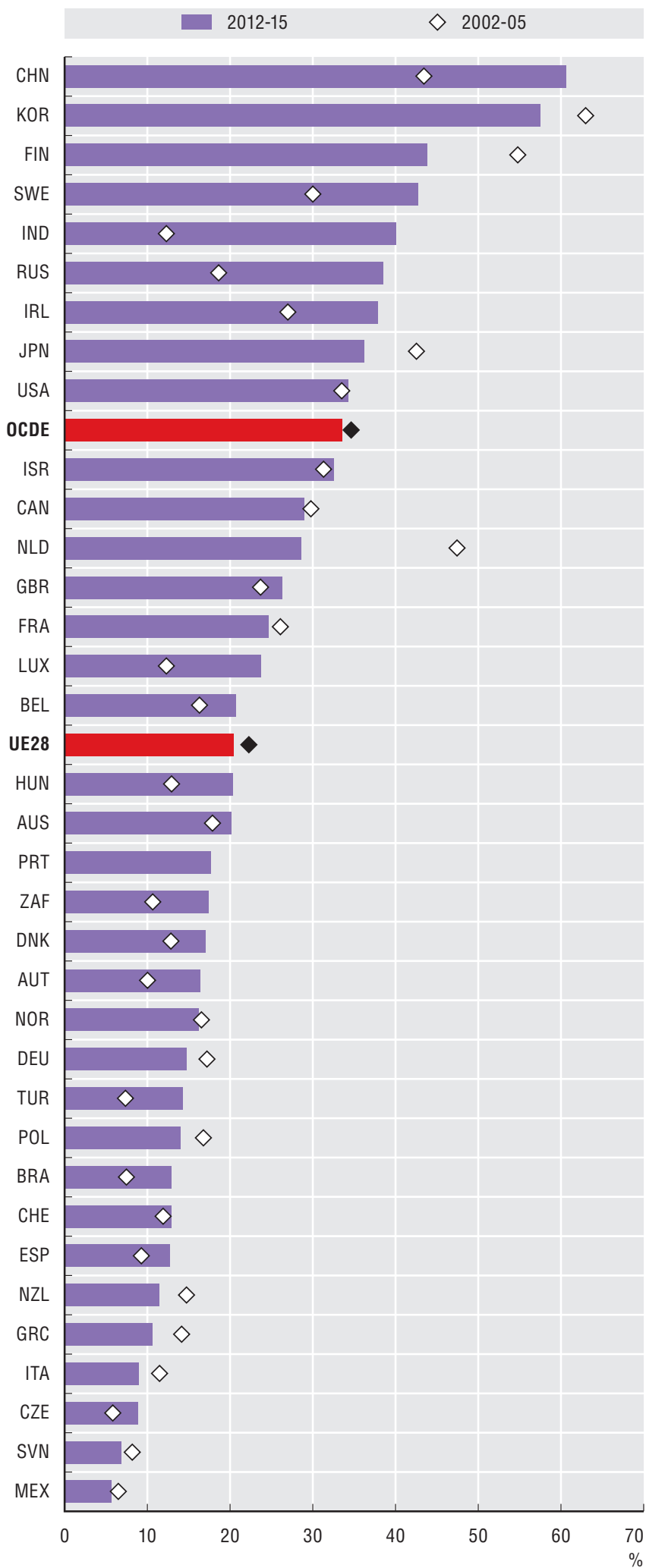

Source : OCDE, STI Microdata Lab : Base de données sur la propriété intellectuelle, http://oe.cd/ipstats, juin 2017. Davantage de données via StatLink. Voir notes de chapitre.

StatLink न्ता Th http://dx.doi.org/10.1787/888933721820

\section{Le saviez-vous?}

La majorité des brevets (57\%), marques (jusqu'à $65 \%$ ) et dessins et modèles (jusqu'à $62 \%$ ) qui composent le portefeuille de propriété intellectuelle de la Corée concernent les TIC.

Soutenir la concurrence sur les marchés mondiaux des technologies de l'information et des communications (TIC) suppose d'allier innovations et progrès technologiques à des dessins ou modèles attractifs, tout en permettant aux consommateurs de reconnaître les produits nouveaux, souvent complexes, qui leur sont proposés.

Au cours de la période 2012-15, les brevets liés aux TIC ont représenté quelque $34 \%$ du total des familles de brevets IP5 déposés par les pays de l'OCDE, soit presqu'autant que dix ans auparavant. À l'inverse, ce chiffre s'est accru de $40 \%$ en Chine, où le portefeuille de brevets IP5 est devenu le plus spécialisé dans les TIC.

Entre 2005 et 2015, les dessins ou modèles de produits TIC déposées par les pays de l'OCDE ont gagné du terrain aux ÉtatsUnis (+14\%) et sur les marchés européens (+9\%), mais en ont perdu au Japon (-32\%). Les BRIICS, eux, ont triplé la part des brevets de dessin ou modèle liés aux TIC qu'ils ont déposés aux États-Unis, accru de plus de $60 \%$ la part des dessins ou modèles liés aux TIC enregistrés en Europe et fait reculer ce chiffre d'environ $17 \%$ au Japon.

À l'inverse, la part des marques liées aux TIC a progressé sur tous les marchés étudiés pour la période 2012-15, en premier lieu en Europe (+35\%), puis au Japon (+27\%) et aux États-Unis (+21\%).

De manière générale, les pays de l'OCDE semblent progressivement se détourner de l'innovation technologique dans leurs stratégies de portefeuille de propriété intellectuelle pour privilégier l'aspect et les fonctionnalités des produits et pour exploiter le poids des marques. A contrario, les BRIICS poursuivent manifestement des stratégies de rattrapage technologique tout en protégeant leurs produits moyennant l'enregistrement de dessins, modèles et marques.

\section{Définitions}

Les brevets protègent des inventions technologiques, c'està-dire des produits ou procédés qui apportent de nouvelles façons de faire ou de nouvelles solutions technologiques à des problèmes. Les familles de brevets IP5 sont composées de brevets déposés auprès des cinq principaux offices de propriété intellectuelle (IP5). Les brevets liés aux TIC sont recensés à l'aide des codes de la Classification internationale des brevets (CIB) (voir Inaba et Squicciarini, 2017). Les dessins ou modèles industriels protègent les éléments nouveaux et/ou originaux qui concernent l'aspect, la configuration ou l'esthétique des produits. Les marques sont des signes distinctifs, par exemple des mots ou des symboles, utilisés pour distinguer les biens ou services d'une entreprise de ceux de ses concurrents. Les modèles, dessins et marques liés aux TIC sont recensés suivant une méthode expérimentale mise au point par l'OCDE sur la base des classifications de Locarno et de Nice, respectivement, et l'exercice repose sur une méthode normative combinée à l'utilisation de mots clés liés aux TIC. 
Dépôts de marques liées aux TIC, 2012-15

En pourcentage du nombre total de marques déposées auprès de l'EUIPO, du JPO et de l'USPTO

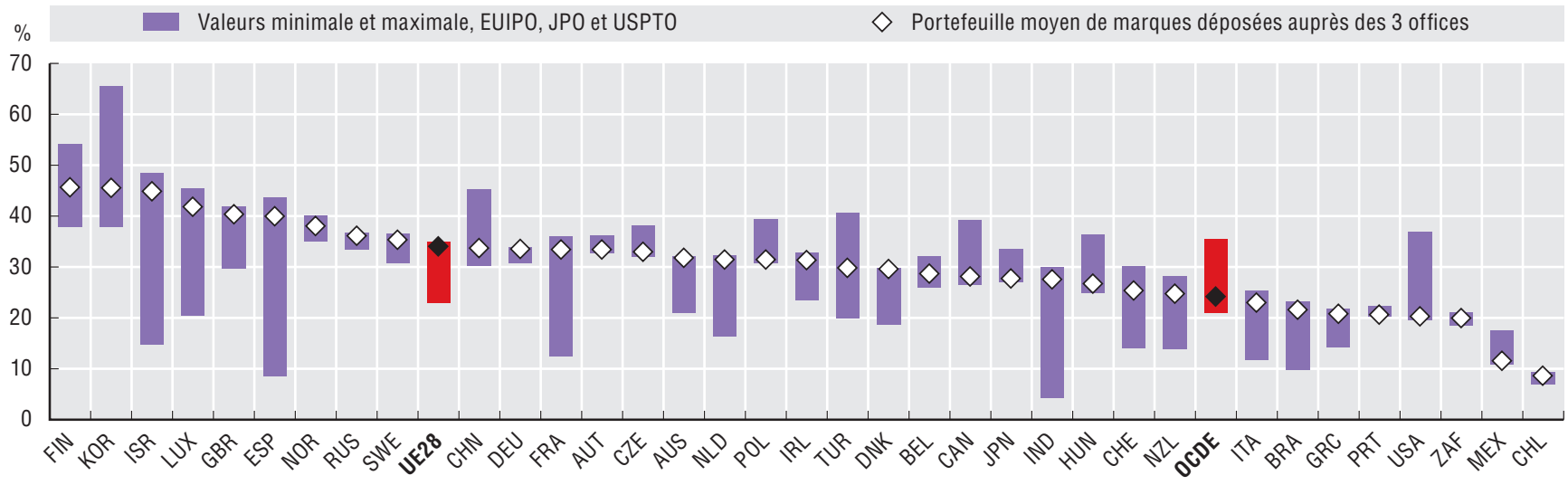

Source : OCDE, STI Microdata Lab : Base de données sur la propriété intellectuelle, http://oe.cd/ipstats, juin 2017. Davantage de données via StatLink. Voir notes de chapitre.

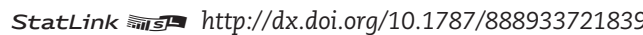

Enregistrement de dessins et modèles liés aux TIC, 2012-15

En pourcentage du nombre total de dessins et modèles déposés auprès de l'EUIPO, du JPO et de l'USPTO

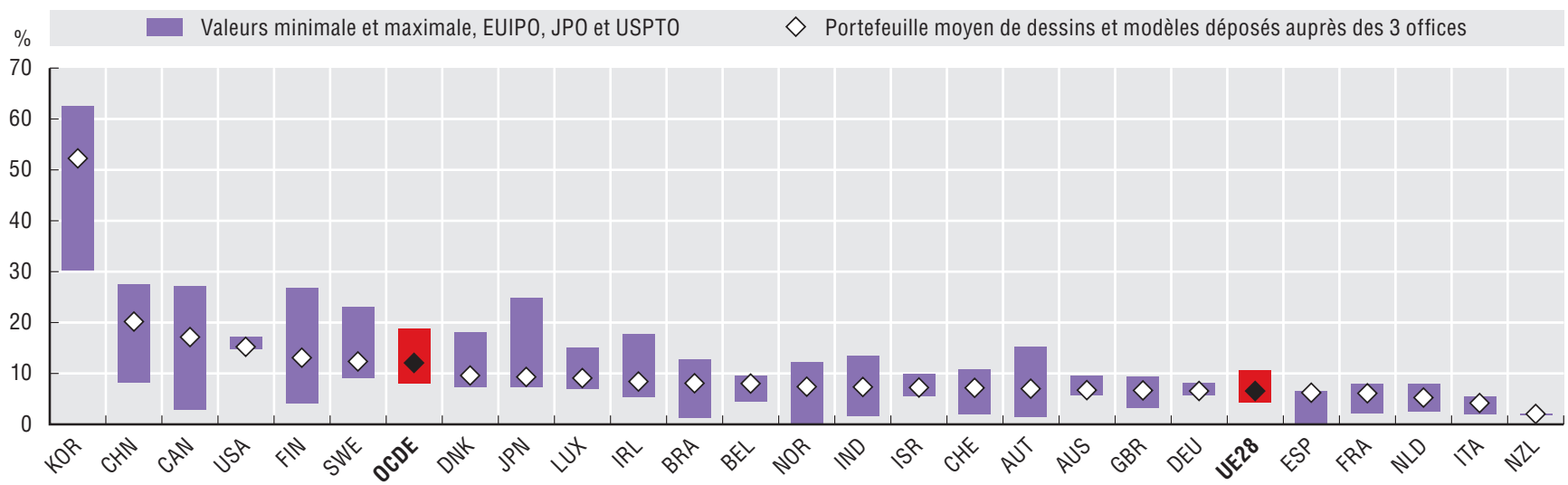

Source : OCDE, STI Microdata Lab : Base de données sur la propriété intellectuelle, http://oe.cd/ipstats, juin 2017. Davantage de données via StatLink. Voir notes de chapitre.

\section{Mesurabilité}

Les droits de propriété intellectuelle sont fondés sur le principe de territorialité. Les brevets, dessins, modèles et marques ne sont protégés que dans les pays où ils ont été déposés et enregistrés. La date de priorité des brevets, c'est-à-dire la date à laquelle un brevet fait l'objet d'une première demande de dépôt avant de voir sa protection étendue à d'autres juridictions de la propriété intellectuelle, permet de reconstituer les familles de brevets et d'éviter le double comptage des actifs intellectuels. Dans le cas des marques, dessins et modèles, il n'est pas possible de procéder de même car pour peu qu'elle existe, l'information sur les cas d'enregistrements multiples n'est que très rarement, voire jamais disponible. Aux États-Unis, les dessins et modèles sont protégés par des brevets dédiés (auprès de l'United States Patents and Trademark Office, USPTO), tandis qu'en Europe (Office de l'Union européenne pour la propriété intellectuelle, EUIPO) et au Japon (Japan Patent Office, JPO), cette protection découle de l'enregistrement des dessins et modèles industriels. Faute de données, il n'est pas possible de reconstituer les portefeuilles de dessins, modèles et marques enregistrés auprès des IP5, comme dans le cas des brevets. La définition de brevets liés aux TIC indiquée par Inaba et Squicciarini (2017) rejoint celles que l'OCDE donne de " secteur des TIC » (2007) et de " produits TIC » (2008). 


\section{Dépenses de R-D dans les équipements TIC et les services d'information, 2015}

En pourcentage des dépenses intérieures de R-D des entreprises
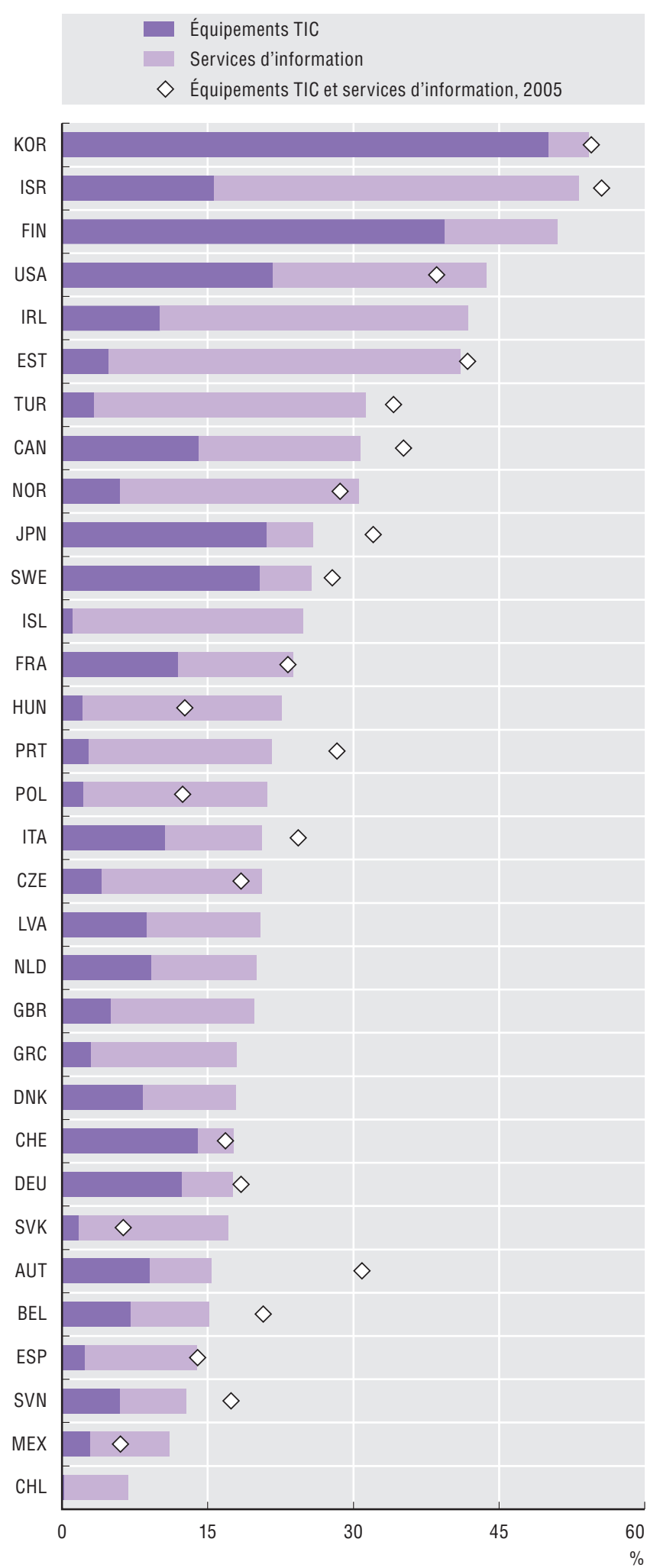

Source : OCDE, d'après les bases de données ANBERD, http://oe.cd/anberd-fr et Statistiques de la recherche et développement, http://oe.cd/srd-fr, juin 2017. Davantage de données via StatLink. Voir notes de chapitre. StatLink ints http://dx.doi.org/10.1787/888933721877

\section{Le saviez-vous ?}

La Chine, la Corée, les États-Unis et le Japon se partagent entre $70 \%$ et $80 \%$ des inventions brevetées liées aux TIC.

Les technologies de l'information et des communications (TIC) font partie des grands ressorts qui animent l'innovation. Dans la plupart des économies de l'OCDE, les «secteurs de l'information " absorbent à peu près un quart des dépenses intérieures de R-D des entreprises (DIRDE). En Corée, aux ÉtatsUnis, en Finlande et en Israël, cette proportion se situe entre $40 \%$ et plus de $50 \%$. Les DIRDE liées aux TIC représentent à elles seules entre $0.8 \%$ et $1.9 \%$ du PIB, ce qui atteste la forte intensité de recherche de ces économies et du secteur des TIC lui-même.

Les brevets permettent de mesurer le pouvoir d'innovation de l'investissement dans la R-D. Les inventions brevetées dans les TIC au cours de la période 2012-15 relèvent en majorité des trois domaines suivants : dispositifs d'information et de communication (environ $27 \%$ ), technologies de l'image et du son (environ $15 \%$ ) et réseaux haut débit (environ $12 \%$ ). Les ÉtatsUnis occupent la première place dans sept des 13 domaines TIC couverts par ces brevets, à savoir le traitement des données à grande vitesse, l'analyse massive d'informations (environ $33 \%$, respectivement) et la sécurité (28\%). Le Japon domine dans cinq domaines, dont les technologies de l'image et du son (37\%), et la Corée dans celui des technologies d'interface humaine (24\%). La Chine, en revanche, représente, selon le domaine considéré, entre $5 \%$ et $17 \%$ des inventions brevetées dans les TIC.

L'innovation couvre un éventail d'activités plus large que la R-D. À la différence des brevets et autres sources de valeur, les innovations (de produit, de procédé et de méthode) ont besoin d'être mises sur le marché ou adoptées par les entreprises. En moyenne, $74 \%$ des fabricants de biens TIC ont été à l'origine d'innovations au cours de la période 2012-14, contre $51 \%$ de l'ensemble des entreprises manufacturières. Les services TIC comptent eux aussi une plus forte proportion d'entreprises innovantes que les activités de services couvertes par les enquêtes sur l'innovation (64\% contre $50 \%$ ).

\section{Définitions}

Les dépenses de R-D des entreprises (DIRDE) recouvrent toutes les dépenses induites par les activités de R-D des entreprises, quelle que soit l'origine des fonds employés. Ces dépenses sont réparties selon la principale source de valeur ajoutée de l'entreprise. Les secteurs de l'information regroupent le secteur des TIC et le secteur des supports et contenus numériques.

Les familles de brevets IP5 regroupent les brevets déposés auprès des cinq principaux offices de propriété intellectuelle (IP5, www.fiveipoffices.org). Les brevets liés aux TIC sont recensés à l'aide des codes de la Classification internationale des brevets (CIB) (voir Inaba et Squicciarini, 2017) et conformément aux définitions de "secteur des TIC " (2007) et " produits TIC " (2008) formulées par l'OCDE.

Une entreprise innovante est une entreprise qui a introduit un produit ou un procédé nouveau ou sensiblement amélioré ou qui a mis en place une nouvelle méthode de commercialisation ou un nouveau mode d'organisation au cours de la période de référence. 
Brevets liés aux TIC et principaux acteurs, 2012-15

Part des cinq principaux acteurs du domaine considéré

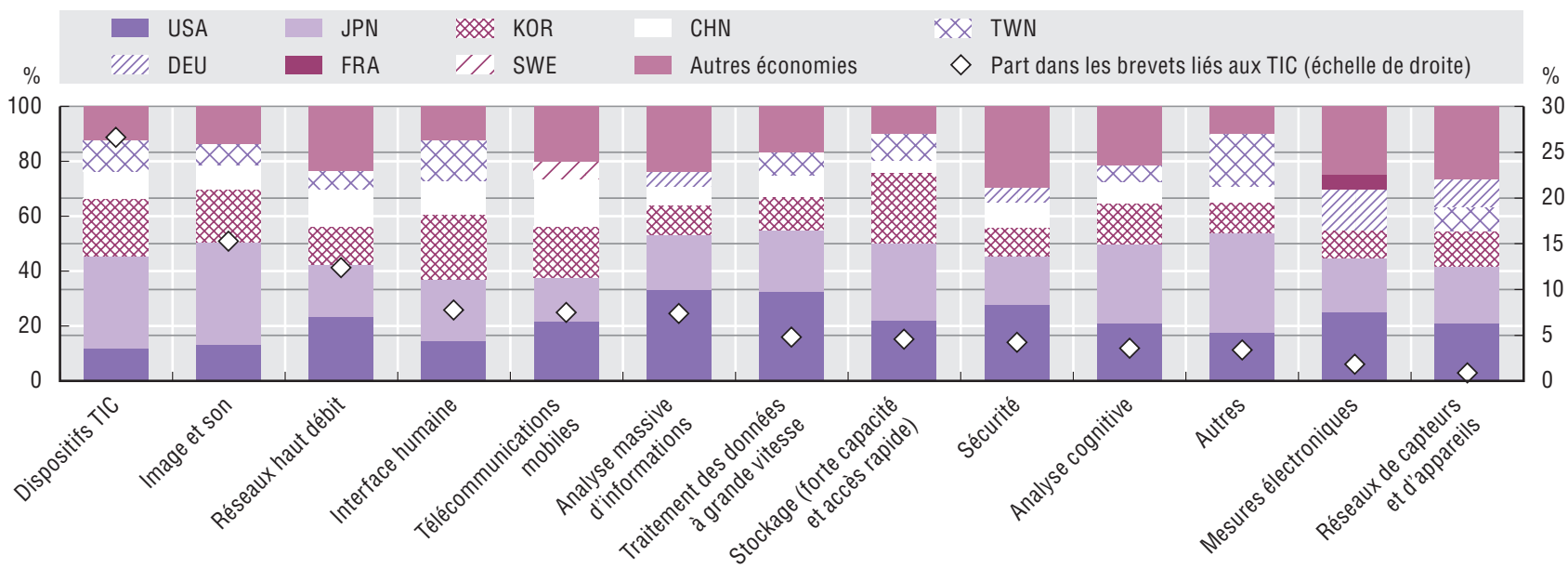

Source : OCDE, STI Microdata Lab : Base de données sur la propriété intellectuelle, http://oe.cd/ipstats, juin 2017. Davantage de données via StatLink. Voir notes de chapitre.

Entreprises innovant dans les produits TIC et les services informatiques, 2012-14

En pourcentage des entreprises appartenant à la catégorie considérée

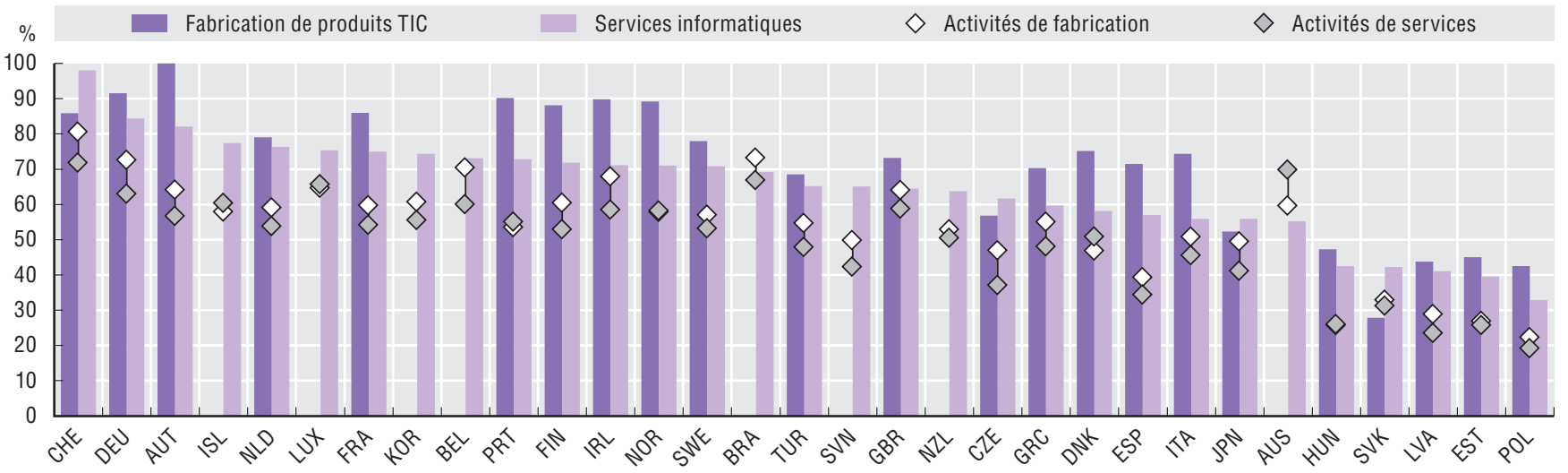

Note : La comparabilité internationale peut être limitée du fait des différences méthodologiques et des profils de réponses propres à chaque économie. Les pays européens suivent le schéma harmonisé de l'Enquête communautaire sur l'innovation.

Source : OCDE, d'après l'enquête 2017 de l'OCDE sur les statistiques nationales de l'innovation et l'Enquête communautaire sur l'innovation (CIS-2014) d'Eurostat, http://oe.cd/inno-stats, juin 2017. Davantage de données via StatLink. Voir notes de chapitre.

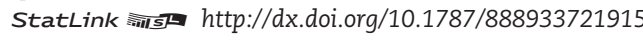

\section{Mesurabilité}

Les méthodes employées pour recueillir et publier des données sur la R-D par activité économique varient d'une économie à l'autre. Ces données pourront donc s'interpréter différemment selon qu'elles sont collectées en fonction de l'activité principale de l'exécutant ou du secteur ou produit ciblé par l'activité de R-D, ou bien sur la base d'une combinaison des deux. Dans le Manuel de Frascati (OCDE, 2015), il est recommandé de séparer ces deux types de données. Il est également prôné d'indiquer à part la R-D logicielle afin de rendre visible le chevauchement des statistiques de la R-D et de l'investissement en logiciels. La propagation de la R-D logicielle dans tous les secteurs (par exemple, le secteur automobile) explique probablement aussi la stabilité apparente du poids des DIRDE dans les secteurs de l'information.

Les brevets liés aux TIC couvrent 13 domaines définis en fonction des caractéristiques techniques et fonctions qui leur sont propres (par exemple, communication mobile, réseau haut débit, traitement des données à grande vitesse et analyse de gros volumes de données). Comme les inventions ne sont généralement protégées que dans certaines économies, les résultats de l'exploitation des données s'y rapportant peuvent varier selon l'office de brevets dont elles proviennent.

Les principales caractéristiques et difficultés présentées par les indicateurs issus des enquêtes sur l'innovation sont détaillées dans les différentes sections du présent chapitre. 


\section{Types d'innovation, par taille d'entreprise, 2012-14}

En pourcentage de l'ensemble des entreprises couvertes par les enquêtes nationales sur l'innovation, par catégorie de taille

Innovation de produit ou de procédé uniquement

Innovation de produit ou de procédé et de commercialisation ou d'organisation

Innovation de commercialisation ou d'organisation uniquement Grandes entreprises

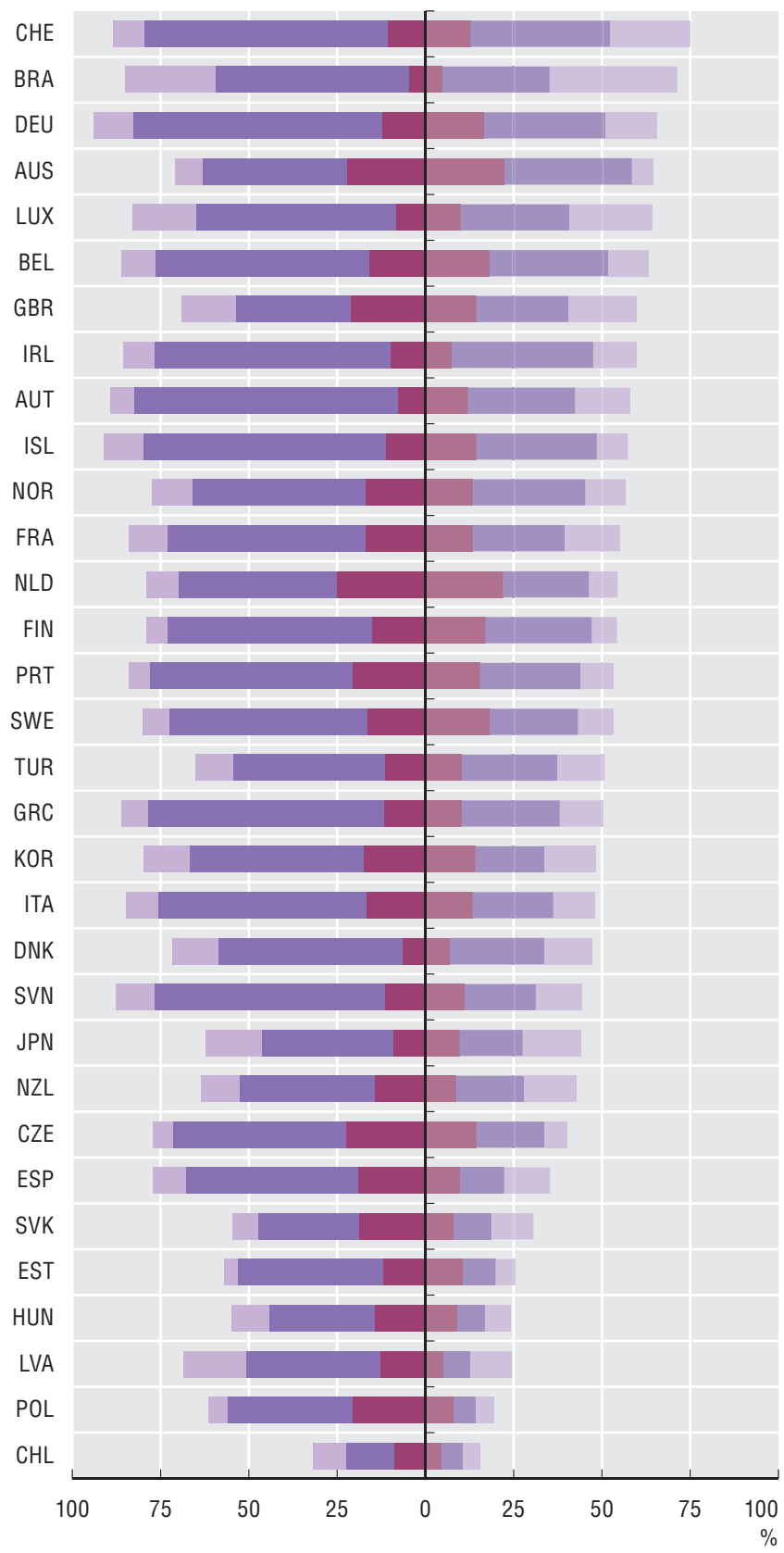

Note : La comparabilité internationale peut être limitée du fait des différences méthodologiques entre les enquêtes sur l'innovation et des profils de réponses propres à chaque pays. Les pays européens suivent le schéma harmonisé de l'Enquête communautaire sur l'innovation.

Source: OCDE, d'après l'enquête 2017 de l'OCDE sur les statistiques nationales de l'innovation et l'Enquête communautaire sur l'innovation (CIS-2014) d'Eurostat, http://oe.cd/inno-stats, juin 2017. Davantage de données via StatLink. Voir notes de chapitre.

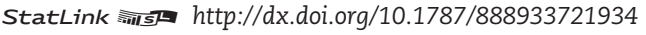

\section{Le saviez-vous?}

Dans la plupart des pays, les grandes entreprises sont deux à trois fois plus susceptibles que les PME de commercialiser des produits nouveaux pour le marché.

Il ressort des données recueillies au niveau des entreprises que leurs stratégies d'innovation mêlent différents types d'innovation complémentaires (panachage). Les entreprises innovantes - qu'il s'agisse de grandes entreprises ou de PME ont tendance à mettre en place de nouvelles méthodes de commercialisation ou de nouveaux modes d'organisation parallèlement aux innovations de produit ou de procédé.

Les indicateurs, qui expriment en pourcentage la part des entreprises ayant introduit au moins une innovation au cours de la période de référence, montrent que, dans tous les pays, il existe un véritable fossé entre PME et grandes entreprises. Le nombre des innovations de produit ou de procédé communiqué est en général plus faible dans le secteur des services que dans le secteur manufacturier.

En considérant à part le sous-ensemble constitué des entreprises qui introduisent des produits nouveaux pour le marché, on obtient une mesure de l'innovation de produit corrigée de la qualité. À quelques exceptions près, les innovations nouvelles pour le marché sont plus fréquentes dans les activités manufacturières que dans les activités de services. En Allemagne, l'écart va du simple au double. De manière générale, l'innovation dans le secteur manufacturier a le vent en poupe dans une majorité de pays de l'OCDE.

Le nombre d'innovations en produits nouveaux pour le marché varie grandement selon qu'elles émanent de grandes entreprises ou de PME (moins de 250 salariés). Dans beaucoup de pays, les innovations nouvelles pour le marché sont rarement le fait des PME. Cela tient probablement aux difficultés que ces entreprises rencontrent quand elles s'efforcent de changer d'échelle, ce qui les empêche de transformer ou de révolutionner les marchés.

\section{Définitions}

L'édition 2005 du Manuel d'Oslo (OCDE et Eurostat, 2005), en cours de révision, recense quatre types d'innovation en fonction de leur objet :

Innovation de produit : introduction d'un bien ou d'un service nouveau ou sensiblement amélioré sur le plan de ses caractéristiques ou de l'usage auquel il est destiné. Cette définition inclut les modifications apportées aux spécifications techniques, aux logiciels ou composants intégrés, à la convivialité ou à d'autres caractéristiques fonctionnelles.

Innovation de procédé : mise en œuvre d'une méthode de production ou de distribution nouvelle ou sensiblement améliorée. Cette définition inclut les modifications apportées aux techniques, au matériel ou les logiciels.

Innovation de commercialisation : mise en œuvre d'une nouvelle méthode de commercialisation impliquant des changements de la conception ou du conditionnement, du placement, de la promotion ou de la tarification d'un produit.

Innovation d'organisation : mise en œuvre d'une nouvelle méthode organisationnelle dans les pratiques, l'organisation du lieu de travail ou les relations extérieures de la firme.

L'innovation en produits nouveaux pour le marché désigne l'introduction par une entreprise d'un produit nouveau ou sensiblement amélioré sur son marché avant n'importe lequel de ses concurrents (le produit en question peut être déjà proposé sur d'autres marchés). 
Innovation en produits nouveaux pour le marché, activités de fabrication et de services, 2012-14

En pourcentage de l'ensemble des entreprises couvertes par les enquêtes nationales sur l'innovation, par type d'activité

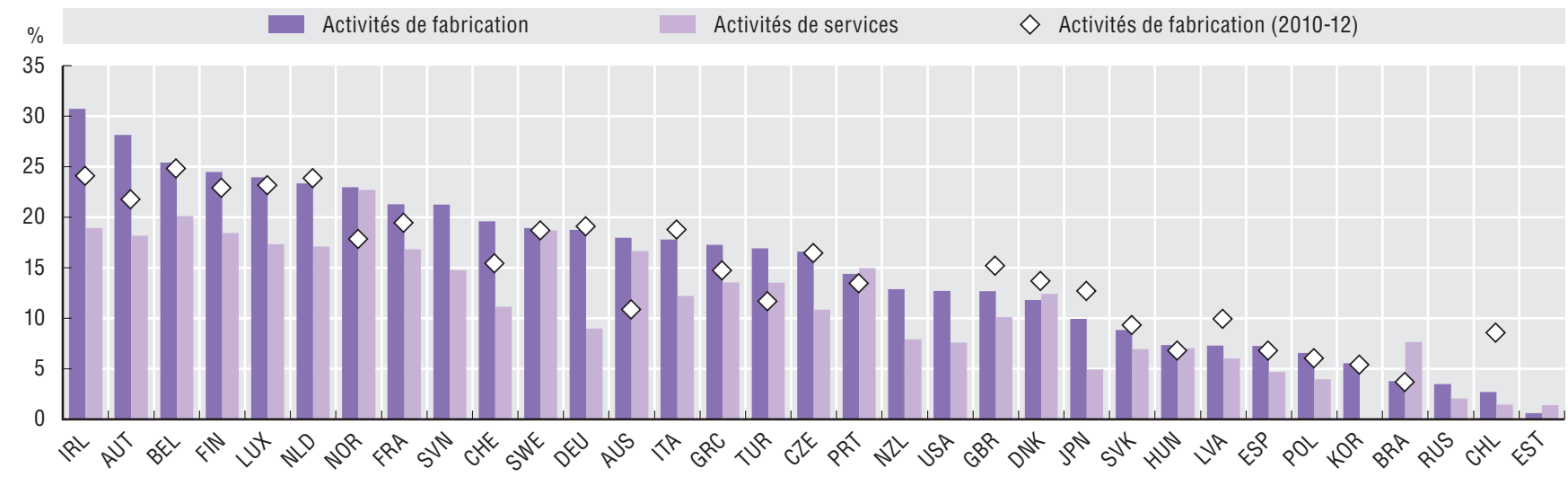

Note : La comparabilité internationale peut être limitée du fait des différences méthodologiques entre les enquêtes sur l'innovation et des profils de réponses propres à chaque pays. Les pays européens suivent le schéma harmonisé de l'Enquête communautaire sur l'innovation.

Source : OCDE, d'après l'enquête 2017 de l'OCDE sur les statistiques nationales de l'innovation et l'Enquête communautaire sur l'innovation (CIS-2014) d'Eurostat, http://oe.cd/inno-stats, juin 2017. Davantage de données via StatLink. Voir notes de chapitre.

StatLink 需正 http://dx.doi.org/10.1787/888933721953

Innovation en produits nouveaux pour le marché, par taille d'entreprise, 2012-14

En pourcentage de l'ensemble des entreprises couvertes par les enquêtes nationales sur l'innovation, par catégorie de taille

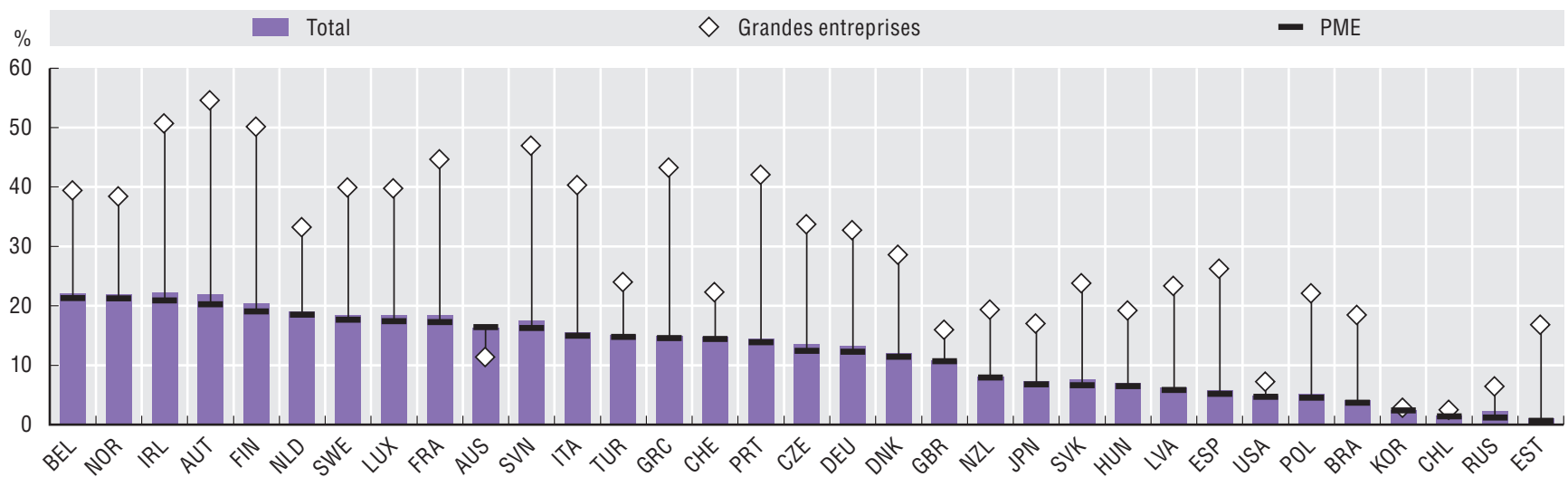

Note : La comparabilité internationale peut être limitée du fait des différences méthodologiques entre les enquêtes sur l'innovation et des profils de réponses propres à chaque pays. Les pays européens suivent le schéma harmonisé de l'Enquête communautaire sur l'innovation.

Source : OCDE, d'après l'enquête 2017 de l'OCDE sur les statistiques nationales de l'innovation et l'Enquête communautaire sur l'innovation (CIS-2014) d'Eurostat, http://oe.cd/inno-stats, juin 2017. Davantage de données via StatLink. Voir notes de chapitre.

StatLink 젶ㄴ http://dx.doi.org/10.1787/888933721972

\section{Mesurabilité}

La comparabilité des indicateurs de l'innovation, s'agissant en particulier de son incidence, est tributaire de facteurs méthodologiques très divers. Les principales entraves à la comparabilité internationale découlent principalement du fait que les pays ne recueillent pas tous des informations sur l'innovation dans le cadre de leurs enquêtes sur la R-D (ce qui influe sur la manière dont les répondants rendent compte des produits ou procédés nouveaux et améliorés) et que ces enquêtes ne revêtent pas nécessairement un caractère obligatoire. Les correspondants nationaux se sont efforcés, parfois sans succès, de rapprocher les périodes de référence, les secteurs et les tailles d'entreprise associés aux pays non européens du champ central de l'Enquête communautaire sur l'innovation. Les indicateurs qualitatifs de l'incidence, fondés sur le concept d'au moins une innovation par type ne donne pas une vue complète de l'intensité d'innovation d'une économie, en particulier si les résultats sont pondérés sur la base de comptages fractionnaires des entreprises. Ces questions sont actuellement à l'étude dans le cadre de la révision du Manuel d'Oslo. 


\section{Incitations fiscales en faveur de la R-D}

\section{Soutien public direct et aide fiscale à la R-D des entreprises, 2015}

En pourcentage du PIB

Soutien public indirect aux DIRDE (incitations fiscales)

Données sur les inctiations fiscales non disponibles

$\diamond$ Total du soutien public aux DIRDE, 2006

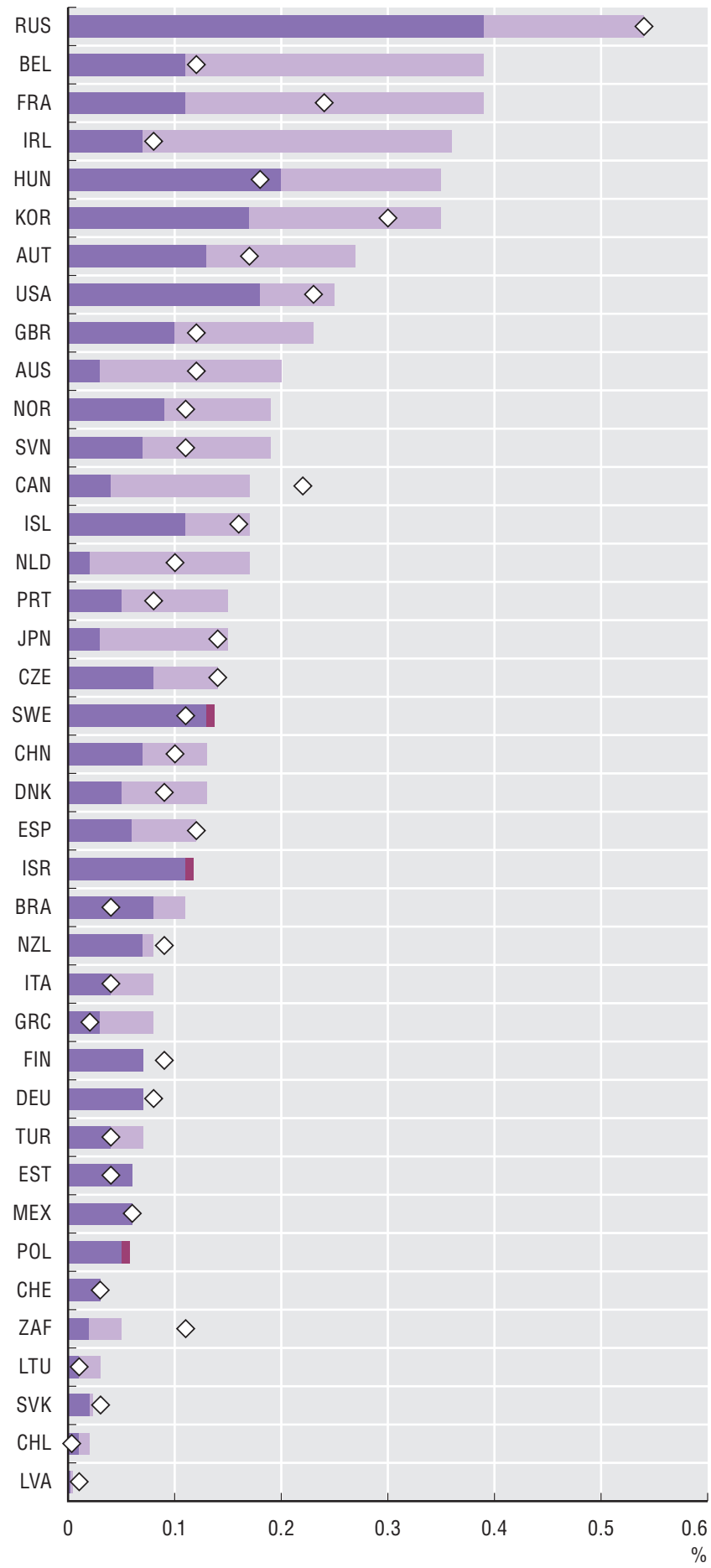

Source : OCDE, Indicateurs des incitations fiscales en faveur de la R-D, http://oe.cd/rdtax, juillet 2017. Davantage de données via StatLink. Voir notes de chapitre.

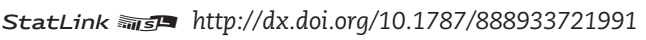

\section{Le saviez-vous ?}

En 2015, les incitations fiscales en faveur de la R-D ont représenté près de la moitié du volume total de l'aide publique à la R-D des entreprises de la zone OCDE, contre un tiers en 2006.

Les incitations fiscales figurent désormais en bonne place dans l'arsenal de mesures déployé par les pays de l'OCDE et les économies partenaires pour promouvoir la R-D des entreprises. En 2017, 30 pays de l'OCDE - contre 16 en 2000 - appliquaient un régime d'imposition préférentiel aux dépenses de R-D des entreprises. Au cours de la période 2006-15, l'aide publique totale allouée à ces dépenses, exprimée en pourcentage du PIB, a augmenté dans 25 des 37 pays pour lesquels des données sont disponibles. En 2015, ce sont la Fédération de Russie, la Belgique et la France qui ont fourni le soutien le plus important en pourcentage du PIB.

Une comparaison du soutien public fourni en 2015 par rapport à 2006 fait apparaître une progression des incitations fiscales, en termes relatifs, dans 22 des 33 pays pour lesquels des données sont disponibles. Le Canada, la Hongrie et le Portugal, qui privilégiaient fortement les aides fiscales, ont rééquilibré leurs mesures de soutien par un recours plus marqué au financement direct. Le Mexique, qui avait abandonné son dispositif d'allègement fiscal en 2009, l'a réintroduit en 2017.

Les différences au niveau du coût des allègements fiscaux en faveur de la R-D tiennent non seulement au taux du crédit d'impôt ou de l'abattement applicable mais aussi aux conditions d'éligibilité à ces avantages et à la propension des entreprises à s'en prévaloir. Il est possible de calculer le niveau théorique de l'aide fiscale, par unité supplémentaire de dépense de $\mathrm{R}-\mathrm{D}$, à laquelle peuvent normalement prétendre des entreprises aux caractéristiques bien déterminées. En Espagne, en France et au Portugal, ce niveau est plus important pour les PME. Des provisions pour crédit ou reports sont parfois utilisés pour soutenir la R-D d'entreprises qui ne pourraient autrement bénéficier des crédits ou abattements auxquels elles sont éligibles. Dix-huit pays de l'OCDE proposent des remboursements ou incitations équivalentes. De tels dispositifs ont tendance à être plus généreux à l'égard des PME et des jeunes entreprises qu'avec les grandes structures : c'est le cas en Australie, au Canada et en France.

\section{Définitions}

Les incitations fiscales en faveur de la R-D des entreprises comprennent les abattements, les crédits d'impôt ainsi que d'autres avantages fiscaux applicables aux dépenses de R-D des entreprises. Les estimations n'incluent ni les mesures d'incitation assises sur les revenus, telles que les " boîtes à brevets ", ni celles visant des contribuables autres que les entreprises. Le taux de subvention fiscale est égal à 1 moins l'indice B, l'indice B correspondant au « revenu avant impôt qu'il faut dégager pour rentabiliser 1 dollar supplémentaire de dépenses de R-D " (Warda, 2001). Cette mesure marginale de l'aide fiscale peut différer du taux moyen de subvention fiscale en présence de plafonds et de seuils et si certaines entreprises ne sont pas autorisées à demander une aide supplémentaire. Chacune de ces mesures peut peser sur les décisions d'investir en R-D : le taux moyen pour la marge extensive (faut-il ou non investir dans un pays ?), le taux marginal pour la marge intensive (combien investir dans un pays ?). 
Évolution du financement public de la R-D des entreprises : financement direct et incitations fiscales, 2006 et 2015 En pourcentage du soutien total

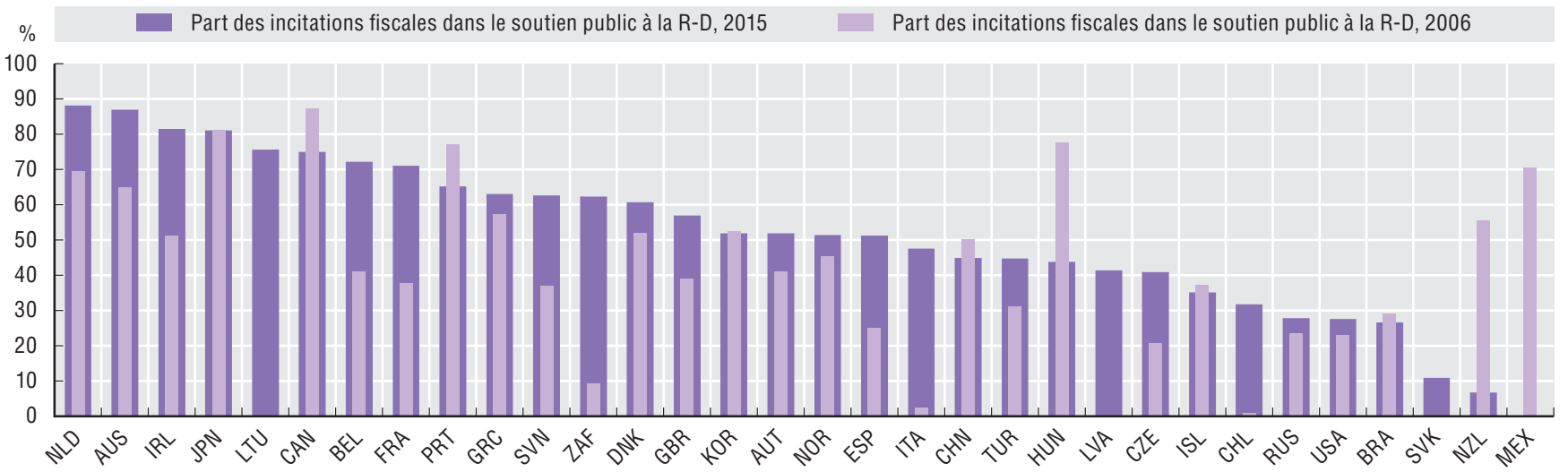

Source : OCDE, Indicateurs des incitations fiscales en faveur de la R-D, http://oe.cd/rdtax, juillet 2017. Davantage de données via StatLink. Voir notes de chapitre.

Taux de subvention fiscale des dépenses de R-D, 2017

1 moins l'indice $B$, selon la taille et la rentabilité de l'entreprise

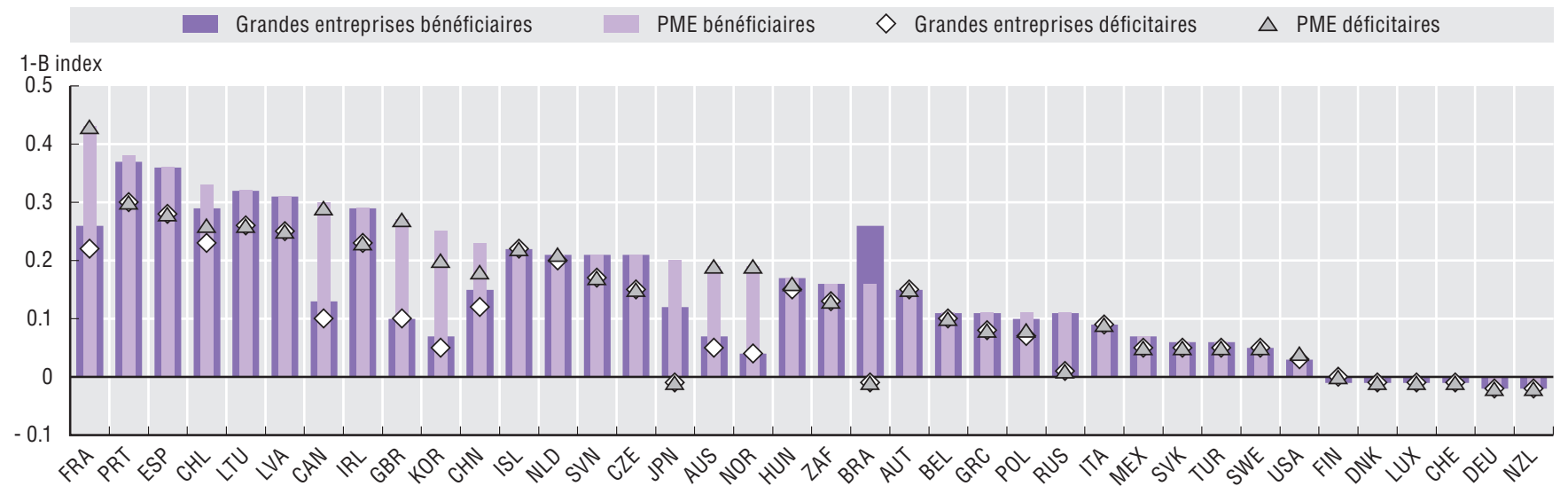

Source : OCDE, Indicateurs des incitations fiscales en faveur de la R-D, http://oe.cd/rdtax, juillet 2017. Voir notes de chapitre.

StatLink न्माड़ा $h t t p: / / d x . d o i . o r g / 10.1787 / 888933722029$

\section{Mesurabilité}

Il existe plusieurs moyens de déterminer la valeur des allègements fiscaux en faveur de la R-D, les dépenses fiscales correspondant à des déviations par rapport à un système fiscal de référence (OCDE, 2010). Les indicateurs retenus posent un cadre de référence commun fondé sur une déductibilité totale des dépenses courantes de R-D et un traitement au niveau national des dépenses d'investissement en capital. Les estimations se fondent en général sur les déclarations fiscales et correspondent au manque à gagner par rapport à l'impôt initial, sans prise en compte, sinon mineure, des effets de comportement. La dernière édition du Manuel de Frascati (OCDE, 2015) récapitule la marche à suivre pour présenter les données sur les allègements fiscaux au titre de la R-D.

Afin de donner une illustration plus précise de différents cas de figure, les valeurs de l'indice B sont calculées pour des entreprises "représentatives ", selon qu'elles peuvent ou non prétendre aux avantages fiscaux au titre de l'impôt dû, pour la période considérée. Lorsque les crédits ou abattements sont remboursables intégralement, la valeur de l'indice B des sociétés éligibles est identique à celle retenue dans le scénario bénéficiaire. Les reports sont modélisés comme des remises en option, permettant de prétendre à l'avantage ultérieurement. Les ajustements relatifs aux plafonds applicables à la R-D ou aux allègements fiscaux ont été modélisés dans la mesure du possible (voir notes de chapitre). 
Investissement en capital-risque, 2016

En pourcentage du PIB

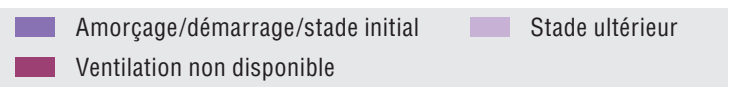

Top 5
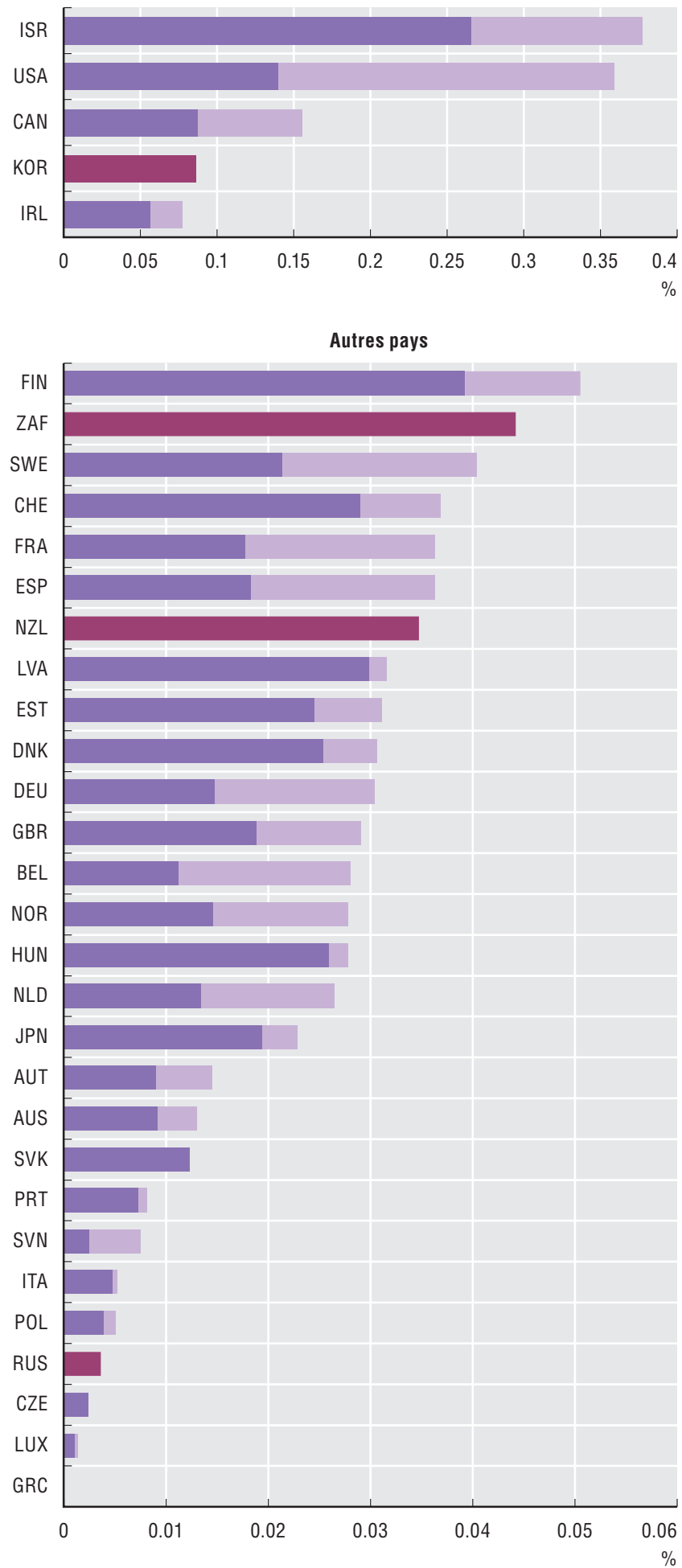

Source : OCDE, d'après OCDE (2017a), Panorama de l'entrepreneuriat 2017, Éditions OCDE, Paris. Voir notes de chapitre.

StatLink -ints http://dx.doi.org/10.1787/888933722048

\section{Le saviez-vous?}

Dans la plupart des pays, les grandes entreprises sont plus susceptibles que les PME de bénéficier d'un soutien public à l'innovation.

Le cadre de la politique économique joue un rôle de premier plan sur les facteurs favorables à l'offre et à la demande d'innovation au sein de l'économie. Les principales difficultés rencontrées par les entreprises désireuses de lancer des produits nouveaux ou d'adopter des pratiques nouvelles ou améliorées tiennent à l'accès au financement et aux marchés.

Pour les jeunes entreprises innovantes, il est difficile d'obtenir des financements d'amorçage et de démarrage du fait d'une rentabilité incertaine et de perspectives de croissance trop aléatoires. Dans la plupart des pays, l'investissement en capitalrisque n'a toujours pas renoué avec les niveaux d'avant la crise malgré quelques signes d'amélioration en 2016. De même, les marchés boursiers ont continué de stagner ces dernières années (OCDE, 2017b). Le volume des activités de capital-risque varie grandement d'un pays à l'autre. Par rapport à la taille de l'économie, il est beaucoup plus élevé au Canada, aux ÉtatsUnis et en Israël qu'en Europe. Il convient de noter également que les apports en capital-risque et en capitaux de croissance constituent des sources de financement nettement moins courantes que, par exemple, le prêt bancaire, le financement par nantissement d'actifs ou le crédit commercial.

La participation aux marchés internationaux et aux marchés du secteur public est une source majeure de la demande d'innovation. Elle est plus fréquente parmi les grandes entreprises et, hormis quelques exceptions notables, nettement plus probable parmi les entreprises innovantes.

En plus d'alimenter la demande en produits nouveaux, les administrations soutiennent l'innovation en subventionnant les activités connexes des entreprises. Dans la plupart des pays, les grandes entreprises ont davantage de chances de bénéficier d'un soutien public à l'innovation que les PME, notamment parce que celles-ci sont moins susceptibles de mener des activités d'innovation fondées sur la R-D.

\section{Définitions}

Le capital-risque se compose de capitaux privés apportés par des sociétés spécialisées dans l'intermédiation entre les sources primaires de financement (compagnies d'assurance, fonds de pensions, banques, etc.) et les start-ups ou entreprises à forte croissance non cotées en bourse.

La participation aux marchés internationaux concerne les entreprises qui vendent des biens ou des services à l'étranger. Les marchés du secteur public renvoient aux organismes publics, tels que les administrations et institutions locales, régionales et nationales, les écoles, les hôpitaux et les prestataires de service public.

Le soutien public à l'innovation comprend les aides financières accordées sous forme de crédits d'impôt ou d'abattements fiscaux, de subventions, de prêts bonifiés et de garanties d'emprunt. La formulation de la question posée à ce sujet varie légèrement suivant les pays. 


\section{Participation des PME innovantes et non innovantes aux marchés internationaux et aux marchés du secteur public, 2012-14}

En pourcentage des entreprises appartenant à la catégorie considérée

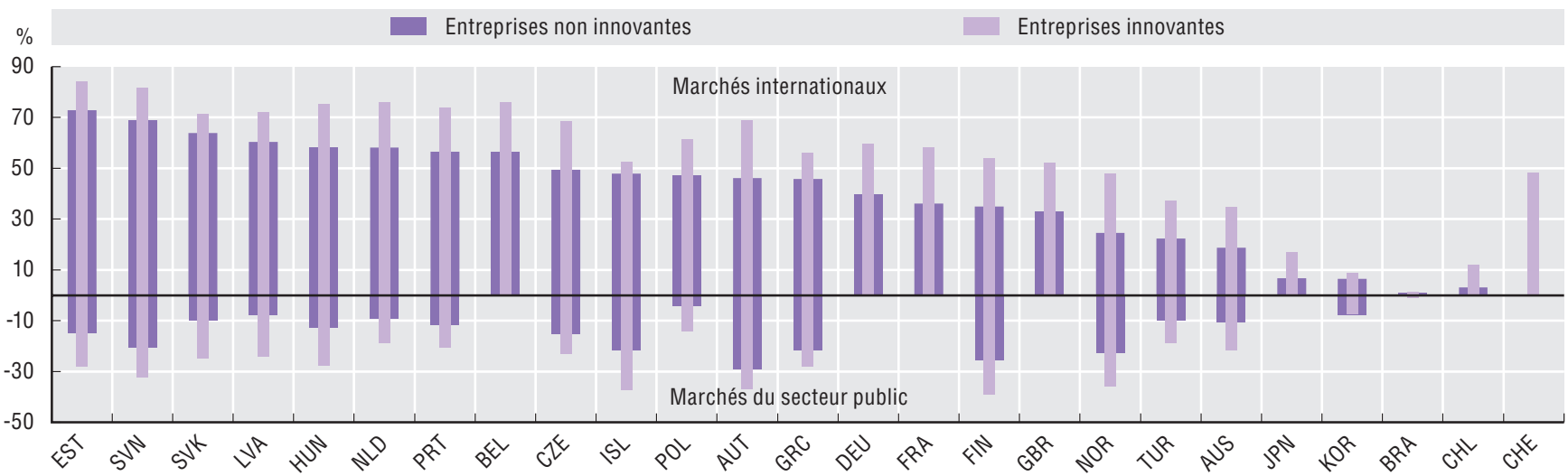

Note : La comparabilité internationale peut être limitée du fait des différences méthodologiques et des profils de réponses propres à chaque pays. Les pays européens suivent le schéma harmonisé de l'Enquête communautaire sur l'innovation.

Source : OCDE, d'après l'enquête 2017 de l'OCDE sur les statistiques nationales de l'innovation et l'Enquête communautaire sur l'innovation (CIS-2014) d'Eurostat, http://oe.cd/inno-stats juin 2017. Davantage de données via StatLink. Voir notes de chapitre.

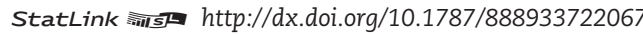

Entreprises bénéficiant du soutien public à l'innovation, par catégorie de taille, 2012-14

En pourcentage des entreprises actives dans l'innovation de produit ou de procédé, par catégorie de taille

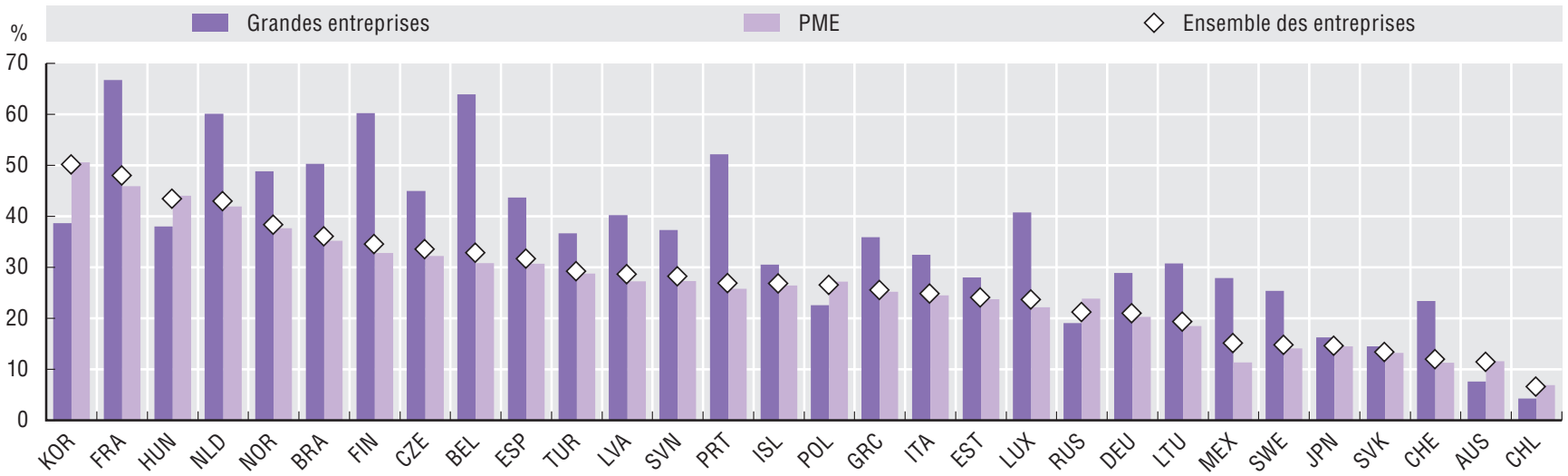

Note : La comparabilité internationale peut être limitée du fait des différences méthodologiques et des profils de réponses propres à chaque pays. Les pays européens suivent le schéma harmonisé de l'Enquête communautaire sur l'innovation.

Source: OCDE, d'après l'enquête 2017 de l'OCDE sur les statistiques nationales de l'innovation et l'Enquête communautaire sur l'innovation (CIS-2014) d'Eurostat, http://oe.cd/inno-stats, juin 2017. Davantage de données via StatLink. Voir notes de chapitre.

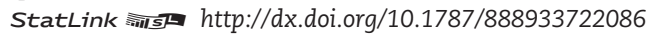

\section{Mesurabilité}

Les données sur le capital-risque proviennent principalement d'associations de capital-risque nationales ou régionales et de fournisseurs privés de données. Il n'existe pas de définition type au niveau international du capital-risque ou de la ventilation des investissements par stade de développement. La base de données de l'OCDE sur le financement de l'entrepreneuriat agrège des données originales pour cadrer avec la classification du capital-risque par stade de développement établie par l'OCDE.

Les questions formulées dans les enquêtes sur l'innovation renseignent sur les débouchés des entreprises et sur l'ampleur du soutien public qu'elles reçoivent (subventions, prêts bonifiés et garanties d'emprunt). L'OCDE a récemment étudié les moyens de mesurer la relation existant entre marchés publics et innovation (Appelt et Galindo-Rueda, 2016) et recommande d'utiliser des questions ciblées ainsi que les micro-données tirées des enquêtes sur l'innovation pour examiner la relation entre demande et innovation. La révision en cours du Manuel d'Oslo vise à fournir les orientations demandées sur la façon de procéder pour recueillir des données comparables concernant les facteurs externes de l'innovation dans les entreprises. 


\section{L'INNOVATION DANS LES ENTREPRISES}

\section{Notes et Références}

\section{Chypre}

La note suivante est incluse à la demande de la Turquie :

"Les informations figurant dans ce document qui font référence à "Chypre” concernent la partie méridionale de l'île. Il n'y a pas d'autorité unique représentant à la fois les Chypriotes turcs et grecs sur l'île. La Turquie reconnaît la République Turque de Chypre Nord (RTCN). Jusqu'à ce qu'une solution durable et équitable soit trouvée dans le cadre des Nations Unies, la Turquie maintiendra sa position sur la "question chypriote". "

La note suivante est ajoutée à la demande de tous les États de l'Union européenne membres de l'OCDE et de l'Union européenne :

"La République de Chypre est reconnue par tous les membres des Nations Unies sauf la Turquie. Les informations figurant dans ce document concernent la zone sous le contrôle effectif du gouvernement de la République de Chypre. »

\section{Israël}

"Les données statistiques concernant Israël sont fournies par et sous la responsabilité des autorités israéliennes ou d'un tiers compétents. L'utilisation de ces données par l'OCDE est sans préjudice du statut des hauteurs du Golan, de JérusalemEst et des colonies de peuplement israéliennes en Cisjordanie aux termes du droit international. »

"Il est à noter que les données statistiques sur les brevets et marques israéliens sont fournies par les offices des brevets et des marques des pays concernés. »

\subsection{R-D des entreprises}

\section{R-D des entreprises, 2005 et 2015}

Ces statistiques se fondent sur la Base de données des Principaux indicateurs de la science et de la technologie de l'OCDE (http://oe.cd/ pist-fr). Se reporter à cette source pour davantage de renseignements sur les données, en particulier sur les ruptures de séries et autres problèmes analogues.

Pour l'Afrique du Sud, les données se rapportent à 2013.

Pour l'Australie, les données se rapportent à 2004 et 2013.

Pour le Chili, les données se rapportent à 2007 et 2015.

Pour l'Irlande, les données se rapportent à 2014.

Pour Israël, la R-D du secteur de la défense est partiellement exclue des estimations disponibles.

Pour la Suisse, les données se rapportent à 2004 et 2015.

\section{R-D des entreprises et aides publiques, par taille d'entreprise, 2015}

S'agissant des DIRDE et de leur financement public, les chiffres indiqués pour les PME concernent les entreprises de moins de 250 salariés.

Ces statistiques se fondent sur la base de données Statistiques de la R-D de l'OCDE (http://oe.cd/srd-fr). Se reporter à cette source pour davantage de renseignements sur les données, en particulier sur les ruptures de séries et autres problèmes analogues.

Pour l'Autriche, les données se rapportent à 2004 et 2013.

Pour la Belgique, le Canada, le Danemark, le Luxembourg et la Suède, les données se rapportent à 2013.

Pour le Chili et la Nouvelle-Zélande, les données se rapportent à 2007 et 2015.

Pour les États-Unis, les chiffres portent sur les dépenses courantes, mais intègrent une composante d'amortissement qui peut différer du niveau réel des dépenses en capital. En outre, les entreprises de moins de cinq salariés et les données relatives aux centres de recherche et développement financés par l'administration fédérale sont exclues de l'Enquête sur la R-D et l'innovation dans les entreprises (BRDIS).

Pour la France, les données se rapportent à 2006 et 2013, année pour laquelle les données sont partiellement disponibles. Les séries ont donc été complétées à l'aide d'estimations fondées sur des ventilations se rapportant à 2012 et sur les totaux se rapportant à 2013.

Pour l'Italie et les Pays-Bas, les données se rapportent à 2003 et 2014.

Pour le Japon, les entreprises dont le capital est inférieur à 10 millions JPY sont exclues du champ des enquêtes sur la R-D. Il en résulte une surévaluation de la part des grandes entreprises dans la R-D.

Pour la Lettonie, la Pologne, le Portugal et la Slovénie, les données se rapportent à 2014.

Pour le Luxembourg, la part des PME dans les DIRDE financées par l'État en 2013 n'est pas disponible pour des raisons de confidentialité.

Pour les Pays-Bas, les entreprises de moins de 10 salariés sont exclues du champ des enquêtes sur la R-D.

Pour le Royaume-Uni, les données se rapportent à 2006 et 2014.

Pour la Suisse, les données se rapportent à 2004 et 2015. 


\section{Intensité de R-D par secteur, 2015}

L'intensité de R-D a été calculée pour chaque secteur pour lequel des données sur la R-D ainsi que sur la valeur ajoutée sont disponibles. Le ratio obtenu est sensible à l'unité statistique employée dans ces deux cas. On trouvera une réflexion plus approfondie sur la cohérence entre le numérateur et le dénominateur dans Galindo-Rueda et Verger (2016). En particulier, selon les pays, un traitement différent est réservé aux grandes entreprises qui mènent un ensemble complexe d'activités et aux entreprises qui fournissent des services de R-D.

Ces chiffres reposent sur des estimations de la R-D des entreprises par secteur, déclarées par activité principale selon la CITI rév. 4. Les secteurs « équipements TIC » et « services d'information » correspondent aux divisions 26 et 58-63, respectivement, de la CITI rév. 4. Pour l'Allemagne, le Danemark, l'Espagne, les États-Unis, la Finlande, la Hongrie, l'Italie, les Pays-Bas, la Pologne, le Portugal, le Royaume-Uni et la Slovénie, les données se rapportent à 2014.

Pour l'Australie, l'Autriche, la Belgique, le Canada, la Corée, la France, la Grèce, l'Irlande et la Suède, les données se rapportent à 2013. La valeur ajoutée est mesurée aux prix de base sauf pour le Japon (prix d'acquisition).

Les données sur la valeur ajoutée sont tirées de la base de données de l'OCDE pour l'analyse structurelle (STAN), sauf pour le Chili, la Corée, la Hongrie, l'Irlande, l'Islande, la Nouvelle-Zélande et la Turquie (statistiques de l'OCDE sur les comptes nationaux), le Canada (source nationale) et l'Australie (statistiques de l'OCDE sur les comptes nationaux et estimations fondées sur les comptes nationaux australiens ABS : tableaux des entrées-sorties, 2013-14, pour les activités de fabrication).

\subsection{Principaux acteurs de la R-D}

\section{Dépenses de R-D par brevet dans les entreprises les plus actives en R-D selon l'implantation du siège, 2012-14}

Les données se rapportent aux économies dont relèvent les sièges de cinq sociétés au moins parmi les 2000 entreprises les plus actives en R-D ayant déposé des brevets au cours de la période 2012-14. Les dépenses de R-D sont indiquées en millions USD. Les données sur les brevets se rapportent aux familles de brevets IP5 détenues par les entreprises les plus actives en R-D, au regard de la date du premier dépôt.

Les branches d'activité sont définies conformément à la CITI rév. 4. Le secteur des TIC comprend les Activités de fabrication dans le secteur des TIC (classes 2610, 2620, 2630, 2640 et 2680), les Activités commerciales du secteur des TIC (4651 et 4652 ), les Activités de services dans le secteur des TIC (5820), les Télécommunications (61), la Programmation informatique (62), les Activités de traitement de données (631) et la Réparation d’ordinateurs et de matériel de communication (951).

\section{Spécialisation sectorielle et technologique des entreprises les plus actives en R-D, 2012-14}

La spécialisation sectorielle (indice de concentration absolue - C4) reflète le poids des quatre branches d'activité prépondérantes parmi les filiales des entreprises les plus actives en R-D dans un secteur donné, exprimé en proportion du nombre total de filiales que comptent ces entreprises. Les branches d'activité sont définies conformément à la CITI rév. 4.

La spécialisation technologique (indice de concentration absolue - C4) reflète la part relative des portefeuilles de brevets intéressant les quatre principaux domaines technologiques dans lesquels les entreprises les plus actives en R-D dans un secteur donné ont déposé des brevets, en proportion du total des brevets déposés par ces entreprises. Les données sur les brevets se rapportent aux familles de brevets IP5 détenues par les entreprises les plus actives en R-D, au regard de la date du premier dépôt. Les brevets sont affectés aux domaines technologiques sur la base des codes de la Classification internationale des brevets (CIB) et suivant la concordance établie par l'OMPI (2013).

Les données se rapportent aux secteurs d'activité dont relèvent les sièges de 10 sociétés au moins parmi les 2000 entreprises les plus actives en R-D.

\section{Ventes nettes par dépôt de marque des entreprises des TIC les plus actives en R-D, selon l'implantation du siège, 2012-14}

Les données se rapportent aux économies dont relèvent les sièges de cinq sociétés au moins parmi les 2000 entreprises les plus actives en R-D ayant enregistré des marques au cours de la période 2012-14. Les ventes nettes sont indiquées en millions USD. Les données sur les marques se rapportent aux nouvelles demandes d'enregistrement déposées auprès de l'EUIPO, du JPO et de l'USPTO, par les entreprises les plus actives en R-D, en fonction de la date de la demande d'enregistrement.

Les branches d'activité sont définies conformément à la CITI rév. 4. Le secteur des TIC comprend les Activités de fabrication dans le secteur des TIC (classes 2610, 2620, 2630, 2640 et 2680), les Activités commerciales du secteur des TIC (4651 et 4652 ), les Activités de services dans le secteur des TIC (5820), les Télécommunications (61), la Programmation informatique (62), les Activités de traitement de données (631) et la Réparation d’ordinateurs et de matériel de communication (951).

\subsection{Portefeuilles de PI}

\section{Brevets liés aux TIC, 2002-05 et 2012-15}

Les données se rapportent aux familles de brevets IP5, par date de dépôt, en fonction du lieu de résidence du déposant, et avec comptages fractionnaires. Les brevets liés aux TIC sont recensés à l'aide des codes de la CIB indiqués dans Inaba et Squicciarini (2017). Seules les économies totalisant plus de 250 familles de brevets pour les périodes considérées sont incluses. Les chiffres de 2014 et 2015 correspondent à des estimations établies sur la base des données disponibles pour ces années. 


\section{Dépôts de marques liées aux TIC, 2012-15}

Les données se rapportent aux marques déposées auprès de l'Office de l'Union européenne pour la propriété intellectuelle (EUIPO), du Japan Patent Office (JPO) et de l'United States Patent and Trademark Office (USPTO), par date de dépôt de la demande, en fonction du lieu de résidence du déposant, et avec comptages fractionnaires. L'enregistrement de marques liées aux TIC renvoie aux demandes d'enregistrement nommant les classes 9, 28, 35, 38, 41 et/ou 42 de la Classification de Nice et contenant des mots clés en relation avec les TIC dans la description des biens et services (la liste complète de ces mots clés est disponible sur demande). Les parts sont calculées pour les pays comptabilisant plus de 250 marques enregistrées auprès de l'EUIPO ou l'USPTO et plus de 25 marques enregistrées auprès du JPO au cours de la période considérée.

\section{Enregistrement de dessins et modèles liés aux TIC, 2012-15}

Les données se rapportent aux dessins et modèles enregistrés auprès de l'Office de l'Union européenne pour la propriété intellectuelle (EUIPO) et du Japan Patent Office (JPO) ainsi qu'aux brevets de dessin et modèle déposés auprès de l'United States Patent and Trademark Office (USPTO), par date de dépôt, en fonction du lieu de résidence du déposant, et avec comptages fractionnaires. L'enregistrement de dessins et modèles liés aux TIC renvoie aux sous-classes 14-01 à 14-04, 14-99, 16-01 à 16-06, 16-99, 18-01 à 1804 et 18-99 de la Classification de Locarno. Les parts sont calculées pour les pays comptabilisant plus de 100 dessins ou modèles enregistrés auprès de l'EUIPO ou plus de 100 brevets de dessin ou modèles déposés auprès de l'USPTO et plus de 25 dessins ou modèles enregistrés auprès du JPO au cours de la période considérée.

\subsection{TIC et innovation}

\section{Dépenses de R-D dans les équipements TIC et les services d'information, 2015}

Ces chiffres reposent sur des estimations de la R-D des entreprises par secteur, déclarées par activité principale selon la CITI rév. 4. Ces statistiques se fondent sur les bases de données Statistiques de la recherche-développement (http://oe.cd/srd-fr) et ANBERD (http:// oe.cd/anberd-fr) de l'OCDE. Se reporter à ces sources pour davantage de renseignements sur les données, en particulier sur les ruptures de séries et autres problèmes analogues.

Pour l'Allemagne, l'Estonie et le Japon, les données se rapportent à 2007 et 2015.

Pour l'Autriche, la Belgique, la Grèce et l'Irlande, les données se rapportent à 2013.

Pour le Canada, le Danemark, les États-Unis, la Finlande, la Hongrie, Israël, l'Italie, les Pays-Bas, la Pologne, le Portugal, le RoyaumeUni et la Slovénie, les données se rapportent à 2014.

Pour la France et la Suède, les données se rapportent à 2007 et 2013.

Pour la Suisse, les données se rapportent à 2004 et 2015.

\section{Brevets liés aux TIC et principaux acteurs, 2012-15}

Les données se rapportent aux familles de brevets IP5, par date de dépôt, en fonction du lieu de résidence du déposant, et avec comptages fractionnaires. Les brevets liés aux TIC sont recensés à l'aide des codes de la CIB indiqués dans Inaba et Squicciarini (2017). Les chiffres de 2014 et 2015 correspondent à des estimations établies sur la base des données disponibles pour ces années.

\section{Entreprises innovant dans les produits TIC et les services informatiques, 2012-14}

La comparabilité internationale peut être limitée du fait des différences méthodologiques entre les enquêtes sur l'innovation et des profils de réponses propres à chaque pays. Les pays européens suivent le schéma harmonisé de l'Enquête communautaire sur l'innovation. Pour en savoir plus, voir www.oecd.org/sti/inno-stats.htm.

La production de biens TIC correspond à la division 26 (Fabrication d'ordinateurs, d'articles électroniques et optiques) de la CITI rév. 4 , les activités de fabrication à la section $\mathrm{C}$, les services d'information et de communication à l'intégralité de la section $\mathrm{J}$ et les activités de services aux sections et divisions B, C, D, E, G46, H, J, K et M71-72-73.

Pour les pays qui suivent le schéma de l'Enquête communautaire sur l'innovation de 2014 d'Eurostat, les données concernent les entreprises menant des activités d'innovation de produit, de procédé, d'organisation ou de commercialisation (y compris activités d'innovation en cours ou abandonnées). Le champ sectoriel principal inclut les sections et divisions B, C, D, E, G46, H, J, K et M71-72-73 de la CITI rév. 4. Seules les entreprises de 10 salariés ou plus sont prises en considération.

Pour l'Australie, les données sont tirées de la Business Characteristics Survey (BCS), se rapportent à l'exercice financier 2014/15 et concernent les entreprises menant des activités d'innovation de produit, de procédé, d'organisation ou de commercialisation (y compris activités d'innovation en cours ou abandonnées). Les secteurs d'activité et tailles d'entreprises pris en considération coïncident avec le champ de l'Enquête communautaire sur l'innovation.

Pour le Brésil, les données sont tirées de l'enquête nationale sur l'innovation (PINTEC) de 2014, se rapportent à la période 2012-14 et concernent les entreprises menant des activités d'innovation de produit, de procédé, d'organisation ou de commercialisation (y compris activités d'innovation en cours ou abandonnées). Les secteurs d'activité retenus pour cette enquête ne coïncident pas avec le champ principal de l'Enquête communautaire sur l'innovation. La section E de la CITI révision 4 est hors étude et seules certaines activités de services sont couvertes (divisions et groupes 592, 61, 62, 631, 71 et 72).

Pour l'Estonie, les données de l'Enquête communautaire sur l'innovation de 2014 ont fait l'objet d'une révision méthodologique. Il y a donc eu une rupture de série par rapport aux campagnes précédentes.

Pour le Japon, les données proviennent de la Japanese National Innovation Survey (J-NIS 2015), se rapportent aux exercices financiers 2012/13, 2013/14 et 2014/15 et concernent les entreprises menant des activités d'innovation de produit, de procédé, d'organisation 
ou de commercialisation (y compris activités d'innovation en cours ou abandonnées). Les secteurs d'activité et tailles d'entreprises pris en considération coïncident avec le champ de l'Enquête communautaire sur l'innovation.

Pour la Corée, les données sont tirées de la Korean Innovation Survey. Cette enquête est menée séparément pour les activités de fabrication et de services, mais les données se rapportent dans les deux cas à la période 2013-15. Elles concernent les entreprises menant des activités d'innovation de produit, de procédé, d'organisation ou de commercialisation (y compris activités d'innovation en cours ou abandonnées). Le champ sectoriel, plus restreint que celui de l'Enquête communautaire sur l'innovation en ce qui concerne le secteur industriel, se limite à la section C (Activités de fabrication) de la CITI rév. 4. Toutes les activités de services sont couvertes par l'enquête, à l'exception de celles relevant de la section O (Administration publique et défense ; sécurité sociale obligatoire).

Pour la Nouvelle-Zélande, les données sont tirées de la Business Operation Survey (BOS), se rapportent aux exercices financiers 2012/13 et 2013/14 et concernent les entreprises de six salariés ou plus réalisant un chiffre d'affaires annuel, tel que calculé aux fins de la taxe sur les produits et services (TPS), supérieur à 30000 NZD. Les données sur les entreprises innovantes se rapportent aux entreprises menant des activités d'innovation de produit, de procédé, d'organisation et de commercialisation (y compris activités d'innovation en cours ou abandonnées).

Pour la Suisse, les données sont tirées de l'Enquête sur l'innovation dans l'économie suisse, se rapportent à la période 2012-14 et concernent les entreprises menant des activités d'innovation de produit, de procédé, d'organisation ou de commercialisation (y compris activités d'innovation en cours ou abandonnées).

\subsection{Panachage des modes d'innovation}

\section{Types d'innovation, par taille d'entreprise, 2012-14}

La comparabilité internationale peut être limitée du fait des différences méthodologiques entre les enquêtes sur l'innovation et des profils de réponses propres à chaque pays. Les pays européens suivent le schéma harmonisé de l'Enquête communautaire sur l'innovation. Pour en savoir plus, voir www.oecd.org/sti/inno-stats.htm.

La taille d'une entreprise est déterminée sur la base du nombre de ses employés. Les PME s'entendent des entreprises de 10 à 249 salariés, et les grandes entreprises des entreprises de 250 salariés ou plus.

Pour les pays qui suivent le schéma de l'Enquête communautaire sur l'innovation de 2012, les données se rapportent aux activités d'innovation en cours ou abandonnées. Le champ sectoriel principal inclut les sections et divisions B, C, D, E, G46, H, J, K et M71-72-73 de la CITI rév. 4. Seules les entreprises de 10 salariés ou plus sont prises en considération.

Pour l'Australie, les données sont tirées de la Business Characteristics Survey (BCS), se rapportent à l'exercice financier 2014/15 et incluent les activités d'innovation en cours ou abandonnées. Les secteurs d'activité et tailles d'entreprises pris en considération coïncident avec le champ de l'Enquête communautaire sur l'innovation.

Pour le Brésil, les données sont tirées de l'enquête nationale sur l'innovation (PINTEC) de 2014, se rapportent à la période 2012-14 et ne prennent pas en considération les activités d'innovation en cours ou abandonnées. Les secteurs d'activité retenus pour cette enquête ne coïncident pas avec le champ principal de l'Enquête communautaire sur l'innovation. La section E de la CITI rév. 4 est hors étude et seules certaines activités de services sont couvertes (divisions et groupes 592, 61, 62, 631, 71 et 72).

Pour le Chili, les données sont tirées de l'enquête nationale sur l'innovation de 2015, se rapportent à la période 2013-14 et ne prennent pas en considération les activités d'innovation en cours ou abandonnées. L'enquête porte sur les entreprises réalisant un chiffre d'affaires annuel supérieur à 2400 UF, sans limite de taille. La couverture sectorielle est plus large pour le secteur industriel et s'étend, au-delà des activités qui forment le champ principal de l'Enquête communautaire sur l'innovation, aux sections A (Agriculture, chasse et sylviculture), B (Pêche) et F (Construction) de la CITI rév. 3. Les activités de services couvertes correspondent aux sections G, I, J et $\mathrm{K}$ de la CITI rév. 3.

Pour l'Estonie, les données de l'Enquête communautaire sur l'innovation de 2014 ont fait l'objet d'une révision méthodologique. Il y a donc eu une rupture de série par rapport aux campagnes précédentes.

Pour le Japon, les données proviennent de la Japanese National Innovation Survey (J-NIS 2015), se rapportent aux exercices financiers 2012/13, 2013/14 et 2014/15 et incluent les activités d'innovation en cours ou abandonnées. Les secteurs d'activité et tailles d'entreprises pris en considération coïncident avec le champ de l'Enquête communautaire sur l'innovation.

Pour la Corée, les données sont tirées de la Korean Innovation Survey. Cette enquête est menée séparément pour les activités de fabrication et de services, mais les données se rapportent dans les deux cas à la période 2013-15. Les données ne prennent pas en considération les activités d'innovation en cours ou abandonnées. Le libellé de la question sur l'innovation de produit se démarque légèrement des principes directeurs énoncés dans le Manuel d'Oslo. Il en résulte que l'introduction de nouveaux services par des entreprises manufacturières, ou celle de nouveaux biens par des entreprises de services, pourraient être sous-déclarées. Le champ sectoriel, plus restreint que celui de l'Enquête communautaire sur l'innovation en ce qui concerne le secteur industriel, se limite à la section C (Activités de fabrication) de la CITI rév. 4. Toutes les activités de services sont couvertes par l'enquête, à l'exception de celles relevant de la section $\mathrm{O}$ (Administration publique et défense ; sécurité sociale obligatoire).

Pour la Nouvelle-Zélande, les données sont tirées de la Business Operation Survey (BOS), se rapportent aux exercices financiers 2012/13 et 2013/14 et concernent les entreprises de six salariés ou plus réalisant un chiffre d'affaires annuel, tel que calculé aux fins de la taxe sur les produits et services (TPS), supérieur à 30000 NZD. Les données ne prennent pas en considération les activités d'innovation en cours ou abandonnées. Les secteurs d'activité et tailles d'entreprises pris en considération coïncident avec le champ de l'Enquête communautaire sur l'innovation.

Pour la Suisse, les données sont tirées de l'Enquête sur l'innovation dans l'économie suisse, se rapportent à la période 2012-14 et incluent les activités d'innovation en cours ou abandonnées. Les secteurs d'activité et tailles d'entreprises pris en considération coïncident avec le champ de l'Enquête communautaire sur l'innovation. 


\section{Innovation en produits nouveaux pour le marché, activités de fabrication et de services, 2012-14}

La comparabilité internationale peut être limitée du fait des différences méthodologiques entre les enquêtes sur l'innovation et des profils de réponses propres à chaque pays. Les pays européens suivent le schéma harmonisé de l'Enquête communautaire sur l'innovation. Pour en savoir plus, voir www.oecd.org/sti/inno-stats.htm.

Les activités de fabrication correspondent à la section C de la CITI rév. 4, et les activités de services aux sections et divisions B, C, D, E, G46, H, J, K et M71-72-73.

Pour les pays qui suivent le schéma de l'Enquête communautaire sur l'innovation de 2014 d'Eurostat, seules les entreprises de 10 salariés ou plus sont prises en considération. Le champ principal des activités de services correspond aux sections et divisions G46, H, J, K et M71-72-73 de la CITI rév. 4.

Pour l'Australie, les données sont tirées de la Business Characteristics Survey (BCS) et se rapportent à l'exercice financier 2014/15. La BCS ne permet pas d'indiquer distinctement les nouveautés pour le marché, mais cette donnée s'obtient en regroupant les produits nouveaux pour le secteur, pour l'Australie et pour le monde (c'est-à-dire en excluant les produits uniquement nouveaux pour l'entreprise). Les secteurs d'activité et tailles d'entreprises pris en considération coïncident avec le champ de l'Enquête communautaire sur l'innovation.

Pour le Brésil, les données sont tirées de l'enquête nationale sur l'innovation (PINTEC) de 2014, se rapportent à la période 201214 et concernent les entreprises menant des activités d'innovation de produit à l'origine de produits nouveaux pour le marché national. Seules certaines activités de services du champ principal de l'Enquête communautaire sur l'innovation sont couvertes (divisions et groupes 592, 61, 62, 631, 71 et 72 de la CITI rév. 4).

Pour le Chili, les données sont tirées de la $9^{e}$ enquête nationale sur l'innovation et se rapportent à la période 2013-14. L'enquête porte sur les entreprises réalisant un chiffre d'affaires annuel supérieur à 2400 UF, sans limite de taille. Les activités de services couvertes correspondent aux sections G, I, J et K de la CITI rév. 3.

Pour l'Estonie, les données de l'Enquête communautaire sur l'innovation de 2014 ont fait l'objet d'une révision méthodologique. Il y a donc eu une rupture de série par rapport aux campagnes précédentes.

Pour le Japon, les données proviennent de la Japanese National Innovation Survey (J-NIS 2015) et se rapportent aux exercices financiers 2012/13, 2013/14 et 2014/15. Les secteurs d'activité et tailles d'entreprises pris en considération coïncident avec le champ de l'Enquête communautaire sur l'innovation.

Pour la Corée, les données sont tirées de la Korean Innovation Survey et se rapportent à la période 2013-15. Le libellé de la question sur l'innovation de produit se démarque légèrement des principes directeurs énoncés dans le Manuel d'Oslo. Il en résulte que l'introduction de nouveaux services par des entreprises manufacturières, ou celle de nouveaux biens par des entreprises de services, pourraient être sous-déclarées. Le champ des activités de services couvert par l'enquête, plus vaste que celui de l'Enquête communautaire sur l'innovation, englobe toutes les activités de services à l'exception de celles relevant de la section $O$ (Administration publique et défense ; sécurité sociale obligatoire).

Pour la Nouvelle-Zélande, les données sont tirées de la Business Operation Survey (BOS), se rapportent aux exercices financiers 2012/13 et 2013/14 et concernent les entreprises de six salariés ou plus réalisant un chiffre d'affaires annuel, tel que calculé aux fins de la taxe sur les produits et services (TPS), supérieur à 30000 NZD. La BOS ne permet pas d'indiquer distinctement les nouveautés pour le marché, mais cette donnée s'obtient en regroupant les produits nouveaux pour le secteur, pour la Nouvelle-Zélande et pour le monde (c'est-à-dire en excluant les produits uniquement nouveaux pour l'entreprise).

Pour la Fédération de Russie, les données se rapportent à la période 2012-14 et concernent les entreprises de 15 salariés et plus. Les secteurs d'activité retenus pour cette enquête ne coïncident pas avec le champ principal de l'Enquête communautaire sur l'innovation. Il s'agit des activités relevant des sections C (Activités extractives), D (Activités de fabrication) et E (Production et distribution d'électricité, de gaz et d'eau) de la CITI rév. 3.1 et, s'agissant des activités de services, des divisions 64, 72, 73 et 74.

Pour la Suisse, les données sont tirées de l'Enquête sur l'innovation dans l'économie suisse, 2013. Les données se rapportent à 2010-12. Les secteurs d'activité et tailles d'entreprises pris en considération coïncident avec le champ de l'Enquête communautaire sur l'innovation.

Pour les États-Unis, les données sont tirées de l'Enquête sur la R-D et l'innovation dans les entreprises (BRDIS) de 2014 et se rapportent à la période 2012-14. Les secteurs d'activité et tailles d'entreprises pris en considération coïncident avec le champ de l'Enquête communautaire sur l'innovation, sauf pour ce qui est des services, qui incluent la totalité des activités non manufacturières comprises dans le champ principal de l'Enquête communautaire sur l'innovation, à savoir les activités relevant des sections et divisions B, D, E, G46, H, J, K et M71-72-73 de la CITI rév. 4.

\section{Innovation en produits nouveaux pour le marché, par taille d'entreprise, 2012-14}

La comparabilité internationale peut être limitée du fait des différences méthodologiques entre les enquêtes sur l'innovation et des profils de réponses propres à chaque pays. Les pays européens suivent le schéma harmonisé de l'Enquête communautaire sur l'innovation. Pour en savoir plus, voir www.oecd.org/sti/inno-stats.htm.

La taille d'une entreprise est déterminée sur la base du nombre de ses employés. Les PME s'entendent des entreprises de 10 à 249 salariés, et les grandes entreprises des entreprises de 250 salariés ou plus.

Pour les pays qui suivent le schéma de l'Enquête communautaire sur l'innovation de 2014 d'Eurostat, le champ sectoriel principal inclut les sections et divisions B, C, D, E, G46, H, J, K et M71-72-73 de la CITI rév. 4. Seules les entreprises de 10 salariés ou plus sont prises en considération.

Pour l'Australie, les données sont tirées de la Business Characteristics Survey (BCS) et se rapportent à l'exercice financier 2014/15. La BCS ne permet pas d'indiquer distinctement les nouveautés pour le marché, mais cette donnée s'obtient en regroupant les 
produits nouveaux pour le secteur, pour l'Australie et pour le monde (c'est-à-dire en excluant les produits uniquement nouveaux pour l'entreprise).

Pour le Brésil, les données sont tirées de l'enquête nationale sur l'innovation (PINTEC) de 2015, se rapportent à la période 2012-14 et concernent les entreprises menant des activités d'innovation de produit à l'origine de produits nouveaux pour le marché national. Seules certaines activités de services du champ principal de l'Enquête communautaire sur l'innovation sont couvertes (divisions et groupes 592, 61, 62, 631, 71 et 72 de la CITI rév. 4).

Pour le Chili, les données sont tirées de la 9 e enquête nationale sur l'innovation et se rapportent à la période 2013-14. L'enquête porte sur les entreprises réalisant un chiffre d'affaires annuel supérieur à 2400 UF, sans limite de taille. La couverture sectorielle est plus large pour le secteur industriel et s'étend, au-delà des activités qui forment le champ principal de l'Enquête communautaire sur l'innovation, aux sections A (Agriculture, chasse et sylviculture), B (Pêche) et F (Construction) de la CITI rév. 3. Les activités de services couvertes correspondent aux sections G, I, J et $\mathrm{K}$ de la CITI rév. 3.

Pour l'Estonie, les données de l'Enquête communautaire sur l'innovation de 2014 ont fait l'objet d'une révision méthodologique. Il y a donc eu une rupture de série par rapport aux campagnes précédentes.

Pour le Japon, les données proviennent de la Japanese National Innovation Survey (J-NIS 2015) et se rapportent aux exercices financiers 2012/13, 2013/14 et 2014/15. Les secteurs d'activité et tailles d'entreprises pris en considération coïncident avec le champ de l'Enquête communautaire sur l'innovation.

Pour la Corée, les données sont tirées de la Korean Innovation Survey. Le libellé de la question sur l'innovation de produit se démarque légèrement des principes directeurs énoncés dans le Manuel d'Oslo. Il en résulte que l'introduction de nouveaux services par des entreprises manufacturières, ou celle de nouveaux biens par des entreprises de services, pourraient être sous-déclarées. L'enquête est menée séparément pour les activités de fabrication et de services, mais les données se rapportent dans les deux cas à la période 2013-15. Le champ sectoriel, plus restreint que celui de l'Enquête communautaire sur l'innovation en ce qui concerne le secteur industriel, se limite à la section C (Activités de fabrication) de la CITI rév. 4. Toutes les activités de services sont couvertes par l'enquête, à l'exception de celles relevant de la section O (Administration publique et défense ; sécurité sociale obligatoire). Pour la Nouvelle-Zélande, les données sont tirées de la Business Operation Survey (BOS), se rapportent aux exercices financiers 2012/13 et 2013/14 et concernent les entreprises de six salariés ou plus réalisant un chiffre d'affaires annuel, tel que calculé aux fins de la taxe sur les produits et services (TPS), supérieur à 30000 NZD. La BOS ne permet pas d'indiquer distinctement les nouveautés pour le marché, mais cette donnée s'obtient en regroupant les produits nouveaux pour le secteur, pour la Nouvelle-Zélande et pour le monde (c'est-à-dire en excluant les produits uniquement nouveaux pour l'entreprise).

Pour la Fédération de Russie, les données se rapportent à la période 2012-14 et concernent les entreprises de 15 salariés et plus. Les secteurs d'activité retenus pour cette enquête ne coïncident pas avec le champ principal de l'Enquête communautaire sur l'innovation. Il s'agit des activités relevant des sections C (Activités extractives), D (Activités de fabrication) et E (Production et distribution d'électricité, de gaz et d'eau) de la CITI rév. 3.1 et, pour les services, des divisions 64, 72, 73 et 74.

Pour la Suisse, les données sont tirées de l'Enquête sur l'innovation dans l'économie suisse et se rapportent à la période 2012-14. Les secteurs d'activité et tailles d'entreprises pris en considération coïncident avec le champ de l'Enquête communautaire sur l'innovation.

Pour les États-Unis, les données sont tirées de l'Enquête sur la R-D et l'innovation dans les entreprises (BRDIS) de 2014 et se rapportent à la période 2012-14. Les secteurs d'activité et tailles d'entreprises pris en considération coïncident avec le champ de l'Enquête communautaire sur l'innovation.

\subsection{Incitations fiscales en faveur de la R-D}

\section{Soutien public direct et aide fiscale à la R-D des entreprises, 2015}

Pour en savoir plus sur les incitations fiscales en faveur de la R-D, voir http://oe.cd/rdtax et pour les notes générales et par pays relatives à cet indicateur, voir http://oe.cd/sb2017_notes_rdtax.

\section{Évolution du financement public de la R-D des entreprises : financement direct et incitations fiscales, 2006 et 2015}

Pour en savoir plus sur les incitations fiscales en faveur de la R-D, voir http://oe.cd/rdtax et pour les notes générales et par pays relatives à cet indicateur, voir http://oe.cd/sb2017_notes_rdtax.

\section{Taux de subvention fiscale des dépenses de R-D, 2017}

Il s'agit d'un indicateur expérimental qui repose sur des informations quantitatives et qualitatives et mesure un taux théorique de subvention fiscale pour différents cas de figure. Il fait intervenir un certain nombre d'hypothèses et de calculs spécifiques pour chaque pays. La comparabilité internationale peut être limitée.

Pour en savoir plus sur les incitations fiscales en faveur de la R-D, voir http://oe.cd/rdtax et pour les notes générales et par pays relatives à cet indicateur, voir http://oe.cd/sb2017_notes_rdtax.

\subsection{Cadre de la politique économique et demande d'innovation}

\section{Investissement en capital-risque, 2016}

Le stade initial recouvre : pour l'Australie, le pré-amorçage, l'amorçage et le démarrage ; pour le Canada et les États-Unis, l'amorçage et le stade initial ; pour les pays européens, l'amorçage et le démarrage ; pour Israël, l'amorçage/démarrage et le stade initial/ expansion et pour le Japon, l'amorçage, le stade initial et le stade d'expansion. 
Le stade ultérieur recouvre : pour l'Australie, le début du stade d'expansion et, pour les États-Unis, le stade d'expansion et ultérieur. L'Afrique du Sud, la Corée, la Fédération de Russie et la Nouvelle-Zélande ne fournissent pas de ventilation du capital-risque par stade de développement permettant d'établir des comparaisons internationales significatives.

Pour Israël et le Japon, les données se rapportent à 2014.

Pour les États-Unis, les données incluent les opérations d'investissement en capital-risque réalisées par d'autres investisseurs que les sociétés de capital-risque, mais excluent celles intégralement financées par des entreprises et/ou des investisseurs providentiels. Les données proviennent des sources suivantes : EVCA (pays européens), ABS (Australie), CVCA (Canada), KVCA (Corée), NVCA (États-Unis), NZVCA (Nouvelle-Zélande), PwCMoneyTree (Israël), RVCA (Fédération de Russie), SAVCA (Afrique du Sud) et VEC (Japon).

\section{Participation des PME innovantes et non innovantes aux marchés internationaux et aux marchés du secteur public, 2012-14}

La comparabilité internationale peut être limitée du fait des différences méthodologiques entre les enquêtes sur l'innovation et des profils de réponses propres à chaque pays. Pour en savoir plus, voir www.oecd.org/sti/inno-stats.htm.

La participation des entreprises couvertes par les enquêtes sur l'innovation aux marchés internationaux et aux marchés du secteur public est comparée selon qu'elles mènent ou non des activités d'innovation. Une entreprise innovante est une entreprise qui a introduit un produit ou un procédé nouveau ou qui a mis en place une nouvelle méthode de commercialisation ou un nouveau mode d'organisation au cours de la période de référence.

La taille d'une entreprise est déterminée sur la base du nombre de ses employés. Les PME s'entendent des entreprises de 10 à 249 salariés, et les grandes entreprises des entreprises de 250 salariés ou plus.

Pour les pays qui suivent le schéma de l'Enquête communautaire sur l'innovation de 2014 d'Eurostat, le champ sectoriel principal inclut les sections et divisions B, C, D, E, G46, H, J, K et M71-72-73 de la CITI rév. 4. Seules les entreprises de 10 salariés ou plus sont prises en considération.

Pour l'Australie, les données sont tirées de la Business Characteristics Survey (BCS) et se rapportent à l'exercice financier 2014/15. Les secteurs d'activité et tailles d'entreprises pris en considération coïncident avec le champ de l'Enquête communautaire sur l'innovation.

Pour le Brésil, les données sont tirées de l'enquête nationale sur l'innovation (PINTEC) de 2015 et se rapportent à la période 2012-14. Les secteurs d'activité retenus pour cette enquête ne coïncident pas avec le champ principal de l'Enquête communautaire sur l'innovation. La section E de la CITI rév. 4 est hors étude et seules certaines activités de services sont couvertes (divisions et groupes 592, 61, 62, 631, 71 et 72).

Pour le Chili, les données sont tirées de la $9^{e}$ enquête nationale sur l'innovation et se rapportent à la période 2013-14. L'enquête porte sur les entreprises réalisant un chiffre d'affaires annuel supérieur à 2400 UF, sans limite de taille. La couverture sectorielle est plus large pour le secteur industriel et s'étend, au-delà des activités qui forment le champ principal de l'Enquête communautaire sur l'innovation, aux sections A (Agriculture, chasse et sylviculture), B (Pêche) et F (Construction) de la CITI rév. 3. Les activités de services couvertes correspondent aux sections G, I, J et K de la CITI rév. 3.

Pour l'Estonie, les données de l'Enquête communautaire sur l'innovation de 2014 ont fait l'objet d'une révision méthodologique. Il y a donc eu une rupture de série par rapport aux campagnes précédentes.

Pour le Japon, les données proviennent de la Japanese National Innovation Survey (J-NIS 2015) et se rapportent aux exercices budgétaires 2012/14, 2010/11 et 2011/12. Les secteurs d'activité et tailles d'entreprises pris en considération coïncident avec le champ de l'Enquête communautaire sur l'innovation.

Pour la Corée, les données sont tirées de la Korean Innovation Survey. Cette enquête est menée séparément pour les activités de fabrication et de services, mais les données se rapportent dans les deux cas à la période 2013-15. Le champ sectoriel, plus restreint que celui de l'Enquête communautaire sur l'innovation en ce qui concerne le secteur industriel, se limite à la section C (Activités de fabrication) de la CITI rév. 4. Toutes les activités de services sont couvertes par l'enquête, à l'exception de celles relevant de la section O (Administration publique et défense ; sécurité sociale obligatoire).

Pour la Suisse, les données sont tirées de l'Enquête sur l'innovation dans l'économie suisse et se rapportent à la période 2012-14. Les secteurs d'activité et tailles d'entreprises pris en considération coïncident avec le champ de l'Enquête communautaire sur l'innovation.

\section{Entreprises bénéficiant du soutien public à l'innovation, par catégorie de taille, 2012-14}

La comparabilité internationale peut être limitée du fait des différences méthodologiques entre les enquêtes sur l'innovation et des profils de réponses propres à chaque pays. Les pays européens suivent le schéma harmonisé de l'Enquête communautaire sur l'innovation. Pour en savoir plus, voir www.oecd.org/sti/inno-stats.htm et les notes de chapitre.

La taille d'une entreprise est déterminée sur la base du nombre de ses employés. Les PME s'entendent des entreprises de 10 à 249 salariés, et les grandes entreprises des entreprises de 250 salariés ou plus.

Pour les pays qui suivent le schéma de l'Enquête communautaire sur l'innovation de 2014 d'Eurostat, les données relatives au soutien public à l'innovation concernent les entreprises menant des activités d'innovation de produit ou de procédé (y compris activités d'innovation en cours ou abandonnées). Le champ sectoriel principal inclut les sections et divisions B, C, D, E, G46, H, J, $\mathrm{K}$ et M71-72-73 de la CITI rév. 4. Seules les entreprises de 10 salariés ou plus sont prises en considération.

Pour l'Australie, les données sont tirées de la Business Characteristics Survey (BCS), se rapportent à l'exercice financier 2014/15 et 2012/13, et concernent les entreprises menant des activités d'innovation de produit, de procédé, de commercialisation et 
d'organisation (y compris activités d'innovation en cours ou abandonnées). La BCS contient une question fermée quant à la réception d'une aide financière publique. Les secteurs d'activité et tailles d'entreprises pris en considération coïncident avec le champ de l'Enquête communautaire sur l'innovation.

Pour le Brésil, les données sont tirées de l'enquête nationale sur l'innovation (PINTEC) de 2015, se rapportent aux périodes 201214 et 2009-11, et concernent les entreprises menant des activités d'innovation de produit ou de procédé (y compris activités d'innovation en cours ou abandonnées). Les secteurs d'activité retenus pour cette enquête ne coïncident pas avec le champ principal de l'Enquête communautaire sur l'innovation. La section E de la CITI rév. 4 est hors étude et seules certaines activités de services sont couvertes (divisions et groupes 592, 61, 62, 631, 71 et 72).

Pour le Chili, les données sont tirées de la 9e enquête nationale sur l'innovation, se rapportent aux périodes 2013-14 et 2009-11, et concernent les entreprises menant des activités d'innovation de produit, de procédé, d'organisation et de commercialisation (les activités d'innovation en cours ou abandonnées ne sont pas mises en évidence). L'enquête porte sur les entreprises réalisant un chiffre d'affaires annuel supérieur à 2400 UF, sans limite de taille. La couverture sectorielle est plus large pour le secteur industriel et s'étend, au-delà des activités qui forment le champ principal de l'Enquête communautaire sur l'innovation, aux sections A (Agriculture, chasse et sylviculture), B (Pêche) et F (Construction) de la CITI rév. 3. Les activités de services couvertes correspondent aux sections G, I, J et $\mathrm{K}$ de la CITI rév. 3.

Pour l'Estonie, les données de l'Enquête communautaire sur l'innovation de 2014 ont fait l'objet d'une révision méthodologique. Il y a donc eu une rupture de série par rapport aux campagnes précédentes.

Pour le Japon, les données proviennent de deux campagnes de la Japanese National Innovation Survey (J-NIS 2015 et J-NIS 2012 ), se rapportent respectivement aux exercices financiers 2012/13, 2013/14 et 2014/15, dans le cas de la première, et 2009/10, 2010/11 et 2011/12, pour la seconde. Les données relatives au soutien public à l'innovation concernent les entreprises menant des activités d'innovation de produit ou de procédé (y compris activités d'innovation en cours ou abandonnées). Les secteurs d'activité et tailles d'entreprises pris en considération coïncident avec le champ de l'Enquête communautaire sur l'innovation.

Pour la Corée, les données sont tirées de la Korean Innovation Survey. Cette enquête est menée séparément pour les activités de fabrication et de services, mais les données se rapportent dans les deux cas aux périodes 2013-15 et 2011-13. Les données relatives au soutien public à l'innovation concernent les entreprises menant des activités d'innovation de produit, de procédé, d'organisation et de commercialisation (y compris activités d'innovation en cours ou abandonnées). Le champ sectoriel, plus restreint que celui de l'Enquête communautaire sur l'innovation en ce qui concerne le secteur industriel, se limite à la section C (Activités de fabrication) de la CITI rév. 4. Toutes les activités de services sont couvertes par l'enquête, à l'exception de celles relevant de la section $O$ (Administration publique et défense ; sécurité sociale obligatoire).

Pour la Fédération de Russie, les données se rapportent aux périodes 2012-14 et 2009-11, et concernent les entreprises de 15 salariés et plus, qui mènent des activités d'innovation de produit ou de procédé (y compris activités d'innovation en cours ou abandonnées). Les secteurs d'activité retenus pour cette enquête ne coïncident pas avec le champ principal de l'Enquête communautaire sur l'innovation. . Il s'agit des activités relevant des sections C (Activités extractives), D (Activités de fabrication) et $\mathrm{E}$ (Production et distribution d'électricité, de gaz et d'eau) de la CITI rév. 3.1 et, s'agissant des activités de services, des divisions 64, 72, 73 et 74 .

Pour la Suisse, les données sont tirées de l'Enquête sur l'innovation dans l'économie suisse et se rapportent aux périodes 2012-14 et 2010-12. Les secteurs d'activité et tailles d'entreprises pris en considération coïncident avec le champ de l'Enquête communautaire sur l'innovation.

\section{Références}

Appelt, S. et F. Galindo-Rueda (2016), « Measuring the link between public procurement and innovation », OECD Science, Technology and Industry Working Papers, ${ }^{\circ}$ 2016/03, Éditions OCDE, Paris, http://dx.doi.org/10.1787/5jluc7sl1w7h-en.

Galindo-Rueda, F. et F. Verger (2016), « OECD Taxonomy of Economic Activities Based on R\&D intensity », OECD Science, Technology and Industry Working Papers, n²016/04, Éditions OCDE, Paris. DOI: http://dx.doi.org/10.1787/5jlu73sqqp8r-en.

Inaba, T. et M. Squicciarini (2017), «ICT: A new taxonomy based on the international patent classification ", OECD Science, Technology and Industry Working Papers, n 2017/01, Éditions OCDE, Paris, http://dx.doi.org/10.1787/ab16c396-en.

OCDE (2017a), Panorama de l'entrepreneuriat 2015, Éditions OCDE, Paris, http://dx.doi.org/10.1787/entrepreneur_aag-2015-fr.

OCDE (2017b), Financing PME and Entrepreneurs 2017: An OECD Scoreboard, Éditions OCDE, Paris, http://dx.doi.org/10.1787/fin_sme_ ent-2017-en.

OCDE (2015), Manuel de Frascati 2015, Lignes directrices pour le recueil et la communication des données sur la recherche et le développement expérimental, Éditions OCDE, Paris, http://dx.doi.org/10.1787/9789264257252-fr.

OCDE (2010), Les dépenses fiscales dans les pays de l'OCDE, Éditions OCDE, Paris, http://dx.doi.org/10.1787/9789264076921-fr.

OCDE, Eurostat (2005), Manuel d'Oslo, Principes directeurs pour le recueil et l'interprétation des données sur l'innovation, $3^{\mathrm{e}}$ édition, Éditions OCDE, Paris, $h t t p: / / d x . d o i . o r g / 10.1787 / 9789264013124-f r$.

Warda, J. (2001), " Measuring the value of R\&D tax treatment in OECD countries ", STI Review, $n^{\circ} 27:$ Special Issue on New Science and Technology Indicators, Éditions OCDE, Paris, www.oecd.org/sti/37124998.pdf. 


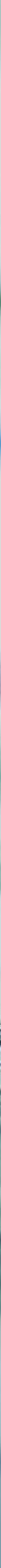


5. LEADERSHIP ET COMPÉTITIVITÉ

1. Spécialisation en R-D

2. Adoption du commerce électronique

3. Le commerce électronique à travers les secteurs et les applications

4. Dynamique des start-ups

5. Avantage technologique

6. Participation aux chaînes de valeur mondiales

7. Échanges et emploi

Notes et références

SCIENCE, TECHNOLOGIE ET INDUSTRIE : TABLEAU DE BORD DE L'OCDE 2017 @ OCDE 2018

169 
Intensité de R-D des entreprises corrigée de la structure d'activités, 2015

En pourcentage de la valeur ajoutée dans le secteur des entreprises

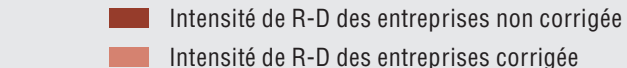

KOR

FIN

SWE

JPN

AUT

DEU

DNK

USA

BEL

SVN

FRA

ISL

AUS

GBR

NLD

NOR

IRL

HUN

CZ

CAN

ITA

EST

ES

PR

TUR

POL

GRC

SVK

LVA

MEX

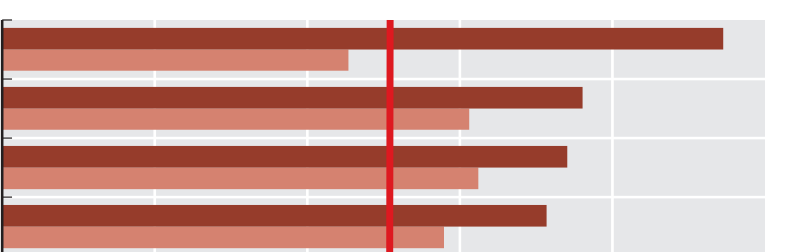

-

US

R

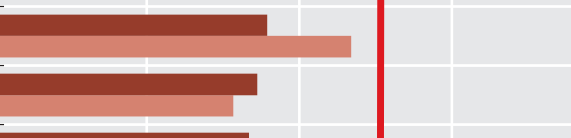

L

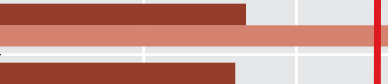

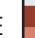

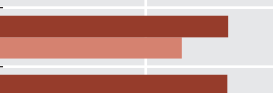

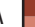

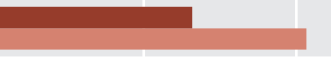

T

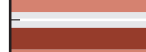



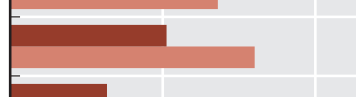

TR

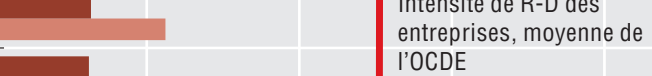

C

K

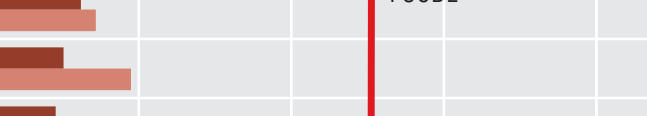

-

Ex

0

1

2

3

4

Source : Calculs de l'OCDE, d'après Base de données ANBERD, http:// oe.cd/anberd, Base de données des Comptes nationaux annuels, Base de données pour l'analyse structurelle (STAN), http://oe.cd/stan, Base de données des principaux indicateurs de la science et de la technologie, $h t t p: / / o e . c d / m s t i$, et Base de données sur les statistiques de la recherchedéveloppement, http://oe.cd/rds, juin 2017. Voir notes de chapitre.

StatLink नiाIst http://dx.doi.org/10.1787/888933722105

\section{Le saviez-vous?}

Les industries manufacturières totalisent plus de $85 \%$ de la R-D des entreprises menée en Allemagne, en Chine, en Corée et au Japon. La moyenne de l'OCDE s'établit à $54 \%$.

L'analyse comparative de l'intensité de R-D des entreprises (dépenses de R-D des entreprises rapportées à la valeur ajoutée) dans les différents pays peut être biaisée par la spécialisation relative des pays dans les différents secteurs d'activités. Pour faciliter la comparaison, on calcule la valeur théorique de l'intensité de R-D des entreprises des pays en supposant que tous ont la structure d'activités qui correspond à la moyenne de la zone OCDE. Si c'était le cas - et en supposant que les pays conservent leurs propres intensités sectorielles de R-D l'intensité de R-D des entreprises corrigée en Allemagne et en Corée serait inférieure à l'intensité moyenne de la zone OCDE qui se situe à $2.5 \%$, car ces deux économies sont relativement spécialisées dans des activités à forte intensité de R-D. En Belgique et en France, des pays plus spécialisés dans des activités à moindre intensité de R-D, l'intensité de R-D des entreprises passerait au-dessus de la moyenne de l'OCDE. Au Canada, en Norvège et au Royaume-Uni, elle se rapprocherait de la moyenne de l'OCDE. Dans la plupart des pays d'Europe du Sud et de l'Est, le changement d'indicateur ne modifierait guère l'intensité de R-D globale. Enfin, aux États-Unis, la structure d'activités est déjà proche de celle qui correspond à la moyenne de l'OCDE.

Les activités à forte intensité de R-D totalisent généralement la majeure partie des dépenses intérieures de R-D des entreprises (DIRDE) dans le secteur manufacturier. Dans la plupart des pays de l'OCDE, plus d'un tiers des DIRDE sont engagées dans le secteur des activités de services. Cette proportion s'est accrue au cours de la dernière décennie, sous l'effet, notamment, du rôle des activités de services liées à l'information et aux communications. Il convient néanmoins de faire preuve de prudence lorsqu'on compare les distributions sectorielles des DIRDE des pays, car les différences au niveau de la structure des entreprises et des pratiques statistiques peuvent avoir un impact sur la mesure dans laquelle les exécutants de la R-D (y compris ceux qui participent à la R-D liée aux activités de fabrication) sont comptabilisés dans le secteur spécifique des activités de services de R-D - ce qui peut représenter plus de la moitié de toute la R-D des activités de services.

\section{Définitions}

L'intensité de R-D corrigée de la structure d'activités correspond, pour un pays, à la moyenne pondérée des intensités de R-D des différentes branches d'activité, la pondération se faisant en fonction de la part de la valeur ajoutée de chaque branche d'activité dans la structure d'activités correspondant à la moyenne de la zone OCDE, alors que dans l'indicateur non corrigé, c'est la part effective dans le pays considéré qui est prise en compte. Les calculs sont fondés sur la CITI rév. 4.

Les catégories d'intensité de R-D sont définies dans GalindoRueda et Verger (2016). Les activités de fabrication à forte et moyenne-forte intensité de R-D sont la fabrication de produits chimiques et de préparations pharmaceutiques (divisions 20 et 21 de la CITI rév. 4) et la fabrication d'ordinateurs, d'articles électroniques et optiques, de matériels électriques, de machines, de véhicules automobiles et d'autres matériels de transport (divisions 26 à 30 de la CITI rév. 4). Les services de R-D correspondent à la division 72 de la CITI rév. 4. 
R-D des entreprises dans les activités de fabrication, par catégorie d'intensité de R-D, 2015

En pourcentage de la R-D dans les activités de fabrication

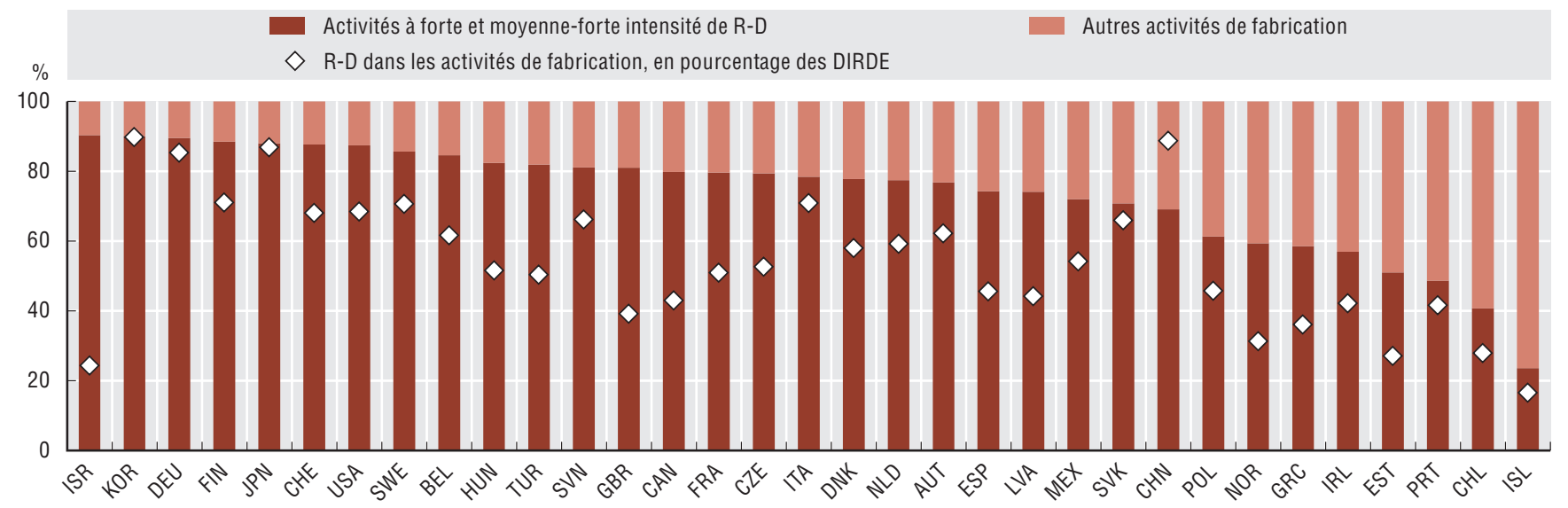

Source : OCDE, Base de données ANBERD http://oe.cd/anberd ; Base de données sur les statistiques de la recherche-développement, http://oe.cd/rds, juin 2017. Davantage de données via StatLink. Voir notes de chapitre.

R-D dans les activités de services, 2015

En pourcentage des dépenses intérieures de $R-D$ des entreprises

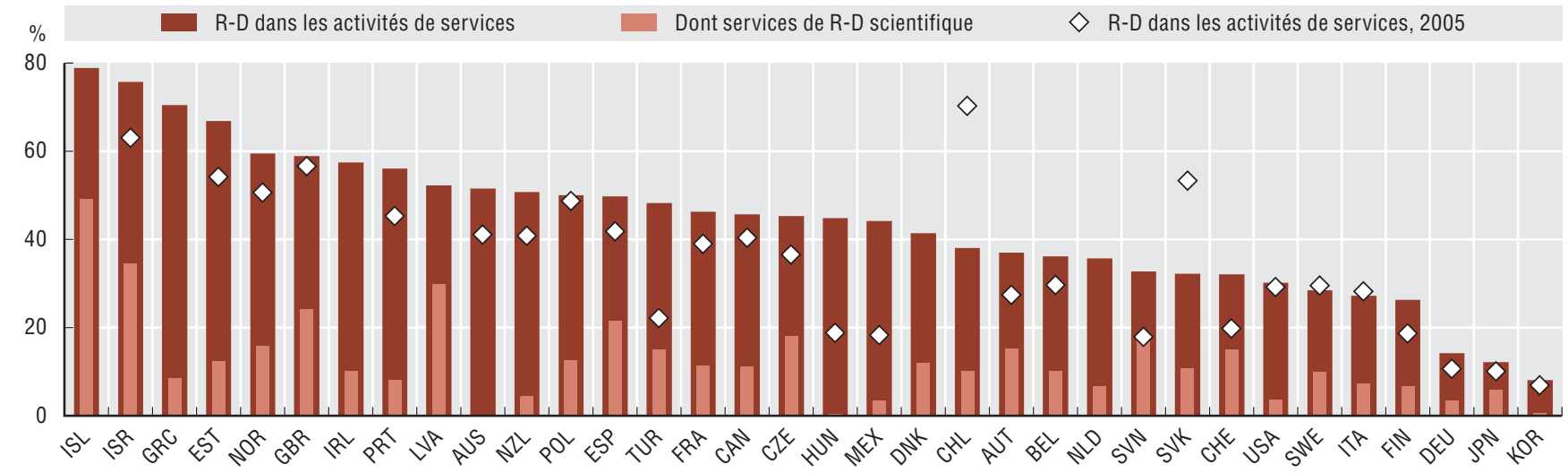

Source : OCDE, Base de données ANBERD http://oe.cd/anberd ; Base de données sur les statistiques de la recherche-développement, http://oe.cd/rds, juin 2017. Davantage de données via StatLink. Voir notes de chapitre.

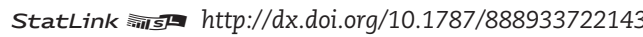

\section{Mesurabilité}

L'allocation de la R-D aux différentes activités ne va pas sans certaines difficultés. Dans la plupart des pays, on impute les dépenses de R-D d'une entreprise à son activité principale, sur la base de la valeur ajoutée (critère de l'« activité principale »). Cependant, certains pays se fondent sur la principale activité de R-D de l'entreprise ou sur le contenu de la R-D (critère de "l'orientation sectorielle "). L'édition 2015 du Manuel de Frascati recommande de classer les unités statistiques en fonction de leur activité principale et propose que leurs activités de R-D soient classées à la fois sur cette base et par orientation sectorielle. Dans la pratique, certains pays appliquent une combinaison de ces deux méthodes. Par ailleurs, la méthodologie utilisée pour corriger l'intensité de R-D de la structure d'activités est sensible à la classification des activités utilisée. Parmi les autres facteurs ayant une incidence, on trouve le niveau d'agrégation auquel les pondérations sectorielles sont calculées, et les pays retenus comme référence. Sont exclus les pays pour lesquels la décomposition de la R-D est limitée et pour lesquels il n'existe pas de données sur la valeur ajoutée par activité qui soient comparables et compilées selon le SCN. 


\section{Diffusion dans les entreprises d'une sélection d'outils et d'activités s'appuyant sur les TIC, par technologie, 2016}

En pourcentage des entreprises de 10 salariés ou plus
- Cloud computing
- Données massives
- PGI
O GRC
O RFID

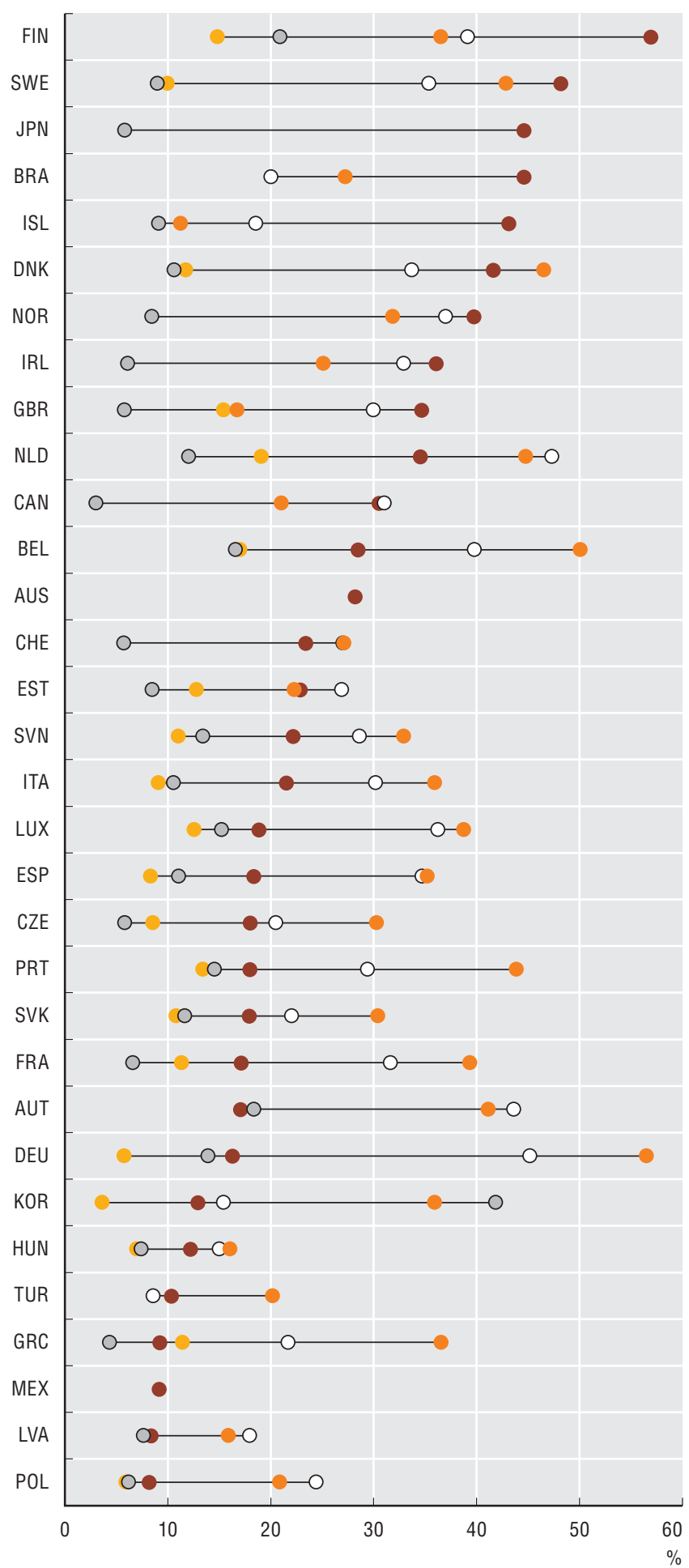

Source : OCDE, Base de données sur l'accès et utilisation des TIC par les entreprises, $h$ ttp://oe.cd/bus, juillet 2017. Davantage de données via StatLink. Voir notes de chapitre.

StatLink ainst http://dx.doi.org/10.1787/888933722162

\section{Le saviez-vous ?}

En 2015-16, 95 \% des entreprises des pays déclarants de l'OCDE disposaient d'une connexion haut débit, mais seulement $22 \%$ pratiquaient la vente en ligne.

Les entreprises ne peuvent plus aujourd'hui exercer leur activité sans les TIC, mais la mesure dans laquelle leurs processus opérationnels intègrent les outils des TIC tend à varier d'un pays à l'autre, en fonction de la composition des entreprises et des secteurs d'activités. En 2016, c'était en Finlande que le pourcentage d'entreprises ayant recours au cloud computing était le plus élevé (57\%), mais l'analyse des données massives y était moins répandue qu'aux Pays-Bas (19\%) et en Belgique (17\%). Inversement, les services de cloud computing sont moins répandus en Allemagne (16\%) que dans la moyenne des pays de l'OCDE (25\%), mais les entreprises allemandes arrivent en tête de classement pour ce qui est des progiciels de gestion intégrés ( $57 \%$ ) et en deuxième position en ce qui concerne les solutions de gestion de la relation client (45\%). La Corée est le pays où les entreprises utilisent le plus (42\%) la radio-identification (RFID) mais le moins (4\%) l'analyse des données massives.

En moyenne, $22 \%$ des entreprises des pays déclarants de l'OCDE ont réalisé des ventes en ligne en 2015, soit seulement 3 points de pourcentage de plus qu'en 2009. Les écarts entre pays sont considérables : plus de la moitié des entreprises en Nouvelle-Zélande sont concernées, mais moins d'une sur dix en Corée et au Mexique.

Si le manque d'harmonisation de la définition des ventes en ligne peut expliquer pour partie ces disparités, la principale cause semble être le poids des entreprises plus petites dans les économies. En moyenne, $40 \%$ des grandes entreprises ont fait du commerce électronique en 2015, contre seulement $20 \%$ des petites entreprises.

\section{Définitions}

Le haut débit renvoie à la fois aux connexions fixes et aux connexions mobiles, avec un débit de téléchargement déclaré d'au moins de 256 kbit/s. La gestion de la chaîne logistique désigne l'utilisation d'applications d'échange automatique de données (EAD). Les progiciels de gestion intégrés (PGI) sont des logiciels de gestion des flux d'informations internes. Les solutions de gestion de la relation client (GRC) sont des logiciels de gestion des interactions d'une entreprise avec ses clients, ses employés et ses fournisseurs. Le cloud computing désigne les services de TIC fournis via l'internet, qui permettent d'accéder à un serveur, des capacités de stockage, des composantes du réseau et des applications logicielles. Les données massives renvoient à l'analyse de grandes quantités de données produites par des activités menées par voie électronique ou au moyen de la communication entre machines (M2M).

Les catégories de taille sont les suivantes : petites entreprises (de 10 à 49 salariés), moyennes entreprises (de 50 à 249 salariés), grandes entreprises (250 salariés ou plus).

Le commerce électronique renvoie à la vente de biens ou de services effectuée via des réseaux informatiques par des méthodes spécifiquement conçues pour la réception ou la passation de commandes (à savoir pages web, extranet ou échange de données informatisé), à l'exclusion des commandes passées par téléphone, télécopie ou courrier électronique composé manuellement. 


\section{Diffusion dans les entreprises d'une sélection d'outils et d'activités s'appuyant sur les TIC, pays de l'OCDE, 2010 et 2016}

En pourcentage des entreprises de 10 salariés ou plus

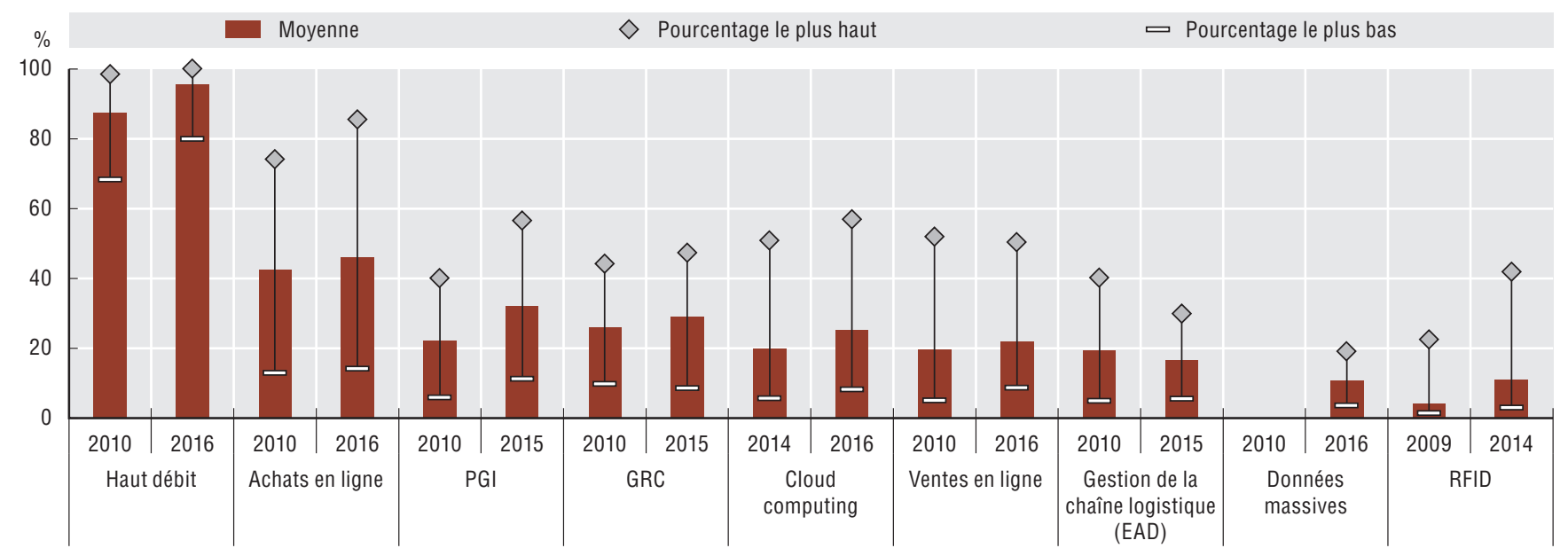

Source : OCDE, Base de données sur l'accès et utilisation des TIC par les entreprises, http://oe.cd/bus, juillet 2017. Davantage de données via StatLink. Voir notes de chapitre.

Entreprises recourant aux ventes en ligne, par taille d'entreprise, 2015

En pourcentage du nombre total d'entreprises dans chaque catégorie de taille

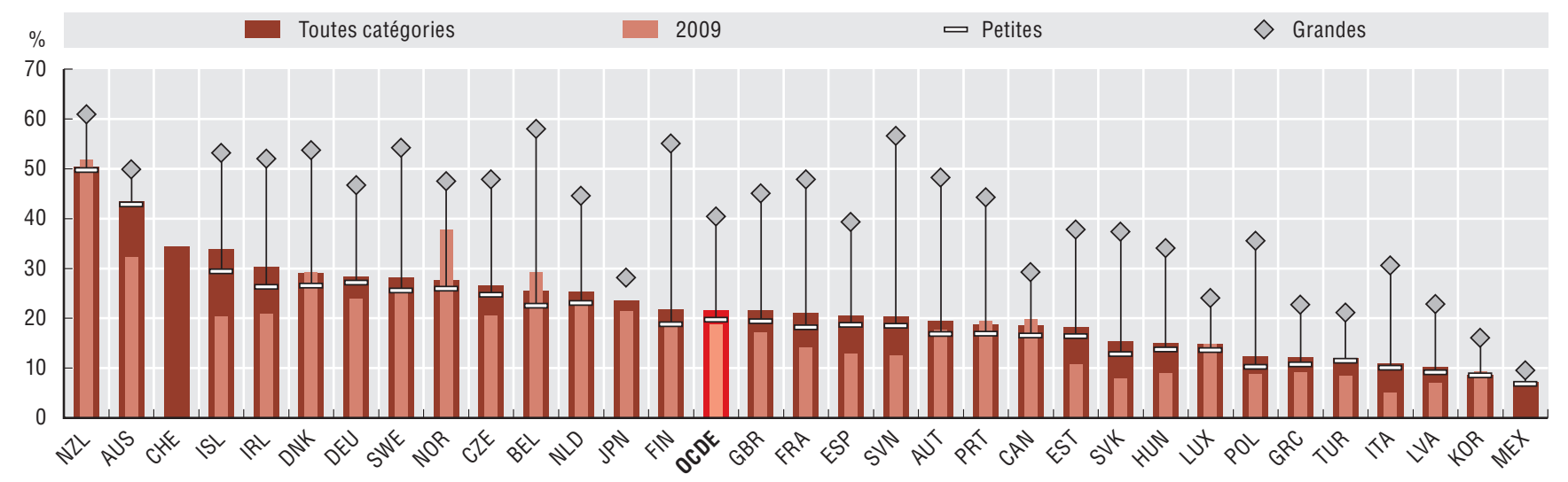

Source : OCDE, Base de données sur l'accès et utilisation des TIC par les entreprises, http://oe.cd/bus, juillet 2017. Davantage de données via StatLink. Voir notes de chapitre.

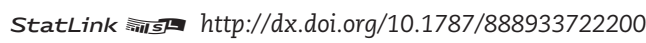

\section{Mesurabilité}

La mesure du commerce électronique pose plusieurs difficultés méthodologiques pouvant nuire à la comparabilité entre les pays, comme l'adoption de pratiques différentes pour la collecte des données et les estimations, le traitement des valeurs aberrantes, ou encore la mesure dans laquelle les entreprises multinationales ont recours au commerce électronique. D'autres problèmes tiennent aux différences dans la couverture sectorielle des enquêtes, et à l'absence de mesure du type d'acteurs impliqué (entre entreprises - B2B, d'entreprise à consommateur - B2C, etc.). La convergence des technologies pose des difficultés supplémentaires pour le traitement des transactions émergentes, notamment par téléphone mobile, par SMS ou à l'aide d'appareils permettant la communication en champ proche, et pour la collecte de données les concernant. Tous les pays de l'OCDE ne mènent pas d'enquête spécifique sur l'utilisation des TIC par les entreprises. Outre les différences dans les outils d'enquête employés, la majorité des indicateurs correspondent à des définitions génériques, qui ne peuvent que donner une mesure approximative des fonctionnalités et des usages possibles des outils exploitant les TIC. Ainsi, les PGI recouvrent divers outils logiciels n'ayant pas les mêmes fonctionnalités : il peut y avoir des écarts substantiels dans le niveau de sophistication des systèmes de PGI et dans leur degré de mise en œuvre. Les services de cloud computing et l'analyse des données massives soulèvent des problèmes analogues. 


\section{Entreprises utilisant des services de cloud computing, par taille d'entreprise, 2016}

En pourcentage du nombre total d'entreprises dans chaque catégorie de taille

$\begin{array}{lll}\checkmark \text { Toutes catégories } & \text { [ Petites } & \text { ○ Moyennes } \\ \diamond \text { Grandes } & 2014\end{array}$

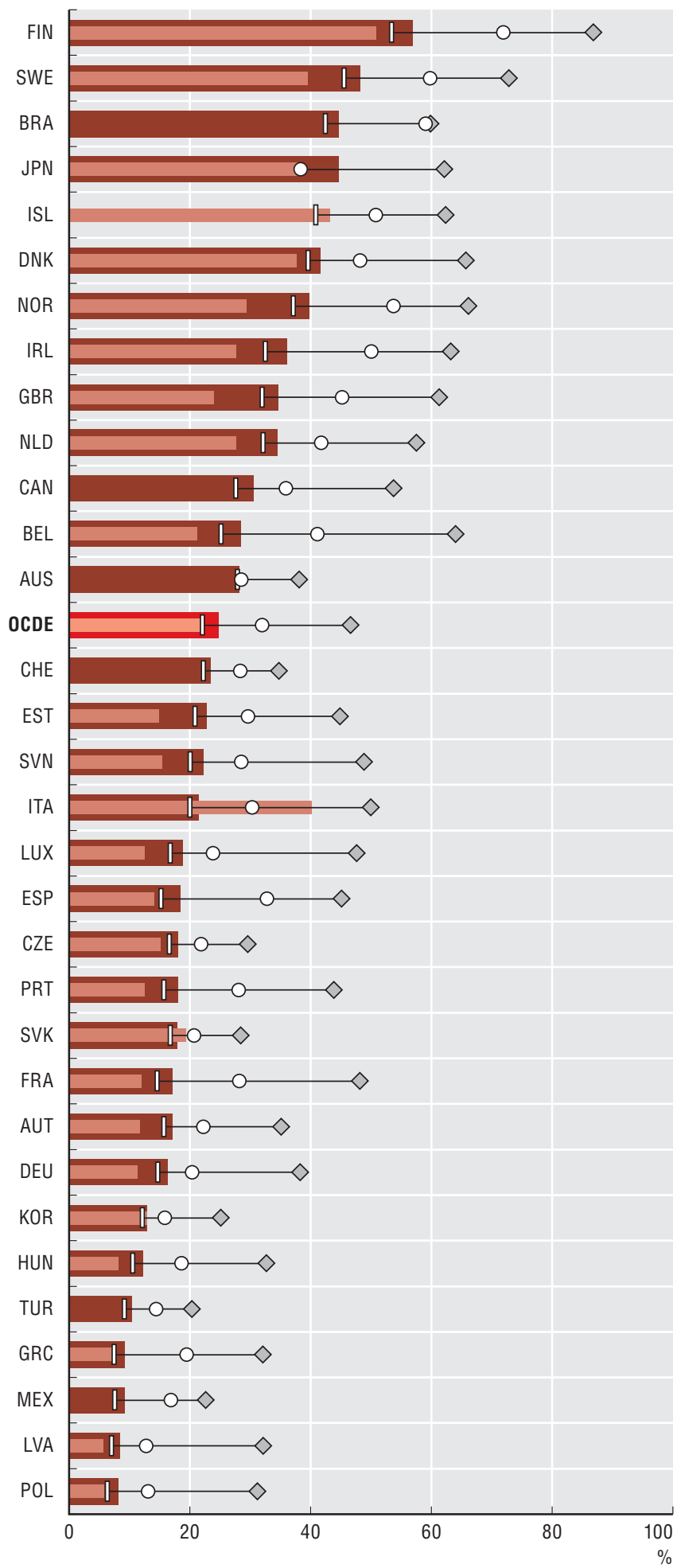

Source : OCDE, Base de données sur l'accès et utilisation des TIC par les entreprises, http://oe.cd/bus, juillet 2017. Davantage de données via StatLink. Voir notes de chapitre.

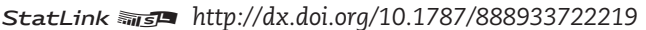

\section{Le saviez-vous ?}

En 2016, plus de $25 \%$ des entreprises des pays de l'OCDE déclaraient utiliser des services de cloud computing, cette proportion variant entre $8 \%$ en Pologne et $57 \%$ en Finlande.

Le commerce électronique peut stimuler la croissance des entreprises en favorisant la diffusion des produits, la réduction des coûts et la prise en compte des demandes personnalisées des clients. Le cloud computing, en particulier, offre tout un éventail de nouveaux processus : les entreprises, et surtout celles qui viennent de démarrer, ont accès à des services informatiques à la demande qu'elles paient en fonction de l'usage qu'elles en font. En 2016, plus de $25 \%$ des entreprises des pays de l'OCDE déclaraient utiliser ces services, contre $22 \%$ en 2014.

L'intensité d'utilisation du cloud computing dépend beaucoup des pays et des secteurs d'activités, ainsi que de la taille des entreprises considérées. En moyenne, les petites entreprises des pays de l'OCDE ne sont que $22 \%$ à utiliser les services de cloud computing, tandis que $32 \%$ des moyennes entreprises et $47 \%$ des grandes entreprises y ont recours.

Les écarts observés d'un secteur à l'autre ou d'un pays à l'autre dans un secteur donné sont également importants. Le secteur où le cloud computing s'est le plus implanté est de loin celui des services d'information et de communication, dans lequel, en moyenne, 51 \% des entreprises de la zone OCDE y ont recours. Le secteur des activités professionnelles, scientifiques et techniques arrive en deuxième position (38\% des entreprises de la zone OCDE). Les secteurs où les entreprises utilisent le moins les services de cloud computing sont le commerce de détail (20\%) et les services d'hébergement et de restauration (17\%). La diffusion des autres outils fondés sur les TIC tend à varier selon les secteurs d'activités. En 2016, les écarts les plus importants entre secteurs étaient observés pour l'utilisation des médias sociaux (46 points de pourcentage) et l'utilisation des solutions de gestion de la relation client (45), tandis que l'écart le plus faible était enregistré pour le haut débit (8). Les secteurs de la construction et de l'hébergement et de la restauration semblent être ceux où la diffusion des outils et activités liés aux TIC est la plus lente.

\section{Définitions}

Le cloud computing désigne les services de TIC fournis via l'internet, qui permettent d'accéder à un serveur, des capacités de stockage, des composantes du réseau et des applications logicielles.

Les catégories de taille sont les suivantes : petites entreprises (de 10 à 49 salariés), moyennes entreprises (de 50 à 249 salariés), grandes entreprises (250 salariés ou plus).

Le haut débit renvoie à la fois aux connexions fixes et aux connexions mobiles, avec un débit de téléchargement déclaré d'au moins de 256 kbit/s. La gestion de la chaîne logistique désigne l'utilisation d'applications d'échange automatique de données (EAD). Les progiciels de gestion intégrés (PGI) sont des logiciels de gestion des flux d'informations internes. Les solutions de gestion de la relation client (GRC) sont des logiciels de gestion des interactions d'une entreprise avec ses clients, ses employés et ses fournisseurs. Les données massives renvoient à l'analyse de grandes quantités de données produites par des activités menées par voie électronique ou au moyen de la communication entre machines (M2M). Le commerce électronique renvoie à la vente de biens ou de services effectuée via des réseaux informatiques par des méthodes spécifiquement conçues pour la réception ou la passation de commandes. 
Adoption des services de cloud computing dans les différents secteurs d'activités, pays de l'OCDE, 2016

En pourcentage des entreprises de 10 salariés ou plus

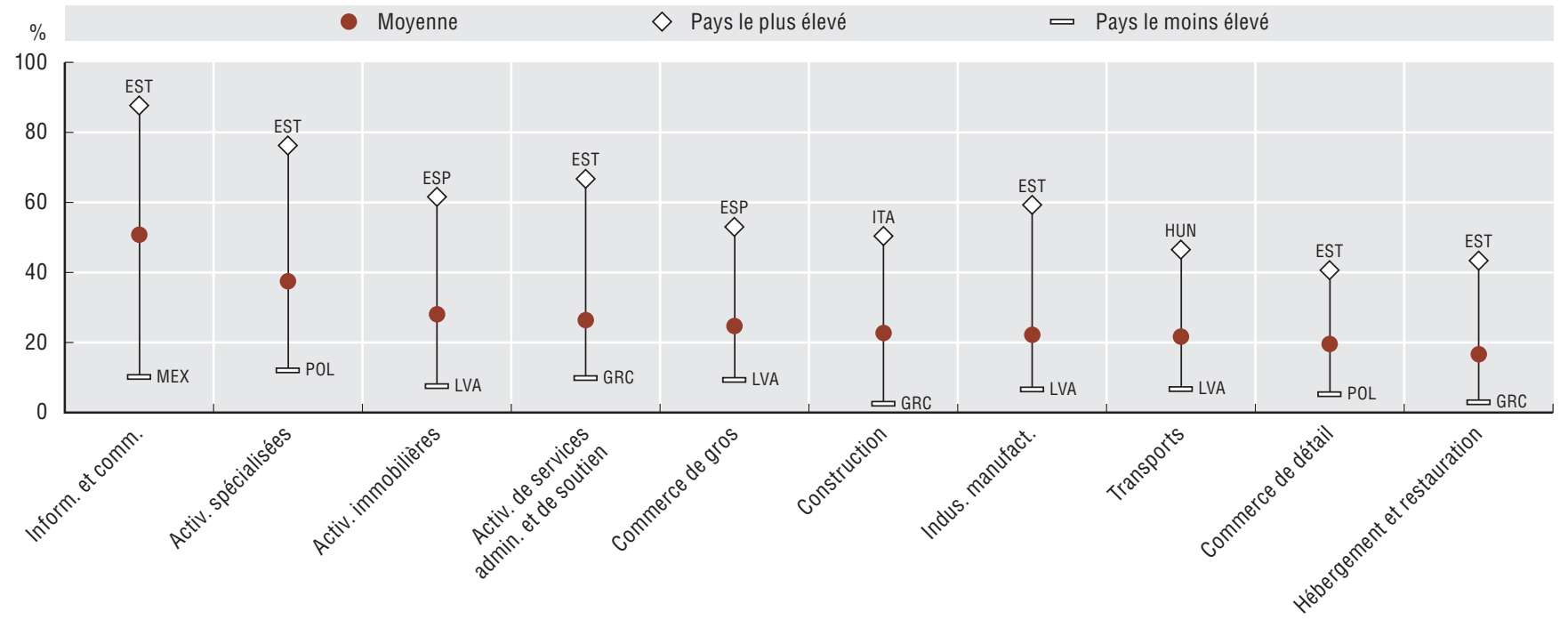

Source : OCDE, Base de données sur l'accès et utilisation des TIC par les entreprises, http://oe.cd/bus, juillet 2017. Davantage de données via StatLink. Voir notes de chapitre.

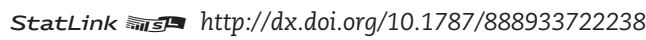

Diffusion dans les secteurs d'activités d'une sélection d'outils et d'activités s'appuyant sur les TIC, pays de l'OCDE, 2016 En pourcentage des entreprises de 10 salariés ou plus

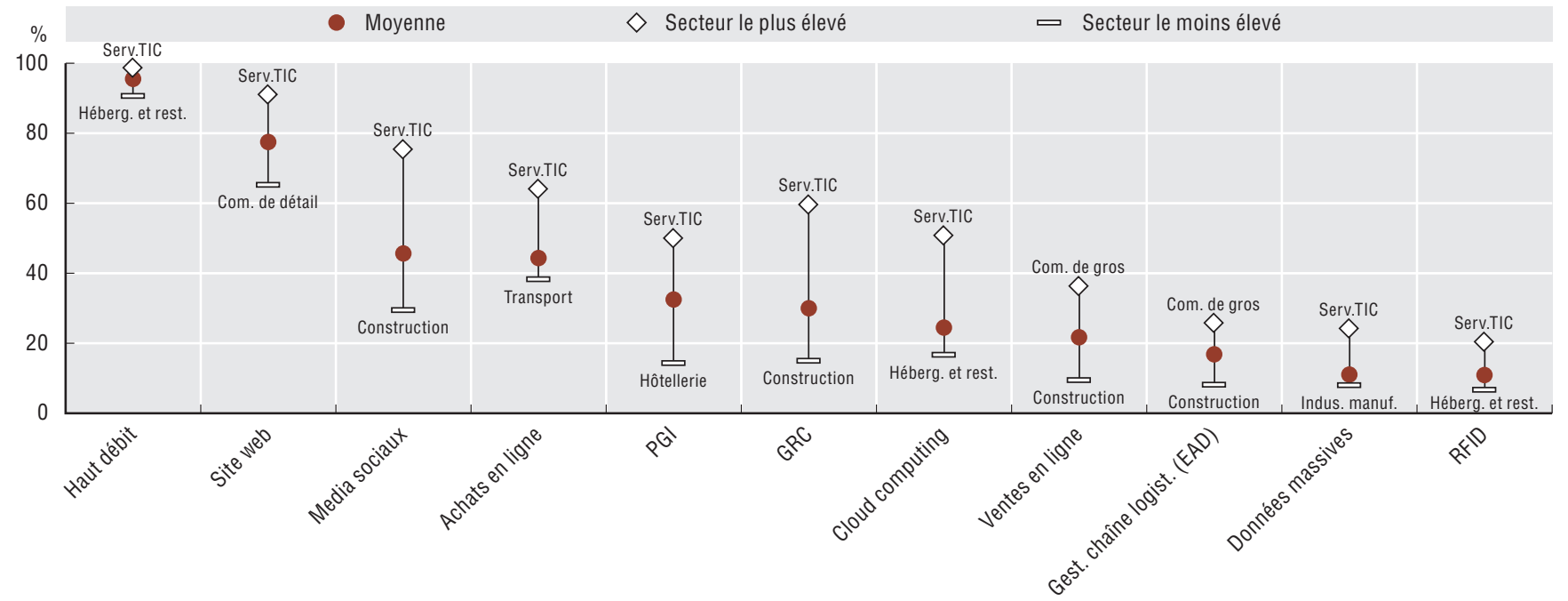

Source : OCDE, Base de données sur l'accès et utilisation des TIC par les entreprises, http://oe.cd/bus, juillet 2017. Voir notes de chapitre.

\section{Mesurabilité}

Tous les pays de l'OCDE ne mènent pas d'enquête spécifique sur l'utilisation des TIC par les entreprises. Outre les différences dans les outils d'enquête employés, la majorité des indicateurs correspondent à des définitions génériques, qui ne peuvent que donner une mesure approximative des fonctionnalités et des usages possibles des outils exploitant les TIC. Ainsi, les PGI recouvrent divers outils logiciels n'ayant pas les mêmes fonctionnalités : il peut y avoir des écarts substantiels dans le niveau de sophistication des systèmes de PGI et dans leur degré de mise en œuvre. Les services de cloud computing sont relativement plus nouveaux que les applications web de GRC ou de PGI, d'où l'importance de bien les distinguer des autres services en ligne lorsqu'on mesure l'utilisation des outils des TIC.

D'autres problèmes tiennent aux différences dans la couverture sectorielle des enquêtes. La convergence des technologies pose des difficultés supplémentaires pour le traitement des transactions émergentes, notamment par téléphone mobile, par SMS ou à l'aide d'appareils permettant la communication en champ proche, et pour la collecte de données les concernant. 
Taux d'entrée et de sortie des entreprises, secteur des TIC et autres secteurs d'activités, 2013-15

Nombre d'unités entrantes/sortantes en pourcentage du nombre total d'unités entrantes/sortantes et existantes

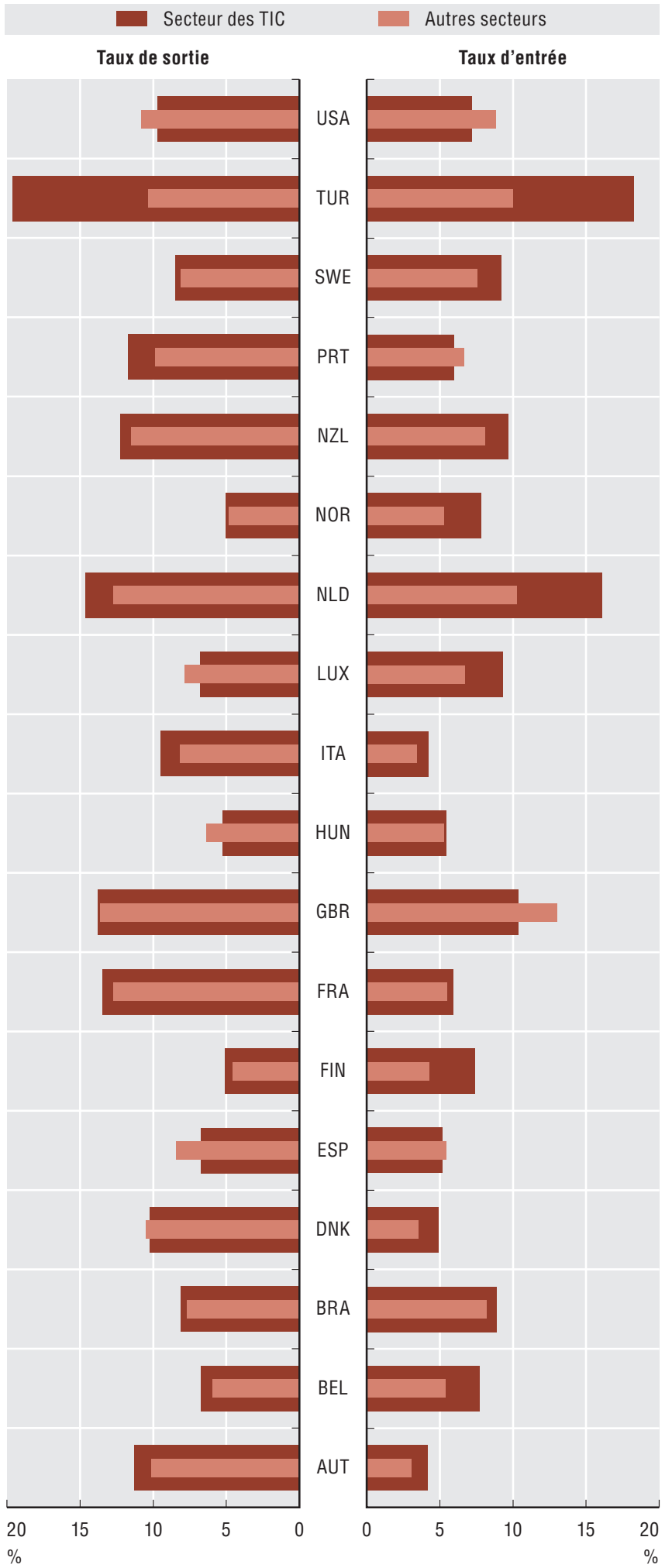

Source : Calculs de l'OCDE, d'après des données préliminaires des bases de données DynEmp v. 2 et v. 3, http://oe.cd/dynemp, juillet 2017. Davantage de données via StatLink. Voir notes de chapitre.

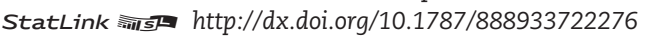

\section{Le saviez-vous ?}

Dans la plupart des pays, le secteur des TIC est plus dynamique que les autres, avec une plus grande proportion de jeunes petites entreprises qui, souvent, connaissent une croissance plus rapide.

Les taux d'entrée sur le marché et de sortie du marché sont plus élevés dans le secteur des TIC que dans les autres secteurs dans la plupart des pays couverts par le projet DynEmp de l'OCDE. Cette tendance doit surtout aux entrées et aux sorties observées dans les secteurs des services de TIC (activités informatiques et services d'information, et télécommunications). Ces taux d'entrée et de sortie plus importants pourraient être associés à une plus grande redistribution susceptible d'améliorer la productivité dans ces secteurs.

Comparativement aux autres secteurs de l'économie, celui des TIC tend à accueillir une proportion plus élevée de jeunes petites et micro-entreprises. Cette observation demeure vraie lorsqu'on compare les services de TIC aux autres services marchands non financiers. Le secteur manufacturier est caractérisé par une dynamique plus hétérogène avec, dans de nombreux pays, de petites différences entre les activités de fabrication de biens TIC et les autres activités de fabrication. Dans le cas de cet indicateur, comme dans celui sur les taux d'entrée, la tendance aux États-Unis et au Royaume-Uni est inverse à la tendance générale observée.

Dans tous les pays, les petites entreprises jeunes croissent plus vite que leurs homologues plus anciennes. Le plus souvent, la différence de croissance moyenne de l'emploi entre les petites entreprises jeunes et les petites entreprises anciennes est plus importante dans le secteur des TIC que dans les autres secteurs de l'économie. Cette observation confirme la perception générale que l'on a d'un secteur des TIC plus dynamique.

\section{Définitions}

Le secteur des TIC recouvre les divisions 26, 61 et 62-63 de la CITI rév. 4 (ordinateurs, articles électroniques et optiques, télécommunications, et activités informatiques et services d'information). Les autres secteurs englobent les activités de fabrication et les services marchands non financiers à l'exclusion du secteur des TIC, de la cokéfaction et fabrication de produits pétroliers raffinés et des activités immobilières.

Le taux d'entrée sur le marché s'obtient en divisant le nombre d'unités entrantes par le nombre total d'unités entrantes et existantes, c'est-à-dire le nombre total d'unités actives pendant l'année en cours. Le taux de sortie du marché s'obtient en divisant le nombre d'unités sortantes par le nombre total d'unités sortantes et existantes, c'est-à-dire le nombre total d'unités actives pendant l'année précédente.

La part des jeunes petites et micro-entreprises existantes s'obtient en divisant le nombre d'entreprises existantes en activité depuis moins de 6 ans et de moins de 50 employés par le nombre total d'entreprises existantes.

Les petites entreprises jeunes sont celles qui sont en activité depuis moins de 6 ans et qui emploient de 10 à 49 salariés. Les petites entreprises anciennes sont celles qui sont en activité depuis 6 ans ou plus et qui emploient de 10 à 49 salariés. La croissance de l'emploi est définie comme la moyenne des taux de croissance de l'emploi au niveau des unités $\mathrm{GR}_{\mathrm{it}}=\mathrm{Emploi}_{\mathrm{t}}-$ Emploi $_{\mathrm{t}-1} / 0.5^{*}$ $\left(\right.$ Emploi $_{t}+$ Emploi $\left._{t-1}\right)$ dans le groupe d'unités du pays et du secteur d'activités considérés. C'est un indicateur indifférent à l'échelle et compris dans l'intervalle $[-200 \% ;+200 \%]$. 
Part des jeunes petites et micro-entreprises existantes dans le secteur des TIC et les autres secteurs d'activités, 2013-15

En pourcentage du nombre total d'entreprises existantes

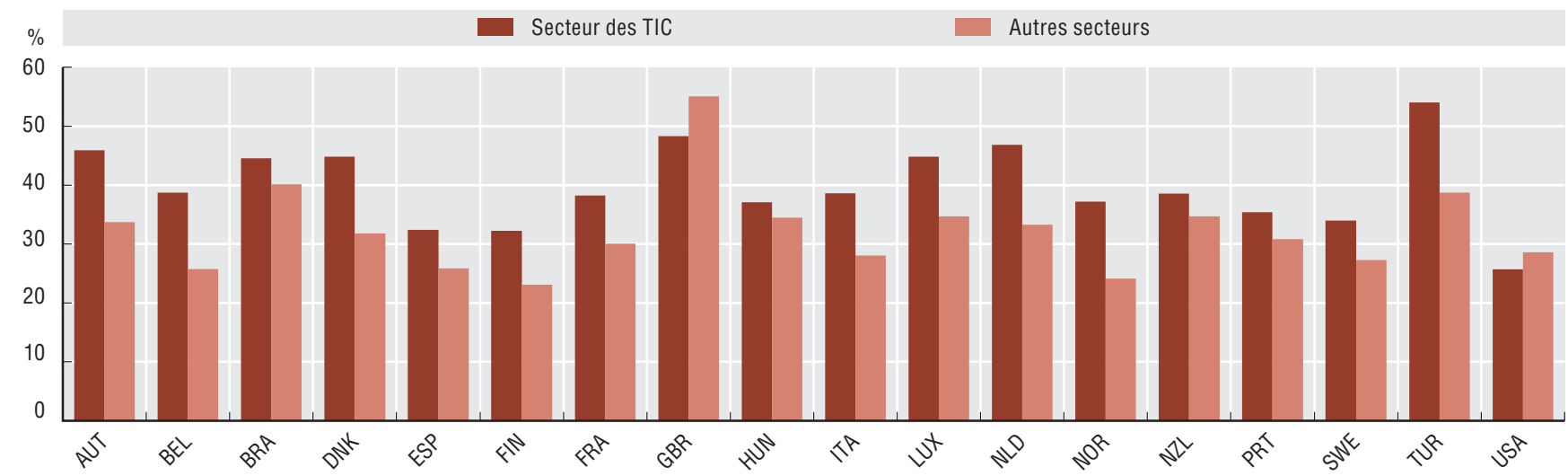

Source : Calculs de l'OCDE, d'après des données préliminaires des bases de données DynEmp v.2 et v.3, http://oe.cd/dynemp, juillet 2017. Davantage de données via StatLink. Voir notes de chapitre.

\section{Différences de croissance de l'emploi entre les petites entreprises jeunes et les petites entreprises anciennes dans le secteur des TIC et les autres secteurs, 2013-15}

Taux de croissance en pourcentage

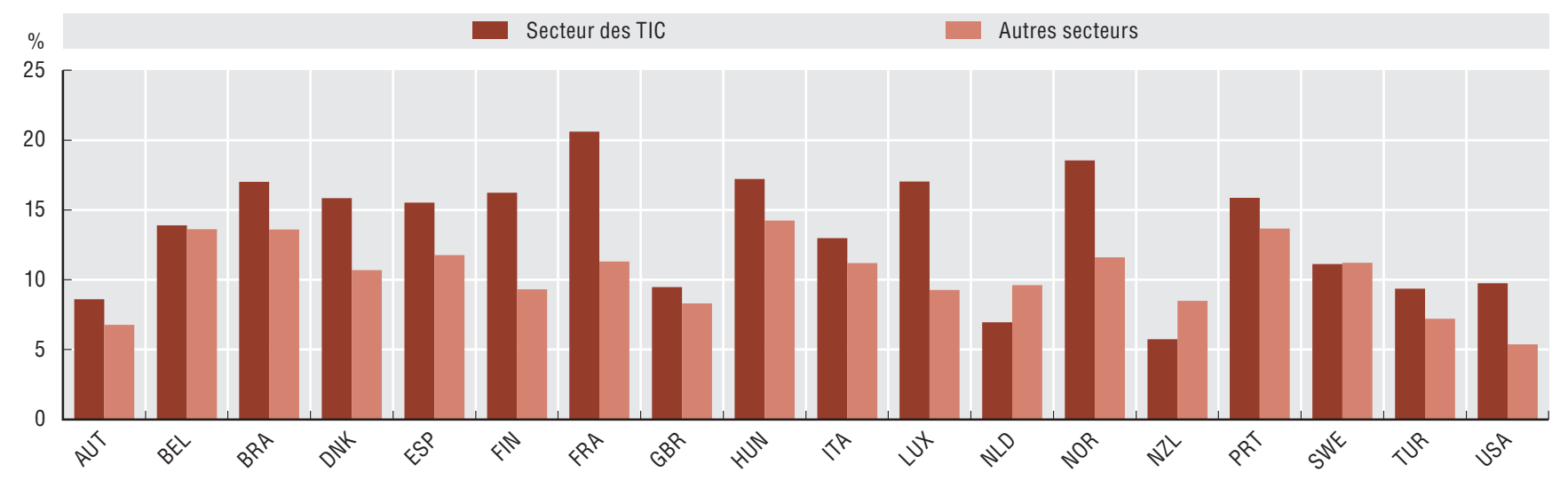

Source : Calculs de l'OCDE, d’après des données préliminaires des bases de données DynEmp v. 2 et v. 3, http://oe.cd/dynemp, juillet 2017. Voir notes de chapitre.

StatLink 제패 http://dx.doi.org/10.1787/888933722314

\section{Mesurabilité}

Le projet DynEmp de l'OCDE s'appuie sur un processus de collecte de données distribuées conçu pour créer une base harmonisée de données micro-agrégées sur la dynamique de l'emploi. Les principales sources de données sont les registres des entreprises. Le projet est soutenu par des experts nationaux qui appliquent la même routine statistique élaborée par l'OCDE aux microdonnées confidentielles auxquelles ils ont accès. Ces experts appliquent également les procédures de divulgation d'information propres à chaque pays de façon à veiller au respect de la confidentialité. Les informations concernant la Belgique, le Brésil, l'Espagne, la Finlande, la Hongrie, la Norvège, la Suède et la Turquie proviennent de la version 3 de la base DynEmp ; celles qui concernent les autres pays proviennent de la version 2. Les chiffres de la version 3 excluent les unités de la catégorie de taille 0-1. De plus, les deux versions de DynEmp appliquent chacune une correction différente à l'année de création lorsqu'elle se situe dans la période examinée. S'agissant du Royaume-Uni, les travaux contiennent des données statistiques de l'Office of National Statistics (ONS), soumis aux droits d'auteur de la Couronne (Crown Copyright). L'utilisation de données statistiques de l'ONS dans ces travaux ne vaut pas approbation par l'ONS de l'interprétation ou de l'analyse de ces données statistiques. Ces travaux utilisent également des ensembles de données de recherche qui pourraient ne pas exactement reproduire les agrégats des statistiques nationales. 
Avantage technologique révélé dans les TIC, 2002-05 et 2012-15

Indice fondé sur les familles de brevets IP5

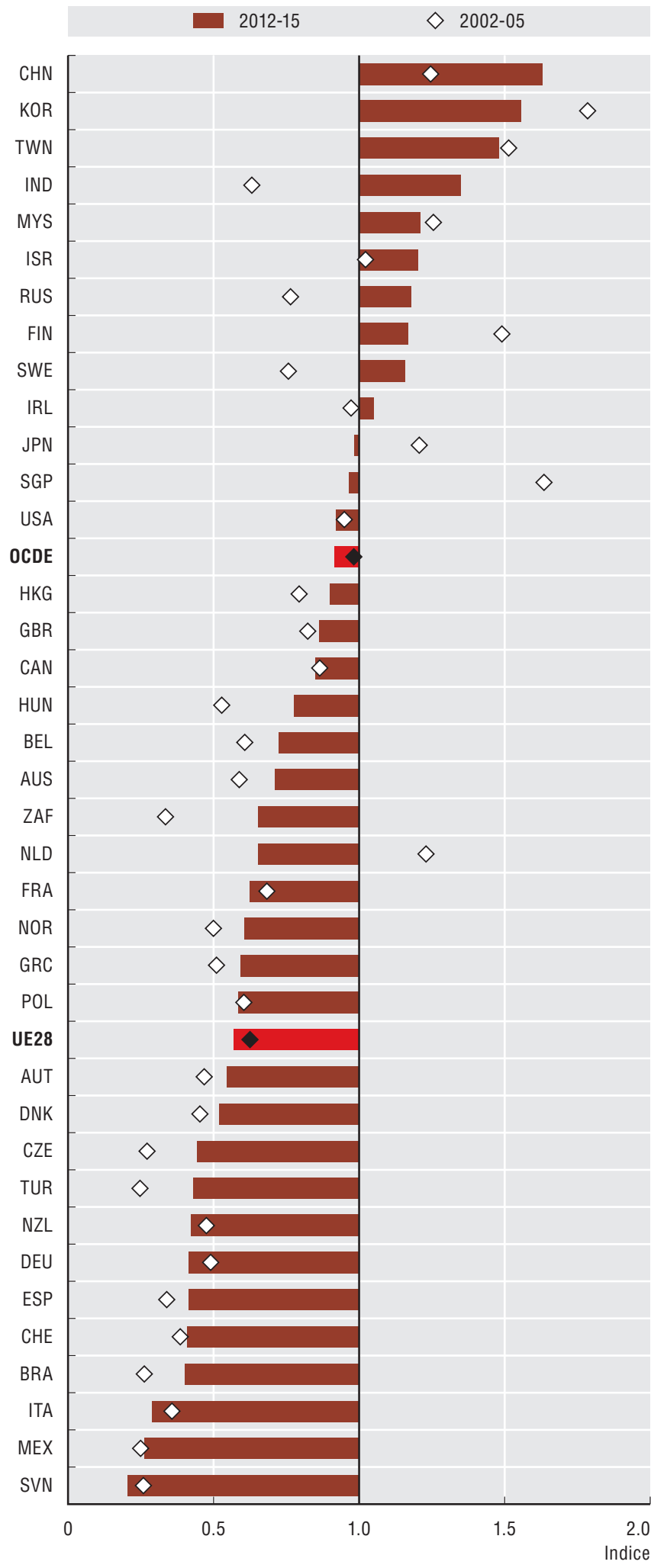

Source : OCDE, STI Micro-data Lab : Base de données sur la propriété intellectuelle, http://oe.cd/ipstats, juin 2017. Davantage de données via StatLink. Voir notes de chapitre.

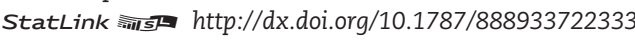

\section{Le saviez-vous ?}

La spécialisation dans les brevets liés aux TIC a plus que doublé en Inde entre 2002 et 2015 et augmenté de plus de $50 \%$ en Chine.

Les innovations dans les technologies génériques comme les technologies de l'information et des communications (TIC) et les technologies destinées à répondre aux besoins de la société dans les domaines de la santé et de l'environnement ont le potentiel de profiter à l'ensemble de la population mondiale. Les informations contenues dans les brevets contribuent à mettre en lumière la spécialisation relative des économies dans ces domaines technologiques.

Entre 2002 et 2015, plusieurs économies, et notamment la Chine et l'Inde, ont augmenté leur spécialisation relative dans les TIC, telle que mesurée par l'indice de l'avantage technologique révélé (ATR). La baisse moyenne (-7\%) de la spécialisation des économies de l'OCDE entre 2002-05 et 2012-15 masque une situation plus hétérogène. Par exemple, la Corée et la Finlande ont vu baisser leur spécialisation dans les technologies brevetées des TIC de plus de $20 \%$, tandis qu'Israël et la Suède l'ont vu augmenter de quelque $20 \%$ et plus.

De même, le fait que les économies de l'OCDE n'aient, dans l'ensemble, pas augmenté leur spécialisation dans les applications liées à la santé ou à l'environnement masque là encore une situation plutôt hétérogène. Dans le domaine de la santé, de nombreuses économies de l'OCDE ont encore augmenté leur ATR au cours de la période de référence. Parmi eux, les Pays-Bas ont plus que doublé leur ATR dans les technologies liées à la santé et la Corée a substantiellement résorbé le déficit relatif de spécialisation qu'elle avait connu durant la décennie précédente. Au contraire, la spécialisation de la Chine a fortement baissé au cours de la même période.

Dans le domaine de l'environnement, l'évolution est quelque peu analogue. Des économies de l'OCDE comme le Danemark et la Nouvelle-Zélande ont augmenté leur ATR de plus de $50 \%$, tandis que les économies BRIICS semblent s'être déspécialisés de plus de $20 \%$ dans l'ensemble.

\section{Définitions}

L'indice d'avantage technologique révélé (ATR) mesure la part des brevets d'une économie dans un domaine technologique donné, rapportée à la part des brevets de cette économie dans l'ensemble des domaines technologiques. Cet indicateur est calculé sur la base des familles de brevets IP5, c'est-à-dire des brevets déposés auprès des cinq principaux offices de propriété intellectuelle, selon la résidence du ou des inventeurs.

Les brevets liés aux TIC sont recensés à l'aide des codes de la Classification internationale des brevets (CIB) indiqués dans Inaba et Squicciarini (2017) et reflètent les récents développements des TIC, notamment s'agissant des réseaux, de la communication mobile, de la sécurité, de l'analyse de données et l'interface humaine.

Les brevets liés à la santé sont recensés sur la base de la table de concordance CIB-technologie établie par l'OMPI (2013) et recouvrent les brevets liés aux technologies pharmaceutiques et médicales.

Les brevets liés aux technologies environnementales sont recensés suivant des stratégies de recherche fondées sur la CIB et la Classification coopérative des brevets (CPC) et prennent appui sur l'expertise des examinateurs de l'Office européen des brevets (OEB), comme décrit dans Haščič et Migotto (2015). 
Avantage technologique révélé dans les technologies de la santé, 2002-05 et 2012-15

Indice fondé sur les familles de brevets IP5

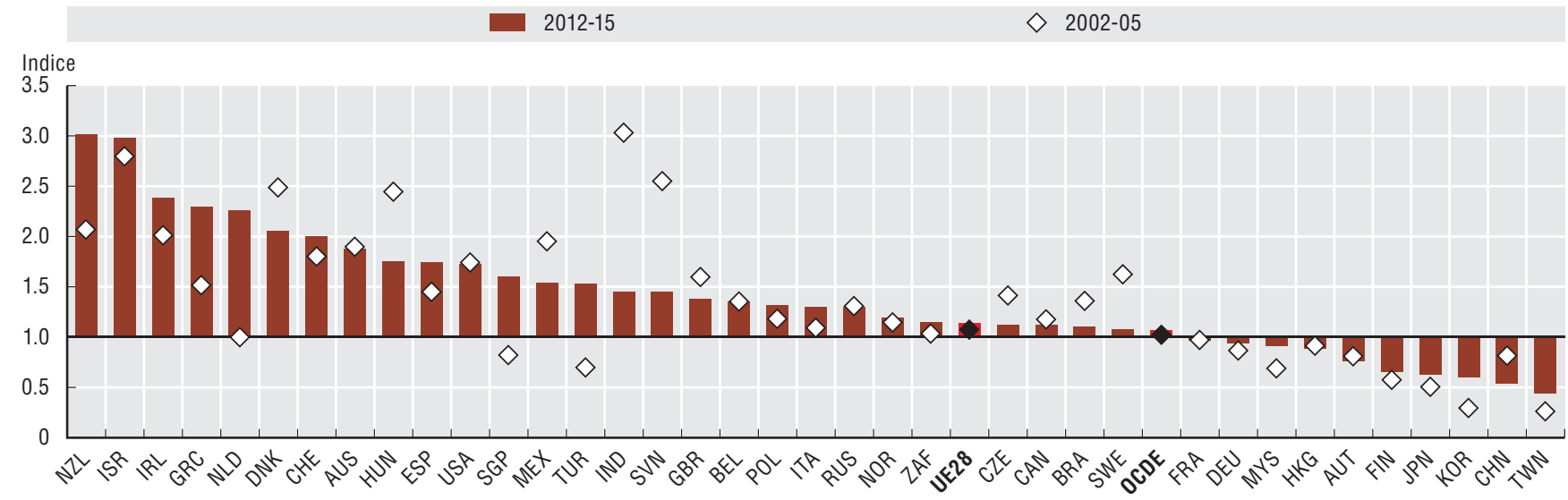

Source : OCDE, STI Micro-data Lab : Base de données sur la propriété intellectuelle, http://oe.cd/ipstats, juin 2017. Davantage de données via StatLink. Voir notes de chapitre.

StatLink तiाइ $h$ ttp://dx.doi.org/10.1787/888933722352

Avantage technologique révélé dans les technologies environnementales, 2002-05 et 2012-15

Indice fondé sur les familles de brevets IP5

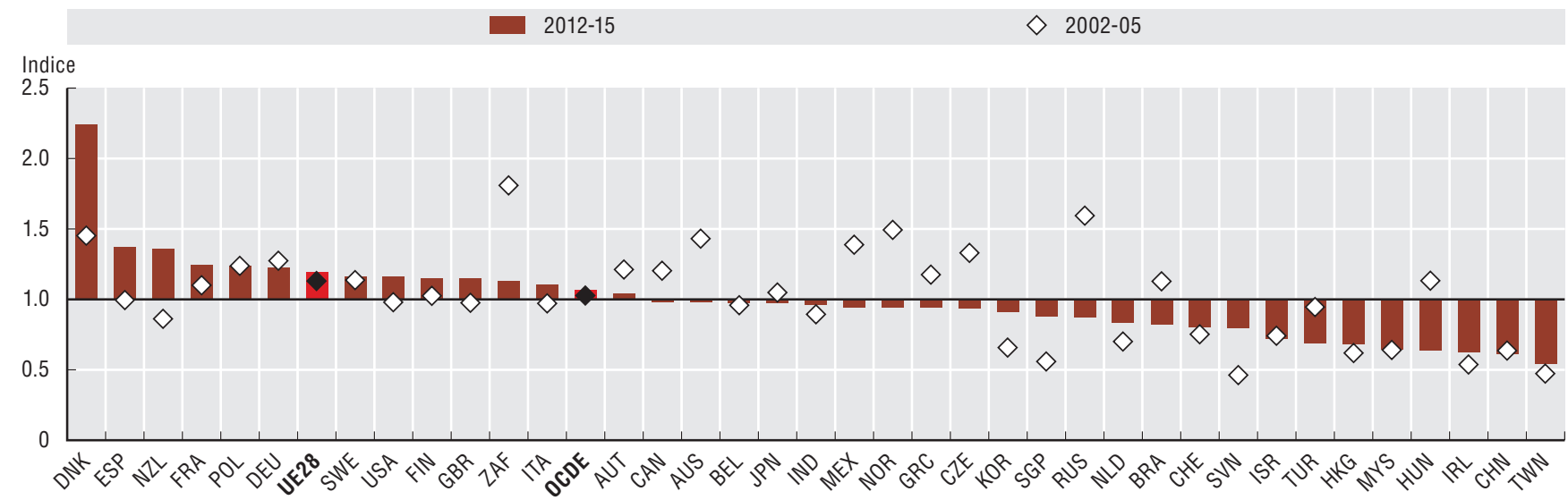

Source : OCDE, STI Micro-data Lab : Base de données sur la propriété intellectuelle, http://oe.cd/ipstats, juin 2017. Davantage de données via StatLink. Voir notes de chapitre.

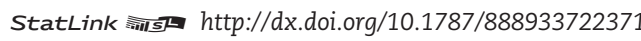

\section{Mesurabilité}

L'indice ATR est nul lorsqu'une économie ne détient aucun brevet dans un domaine donné ; il est égal à 1 si la part de l'économie dans le domaine technologique examiné est équivalente à sa part dans l'ensemble des domaines (pas de spécialisation); il est supérieur à 1 en cas de spécialisation. Compte tenu du mode de calcul de l'ATR, les économies dans lesquelles relativement peu de brevets sont déposés peuvent sembler hautement spécialisées dans certaines technologies du fait que leurs activités sont plus probablement concentrées dans seulement quelques domaines.

Les codes de la Classification internationale des brevets attribués par les examinateurs au cours de l'examen de la demande de brevet précisent le domaine technologique dont relève l'invention. La CIB est révisée périodiquement pour tenir compte de l'émergence de nouvelles technologies et de l'évolution des technologies existantes, ce qui peut induire une réaffectation de brevets dans des classes différentes.

L'utilisation de données provenant d'autres bureaux de brevets pourrait donner des résultats différents, étant donné que les entreprises intervenant dans un ou plusieurs domaines technologiques peuvent adopter un comportement et une stratégie d'innovation différenciés selon le marché. 


\section{Contenu en valeur ajoutée étrangère des exportations brutes, par région d'origine, 2014}

En pourcentage des exportations brutes nationales
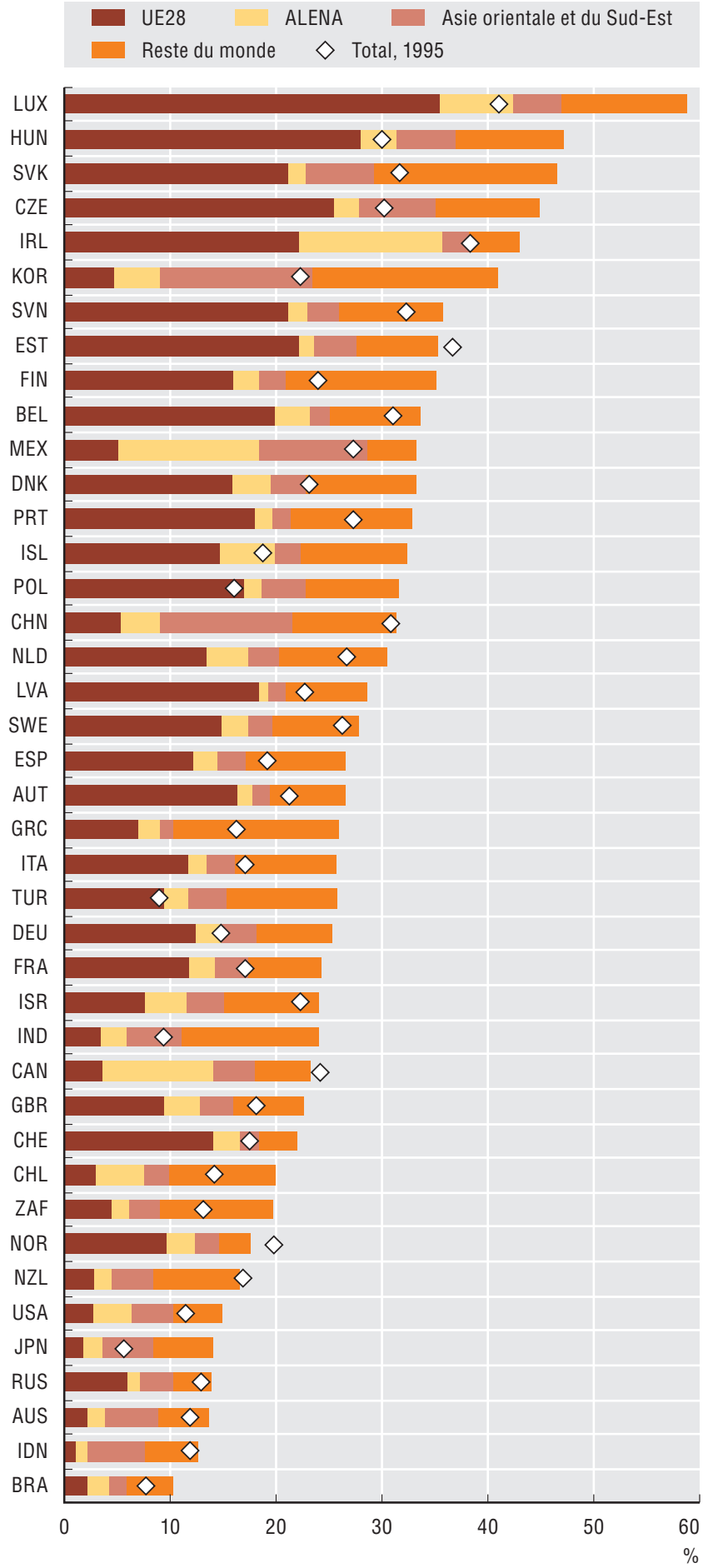

Source : Estimations de l'OCDE d'après OCDE, Base de données sur les échanges en valeur ajoutée (TiVA), http://oe.cd/tiva, Tableaux internationaux des entrées-sorties (TIES), http://oe.cd/icio, Base de données sur les Échanges bilatéraux par industrie et catégorie d'utilisation finale (BTDIxE), http:// oe.cd/btd, Base de données des Comptes nationaux annuels, www.oecd.org/ std/na, et versions les plus récentes des Tableaux des entrées-sorties et des Tableaux des ressources et des emplois nationaux, avril 2017. Davantage de données via StatLink. Voir notes de chapitre.

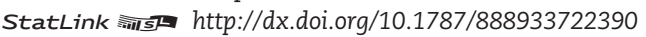

\section{Le saviez-vous ?}

Les pays de l'OCDE demeurent fortement intégrés aux systèmes de production globaux : le contenu en valeur ajoutée étrangère de leurs exportations allant d'environ $15 \%$ au Japon et aux États-Unis à plus de $40 \%$ dans les plus petits pays de l'UE.

L'intégration des pays dans les chaînes de valeur mondiales (CVM) peut se mesurer en déterminant l'origine de la valeur ajoutée contenue dans les exportations. La teneur en valeur ajoutée étrangère des exportations souligne le rôle des importations dans les performances à l'exportation, tandis que la valeur ajoutée locale d'un pays contenue dans les exportations de ses pays partenaires montre les effets des activités économiques de ce pays sur les consommateurs de pays tiers, même si aucune relation commerciale directe n'existe entre eux.

Une majeure partie de la valeur ajoutée étrangère enregistrée dans la plupart des pays provient de leur région d'appartenance, ce qui souligne l'importance de la proximité géographique et des accords commerciaux régionaux. Entre 1995 et 2014, à mesure que les chaînes de valeur mondiales se développaient, de nombreux pays ont vu augmenter notablement la part de la valeur ajoutée étrangère dans leurs exportations. Le Japon, l'Inde, la Corée, la Pologne et la Turquie ont enregistré les plus fortes hausses.

Toujours entre 1995 et 2014, le poids de la valeur ajoutée produite localement et absorbée dans les exportations de partenaires commerciaux (" intégration en aval dans les CVM ») a lui aussi nettement augmenté dans beaucoup de pays, en particulier en Afrique du Sud, en Australie, au Brésil, au Canada, au Chili et en Norvège ; cette tendance témoigne de la forte demande de produits minéraux dans les chaînes de valeur mondiales.

L'origine sectorielle de la valeur ajoutée locale dans les exportations brutes révèle l'importance de la contribution des services locaux qui, en 2014, ont totalisé plus de la moitié du contenu en valeur ajoutée du total des exportations de pays comme la France, Israël, le Royaume-Uni et la Suisse. Si les exportations directes de produits de services ont augmenté ces dernières années, une proportion importante des activités de services locales continue d'être stimulée par la demande étrangère d'exportations de matières premières et de produits manufacturés. Les activités de fabrication locales continuent d'être une source majeure de valeur ajoutée dans les exportations totales (plus de $30 \%$ ) de l'Allemagne, du Chili, de la Chine, de la Corée et du Japon.

\section{Définitions}

La base de données sur les échanges en valeur ajoutée (TiVA) permet d'établir des indicateurs sur l'origine, à la fois locale et étrangère, de la valeur ajoutée contenue dans les exportations et dans la demande finale. Ces indicateurs sont calculés à partir des Tableaux internationaux des entrées-sorties (TIES) de l'OCDE, lesquels renseignent sur les flux de biens et services entre 63 pays et 34 branches d'activités pour les années de 1995 à 2011. Retracer les flux mondiaux de valeur ajoutée permet d'étudier les CVM sous un nouvel angle, ce qui n'est pas toujours possible avec les statistiques classiques sur les échanges. Les estimations pour 2014 sont des projections fondées sur la toute dernière version des TIES, prolongées à l'aide de séries temporelles plus récentes, elles-mêmes tirées des comptes nationaux annuels et des statistiques sur le commerce de marchandises. 
Contenu en valeur ajoutée locale des exportations de pays tiers, 2014

En pourcentage des exportations brutes nationales

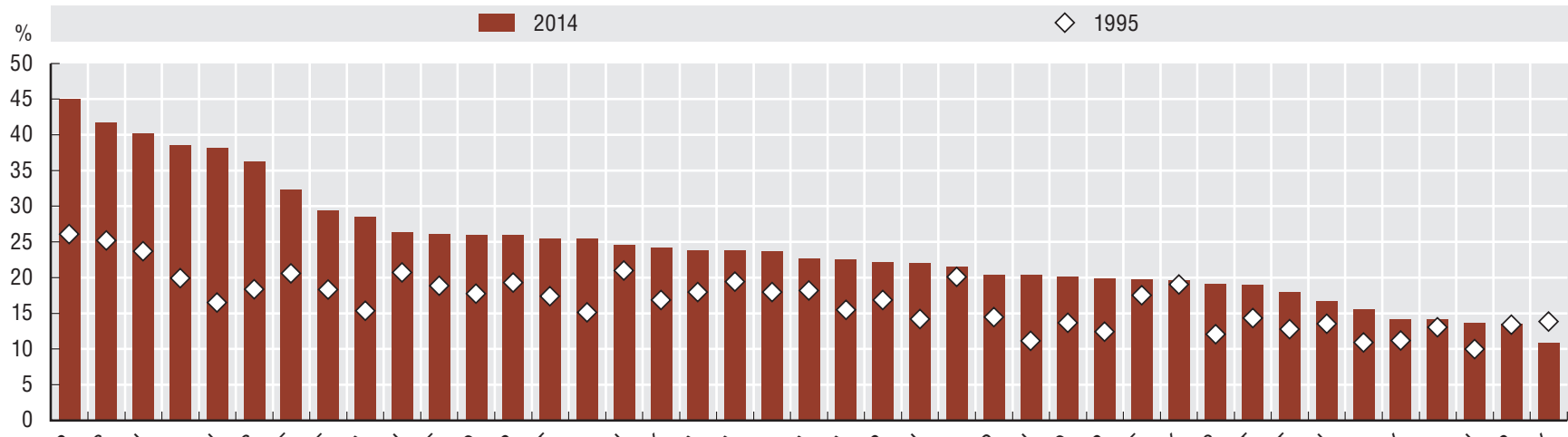

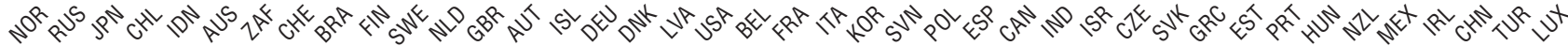

Source : Estimations de l'OCDE d'après la Base de données sur les échanges en valeur ajoutée (TiVA), http://oe.cd/tiva-fr, les Tableaux internationaux des entrées-sorties (TIES), http://oe.cd/icio, la Base de données sur les Échanges bilatéraux par industrie et catégorie d'utilisation finale (BTDIxE), http://oe.cd/ btd-fr, Base de données des Comptes nationaux annuels, www.oecd.org/std/na, et les dernières versions des Tableaux des entrées-sorties et des Tableaux des ressources et des emplois nationaux, avril 2017. Davantage de données via StatLink.

Origine sectorielle de la valeur ajoutée locale créée par les exportations brutes, 2014 En pourcentage des exportations brutes nationales

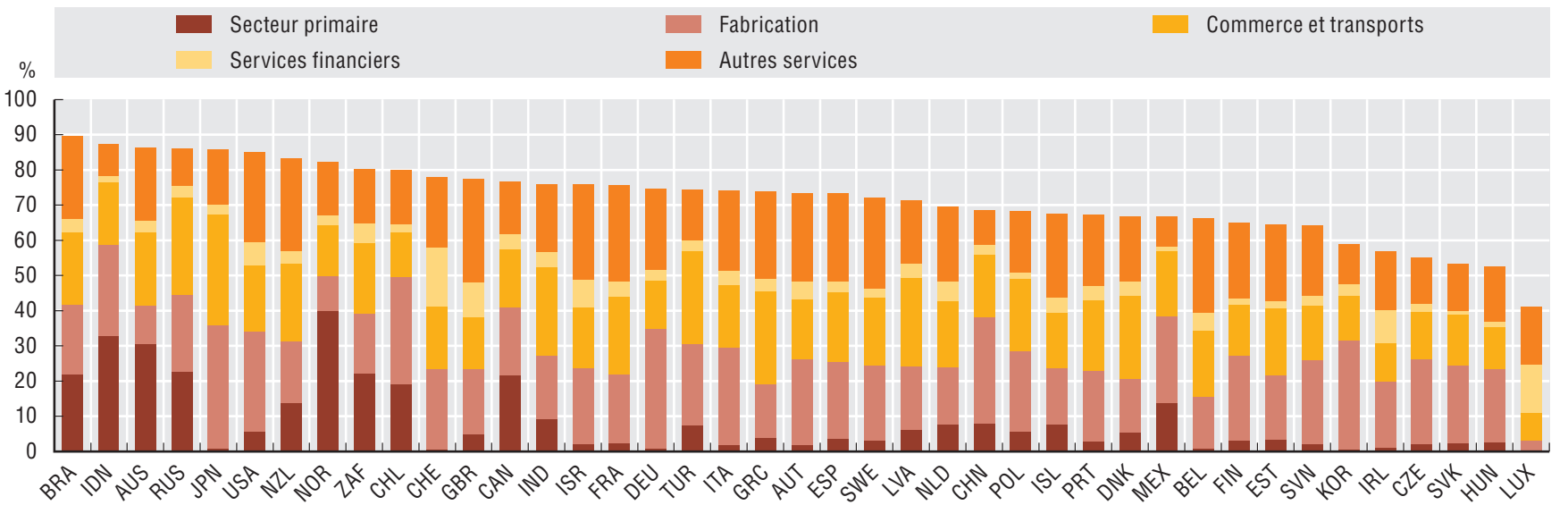

Source : Estimations de l'OCDE d'après la Base de données sur les échanges en valeur ajoutée (TiVA), http://oe.cd/tiva-fr, les Tableaux internationaux des entrées-sorties (TIES), http://oe.cd/icio, la Base de données sur les Échanges bilatéraux par industrie et catégorie d'utilisation finale (BTDIxE), http://oe.cd/btd-fr, la Base de données des Comptes nationaux annuels, www.oecd.org/std/na, et les dernières versions des Tableaux des entrées-sorties et des Tableaux des ressources et des emplois nationaux, avril 2017. Davantage de données via StatLink.

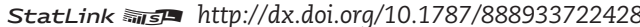

\section{Mesurabilité}

L'intégration en amont dans les CVM fait souvent référence au contenu en valeur ajoutée étrangère des exportations d'un pays ; son pendant est l'intégration en aval, qui désigne le contenu en valeur ajoutée locale des exportations de pays tiers (calculée comme la valeur ajoutée totale des intrants intermédiaires produits localement puis incorporés dans les exportations de pays tiers). Ces deux indicateurs permettent d'évaluer la participation aux chaînes de valeur mondiales et, selon l'approche choisie (valeur ajoutée étrangère ou locale), sont de préférence analysées séparément.

Une évolution dans la participation aux CVM peut refléter non seulement une plus grande spécialisation dans des activités situées vers le début ou vers la fin des chaînes de valeur, mais aussi une évolution du cours des matières premières ; ainsi, une flambée des prix du pétrole pourrait se traduire par une augmentation de la teneur en importations des exportations de nombreux pays. La même valeur ajoutée produite localement peut être incorporée aux exportations de nombreux pays tiers, si elle se déplace le long de chaînes de production internationales. Il convient donc d'interpréter avec prudence les indicateurs sur l'évolution de la participation aux CVM.

Les dépenses finales des non-résidents (par exemple les touristes) sont affectées au pays de résidence du consommateur, c'est-à-dire qu'elles sont traitées comme des exportations du pays dans lequel les biens et services sont achetés. 
Part des emplois du secteur des entreprises soutenus par la demande finale étrangère, 2005 et 2014

En pourcentage de l'emploi total du secteur des entreprises

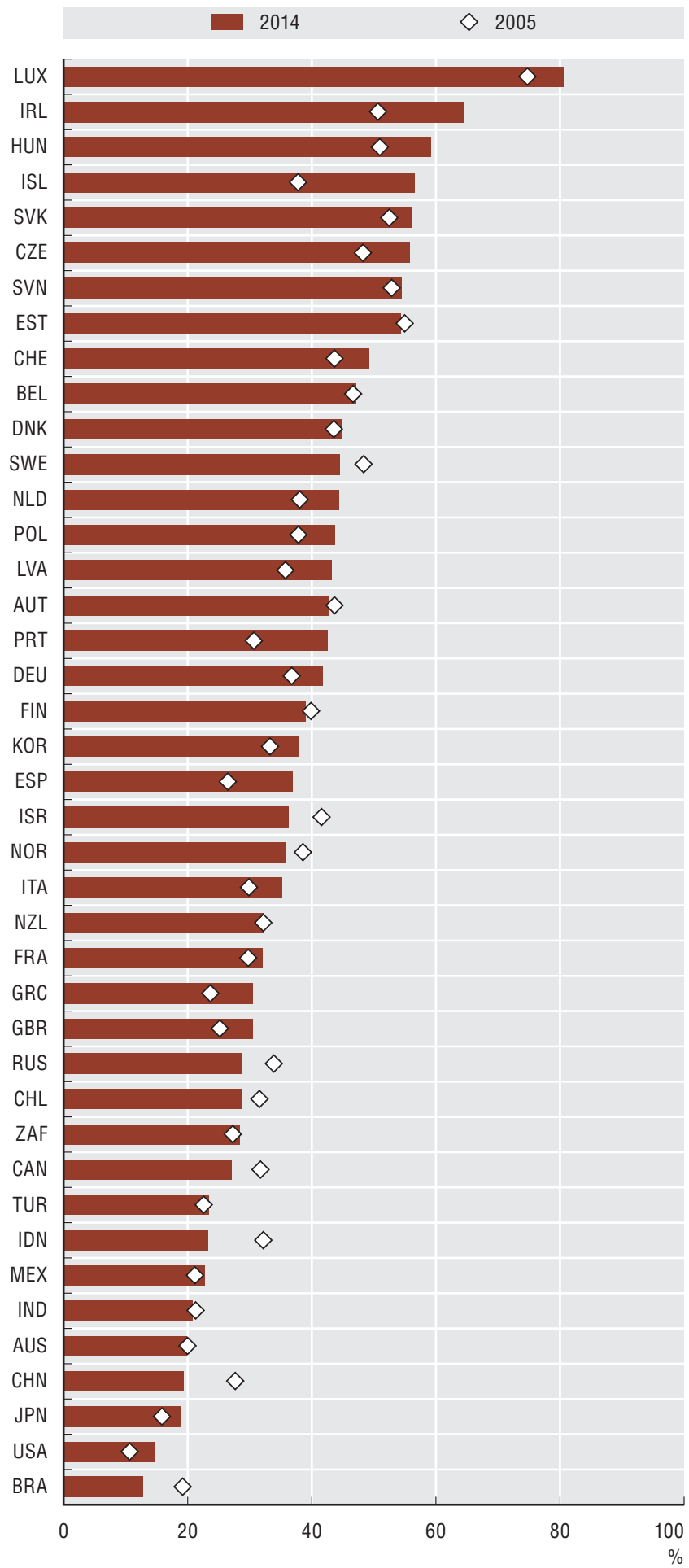

Source : Calculs de l'OCDE d'après les Tableaux internationaux des entrées-sorties (TIES), http://oe.cd/icio, la Base de données des Comptes nationaux annuels, www.oecd.org/std/na, la Base de données pour l'analyse structurelle (STAN), http://oe.cd/stan-fr, le Contenu en emploi des échanges, $h t t p: / / o e . c d / i o-e m p n$, la Base de données mondiales des entrées-sorties (WIOD) et des sources nationales, juin 2017. Davantage de données via StatLink. Voir notes de chapitre.

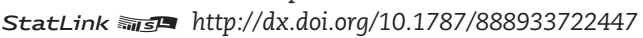

\section{Le saviez-vous ?}

En 2014 , dans la plupart des pays d'Europe, entre $30 \%$ et $60 \%$ des emplois du secteur des entreprises étaient soutenus par des consommateurs situés sur des marchés étrangers. Aux États-Unis, le chiffre s'élevait à $15 \%$ seulement, soit, tout de même, 13 millions d'emplois.

Les estimations de l'emploi soutenu par la demande finale étrangère peuvent révéler le degré d'intégration d'un pays dans l'économie mondiale. Plus il y a d'entreprises spécialisées dans des processus spécifiques de la production mondiale et plus les économies sont interdépendantes. La capacité des économies à répondre à la demande finale étrangère détermine de plus en plus l'évolution des marchés de l'emploi. Les statistiques classiques ne permettent pas de révéler la véritable nature de ces interdépendances - en particulier la manière dont les consommateurs dans un pays peuvent influer sur la production et, donc, soutenir les emplois dans des pays situés plus en amont de la chaîne de valeur. De nouveaux indicateurs, fondés sur les Tableaux internationaux des entrées-sorties (TIES) de l'OCDE, peuvent illustrer ces relations.

Par exemple, en 2014, dans la plupart des pays d'Europe, entre $40 \%$ et $60 \%$ des emplois du secteur des entreprises étaient soutenus par des consommateurs de marchés étrangers. $\mathrm{Au}$ Japon et aux États-Unis, les pourcentages étaient plus modestes du fait de la taille relativement importante de ces économies et de leur moindre dépendance vis-à-vis des exportations/importations. Néanmoins, l'OCDE estime qu'en 2014, le nombre d'emplois soutenus par la demande étrangère avoisinait 13 millions aux États-Unis et dépassait 8 millions au Japon.

Dans les pays comme la Chine, l'Inde, Israël et le Mexique, la part des emplois soutenus par la demande étrangère était beaucoup plus élevée dans les secteurs de l'information et de la communication que dans les autres secteurs. Entre 2005 et 2014, la Chine a vu ce chiffre fortement augmenter pour atteindre $64 \%$.

Pour illustrer l'impact des CVM sur les marchés du travail, on peut également comparer la part de l'emploi (la part de la valeur ajoutée dédiée à la rémunération des employés) dans la production destinée à répondre à la demande locale à la part correspondante devant satisfaire la demande finale étrangère. Les premières estimations suggèrent que, dans la plupart des pays de l'OCDE, les branches d'activités du secteur des entreprises plus orientées vers la demande finale étrangère (y compris, en amont, les fournisseurs locaux non exportateurs qui produisent des biens et services intermédiaires, ainsi que les exportateurs directs) ont une part de l'emploi supérieure aux branches d'activités plus axées sur la demande locale.

\section{Définitions}

L'emploi désigne le nombre total de personnes exerçant une activité et concourant à la production. Les coûts de la main d'œuvre correspondent à la rémunération des employés telle que définie dans le Système de comptabilité nationale. Les secteurs de l'information et de la communication renvoient aux divisions 30, 32, 33 et 64 de la CITI rév. 3. 
Emplois des secteurs de l'information et de la communication soutenus par la demande finale étrangère, 2005 et 2014 En pourcentage de l'emploi total dans les secteurs de l'information et de la communication

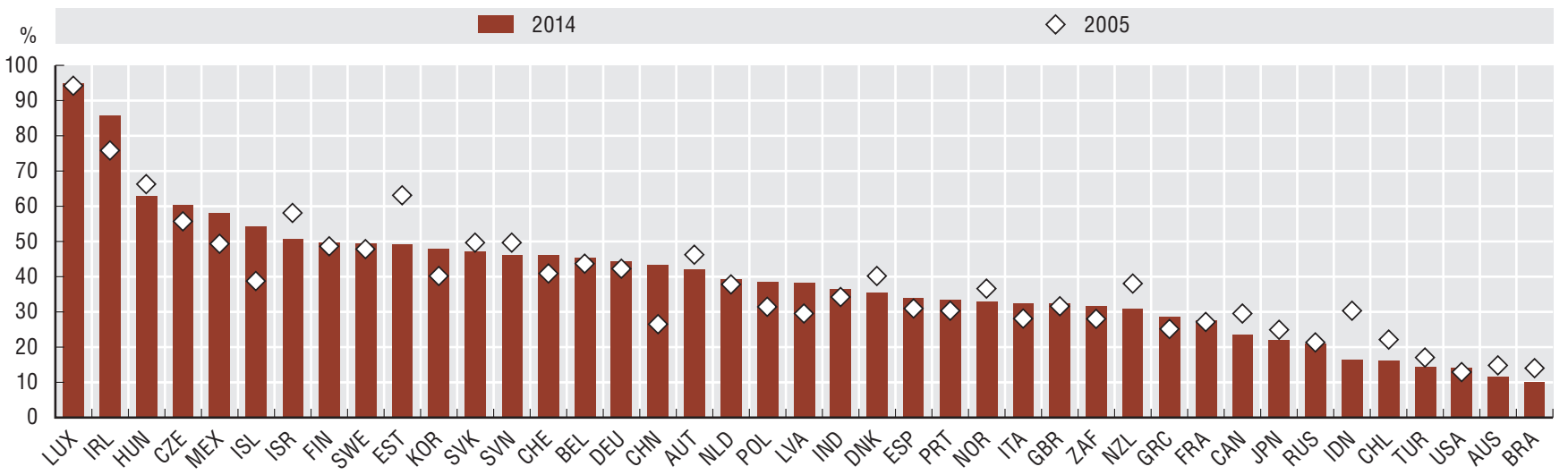

Source : Calculs de l'OCDE d'après les Tableaux internationaux des entrées-sorties (TIES), http://oe.cd/icio, la Base de données des Comptes nationaux annuels, www.oecd.org/std/na, le Contenu en emploi des échanges, http://oe.cd/io-empn, la Base de données mondiales des entrées-sorties (WIOD) et des sources nationales, juin 2017. Davantage de données via StatLink. Voir notes de chapitre.

Part de la rémunération des employés du secteur des entreprises soutenue par la demande finale locale et étrangère, 2014

En pourcentage de la valeur ajoutée dans le secteur des entreprises

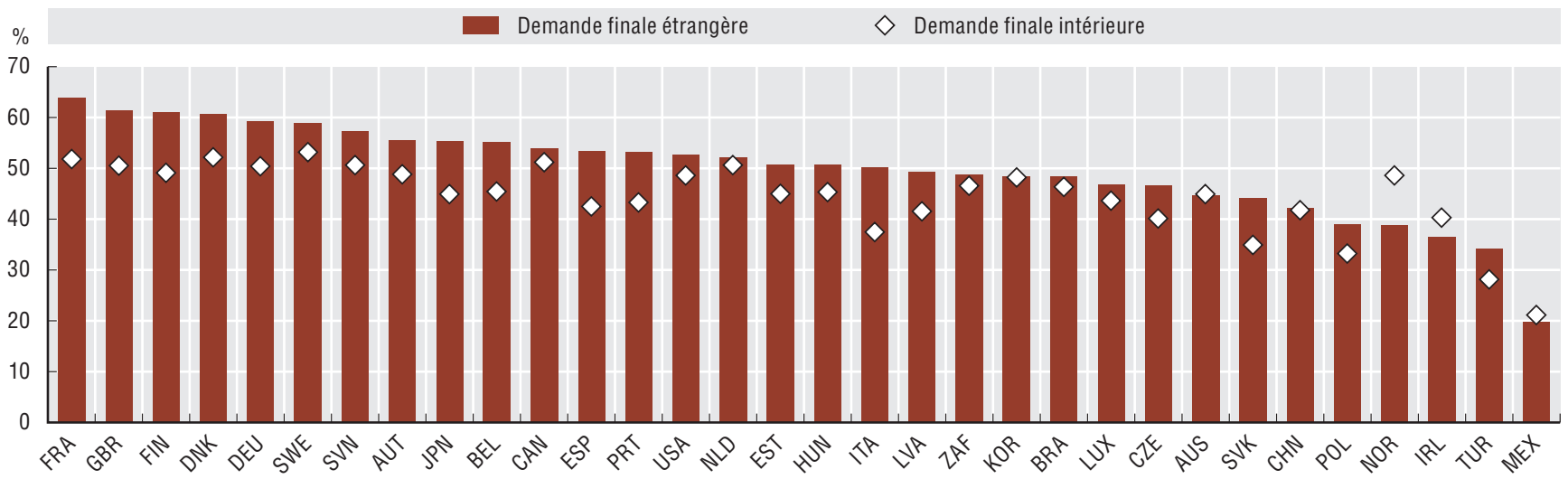

Source : Calculs de l'OCDE d'après les Tableaux internationaux des entrées-sorties (TIES), http://oe.cd/icio, la Base de données des Comptes nationaux annuels, www.oecd.org/std/na, la Base de données pour l'analyse structurelle (STAN), http://oe.cd/stan-fr, le Contenu en emploi des échanges, http://oe.cd/ io-empn et des sources nationales, juin 2017. Davantage de données via StatLink. Voir notes de chapitre.

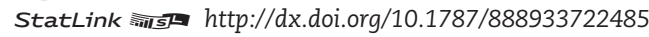

\section{Mesurabilité}

Les outils de comptabilité sur les entrées-sorties sont sensibles à certaines hypothèses, par exemple le fait que les entreprises exportatrices affichent la même productivité du travail et la même proportion d'importations, par rapport à la production, que les entreprises produisant des biens et services pour leur marché intérieur. Toutefois, des éléments factuels indiquent que les entreprises exportatrices pourraient avoir une productivité du travail et une proportion d'importations pour une production donnée plus élevées. Il se pourrait donc que les résultats présentés ici soient surestimés. De plus, l'emploi n'est pas estimé en équivalent temps plein, et les résultats portent sur des emplois, non pas créés, mais soutenus : en effet, peutêtre ces emplois existaient-ils auparavant pour servir les consommateurs locaux.

Pour la plupart des pays, au niveau détaillé des secteurs d'activités utilisé dans les TIES, la part de l'emploi dans la valeur ajoutée est la même, que la production soit destinée à la consommation intérieure ou étrangère, et ce en raison des hypothèses utilisées dans la construction des TIES (les exceptions sont la Chine et le Mexique où, respectivement, les « exportateurs ayant une activité de transformation » et les « entreprises manufacturières mondiales » sont distinguées des autres entreprises). Les différences de parts de l'emploi, au niveau agrégé, du secteur des entreprises reflètent donc les différences de poids des secteurs d'activités soutenus par la demande intérieure ou étrangère. 


\begin{abstract}
Chypre
La note suivante est ajoutée à la demande de la Turquie :

"Les informations figurant dans ce document qui font référence à "Chypre” concernent la partie méridionale de l'île. Il n'y a pas d'autorité unique représentant à la fois les Chypriotes turcs et grecs sur l'île. La Turquie reconnaît la République turque de Chypre Nord (RTCN). Jusqu'à ce qu'une solution durable et équitable soit trouvée dans le cadre des Nations Unies, la Turquie maintiendra sa position sur la "question chypriote". "

La note suivante est ajoutée à la demande de tous les États de l'Union européenne membres de l'OCDE et de l'Union européenne :

"La République de Chypre est reconnue par tous les membres des Nations Unies sauf la Turquie. Les informations figurant dans ce document concernent la zone sous le contrôle effectif du gouvernement de la République de Chypre. »

Israël

"Les données statistiques concernant Israël sont fournies par et sous la responsabilité des autorités israéliennes ou d'un tiers compétents. L'utilisation de ces données par l'OCDE est sans préjudice du statut des hauteurs du Golan, de JérusalemEst et des colonies de peuplement israéliennes en Cisjordanie aux termes du droit international. "

" Il est à noter que les données statistiques sur les brevets et marques israéliens sont fournies par les offices des brevets et des marques des pays concernés. ”
\end{abstract}

\title{
5.1. Spécialisation de la R-D
}

\section{Intensité de R-D des entreprises corrigée de la structure d'activités, 2015}

L'indicateur de l'intensité de R-D corrigée de la structure d'activités correspond, pour un pays, à la moyenne pondérée des intensités de R-D (ratio R-D sur valeur ajoutée), la structure d'activités de l'OCDE (part des différentes activités dans la valeur ajoutée de la zone OCDE en 2015) servant de pondération pour tous les pays. L'indicateur non corrigé de l'intensité de DIRDE est, par définition, une moyenne prenant en compte la part effective de chaque activité dans le pays examiné.

Les séries relatives à la R-D sont présentées en pourcentage de la valeur ajoutée estimée comme étant la valeur ajoutée dans l'ensemble des secteurs d'activités hors : Activités immobilières (CITI rév. 4,68) ; Administration publique et défense, sécurité sociale obligatoire et éducation (CITI rév. 4,84-85) ; Activités relatives à la santé et activités d'action sociale (CITI rév. 4, 86-88) ; et Activités des ménages employant du personnel (CITI rév. 4, 97-98). La R-D réalisée dans ces secteurs dans les pays de l'OCDE est supposée négligeable.

Ces chiffres reposent sur des estimations de la R-D des entreprises par secteur déclarées par activité principale selon la CITI rév. 4. Pour l'Australie, l'Autriche, la Belgique, le Canada, la Corée, la France, la Grèce, l'Irlande et la Suède, les données se rapportent à 2013. Pour le Danemark, les États-Unis, la Finlande, la Hongrie, l'Italie, les Pays-Bas, la Pologne, le Portugal, le Royaume-Uni et la Slovénie, les données se rapportent à 2014.

La valeur ajoutée est mesurée aux prix de base sauf pour le Japon (prix d'acquisition).

Les données sur la valeur ajoutée proviennent de la Base de données pour l'analyse structurelle (STAN) de l'OCDE, sauf en ce qui concerne la Corée, la Hongrie, l'Irlande, l'Islande et la Turquie (statistiques de l'OCDE sur les comptes nationaux), le Canada (source nationale) et l'Australie (statistiques de l'OCDE sur les comptes nationaux et estimations fondées sur les comptes nationaux australiens ABS : tableaux des entrées-sorties, 2013-14, pour les activités de fabrication).

\section{R-D des entreprises dans les activités de fabrication, par catégorie d'intensité de R-D, 2015}

Les catégories d'intensité de R-D sont définies selon la classification de l'intensité de R-D de l'OCDE, avec une précision de deux chiffres (voir Galindo-Rueda et Verger, 2016).

Les activités de fabrication à forte et moyenne-forte intensité de R-D sont la fabrication de produits chimiques et de préparations pharmaceutiques (divisions 20 et 21 de la CITI rév. 4) et la fabrication d'ordinateurs, d'articles électroniques et optiques, de matériels électriques, de machines, de véhicules automobiles et d'autres matériels de transport (divisions 26 à 30 de la CITI rév. 4).

Ces chiffres reposent sur des estimations de la R-D des entreprises par secteur déclarées par activité principale selon la CITI rév. 4. Pour l'Autriche, la Belgique, la France, la Grèce, l'Irlande et la Suède, les données se rapportent à 2013.

Pour le Canada, le Danemark, les États-Unis, la Finlande, la Hongrie, Israël, l'Italie, les Pays-Bas, la Pologne, le Portugal, le RoyaumeUni et la Slovénie, les données se rapportent à 2014.

Pour le Danemark, Israël, la Suède et la Suisse, la « Fabrication de produits chimiques » (division 20 de la CITI rév. 4) est incluse dans les « Autres activités de fabrication ».

\section{R-D dans les activités de services, 2015}

Ces chiffres reposent sur des estimations de la R-D des entreprises par secteur déclarées par activité principale selon la CITI rév. 4. Ces statistiques sont fondées sur les Statistiques de la R-D de l'OCDE (http://oe.cd/rds) et les données de la base ANBERD (http:// oe.cd/anberd). Se reporter à ces sources pour davantage de renseignements sur les données, en particulier sur les ruptures de séries et autres problèmes analogues. 
Pour l'Australie, l'Autriche, la Belgique, l'Irlande et la Nouvelle-Zélande, les données se rapportent à 2013.

Pour le Canada, le Danemark, les États-Unis, la Finlande, la Hongrie, Israël, l'Italie, les Pays-Bas, la Pologne, le Portugal et la Slovénie, les données se rapportent à 2014.

Pour le Chili, les données se rapportent à 2007 et 2015.

Pour la France et la Suède, les données se rapportent à 2007 et 2013.

Pour la Grèce, l'estimation de 2015 qui concerne le secteur de services de R-D est fondée sur des données de 2013.

Pour le Royaume-Uni, les données se rapportent à 2007 et 2014.

Pour la Suisse, les données se rapportent à 2004 et 2015.

\subsection{Adoption du commerce électronique}

\section{Diffusion dans les entreprises d'une sélection d'outils et d'activités s'appuyant sur les TIC, par technologie, 2016}

Sauf mention contraire, seules les entreprises de 10 salariés ou plus sont prises en compte.

Les données relatives aux PGI se rapportent à 2015 pour tous les pays à l'exception du Canada (2013), de l'Islande (2014) et de la Suède (2014).

Les données relatives à la GRC se rapportent à 2015.

Les données relatives à la RFID se rapportent à 2014.

Cloud computing : Pour le Canada, les données se rapportent à 2012 et aux entreprises qui ont effectué des dépenses en logicielservice (par exemple, cloud computing). Pour le Mexique, les données se rapportent à 2012.

Pour les pays du Système statistique européen, les données relatives aux achats en ligne et aux ventes en ligne se rapportent à 2015. Pour l'Australie, les données se rapportent à l'exercice 2014/15 clos au 30 juin.

Pour le Canada, les données se rapportent à 2013, sauf celles qui concernent le cloud computing (2012).

Pour l'Islande, les données se rapportent à 2014.

Pour le Japon, les données se rapportent à 2015 et aux entreprises d'au moins 100 salariés (au lieu de 10).

Pour la Corée, les données se rapportent à 2015, sauf celles qui concernent le cloud computing (2013).

Pour la Suisse, les données se rapportent à 2015 et aux entreprises d'au moins 5 salariés (au lieu de 10).

\section{Diffusion dans les entreprises d'une sélection d'outils et d'activités s'appuyant sur les TIC, pays de l'OCDE, 2010 et 2016}

Les achats en ligne et les ventes en ligne se rapportent à l'achat et à la vente de biens ou de services effectués via des réseaux informatiques par des méthodes spécifiquement conçues pour la réception ou la passation de commandes (à savoir pages web, extranet ou échange de données informatisé [EDI]), à l'exclusion des commandes passées par téléphone, télécopie ou courrier électronique composé manuellement. Les méthodes de paiement et de livraison ne sont pas prises en considération.

Les progiciels de gestion intégrés (PGI) sont des logiciels qui peuvent intégrer la gestion des flux d'informations internes et externes, allant des ressources matérielles et humaines aux finances, à la comptabilité et aux relations avec la clientèle. Ici, seul l'échange d'informations au sein de l'entreprise est pris en considération. Pour les PGI, les données se rapportent à 2015.

Le cloud computing désigne les services de TIC fournis via l'internet, qui permettent d'accéder à un serveur, des capacités de stockage, des composantes du réseau et des applications logicielles.

La gestion de la chaîne logistique désigne l'utilisation d'applications d'échange automatique de données (EAD). Pour la gestion de la chaîne logistique, les données se rapportent à 2015.

Les solutions de gestion de la relation client/fournisseur (GRC) sont des logiciels de gestion des interactions d'une entreprise avec ses clients, ses clients potentiels, ses partenaires, ses employés et ses fournisseurs. Pour la GRC, les données se rapportent à 2015. Les médias sociaux désignent des applications prenant appui sur la technologie de l'internet ou les plateformes de communication, qui permettent d'entrer en relation et de créer et d'échanger du contenu en ligne avec des clients, fournisseurs ou partenaires, ou des interlocuteurs au sein de l'entreprise. Les médias sociaux peuvent inclure des réseaux sociaux (hors publicité payante), des blogs, du partage de fichiers et des outils de partage de connaissances de type " wiki ».

La radio-identification (RFID) est une technologie qui permet la transmission d'informations sans contact via les ondes radio. Elle peut être utilisée pour des finalités très diverses, notamment l'identification personnelle ou le contrôle d'accès, la logistique, le commerce de détail ou le suivi des processus dans les activités de fabrication. Pour la RFID, les données se rapportent à 2014.

\section{Entreprises recourant aux ventes en ligne, par taille d'entreprise, 2015}

Sauf mention contraire, seules les entreprises de 10 salariés ou plus sont prises en compte. Les petites entreprises emploient de 10 à 49 salariés, les grandes, 250 ou plus.

Pour l'Australie, les données se rapportent aux exercices 2010/11 et 2014/15 clos au 30 juin.

Pour le Canada, les données se rapportent à 2012 et 2013. Les grandes entreprises emploient 300 salariés ou plus. Les ventes en ligne par internet peuvent inclure les ventes par EDI via l'internet ainsi que les ventes via des sites web, mais elles n'incluent pas les ventes par courrier électronique composé manuellement ou par mailing.

Pour l'Islande, les données se rapportent à 2013 au lieu de 2015. 
Pour le Japon, les données se rapportent à 2010 au lieu de 2009 et aux entreprises d'au moins 100 salariés (au lieu de 10). Les grandes entreprises emploient 300 salariés ou plus.

Pour la Corée, les données se rapportent à 2010 au lieu de 2009.

Pour le Mexique, les données se rapportent à 2012 et aux entreprises qui reçoivent des commandes via l'internet plutôt que via des réseaux informatiques.

Pour la Nouvelle-Zélande, les données se rapportent aux exercices 2010/11 et 2015/16 clos au 30 juin.

Pour la Suisse, les données se rapportent à 2011.

\subsection{Le commerce électronique à travers les secteurs et les applications}

\section{Entreprises utilisant des services de cloud computing, par taille d'entreprise, 2016}

Sauf mention contraire, seules les entreprises de 10 salariés ou plus sont prises en compte. Les catégories de taille sont les suivantes : petites entreprises (de 10 à 49 salariés), moyennes entreprises (de 50 à 249 salariés), grandes entreprises (250 salariés ou plus).

Pour l'Australie, les données se rapportent à l'exercice 2014/15 clos au 30 juin.

Pour le Brésil, les données se rapportent à 2015.

Pour le Canada, les données se rapportent à 2012 et aux entreprises qui ont effectué des dépenses en logiciel-service (par exemple, cloud computing). Sont considérées comme moyennes entreprises celles de 50 à 299 salariés, et comme grandes entreprises celles de 300 salariés ou plus.

Pour la Corée, les données se rapportent à 2015, au lieu de 2016.

Pour l'Islande, les données se rapportent à 2014.

Pour l'Italie, il y a une rupture de série entre 2014 et 2016.

Pour le Japon, les données se rapportent à 2015 au lieu de 2016 et aux entreprises d'au moins 100 salariés. Sont considérées comme moyennes entreprises celles de 100 à 299 salariés, et comme grandes entreprises celles de 300 salariés ou plus.

Pour le Mexique, les données se rapportent à 2012.

Pour la Suisse, les données se rapportent à 2015 et aux entreprises d'au moins 5 salariés (au lieu de 10).

\section{Adoption des services de cloud computing dans les différents secteurs d'activités, pays de l'OCDE, 2016}

Les données se rapportent à 2016 ou à l'année la plus récente disponible.

Sauf mention contraire, les données se rapportent aux entreprises de 10 salariés ou plus.

Les secteurs d'activités suivants ont été pris en compte : Industrie manufacturière; construction ; commerce de gros, sauf véhicules automobiles et motocycles ; commerce de détail, sauf véhicules automobiles et motocycles ; transports et entreposage ; hébergement et restauration ; information et communication ; activités immobilières ; activités professionnelles, scientifiques et techniques; activités de services administratifs et de soutien. Pour chacun de ces secteurs d'activités, les indicateurs suivants sont mentionnés : (i) la moyenne simple de la zone OCDE calculée sur la base des données disponibles pour les pays de l'OCDE ; (ii) les pays dans lesquels la valeur est maximale ou minimale.

\section{Diffusion dans les secteurs d'activités d'une sélection d'outils et d'activités s'appuyant sur les TIC, pays de l'OCDE, 2016}

Les données se rapportent à 2016 ou à l'année la plus récente disponible.

Pour chaque outil ou activité lié aux TIC, les secteurs d'activités suivants ont été pris en compte : Industrie manufacturière; construction ; commerce de gros, sauf véhicules automobiles et motocycles ; commerce de détail, sauf véhicules automobiles et motocycles ; transports et entreposage ; hébergement et restauration ; information et communication ; activités immobilières ; activités professionnelles, scientifiques et techniques; activités de services administratifs et de soutien. Pour chaque outil ou activité lié aux TIC : (i) on a calculé, pour chaque secteur d'activités, la moyenne simple de la zone OCDE sur la base des données disponibles pour les pays de l'OCDE; (ii) on a classé par ordre décroissant l'ensemble de ces moyennes simples obtenues pour les secteurs, et reporté sur le graphique les moyennes maximale et minimale en indiquant à quel secteur elles correspondent. Pour chaque outil ou activité lié aux TIC, la valeur reportée dans le graphique est celle de la moyenne de la zone OCDE calculée pour tous les pays pour lesquels on dispose de données et pour tous les secteurs d'activité. Elle peut différer légèrement de la valeur moyenne indiquée dans le graphique 5.2.2.

Les achats en ligne et les ventes en ligne se rapportent à l'achat et à la vente de biens ou de services effectués via des réseaux informatiques par des méthodes spécifiquement conçues pour la réception ou la passation de commandes (à savoir pages web, extranet ou échange de données informatisé), à l’exclusion des commandes passées par téléphone, télécopie ou courrier électronique composé manuellement. Les méthodes de paiement et de livraison ne sont pas prises en considération.

Les progiciels de gestion intégrés (PGI) sont des logiciels qui peuvent intégrer la gestion des flux d'informations internes et externes, allant des ressources matérielles et humaines aux finances, à la comptabilité et aux relations avec la clientèle. Ici, seul l'échange d'informations au sein de l'entreprise est pris en considération. Pour les PGI, les données se rapportent à 2015.

La gestion de la chaîne logistique désigne l'utilisation d'applications d'échange automatique de données (EAD). Pour la gestion de la chaîne logistique, les données se rapportent à 2015. 
Les solutions de gestion de la relation client/fournisseur (GRC) sont des logiciels de gestion des interactions d'une entreprise avec ses clients, ses clients potentiels, ses partenaires, ses employés et ses fournisseurs. Pour la GRC, les données se rapportent à 2015. Les médias sociaux désignent des applications prenant appui sur la technologie de l'internet ou les plateformes de communication, qui permettent d'entrer en relation et de créer et d'échanger du contenu en ligne avec des clients, fournisseurs ou partenaires, ou des interlocuteurs au sein de l'entreprise. Les médias sociaux peuvent inclure des réseaux sociaux (hors publicité payante), des blogs, du partage de fichiers et des outils de partage de connaissances de type " wiki ».

La radio-identification (RFID) est une technologie qui permet la transmission d'informations sans contact via les ondes radio. Elle peut être utilisée pour des finalités très diverses, notamment l'identification personnelle ou le contrôle d'accès, la logistique, le commerce de détail ou le suivi des processus dans les activités de fabrication. Pour la RFID, les données se rapportent à 2014.

\subsection{Dynamique des start-ups}

\section{Taux d'entrée et de sortie des entreprises, secteur des TIC et autres secteurs d'activités, 2013-15}

Les chiffres rendent compte de moyennes des trois années disponibles les plus récentes, sous réserve de la disponibilité des données.

Le secteur des TIC recouvre les divisions 26, 61 et 62-63 de la CITI rév. 4 (ordinateurs, articles électroniques et optiques, télécommunications, activités informatiques et services d'information).

Les autres secteurs englobent les activités de fabrication et les services marchands non financiers à l'exclusion du secteur des TIC, de la cokéfaction et fabrication de produits pétroliers raffinés et des activités immobilières.

Les chiffres de DynEmp v.3 excluent les unités de la catégorie de taille 0-1.

Les versions 2 et 3 de DynEmp appliquent chacune une correction différente à l'année de création lorsqu'elle se situe dans la période examinée.

Les données relatives à certains pays sont encore préliminaires.

En raison de divergences méthodologiques, les chiffres indiqués peuvent s'écarter des statistiques nationales officielles publiées par les offices statistiques nationaux.

Pour l'Autriche, le Danemark, le Luxembourg, les Pays-Bas et le Portugal, les données se rapportent à 2010-12.

Pour la Belgique et les États-Unis, les données se rapportent à 2011-13.

Pour la France et la Nouvelle-Zélande, les données se rapportent à 2009-11.

Pour l'Italie et le Royaume-Uni, les données se rapportent à 2008-10.

Pour la Norvège, les données se rapportent à 2010-11 et 2014.

S'agissant du Royaume-Uni, les travaux contiennent des données statistiques de l'Office for National Statistics (ONS), soumis aux droits d'auteur de la Couronne (Crown Copyright). L'utilisation de données statistiques de l'ONS dans ces travaux ne vaut pas approbation par l'ONS de l'interprétation ou de l'analyse de ces données statistiques. Ces travaux utilisent également des ensembles de données de recherche qui pourraient ne pas exactement reproduire les agrégats des statistiques nationales.

\section{Part des jeunes petites et micro-entreprises existantes dans le secteur des TIC et les autres secteurs d'activités, 2013-15}

Le graphique rend compte de la part des jeunes (moins de 6 ans d'ancienneté), micro et petites (moins de 50 salariés) entreprises existantes.

Les chiffres rendent compte de moyennes des trois années disponibles les plus récentes, sous réserve de la disponibilité des données.

Le secteur des TIC recouvre les divisions 26, 61 et 62-63 de la CITI rév. 4 (ordinateurs, articles électroniques et optiques, télécommunications, activités informatiques et services d'information).

Les autres secteurs englobent les activités de fabrication et les services marchands non financiers à l'exclusion du secteur des TIC, de la cokéfaction et fabrication de produits pétroliers raffinés et des activités immobilières.

Les chiffres de DynEmp v.3 excluent les unités de la catégorie de taille 0-1.

Les versions 2 et 3 de DynEmp appliquent chacune une correction différente à l'année de création lorsqu'elle se situe dans la période examinée.

Les données relatives à certains pays sont encore préliminaires.

En raison de divergences méthodologiques, les chiffres indiqués peuvent s'écarter des statistiques nationales officielles publiées par les offices statistiques nationaux.

Pour l'Autriche, le Danemark, le Luxembourg, les Pays-Bas et le Portugal, les données se rapportent à 2010-12.

Pour la Belgique et les États-Unis, les données se rapportent à 2011-13.

Pour la France et la Nouvelle-Zélande, les données se rapportent à 2009-11.

Pour l'Italie et le Royaume-Uni, les données se rapportent à 2008-10.

Pour la Norvège, les données se rapportent à 2010-11 et 2014. 
S'agissant du Royaume-Uni, les travaux contiennent des données statistiques de l'Office for National Statistics (ONS), soumis aux droits d'auteur de la Couronne (Crown Copyright). L'utilisation de données statistiques de l'ONS dans ces travaux ne vaut pas approbation par l'ONS de l'interprétation ou de l'analyse de ces données statistiques. Ces travaux utilisent également des ensembles de données de recherche qui pourraient ne pas exactement reproduire les agrégats des statistiques nationales.

\section{Différences de croissance de l'emploi entre les petites entreprises jeunes et les petites entreprises anciennes dans le secteur des TIC et les autres secteurs, 2013-15}

Le graphique montre la différence entre le taux moyen de croissance de l'emploi dans les petites entreprises (de 10 à 49 salariés) jeunes (moins de 6 ans d'ancienneté) et le taux moyen de croissance de l'emploi dans les petites entreprises (de 10 à 49 salariés) anciennes (6 ans d'ancienneté ou plus).

Les micro-entreprises (moins de 10 salariés) sont exclues du calcul.

Les chiffres rendent compte de moyennes des trois années disponibles les plus récentes, sous réserve de la disponibilité des données.

Le secteur des TIC recouvre les divisions 26, 61 et 62-63 de la CITI rév. 4 (ordinateurs, articles électroniques et optiques, télécommunications, activités informatiques et services d'information).

Les autres secteurs englobent les activités de fabrication et les services marchands non financiers à l'exclusion du secteur des TIC, de la cokéfaction et fabrication de produits pétroliers raffinés et des activités immobilières.

Les données relatives à certains pays sont encore préliminaires.

En raison de divergences méthodologiques, les chiffres indiqués peuvent s'écarter des statistiques nationales officielles publiées par les offices statistiques nationaux.

Pour l'Autriche, le Danemark, le Luxembourg, les Pays-Bas et le Portugal, les données se rapportent à 2010-12.

Pour la Belgique et les États-Unis, les données se rapportent à 2011-13.

Pour la France et la Nouvelle-Zélande, les données se rapportent à 2009-11.

Pour l'Italie et le Royaume-Uni, les données se rapportent à 2008-10.

Pour la Norvège, les données se rapportent à 2010-11 et 2014.

S'agissant du Royaume-Uni, les travaux contiennent des données statistiques de l'Office for National Statistics (ONS), soumis aux droits d'auteur de la Couronne (Crown Copyright). L'utilisation de données statistiques de l'ONS dans ces travaux ne vaut pas approbation par l'ONS de l'interprétation ou de l'analyse de ces données statistiques. Ces travaux utilisent également des ensembles de données de recherche qui pourraient ne pas exactement reproduire les agrégats des statistiques nationales.

\subsection{Avantage technologique}

\section{Avantage technologique révélé dans les TIC, 2002-05 et 2012-15}

L'indice d'avantage technologique révélé (ATR) mesure la part des brevets d'une économie dans un domaine technologique donné, rapportée à la part des brevets de cette économie dans l'ensemble des domaines technologiques. Les données se rapportent aux familles de brevets IP5, par date de dépôt, en fonction du lieu de résidence des inventeurs, et avec comptages fractionnaires. Les brevets liés aux TIC sont recensés à l'aide des codes de la CIB indiqués dans Inaba et Squicciarini (2017). Seules les économies totalisant plus de 250 familles de brevets pour les périodes considérées sont incluses. Les chiffres de 2014 et 2015 sont estimés sur la base des données disponibles pour ces années.

\section{Avantage technologique révélé dans les technologies de la santé, 2002-05 et 2012-15}

L'indice d'avantage technologique révélé (ATR) mesure la part des brevets d'une économie dans un domaine technologique donné, rapportée à la part des brevets de cette économie dans l'ensemble des domaines technologiques. Les données se rapportent aux familles de brevets IP5, par date de dépôt, en fonction du lieu de résidence des inventeurs, et avec comptages fractionnaires. Les brevets sont affectés aux domaines liés à la santé sur la base de leurs codes dans la Classification internationale des brevets (CIB), suivant la concordance établie par l'OMPI (2013). Seules les économies totalisant plus de 250 familles de brevets pour les périodes considérées sont incluses. Les chiffres de 2014 et 2015 sont estimés sur la base des données disponibles pour ces années.

\section{Avantage technologique révélé dans les technologies environnementales, 2002-05 et 2012-15}

L'indice d'avantage technologique révélé (ATR) mesure la part des brevets d'une économie dans un domaine technologique donné, rapportée à la part des brevets de cette économie dans l'ensemble des domaines technologiques. Les données se rapportent aux familles de brevets IP5, par date de dépôt, en fonction du lieu de résidence des inventeurs, et avec comptages fractionnaires. Les brevets sont associés à l'environnement sur la base des codes de la Classification internationale des brevets (CIB) ou de la Classification coopérative des brevets (CPC), comme décrit par Haščič et Migotto (2015). Seules les économies totalisant plus de 250 familles de brevets pour les périodes considérées sont incluses. Les chiffres de 2014 et 2015 sont estimés sur la base des données disponibles pour ces années. 


\subsection{Participation aux chaînes de valeur mondiales}

\section{Contenu en valeur ajoutée étrangère des exportations brutes, par région d'origine, 2014}

L'Asie orientale et du Sud-Est comprend le Brunei Darussalam, le Cambodge, la Chine, la Corée, Hong Kong (Chine), l'Indonésie, le Japon, la Malaisie, les Philippines, Singapour, le Taipei chinois, la Thaïlande et le Viet Nam.

\section{7. Échanges et emploi}

Part des emplois du secteur des entreprises soutenus par la demande finale étrangère, 2005 et 2014

Le secteur des entreprises correspond aux divisions 10 à 74 de la CITI rév. 3, c'est-à-dire l'ensemble des activités économiques hors Agriculture, sylviculture et pêche (divisions 01 à 05), Administration publique (75), Éducation (80), Santé (85) et Autres activités de services collectifs, sociaux et personnels (90 à 95).

Emplois des secteurs de l'information et de la communication soutenus par la demande finale étrangère, 2005 et 2014 Les secteurs de l'information et de la communication renvoient aux divisions 30, 32, 33 et 64 de la CITI rév. 3.

Part de la rémunération des employés du secteur des entreprises soutenue par la demande finale locale et étrangère, 2014

Le secteur des entreprises correspond aux divisions 10 à 74 de la CITI rév. 3, c'est-à-dire l'ensemble des activités économiques hors Agriculture, sylviculture et pêche (divisions 01 à 05), Administration publique (75), Éducation (80), Santé (85) et Autres activités de services collectifs, sociaux et personnels (90 à 95).

La production d'un secteur peut être stimulée à la fois par la demande finale intérieure et par la demande finale étrangère. À l'aide d'un cadre TIES/TiVA, la valeur ajoutée générée pour répondre à la demande étrangère peut être séparée de la valeur ajoutée générée pour répondre à la demande intérieure. La même distinction peut être faite pour les coûts de main d'œuvre. Ici, les coûts de main d'œuvre qui font partie de la demande intérieure sous la forme d'une part de la valeur ajoutée intégrée à la demande intérieure sont comparés aux coûts de main d'œuvre qui font partie de la demande étrangère sous la forme d'une part de la valeur ajoutée intégrée à la demande étrangère. On notera que, pour la plupart des pays, au niveau le plus détaillé des secteurs d'activités, la part de l'emploi dans la valeur ajoutée est la même, que la production soit destinée à la consommation intérieure ou étrangère, et ce en raison des hypothèses utilisées dans la construction des TIES (les exceptions sont la Chine et le Mexique où les exportateurs qui ont une activité de transformation sont distingués des autres entreprises). Les différences de parts de l'emploi au niveau agrégé du secteur des entreprises reflètent principalement les différences de poids des secteurs d'activités soutenus par la demande intérieure ou étrangère.

\section{Références}

Galindo-Rueda, F. and F. Verger (2016), “ OECD Taxonomy of Economic Activities Based on R\&D Intensity », OECD Science, Technology and Industry Working Papers, No. 2016/04, Éditions OCDE, Paris, http://dx.doi.org/10.1787/5jlv73sqqp8r-en.

Haščič, I. et M. Migotto (2015), « Measuring environmental innovation using patent data ", OECD Environment Working Papers, $\mathrm{n}^{\circ} 89$, Éditions OCDE, Paris, http://dx.doi.org/10.1787/5js009kf48xw-en.

Inaba, T. et M. Squicciarini (2017), "ICT: A new taxonomy based on the international patent classification", Documents de travail de l'OCDE sur la science, la technologie et l’industrie, n 2017/01, Éditions OCDE, Paris, http://dx.doi.org/10.1787/ab16c396-en.

OMPI (2013), Table de concordance CIB - technologie, www.wipo.int/ipstats/fr/statistics/technology_concordance.html, consulté le 1er juin 2017). 


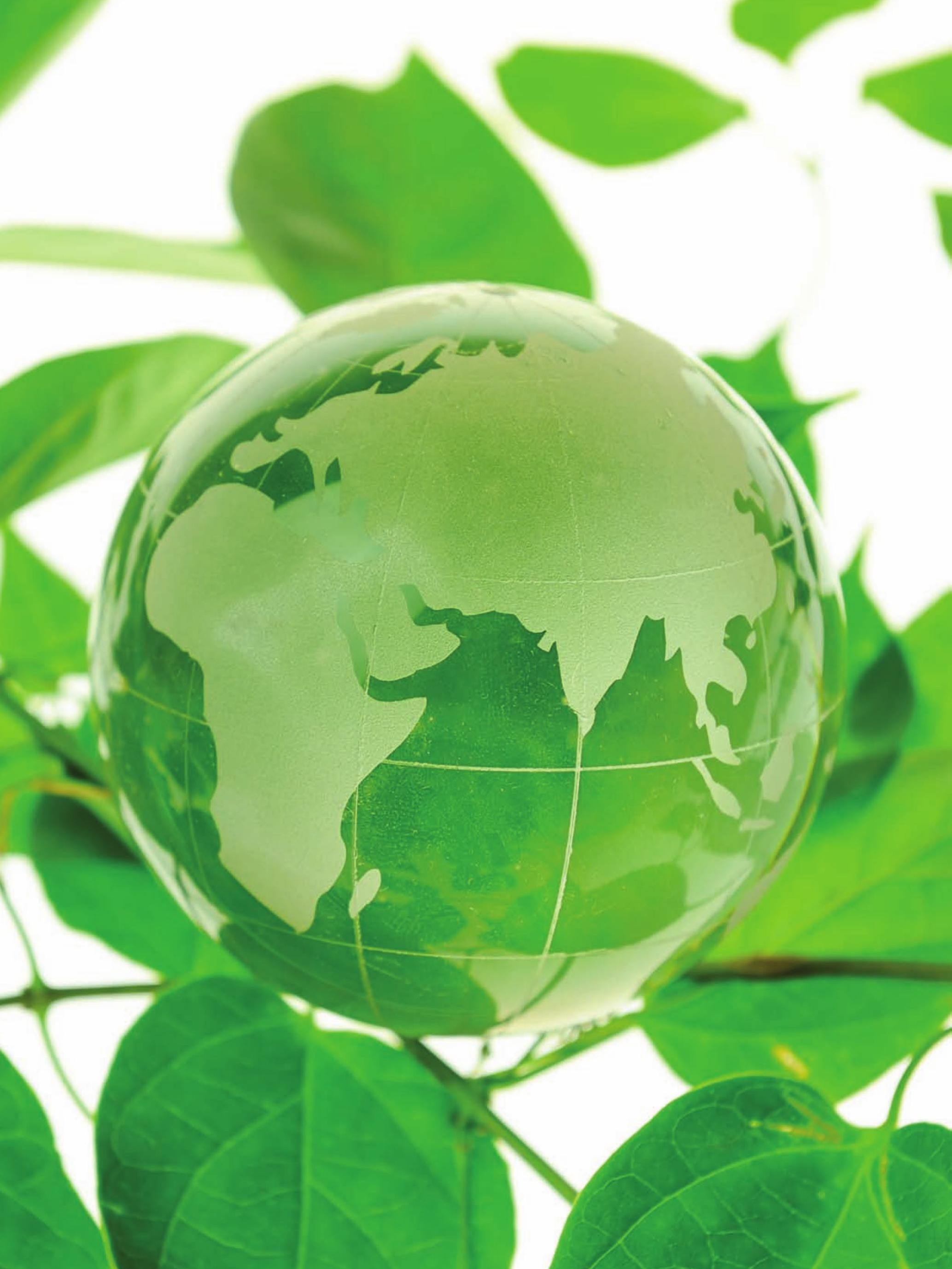




\section{SOCIÉTÉ ET TRANSFORMATION NUMÉRIQUE}

1. Connectivité

2. Les enfants du numérique

3. Internautes

4. Niveau de cyberactivité

5. Cyberconsommateurs sans frontières

6. Administration électronique

7. Confiance

Notes et références 


\section{Connectivité}

\section{Pénétration du haut débit mobile par technologie, décembre 2016}

Pour 100 habitants

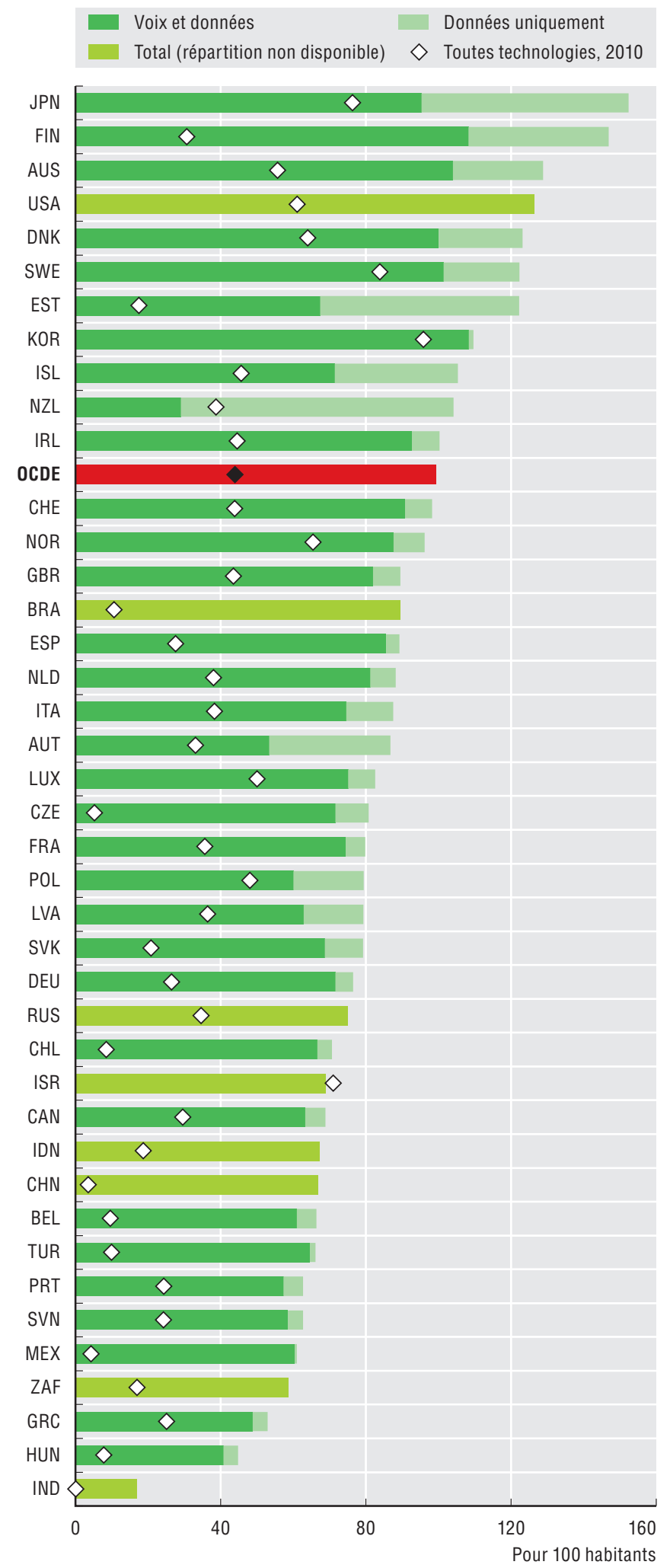

Source : OCDE, Portail de l'OCDE sur le haut débit, www.oecd.org/fr/sti/ hautdebit/portaildelocdesurlehautdebit.htm, juillet 2017. Davantage de données via StatLink. Voir notes de chapitre.

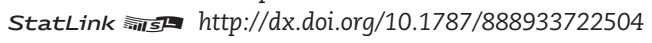

\section{Le saviez-vous ?}

Le nombre d'abonnements au haut débit mobile a plus que doublé dans la zone OCDE entre 2010 et 2016, pour atteindre environ 1.3 milliard - soit près d'un abonnement par habitant.

Les réseaux de communication haut débit et les services fournis à travers eux soutiennent les activités économiques et sociales existantes et affichent un formidable potentiel d'innovation. Le haut débit continue de gagner du terrain dans l'ensemble de la zone OCDE, mais à des degrés variables selon les pays. En décembre 2016, on y recensait 387 millions d'abonnements au haut débit fixe, ce qui représente un taux de pénétration moyen de $30 \%$, contre $25 \%$ fin 2010. La Suisse affichait le taux le plus élevé (50\%), suivie du Danemark, des Pays-Bas et de la France (qui présentaient tous des chiffres supérieurs à $40 \%$ ).

La progression du haut débit mobile a été particulièrement fulgurante. De fait, le taux de pénétration dans la zone OCDE est passé de $44 \%$ en 2010 à $99 \%$ en décembre 2016 - soit près d'un abonnement par habitant. Au cours de la même période, le nombre total d'abonnements au haut débit mobile a bondi de 544 millions à 1.275 milliard. Ils représentent désormais $77 \%$ de l'ensemble des accès haut débit de la zone OCDE. Les taux de pénétration ont connu, dans les pays membres de l'Organisation, une croissance spectaculaire entre 2010 et 2016, en particulier en République tchèque (où ils ont été multipliés par 16) et au Mexique (multipliés par 14).

Les connexions haut débit des ménages renseignent sur l'accès des particuliers aux informations et aux services. Les disparités en termes d'accès haut débit s'expliquent en partie par les clivages infranationaux entre zones urbaines et zones rurales. Les écarts les plus importants ont été constatés en Grèce (21 points de pourcentage), au Chili (19 points) et au Portugal (15 points).

La progression de la pénétration du haut débit s'est traduite par une réduction des écarts entre petites et moyennes entreprises. Ceux-ci restent toutefois notables au Mexique (17 points de pourcentage), en Grèce (14 points), en Pologne (7 points) et au Royaume-Uni (6 points).

\section{Définitions}

La pénétration du haut débit correspond au rapport entre, d'une part, le nombre d'abonnements aux services de haut débit fixe et mobile (caractérisé par des débits de données annoncés de $256 \mathrm{kbit} / \mathrm{s}$ ou plus), et, d'autre part, le nombre de résidents dans chaque pays. Le haut débit fixe englobe les technologies suivantes: DSL, câble, FTTH (fibre jusqu'au domicile) et FTTB (fibre jusqu'à l'immeuble), hertzien satellite, hertzien fixe terrestre, et autres technologies fixes filaires. Le haut débit mobile comprend à la fois les abonnements voix/données et les abonnements portant exclusivement sur la transmission de données.

Selon la Typologie régionale de l'OCDE, une région est considérée comme rurale si plus de $50 \%$ de la population vit dans des unités locales présentant une densité inférieure à 150 habitants par kilomètre carré. Elle est considérée comme urbaine si moins de $15 \%$ de la population vit dans de telles unités locales. Au Japon et en Corée, le seuil est établi à 500 habitants, la densité de population nationale étant supérieure à 300 habitants par kilomètre carré.

Les classes de taille des entreprises sont les suivantes : petites entreprises (de 10 à 49 salariés), entreprises moyennes (de 50 à 249 salariés) et grandes entreprises (plus de 250 salariés). 
Ménages équipés d'une connexion haut débit, régions urbaines et rurales, 2010 et 2016

En pourcentage du nombre de ménages dans chaque catégorie

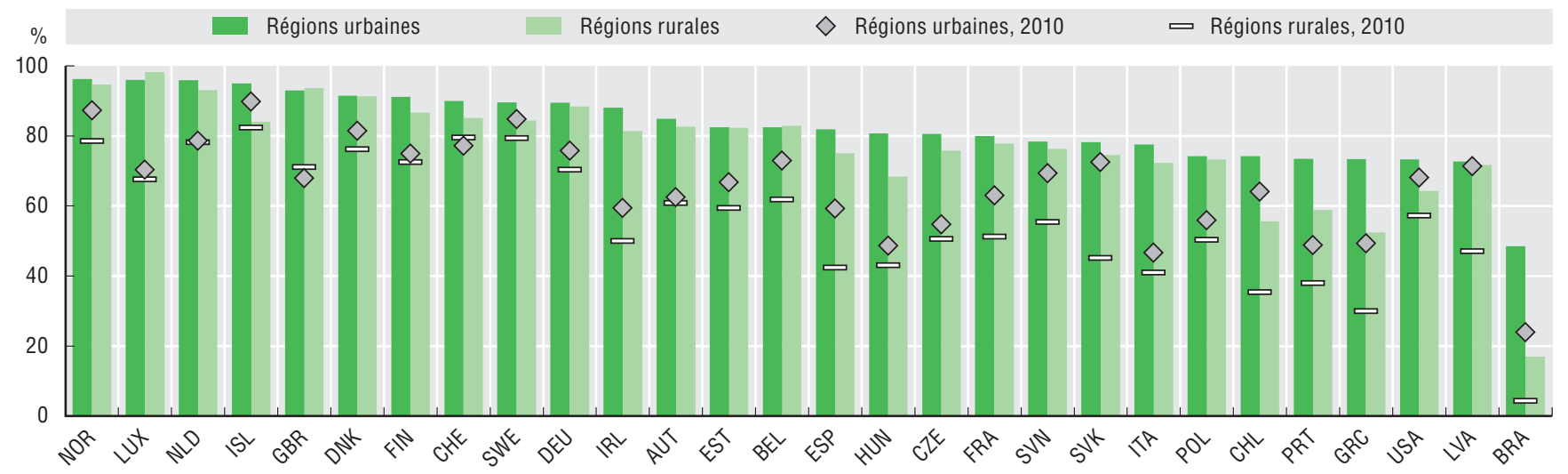

Source : OCDE, Base de données sur l'accès et utilisation des TIC par les ménages et les individus, http://oe.cd/hhind, juin 2017. Davantage de données via StatLink. Voir notes de chapitre.

StatLink त्ताइ $h$ ttp://dx.doi.org/10.1787/888933722523

Petites et moyennes entreprises dotées d'un accès haut débit, fixe ou mobile, 2016

En pourcentage du nombre total d'entreprises dans chaque classe de taille

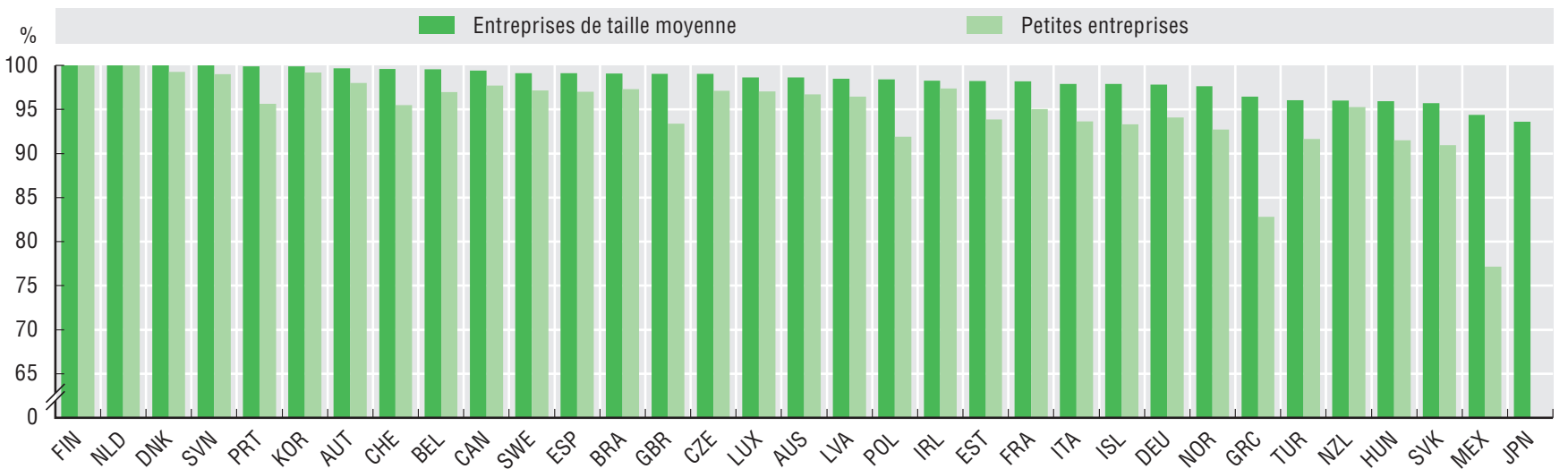

Source : OCDE, Base de données sur l'accès et utilisation des TIC par les entreprises, http://oe.cd/bus, juillet 2017. Davantage de données via StatLink. Voir notes de chapitre.

StatLink त्ताइ $h$ ttp://dx.doi.org/10.1787/888933722542

\section{Mesurabilité}

Dans les pays de l'OCDE, les données relatives aux abonnements au haut débit fixe et mobile sont recueillies sur la base de définitions communes et présentent un niveau élevé de comparabilité (OCDE, 2015a). Les données afférentes aux abonnements au haut débit mobile gagnent fortement en qualité depuis quelques années, notamment pour ce qui est des abonnements voix/données et des abonnements dédiés à la transmission de données. S'agissant des abonnements voix/données, seuls ceux qui étaient actifs dans les trois mois précédant la date du recensement sont comptabilisés, ce qui peut soulever des problèmes. Des données conformes à ces critères sont à présent disponibles pour la plupart des pays de l'OCDE.

La typologie régionale de l'OCDE peut aider à expliquer les écarts régionaux en matière de résultats économiques et de comportement du marché du travail. En revanche, dans la mesure où elle se fonde sur la densité de la population, elle ne permet pas d'opérer une distinction entre les régions proches d'une grande agglomération à forte densité et les régions éloignées. Pour y remédier, on y a intégré un critère supplémentaire, basé sur le temps de déplacement nécessaire pour que $50 \%$ de la population d'une région rejoigne une agglomération (Brezzi et al., 2011). Pour l'heure, la typologie étendue a été appliquée uniquement aux calculs effectués pour les régions Amérique du Nord (Canada, Mexique et États-Unis) et Europe. 
Élèves ayant accédé à l'internet pour la première fois à l'âge de six ans ou avant, 2012 et 2015

En pourcentage des élèves de 15 ans

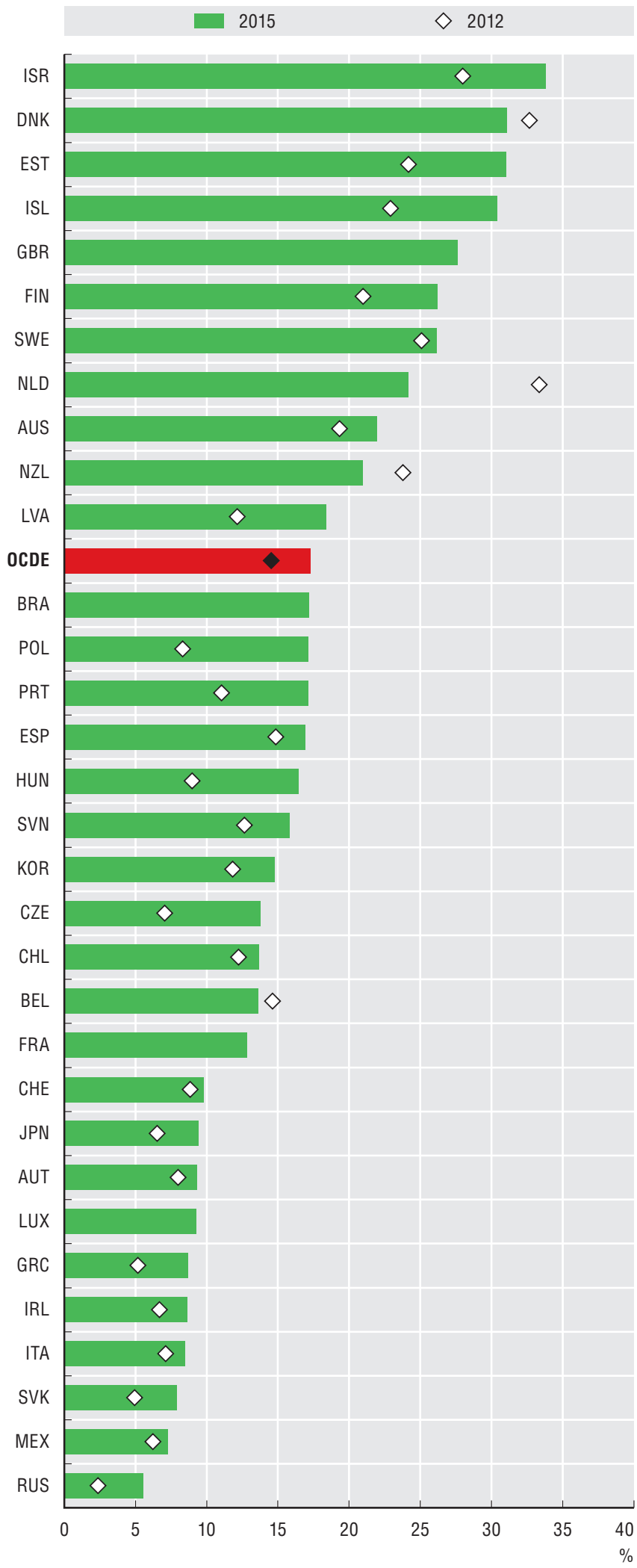

\section{Le saviez-vous ?}

En moyenne, 56 \% des garçons de 15 ans vivant dans les pays de l'OCDE jouent à des jeux en ligne quotidiennement ou quasi quotidiennement, contre seulement $13 \%$ des filles du même âge ; celles-ci sont en revanche plus nombreuses à dialoguer en ligne.

Omniprésent dans l'économie et la société, l'internet occupe peu à peu une place prépondérante dans la vie des jeunes. Les décideurs publics requièrent de plus en plus de preuves tangibles de l'incidence des TIC sur les résultats scolaires des élèves. Or les conclusions des recherches demeurent pour l'heure contrastées et appellent des mesures supplémentaires. Selon les résultats de l'enquête menée en 2015 au titre du programme international de l'OCDE pour le suivi des acquis des élèves (PISA), $17 \%$ des élèves de la zone OCDE ont accédé à l'internet pour la première fois à l'âge de six ans ou avant. Dans les pays pour lesquels on dispose de données, moins de $0.3 \%$ des jeunes de 15 ans ont déclaré n'y avoir jamais eu accès.

L'âge auquel les jeunes accèdent pour la première fois à l'internet varie d'un pays à l'autre. Au Danemark, en Estonie, en Islande et en Israël, plus de $30 \%$ des élèves ont commencé à l'utiliser à l'âge de six ans ou avant. Dans environ deux tiers des pays ayant participé à l'enquête PISA, la plupart des élèves ont accédé pour la première fois à l'internet entre sept et neuf ans ; dans le tiers des pays restant, ils étaient généralement âgés de dix ans ou plus.

En 2015, dans les pays de l'OCDE 43 \% des jeunes de 15 ans passaient entre deux et six heures par jour sur l'internet en dehors du temps scolaire - soit une augmentation notable par rapport à 2012, où ils étaient moins de $30 \%$. Le Brésil et le Chili étaient les pays qui comptaient la plus forte proportion d'élèves (supérieure à $30 \%$ ) passant plus de six heures par jour sur l'internet en dehors de l'école.

Aujourd'hui, $62 \%$ des adolescents de 15 ans vivant dans la zone OCDE dialoguent en ligne et $73 \%$ prennent part à un réseau social quotidiennement ou quasi quotidiennement. Les écarts entre les sexes sont particulièrement marqués dans certaines activités comme les jeux en ligne (surtout prisés des garçons), ou le dialogue en ligne (auquel s'adonnent davantage les filles).

\section{Définitions}

L'âge des élèves évalués dans le cadre de l'enquête PISA s'échelonne entre 15 ans et trois mois, et 16 ans et deux mois. Ils doivent être scolarisés et avoir reçu au moins six années d'enseignement structuré, indépendamment du type d'établissement fréquenté, du programme suivi ou des modalités de l'enseignement (temps plein ou temps partiel).

Les jeux en ligne sont les jeux auxquels les internautes peuvent jouer seuls ou à plusieurs.

Tous les chiffres de l'enquête PISA sont exprimés en pourcentage du nombre de répondants. Les résultats sont le reflet des déclarations des élèves sondés. 


\section{SOCIÉTÉ ET TRANSFORMATION NUMÉRIQUE}

Temps passé sur l'internet en dehors de l'école, 2012 et 2015

En pourcentage des élèves de 15 ans passant deux à six heures en ligne, durant un jour de semaine type

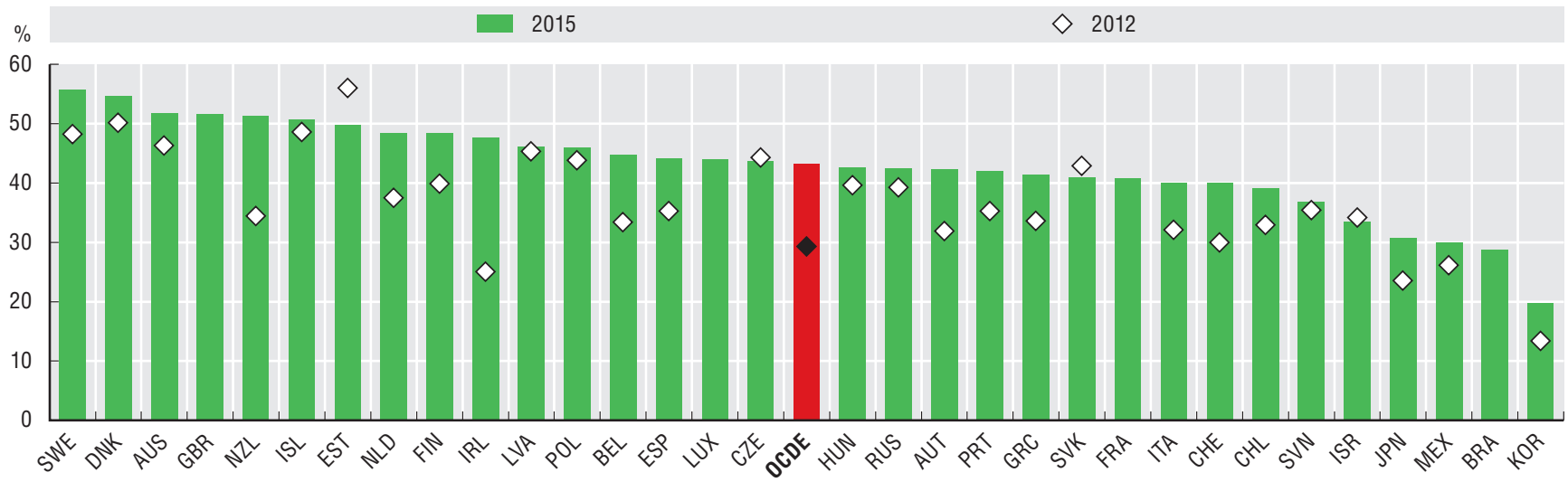

Source : Calculs de l'OCDE d'après OCDE, Base de données PISA 2015, juillet 2017. Davantage de données via StatLink.

StatLink - तilst http://dx.doi.org/10.1787/888933722580

Diffusion d'une sélection d'activités en ligne parmi les élèves des pays de l'OCDE, par sexe, 2015

En pourcentage des élèves de 15 ans pratiquant chaque activité quotidiennement ou quasi quotidiennement

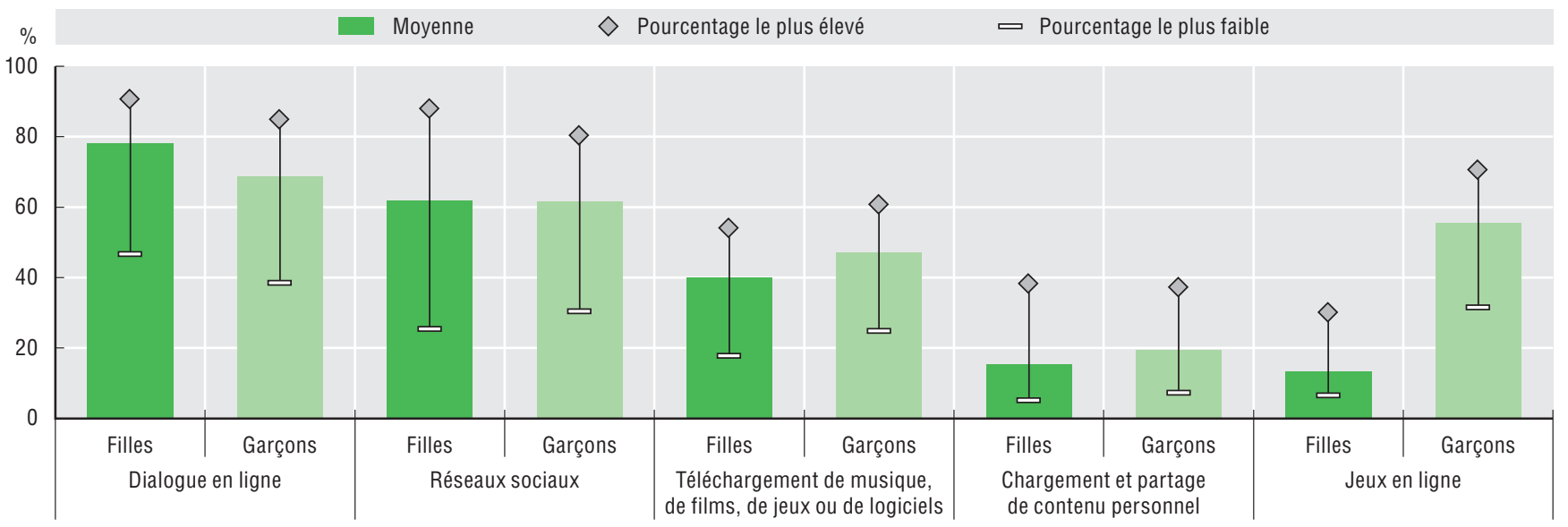

Source : Calculs de l'OCDE d'après OCDE, Base de données PISA 2015, juillet 2017. Davantage de données via StatLink.

StatLink न्माड़ा $h t t p: / / d x . d o i . o r g / 10.1787 / 888933722599$

\section{Mesurabilité}

L'enquête PISA 2015, menée dans 72 économies, vise à évaluer les capacités des jeunes de 15 ans. Plus d'un demi-million d'élèves d'une tranche d'âge allant de 15 ans et trois mois à 16 ans et deux mois, représentatifs des 28 millions d'adolescents de 15 ans recensés à l'échelle mondiale, ont passé un test de deux heures, convenu à l'échelle internationale.

Module optionnel, le questionnaire sur les habitudes d'utilisation des TIC compte des questions portant sur les TIC auxquelles les élèves ont accès à domicile et à l'école, la fréquence d'utilisation de différents appareils et technologies, la capacité des élèves à réaliser des tâches informatiques et leur attitude à l'égard de l'utilisation d'un ordinateur. En 2015 , 47 des 72 économies qui ont pris part à l'enquête PISA ont mis en œuvre ce module. Bien qu'il permettre de recueillir de précieuses informations, plusieurs pays de l'OCDE (Canada, Norvège, Turquie et États-Unis) n'ont pas exploité le questionnaire sur les TIC en 2015, du fait principalement du coût élevé inhérent à l'ajout de ces questions supplémentaires à l'enquête.

Néanmoins, la disponibilité croissante des données issues des différentes vagues PISA permet d'évaluer l'évolution dans le temps de l'utilisation des TIC à l'école et en dehors du temps scolaire, et d'en examiner les incidences sur les résultats scolaires, préoccupation majeure des responsables des politiques d'éducation. 


\section{Population totale d'individus utilisant l'internet mobile quotidiennement, 2016}

En pourcentage des 16-74 ans
Total internautes
$\diamond$ Total internautes, 2006
Utilisateurs quotidiens

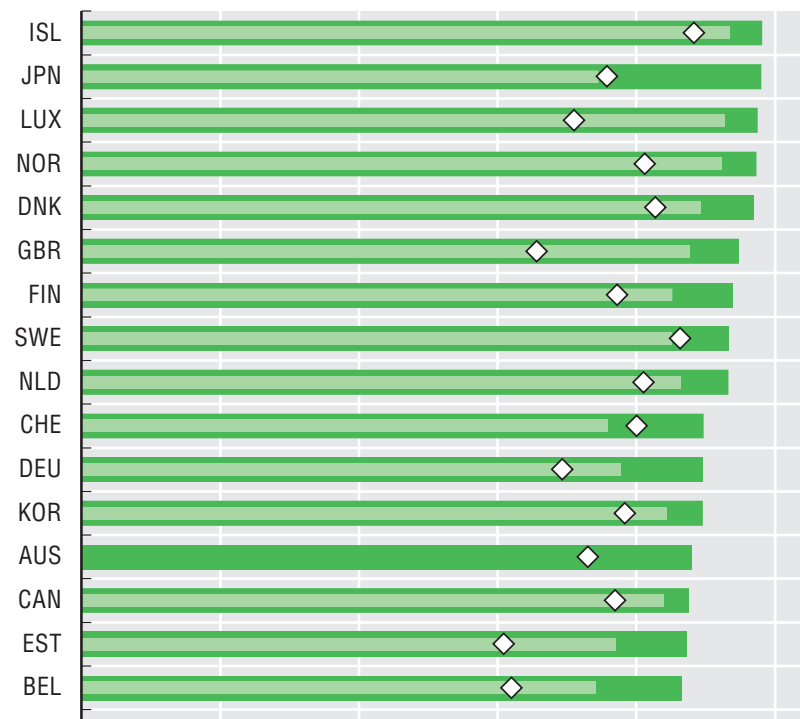

FRA

AUT

NZL

OCD

CZ

IR

\section{ESP}

SVK

HUN

USA

CHL

SVN

RUS

POL

PRT

GRC

ITA
MEX

TUR

BRA

ZAF

$\mathrm{CHN}$

IND

IDN

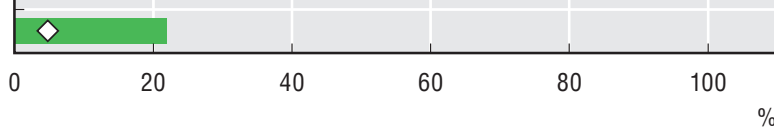

Sources : OCDE, Base de données sur l'accès et utilisation des TIC par les ménages et les individus, http://oe.cd/hhind ; UIT, Base de données Indicateurs des télécommunications/TIC dans le monde, et sources nationales, juin 2017. Davantage de données via StatLink. Voir notes de chapitre.

StatLink तils $h t t p: / / d x . d o i . o r g / 10.1787 / 888933722618$

\section{Le saviez-vous ?}

Plus de $60 \%$ de la population adulte des pays de l'OCDE utilisent un mobile ou un smartphone pour accéder à l'internet.

L'internet fait désormais partie du quotidien du plus grand nombre. Entre 2006 et 2016, le taux de pénétration total est passé de $59 \%$ à $84 \%$, soit une hausse de 25 points de pourcentage à l'échelle de la zone OCDE. En moyenne, $74 \%$ des individus vivant dans les pays de l'OCDE pour lesquels on dispose de données accèdent quotidiennement à l'internet $62 \%$ de la population adulte le font à partir d'un terminal mobile ou d'un smartphone.

L'utilisation de l'internet varie considérablement selon les pays de l'OCDE et les catégories sociales. En 2016, les internautes ne représentaient pas moins de $97 \%$ des adultes au Danemark, en Islande, au Japon, au Luxembourg et en Norvège. En revanche, ils n'étaient que $60 \%$ au Mexique, et encore moins en Turquie (58\%). Dans les pays non membres de l'Organisation, cette part varie entre $22 \%$ en Indonésie et $58 \%$ au Brésil.

Les écarts observés dans l'adoption de l'internet sont principalement liés à l'âge et au niveau d'instruction, souvent combinés aux revenus. Dans tous les pays de l'OCDE à l'exception des États-Unis, la proportion d'internautes parmi les diplômés de l'enseignement supérieur dépassait 90 \% en 2016, tandis que l'on observe des disparités importantes parmi les personnes moins instruites. De fait, la part des internautes parmi les individus affichant un niveau d'instruction faible ou n'ayant pas bénéficié d'un enseignement structuré va de moins de $40 \%$ au Chili et en Grèce, à plus de $90 \%$ au Danemark, en Islande, au Luxembourg et en Norvège. Au Mexique et en Turquie, les taux d'adoption de l'internet par les individus les plus instruits et les moins instruits présentent des écarts de 35 points de pourcentage et plus. Les personnes disposant d'un niveau d'instruction peu élevé sont donc susceptibles de faire l'objet d'une attention particulière dans le cadre de l'élaboration des stratégies en faveur de l'inclusion numérique. En 2016, le taux d'utilisation de l'internet par les femmes vivant dans la zone OCDE (83\%) était légèrement inférieur à celui des hommes (85\%). Les écarts les plus marqués ont été observés en Turquie (18 points de pourcentage), en Autriche (7 points) et en Italie (6 points). Dans l'ensemble, à l'échelle de la zone OCDE, le clivage est particulièrement important entre les femmes jeunes (96\%) et les plus âgées (61\%).

\section{Définitions}

On entend par internautes les personnes qui ont accédé à l'internet au cours des trois mois précédant l'enquête. Des périodes de référence différentes ont été utilisées pour certains pays (voir notes de chapitre).

Les utilisateurs quotidiens sont les personnes qui consultent l'internet tous les jours ou presque, au cours d'une semaine type (hors vacances, etc.).

L'internet mobile renvoie à la consultation de l'internet en dehors du domicile ou du lieu de travail de l'internaute, qui utilise pour ce faire un ordinateur ou autre appareil portables. Dans le cas des pays participant au Système statistique européen, les chiffres relatifs aux internautes connectés depuis un appareil mobile ou un smartphone incluent ceux qui sont raccordés à un réseau mobile ou hertzien ; pour les autres pays, on se reportera aux notes de chapitre. 


\section{Écarts d'adoption de l'internet selon le niveau d'instruction, 2016}

En pourcentage de la population dans chaque catégorie

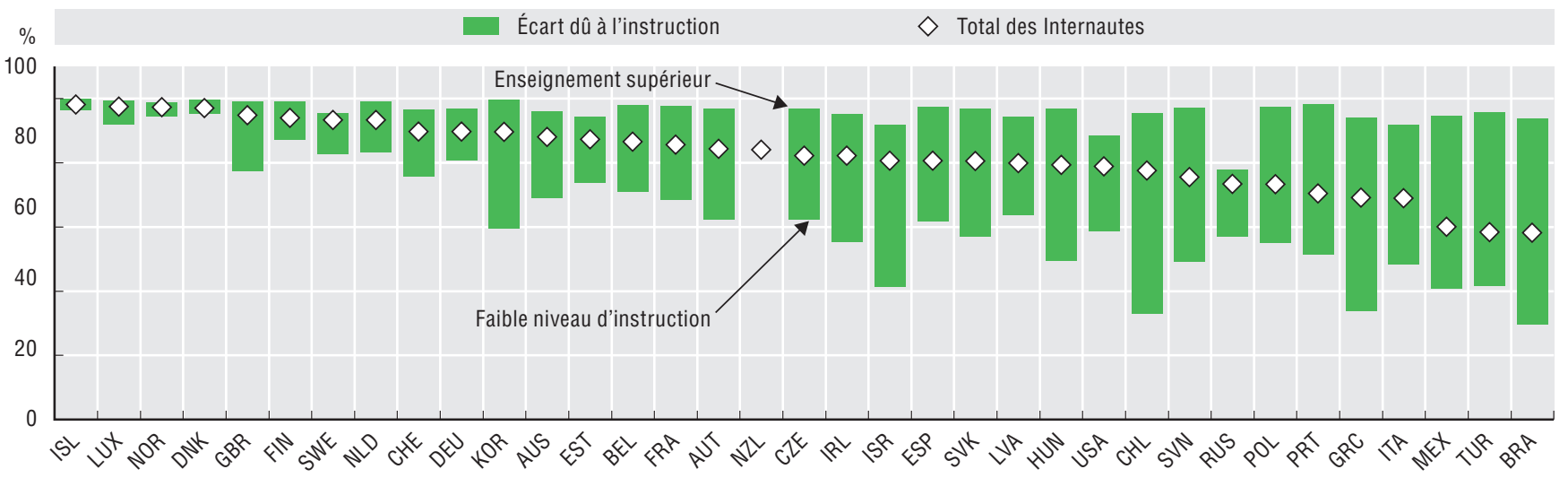

Sources : OCDE, Base de données sur l'accès et utilisation des TIC par les ménages et les individus, http://oe.cd/hhind et UIT, Base de données Indicateurs des télécommunications/TIC dans le monde, juin 2017. Davantage de données via StatLink. Voir notes de chapitre.

StatLink ails $h$ ttp://dx.doi.org/10.1787/888933722637

Utilisatrices de l'internet, par âge, 2016

En pourcentage de la population dans chaque groupe d'âges

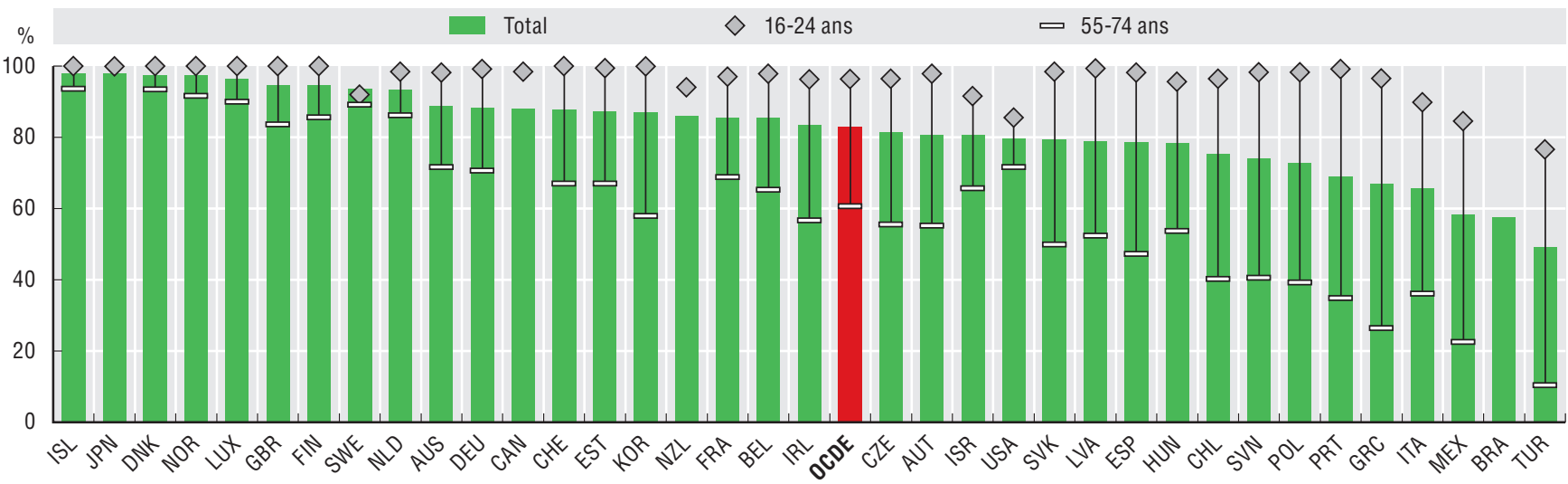

Sources : OCDE, Base de données sur l'accès et utilisation des TIC par les ménages et les individus, http://oe.cd/hhind et UIT, Base de données Indicateurs des télécommunications/TIC dans le monde, juin 2017. Davantage de données via StatLink. Voir notes de chapitre.

StatLink त्ताज़ http://dx.doi.org/10.1787/888933722656

\section{Mesurabilité}

Tous les pays de l'OCDE ne réalisent pas des enquêtes sur l'utilisation des TIC par les ménages et les particuliers. De plus, les données entrant dans le calcul de certains indicateurs ne sont pas toujours disponibles (voir notes de chapitre). En Australie, au Canada, au Chili, en Israël et en Nouvelle-Zélande, les enquêtes sont pluriannuelles ou ponctuelles, tandis que d'autres pays procèdent à des enquêtes annuelles. Celle de l'Union européenne est obligatoire dans huit pays seulement. La répartition des indicateurs par groupe d'âges et niveau d'instruction peut également soulever des questions quant à la robustesse des informations, en particulier dans le cas des petits pays, en raison de la taille des échantillons et des modalités de réalisation des enquêtes.

L'OCDE s'emploie activement à faciliter la collecte d'informations comparables dans ce domaine ; pour ce faire, elle a mis au point une enquête type sur l'accès aux TIC et leur utilisation par les ménages et les individus (OCDE, 2015b) et promeut la coordination des collectes de statistiques sur l'utilisation des TIC en général. 


\section{Niveau de cyberactivité}

\section{Diffusion d'une sélection d'activités en ligne parmi les internautes des pays de l'OCDE, par groupe d'âges et niveau d'instruction, 2016}

Internautes pratiquant chaque activité considérée, en pourcentage du nombre d'individus dans les groupes respectifs

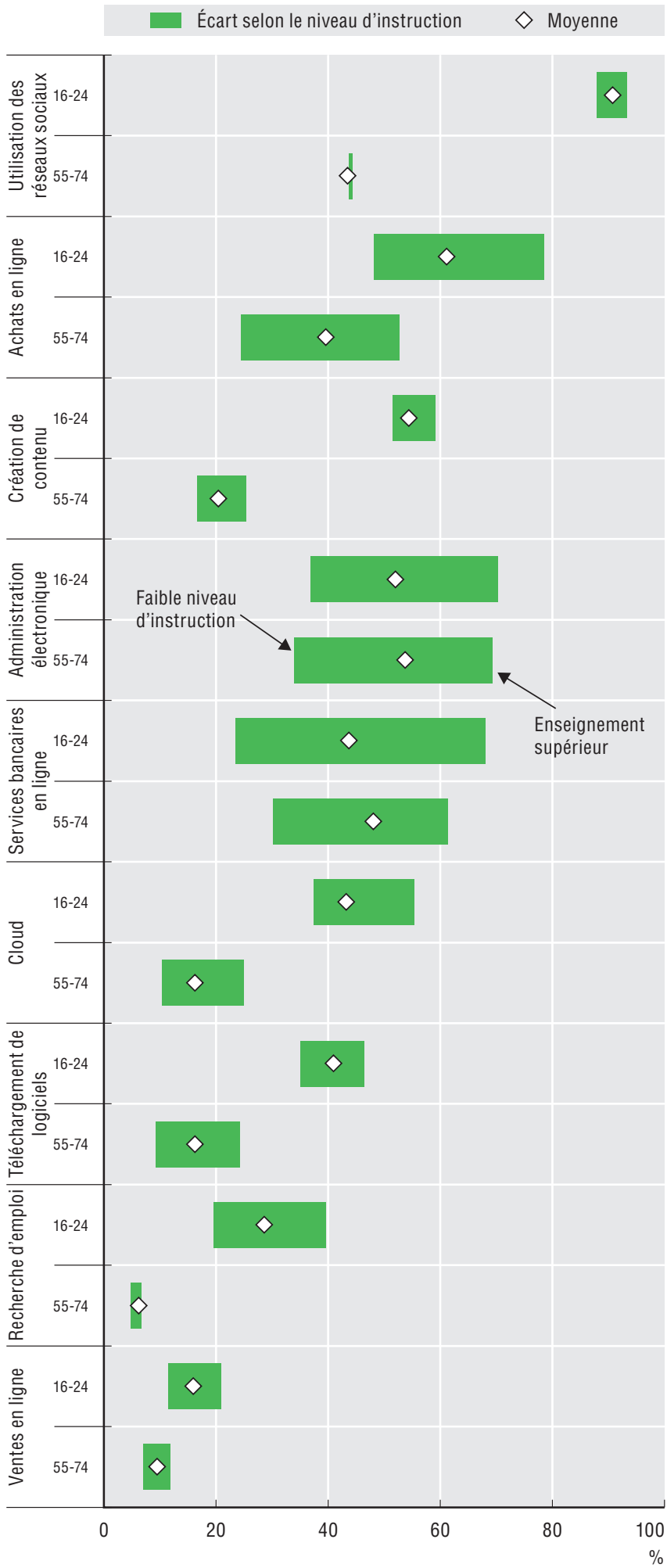

Source : OCDE, Base de données sur l'accès et utilisation des TIC par les ménages et les individus, http://oe.cd/hhind, juin 2017. Voir notes de chapitre.

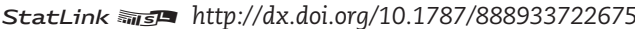

\section{Le saviez-vous ?}

En 2016, 70 \% des internautes de la zone OCDE étaient actifs sur les réseaux sociaux, quel que soit leur niveau d'instruction.

L'usage qui est fait de l'internet varie grandement d'un pays à l'autre, sous l'effet de facteurs institutionnels, culturels ou économiques. L'éventail d'activités pratiquées par les internautes donne une indication de leur niveau de cyberactivité.

L'âge et le niveau d'instruction font partie des principaux facteurs susceptibles d'expliquer les écarts entre les pays. La plupart des activités sont davantage pratiquées par les jeunes, à l'exception du recours aux services de l'administration électronique et aux services bancaires en ligne, où la part des plus âgés est supérieure. Le niveau d'instruction interagit avec l'âge de différentes manières selon le type d'activités pratiquées en ligne. Parmi les plus âgés, les écarts d'adoption selon le niveau d'instruction sont plus importants pour certaines activités, comme le téléchargement de logiciels et, dans une moindre mesure, la création de contenu. Parmi les jeunes, les écarts de niveau d'instruction jouent un rôle plus important dans la réalisation de toutes les autres activités (utilisation des services bancaires en ligne, des services de l'administration électronique, des services de cloud et des réseaux sociaux, achats et ventes en ligne, ou recherche d'emploi).

Dans la quasi-totalité des pays, la part des internautes effectuant des achats en ligne a augmenté entre 2010 et 2016 . Elle a même plus que doublé dans des pays qui affichaient, au départ, des taux d'adoption particulièrement bas, comme l'Estonie et le Mexique. En 2016, 60 \% de la population totale d'internautes de la zone OCDE ont effectué au moins un achat en ligne, sachant néanmoins que la proportion d'acheteurs parmi les internautes âgés de 16 à 24 ans était, en moyenne, supérieure de plus de 20 points de pourcentage à celle de la tranche d'âges 55-74 ans. Au cours des dernières années, les TIC ont contribué à un élargissement des possibilités d'apprentissage, avec l'essor des cours sur l'internet, comme les cours en ligne ouverts à tous (MOOCs). En 2016, dans la zone OCDE, plus de $14 \%$ des internautes âgés de 16 à 24 ans ont suivi un cours sur le web, contre $13 \%$ en 2009. On observe toutefois des disparités marquées, puisque les taux vont de moins de $3 \%$ en République slovaque à $77 \%$ au Canada.

\section{Définitions}

On entend par internautes les personnes qui ont accédé à l'internet au cours des trois mois précédant l'enquête. D'autres périodes de référence ont été utilisées pour certains pays (voir notes de chapitre).

Les diplômés de l'enseignement supérieur sont les personnes titulaires d'un diplôme de niveau 5, 6 ou plus de la CITE. Les personnes présentant un niveau d'instruction faible correspondent aux niveaux 0 à 2 de la CITE.

Les achats en ligne ou les ventes en ligne (transactions réalisées dans le cadre du commerce électronique) désignent les opérations d'achat ou de vente de biens ou de services réalisées sur des réseaux informatiques par des méthodes spécifiquement conçues pour la réception ou la passation de commandes (OCDE, 2011).

Un cours en ligne est un cours dont une partie du contenu est fournie au format électronique (via le réseau internet ou par d'autres méthodes informatiques) et/ou dont une partie de l'enseignement est dispensé à distance, à l'aide d'outils en ligne ou électroniques. 


\section{SOCIÉTÉ ET TRANSFORMATION NUMÉRIQUE}

4. Niveau de cyberactivité

Individus ayant effectué des achats en ligne au cours des 12 derniers mois, par groupe d'âges, 2016 En pourcentage de la population d'internautes dans chaque groupe d'âges

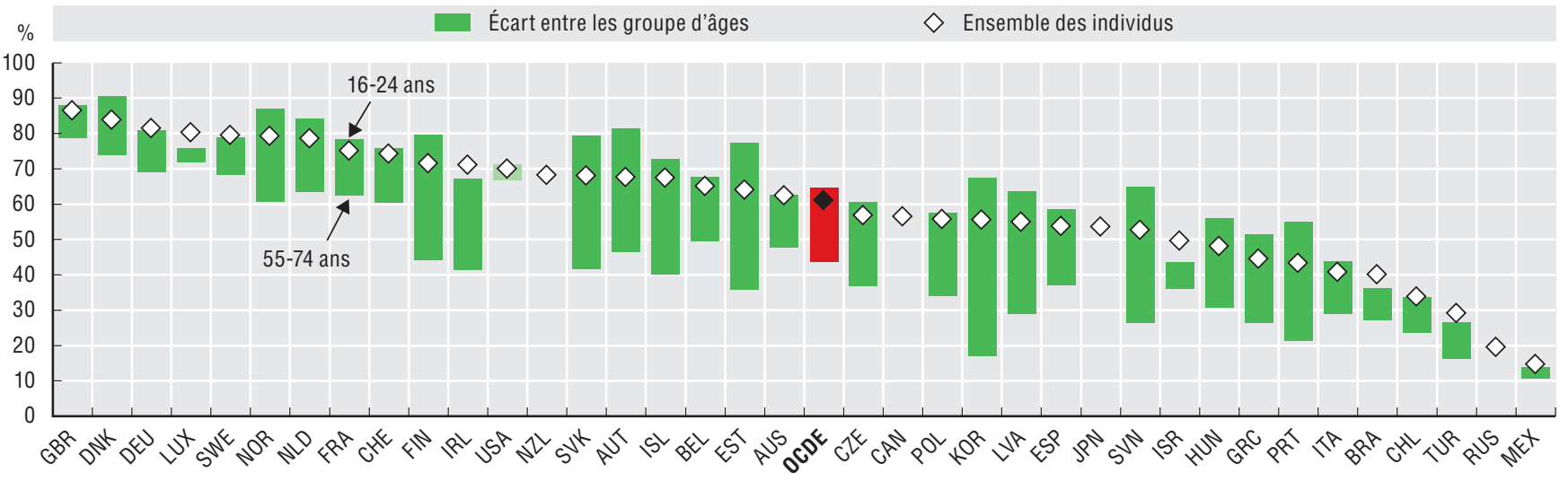

Note : Pour les États-Unis, l'écart entre les groupes d'âges, représenté en bleu clair, est inversé. Les individus âgés de 55 à 74 ans affichent en effet une propension à effectuer des achats en ligne légèrement supérieure à celle de la tranche d'âges 16-24 ans.

Sources : OCDE, Base de données sur l'accès et utilisation des TIC par les ménages et les individus, http://oe.cd/hhind et UIT, Base de données Indicateurs des télécommunications/TIC dans le monde, juin 2017. Davantage de données via StatLink. Voir notes de chapitre.

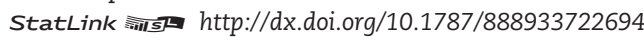

Individus âgés de 16 à 24 ans ayant suivi un cours en ligne, 2009 et 2016

En pourcentage des individus âgés de 16 à 24 ans

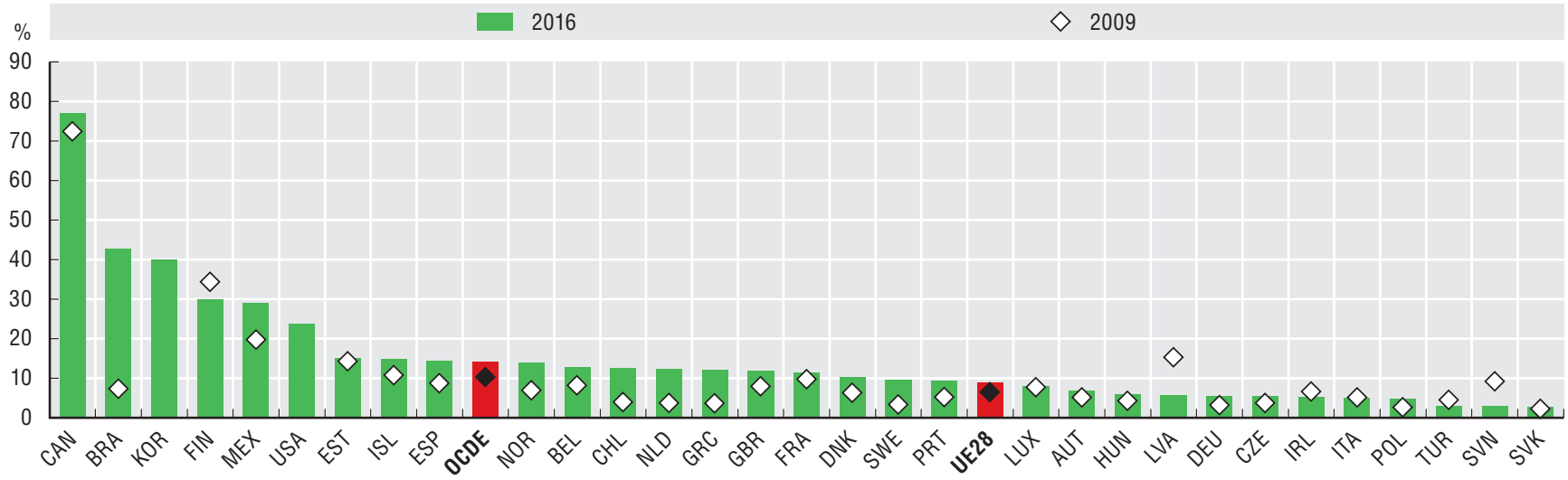

Source : OCDE, Base de données sur l'accès et utilisation des TIC par les ménages et les individus, http://oe.cd/hhind, juin 2017. Davantage de données via StatLink. Voir notes de chapitre.

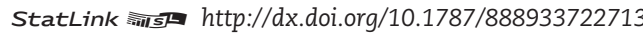

\section{Mesurabilité}

La collecte de données sur l'utilisation des TIC par les individus varie selon les pays de l'OCDE, en termes à la fois de fréquence et de nature des enquêtes réalisées. En particulier, les données relatives aux différentes activités pratiquées - dont l'éventail est potentiellement large et qui ne cessent de se développer - se limitent souvent à des informations de base.

Pour les achats en ligne, plusieurs facteurs peuvent expliquer les problèmes de comparabilité. Prenons les critères d'âge, par exemple : les données pour le Japon et les États-Unis portent sur tous les individus âgés de 6 ans ou plus, et non pas de 16 à 74 ans, ce qui peut donner lieu à des taux globaux moins élevés. Les différences de périodes de référence jouent également un rôle déterminant : pour Israël, la période utilisée est de 3 mois au lieu de 12 ; pour le Chili et les États-Unis, aucune période de référence n'est précisée. À cela s'ajoutent des divergences au niveau de la définition elle-même : pour la Nouvelle-Zélande, seuls les achats en ligne donnant lieu à un paiement électronique sont pris en compte. Enfin, il arrive que la méthodologie employée pour réaliser l'enquête diffère (techniques, période de l'année où elle est menée, etc.). 


\section{Entreprises ayant pratiqué la vente par commerce électronique à l'étranger, 2014}

En pourcentage de l'ensemble des entreprises pratiquant le commerce électronique

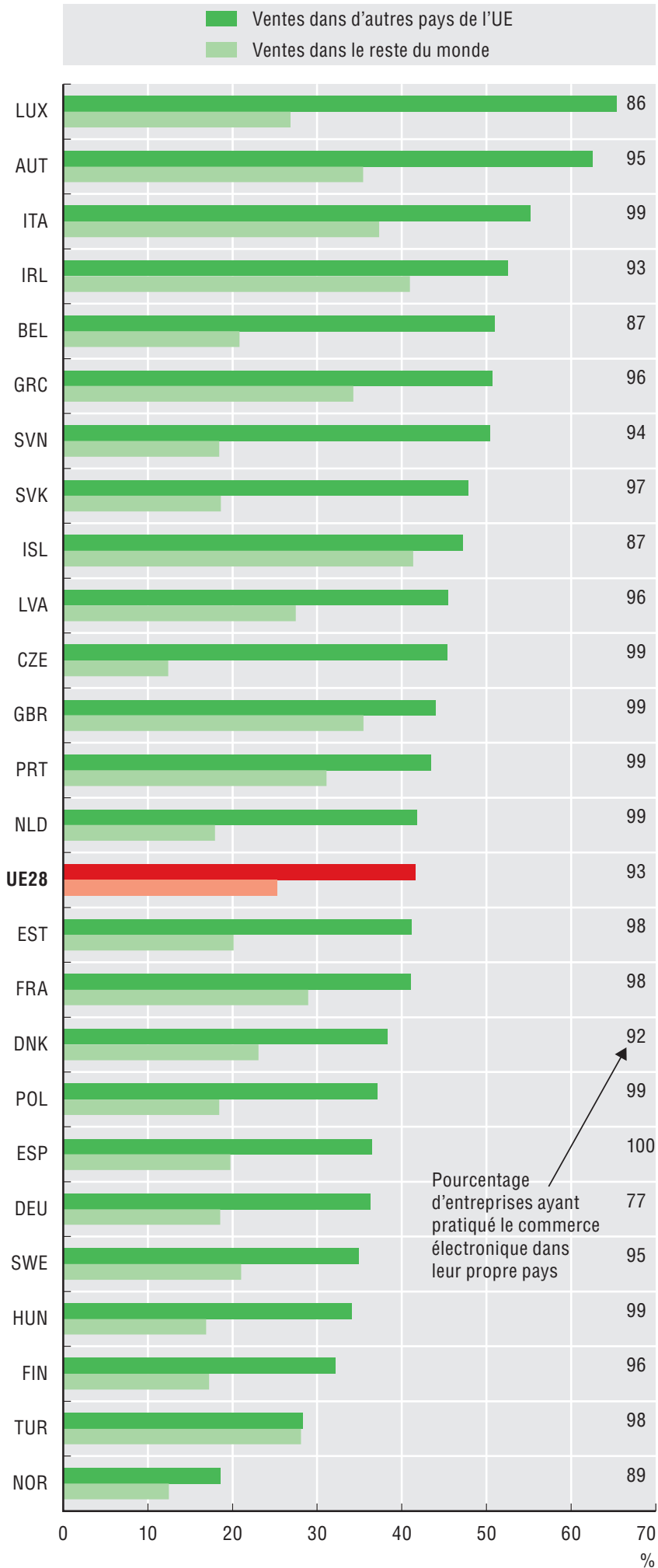

Source : OCDE, d'après Eurostat, Économie et société numériques, Base de données complète, juillet 2017. Davantage de données via StatLink. Voir notes de chapitre.

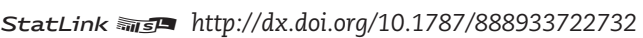

\section{Le saviez-vous ?}

En 2016, 20 \% des individus ayant réalisé des achats sur l'internet en Europe ont passé commande auprès de vendeurs basés hors d'Europe.

L'internet a facilité l'accès aux marchés mondiaux, créant par là même de nouvelles opportunités pour les consommateurs et les entreprises. L'essor du commerce électronique transfrontière est principalement lié à l'infrastructure informatique, aux cadres réglementaires et à l'intégration économique.

En 2014, 42 \% des entreprises européennes pratiquant le commerce électronique ont vendu des produits ou services en ligne dans d'autres pays européens, et $25 \%$ dans des pays non européens. La part des entreprises ayant vendu des produits ou services à destination d'autres pays européens était la plus élevée au Luxembourg (65\%) et en Autriche (62\%). Pour ce qui est des ventes en ligne transfrontières à destination de pays hors d'Europe, ce sont l'Islande et l'Irlande qui arrivent en tête (avec $41 \%$ ).

Les consommateurs canadiens et européens qui effectuent des achats en ligne privilégient les vendeurs nationaux, plutôt qu'étrangers. Seules font exception les petites économies ouvertes, comme l'Autriche et le Luxembourg. Dans tous les pays sauf l'Islande, les consommateurs achetant des biens ou des services en ligne auprès d'un vendeur étranger privilégient ceux qui sont installés dans des pays partenaires. En 2016, 90 \% des personnes qui ont effectué des achats en ligne depuis le Luxembourg ont passé commande auprès d'un vendeur implanté dans un pays partenaire, contre $7 \%$ en Turquie.

Aux États-Unis, le chiffre d'affaires du commerce électronique entre entreprises et consommateurs représentait à peine plus de $7 \%$ de celui de la vente de détail, un taux proche de la moyenne des pays européens (8.7\%). La moyenne européenne cache toutefois de fortes disparités, puisque les chiffres allaient, en 2015, de moins de $3 \%$ au Portugal à $15 \%$ au Danemark et $20 \%$ en Suède. La pénétration du commerce électronique de détail ne semble pas corrélée à la taille du secteur ; de fait, les pays affichant la part la plus élevée de recettes issues du commerce de détail dans le total des recettes du secteur des entreprises sont également ceux qui enregistrent la plus faible part de transactions liées au commerce de détail en ligne.

\section{Définitions}

Les achats en ligne ou les ventes en ligne (transactions réalisées dans le cadre du commerce électronique) désignent les opérations d'achat ou de vente de biens ou de services réalisées sur des réseaux informatiques par des méthodes spécifiquement conçues pour la réception ou la passation de commandes (OCDE, 2011). Pour les particuliers (vendeurs ou acheteurs), ces transactions s'effectuent généralement sur l'internet. S'agissant des entreprises, les ventes électroniques correspondent à l'ensemble des transactions réalisées par le biais de pages web, d'un réseau extranet ou d'un système d'échange de données informatisé (EDI).

On entend par transactions entre entreprises et consommateurs les ventes réalisées auprès de consommateurs soit par des entreprises présentes exclusivement sur le web, soit par des entreprises de vente au détail ou des fabricants traditionnels ayant élargi leurs circuits de distribution au commerce électronique. Les ventes peuvent porter sur des biens physiques ou des produits et services numériques.

Les classes de taille des entreprises sont les suivantes : petites entreprises (de 10 à 49 salariés), entreprises moyennes (de 50 à 249 salariés) et grandes entreprises (plus de 250 salariés). On entend par pays partenaires les pays membres de l'UE dans le cas des pays appartenant au Système statistique européen, et les États-Unis dans le cas du Canada. 


\section{SOCIÉTÉ ET TRANSFORMATION NUMÉRIQUE}

5. Cyberconsommateurs sans frontières

Individus ayant effectué des achats en ligne auprès de vendeurs nationaux et étrangers, 2016

En pourcentage des individus ayant commandé des biens ou des services sur l'internet au cours des 12 derniers mois

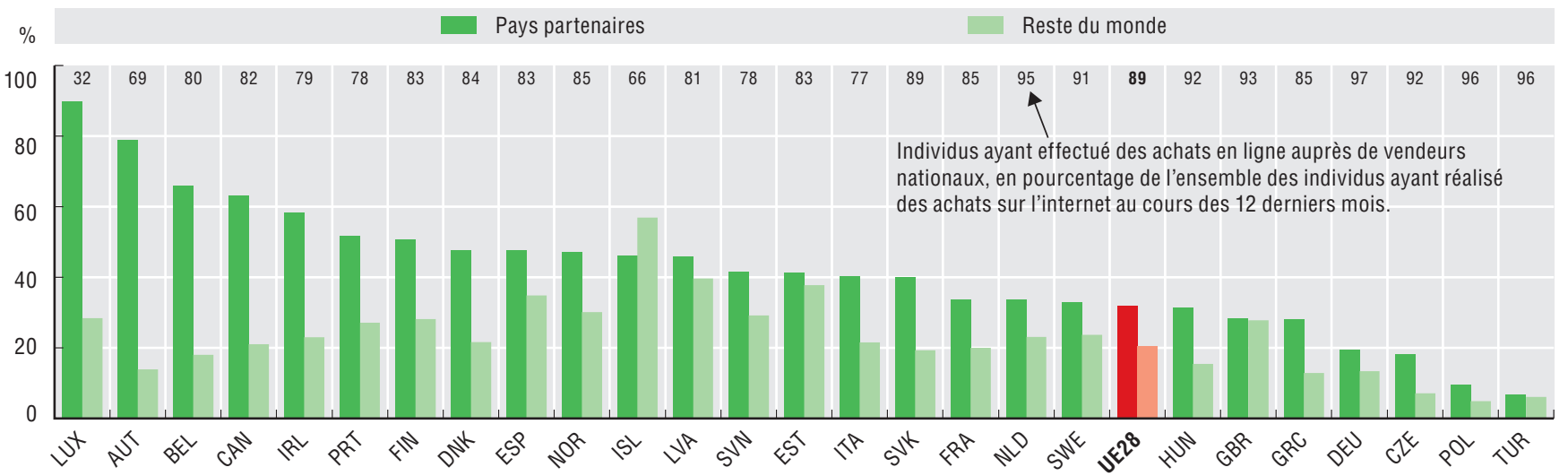

Sources : OCDE, d'après Eurostat, Économie et société numériques, Base de données complète, et sources nationales, juillet 2017. Davantage de données via StatLink. Voir notes de chapitre.

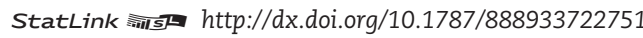

Transactions entre entreprises et consommateurs (B2C), 2009 et 2015

Chiffre d'affaires du commerce électronique de détail en pourcentage du chiffre d'affaires total du secteur du commerce de détail

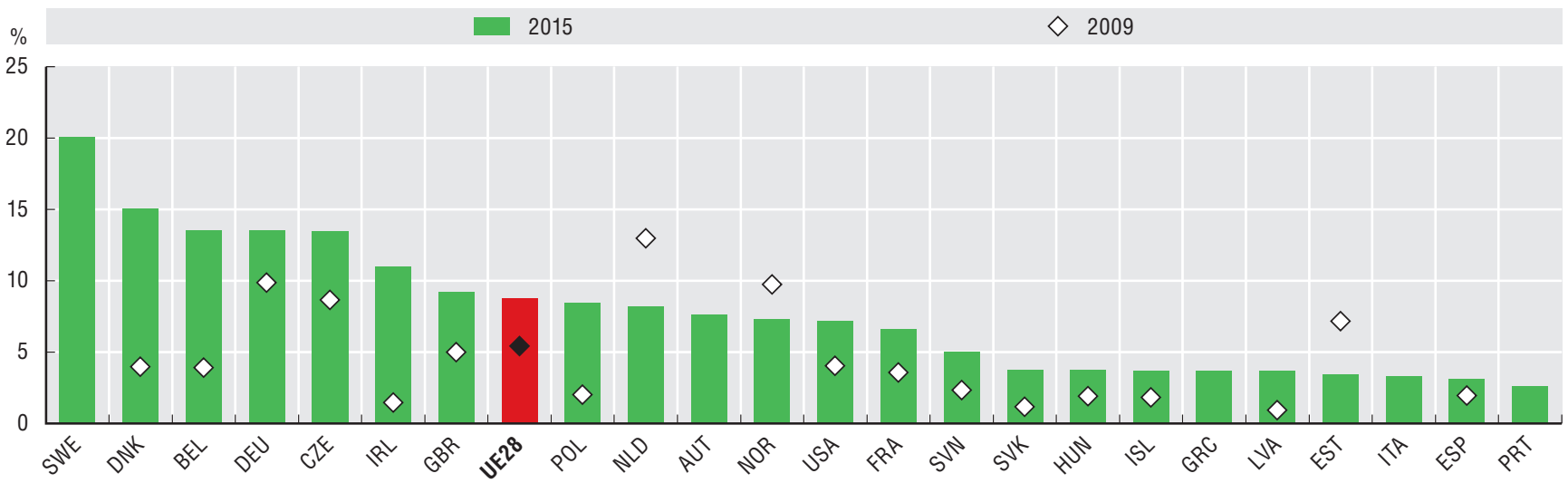

Sources : OCDE, d'après Eurostat, Économie et société numériques, Base de données complète, et US Census Bureau, Quarterly Retail E-Commerce Sales, 1st Quarter 2017, juillet 2017. Davantage de données via StatLink. Voir notes de chapitre.

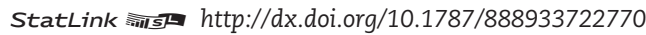

\section{Mesurabilité}

La mesure du commerce électronique présente des difficultés méthodologiques susceptibles d'avoir des incidences sur la comparabilité des estimations : adoption de pratiques diverses pour la collecte des données et l'établissement des estimations, traitement des cas déviants et des activités de commerce électronique des multinationales, imputation des valeurs à partir des fourchettes utilisées dans les enquêtes, ou encore hétérogénéité du champ sectoriel considéré. Dans le cas des enquêtes réalisées du côté de la demande, les consommateurs ne sont pas toujours en mesure de répondre à certains types de questions, notamment sur la provenance des produits ou services achetés. Sans compter qu'une part non négligeable d'internautes ignore le pays d'origine des sites web sur lesquels ils réalisent leurs achats, ou ne se souviennent pas nécessairement du montant de leurs dépenses. En outre, certaines transactions entre entreprises et consommateurs portent sur l'achat de produits numériques, dont une part de plus en plus importante est téléchargée ou consultée en streaming sur l'internet ; il est alors particulièrement difficile pour les consommateurs d'en identifier le pays d'origine.

Les données relatives aux transactions réalisées dans le cadre du commerce électronique transfrontière sont collectées par le biais des enquêtes sur l'utilisation des TIC ; elles couvrent à la fois les entreprises et les particuliers pour les pays appliquant le Système statistique européen, mais uniquement les particuliers pour le Canada. Au vu des incidences croissantes de l'économie numérique sur la société, d'autres pays ont l'intention de collecter des données comparables. 
Individus utilisant l'internet pour communiquer avec les pouvoirs publics, par âge, 2016

En pourcentage de la population dans chaque groupe d'âges

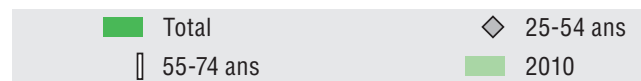

DNK

ISL

NOR

FIN

SWE

EST

LUX

NLD

CHE

LVA

FRA

AUT

CAN

BEL
DEU

OCDE

GBR

IRL

NZ

ESP

AUS

GRC

HUN

SVK

SVN

PR

TUR
CZE

ISR

POL

ITA

BRA

CHL

MEX

JPN

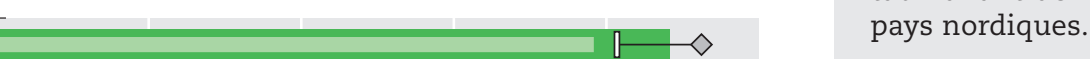

\section{Le saviez-vous?}

Plus de $50 \%$ des individus ont recours aux services de l'administration électronique dans les pays de l'OCDE. Toutefois, ce chiffre cache de fortes disparités, avec des taux allant de moins de $10 \%$ au Japon à $80 \%$ dans les pays nordiques.

La part des individus utilisant l'internet pour interagir avec les pouvoirs publics dans les pays de l'OCDE a augmenté au cours des dernières années, passant de $46 \%$ en 2010 à $54 \%$ en 2016. Les écarts d'un pays à l'autre demeurent toutefois conséquents, puisque les taux oscillent entre $5 \%$ au Japon et plus de $80 \%$ au Danemark, en Islande, en Norvège et en Finlande. Dans tous les pays, le groupe des 55-74 ans affiche des chiffres très en-deçà de la moyenne.

Les écarts observés entre les pays peuvent être liés à la diversité des taux d'utilisation de l'internet, à l'offre de services de l'administration électronique, à la propension des individus à effectuer les formalités administratives en ligne, ou encore à la faible comparabilité des données. En moyenne, en 2016, moins de $4 \%$ des citoyens de l'UE amenés à transmettre à l'administration un formulaire officiel rempli par leurs soins ont déclaré ne pas l'avoir fait en ligne du fait de l'indisponibilité d'un tel service. Le taux était bien plus élevé en Allemagne (13\%).

Un autre motif est fréquemment invoqué : les inquiétudes quant à la protection et la sécurité des données à caractère personnel. En 2016, 21 \% des citoyens européens ont choisi de ne pas soumettre leurs formulaires officiels en ligne ; $22 \%$ d'entre eux en moyenne ont justifié leur décision par des craintes quant au respect de leur vie privée et à la sécurité. Des taux particulièrement élevés ont été enregistrés en Allemagne (38\%), au Portugal (34 \%) et en Hongrie (33 \%).

Les entreprises sont plus nombreuses que les particuliers à utiliser l'internet pour transmettre des documents aux pouvoirs publics, et pour cause : elles sont plus souvent amenées à effectuer des formalités administratives. Dans certains cas, la voie électronique est imposée par la loi. En 2015, 36 \% des entreprises de l'UE ont adressé une facture électronique aux pouvoirs publics ; le taux varie toutefois entre $17 \%$ en République tchèque et $70 \%$ au Danemark. Dans tous les pays de l'UE à l'exception du Danemark, les grandes entreprises sont plus nombreuses que les petites à utiliser l'internet à cette fin.

\section{Définitions}

L'utilisation de l'internet pour interagir avec les pouvoirs publics va de la simple consultation d'informations sur les sites web des administrations publiques à l'exécution de procédures interactives impliquant la soumission de formulaires officiels par l'internet, à l'exclusion des échanges par courriel (dans le cas des entreprises) ou de l'envoi de courriels saisis manuellement (dans le cas des particuliers).

Les pouvoirs publics englobent à la fois les services publics et les activités des administrations. Sont donc concernées les autorités locales, régionales ou nationales.

Les signatures électroniques et identifiants/certificats électroniques fournissent aux utilisateurs un mode d'identification sécurisé et fiable pour la transmission des formulaires au format électronique. Les classes de taille des entreprises sont les suivantes : petites entreprises (de 10 à 49 salariés) et grandes entreprises (plus de 250 salariés). 
Individus n'ayant pas soumis de formulaires officiels en ligne du fait de craintes quant à la sécurité et au respect de leur vie privée, 2016

En pourcentage des individus ayant choisi de ne pas soumettre leurs formulaires en ligne

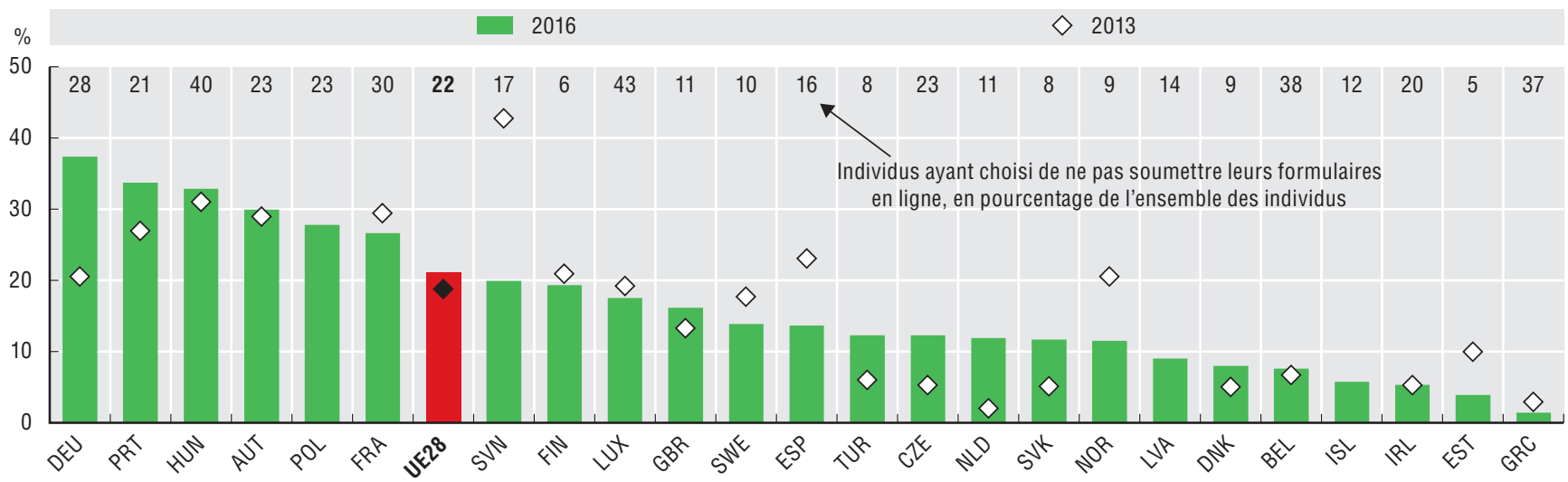

Source : OCDE, d'après Eurostat, Économie et société numériques, Base de données complète, juillet 2017. Voir notes de chapitre.

Entreprises utilisant l'internet pour transmettre des factures aux pouvoirs publics, par taille, 2015

En pourcentage des entreprises dans chaque classe de taille

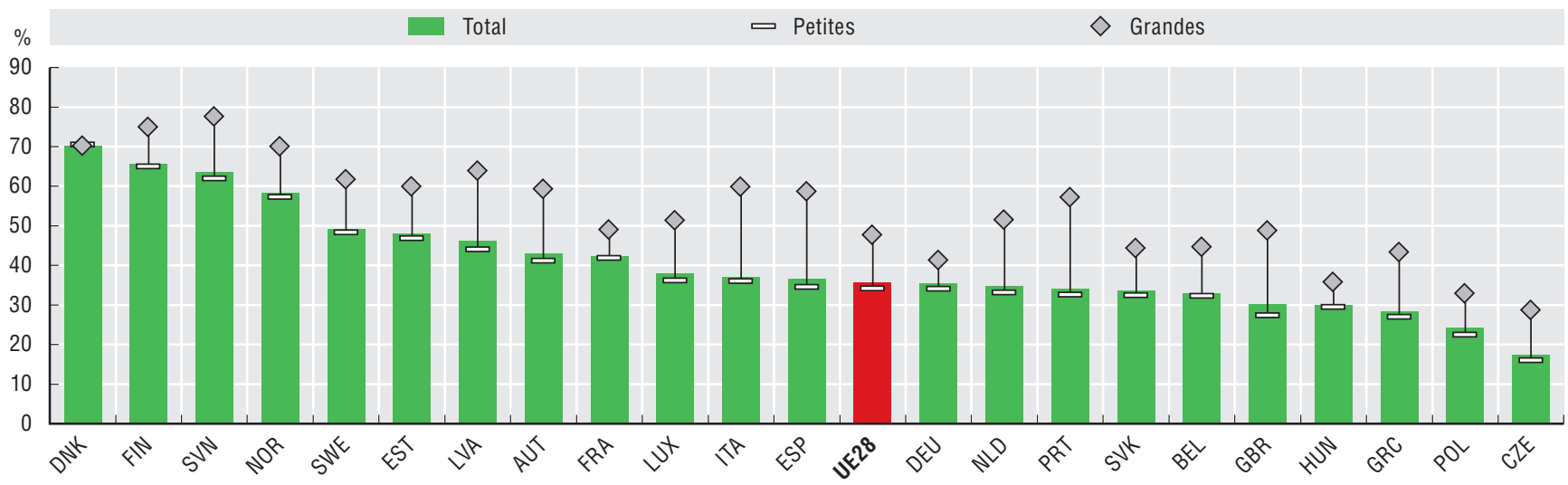

Source : OCDE, d'après Eurostat, Économie et société numériques, Base de données complète, juillet 2017. Davantage de données via StatLink. Voir notes de chapitre.

\section{Mesurabilité}

La mesure des services de l'administration électronique peut se faire à partir des informations recueillies sur les services électroniques proposés par les entités publiques (méthode axée sur l'offre) ou sur l'utilisation de ces services par les entreprises et les particuliers (méthode axée sur la demande). Compte tenu des difficultés d'ordre statistique posées par la première approche, l'OCDE et d'autres organisations internationales tendent à privilégier la deuxième, ce qui n'est pas non plus sans soulever des problèmes. De fait, certains services (par exemple, dans les domaines des transports, de l'éducation et de la santé) sont fournis, selon les pays, tantôt exclusivement par l'État, tantôt par des entreprises publiques ou privées ; par conséquent, le champ d'étude de l'utilisation des services de l'administration électronique par les particuliers et les entreprises diffère. Ces disparités structurelles sont susceptibles d'avoir des incidences non seulement sur les comparaisons internationales, mais aussi sur les comparaisons chronologiques pour un pays donné.

L'OCDE s'emploie activement à recueillir des informations comparables et plus détaillées dans ce domaine, grâce à ses enquêtes types sur l'utilisation des TIC par les ménages et les individus (OCDE, 2015b), et par les entreprises (OCDE, 2015c). D'autres modes de collecte complémentaires sont à l'étude, notamment via l'exploitation des informations disponibles sur les portails web des administrations publiques. 
Entreprises disposant d'une stratégie de sécurité formelle, par classe de taille, 2015

En pourcentage du nombre total d'entreprises dans chaque classe de taille

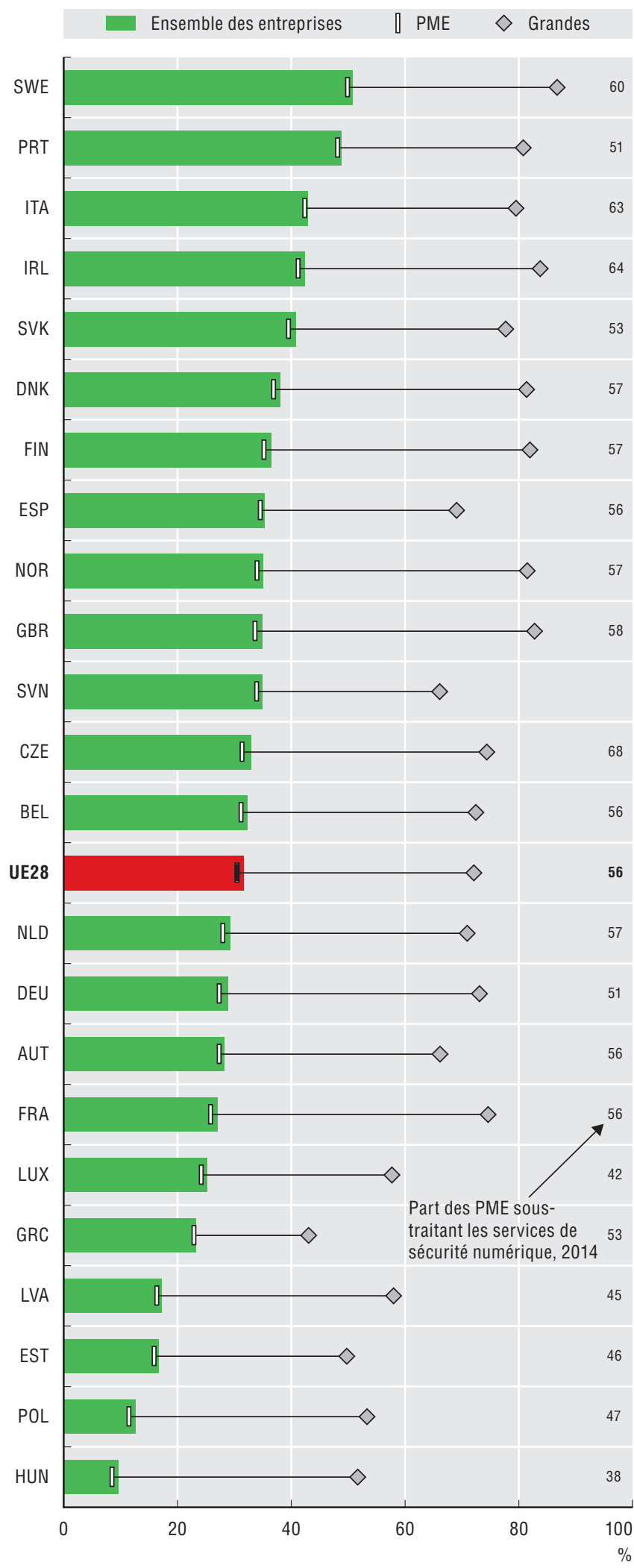

Source : OCDE, d'après Eurostat, Économie et société numériques, Base de données complète, juillet 2017. Voir notes de chapitre.

StatLink anist http://dx.doi.org/10.1787/888933722846

\section{Le saviez-vous ?}

En 2015, en Europe, 70 \% des grandes entreprises disposaient d'une stratégie formelle de sécurité numérique, contre seulement 30 \% des PME.

La progression du numérique et de la connectivité créent de nouveaux défis en termes de protection des données sensibles et des communications réseau. L'adoption d'une stratégie formelle de sécurité informatique est un signe que l'entreprise a été exposée à des risques de sécurité numérique ou y est sensibilisée.

En 2015, environ 32 \% des entreprises européennes s'étaient dotées d'une stratégie formelle de sécurité numérique. Toutefois, ce taux masque d'importantes disparités selon les pays et la taille des entreprises. Si $30 \%$ des PME européennes disposaient d'une telle stratégie en 2015, elles n'étaient que $23 \%$ aux ÉtatsUnis (National Cyber Security Alliance et Symantec, 2012).

Par ailleurs, les PME tendent à faire davantage appel à des services extérieurs pour assurer leur sécurité numérique et la protection des données, du fait vraisemblablement d'un accès limité aux ressources financières et aux compétences spécialisées. En 2015, les fonctions de la sécurité numérique et de la protection des données étaient assurées en interne dans plus de $64 \%$ des grandes entreprises, contre $14 \%$ des PME.

En 2016, en Europe, plus de 70 \% des internautes ont transmis des informations à caractère personnel par voie électronique ; la plupart avaient pris des mesures pour contrôler l'accès à ces données sur l'internet. Toujours en Europe, $46 \%$ de la population totale d'internautes ont refusé que leurs données personnelles soient utilisées à des fins publicitaires, et $40 \%$ ont limité l'accès à leur profil ou aux contenus qu'ils ont publiés sur les réseaux sociaux. Plus d'un tiers des internautes ont pris connaissance des déclarations de confidentialité avant de fournir des informations à caractère personnel et ont restreint l'accès aux données afférentes à leur localisation géographique. Les jeunes affichent une plus forte propension pour partager les informations à caractère personnel qui les concernent, mais ils prennent aussi plus souvent des mesures pour y contrôler l'accès. Dans plus des deux tiers des pays ayant participé à l'enquête, les hommes semblent être plus enclins que les femmes à partager des informations privées en ligne.

\section{Définitions}

Les stratégies de sécurité informatique couvrent les mesures, contrôles et procédures appliqués aux systèmes TIC pour garantir l'intégrité, l'authenticité, la disponibilité et la confidentialité des données et des systèmes. Elles sont notamment destinées à contrer les risques de sécurité suivants : destruction ou corruption des données suite à des pannes matérielles ou logicielles ; indisponibilité des services TIC due à des attaques extérieures ; et divulgation de données confidentielles suite à des attaques de type intrusion, dévoiement ou hameçonnage.

Les classes de taille des entreprises sont les suivantes : PME (de 10 à 249 salariés) et grandes entreprises (plus de 250 salariés). Les informations à caractère personnel désignent les informations que les utilisateurs considèrent comme revêtant un caractère privé et ne seraient pas nécessairement disposés à divulguer publiquement, à l'instar des coordonnées personnelles et bancaires, ou d'autres informations de même ordre. 
Individus ayant fourni des informations à caractère personnel via l'internet au cours des 12 derniers mois, par sexe et par groupe d'âges, 2016

En pourcentage de la population d'internautes dans chaque groupe d'âges

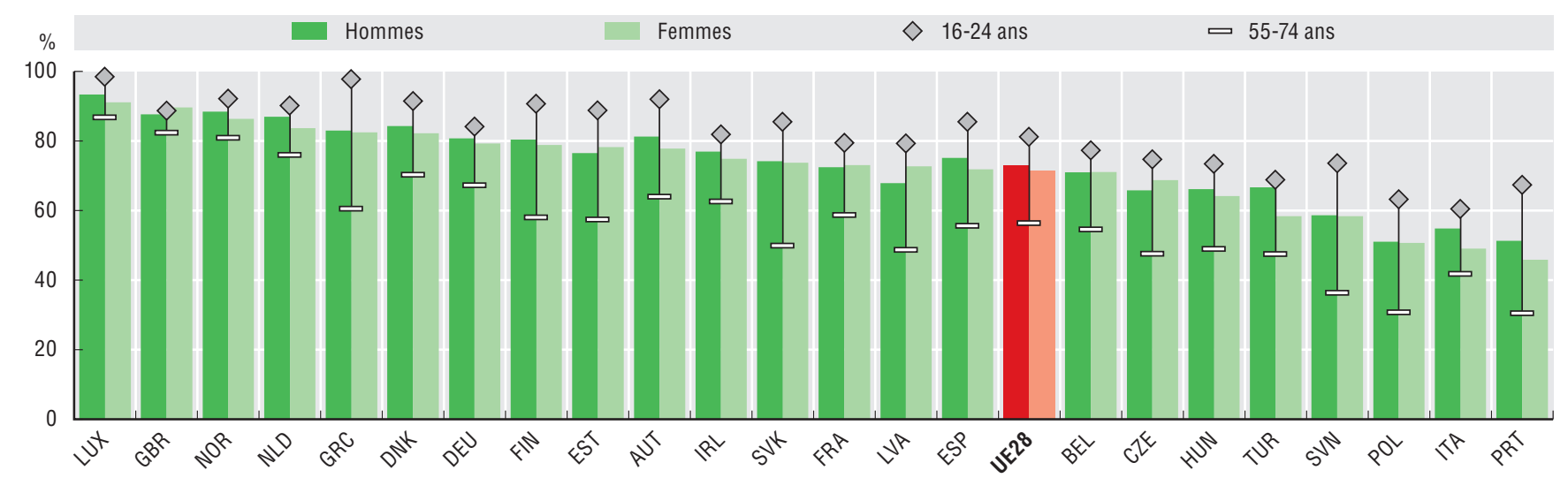

Source : OCDE, d'après Eurostat, Économie et société numériques, Base de données complète, juillet 2017. Davantage de données via StatLink.

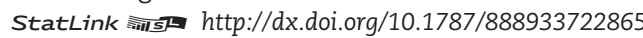

Individus gérant l'accès à leurs informations à caractère personnel sur l'internet, par groupe d'âges, 2016

En pourcentage de la population d'internautes dans chaque groupe d'âges

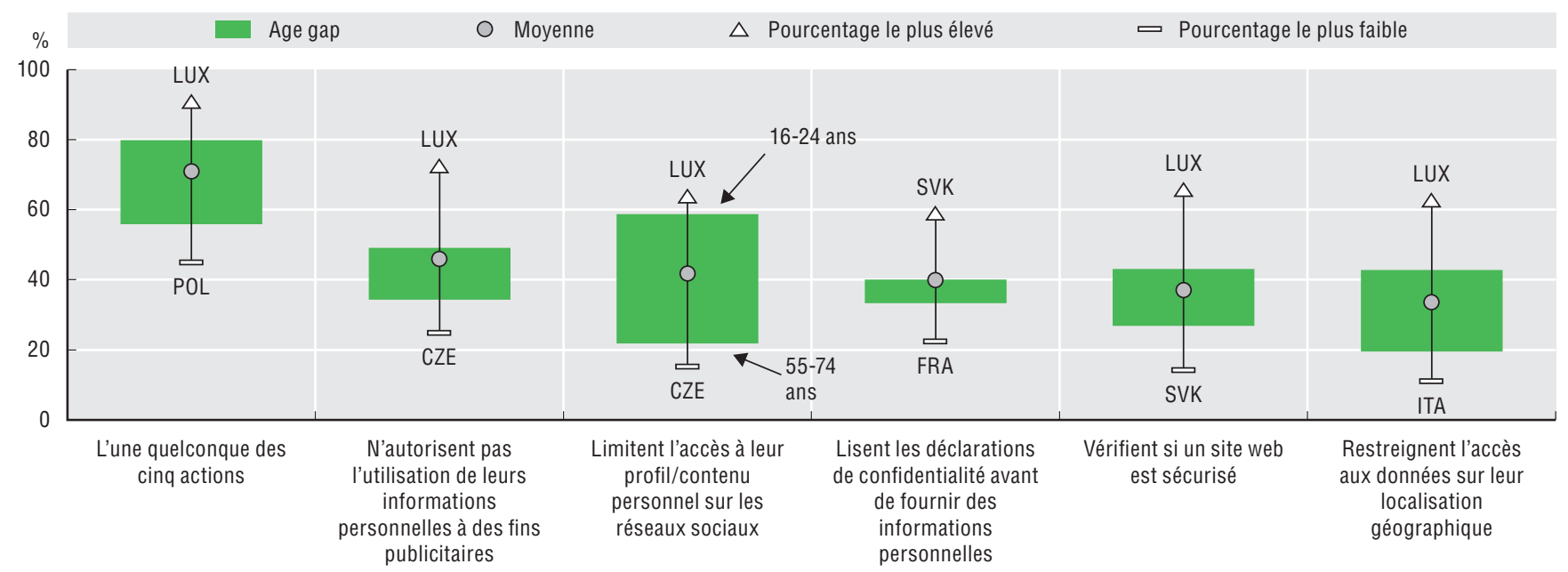

Source : OCDE, d'après Eurostat, Économie et société numériques, Base de données complète, juillet 2017. Davantage de données via StatLink.

\section{Mesurabilité}

Les données relatives aux stratégies de sécurité informatique sont issues de l'enquête d'Eurostat sur l'utilisation des TIC et du commerce électronique dans les entreprises. Celles afférentes à la divulgation et la protection des informations à caractère personnel sont tirées de l'enquête sur l'utilisation des TIC par les ménages et les particuliers.

Les enquêtes types d'Eurostat et de l'OCDE sur l'utilisation des TIC intègrent des questions directes sur la sécurité et la protection de la vie privée, y compris sur le recours à des solutions de protection contre les menaces informatiques, la fréquence des bulletins de sécurité et les incidents de sécurité.

La révision 2014 de l'enquête type de l'OCDE sur l'accès aux TIC et leur utilisation par les ménages et les individus comprenait un module spécifique sur la sécurité et la protection de la vie privée, élaboré conformément aux priorités de l'action publique telles que formulées par le Groupe de travail de l'OCDE sur la sécurité et la vie privée dans l'économie numérique.

La capacité des sondés à répondre à des questions techniques sur la sécurité informatique fait débat parmi les statisticiens. Pour minimiser le problème, les questions du module sur la sécurité de l'enquête de l'OCDE portent uniquement sur l'utilisation à domicile; de fait, il s'agit là de l'environnement TIC sur lequel les utilisateurs sont davantage susceptibles de disposer d'informations, contrairement aux environnements utilisés dans le cadre professionnel ou scolaire. 


\section{Notes et Références}

\section{Chypre}

La note suivante est ajoutée à la demande de la Turquie :

"Les informations figurant dans ce document qui font référence à “Chypre” concernent la partie méridionale de lîle. Il n’y a pas d'autorité unique représentant à la fois les Chypriotes turcs et grecs sur l'île. La Turquie reconnaît la République turque de Chypre Nord (RTCN). Jusqu'à ce qu'une solution durable et équitable soit trouvée dans le cadre des Nations Unies, la Turquie maintiendra sa position sur la "question chypriote". "

La note suivante est ajoutée à la demande de tous les États de l'Union européenne membres de l'OCDE et de l'Union européenne :

"La République de Chypre est reconnue par tous les membres des Nations Unies sauf la Turquie. Les informations figurant dans ce document concernent la zone sous le contrôle effectif du gouvernement de la République de Chypre. »

\section{Israël}

"Les données statistiques concernant Israël sont fournies par et sous la responsabilité des autorités israéliennes ou d'un tiers compétents. L'utilisation de ces données par l'OCDE est sans préjudice du statut des hauteurs du Golan, de JérusalemEst et des colonies de peuplement israéliennes en Cisjordanie aux termes du droit international.

Il est à noter que les données statistiques sur les brevets et marques israéliens sont fournies par les offices des brevets et des marques des pays concernés. "

\subsection{Connectivité}

\section{Pénétration du haut débit mobile par technologie, décembre 2016}

Pour l'Afrique du Sud, l'Arabie saoudite, le Brésil, la Chine, la Fédération de Russie, l'Inde et l'Indonésie, les données sont issues de la base des Indicateurs des télécommunications/TIC dans le monde de l'UIT, consultée en juillet 2017.

Pour les États-Unis et la Suisse, les données pour décembre 2016 sont des estimations.

Pour Israël, les données proviennent de GSMA Intelligence.

\section{Ménages équipés d'une connexion haut débit, régions urbaines et rurales, 2010 et 2016}

Pour le Brésil et les États-Unis, les données se rapportent à 2015 au lieu de 2016.

Pour le Chili, elles concernent 2012 et 2015.

Pour l'Islande, elles se rapportent à 2010 et 2014.

Pour le Royaume-Uni, elles se rapportent à 2009 au lieu de 2010.

Pour la Suisse, elles correspondent à 2012 et 2014.

Pour le Brésil, la définition des zones urbaines et rurales se fonde sur la législation locale ; les données sont compilées par l'office statistique national (OSN). Les données communiquées correspondent aux zones urbaines (à forte densité de population) et aux zones rurales (à faible densité).

Concernant le Chili, pour l'année 2012, les données afférentes aux grandes zones urbaines correspondent à un ensemble contigu de localités présentant chacune une densité supérieure à 500 habitants par kilomètre carré, et dont la population totale est d'au moins 50000 habitants. Les zones rurales correspondent quant à elles à un ensemble contigu de localités n'appartenant ni à une zone à forte densité de population, ni à une zone intermédiaire. Une zone intermédiaire est un ensemble contigu de localités ne correspondant pas à une zone densément peuplée, dont chacune présente une densité supérieure à 100 habitants par kilomètre carré, et dont la population totale est d'au moins 50000 habitants, ou qui jouxte une zone à forte densité de population.

Pour les États-Unis, les catégories de densité de population sont estimées à partir de la localisation des ménages dans une ville principale, des données relatives à une zone statistique métropolitaine (MSA), ou d'aucun des deux critères. Pour assurer la confidentialité des données des répondants, les informations s'appuient sur certaines observations issues des ensembles de données en libre accès.

Pour la France, la Lettonie, les Pays-Bas et la Suède, il y a, en 2016, une rupture de série par rapport aux années précédentes pour les données afférentes aux zones rurales et urbaines.

\section{Petites et moyennes entreprises dotées d'un accès haut débit, fixe ou mobile, 2016}

Seules les entreprises de dix salariés ou plus sont prises en considération. Sauf indication contraire, les classes de taille sont les suivantes : petites entreprises (de 10 à 49 salariés) et entreprises moyennes (de 50 à 249 salariés).

Pour l'Australie, les données se rapportent à l'exercice 2014/15 clos au 30 juin.

Pour le Brésil, la définition du haut débit se fonde sur le type de connexion et non sur le débit de téléchargement ; les valeurs possibles sont : DSL, modem câble, fibre, radio, satellite et 3G/4G. Les données se rapportent à 2015.

Pour le Canada, les données couvrent tous les types de connexion à l'exception des connexions par ligne commutée. Les réponses indiquant que les utilisateurs ignorent le type de connexion employé ont été déduites du numérateur comme du dénominateur. Les données se rapportent à 2013, et les entreprises de taille moyenne comptent entre 50 et 299 salariés. 
Pour la Corée, les données se rapportent à 2015.

Pour le Japon, les données se rapportent à 2015 et concernent les entreprises de 100 salariés ou plus ; les entreprises de taille moyenne comptent entre 100 et 299 salariés. Les données incluent les lignes louées et le haut débit mobile.

Pour le Mexique, elles concernent 2012.

Pour la Nouvelle-Zélande, les données portent sur l'exercice 2015/16, clos au 30 juin.

Pour la Suisse, les données se rapportent à 2015, et les petites entreprises comptent entre 5 et 49 salariés, au lieu de 10 à 49 .

\subsection{Internautes}

\section{Population totale d'individus utilisant l'internet mobile quotidiennement, 2016}

Sauf indication contraire, la période de référence prise en compte pour recenser les internautes est de 3 mois. Pour l'Australie, le Canada et le Japon, elle est de 12 mois. Pour les États-Unis, elle est de 6 mois pour 2015 ; en revanche, aucune période de référence n'est précisée pour 2006. Pour la Corée et la Nouvelle-Zélande, elle est de 12 mois pour 2006. Pour l'Afrique du Sud, le Chili en 2009 , la Chine, la Fédération de Russie, l’Inde et l'Indonésie, aucune période de référence n'est spécifiée.

Pour l'Australie, les données se rapportent aux exercices 2006/07 et 2014/15 clos au 30 juin.

Pour le Brésil, les données correspondent à 2007 et 2015.

Pour le Canada, elles se rapportent à 2007 et 2012. Pour 2007, elles concernent les individus âgés de 16 ans ou plus, au lieu de 16 à 74 ans.

Pour la Corée, elles se rapportent à 2015 au lieu de 2016.

Pour l'Islande, les données se rapportent à 2014 au lieu de 2016.

Pour Israël, elles concernent 2015 au lieu de 2016, et couvrent les individus âgés de 20 ans ou plus, au lieu de 16 à 74 ans.

Pour le Japon, les données se rapportent à 2015 au lieu de 2016, et portent sur les individus âgés de 15 à 69 ans.

Pour la Suisse, elles correspondent à 2014 au lieu de 2016.

Notes concernant l'ensemble des internautes :

Pour l'Afrique du Sud, la Chine, la Fédération de Russie, l'Inde et l'Indonésie, les données proviennent de la base des Indicateurs des télécommunications/TIC dans le monde de l'UIT et se rapportent à 2015 au lieu de 2016.

Pour le Chili, les données concernent 2009 et 2015.

Pour les États-Unis, elles se rapportent à 2007 et 2015.

Pour l'Indonésie, les données concernent les individus âgés de 5 ans ou plus.

Pour la Nouvelle-Zélande, les données se rapportent à 2012 au lieu de 2016.

Pour la Turquie, elles concernent 2007 au lieu de 2006.

Notes concernant les utilisateurs quotidiens :

Les données relatives à la Fédération de Russie sont issues de la base des Indicateurs des télécommunications/TIC dans le monde de l'UIT et se rapportent à 2014 au lieu de 2016.

Notes concernant les individus utilisant l'internet mobile :

Pour les États-Unis, les données proviennent la NTIA et se rapportent à 2015. Elles correspondent au rapport entre, d'une part, les individus âgés de 15 ans ou plus qui accèdent à l'internet lors de leurs déplacements, et, d'autre part, le nombre total d'individus âgés de 15 ans ou plus qui se connectent à l'internet, indépendamment du lieu.

Pour Israël, les données concernent les individus accédant à l'internet via un téléphone mobile, où qu'ils soient.

Pour la Nouvelle-Zélande, les données proviennent de Statistics New Zealand. Elles se rapportent à 2012 et couvrent les individus âgés de 15 à 74 ans utilisant une connexion cellulaire, sans fil, ou les deux.

Pour la Suisse, les données concernent les internautes utilisant un appareil mobile à des fins personnelles pour accéder à l'internet en dehors de leur domicile ou de leur lieu de travail.

\section{Écarts d'adoption de l'internet selon le niveau d'instruction, 2016}

Sauf indication contraire, la période de référence prise en compte pour recenser les internautes est de 3 mois. Pour l'Australie, elle est de 12 mois. Pour la Fédération de Russie, aucune période de référence n'est spécifiée. Pour les États-Unis, elle est de 6 mois pour 2015.

Pour l'Australie, les données se rapportent à l'exercice 2014/15 clos au 30 juin.

Pour le Brésil, le Chili, la Corée, les États-Unis et Israël, les données se rapportent à 2015.

Pour l'Islande et la Suisse, les données se rapportent à 2014.

Pour Israël, les données concernent les individus âgés de 20 ans ou plus, et non de 16 à 74 ans.

Pour la Nouvelle-Zélande, les données se rapportent à 2012. 
Pour la Fédération de Russie, les données sont tirées de la base des Indicateurs des télécommunications/TIC dans le monde de l'UIT. Elles se rapportent à 2015 pour tous les internautes, et à 2014 pour la répartition en fonction du niveau d'instruction.

\section{Utilisatrices de l'internet, par âge, 2016}

Sauf indication contraire, la période de référence prise en compte pour recenser les internautes est de 3 mois. Pour le Canada et le Japon, elle est de 12 mois, et pour les États-Unis, de 6 mois.

Pour l'Australie, les données se rapportent à l'exercice 2014/15 clos au 30 juin.

Pour le Brésil, le Chili, la Corée, les États-Unis et Israël, les données se rapportent à 2015.

Pour le Canada et la Nouvelle-Zélande, les données se rapportent à 2012.

Pour l'Islande et la Suisse, elles se rapportent à 2014.

Pour Israël, les données concernent les femmes âgées de 20 ans ou plus, au lieu de 16 à 74 ans, et les femmes de 20 à 24 ans au lieu de 16 à 24 ans.

Pour le Japon, les données concernent les femmes âgées de 15 à 69 ans, au lieu de 16 à 74 ans, et les femmes de 15 à 29 ans, au lieu de 16 à 24 ans.

\subsection{Niveau de cyberactivité}

\section{Diffusion d'une sélection d'activités en ligne parmi les internautes des pays de l'OCDE, par groupe d'âges et niveau d'instruction, 2016}

Pour une activité donnée :

(i) Les données sont calculées en s'appuyant sur le même groupe de pays de l'OCDE pour les deux tranches d'âges.

(ii) Pour les deux tranches d'âges, les données correspondent à la moyenne de tous les individus (" Moyenne "), à la moyenne de tous les individus présentant un niveau d'instruction faible, et à la moyenne de tous les individus diplômés de l'enseignement supérieur.

Pour toutes les activités, la moyenne de tous les individus couvre un certain nombre de pays de l'OCDE (entre 20 et 24), selon les données disponibles pour les deux tranches d'âges. Par conséquent, la moyenne de l'OCDE pour une activité donnée figurant dans ce graphique peut différer des valeurs affichées dans d'autres graphiques.

Les diplômés de l'enseignement supérieur désignent les personnes titulaires d'un diplôme de niveau 5, 6 ou supérieur de la CITE. Les personnes présentant un niveau d'instruction faible correspondent aux niveaux 0 à 2 de la CITE.

\section{Individus ayant effectué des achats en ligne au cours des 12 derniers mois, par âge, 2016}

Sauf indication contraire, la période de référence prise en compte pour recenser les internautes est de 3 mois. Pour l'Australie, le Canada et le Japon, elle est de 12 mois. Pour le Chili et la Fédération de Russie, aucune période de référence n'est spécifiée. Pour les États-Unis, elle est de 6 mois.

Pour l'Australie, les données se rapportent à l'exercice 2014/15 clos au 30 juin. Les informations fournies reflètent les réponses à une question dont la formulation diffère légèrement de celle utilisée dans les autres pays, à savoir : «Au cours des trois derniers mois, avez-vous accédé à titre personnel à l'internet pour l'une des raisons suivantes : Achat de biens ou de services ? ”.

Pour le Brésil, les données se rapportent à 2015.

Pour le Canada, les données se rapportent à 2012.

Pour le Chili, les données se rapportent à 2015.

Pour la Corée, les données se rapportent à 2015.

Pour les États-Unis, les données se rapportent à 2015. L'écart entre les groupes d'âges, représenté en bleu clair, est inversé. Les individus âgés de 55 à 74 ans affichent en effet une propension à effectuer des achats en ligne légèrement supérieure à celle de la tranche d'âges 16-24 ans.

Pour l'Islande, les données se rapportent à 2014.

Pour Israël, les données se rapportent à 2015 et concernent les individus âgés de 20 ans ou plus, au lieu de 16 à 74 ans, et de 20 à 24 ans, au lieu de 16 à 24 ans. Les données couvrent les individus ayant utilisé l'internet pour acheter des biens ou des services, quel qu'en soit le type, au cours des trois derniers mois.

Pour le Japon, les données se rapportent à 2015 et concernent les individus âgés de 15 à 69 ans.

Pour la Nouvelle-Zélande, les données se rapportent à 2012.

Pour la Suisse, les données se rapportent à 2014.

Pour la Fédération de Russie, les données proviennent de la base des Indicateurs des télécommunications/TIC dans le monde de l'UIT, se rapportent à 2014 et concernent les individus âgés de 15 à 72 ans. 
Individus âgés de 16 à 24 ans ayant suivi un cours en ligne, 2009 et 2016

Pour l'Autriche, les données se rapportent à 2011 au lieu de 2009.

Pour le Brésil et le Danemark, les données se rapportent à 2015 au lieu de 2016.

Pour le Canada, les données se rapportent à 2010 et 2012.

Pour le Chili, les données se rapportent à 2012 et 2015.

Pour la Corée, les données se rapportent à 2015.

Pour les États-Unis, les données se rapportent à 2015, avec une période de référence de 6 mois.

Pour l'Islande, les données se rapportent à 2013 au lieu de 2016.

Pour le Mexique, les données se rapportent à 2014 au lieu de 2016. Les données pour 2009 incluent la catégorie « Soutien aux efforts en faveur de l'éducation et de l'apprentissage »; celles pour 2014 sont intégrées à la catégorie « Soutien à l'éducation/la formation ".

\subsection{Cyberconsommateurs sans frontières}

\section{Entreprises ayant pratiqué la vente par commerce électronique à l'étranger, 2014}

Les ventes par commerce électronique désignent les ventes sur l'internet (commandes reçues via les sites web).

Pour l'Islande, les données se rapportent à 2012.

\section{Individus ayant effectué des achats en ligne auprès de vendeurs nationaux et étrangers, 2016}

On entend par pays partenaires les autres membres de l'UE dans le cas des pays appartenant au Système statistique européen, et les États-Unis dans le cas du Canada.

Pour le Canada, les données se rapportent à 2012.

\section{Transactions entre entreprises et consommateurs (B2C), 2009 et 2015}

Pour les États-Unis, les données proviennent du US Census Bureau (Quarterly Retail E-commerce sales, 1st Quarter 2017, https:// www.census.gou/retail/mrts/www/data/pdf/ec_current.pdf). Les ratios ont été calculés à partir des valeurs trimestrielles des années respectives - valeurs ajustées - (tableau 1).

Pour l'Islande, les données se rapportent à 2011 au lieu de 2015.

Pour la Lettonie, les données concernent 2013 au lieu de 2015.

Pour le Portugal, les données se rapportent à 2014 au lieu de 2015.

\subsection{Administration électronique}

\section{Individus utilisant l'internet pour communiquer avec les pouvoirs publics, par âge, 2016}

Sauf indication contraire, les données se rapportent aux activités en ligne respectives, au cours des 12 derniers mois.

Pour l'Australie, les données se rapportent aux exercices 2010/11 et 2012/13 clos au 30 juin. Elles couvrent les « individus ayant utilisé l'internet pour télécharger des formulaires officiels à partir des sites web des organisations gouvernementales, au cours des 12 derniers mois " et les « individus ayant utilisé l'internet pour compléter/soumettre des formulaires mis à disposition sur les sites web des organisations gouvernementales, au cours des 12 derniers mois ».

Pour le Brésil et le Chili, les données se rapportent à 2015.

Pour le Canada, les données se rapportent à 2012.

Pour l'Islande et la Suisse, les données se rapportent à 2014.

Pour Israël, les données se rapportent à 2015 et concernent les individus âgés de 20 ans ou plus, et non de 16 à 74 ans. Elles couvrent l'utilisation de l'internet, au cours des 3 derniers mois, pour bénéficier des services en ligne des organismes gouvernementaux, dans le but notamment de télécharger ou de remplir des formulaires officiels.

Pour le Japon, les données se rapportent à 2015 et aux individus âgés de 15 à 69 ans, et non de 16 à 74 ans, utilisant l'internet pour envoyer des formulaires remplis par leurs soins via le site web des autorités publiques, au cours des 12 derniers mois.

Pour le Mexique, l'utilisation des services de l'administration électronique couvre les catégories suivantes : communiquer avec les pouvoirs publics, consulter des informations mises à disposition par l'administration, télécharger des formulaires officiels, remplir ou soumettre des formulaires officiels, effectuer des démarches administratives, et participer à des consultations publiques. Pour l'envoi de formulaires, les données correspondent à l'utilisation de l'internet au cours des 3 derniers mois.

Pour la Nouvelle-Zélande, les données se rapportent à 2012 et concernent les individus ayant utilisé l'internet pour obtenir des informations auprès des pouvoirs publics au cours des 12 derniers mois.

Pour la Suisse, seules sont prises en compte les interactions avec les administrations publiques locales, régionales ou nationales, regroupées sous l'appellation « administration ou autorités publiques ». En sont exclus les établissements de santé ou d'enseignement. 
Individus n'ayant pas soumis de formulaires officiels en ligne du fait de craintes quant à la sécurité et au respect de leur vie privée, 2016

Pour l'Islande, les données se rapportent à 2014.

Pour le Royaume-Uni, elles concernent 2014 au lieu de 2013.

\subsection{Confiance}

\section{Entreprises disposant d'une stratégie de sécurité formelle par taille, 2015}

Les données relatives aux PME qui sous-traitent les services de sécurité numérique correspondent à la part des PME disposant d'une stratégie de sécurité TIC formelle et confiant à des prestataires extérieurs l'essentiel de la gestion de la sécurité et de la protection des données.

\section{Références}

Brezzi, M., L. Dijkstra et V. Ruiz (2011), « OECD extended regional typology: The economic performance of remote rural regions », OECD Regional Development Working Papers, n²011/06, Éditions OCDE, Paris, http://dx.doi.org/10.1787/5kg6z83tw7f4-en.

Kaiser, M. (2011), Prepared testimony of the National Cyber Security Alliance on the State of Cybersecurity and Small Business before the Committee on House Small Business Subcommittee on Healthcare and Technology, United States House of Representatives, $1^{\mathrm{er}}$ décembre, http://smallbusiness.house.gov/uploadedfiles/kaiser_testimony.pdf.

OCDE (2011), OECD Guide to Measuring the Information Society 2011, Éditions OCDE, Paris, http://dx.doi.org/10.1787/9789264113541-en.

OCDE (2015a), Perspectives de l'économie numérique de l'OCDE 2015, Éditions OCDE, Paris, http://dx.doi.org/10.1787/9789264243767-fr.

OCDE (2015b), The OECD Model Survey on ICT Access and Usage by Households and Individuals, 2nd Revision, Groupe de travail sur la mesure et l'analyse de l'économie numérique, OCDE, Paris, https://www.oecd.org/sti/ieconomy/ICT-Model-Survey-Access-Usage-HouseholdsIndividuals.pdf.

OCDE (2015c), The OECD Model Survey on ICT Access and Usage by Businesses, 2 ème révision, Groupe de travail sur la mesure et l'analyse de l'économie numérique, OCDE, Paris, https://www.oecd.org/sti/ieconomy/ICT-Model-Survey-Usage-Businesses.pdf. 
Science, technologie et industrie : Tableau de bord de l'OCDE 2017

La transformation numérique

(c) OECD 2018

\section{Sources des données}

Pour en savoir plus sur les méthodes employées à l'OCDE pour mesurer la science, la technologie et l'innovation, consulter http://oe.cd/sti-stats.

\section{Sources des données de l'OCDE}

OCDE, Base de données l'accès et utilisation des TIC par les entreprises, http://oe.cd/bus

OCDE, Base de données l'accès et utilisation des TIC par les ménages et les individus, http://oe.cd/hhind

OCDE, Base de données ANBERD, http://oe.cd/anberd-fr

OCDE, Base de données Comptes nationaux annuels, http://www.oecd.org/fr/sdd/cn/

OCDE, Base de données du Programme pour l'évaluation internationale des compétences des adultes (PIAAC), http://www.oecd.org/fr/competences/piaac/donneespubliquesetanalyses.htm

OCDE, Base de données PISA 2015, http://www.oecd.org/pisa/data/2015database

OCDE, Base de données pour l'Analyse structurelle (STAN), http://oe.cd/stan-fr

OCDE, Base de données Principaux indicateurs de la science et de la technologie, http://oe.cd/pist-fr

OCDE, Base de données Statistiques de la recherche et développement, http://oe.cd/srd-fr

OCDE, STI Micro-data Lab : Base de données sur la propriété intellectuelle, http://oe.cd/ipstats

OCDE, Base de données sur le Contenu en emploi des échanges (TiM), http://oe.cd/io-emp-fr

OCDE, Base de données sur le financement de l'entrepreneuriat

OCDE, Base de données sur les activités des entreprises multinationales, http://oe.cd/amne

OCDE, Base de données sur les échanges en valeur ajoutée (TiVA), http://oe.cd/tiva-fr

OCDE, Bases de données DynEmp v.2 et v.3, données préliminaires, http://oe.cd/dynemp

OCDE, Statistiques et bases de données sur l'éducation, http://www.oecd.org/fr/education/ base-de-donnees.htm

OCDE, Carrières des titulaires de doctorat, 2017, http://oe.cd/cdh-fr

OCDE, Commerce bilatéral des biens par industrie et catégorie d'utilisation finale (BTDIxE), http://oe.cd/btd

OCDE, Enquête internationale auprès des auteurs scientifiques, http://oe.cd/issa

OCDE, Enquête sur les statistiques nationales de l'innovation, http://oe.cd/inno-stats

OCDE, Indicateurs des incitations fiscales en faveur de la R-D, http://oe.cd/rdtax

OCDE, Portail sur le haut débit, http://oe.cd/haut-debit

OCDE, Projet microBeRD, http://oe.cd/microberd

OCDE, Scopus Custom Data, Elsevier, version 4.2017, http://oe.cd/scientometrics 
OCDE, Base de données de l'OCDE sur la productivité, http://www.oecd.org/fr/std/statsproductivite/

OCDE, Tableaux internationaux des entrées-sorties (TIES), http://oe.cd/icio-fr

\section{Autres sources de données}

Banque mondiale, Base de données World Development Indicators, https://data.worldbank.org/ data-catalog/world-development-indicators

Base de données sur l'investissement immatériel international (INTAN-Invest, Cross-Country Intangible Investment), http://www.intan-invest.net/

CCR-OCDE, Base de données COR\&DIP@ v.1., http://oe.cd/ipstats

Commission européenne, Base de données mondiales des entrées-sorties (WIOD), http://www.wiod.org/home

Comptes satellites du Bureau d'analyse économique (BEA, Bureau of Economic Analysis) des États-Unis, https://www.bea.gov/industry/satdef.htm

Eurostat, Base de données Entreprises sous contrôle étranger dans l'UE (FATS entrantes), http://ec.europa.eu/eurostat/web/structural-business-statistics/global-value-chains/foreignaffiliates

Eurostat, Base de données EU KLEMS, http://www.euklems.net/

Eurostat, Enquête communautaire sur l'innovation (CIS-2014), http://ec.europa.eu/eurostat/ web/science-technology-innovation/data/database

Eurostat, Enquêtes sur les forces de travail (EFT-UE), http://ec.europa.eu/eurostat/web/lfs/data/ database

Eurostat, Statistiques sur l'économie et la société numériques, Base de données complète, http://ec.europa.eu/eurostat/web/digital-economy-and-society/data/comprehensive-database

Eurostat, Statistiques sur la recherche et développement, http://ec.europa.eu/eurostat/web/ science-technology-innovation/data/database

GSMA Intelligence, https://www.gsmaintelligence.com

Institut de statistique de l'UNESCO, Recherche et développement expérimental (base de données complète), http://data.uis.unesco.org/Index.aspx?DataSetCode=SCN_DS

Fédération internationale de la robotique, https://ifr.org

oaDOI API, https://oaDOI.org

Smart public intangibles (SPINTAN), http://www.spintan.net

UIT, Base de données Indicateurs des télécommunications/TIC dans le monde, http://www. itu.int/en/ITU-D/Statistics/Pages/stat/default.aspx

US Census Bureau, Quarterly Retail E-Commerce Sales, 1st Quarter 2017, https://www2.census. gov/retail/releases/historical/ecomm/17q1.pdf

US Census Bureau, United States Current Population Survey (CPS), http://www.census.gov/cps

\section{STI Micro-data Lab}

Le projet d'infrastructure de données STI Micro-data Lab, exécuté par la Direction de la science, de la technologie et de l'innovation (DSTI) de l'OCDE, regroupe et rapproche de volumineux ensembles de microdonnées de nature administrative et commerciale. Ces microdonnées, qui complètent et consolident les statistiques officielles (par exemple, 
données micro-agrégées ou données d'enquêtes), présentent l'intérêt d'être détaillées et de couvrir un vaste champ chronologique et géographique.

Il s'agit notamment des données administratives concernant les actifs intellectuels (brevets, marques et dessins/modèles enregistrés) qui sont recueillies dans le cadre des travaux du groupe spécial sur les statistiques de la propriété intellectuelle, piloté par l'OCDE et composé des représentants des offices de propriété intellectuelle du monde entier. S'y ajoutent des données bibliométriques (publications scientifiques et informations des entreprises) d'origine privée.

Les séries de microdonnées ainsi constituées du STI Microdata Lab peuvent s'employer de manière indépendante - par exemple, pour élaborer des indicateurs à des fins analytiques particulières - ou combinée, pour produire des informations nouvelles concernant un large éventail de questions ou une dynamique plus complexe. En éclairant dans le détail le comportement des agents économiques et les modalités d'évolution de la science et de la technologie, ces données permettent d'apporter des réponses aux questions intéressant les pouvoirs publics, s'agissant notamment de la création et de la diffusion des nouvelles technologies, des différents modes d'innovation des entreprises, des liens entre science et industrie, des modèles de mobilité des chercheurs et du rôle des actifs intellectuels dans les performances économiques des entreprises.

Le STI Micro-data Lab est accessible aux chercheurs invités. Cet accès est gratuit sur présentation d'une demande officielle, sous réserve du respect des règles de confidentialités et du caractère mutuellement bénéfique du projet pour l'OCDE et le(s) chercheur(s) invité(s). 


\section{Liste des graphiques}

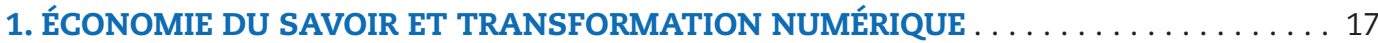

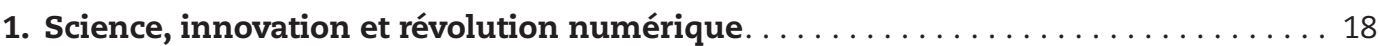

1. Pénétration du haut débit fixe et mobile dans le monde, 2010 et 2016 . . . . . . . 18

2. Pénétration du haut débit mobile, OCDE, G20 et BRIICS, $2016 \ldots \ldots \ldots \ldots \ldots \ldots$

3. Pénétration des cartes SIM M2M, OCDE, Monde et pays du G20, juin 2017 . . . . . 19

4. Pays comptant le plus de connexions par carte SIM M2M, juin 2017 . . . . . . . . 19

5. Principaux acteurs des TIC émergentes, $2012-15 \ldots \ldots \ldots \ldots \ldots \ldots \ldots \ldots \ldots$

6. Intensité et rapidité de développement des technologies liées

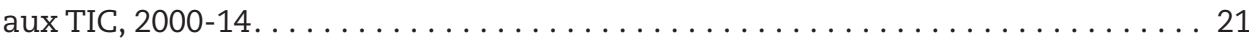

7. Brevets portant sur des technologies liées à l'intelligence

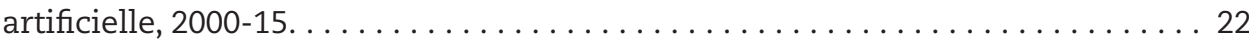

8. Brevets portant sur les principales technologies intégrant des fonctions d'intelligence artificielle, $2000-05$ et $2010-15 \ldots \ldots \ldots \ldots \ldots \ldots \ldots \ldots . \ldots \ldots$

9. Dix principales technologies médicales couplées à l'intelligence artificielle, 2000-05 et 2010-15 . . . . . . . . . . . . . . . . . . . . . . . . . . . . . . . . . . 23

10. La R-D dans la zone OCDE et les principales économies partenaires, 2015 . . . . 24

11. Économies en tête des volumes de publications scientifiques parmi

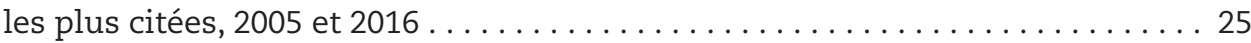

12. Évolution récente de l'excellence scientifique dans une sélection

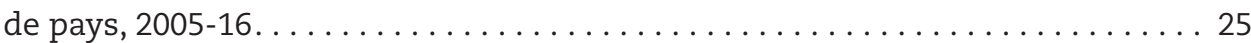

13. Dépenses de R-D par secteur d'exécution, zone OCDE, 1995-2015 . . . . . . . . . 26

14. Évolution des activités de R-D dans la zone OCDE et une sélection

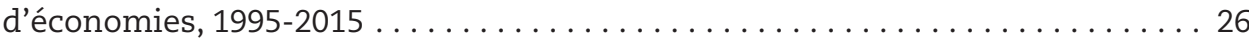

15. Évolution conjoncturelle des dépenses de R-D, par source de financement, zone OCDE, 1995-2016. . . . . . . . . . . . . . . . . . . . . . . . . . . . 27

16. Évolution de la recherche fondamentale, de la recherche appliquée et du développement expérimental dans la zone OCDE, 1985-2015 . . . . . . . . 27

17. Concentration de la R-D des entreprises : 50 et 100 principaux

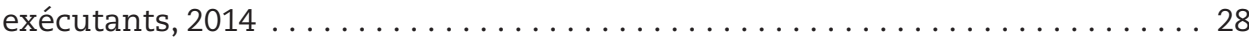

18. Activités de R-D par taille et ancienneté des entreprises, 2014 . . . . . . . . . . 29

19. Sources externes de financement de la R-D par taille et ancienneté

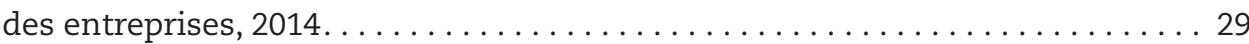

20. Dépenses de R-D et portefeuille de PI des entreprises les plus actives

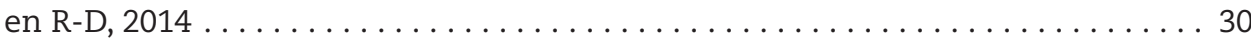

21. Portefeuille de brevets détenus par les entreprises les plus actives en R-D,

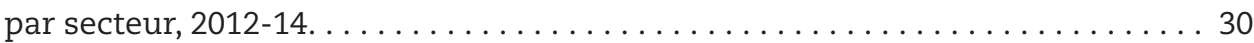

22. Principaux acteurs de la R-D détenant des actifs de propriété intellectuelle, $2012-14 \ldots$. . . . . . . . . . . . . . . . . . . . . . . . . . . . . . . . . . . 31 
23. Classement des 20 premières technologies émergentes développées par les principaux acteurs de la $\mathrm{R}-\mathrm{D}, 2012-14 \ldots \ldots \ldots \ldots \ldots \ldots \ldots \ldots \ldots \ldots$

24. Brevets liés à l'intelligence artificielle déposés par les 2000 entreprises les plus actives en $\mathrm{R}-\mathrm{D}$, par secteur, $2012-14 \ldots \ldots \ldots \ldots \ldots \ldots \ldots$

25. Brevets liés à l'intelligence artificielle déposés par les entreprises les plus actives en $\mathrm{R}-\mathrm{D}$, selon l'implantation du siège, $2012-14$. . . . . . . . 33

26. Évolution des publications scientifiques liées à l’apprentissage

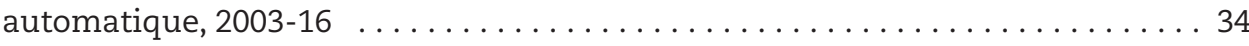

27. Articles scientifiques sur l'apprentissage automatique parmi les publications

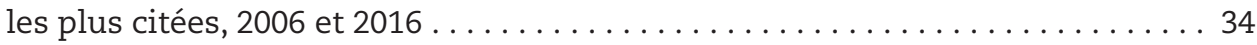

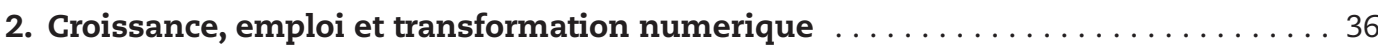

28. Économies et BRICS les plus robotisés, 2005 et 2015 . . . . . . . . . . . . . . 36

29. Intensité robotique et intensité des tâches liées aux TIC dans les emplois manufacturiers, 2012 ou 2015 . . . . . . . . . . . . . . . . . . . . . . . . 37

30. Dispersion des secteurs pour chaque dimension du numérique

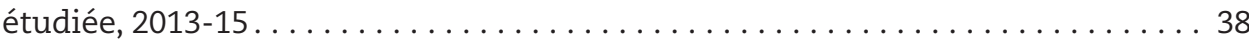

31. Taxonomie des secteurs par quartile d'intensité numérique, 2013-15 . . . . . . 39

32. Niveaux de compétences dans les secteurs à plus ou moins forte intensité numérique, 2012 ou 2015 . . . . . . . . . . . . . . . . . . . . 40

33. Rendements additionnels des compétences sur le marché du travail dans les secteurs à forte intensité numérique, 2012 ou 2015 . . . . . . . . . . . 41

34. Géographie des pertes et des créations d'emplois, $2010-16 \ldots \ldots$. . . . . . . 42

35. Croissance de l'emploi dans les secteurs de l'information,

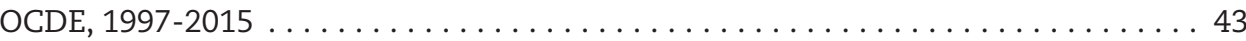

36. Origine de la demande soutenant les emplois dans le secteur des entreprises, zone OCDE, $1995-2014 \ldots \ldots \ldots \ldots \ldots \ldots \ldots \ldots$. . . . . . . . . 44

37. Origine de la demande soutenant les emplois dans les secteurs de l'information, zone OCDE, $1995-2014 \ldots \ldots \ldots \ldots \ldots \ldots \ldots \ldots \ldots 4$

38. Emplois du secteur des entreprises soutenus par la demande finale extérieure, par intensité de qualification, $2014 \ldots \ldots \ldots \ldots \ldots$. . . . . . 45

39. Part de l'emploi non répétitif et de l'intensité des tâches liées aux TIC, 2012 ou $2015 \ldots . \ldots \ldots \ldots \ldots$. . . . . . . . . . . . . . . . . . . . . . . . . 46

40. Travailleurs ayant bénéficié d'une formation en entreprise, par niveau

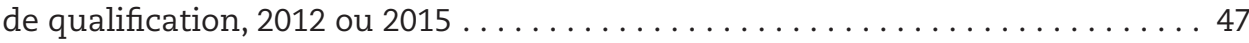

41. Écarts salariaux hommes-femmes, par pays, 2012 ou 2015 . . . . . . . . . . . . 48

42. Rendement de la composante TIC sur le marché du travail, par sexe, 2012 ou $2015 \ldots \ldots \ldots \ldots \ldots$. . . . . . . . . . . . . . . . . . . . . . . . . . . . 48

43. Employés ayant suivi des formations en cours d'emploi, par sexe,

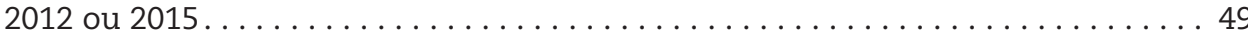

44. Décomposition sectorielle de la croissance de la productivité du travail, 2001-07 et $2009-15 \ldots \ldots \ldots$. . . . . . . . . . . . . . . . . . . . . . . . . . . . 50

45. Productivité du travail des secteurs de l'information, 2015 . . . . . . . . . . 51

46. Croissance de la productivité totale des facteurs, $1995-2015$. . . . . . . . . . 51

47. Empreinte élargie des TIC de la valeur ajoutée locale, 2011 . . . . . . . . . . 52

48. Valeur ajoutée locale liée aux TIC, $2011 \ldots \ldots \ldots \ldots \ldots \ldots \ldots \ldots \ldots$

49. Valeur ajoutée étrangère liée aux TIC contenue dans la demande finale

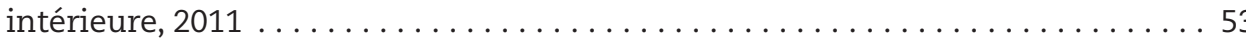


50. Contribution des équipements informatiques et des actifs intellectuels à la croissance de la productivité du travail augmentée

du capital intellectuel, $2000-14 \ldots \ldots \ldots \ldots \ldots \ldots \ldots \ldots \ldots \ldots \ldots . \ldots \ldots$

51. Contribution du capital intellectuel et de la PTF à la croissance de la productivité du travail augmentée du capital intellectuel, 2000-14 . . . . 54

52. Intensité en capital intellectuel des secteurs marchand

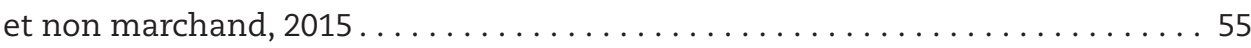

53. Évolution de la centralité de la fabrication de matériel informatique

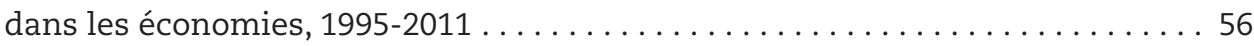

54. Évolution de la centralité des services informatiques dans les économies,

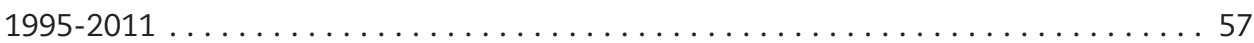

55. Variations les plus fortes de la centralité étrangère et locale :

fabrication et services informatiques, $1995-2011 \ldots \ldots \ldots \ldots \ldots \ldots \ldots$

56. Les dix pôles informatiques les plus centraux, 1995 et 2011 . . . . . . . . . . 59

57. Évolution de l'utilisation de l'internet, $2005-16 \ldots \ldots \ldots \ldots \ldots \ldots \ldots \ldots \ldots$

58. Évolution de l'utilisation de l'internet, par âge, 2005-16 . . . . . . . . . . 61

3. Agir maintenant au service de l'innovation $\ldots \ldots \ldots \ldots \ldots \ldots \ldots \ldots \ldots \ldots \ldots \ldots \ldots \ldots$

59. Diplômées de l'enseignement supérieur en sciences naturelles,

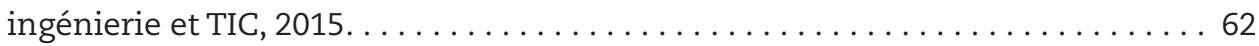

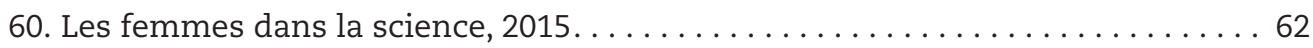

61. Contribution des femmes aux inventions brevetées, 2012-15 . . . . . . . . . 63

62. Budgets publics destinés à la R-D dans une sélection d'économies,

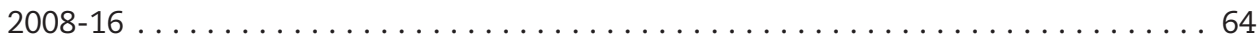

63. Crédits budgétaires publics de R-D, par objectif socio-économique, 2016 . . . 65

64. Recherche scientifique sur la démence et les maladies neurodégénératives dans une sélection de pays, $1996-2016 \ldots \ldots \ldots \ldots \ldots \ldots \ldots \ldots \ldots 66 \ldots \ldots \ldots \ldots \ldots \ldots$

65. Disciplines contribuant à la recherche scientifique sur la démence et les maladies neurodégénératives, $1996-2016 \ldots \ldots \ldots \ldots \ldots \ldots \ldots 67$

66. Documents scientifiques publiés en libre accès, $2017 \ldots \ldots \ldots \ldots \ldots \ldots .68$

67. Documents scientifiques les plus cités, par modalité d'accès, 2017 . . . . . . . 69

68. Collaboration internationale en science et innovation, $2005-16 \ldots \ldots \ldots \ldots 70$

69. Flux internationaux nets d'auteurs scientifiques dans une sélection

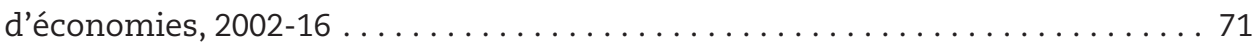

70. Aides directes et fiscales à la R-D des PME, $2015 \ldots \ldots \ldots \ldots \ldots \ldots \ldots \ldots \ldots$

71. Intensité de la R-D des entreprises et soutien public à la R-D

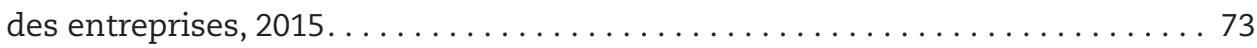

72. Évolution du soutien public à la R-D des entreprises et des DIRDE, 2006-15 . . . 73

73. Investissement en capital-risque dans une sélection de pays,

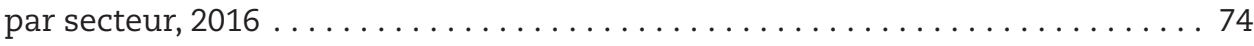

74. Accords conclus avec des investisseurs providentiels par secteur, Europe, 2015 et États-Unis, 2016 . . . . . . . . . . . . . . . . . . . . . . 74

75. Start-ups axées sur le numérique ayant attiré des fonds propres dans les économies de l'OCDE et BRIICS, 2011-16 . . . . . . . . . . . . . 75

76. Activités liées au numérique ayant attiré le plus de financements

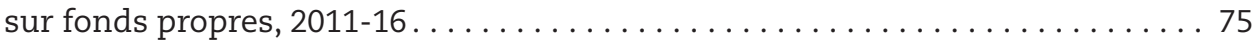

77. Publications scientifiques avec remerciements pour financement

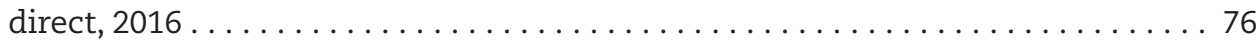


78. Impact de citation des publications scientifiques avec ou sans remerciements

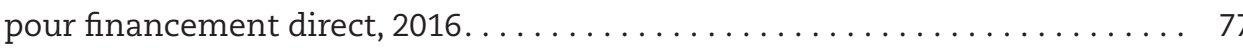

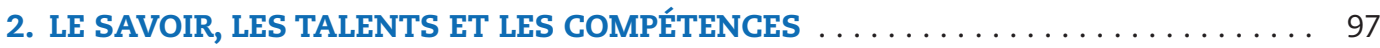

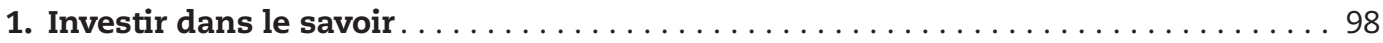
Dépenses consacrées à l'enseignement supérieur et à l'enseignement

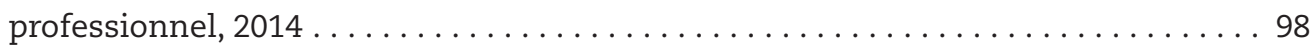
Dépenses intérieures brutes de R-D, par type d'activité, 2015 . . . . . . . . . . . 99 Investissement en TIC, par type d'actifs, $2015 \ldots \ldots \ldots \ldots \ldots \ldots \ldots \ldots$

2. Enseignement supérieur et recherche fondamentale . . . . . . . . . . . . 100 Dépenses de R-D de l'enseignement supérieur, 2015 . . . . . . . . . . . . . . . 100 Recherche fondamentale dans les secteurs de l'enseignement supérieur et de l'État, $2015 \ldots \ldots \ldots \ldots \ldots \ldots \ldots$. . . . . . . . . . . . . . . . . . . . . . . . . . . . . . . 101 Financement de la R-D dans l'enseignement supérieur, 2015 . . . . . . . . . . 101

3. Scientifiques et ingénieurs. . . . . . . . . . . . . . . . . . . . . . . . . 102 Diplômés de l'enseignement supérieur en sciences naturelles, ingénierie et TIC, 2005 et 2015 . . . . . . . . . . . . . . . . . . . . . . . . . . . . . . . . . . . . 102 Diplômés de l'enseignement supérieur en technologies de l'information et de la communication, selon le sexe, $2015 \ldots \ldots \ldots \ldots \ldots \ldots$ Titulaires de doctorat dans la population en âge de travailler, 2016 . . . . . . . 103

4. Chercheurs . . . . . . . . . . . . . . . . . . . . . . . . . . . . . . . . . . . . . 104

Personnel de R-D, 2015 . . . . . . . . . . . . . . . . . . . . . . . . . . . . . 104

Chercheurs, par secteur d'emploi, $2015 \ldots \ldots \ldots \ldots \ldots \ldots \ldots \ldots \ldots \ldots$. . . . . . . . . . . . . . . . . . . . . . . . . . 105

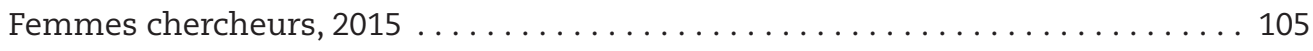

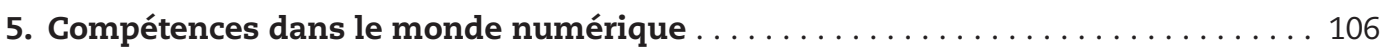
Élèves forts et élèves faibles en sciences et mathématiques,

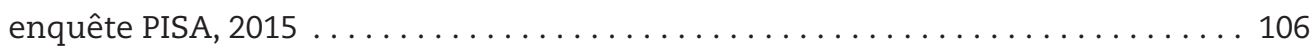
Résolution de problèmes en environnement à forte composante technologique, 2012 ou $2015 \ldots \ldots \ldots \ldots \ldots$. . . . . . . . . . . . . . . . . . . . . . . 107 Disposition à l'apprentissage et créativité, 2012 ou 2015 . . . . . . . . . . . . . . 107

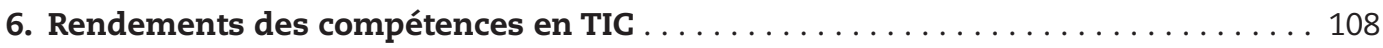
Composante TIC des emplois, 2012 ou 2015 . . . . . . . . . . . . . . . . . . . 108 Rendements de la composante TIC sur le marché du travail, 2012 ou 2015 . . . . . 109 Rendements de la composante gestion et communication des emplois :

la prime TIC, 2012 ou 2015 . . . . . . . . . . . . . . . . . . . . . . . . . . 109

7. Capital intellectuel . . . . . . . . . . . . . . . . . . . . . . . . . 110 Travailleurs suivant une formation, selon le type de formation, 2012 ou 2015 . . 110 Investissement des entreprises en capital fixe et en capital intellectuel, 2015 . . . 111 Investissement en capital intellectuel des secteurs marchand

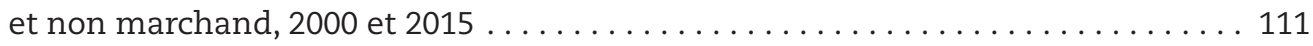

3. EXCELLENCE DE LA RECHERCHE ET COLLABORATION . . . . . . . . . . . . . . 121

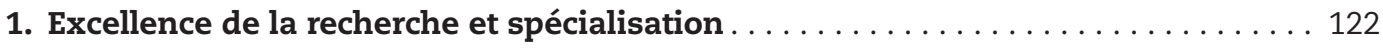

Quantité et qualité de la production scientifique, 2005 et 2015 . . . . . . . . . . . 122 Spécialisation et impact de citation dans quelques disciplines scientifiques, $2015 \ldots \ldots \ldots \ldots \ldots \ldots \ldots \ldots \ldots \ldots \ldots \ldots \ldots \ldots \ldots$ 


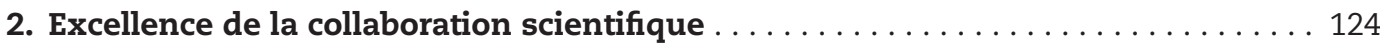

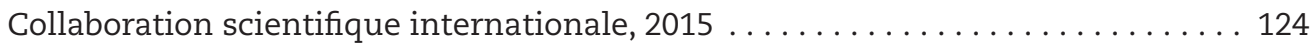

Impact de citation de la production scientifique et degré de collaboration

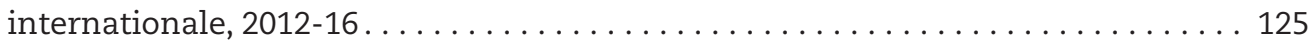

Production parmi les $10 \%$ de publications les plus citées et profils

de collaboration internationale, 2015 . . . . . . . . . . . . . . . . . . . . . . . . . 125

3. Mobilité internationale des personnes hautement qualifiées. . . . . . . . . . 126

Mobilité internationale des étudiants inscrits dans l'enseignement

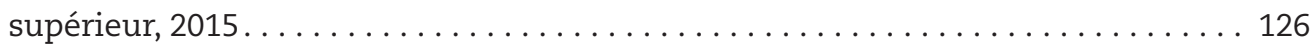

Doctorants internationaux et locaux dans les domaines des sciences naturelles,

de l'ingénierie et des TIC, 2015 . . . . . . . . . . . . . . . . . . . . . . . 127

Personnes dotées d'un niveau d'instruction élevé dans la population en âge

de travailler, par pays de naissance, $2015 \ldots \ldots \ldots \ldots \ldots \ldots \ldots \ldots \ldots \ldots \ldots \ldots \ldots \ldots \ldots \ldots \ldots$

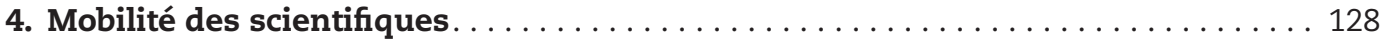

Flux bilatéraux internationaux d'auteurs scientifiques, $2006-16 \ldots \ldots \ldots \ldots \ldots 128$

Mobilité internationale des auteurs scientifiques, 2016 . . . . . . . . . . . . 129

Impact attendu des citations des auteurs scientifiques, par profil

de mobilité, 2016 . . . . . . . . . . . . . . . . . . . . . . . . . . . . . . . . . . . . . . . . . . . 129

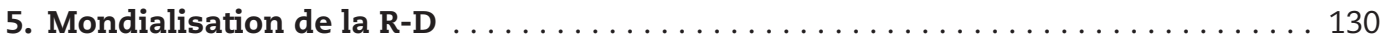

R-D des entreprises financée par des fonds étrangers, par source

de financement, 2015 . . . . . . . . . . . . . . . . . . . . . . . . . . . . . . . 130

Dépenses de R-D engagées par des entreprises affiliées sous contrôle étranger,

échantillon de pays, 2015 ou année disponible la plus récente . . . . . . . . . . 131

Financement de la R-D de l'État et de l'enseignement supérieur en Europe

par la Commission européenne, 2015 . . . . . . . . . . . . . . . . . . . . 131

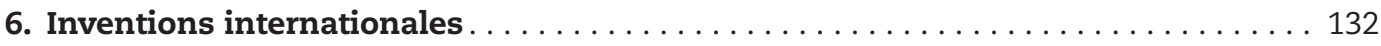

Co-inventions internationales dans le domaine des TIC, 2012-15 . . . . . . . 132

Nombre d'économies de résidence des inventeurs, par technologie, 2012-15 . . . . 133

Inventions TIC étrangères détenues par les économies, $2012-15 \ldots \ldots \ldots \ldots \ldots 133$

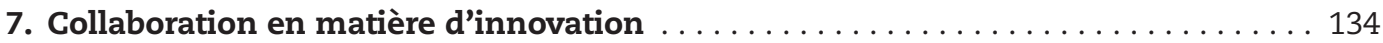

Entreprises menant des activités d'innovation en collaboration avec

des établissements d'enseignement supérieur ou de recherche,

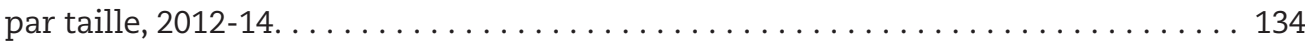

Entreprises menant des activités d'innovation en collaboration

avec des fournisseurs ou des clients, par taille, 2012-14 . . . . . . . . . . . . . . 135

Entreprises engagées dans des collaborations internationales

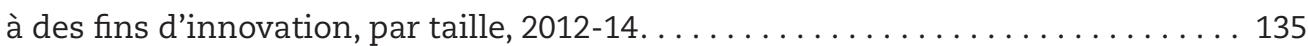

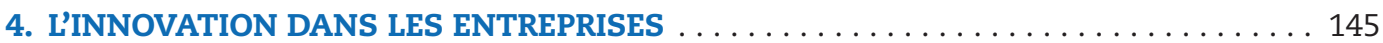

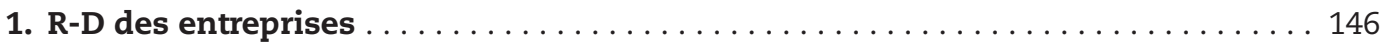

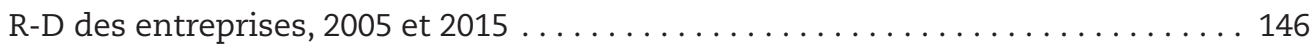

R-D des entreprises et aides publiques, par taille d'entreprise, 2015 . . . . . . . 147

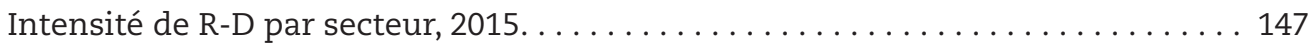

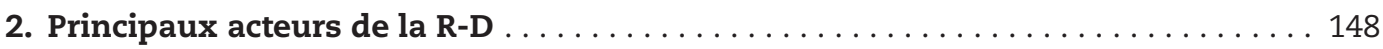

Dépenses de R-D par brevet dans les entreprises les plus actives en R-D,

selon l'implantation du siège, $2012-14 \ldots \ldots \ldots \ldots \ldots \ldots \ldots \ldots \ldots \ldots \ldots . \ldots \ldots$ 
Spécialisation sectorielle et technologique des entreprises les plus actives en R-D, 2012-14 . . . . . . . . . . . . . . . . . . . . . . . . . . . 149 Ventes nettes par dépôt de marques des entreprises des TIC les plus actives en R-D, selon l'implantation du siège, $2012-14 \ldots \ldots \ldots \ldots \ldots \ldots \ldots$. . . . . . . . 149

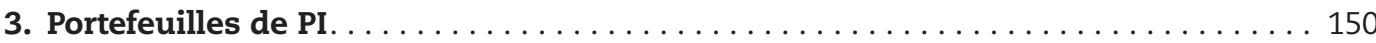

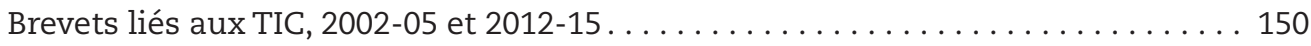

Dépôts de marques liées aux TIC, $2012-15 \ldots \ldots \ldots \ldots \ldots \ldots \ldots \ldots \ldots 1$

Enregistrement de dessins et modèles liés aux TIC, 2012-15 . . . . . . . . . . . 151

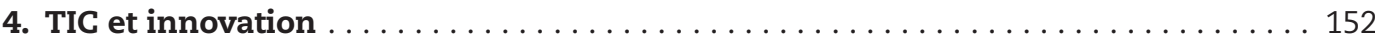

Dépenses de R-D dans les équipements TIC et les services d'information, 2015 . . 152

Brevets liés aux TIC et principaux acteurs, 2012-15 . . . . . . . . . . . . . . 153

Entreprises innovant dans les produits TIC et les services

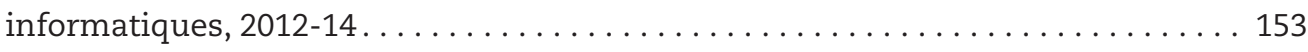

5. Panachage des modes d'innovation . . . . . . . . . . . . . . . . . . . . . . . . . 154

Types d'innovation, par taille d'entreprise, $2012-14 \ldots \ldots \ldots \ldots \ldots \ldots$. . . . . . . . 154 Innovation en produits nouveaux pour le marché, activités de fabrication

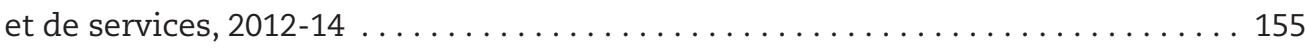
Innovation en produits nouveaux pour le marché, par taille d'entreprise,

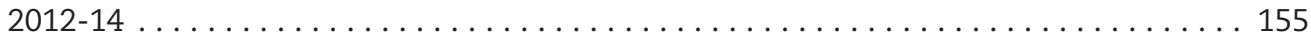

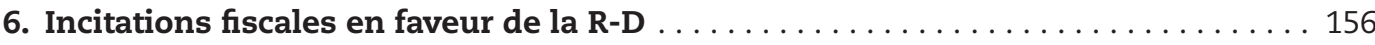

Soutien public direct et aide fiscale à la R-D des entreprises, 2015 . . . . . . . . . 156 Évolution du financement public de la R-D des entreprises : financement direct

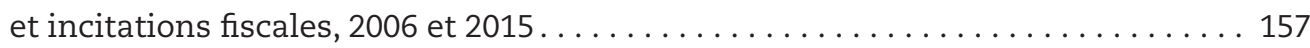

Taux de subvention fiscale des dépenses de R-D, 2017 . . . . . . . . . . . . . . 157

7. Cadre de la politique économique et demande d'innovation . . . . . . . . . . . 158 Investissement en capital-risque, $2016 \ldots \ldots \ldots \ldots \ldots \ldots \ldots \ldots$ Participation des PME innovantes et non innovantes aux marchés internationaux et aux marchés du secteur public, 2012-14 . . . . . . . . . . . . 159 Entreprises bénéficiant du soutien public à l'innovation, par catégorie

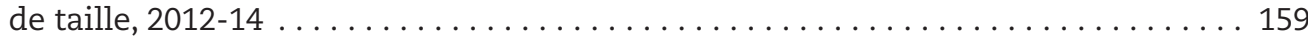

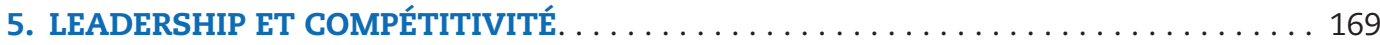

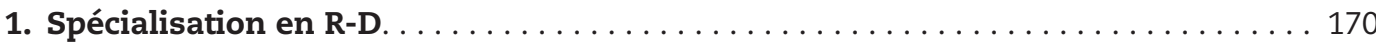
Intensité de R-D des entreprises corrigée de la structure d'activités, 2015 . . . . 170 R-D des entreprises dans les activités de fabrication, par catégorie d'intensité

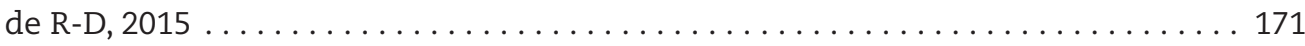

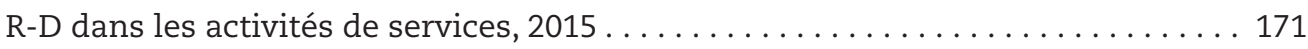

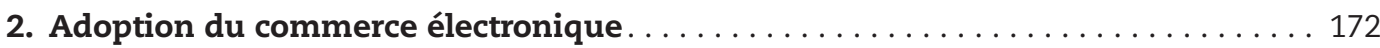
Diffusion dans les entreprises d'une sélection d'outils et d'activités s'appuyant sur les TIC, par technologie, $2016 \ldots \ldots \ldots \ldots \ldots \ldots \ldots \ldots \ldots \ldots \ldots \ldots$ Diffusion dans les entreprises d'une sélection d'outils et d'activités s'appuyant sur les TIC, pays de l'OCDE, 2010 et $2016 \ldots \ldots \ldots \ldots \ldots \ldots \ldots \ldots \ldots$ Entreprises recourant aux ventes en ligne, par taille d'entreprise, 2015 . . . . . . 173

3. Le commerce électronique à travers les secteurs et les applications. . . . . . . . . . . 174 Entreprises utilisant des services de cloud computing, par taille d'entreprise, 2016 
Adoption des services de cloud computing dans les différents secteurs

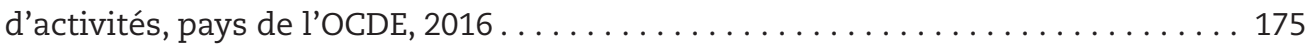

Diffusion dans les secteurs d'activités d'une sélection d'outils et d'activités s'appuyant sur les TIC, pays de l'OCDE, 2016 . . . . . . . . . . . . . 175

4. Dynamique des start-ups................................. 176

Taux d'entrée et de sortie des entreprises, secteur des TIC et autres secteurs

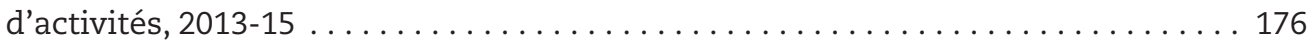

Part des jeunes petites et micro-entreprises existantes dans le secteur des TIC et les autres secteurs d'activités, $2013-15 \ldots \ldots \ldots \ldots \ldots \ldots \ldots \ldots \ldots$ Différences de croissance de l'emploi entre les petites entreprises jeunes et les petites entreprises anciennes dans le secteur des TIC et les autres

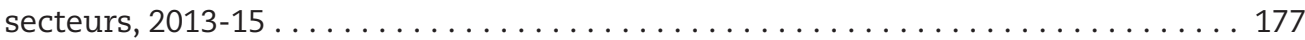

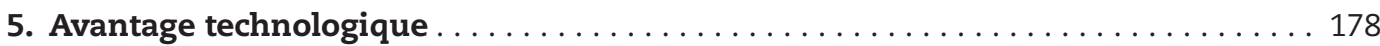
Avantage technologique révélé dans les TIC, 2002-05 et 2012-15 . . . . . . . . . . 178 Avantage technologique révélé dans les technologies de la santé, 2002-05

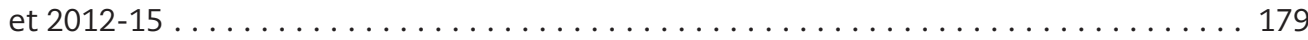
Avantage technologique révélé dans les technologies environnementales, 2002-05 et 2012-15 . . . . . . . . . . . . . . . . . . . . . . . . . . . . . . . . . . . 179

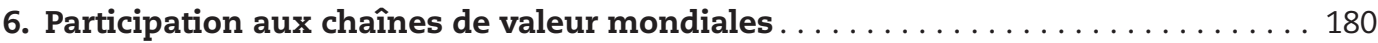
Contenu en valeur ajoutée étrangère des exportations brutes,

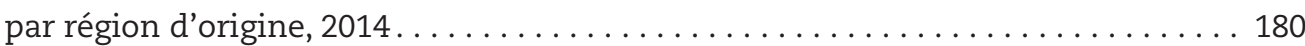
Contenu en valeur ajoutée locale des exportations de pays tiers, 2014 . . . . . . 181 Origine sectorielle de la valeur ajoutée locale créée par les exportations

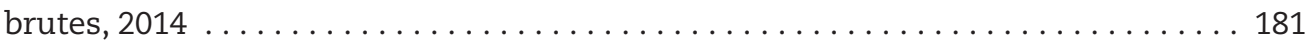

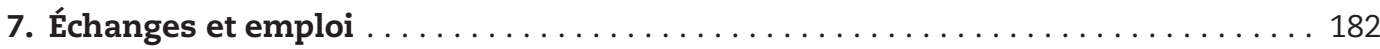

Part des emplois du secteur des entreprises soutenus par la demande

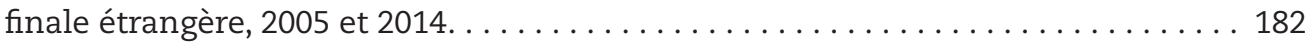
Emplois des secteurs de l'information et de la communication soutenus par la demande finale étrangère, 2005 et 2014 . . . . . . . . . . . . . . . . . . 183 Part de la rémunération des employés du secteur des entreprises soutenue par la demande finale locale et étrangère, 2014 . . . . . . . . . . . . . . . . . . . 183

6. SOCIÉTÉ ET TRANSFORMATION NUMÉRIQUE $\ldots \ldots \ldots \ldots \ldots \ldots \ldots \ldots \ldots \ldots \ldots \ldots$

1. Connectivité . . . . . . . . . . . . . . . . . . . . . . . . . . . . . . . . . . . 192

Pénétration du haut débit mobile par technologie, décembre 2016. . . . . . . . . . 192

Ménages équipés d'une connexion haut débit, régions urbaines et rurales, 2010 et 2016 . . . . . . . . . . . . . . . . . . . . . . . . . . . . . . . . . . . . . . 193 Petites et moyennes entreprises dotées d'un accès haut débit,

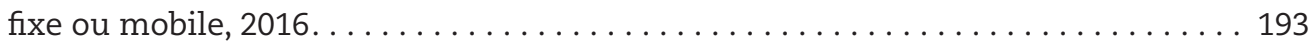

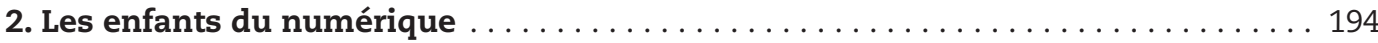
Élèves ayant accédé à l'internet pour la première fois à l'âge de six ans ou avant, 2012 et 2015 . . . . . . . . . . . . . . . . . . . . . . . . . . . 194 Temps passé sur l'internet en dehors de l'école, 2012 et 2015 . . . . . . . . . . 195 Diffusion d'une sélection d'activités en ligne parmi les élèves des pays de l'OCDE, par sexe, 2015 . . . . . . . . . . . . . . . . . . . . . . . . . . . . . 195 
3. Internautes . . . . . . . . . . . . . . . . . . . . . . . . . . . . . . . 196

Population totale d'individus utilisant l'internet mobile

quotidiennement, 2016. . . . . . . . . . . . . . . . . . . . . . . . 196

Écarts d'adoption de l'internet selon le niveau d'instruction, 2016 . . . . . . . . . . 197

Utilisatrices de l'internet, par âge, 2016 . . . . . . . . . . . . . . . . . . . . . . . . 197

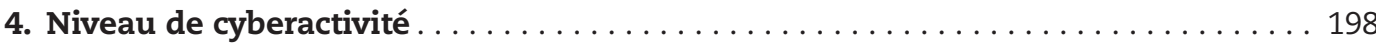

Diffusion d'une sélection d'activités en ligne parmi les internautes

des pays de l'OCDE, par groupe d'âges et niveau d'instruction, 2016 . . . . . . . . 198

Individus ayant effectué des achats en ligne au cours des 12 derniers mois,

par groupe d'âges, $2016 \ldots \ldots \ldots \ldots \ldots \ldots \ldots \ldots \ldots$

Individus âgés de 16 à 24 ans ayant suivi un cours en ligne, 2009 et 2016 . . . . . . 199

5. Cyberconsommateurs sans frontières . . . . . . . . . . . . . . . . . . . . . 200

Entreprises ayant pratiqué la vente par commerce électronique

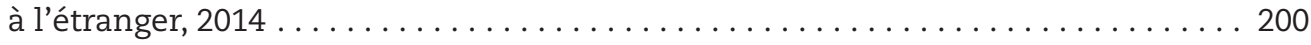

Individus ayant effectué des achats en ligne auprès de vendeurs nationaux

et étrangers, 2016. . . . . . . . . . . . . . . . . . . . . . . . . . . . 201

Transactions entre entreprises et consommateurs (B2C), 2009 et 2015 . . . . . . . 201

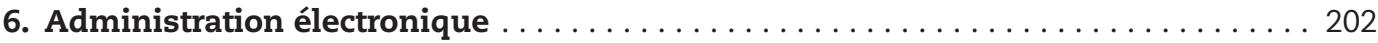

Individus utilisant l'internet pour communiquer avec les pouvoirs publics,

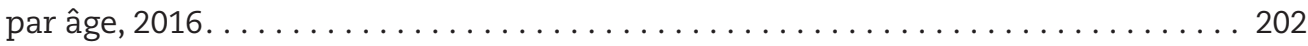

Individus n'ayant pas soumis de formulaires officiels en ligne du fait de craintes quant à la sécurité et au respect de leur vie privée, 2016 . . . . . . . 203

Entreprises utilisant l'internet pour transmettre des factures aux pouvoirs

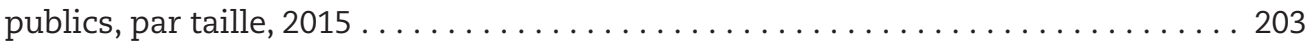

7. Confiance . . . . . . . . . . . . . . . . . . . . . . . . . . . . . . . . . . . . . . 204

Entreprises disposant d'une stratégie de sécurité formelle, par classe

de taille, $2015 \ldots \ldots \ldots \ldots \ldots \ldots$. . . . . . . . . . . . . . . . . . . 204

Individus ayant fourni des informations à caractère personnel via l'internet au cours des 12 derniers mois, par sexe et par groupe d'âges, 2016 . . . . . . . . 205

Individus gérant l'accès à leurs informations à caractère personnel sur l'internet, par groupe d'âges, $2016 \ldots \ldots \ldots \ldots \ldots \ldots \ldots$ 


\section{ORGANISATION DE COOPÉRATION ET DE DÉVELOPPEMENT ÉCONOMIQUES}

L'OCDE est un forum unique en son genre où les gouvernements oeuvrent ensemble pour relever les défis économiques, sociaux et environnementaux que pose la mondialisation. L'OCDE est aussi à l'avantgarde des efforts entrepris pour comprendre les évolutions du monde actuel et les préoccupations qu'elles font naître. Elle aide les gouvernements à faire face à des situations nouvelles en examinant des thèmes tels que le gouvernement d'entreprise, l'économie de l'information et les défis posés par le vieillissement de la population. L'Organisation offre aux gouvernements un cadre leur permettant de comparer leurs expériences en matière de politiques, de chercher des réponses à des problèmes communs, d'identifier les bonnes pratiques et de travailler à la coordination des politiques nationales et internationales.

Les pays membres de l'OCDE sont : l'Allemagne, l'Australie, l'Autriche, la Belgique, le Canada, le Chili, la Corée, le Danemark, l'Espagne, l'Estonie, les États-Uunis, la Finlande, la France, la Grèce, la Hongrie, l'Irlande, l'Islande, Israël, l'Italie, le Japon, la Lettonie, le Luxembourg, le Mexique, la Norvège, la Nouvelle-Zélande, les Pays-Bas, la Pologne, le Portugal, la Rrépublique slovaque, la République tchèque, le Rroyaume-Uuni, la Slovénie, la Suède, la Suisse et la Tturquie. L'Uunion européenne participe aux travaux de l'OCDE.

Les Éditions OCDE assurent une large diffusion aux travaux de l'Organisation. Ces derniers comprennent les résultats de l'activité de collecte de statistiques, les travaux de recherche menés sur des questions économiques, sociales et environnementales, ainsi que les conventions, les principes directeurs et les modèles développés par les pays membres. 


\section{Science, technologie et industrie : Tableau de bord de l'OCDE 2017}

\section{LA TRANSFORMATION NUMÉRIQUE}

La publication biennale Science, technologie et industrie (STI) : Tableau de bord de l'OCDE présente les indicateurs traditionnels relatifs aux avancées de la science, de la technologie, de l'innovation et de l'industrie. Elle comprend également de nouveaux indicateurs expérimentaux qui abordent, sous un nouvel angle, des domaines intéressant l'action publique : l'investissement dans le capital intellectuel, les compétences à l'ère du numérique, la mobilité internationale des chercheurs, la transformation numérique dans les entreprises et les industries, les stratégies d'innovation, l'internationalisation de la recherche, l'évolution de la compétitivité dans les échanges et de la productivité, ainsi que l'utilisation des technologies dans la vie de tous les jours.

Avec environ 200 indicateurs, l'édition 2017 du rapport Science, Technologie et Industrie (STI) : Tableau de bord de l'OCDE montre comment la transformation numérique rejaillit sur la science, l'innovation, l'économie, l'organisation du travail et les modes de vie. Son objectif est d'aider les pouvoirs publics à concevoir des politiques de la science, de l'innovation et de l'industrie plus efficaces compte tenu des mutations de l'ère numérique.

Les graphiques qui figurent dans la présente publication et les données connexes sont téléchargeables, de même que des données complémentaires permettant, pour plus de la moitié des indicateurs, d'élargir la couverture chronologique et/ou géographique retenue dans la version papier.

La page web du Tableau de bord STI de l'OCDE (http://www.oecd.org/sti/scoreboard.htm) contient des synthèses thématiques, des notes par pays ainsi que des outils en ligne permettant de visualiser les indicateurs.

Cette publication s'inscrit dans le cadre du projet « Going Digital » de l'OCDE. Dans un monde résolument tourné vers le numérique et les données, ce projet vise à fournir aux décideurs les outils dont ils ont besoin pour aider leurs économies et leurs sociétés à prospérer.

Pour plus d'informations, consultez www.oecd.org/going-digital

\#GoingDigital

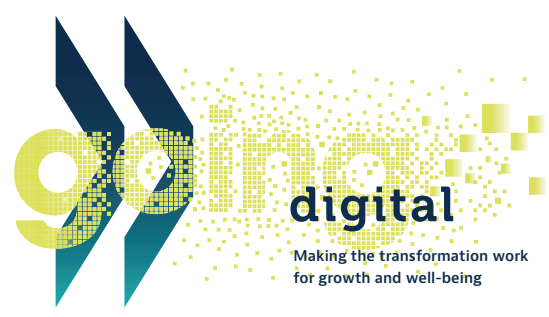

Veuillez consulter cet ouvrage en ligne : http://dx.doi.org/10.1787/sti_scoreboard-2017-fr.

Cet ouvrage est publié sur OECD iLibrary, la bibliothèque en ligne de l'OCDE, qui regroupe tous les livres, périodiques et bases de données statistiques de l'Organisation.

Rendez-vous sur le site www.oecd-ilibrary.org pour plus d'informations.

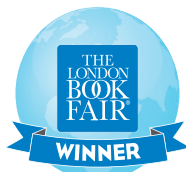

INTERNATIONAL EXCELLENCE Awards 2017

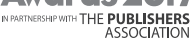

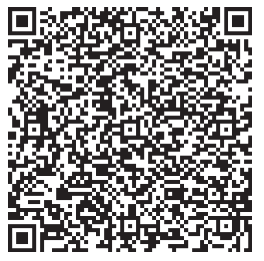

ISBN 978-92-64-29724-1 $922017082 \mathrm{P}$

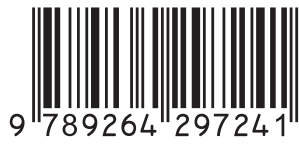

